\title{
Iterative Reductive Aromatization/Ring-Closing Metathesis Strategy toward the Synthesis of Strained Aromatic Belts
}

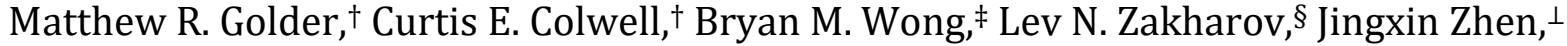 \\ and Ramesh Jasti*,† \\ ${ }^{\dagger}$ Department of Chemistry \& Biochemistry and Material Science Institute, University of Oregon, Eugene, OR 97403 \\ *Department of Chemical \& Environmental Engineering and Materials Science \& Engineering Program, University \\ of California, Riverside, Riverside, CA 92521 \\ ${ }^{\S}$ CAMCOR - Center for Advanced Materials Characterization in Oregon, University of Oregon, Eugene, OR 97403 \\ ${ }^{\perp}$ Department of Chemistry, Boston University, Boston, MA 02215
}

\section{Table of Contents}

1) General Considerations and Synthesis (S2 - S27)

2) X-ray Crystallography (S27 - S30)

3) Optical and Electrochemical Analysis (S31 - S39)

4) Computational Analysis of Macrocycle Atropisomers (S40 - S46)

5) Homodesmotic Strain Energy Analysis (S47 - S51)

6) VT NMR Spectra (S52)

7) ${ }^{1} \mathrm{H} /{ }^{13} \mathrm{C}$ NMR Spectra (S53 - S77)

8) Cartesian Coordinates of Select Compounds (S78 - S234)

9) References (S234 - S235) 
1) General Experimental Considerations. All glassware was oven $\left(140{ }^{\circ} \mathrm{C}\right)$ or flame dried and cooled under an inert atmosphere of nitrogen unless otherwise noted. Moisture sensitive reactions were carried out under an inert atmosphere of nitrogen using standard syringe/septa techniques. Tetrahydrofuran, dichloromethane, 1,4-dioxane, and dimethylformamide were dried by filtration through alumina according to the methods described by Grubbs (JC Meyer). ${ }^{1}$ Silica column chromatography was conducted with Zeochem Zeoprep 60 Eco 40-63 $\mu \mathrm{m}$ silica gel. Thin Layer Chromatography (TLC) was performed using Sorbent Technologies Silica Gel XHT TLC plates. Developed plates were visualized using UV light at wavelengths of 254 and $365 \mathrm{~nm}$ or by staining with iodine vapor. ${ }^{1} \mathrm{H}$ NMR spectra were recorded at $600 \mathrm{MHz}$ on a Bruker Avance-III, $500 \mathrm{MHz}$ on a Bruker Avance-III, $500 \mathrm{MHz}$ on a Varian VNMRS, $500 \mathrm{MHz}$ on a Varian INOVA, $400 \mathrm{MHz}$ on a Varian VNMRS, or $300 \mathrm{MHz}$ on a Varian INOVA. ${ }^{13} \mathrm{C}$ NMR spectra were recorded at $150 \mathrm{MHz}$ on a Bruker Avance-III, $125 \mathrm{MHz}$ on a Bruker Avance-III, $125 \mathrm{MHz}$ on a Varian VNMRS, $125 \mathrm{MHz}$ on a Varian INOVA, or $100 \mathrm{MHz}$ on a Varian VNMRS. All ${ }^{1} \mathrm{H}$ NMR spectra were taken in $\mathrm{CDCl}_{3}$ (referenced to TMS, $\delta 0.00 \mathrm{ppm}$ ), benzene- $d_{6}$ (referenced to residual $\mathrm{C}_{6} \mathrm{H}_{6}, \delta 7.16 \mathrm{ppm}$, or DMSO- $d_{6}$ (referenced to residual DMSO, $\delta 2.50 \mathrm{ppm}$ ). All ${ }^{13} \mathrm{C}$ NMR spectra were taken in $\mathrm{CDCl}_{3}$ (referenced to chloroform, $\delta 77.16 \mathrm{ppm}$ ), benzene- $d_{6}$ (referenced to benzene, $\delta 128.06 \mathrm{ppm}$ ), or DMSO- $d_{6}$ (referenced to DMSO, $\delta 39.52 \mathrm{ppm}$ ). The MALDI matrix, trans-2-[3-(4-tert-butylphenyl)-2-methyl-2-propenylidene]malononitrile (DCTB), was obtained from Sigma-Aldrich, and was used as a solution in $\mathrm{CH}_{2} \mathrm{Cl}_{2}$. IR spectra were recorded on a Thermo Nicolet 6700 FT-IR. Absorbance and fluorescence spectra were obtained in a $1 \mathrm{~cm}$ Quartz cuvette with dichloromethane using a Varian Cary 60 UV-vis spectrometer and a Horiba Jobin Yvon Fluoromax-4 Fluorimeter. Quantum yields were determined using an integrating sphere accessory. Cyclic voltammetry experiments (scan rate $=$ 
$100 \mathrm{mV} / \mathrm{s}$ ) were performed using a CH Instruments 1200B potentiostat running $\mathrm{CH}$ Instruments software. Measurements were conducted in degassed $0.1 \mathrm{M} n \mathrm{Bu}_{4} \mathrm{PF}_{6}$ (recrystallized $3 \mathrm{x}$ from methanol) in dichloromethane or tetrahydrofuran under an $\mathrm{N}_{2}$ atmosphere with a glassy carbon working electrode, platinum counter electrode, and an Ag reference electrode. The ferrocene/ferrocenium couple was used as an internal reference. Recycling gel permeation chromatography (GPC) was performed using a Japan Analytical Industry LC-9101 preparative HPLC with JAIGEL-1H/JAIGEL-2H columns in series using $\mathrm{CHCl}_{3}$. Automated flash chromatography was performed using a Biotage Isolera One. NBS was recrystallized from water and dried under high vacuum before use. 4 was synthesized as previously described. ${ }^{2}$ All reagents were obtained commercially unless otherwise noted.

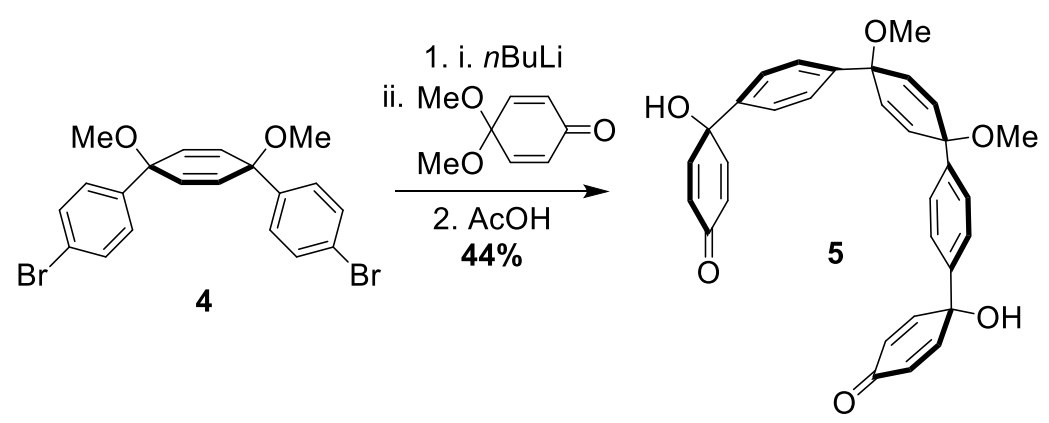

To a solution of syn-dibromide 4 (9.0 g, $20 \mathrm{mmol}, 1.0$ equiv) in anhydrous THF (175 mL) at -78 ${ }^{\circ} \mathrm{C}$ was added $n \mathrm{BuLi}(17 \mathrm{~mL}, 42 \mathrm{mmol}, 2.1$ equiv, $2.5 \mathrm{M}$ in hexanes) over ca. $5 \mathrm{~min}$. Upon completion of $n \mathrm{BuLi}$ addition, benzoquinone monoketal ( $6.8 \mathrm{~g}, 44 \mathrm{mmol}, 2.2$ equiv) was added immediately as a single stream. The reaction mixture became extremely viscous and stirring stopped at this time. The bath was removed and the reaction mixture was allowed to warm to $\mathrm{rt}$ over $3 \mathrm{~h}$. The reaction was then quenched with water $(50 \mathrm{~mL})$ and diluted with DCM $(100 \mathrm{~mL})$. The layers were separated and the aqueous phase was extracted with DCM (2 x $100 \mathrm{~mL})$. The 
combined organics were washed with water $(1 \times 50 \mathrm{~mL})$ and brine $(1 \times 50 \mathrm{~mL})$ then dried over sodium sulfate and concentrated under vacuum to a dark yellow oil. To the crude oil was added acetone $(150 \mathrm{~mL})$ and $10 \%$ aq acetic acid $(55 \mathrm{~mL})$. The solution was stirred at $\mathrm{rt}$ and became cloudy after $20 \mathrm{~min}$. After stirring for $30 \mathrm{~min}$, the solid was collected by vacuum filtration and was washed with $\mathrm{MeOH}(3 \times 50 \mathrm{~mL})$. The resulting solid was dried under high vacuum to afford diquinol 5 as an off-white solid (4.5 g, 44\%). ${ }^{1} \mathrm{H}$ NMR (500 MHz, DMSO- $\left.d_{6}\right): \delta(p p m) 7.38(\mathrm{~d}, J$ $=8.7 \mathrm{~Hz}, 4 \mathrm{H}), 7.34(\mathrm{~d}, J=8.7 \mathrm{~Hz}, 4 \mathrm{H}), 6.91(\mathrm{~d}, J=10.0 \mathrm{~Hz}, 4 \mathrm{H}), 6.55(\mathrm{br} \mathrm{s}, 2 \mathrm{H}), 6.13(\mathrm{~d}, J=$ $10.0 \mathrm{~Hz}, 4 \mathrm{H}), 6.07$ (s, 4H), $3.31(\mathrm{~s}, 6 \mathrm{H}) ;{ }^{13} \mathrm{C}$ NMR (150 MHz, DMSO-d $): \delta(\mathrm{ppm})$ 185.94, 152.97, 143.41, 139.99, 133.47, 126.55, 125.98, 125.838, 74.29, 70.34, 51.939. HRMS (FAB+) $(m / z):[M]^{+}$calcd. for $\mathrm{C}_{32} \mathrm{H}_{28} \mathrm{O}_{6}, 508.1886$; found, 508.1897. IR (neat): 665.96, 700.79, 735.09, 764.23, 833.02, 859.16, 948.54, 1015.94, 1027.50, 1078.34, 1171.62, 1227.79, 1226.22, 1395.25, $1448.72,1461.42,1500.16,1621.32,1665.36,2823.69,2936.05,3400.15 \mathrm{~cm}^{-1}$.

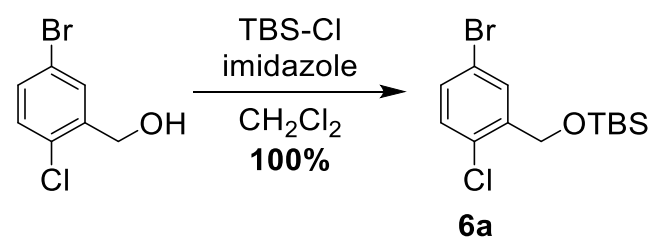

To a solution of (5-bromo-2-chlorophenyl)methanol (13 g, $57 \mathrm{mmol}, 1.0$ equiv) and imidazole (6.2 g, $91 \mathrm{mmol}, 1.6$ equiv) in anhydrous DCM $(50 \mathrm{~mL})$ at $0{ }^{\circ} \mathrm{C}$ was added solid TBS-Cl $(11 \mathrm{~g}$, $74 \mathrm{mmol}, 1.3$ equiv). The resulting slurry was allowed to slowly warm to $\mathrm{rt}$. After stirring at $\mathrm{rt}$ for $14 \mathrm{~h}$, the reaction mixture was filtered through a short plug of silica gel (DCM). The filtrate was concentrated under vacuum to afford $6 \mathbf{a}$ as a colorless oil (19 g, quant). ${ }^{1} \mathrm{H}$ NMR (300 MHz, $\left.\mathrm{CDCl}_{3}\right): \delta(\mathrm{ppm}) 7.69(\mathrm{dd}, J=2.4,1.1 \mathrm{~Hz}, 1 \mathrm{H}), 7.31(\mathrm{dd}, J=8.4,2.4 \mathrm{~Hz}, 1 \mathrm{H}), 7.16(\mathrm{~d}, J=8.4 \mathrm{~Hz}$, 1H), $4.74(\mathrm{~d}, J=1.0 \mathrm{~Hz}, 2 \mathrm{H}), 0.97(\mathrm{~s}, 9 \mathrm{H}), 0.14(\mathrm{~s}, 6 \mathrm{H}) ;{ }^{13} \mathrm{C} \mathrm{NMR}\left(125 \mathrm{MHz}, \mathrm{CDCl}_{3}\right) \delta(\mathrm{ppm})$ $141.12,130.87,130.52,130.30,130.18,120.88,62.04,26.07,18.54,-5.22$. HRMS (FAB+) 
(m/z): $[\mathrm{M}]^{+}$calcd. for $\mathrm{C}_{13} \mathrm{H}_{19} \mathrm{OSiBrCl}, 333.0077$; found, 333.0102. IR (neat): 620.65, 668.89, $775.35,807.69,833.33,938.45,1005.08,1039.84,1079.25,1105.59,1195.10,1253.26,1395.05$, $1470.97,2856.37,2928.26,2954.01 \mathrm{~cm}^{-1}$.

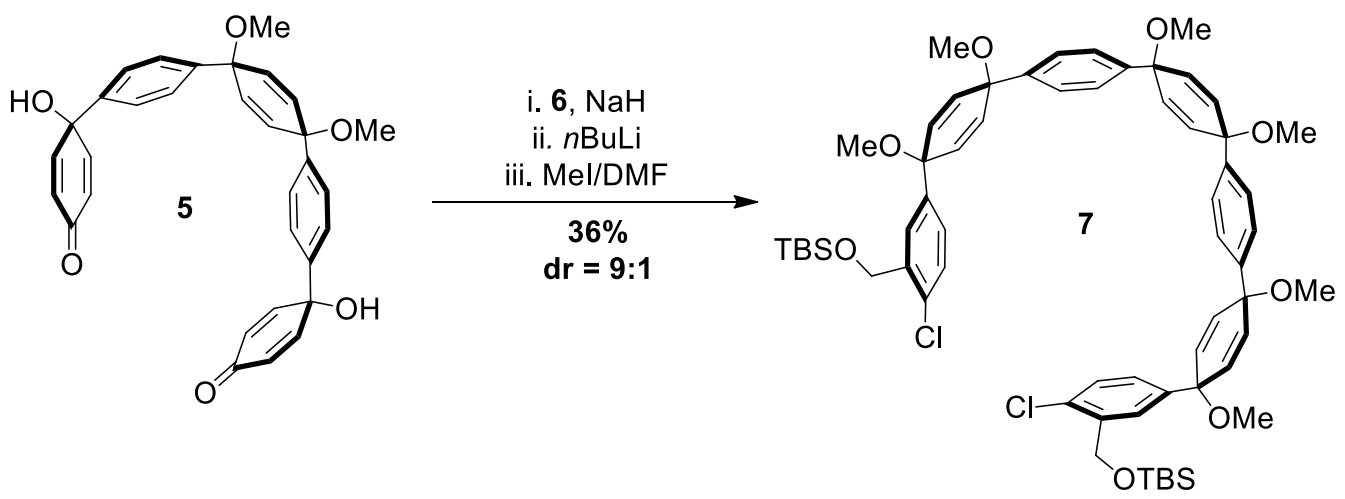

To a dry $500 \mathrm{~mL}$ RBF charged with a magnetic stir bar was added solid diquinol 5 (1.9 g, 3.7 mmol, 1.0 equiv) and $6 \mathbf{6}(8.7 \mathrm{~g}, 26 \mathrm{mmol}, 7.0$ equiv). The mixture was evacuated/backfilled with $\mathrm{N}_{2}(3 \mathrm{x})$ and fitted with a rubber septum under $\mathrm{N}_{2}$. Anhydrous THF (50 mL) was added and the slurry was cooled to $-78{ }^{\circ} \mathrm{C}$. Solid sodium hydride $(0.74 \mathrm{~g}, 19 \mathrm{mmol}, 5.0$ equiv, $60 \%$ suspension in mineral oil) was added and the reaction mixture was stirred $1 \mathrm{~h}$ at $-78{ }^{\circ} \mathrm{C}$. Next, $n \mathrm{BuLi}$ was added dropwise (10 mL, $26 \mathrm{mmol}, 7.0$ equiv, $2.5 \mathrm{M}$ in hexanes). The reaction mixture became extremely viscous and stirring became sluggish. After $10 \mathrm{~min}$, the tan suspension was slowly warmed to rt and stirred for $90 \mathrm{~min}$ before quenching with $\mathrm{MeI}(5.2 \mathrm{~g}, 2.3 \mathrm{~mL}, 37 \mathrm{mmol}, 10$ equiv) and anhydrous DMF (10 mL). The solution was stirred for $16 \mathrm{~h}$ before quenching with water $(25 \mathrm{~mL})$ and diluting with $\mathrm{Et}_{2} \mathrm{O}(30 \mathrm{~mL})$. The layers were separated and the aqueous phase was extracted with $\mathrm{Et}_{2} \mathrm{O}(2 \times 30 \mathrm{~mL})$. The combined organics were washed with water $(10 \times 10$ $\mathrm{mL})$ and brine $(10 \mathrm{~mL})$ then were dried over sodium sulfate and concentrated onto Celite. 
Purification on silica gel $(4 \mathrm{~cm} \times 9 \mathrm{~cm}, 0-20 \%$ EtOAc/hexanes) afforded a semi-pure off-white foam that was concentrated onto Celite again. Purification on silica gel $(3 \mathrm{~cm} \times 9 \mathrm{~cm}, 5-20 \%$ EtOAc/hexanes) afforded 7 as a white foam $(1.7 \mathrm{~g}, 43 \%) .{ }^{1} \mathrm{H}$ NMR (300 MHz, $\mathrm{CDCl}_{3}$, major diastereomer): $\delta(\mathrm{ppm}) 7.77$ (d, $J=2.3 \mathrm{~Hz}, 2 \mathrm{H}), 7.33-7.29$ (overlap, 8H), $7.22(\mathrm{~d}, J=8.3 \mathrm{~Hz}$, 2H), $7.10(\mathrm{dd}, J=8.3,2.3 \mathrm{~Hz}, 2 \mathrm{H}), 6.11-6.02$ (overlap, 12H), $4.76(\mathrm{~s}, 4 \mathrm{H}), 3.41(\mathrm{~s}, 6 \mathrm{H}), 3.41(\mathrm{~s}$, $6 \mathrm{H}), 3.40(\mathrm{~s}, 6 \mathrm{H}), 0.92(\mathrm{~s}, 18 \mathrm{H}), 0.08(\mathrm{~s}, 12 \mathrm{H}) ;{ }^{13} \mathrm{C} \mathrm{NMR}\left(125 \mathrm{MHz}, \mathrm{CDCl}_{3}\right): \delta(\mathrm{ppm})$ 142.57, $142.31,142.04,138.60,133.40,133.05,132.72,130.42,128.47,125.84,125.81,125.71,125.52$, $125.32,74.39,74.29,74.16,62.19,51.69,31.50,25.67,18.09,-5.54$. MALDI-TOF $(\mathrm{m} / \mathrm{z}):[\mathrm{M}-$ OMe $]^{+}$calcd. for $\mathrm{C}_{61} \mathrm{H}_{75} \mathrm{O}_{7} \mathrm{Cl}_{2} \mathrm{Si}_{2}, 1045.44$, found 1044.98. IR (neat): 663.90, 814.18, 834.94, $1041.67,1085.95,1196.50,1256.21,1462.86,2855.45,2933.08,2954.67 \mathrm{~cm}^{-1}$.

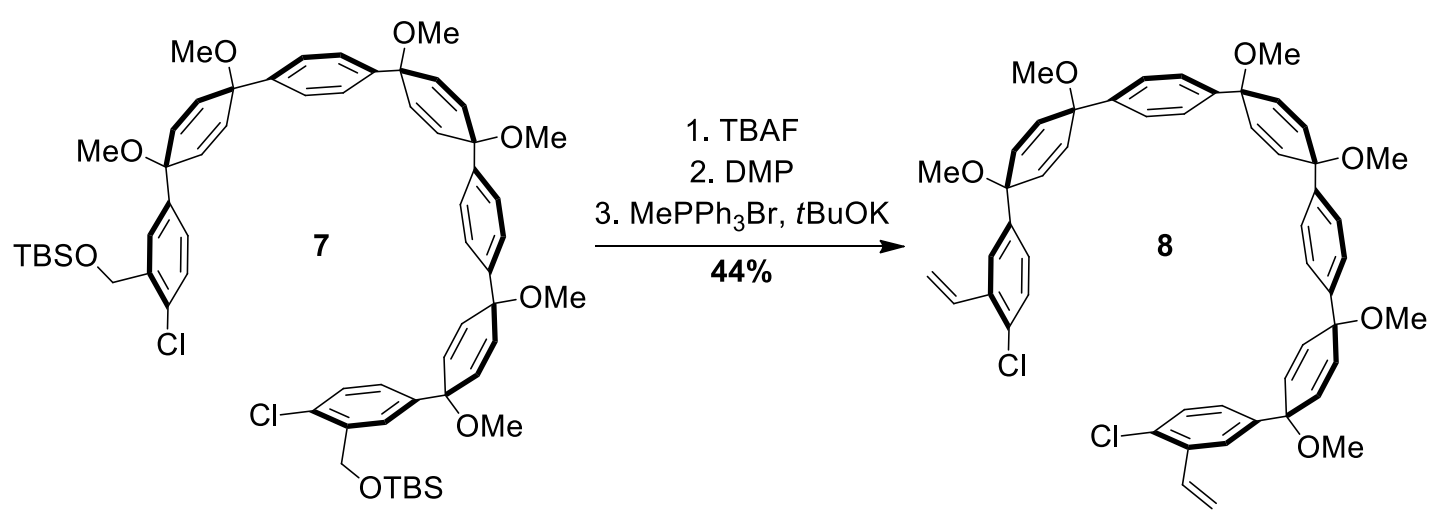

To a dry RBF charged with a magnetic stirbar was added 7 ( $0.93 \mathrm{~g}, 0.86 \mathrm{mmol}, 1.0$ equiv). The flask was purged with $\mathrm{N}_{2}$ and anhydrous THF ( $8 \mathrm{~mL}$ ) was added under an $\mathrm{N}_{2}$ atmosphere. The reaction mixture was cooled to $0{ }^{\circ} \mathrm{C}$ whereupon $\mathrm{TBAF}(2.2 \mathrm{~mL}, 2.2 \mathrm{mmol}, 2.5$ equiv, $1.0 \mathrm{M}$ in THF) was added dropwise. The resulting solution was allowed to slowly warm to rt over $2 \mathrm{~h}$ before quenching with sat. aq $\mathrm{NH}_{4} \mathrm{Cl}(5 \mathrm{~mL})$ and diluting with EtOAc $(20 \mathrm{~mL})$. The layers were separated and the aqueous phase was extracted with EtOAc $(2 \times 20 \mathrm{~mL})$. The combined organics 
were washed with water $(3 \times 10 \mathrm{~mL})$ and brine $(10 \mathrm{~mL})$ then were dried over sodium sulfate and concentrated under vacuum. The resulting crude foam was passed through a plug of silica gel (30\% EtOAc/DCM) to afford a semi-pure diol $(0.42 \mathrm{~g}, 0.49 \mathrm{mmol})$ that was added to a dry RBF charged with a magnetic stirbar and $\mathrm{NaHCO}_{3}(0.25 \mathrm{~g}, 3.0 \mathrm{mmol}, 6$ equiv). The flask was purged with $\mathrm{N}_{2}$ and anhydrous DCM $(25 \mathrm{~mL})$ was added under an $\mathrm{N}_{2}$ atmosphere. The reaction mixture was cooled to $0{ }^{\circ} \mathrm{C}$ whereupon Dess-Martin Periodinane $(0.48 \mathrm{~g}, 1.1 \mathrm{mmol}, 2.3$ equiv) was added. The resulting slurry was allowed to slowly warm to rt over $90 \mathrm{~min}$ and was then quenched by the addition sat. aq $\mathrm{NaHCO}_{3}(10 \mathrm{~mL})$ and sat. aq $\mathrm{Na}_{2} \mathrm{~S}_{2} \mathrm{O}_{3}(10 \mathrm{~mL})$. The biphasic mixture was stirred for $1 \mathrm{~h}$ and the layers were separated. The aqueous phase was extracted with DCM $(2 \times 20$ $\mathrm{mL})$ and the combined organic were washed with brine $(10 \mathrm{~mL})$, dried over sodium sulfate, and concentrated under vacuum. The resulting crude white solid dialdehyde $(0.41 \mathrm{~g})$ was used immediately without purification by first dissolving it in anhydrous THF $(10 \mathrm{~mL})$. In a separate dry RBF, methyltriphenylphosphonium bromide ( $0.67 \mathrm{~g}, 1.9 \mathrm{mmol}, 4.0$ equiv) was suspended in anhydrous THF $(10 \mathrm{~mL})$ and cooled to $0{ }^{\circ} \mathrm{C}$. Solid potassium tert-butoxide $(0.32 \mathrm{~g}, 2.9 \mathrm{mmol}$, 6.0 equiv) was added and the resulting bright yellow ylide solution was stirred for $10 \mathrm{~min}$. The solution of dialdehyde was added to the solution of ylide via cannula and the reaction mixture was allowed to stir at $\mathrm{rt}$ for $15 \mathrm{~min}$. Acetone $(1 \mathrm{~mL})$ was added and the reaction mixture was concentrated under vacuum. The crude material was loaded onto silica gel in DCM; purification on silica gel $(3 \mathrm{~cm} \times 7 \mathrm{~cm}, 0-5 \%$ EtOAc/DCM) afforded dichloride 8 as a white foam $(0.32 \mathrm{~g}$, 44\%). ${ }^{1} \mathrm{H}$ NMR (500 MHz, $\left.\mathrm{CDCl}_{3}\right): \delta(\mathrm{ppm}) 7.53(\mathrm{~d}, J=2.2 \mathrm{~Hz}, 2 \mathrm{H}), 7.40-7.35$ (overlap, 8H), $7.30(\mathrm{~d}, J=8.6 \mathrm{~Hz}, 2 \mathrm{H}), 7.27(\mathrm{dd}, J=8.6,2.2 \mathrm{~Hz}, 2 \mathrm{H}), 7.04(\mathrm{dd}, J=17.5,10.9 \mathrm{~Hz}, 2 \mathrm{H}), 6.17(\mathrm{~d}$, $J=10.2 \mathrm{~Hz}, 4 \mathrm{H}), 6.09(\mathrm{~s}, 3 \mathrm{H}), 6.07(\mathrm{~d}, J=10.2 \mathrm{~Hz}, 4 \mathrm{H}), 5.51(\mathrm{~d}, J=17.5 \mathrm{~Hz}, 2 \mathrm{H}), 5.25(\mathrm{~d}, J=$ $10.9 \mathrm{~Hz}, 2 \mathrm{H}), 3.46(\mathrm{~s}, 6 \mathrm{H}), 3.44(\mathrm{~s}, 12 \mathrm{H}) ;{ }^{13} \mathrm{C} \mathrm{NMR}\left(125 \mathrm{MHz}, \mathrm{CDCl}_{3}\right) \delta(\mathrm{ppm}) \quad 143.01,142.53$, 
$142.29,135.40,133.65,133.24,133.15,132.87,132.17,129.56,126.43,126.18,126.06,124.20$

116.74, 74.60, 74.55, 74.48, 52.05, 52.03, 51.98. MALDI-TOF $(\mathrm{m} / \mathrm{z}):[\mathrm{M}-\mathrm{OMe}+\mathrm{H}]^{+}$calcd. for $\mathrm{C}_{51} \mathrm{H}_{48} \mathrm{O}_{5} \mathrm{Cl}_{2}, 810.29$, found 810.55. IR (neat): 752.26, 831.60, 1079.88, 1228.92, 1365.36, $1467.32,1737.30,2821.77,2937.70 \mathrm{~cm}^{-1}$.

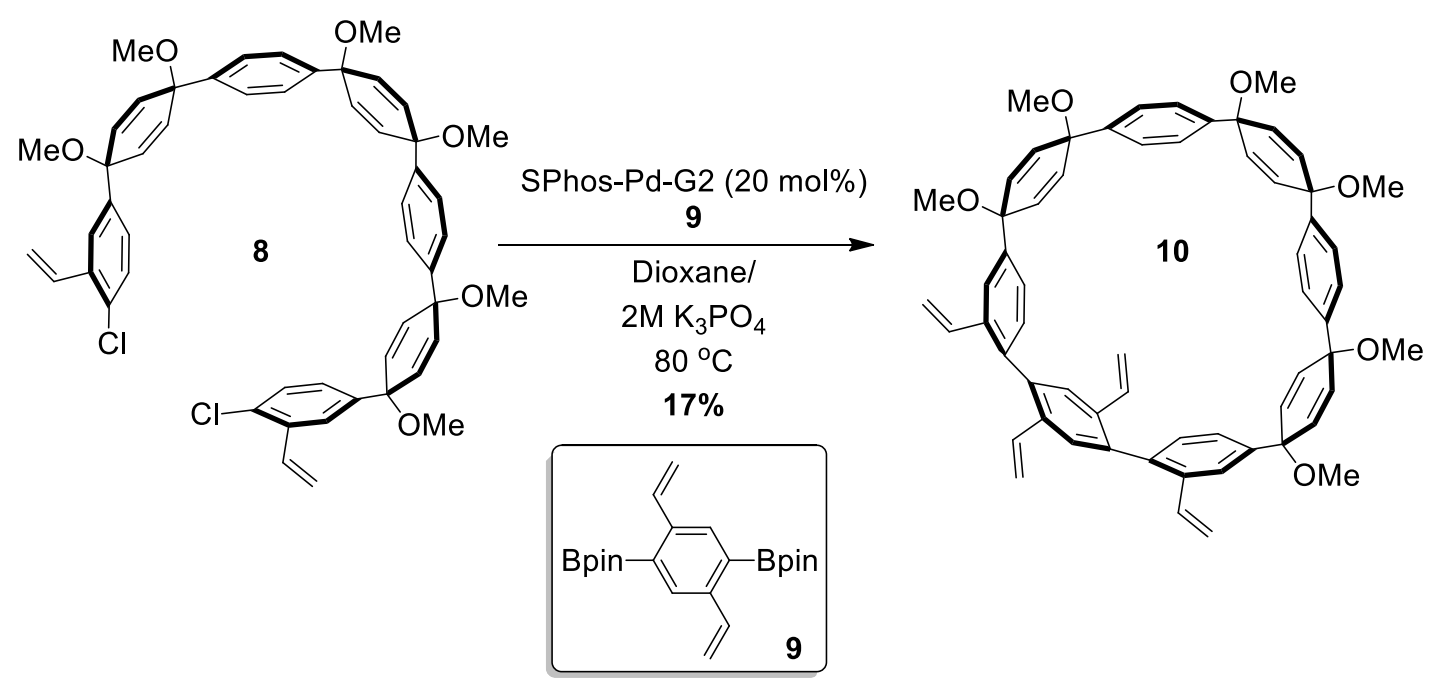

To a dry $250 \mathrm{~mL}$ Schlenk flask charged with a magnetic stirbar was added dichloride $8(0.14 \mathrm{~g}$, $0.16 \mathrm{mmol}, 1.0$ equiv), diboronate 9 (76 mg, $0.20 \mathrm{mmol}, 1.2$ equiv), and SPhos-Pd-G2 (24 mg, $0.033 \mathrm{mmol}, 20 \mathrm{~mol} \%)$. The vessel was fitted with a rubber septum and evacuated/backfilled with $\mathrm{N}_{2}(3 \mathrm{x})$ before the addition of anhydrous dioxane $(150 \mathrm{~mL})$ under an $\mathrm{N}_{2}$ atmosphere. The reaction mixture was heated to $85{ }^{\circ} \mathrm{C}$ for ca. 10 min whereupon degassed (sparged $1 \mathrm{~h}$ ) $2 \mathrm{M}$ aq $\mathrm{K}_{3} \mathrm{PO}_{4}(17 \mathrm{~mL})$ was added via cannula. The resulting dark yellow solution was heated under $\mathrm{N}_{2}$ for $19.5 \mathrm{~h}$. Upon cooling to rt, the brown reaction mixture was filtered through a short plug of Celite (EtOAc) and the layers were separated. The aqueous phase was extracted with EtOAc $(2 \mathrm{x}$ $50 \mathrm{~mL})$ and the combined organics were washed with brine $(20 \mathrm{~mL})$, dried over sodium sulfate, and concentrated under vacuum to a brown foam. This brown foam was redissolved in DCM and pass through a silica plug $(4 \mathrm{~cm} \times 3 \mathrm{~cm})$ topped with Celite $(50 \%$ EtOAc/DCM). The filtrate was concentrated under vacuum and purified by preparative recycling GPC to afford an off-white 
solid that could be washed with minimal acetone to afford tetravinyl macrocycle $\mathbf{1 0}$ as a while solid (25 mg, 17\%). ${ }^{1} \mathrm{H}$ NMR (400 MHz, $\left.\mathrm{CDCl}_{3}\right): \delta(\mathrm{ppm}) 8.03(\mathrm{~d}, J=1.8 \mathrm{~Hz}, 2 \mathrm{H}), 7.49-7.32$ (overlap, 10H), $6.98(\mathrm{dd}, J=17.6,11.0 \mathrm{~Hz}, 2 \mathrm{H}), 6.78(\mathrm{dd}, J=8.2,1.8 \mathrm{~Hz}, 2 \mathrm{H}), 6.52-6.40$ (overlap, 4H), $6.32-6.16$ (overlap, 8H), $6.10(\mathrm{dd}, J=10.2,2.2 \mathrm{~Hz}, 2 \mathrm{H}), 6.04(\mathrm{dd}, J=10.2,2.2$ $\mathrm{Hz}, 2 \mathrm{H}), 5.89(\mathrm{~d}, J=17.5 \mathrm{~Hz}, 2 \mathrm{H}), 5.58(\mathrm{~d}, J=17.5 \mathrm{~Hz}, 2 \mathrm{H}), 5.30(\mathrm{~d}, J=11.0 \mathrm{~Hz}, 2 \mathrm{H}), 5.07(\mathrm{~d}$, $J=11.1 \mathrm{~Hz}, 2 \mathrm{H}), 3.54(\mathrm{~s}, 6 \mathrm{H}), 3.39(\mathrm{~s}, 6 \mathrm{H}), 3.18(\mathrm{~s}, 6 \mathrm{H}) ;{ }^{13} \mathrm{C} \mathrm{NMR}\left(125 \mathrm{MHz}, \mathrm{CDCl}_{3}\right): \delta(\mathrm{ppm})$ $143.51,142.89,142.13,139.83,139.19,135.88,135.36,135.16,135.03,134.87,134.27,134.11$, $133.74,133.56,133.17,131.24,128.23,126.83,126.66,125.62,121.27,115.30,114.77,75.95$, 74.65, 72.43, 52.66, 51.79, 51.47; MALDI-TOF $(m / z):[\mathrm{M}-\mathrm{OMe}]^{+}$calcd. for $\mathrm{C}_{61} \mathrm{H}_{55} \mathrm{O}_{5}, 867.40$; found, 867.92. IR (neat): 754.83, 828.35, 907.32, 948.53, 1084.65, 1226.47, 1377.55, 1410.43, $1466.49,1661.06,1737.12,2853.29,2924.08,3363.89 \mathrm{~cm}^{-1}$.

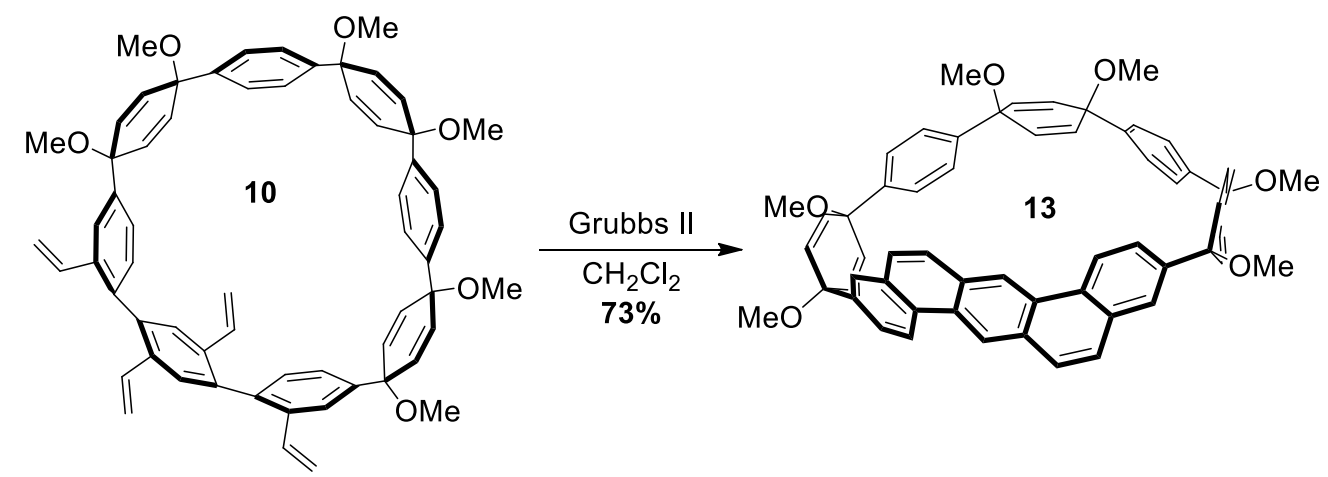

To a dry 2 dram vial charged with a septum cap and magnetic stirbar was added tetravinyl macrocycle 10 (16 mg, $0.018 \mathrm{mmol}, 1.0$ equiv) and Grubbs II (2 mg, $0.0024 \mathrm{mmol}, 10 \mathrm{~mol} \%)$. The vial was sealed and evacuated/backfilled with $\mathrm{N}_{2}(3 \mathrm{x})$ before the addition of anhydrous DCM $(1 \mathrm{~mL})$ under an $\mathrm{N}_{2}$ atmosphere. The reaction mixture was heated to $40{ }^{\circ} \mathrm{C}$ for $30 \mathrm{~min}$ then concentrated under reduced pressure onto Celite. Purification on silica gel $(1 \mathrm{~cm} \times 5 \mathrm{~cm}, 25 \%$ EtOAc/DCM) afforded an off-white solid that was washed several times with minimal acetone to 
afford benzo[k]tetraphene macrocycle $\mathbf{1 3}$ as a white solid $(11 \mathrm{mg}, 73 \%) .{ }^{1} \mathrm{H}$ NMR (400 MHz, $\left.\mathrm{CDCl}_{3}\right): \delta(\mathrm{ppm}) 9.04(\mathrm{~s}, 2 \mathrm{H}), 8.59(\mathrm{~d}, J=8.8 \mathrm{~Hz}, 2 \mathrm{H}), 7.92(\mathrm{~d}, J=9.0 \mathrm{~Hz}, 2 \mathrm{H}), 7.64(\mathrm{~s}, 2 \mathrm{H})$, $7.57-7.39$ (overlap, 4H), $6.88(\mathrm{~d}, J=8.2 \mathrm{~Hz}, 4 \mathrm{H}), 6.62(\mathrm{~d}, J=8.2 \mathrm{~Hz}, 4 \mathrm{H}), 6.33$ (overlap, 4H), $6.13(\mathrm{~d}, J=10.3 \mathrm{~Hz}, 2 \mathrm{H}), 6.09$ (d, $J=10.3 \mathrm{~Hz}, 2 \mathrm{H}), 6.04-5.94$ (overlap, 4H), 3.56 (s, 6H), 3.36 $(\mathrm{s}, 12 \mathrm{H}) ;{ }^{13} \mathrm{C} \mathrm{NMR}\left(125 \mathrm{MHz}, \mathrm{CDCl}_{3}\right): \delta(\mathrm{ppm}) 142.96,140.40,139.70,133.57,133.50,133.30$, $133.17,133.06,131.89,131.27,130.09,128.90,127.33,127.12,126.73,126.30,125.91,125.68$, $123.13,122.16,76.08,75.56,72.60,52.58,51.79,51.40$. MALDI-TOF $(\mathrm{m} / \mathrm{z}):[\mathrm{M}-3 \mathrm{OMe}]^{+}$ calcd. for $\mathrm{C}_{55} \mathrm{H}_{41} \mathrm{O}_{3}, 749.03$; found, 749.81. IR (neat): 752.34, 1082.39, 1217.14, 1365.34, $1736.81,3600.67,3626.27,3702.45,3726.69 \mathrm{~cm}^{-1}$.

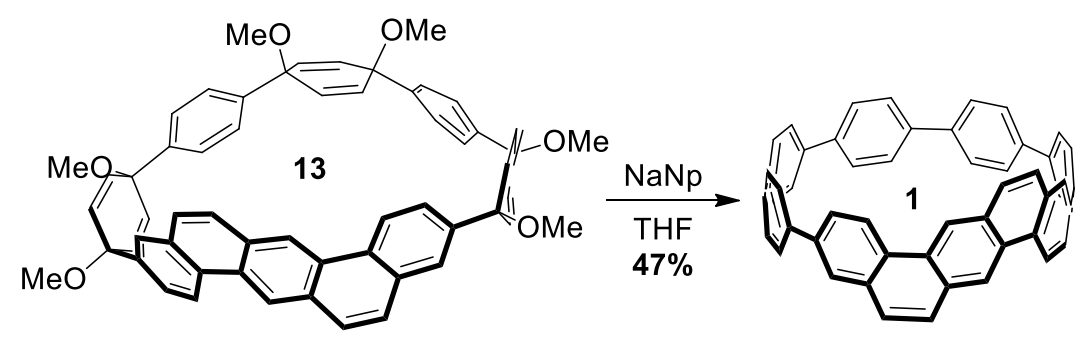

To a dry $25 \mathrm{~mL}$ RBF charged with a magnetic stirbar was added benzo[ $k]$ tetraphene macrocycle 13 (9.5 mg, $0.013 \mathrm{mmol}, 1.0$ equiv). The vessel was sealed with a rubber septum and evacuated/backfilled with $\mathrm{N}_{2}(3 \mathrm{x})$ before adding anhydrous THF $(1.5 \mathrm{~mL})$. The solution was cooled to $-78{ }^{\circ} \mathrm{C}$, whereupon sodium naphthalenide $(2.3 \mathrm{~mL}, 0.56 \mathrm{mmol}$, 50 equiv, $0.25 \mathrm{M}$ in THF) was added dropwise via syringe. During the addition process, the reaction mixture first became dark red, then eventually purple. After stirring for $30 \mathrm{~min}$, a solution of $\mathrm{I}_{2}$ in anhydrous THF $(1 \mathrm{~mL}, 1 \mathrm{M})$ was added via syringe and the resulting dark red solution was stirred at $-78{ }^{\circ} \mathrm{C}$ for 15 min. Sat. aq $\mathrm{Na}_{2} \mathrm{~S}_{2} \mathrm{O}_{3}(3 \mathrm{~mL})$ was then added and the reaction mixture was allowed to warm to rt. The yellow biphasic mixture was extracted with DCM $(2 \times 25 \mathrm{~mL})$ and the combined organics were washed with brine, dried over sodium sulfate, concentrated under vacuum onto 
Celite, and purified on silica gel $(1 \mathrm{~cm} \times 9 \mathrm{~cm}, 5-30 \%$ DCM/hexanes) to afford cyclo(3,10)benzo[ $k]$ tetraphene-penta( $p$-phenylene) $1(3.5 \mathrm{mg}, 47 \%) .{ }^{1} \mathrm{H}$ NMR $\left(500 \mathrm{MHz}, \mathrm{CDCl}_{3}\right): \delta(\mathrm{ppm})$ $8.63(\mathrm{~s}, 2 \mathrm{H}), 8.42(\mathrm{~d}, J=9.1 \mathrm{~Hz}, 2 \mathrm{H}), 7.88(\mathrm{dd}, J=9.1,2.1 \mathrm{~Hz}, 2 \mathrm{H}), 7.86(\mathrm{~d}, J=2.1 \mathrm{~Hz}, 2 \mathrm{H})$, $7.72(\mathrm{~d}, J=9.2 \mathrm{~Hz}, 2 \mathrm{H}), 7.66(\mathrm{~d}, J=8.4 \mathrm{~Hz}, 4 \mathrm{H}), 7.54(\mathrm{~d}, J=9.2 \mathrm{~Hz}, 2 \mathrm{H}), 7.49$ (overlap, 16H); ${ }^{13} \mathrm{C}$ NMR $\left(125 \mathrm{MHz}, \mathrm{CDCl}_{3}\right): \delta(\mathrm{ppm}) 137.78,137.58,137.56,136.66,135.57,132.83,132.11$, $129.58,129.06,127.72,127.65,127.43,127.39,127.26,127.17,126.69,126.40,125.32,123.84$, 122.71. MALDI-TOF $(\mathrm{m} / \mathrm{z})$ : $[\mathrm{M}]^{+}$calcd. for $\mathrm{C}_{52} \mathrm{H}_{32}, 656.81$; found, 656.74. IR (neat): 720.43, $810.94,886.03,1217.18,1365.34,1485.57,1583.43,1737.78,3021.78 \mathrm{~cm}^{-1}$.

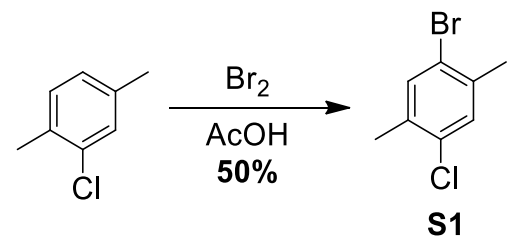

To a 3N $500 \mathrm{~mL}$ RBF charged with a magnetic stirbar and fitted with a septum, a condenser, and an addition funnel was added a solution of 2-chloro- $p$-xylene (56 g, $0.40 \mathrm{~mol}, 1.0$ equiv) in glacial $\mathrm{AcOH}(180 \mathrm{~mL})$. The solution was cooled to $0{ }^{\circ} \mathrm{C}$ and a solution of bromine $(71 \mathrm{~g}, 23$ $\mathrm{mL}, 0.44 \mathrm{~mol}, 1.1$ equiv) in glacial $\mathrm{AcOH}(180 \mathrm{~mL})$ was added over ca. $1 \mathrm{~h}$ via addition funnel. Upon completion of the addition, the reaction mixture was heated to $60{ }^{\circ} \mathrm{C}$ for $21 \mathrm{~h}$. The reaction mixture was cooled to $\mathrm{rt}$ whereupon $1 \mathrm{M}$ aq $\mathrm{NaOH}(400 \mathrm{~mL})$ was slowly added. When gas evolution stopped, sat. aq $\mathrm{Na}_{2} \mathrm{~S}_{2} \mathrm{O}_{3}(50 \mathrm{~mL})$ was added to quench excess bromine. The solution was extracted with $\mathrm{Et}_{2} \mathrm{O}(3 \times 200 \mathrm{~mL})$ and the combined organic were dried over sodium sulfate. Concentration under vacuum gave a white solid that was washed with ice cold $\mathrm{MeOH}$ to afford S1 as a crystalline white sold $(44 \mathrm{~g}, 50 \%) .{ }^{1} \mathrm{H}$ NMR $\left(500 \mathrm{MHz}, \mathrm{CDCl}_{3}\right) \delta(\mathrm{ppm}) 7.37(\mathrm{~s}, 1 \mathrm{H})$, $7.19(\mathrm{~s}, 1 \mathrm{H}), 2.32(\mathrm{~s}, 3 \mathrm{H}), 2.30(\mathrm{~s}, 3 \mathrm{H}) ;{ }^{13} \mathrm{C} \mathrm{NMR}\left(125 \mathrm{MHz}, \mathrm{CDCl}_{3}\right) \delta(\mathrm{ppm}) 136.82,135.22$ 134.22, 133.20, 130.91, 122.56, 22.36, 19.43. HRMS (EI+) $(\mathrm{m} / \mathrm{z})$ : $[\mathrm{M}]^{+}$calcd. for $\mathrm{C}_{8} \mathrm{H}_{8} \mathrm{BrCl}$, 
217.9498; found, 217.9493. IR(neat): 741.48, 755.97, 877.81, 987.66, 1037.51, 1185.14, 1265.43, 1348.02, 1379.99, 1451.71, 1477.79, 1745.77, 2851.62, 2920.24, 2952.89, 2984.20 $\mathrm{cm}^{-1}$.

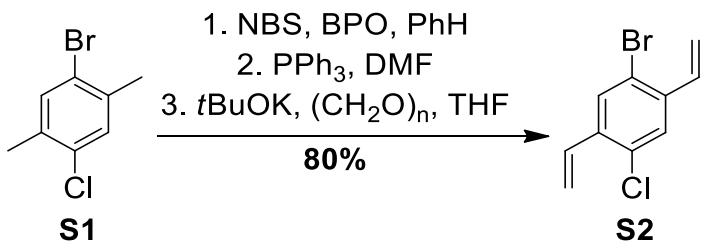

To a solution of $\mathbf{S 1}$ (44 g, $0.20 \mathrm{~mol}, 1.0$ equiv) in benzene $(1000 \mathrm{~mL})$ was added $N$ bromosuccinimide (78 g, $0.44 \mathrm{~mol}, 2.2$ equiv) and benzoyl peroxide $(2.2 \mathrm{~g}, 10 \mathrm{mmol}, 0.050$ equiv, wet with $25 \%$ water). The reaction mixture was heated to $80{ }^{\circ} \mathrm{C}$ for $20 \mathrm{~h}$, then another portion of benzoyl peroxide $(1.0 \mathrm{~g}, 4.5 \mathrm{mmol}, 0.023$ equiv, wet with $25 \%$ water) was added and stirring was continued for another $20 \mathrm{~h}$. Upon cooling to $\mathrm{rt}$, the reaction mixture was diluted with EtOAc $(300 \mathrm{~mL})$ and was washed with $1 \mathrm{M}$ aq $\mathrm{NaOH}(3 \times 200 \mathrm{~mL})$, water $(200 \mathrm{~mL})$ and brine $(100 \mathrm{~mL})$. The organics were dried over sodium sulfate and concentrated to afford an off white solid that was crystallized from $95 \%$ EtOH to yield crystalline white tetrabromide (21 g) that was then dissolved in DMF (250 mL). To this solution was added triphenylphosphine (37 g, 139 mmol, 2.5 equiv). The resulting mixture was heated to $120{ }^{\circ} \mathrm{C}$ for $16 \mathrm{~h}$. Upon cooling to room temperature, the solid white precipitate was collected by vacuum filtration and was washed with EtOAc $(2 \times 50 \mathrm{~mL})$ and hexanes $(2 \times 50 \mathrm{~mL})$. The diphosphonium salt was then dried under high vacuum and suspended in THF (200 mL). p-Formaldehyde (22 g, $748 \mathrm{mmol}, 15$ equiv) was added to the suspension, followed by the portion-wise addition of potassium tert-butoxide $(11 \mathrm{~g}$, $200 \mathrm{mmol}, 4.0$ equiv). The reaction mixture turned brown initially, and slowly became yellow. After 90 min, the reaction mixture was milky white and was filtered through a short plug of 
Celite (hexanes). The filtrate was concentrated under vacuum and purified on silica gel by loading the crude product as a slurry in hexanes $(4 \mathrm{~cm} \times 6 \mathrm{~cm}$, hexanes) to afford $\mathbf{S} 2$ as a white solid (9.7 g, 20\%). ${ }^{1} \mathrm{H}$ NMR (300 MHz, $\left.\mathrm{CDCl}_{3}\right): \delta(\mathrm{ppm}) 7.74(\mathrm{~s}, 1 \mathrm{H}), 7.53(\mathrm{~s}, 1 \mathrm{H}), 7.00(\mathrm{dd}, J=$ 17.5, 11.2 Hz, 1H), $6.96(\mathrm{dd}, J=17.4,11.2 \mathrm{~Hz}, 1 \mathrm{H}), 5.75(\mathrm{~d}, J=17.5 \mathrm{~Hz}, 1 \mathrm{H}), 5.72(\mathrm{~d}, J=17.4$ $\mathrm{Hz}, 1 \mathrm{H}), 5.42(\mathrm{~d}, J=11.2 \mathrm{~Hz}, 1 \mathrm{H}), 5.42(\mathrm{~d}, J=11.2 \mathrm{~Hz}, 1 \mathrm{H}) ;{ }^{13} \mathrm{C} \mathrm{NMR}\left(125 \mathrm{MHz}, \mathrm{CDCl}_{3}\right)$ $\delta(\mathrm{ppm}) 137.92,136.46,134.61,132.46,131.90,130.52,127.46,121.79,117.78,117.72$. HRMS (EI+) $(\mathrm{m} / z):[\mathrm{M}]^{+}$calcd. for $\mathrm{C}_{10} \mathrm{H}_{8} \mathrm{BrCl}, 241.9498$; found, 241.9489. IR (neat): 720.10, 741.59, $886.65,988.01,1032.20,1069.82,1365.54,1416.16,1469.64,1620.32,1843.07,3094.60 \mathrm{~cm}^{-1}$.

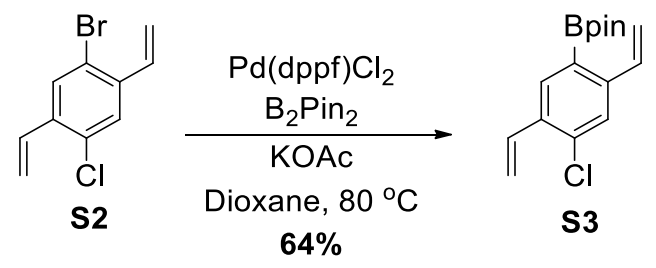

To a dry $100 \mathrm{~mL}$ Schlenk flask charged with a large magnetic stirbar was added freshly ground anhydrous potassium acetate $(3.1 \mathrm{~g}, 32 \mathrm{mmol}, 3.0$ equiv), $\mathbf{S 3}$ ( $2.6 \mathrm{~g}, 11 \mathrm{mmol}, 1.0$ equiv), freshly ground bis(pinacolato)diboron $\left(3 \mathrm{~g}, 12 \mathrm{mmol}, 1.1\right.$ equiv), and $\mathrm{Pd}(\mathrm{dppf}) \mathrm{Cl}_{2}(0.26 \mathrm{~g}, 0.30 \mathrm{mmol}$, $3.0 \mathrm{~mol} \%)$. The vessel was fitted with a rubber septum and evacuated/backfilled with $\mathrm{N}_{2}(3 \mathrm{x})$ before the addition of anhydrous dioxane $(30 \mathrm{~mL})$ under an $\mathrm{N}_{2}$ atmosphere. The resulting dark red suspension was heated to $80{ }^{\circ} \mathrm{C}$ for $20 \mathrm{~h}$. Upon cooling to $\mathrm{rt}$, the reaction mixture was concentrated onto Celite. Automated flash chromatography (100 g KP-Sil, $3-5 \%$ EtOAc/hexanes) afforded $\mathbf{S 3}$ as a pale yellow oil that solidified upon standing $(2.0 \mathrm{~g}, 64 \%) .{ }^{1} \mathrm{H}$ NMR (300 MHz, CDCl $): \delta(\mathrm{ppm}) 7.98(\mathrm{~s}, 1 \mathrm{H}), 7.61(\mathrm{~s}, 1 \mathrm{H}), 7.46(\mathrm{dd}, J=17.6,10.9 \mathrm{~Hz}, 1 \mathrm{H})$, $7.06(\mathrm{dd}, J=17.6,11.1 \mathrm{~Hz}, 1 \mathrm{H}), 5.83(\mathrm{dd}, J=17.6,1.2 \mathrm{~Hz}, 1 \mathrm{H}), 5.70(\mathrm{dd}, J=17.6,1.2 \mathrm{~Hz}, 1 \mathrm{H})$, $5.37(\mathrm{dd}, J=11.1,1.2 \mathrm{~Hz}, 1 \mathrm{H}), 5.30(\mathrm{dd}, J=10.9,1.2 \mathrm{~Hz}, 1 \mathrm{H}), 1.35(\mathrm{~s}, 12 \mathrm{H}) ;{ }^{13} \mathrm{C}$ NMR $(125$ 
$\left.\mathrm{MHz}, \mathrm{C}_{6} \mathrm{D}_{6}\right) \delta(\mathrm{ppm}) 145.07,136.52,136.20,135.00,134.24,132.58,125.78,116.30,115.49$, 83.52, 24.45 (C-B not observed). HRMS (FAB+) $(m / z)$ : $[\mathrm{M}]^{+}$calcd. for $\mathrm{C}_{16} \mathrm{H}_{20} \mathrm{O}_{2} \mathrm{BCl}, 290.1245$; found, 290.1256. IR (neat): 668.17, 731.73, 855.35, 912.12, 966.99, 987.91, 1040.83, 1083.18, $1110.69,1212.77,1256.31,1299.87,1314.58,1329.48,1388.76,1477.54,1529.56,1586.82$, $1623.78,2929.50,2977.71,3086.38 \mathrm{~cm}^{-1}$.

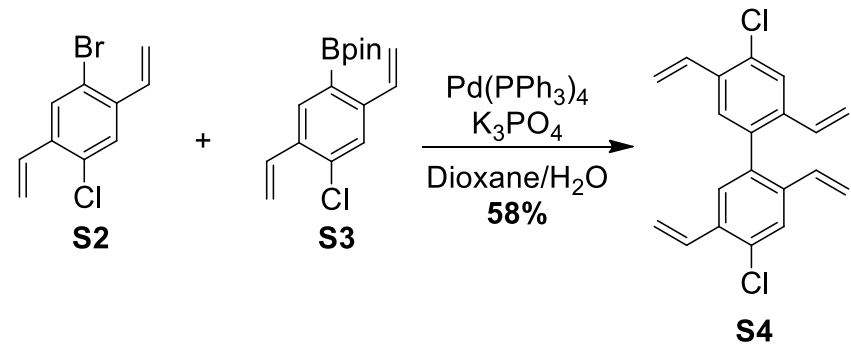

To a $50 \mathrm{~mL}$ Schlenk flask charged with a magnetic stirbar was added $\mathbf{S 2}(1.8 \mathrm{~g}, 7.3 \mathrm{mmol}, 1.0$ equiv), $\mathbf{S 3}$ (2.3 g, $8.0 \mathrm{mmol}, 1.1$ equiv), $\mathrm{Pd}\left(\mathrm{PPh}_{3}\right)_{4}(0.37 \mathrm{~g}, 0.32 \mathrm{mmol}, 4.5 \mathrm{~mol} \%)$, and freshly ground $\mathrm{K}_{3} \mathrm{PO}_{4}$ (4.6 g, $22 \mathrm{mmol}, 3.0$ equiv). The vessel was sealed with a rubber septum and evacuated/backfilled with $\mathrm{N}_{2}(3 \mathrm{x})$ before adding degassed (sparged $1 \mathrm{~h}$ ) dioxane $(12 \mathrm{~mL})$ and water ( $2 \mathrm{~mL}$ ) under an $\mathrm{N}_{2}$ atmosphere. The bright yellow reaction mixture was heated in the dark to $70{ }^{\circ} \mathrm{C}$. After stirring for $20 \mathrm{~h}$, the reaction mixture was cooled to rt and diluted with EtOAc (20 $\mathrm{mL})$. The layers were separated and the aqueous layer was extracted with EtOAc $(2 \times 20 \mathrm{~mL})$. The combined organics were washed with brine $(20 \mathrm{~mL})$, dried over sodium sulfate, and concentrated onto Celite. Purification on silica gel $(3 \mathrm{~cm} \times 10 \mathrm{~cm}$, hexanes) afforded $\mathbf{S 4}$ as a viscous, colorless oil that solidified to a white solid upon standing (1.0 g, 42\%). ${ }^{1} \mathrm{H}$ NMR (500 $\left.\mathrm{MHz}, \mathrm{CDCl}_{3}\right): \delta(\mathrm{ppm}) 7.65(\mathrm{~s}, 1 \mathrm{H}), 7.36(\mathrm{~s}, 1 \mathrm{H}), 7.11(\mathrm{dd}, J=17.6,11.1 \mathrm{~Hz}, 1 \mathrm{H}), 6.31(\mathrm{dd}, J=$ 17.5, 11.0 Hz, 1H), $5.72(\mathrm{~d}, J=17.5 \mathrm{~Hz}, 1 \mathrm{H}), 5.67(\mathrm{~d}, J=17.6 \mathrm{~Hz}, 1 \mathrm{H}), 5.37(\mathrm{~d}, J=11.1 \mathrm{~Hz}$, $1 \mathrm{H}), 5.16(\mathrm{~d}, J=11.0 \mathrm{~Hz}, 1 \mathrm{H}) ;{ }^{13} \mathrm{C}$ NMR $\left(125 \mathrm{MHz}, \mathrm{CDCl}_{3}\right): \delta(\mathrm{ppm}) 137.26,137.21,134.70$, 
133.55, 133.03, 132.59, 128.35, 126.08, 117.01, 116.36; LRMS (FAB+) $(\mathrm{m} / z):[\mathrm{M}]^{+}$calcd. for $\mathrm{C}_{20} \mathrm{H}_{16} \mathrm{Cl}_{2}$, 326.06; found, 326.10. IR(neat): 681.12, 880.49, 898.06, 985.63, 1029.19, 1041.91, 1216.46, 1260.44, 1372.48, 1415.59, 1472.13, 1622.27, 2987.93, 3017.88, $3086.40 \mathrm{~cm}^{-1}$.

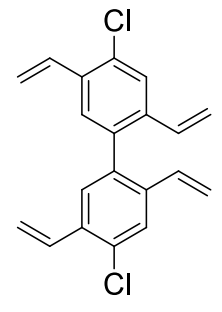

S4

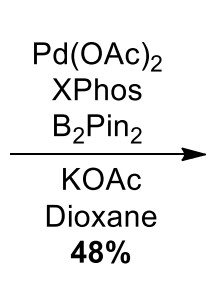

$48 \%$

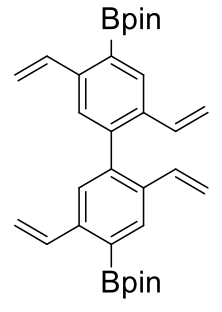

11

To a dry $50 \mathrm{~mL}$ RBF was added $\mathbf{S 4}(0.46 \mathrm{~g}, 1.4 \mathrm{mmol}, 1.0$ equiv), freshly ground anhydrous potassium acetate $(0.84 \mathrm{~g}, 8.5 \mathrm{mmol}, 6.0$ equiv), freshly ground bis(pinacolato)diboron $(2.2 \mathrm{~g}$, $8.5 \mathrm{mmol}, 6.0$ equiv), XPhos (81 mg, $0.17 \mathrm{mmol}, 12 \mathrm{~mol} \%)$, and $\mathrm{Pd}(\mathrm{OAc})_{2}(19 \mathrm{mg}, 0.028 \mathrm{mmol}$, 2.0 mol\%). The vessel was fitted with a rubber septum and evacuated/backfilled with $\mathrm{N}_{2}(3 \mathrm{x})$ before the addition of anhydrous dioxane $(10 \mathrm{~mL})$ under an $\mathrm{N}_{2}$ atmosphere. The brown reaction mixture was heated to $75{ }^{\circ} \mathrm{C}$ for $19 \mathrm{~h}$. Upon cooling to $\mathrm{rt}$, the crude reaction mixture was filtered through a short pad of Celite (EtOAc) then concentrated onto Celite. Purification on silica gel (3 $\mathrm{cm} \times 9 \mathrm{~cm}, 3 \%$ EtOAc/hexanes) afforded $\mathbf{1 1}$ as a white solid after washing with minimal hexanes (350 mg, 48\%). ${ }^{1} \mathrm{H}$ NMR (300 MHz, $\left.\mathrm{CDCl}_{3}\right): \delta(\mathrm{ppm}) 8.09$ (s, $\left.1 \mathrm{H}\right), 7.55(\mathrm{dd}, J=17.5,11.0 \mathrm{~Hz}$, 1H), $7.44(\mathrm{~s}, 1 \mathrm{H}), 6.37(\mathrm{dd}, J=17.6,11.1 \mathrm{~Hz}, 1 \mathrm{H}), 5.72(\mathrm{dd}, J=17.6,1.3 \mathrm{~Hz}, 1 \mathrm{H}), 5.66(\mathrm{dd}, J=$ 17.5, $1.4 \mathrm{~Hz}, 1 \mathrm{H}), 5.25(\mathrm{dd}, J=11.1,1.3 \mathrm{~Hz}, 1 \mathrm{H}), 5.07(\mathrm{dd}, J=11.0,1.4 \mathrm{~Hz}, 1 \mathrm{H}), 1.38(\mathrm{~s}, 12 \mathrm{H})$; ${ }^{13} \mathrm{C}$ NMR (125 MHz, $\left.\mathrm{CDCl}_{3}\right): \delta(\mathrm{ppm}) 142.70,142.22,137.02,134.81,134.61,132.86,126.32$, 115.04, 114.97, 83.98, 25.06 (C-B not observed). HRMS (FAB+) $[M]^{+}$calcd. for $\mathrm{C}_{32} \mathrm{H}_{40} \mathrm{~B}_{2} \mathrm{O}_{4}$, 510.3113; found, 510.3141. IR(neat): 854.14, 909.77, 964.87, 991.92, 1041.91, 1084.58, 
$1110.49,1143.47,1214.40,1273.43,1329.85,1389.91,1590.34,1737.03,2930.22,2977.37$, $3085.05 \mathrm{~cm}^{-1}$.
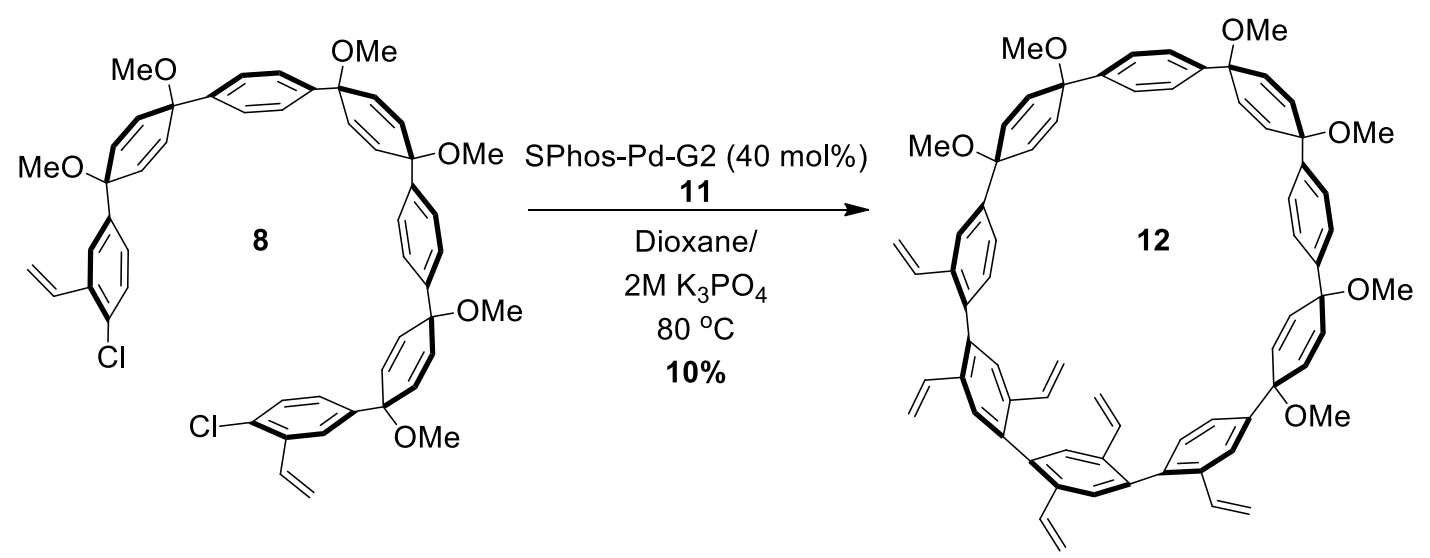

To a dry $200 \mathrm{~mL}$ Schlenk flask charged with a magnetic stirbar was added dichloride 8 (95 mg, $0.11 \mathrm{mmol}, 1.0$ equiv), diboronate 11 (63 mg, $0.12 \mathrm{mmol}, 1.1$ equiv), and SPhos-Pd-G2 (75 mg, $0.33 \mathrm{mmol}, 40 \mathrm{~mol} \%)$. The vessel was fitted with a rubber septum and evacuated/backfilled with $\mathrm{N}_{2}$ (3x) before the addition of anhydrous dioxane $(160 \mathrm{~mL})$ under an $\mathrm{N}_{2}$ atmosphere. The reaction mixture was heated to $80{ }^{\circ} \mathrm{C}$ for ca. 10 min whereupon degassed (sparged $1 \mathrm{~h}$ ) $2 \mathrm{M}$ aq $\mathrm{K}_{3} \mathrm{PO}_{4}(20 \mathrm{~mL})$ was added via cannula. The resulting dark yellow solution was heated under $\mathrm{N}_{2}$ for $15 \mathrm{~h}$. Upon cooling to rt, the brown reaction mixture was diluted with EtOAc and water. The aqueous phase was extracted with EtOAc $(2 \times 50 \mathrm{~mL})$ and the combined organics were washed with brine $(20 \mathrm{~mL})$, dried over sodium sulfate, and concentrated onto Celite. Purification on silica gel ( $3 \mathrm{~cm}$ x $6 \mathrm{~cm}, 15-20 \%$ THF/hexanes) afforded hexavinyl macrocycle $\mathbf{1 2}$ as a white solid (27 mg, 10\%). ${ }^{1} \mathrm{H}$ NMR $\left(500 \mathrm{MHz}, \mathrm{C}_{6} \mathrm{D}_{6}, 70{ }^{\circ} \mathrm{C}\right) \delta(\mathrm{ppm}) 8.26(\mathrm{br} \mathrm{s}, 2 \mathrm{H}), 7.62(\mathrm{~s}, 2 \mathrm{H}), 7.53$ - 7.39 (overlap, 10H), 7.10 - 6.97 (overlap, 4H), 6.87 - 6.78 (overlap, 4H), 6.53 - 6.40 (br m, 2H), $6.18(\mathrm{~s}, 4 \mathrm{H}), 6.01-5.92$ (overlap, $8 \mathrm{H}), 5.78(\mathrm{~d}, J=17.7 \mathrm{~Hz}, 2 \mathrm{H}), 5.59(\mathrm{~d}, J=17.6 \mathrm{~Hz}, 2 \mathrm{H})$, $5.41(\mathrm{~d}, J=17.5 \mathrm{~Hz}, 2 \mathrm{H}), 5.11(\mathrm{~d}, J=11.0 \mathrm{~Hz}, 2 \mathrm{H}), 5.04(\mathrm{~d}, J=11.1 \mathrm{~Hz}, 2 \mathrm{H}), 4.86(\mathrm{~d}, J=11.0$ 
$\mathrm{Hz}, 2 \mathrm{H}), 3.37(\mathrm{~s}, 6 \mathrm{H}), 3.23(\mathrm{~s}, 6 \mathrm{H}), 3.10(\mathrm{~s}, 6 \mathrm{H}) ;{ }^{13} \mathrm{C} \mathrm{NMR}\left(125 \mathrm{MHz}, \mathrm{C}_{6} \mathrm{D}_{6}, 70{ }^{\circ} \mathrm{C}\right) \delta(\mathrm{ppm})$ 144.30, 143.63, 143.40, 140.18, 140.01, 139.62, 136.67, 136.46, 136.07, 135.71, 135.31, 134.49, $133.11,127.28,126.94,126.39,122.88,115.62,115.46,115.39,76.17,75.00,72.70,52.27$ $51.48,51.20$ ( 5 carbons are not observed due to overlap and/or broadening; attempts to collect a ${ }^{13} \mathrm{C}$ NMR spectrum at higher temperatures in 1,1,2,2-tetrachloroethane- $d_{2}$ led to decomposition of 12). MALDI-TOF $(\mathrm{m} / \mathrm{z})$ : $[\mathrm{M}+\mathrm{H}]^{+}$calcd. for $\mathrm{C}_{72} \mathrm{H}_{66} \mathrm{O}_{6}, 1027.50$, found 1027.10. IR(neat): $668.11,828.08,904.69,1083.50,1226.97,1365.37,1462.32,1737.13,2854.81,2926.83 \mathrm{~cm}^{-1}$.

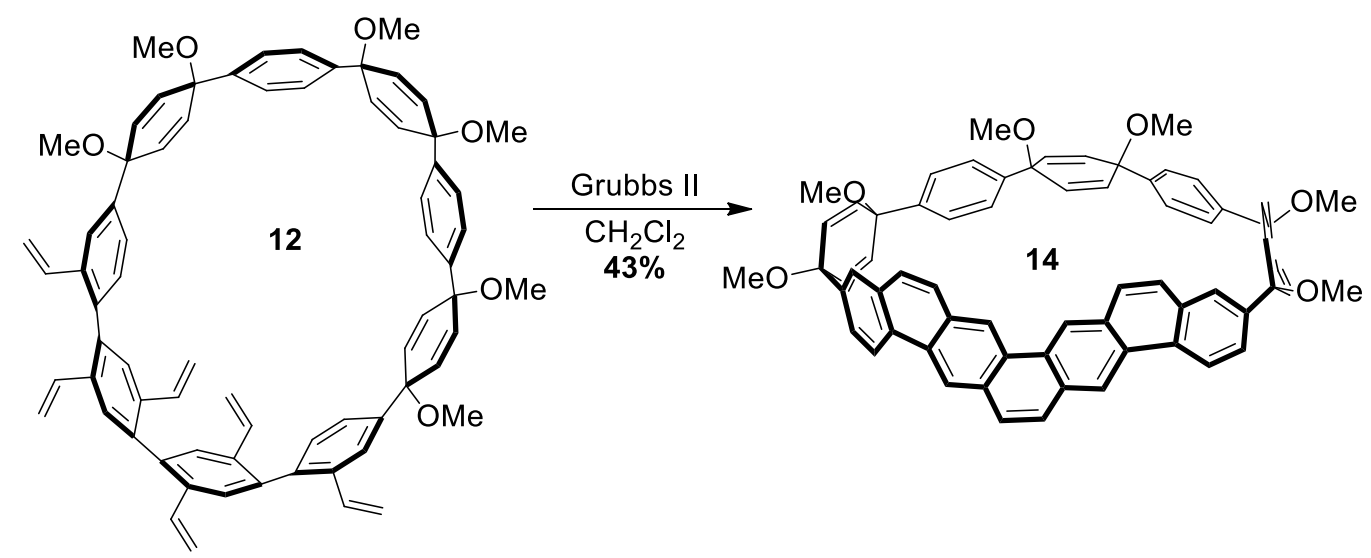

To a dry 4 dram vial with a septum cap and magnetic stirbar was added hexavinyl macrocycle $\mathbf{1 2}$ (32 mg, $0.031 \mathrm{mmol}, 1.0$ equiv) and Grubbs II (4.0 mg, $0.0047 \mathrm{mmol}, 15 \mathrm{~mol} \%)$. The vial was sealed and evacuated/backfilled with $\mathrm{N}_{2}(3 \mathrm{x})$ before the addition of anhydrous DCM $(1.5 \mathrm{~mL})$ under an $\mathrm{N}_{2}$ atmosphere. The reaction mixture was heated to $40{ }^{\circ} \mathrm{C}$ for 90 min then concentrated onto Celite. Purification on silica gel $(1 \mathrm{~cm} \times 7 \mathrm{~cm}, 25-35 \% \mathrm{THF} / \mathrm{hexanes})$ afforded dibenzo[c,m]pentaphene macrocycle $\mathbf{1 4}$ as a pale yellow solid, that was further purified by washing with minimal acetone (12.5 mg, 43\%). ${ }^{1} \mathrm{H}$ NMR $\left(500 \mathrm{MHz}, \mathrm{C}_{6} \mathrm{D}_{6}, 70{ }^{\circ} \mathrm{C}\right): \delta(\mathrm{ppm}) 9.15$ (s, 2H), $8.93(\mathrm{~s}, 2 \mathrm{H}), 8.64(\mathrm{~d}, J=8.9 \mathrm{~Hz}, 2 \mathrm{H}), 7.89(\mathrm{~s}, 2 \mathrm{H}), 7.81(\mathrm{~d}, J=8.9 \mathrm{~Hz}, 2 \mathrm{H}), 7.77(\mathrm{dd}, J$ $=8.8,1.9 \mathrm{~Hz}, 2 \mathrm{H}), 7.25-7.21$ (overlap, $4 \mathrm{H}), 6.60(\mathrm{~d}, J=8.5 \mathrm{~Hz}, 4 \mathrm{H}), 6.53(\mathrm{~d}, J=8.5 \mathrm{~Hz}, 4 \mathrm{H})$, 
$6.52-6.48$ (overlap, 2H), $6.33(\mathrm{dd}, J=10.3,2.2 \mathrm{~Hz}, 2 \mathrm{H}), 6.29(\mathrm{dd}, J=10.2,2.2 \mathrm{~Hz}, 2 \mathrm{H}), 5.91$ $(\mathrm{d}, J=2.2 \mathrm{~Hz}, 2 \mathrm{H}), 5.81(\mathrm{~d}, J=2.2 \mathrm{~Hz}, 2 \mathrm{H}), 5.74(\mathrm{dd}, J=10.2,2.2 \mathrm{~Hz}, 2 \mathrm{H}), 3.53(\mathrm{~s}, 6 \mathrm{H}), 3.27$ (s, 6H), $3.26(\mathrm{~s}, 6 \mathrm{H}) ;{ }^{13} \mathrm{C}$ NMR $\left(125 \mathrm{MHz}, \mathrm{C}_{6} \mathrm{D}_{6}, 70{ }^{\circ} \mathrm{C}\right) \delta(\mathrm{ppm}) 142.18,140.73,139.36,136.25$, $136.18,133.51,132.59,131.90,131.57,131.36,130.69,130.41,130.03,129.39,128.91,127.56$, $127.48,126.84,126.13,126.11,123.62,122.70,122.58,75.95,75.42,71.87,52.77,51.85,51.47$. MALDI-TOF $(\mathrm{m} / \mathrm{z})$ : $[\mathrm{M}-\mathrm{OMe}]^{+}$calcd. for $\mathrm{C}_{72} \mathrm{H}_{51} \mathrm{O}_{5}, 911.37$, found 911.74. IR(neat): 824.49, $954.80,1076.42,1217.26,1375.31,1458.12,1738.04,2853.55,2923.05,2953.07 \mathrm{~cm}^{-1}$.

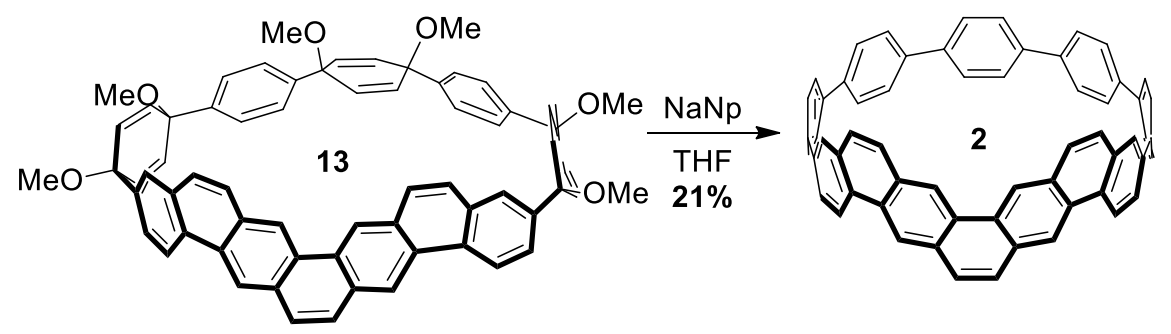

To a dry $50 \mathrm{~mL} \mathrm{RBF}$ charged with a magnetic stirbar was added dibenzo[c,m]pentaphene macrocycle 13 (11 mg, $0.011 \mathrm{mmol}, 1.0$ equiv). The vessel was sealed with a rubber septum and evacuated/backfilled with $\mathrm{N}_{2}(3 \mathrm{x})$ before adding anhydrous THF ( $\left.3 \mathrm{~mL}\right)$. The solution was cooled to $-78{ }^{\circ} \mathrm{C}$, whereupon sodium naphthalenide $(0.90 \mathrm{~mL}, 0.44 \mathrm{mmol}, 0.50 \mathrm{M}$ in THF, 40 equiv) was added dropwise via syringe. After stirring for $45 \mathrm{~min}$, a solution of $\mathrm{I}_{2}(1 \mathrm{~mL}, 1 \mathrm{M}$ in THF) was added via syringe and the resulting dark red solution was stirred at $-78{ }^{\circ} \mathrm{C}$ for $15 \mathrm{~min}$. Sat. aq $\mathrm{Na}_{2} \mathrm{~S}_{2} \mathrm{O}_{3}(2 \mathrm{~mL})$ was then added and the reaction mixture was allowed to warm to $\mathrm{rt}$. The yellow biphasic mixture was extracted with DCM $(2 \times 20 \mathrm{~mL})$ and the combined organics were washed with brine $(10 \mathrm{~mL})$, dried over sodium sulfate, and concentrated onto Celite. Purification was performed using automated flash chromatography (KP-Sil $10 \mathrm{~g}$, hexanes then $30-50 \%$ DCM/hexanes) followed by preparative recycling GPC to afford cyclo(2,11)dibenzo[c,m]pentaphene-penta(p-phenylene) 2 (1.8 mg, 21\%). ${ }^{1} \mathrm{H}$ NMR $\left(600 \mathrm{MHz}, \mathrm{CDCl}_{3}\right)$ : 
$\delta(\mathrm{ppm}) 8.73(\mathrm{~s}, 2 \mathrm{H}), 8.5(\mathrm{~s}, 2 \mathrm{H}), 8.42(\mathrm{~d}, J=9.1 \mathrm{~Hz}, 2 \mathrm{H}), 7.84(\mathrm{dd}, J=9.1,2.0 \mathrm{~Hz}, 2 \mathrm{H}), 7.80(\mathrm{~d}$, $J=2.0 \mathrm{~Hz}, 2 \mathrm{H}), 7.73(\mathrm{~d}, J=9.3 \mathrm{~Hz}, 2 \mathrm{H}), 7.65(\mathrm{~s}, 2 \mathrm{H}), 7.59(\mathrm{~d}, J=8.7 \mathrm{~Hz}, 4 \mathrm{H}), 7.53(\mathrm{~d}, J=9.3$ $\mathrm{Hz}, 2 \mathrm{H}), 7.47$ - 7.39 (overlap, $16 \mathrm{H}) ;{ }^{13} \mathrm{C} \mathrm{NMR}\left(150 \mathrm{MHz}, \mathrm{CDCl}_{3}\right) \delta(\mathrm{ppm})$ 138.17, 138.00, $137.78,137.75,137.10,135.93,132.66,131.90,131.55,129.51,129.07,128.94,127.60,127.50$, $127.23,127.21,127.15,127.11,126.97,126.73,126.70,124.87,123.85,122.73,122.36$. MALDI-TOF $(\mathrm{m} / \mathrm{z})$ : $[\mathrm{M}]^{+}$calcd. for $\mathrm{C}_{60} \mathrm{H}_{36}, 756.28$, found 756.89. IR(neat): 703.43, 811.72, 891.82, 1012.39, 1263.66, 1377.32, 1458.54, 1662.05, 1737.14, 2853.18, $2923.55 \mathrm{~cm}^{-1}$.

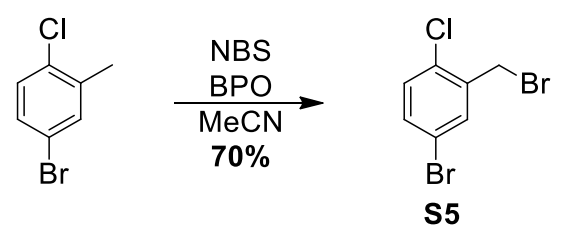

5-bromo-2-chlorotoluene (13 mL, $21 \mathrm{~g}, 100 \mathrm{mmol}, 1.0$ equiv) was dissolved in acetonitrile (500 $\mathrm{mL}$ ) in a $1 \mathrm{~L}$ flask open to the atmosphere. $\mathrm{N}$-bromosuccinimide (20 g, $110 \mathrm{mmol}, 1.1$ equiv) and benzoyl peroxide $(0.50 \mathrm{~g}, 2.0 \mathrm{mmol}, 0.020$ equiv) were added and the reaction was refluxed overnight. The next morning, the reaction was quenched by adding solid sodium sulfite $(4.0 \mathrm{~g})$. The mixture was concentrated and dissolved in $\mathrm{CH}_{2} \mathrm{Cl}_{2}$. The organics were washed with water and brine. The solvent was then removed under vacuum to yield a solid that was recrystallized from methanol $(30 \mathrm{~mL})$ to yield $\mathbf{S 5}$ as a white crystalline solid $(20 \mathrm{~g}, 70 \%) .{ }^{1} \mathrm{H} \mathrm{NMR}(300 \mathrm{MHz}$, $\left.\mathrm{CDCl}_{3}\right) \delta(\mathrm{ppm}) 7.58(\mathrm{~d}, J=2.4 \mathrm{~Hz}, 1 \mathrm{H}), 7.38(\mathrm{dd}, J=8.5,2.5 \mathrm{~Hz}, 1 \mathrm{H}), 7.26(\mathrm{~d}, J=8.5 \mathrm{~Hz}, 1 \mathrm{H})$, $4.52(\mathrm{~s}, 2 \mathrm{H}) ;{ }^{13} \mathrm{C} \mathrm{NMR}\left(150 \mathrm{MHz}, \mathrm{CDCl}_{3}\right) \delta(\mathrm{ppm}) 137.07,133.71,132.99,132.69,131.17$, 120.35, 29.09. HRMS (CI) $(\mathrm{m} / \mathrm{z})$ : $[\mathrm{M}]^{+}$calcd for $\mathrm{C}_{7} \mathrm{H}_{5} \mathrm{Br}_{2} \mathrm{Cl}, 281.8446$; found, 281.8447. IR (neat) $1903,1770,1643,1464,1215,1082,1049 \mathrm{~cm}^{-1}$. 


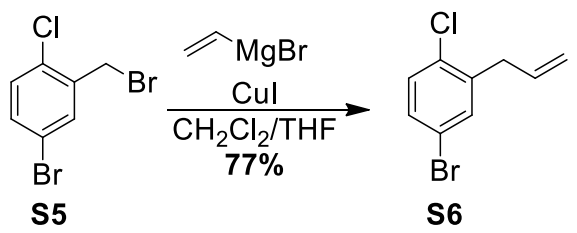

4-bromo-2-(bromomethyl)-1-chlorobenzene $\mathbf{S 5}$ (15 g, $51 \mathrm{mmol})$ and copper (I) bromide (4.9 g, $26 \mathrm{mmol})$ was suspended in $\mathrm{CH}_{2} \mathrm{Cl}_{2}(260 \mathrm{~mL})$ and cooled to $-78{ }^{\circ} \mathrm{C}$. A solution of vinyl magnesium bromide (250 mL, $110 \mathrm{mmol}, 0.43 \mathrm{M}$ in THF) was then added as a slow stream to the mixture. The reaction was stirred for $3 \mathrm{~h}$ at $-78{ }^{\circ} \mathrm{C}$ and warmed to room temperature by allowing the cooling bath to expire. The mixture was then quenched by adding saturated aq ammonium chloride solution $(100 \mathrm{~mL})$. The biphasic solution was filtered through Celite and extracted into EtOAc $(2 \times 200 \mathrm{~mL})$. The combined organics were washed with brine $(100 \mathrm{~mL})$, dried over anhydrous sodium sulfate, and concentrated under vacuum to yield a white solid under an oil. The oil was decanted off and the solid was washed with EtOAc (100 mL). This solution was then concentrated and purified by vacuum distillation (200 mTorr, $\left.120{ }^{\circ} \mathrm{C}\right)$. Anhydrous sodium sulfate was then added to the distillate which was subsequently filtered and washed with EtOAc. The filtrate was concentrated to yield $\mathbf{S 6}$ as a clear colorless oil $(9.2 \mathrm{~g}$, 77\%). ${ }^{1} \mathrm{H}$ NMR $\left(300 \mathrm{MHz}, \mathrm{CDCl}_{3}\right) \delta(\mathrm{ppm}) 7.36(\mathrm{~d}, J=2.5 \mathrm{~Hz}, 1 \mathrm{H}), 7.29(\mathrm{dd}, J=8.5,2.5 \mathrm{~Hz}$, 1H), $7.22(\mathrm{~d}, J=8.5 \mathrm{~Hz}, 1 \mathrm{H}), 5.93(\mathrm{ddt}, J=16.8,10.1,6.5 \mathrm{~Hz}, 1 \mathrm{H}), 5.15(\mathrm{dd}, J=10.1,1.6 \mathrm{~Hz}$, $1 \mathrm{H}), 5.09(\mathrm{dd}, J=16.8,1.6 \mathrm{~Hz}, 1 \mathrm{H}), 3.46(\mathrm{~d}, J=6.5 \mathrm{~Hz}, 2 \mathrm{H}) ;{ }^{13} \mathrm{C} \mathrm{NMR}\left(125 \mathrm{MHz}, \mathrm{CDCl}_{3}\right)$ $\delta(\mathrm{ppm}) 139.87,134.55,133.15,130.79,120.42,117.32,37.41$. HRMS $(\mathrm{CI})(\mathrm{m} / \mathrm{z})$ calcd for $\mathrm{C}_{9} \mathrm{H}_{8} \mathrm{BrCl}[\mathrm{M}]^{+} 229.9498$, found 229.9497. IR (neat) 1638, 1582, 1463, 1081, $1041917,807 \mathrm{~cm}^{-}$ 


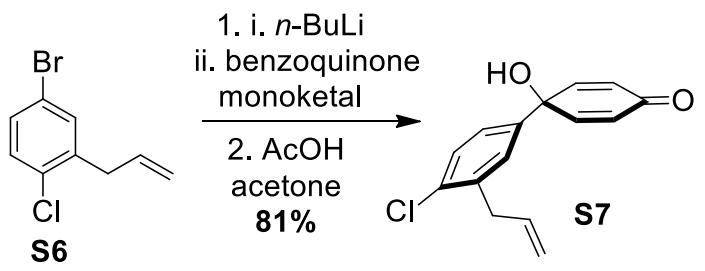

2-allyl-4-bromo-1-chlorobenzene S6 (5.2 g, $23 \mathrm{mmol})$ was stirred under vacuum to remove dissolved gases. Tetrahydrofuran $(40 \mathrm{~mL})$ was added to the reaction vessel and the solution was cooled to $-78{ }^{\circ} \mathrm{C}$. A solution of $n \mathrm{BuLi}(8.6 \mathrm{~mL}, 21 \mathrm{mmol}, 2.5 \mathrm{M}$ in hexanes) was added slowly. The resulting solution was then stirred for $5 \mathrm{~min}$ and benzoquinone monoketal $(2.7 \mathrm{~mL}, 3.2 \mathrm{~g}, 20$ mmol) was added slowly. The reaction was stirred at $-78^{\circ} \mathrm{C}$ for 30 min before quenching with water $(50 \mathrm{~mL})$ and warming to room temperature. The mixture was extracted with EtOAc $(2 \times 50$ $\mathrm{mL})$. The combined organics were washed with brine $(40 \mathrm{~mL})$, dried over anhydrous sodium sulfate, and concentrated under vacuum to yield a crude yellow oil. To a solution of the crude yellow oil in acetone $(25 \mathrm{~mL})$ was added $10 \%$ aq acetic acid $(25 \mathrm{~mL})$. The reaction mixture was stirred for $1 \mathrm{~h}$ at room temperature, quenched with sat. sodium bicarbonate solution $(25 \mathrm{~mL})$, and extracted into EtOAc $(2 \times 30 \mathrm{~mL})$. The extract was washed with sat. sodium bicarbonate solution $(25 \mathrm{~mL})$, water $(25 \mathrm{~mL})$ and brine $(15 \mathrm{~mL})$. The organics were dried over anhydrous sodium sulfate and were concentrated to yield a crude brown oil. This oil was loaded onto silica gel and purified on $\mathrm{SiO}_{2}\left(20-50 \%\right.$ EtOAc/hexanes) to yield $\mathbf{S 7}$ as a yellow solid $(4.3 \mathrm{~g}, 81 \%) .{ }^{1} \mathrm{H}$ NMR $\left(300 \mathrm{MHz}, \mathrm{CDCl}_{3}\right) \delta(\mathrm{ppm}) 7.39-7.37$ (overlap, 2H), $7.29-7.25(\mathrm{~m}, 1 \mathrm{H}), 6.87(\mathrm{~d}, J=9.8 \mathrm{~Hz}$, 2H), $6.25(\mathrm{~d}, J=9.8 \mathrm{~Hz}, 2 \mathrm{H}), 5.96(\mathrm{ddt}, J=16.9,10.1,6.5 \mathrm{~Hz}, 1 \mathrm{H}), 5.13(\mathrm{dd}, J=10.1,1.7 \mathrm{~Hz}$, 1H), $5.09(\mathrm{dd}, J=16.9,1.7 \mathrm{~Hz}, 1 \mathrm{H}), 3.53(\mathrm{~d}, J=6.5 \mathrm{~Hz}, 2 \mathrm{H}), 2.47(\mathrm{~s}, 1 \mathrm{H}) ;{ }^{13} \mathrm{C} \mathrm{NMR}(150 \mathrm{MHz}$, $\left.\mathrm{CDCl}_{3}\right) \delta(\mathrm{ppm}) 185.71,150.73,138.26,137.25,134.79,133.97,129.68,127.08,126.62,124.44$ 116.67, 70.38, 37.56. HRMS (CI) $(m / z)$ calcd for $\mathrm{C}_{15} \mathrm{H}_{13} \mathrm{ClO}_{2}[\mathrm{M}]^{+} 260.0604$, found 260.0597. IR (neat) $3500-3300(\mathrm{br}), 1661,1622,1473,1390,1039 \mathrm{~cm}^{-1}$. 


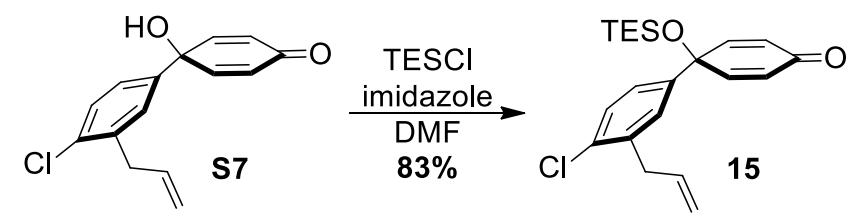

Alcohol S7 (4.6 g, $18 \mathrm{mmol})$ and imidazole (2.4 g, $36 \mathrm{mmol})$ were dissolved in DMF (90 mL). To this solution was added triethylsilyl chloride $(4.5 \mathrm{~mL}, 4.0 \mathrm{~g}, 27 \mathrm{mmol})$ and the resulting reaction mixture was stirred at $40{ }^{\circ} \mathrm{C}$ for $16 \mathrm{~h}$. The reaction was quenched with water $(60 \mathrm{~mL})$ and extracted with EtOAc $(2 \times 80 \mathrm{~mL})$. The extract was then washed with brine $(40 \mathrm{~mL})$, dried over anhydrous sodium sulfate, and concentrated under vacuum to yield a crude oil. Purification on $\mathrm{SiO}_{2}\left(0-10 \%\right.$ EtOAc/hexanes) yielded 15 as a yellow oil $(5.5 \mathrm{~g}, 83 \%) .{ }^{1} \mathrm{H}$ NMR (500 MHz, $\left.\mathrm{CDCl}_{3}\right) \delta(\mathrm{ppm}) 7.33-7.32(\mathrm{~m}, 1 \mathrm{H}), 7.32(\mathrm{~d}, J=8.4 \mathrm{~Hz}, 1 \mathrm{H}), 7.21(\mathrm{dd}, J=8.4,2.3 \mathrm{~Hz}, 1 \mathrm{H}), 6.79$ (d, $J=10.0 \mathrm{~Hz}, 2 \mathrm{H}), 6.22(\mathrm{~d}, J=10.0 \mathrm{~Hz}, 2 \mathrm{H}), 5.92(\mathrm{ddt}, J=16.9,10.1,6.6 \mathrm{~Hz}, 1 \mathrm{H}), 5.11(\mathrm{dd}, J$ $=10.1,1.6 \mathrm{~Hz}, 1 \mathrm{H}), 5.06(\mathrm{dd}, J=16.9,1.6 \mathrm{~Hz}, 1 \mathrm{H}), 3.48(\mathrm{~d}, J=6.6 \mathrm{~Hz}, 2 \mathrm{H}), 0.97(\mathrm{t}, J=7.9$, 9H), $0.65(\mathrm{q}, J=7.9,6 \mathrm{H}) ;{ }^{13} \mathrm{C} \mathrm{NMR}\left(125 \mathrm{MHz}, \mathrm{CDCl}_{3}\right) \delta(\mathrm{ppm}) 185.64,151.56,138.80,138.13$, 135.04, 133.66, 129.60, 127.29, 126.63, 124.60, 116.80, 72.72, 37.68, 6.86, 6.23. HRMS (CI) $(\mathrm{m} / \mathrm{z})$ calcd for $\mathrm{C}_{21} \mathrm{H}_{27} \mathrm{ClO}_{2} \mathrm{Si}[\mathrm{M}]^{+} 374.1469$, found 374.1482. IR (neat) 1671, 1094, 1041, 1001 $\mathrm{cm}^{-1}$.

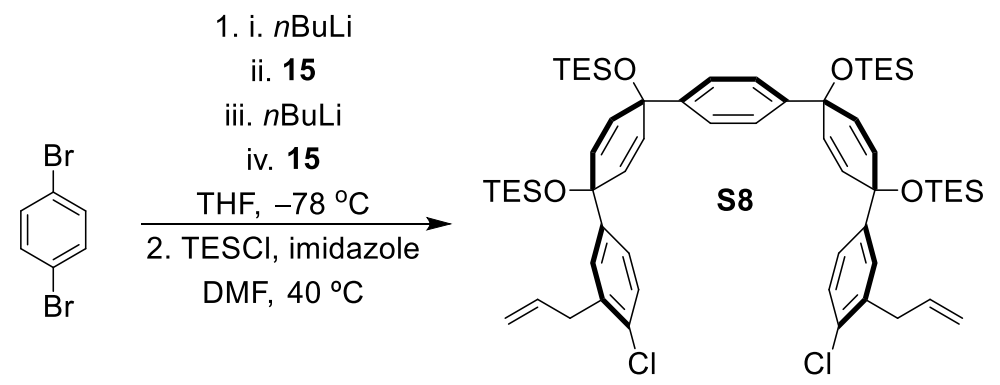

Dibromobenzene (320 mg, $1.4 \mathrm{mmol})$ was dissolved in THF $(14 \mathrm{~mL})$ and cooled to $-78{ }^{\circ} \mathrm{C}$. A solution of $n \mathrm{BuLi}(580 \mu \mathrm{L}, 1.4 \mathrm{mmol}, 2.4 \mathrm{M}$ in hexanes) was added followed immediately by TES-ketone 15 (500 $\mu \mathrm{L}, 520 \mathrm{mg}, 1.4 \mathrm{mmol})$. The reaction mixture was stirred for $30 \mathrm{~min}$ before 
an additional portion of $n \mathrm{BuLi}(580 \mu \mathrm{L}, 1.4 \mathrm{mmol}, 2.4 \mathrm{M}$ in hexanes) and $15(500 \mu \mathrm{L}, 515 \mathrm{mg}$, $1.37 \mathrm{mmol}$ ) were added. The final reaction mixture was stirred for $30 \mathrm{~min}$, quenched with water $(20 \mathrm{~mL})$, warmed to room temperature, and extracted with EtOAc $(2 \times 20 \mathrm{~mL})$. The extract was washed with brine $(20 \mathrm{~mL})$, dried over anhydrous sodium sulfate, and concentrated under vacuum to yield the crude diol. The crude oil was added to a flask with imidazole ( $380 \mathrm{mg}, 5.5$ mmol) under nitrogen. DMF (7.0 mL) was added followed by triethylsilyl chloride ( $690 \mu \mathrm{L}, 620$ $\mathrm{mg}, 4.1 \mathrm{mmol})$. The reaction mixture was then stirred at $40{ }^{\circ} \mathrm{C}$ for $16 \mathrm{~h}$. The reaction was quenched with water $(10 \mathrm{~mL})$ and extracted using EtOAc $(2 \times 10 \mathrm{~mL})$. The extract was then washed with brine $(5 \mathrm{~mL})$, dried over anhydrous sodium sulfate, and concentrated under vacuum to afford a crude oil. Purification on $\mathrm{SiO}_{2}(0-10 \%$ EtOAc/hexanes) yielded $\mathbf{S 8}$ as a yellow oil $(1.1 \mathrm{~g}, 78 \%)$. NOTE: When this reaction was run on larger scale, the product often contained impurities that were carried through to the following step. ${ }^{1} \mathrm{H}$ NMR $\left(300 \mathrm{MHz}, \mathrm{CDCl}_{3}\right) \delta(\mathrm{ppm})$ $7.22(\mathrm{~s}, 4 \mathrm{H}), 7.22(\mathrm{~d}, J=8.3 \mathrm{~Hz}, 2 \mathrm{H}), 7.16(\mathrm{~d}, J=2.3 \mathrm{~Hz}, 2 \mathrm{H}), 7.11(\mathrm{dd}, J=8.3,2.3 \mathrm{~Hz}, 2 \mathrm{H})$, $5.97(\mathrm{~d}, J=10.3 \mathrm{~Hz}, 4 \mathrm{H}), 5.91(\mathrm{~d}, J=10.3 \mathrm{~Hz}, 4 \mathrm{H}), 5.93-5.76$ (overlap, 2H), $5.01-4.90$ (overlap, 4H), $3.37(\mathrm{~d}, J=6.6 \mathrm{~Hz}, 4 \mathrm{H}), 0.97-0.86(\mathrm{~m}, 36 \mathrm{H}), 0.64-0.53(\mathrm{~m}, 24 \mathrm{H}) ;{ }^{13} \mathrm{C}$ NMR $\left(150 \mathrm{MHz}, \mathrm{CDCl}_{3}\right) \delta(\mathrm{ppm}) 144.76,144.75,137.10,135.16,132.57,131.53,130.98,128.86$, 127.76, 125.50, 124.91, 116.21, 71.03, 71.01, 37.51, 6.87, 6.29. MALDI-TOF $(\mathrm{m} / \mathrm{z})$ calcd for $\mathrm{C}_{60} \mathrm{H}_{88} \mathrm{Cl}_{2} \mathrm{O}_{4} \mathrm{Si}_{4}[\mathrm{M}+\mathrm{Na}]^{+}$1077.50, found 1077.40. IR (neat) 2953, 2875, 1698, $1532 \mathrm{~cm}^{-1}$.
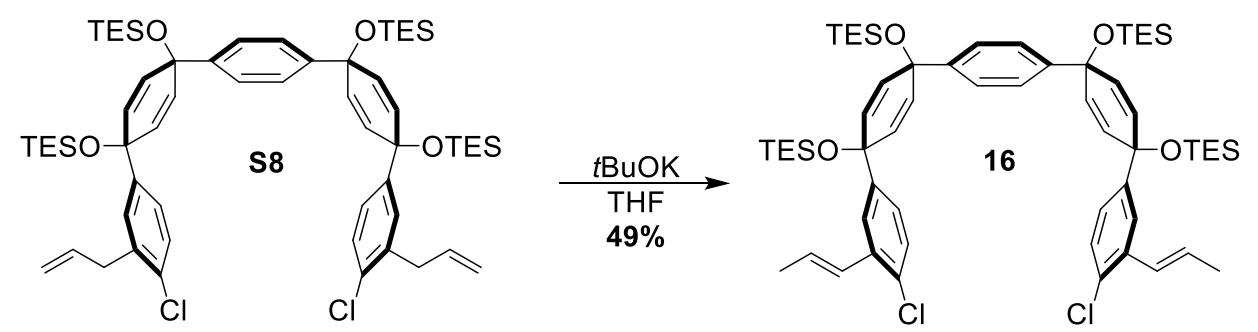

Diallyl S8 (1.1 g, $1.1 \mathrm{mmol})$ was stirred under vacuum to remove dissolved gases before 
dissolving in THF $(80 \mathrm{~mL})$. Solid potassium tert-butoxide $(270 \mathrm{mg}, 2.4 \mathrm{mmol})$ was then added and the reaction was stirred for $5 \mathrm{~min}$ at room temperature. The reaction was then quenched with water $(80 \mathrm{~mL})$ and extracted in to EtOAc $(2 \times 60 \mathrm{~mL})$. The extract was washed with brine (40 $\mathrm{mL}$ ) and dried over anhydrous sodium sulfate. Solvent was removed to yield a yellow oil. Purification on $\mathrm{SiO}_{2}\left(10 \% \mathrm{CH}_{2} \mathrm{Cl}_{2} /\right.$ hexanes $)$ yielded 16 as a colorless oil $(560 \mathrm{mg}, 49 \%) .{ }^{1} \mathrm{H}$ NMR $\left(300 \mathrm{MHz}, \mathrm{CDCl}_{3}\right) \delta(\mathrm{ppm}) 7.45(\mathrm{~d}, J=2.2 \mathrm{~Hz}, 2 \mathrm{H}) 7.25(\mathrm{~s}, 4 \mathrm{H}), 7.20(\mathrm{~d}, J=8.4 \mathrm{~Hz}, 2 \mathrm{H})$, $7.09(\mathrm{dd}, J=8.4,2.2 \mathrm{~Hz}, 2 \mathrm{H}), 6.71(\mathrm{dq}, J=15.7,1.8 \mathrm{~Hz}, 2 \mathrm{H}), 6.05-5.95(\mathrm{~m}, 2 \mathrm{H}), 5.98(\mathrm{~d}, J=$ $10.3 \mathrm{~Hz}, 4 \mathrm{H}), 5.91(\mathrm{~d}, J=10.3 \mathrm{~Hz}, 4 \mathrm{H}), 1.82(\mathrm{dd}, J=6.7,1.8 \mathrm{~Hz}, 6 \mathrm{H}), 0.99-0.86(\mathrm{~m}, 36 \mathrm{H})$, $0.66-0.52(\mathrm{~m}, 24 \mathrm{H}) ;{ }^{13} \mathrm{C}$ NMR $\left(125 \mathrm{MHz}, \mathrm{CDCl}_{3}\right) \delta(\mathrm{ppm})$ 144.99, 144.76, 135.37, 131.73, $131.13,129.24,128.44,127.29,125.66,125.34,123.94,71.19,71.16,18.75,7.05,7.03,6.45$ MALDI-TOF $(m / z)$ calcd for $\mathrm{C}_{60} \mathrm{H}_{88} \mathrm{Cl}_{2} \mathrm{O}_{4} \mathrm{Si}_{4}[\mathrm{M}+\mathrm{Na}]^{+}$1077.50, found 1077.30. IR (neat) 2953, $2875,1465,1073 \mathrm{~cm}^{-1}$.

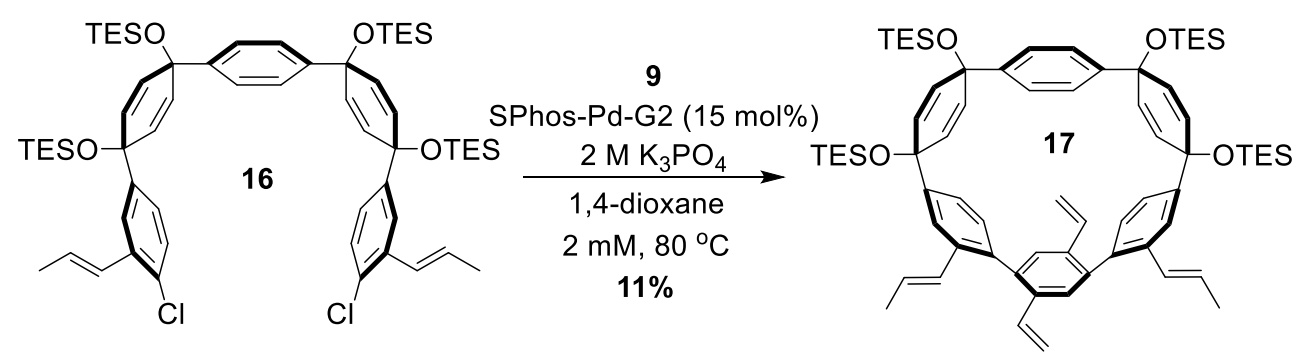

2,5-vinylbenzene-1,4-diboronic acid bis(pinacol) ester 9 (200 mg, $520 \mu \mathrm{mol})$ and SPhos-Pd-G2 (56 mg, $78 \mu \mathrm{mol}$ ) were placed in a round bottom flask. The flask was evacuated and refilled with nitrogen. 16 (546 mg, $517 \mu \mathrm{mol})$ was dissolved in 1,4-dioxane $(50 \mathrm{~mL})$, sparged with nitrogen, and added to the flask. Additional dioxane $(183 \mathrm{~mL})$ was added and the mixture was warmed to $80{ }^{\circ} \mathrm{C}$. A $2 \mathrm{M}$ aq solution of $\mathrm{K}_{3} \mathrm{PO}_{4}(25 \mathrm{~mL})$ was then added. The reaction was stirred for $3 \mathrm{~h}$ before cooling to room temperature and filtering through Celite. The filtrate was extracted into 
EtOAc $(2 \times 100 \mathrm{~mL})$ and the combined organics were washed with brine $(60 \mathrm{~mL})$ and dried over anhydrous sodium sulfate. Solvent was removed to yield a brown oil. Purification on $\mathrm{SiO}_{2}(10 \%$ EtOAc/hexanes) to afforded a semi-pure golden oil that was further purified by preparative recycling $\mathrm{GPC}\left(\mathrm{CHCl}_{3}\right)$ to yield $\mathbf{1 7}$ as a yellow oil $(65 \mathrm{mg}, 11 \%) .{ }^{1} \mathrm{H} \mathrm{NMR}\left(500 \mathrm{MHz}, \mathrm{CDCl}_{3}\right)$ $\delta(\mathrm{ppm}) 7.80(\mathrm{~d}, J=1.9 \mathrm{~Hz}, 2 \mathrm{H}) 7.44(\mathrm{~s}, 2 \mathrm{H}), 6.99(\mathrm{dd}, J=15.7,1.9 \mathrm{~Hz}, 2 \mathrm{H}), 6.95(\mathrm{~s}, 4 \mathrm{H}), 6.95-$ $6.89(\mathrm{~m}, 2 \mathrm{H}), 6.64(\mathrm{dd}, J=8.4,1.9 \mathrm{~Hz}, 2 \mathrm{H}), 6.50(\mathrm{dq}, J=15.7,6.6 \mathrm{~Hz}, 2 \mathrm{H}), 6.12(\mathrm{~d}, J=8.3 \mathrm{~Hz}$, 2H), $6.00(\mathrm{dd}, J=10.2,2.4 \mathrm{~Hz}, 2 \mathrm{H}), 5.95(\mathrm{dd}, J=10.2,2.4 \mathrm{~Hz}, 2 \mathrm{H}), 5.77(\mathrm{dd}, J=10.0,2.4 \mathrm{~Hz}$, 2H), $5.66(\mathrm{dd}, J=17.5,1.2 \mathrm{~Hz}, 2 \mathrm{H}), 5.52(\mathrm{dd}, J=10.0,2.3 \mathrm{~Hz}, 2 \mathrm{H}), 5.20(\mathrm{~d}, J=10.8 \mathrm{~Hz}, 2 \mathrm{H})$, $2.00(\mathrm{dd}, J=6.7,1.7 \mathrm{~Hz}, 6 \mathrm{H}), 0.99(\mathrm{t}, J=7.9 \mathrm{~Hz}, 18 \mathrm{H}), 0.93(\mathrm{t}, J=7.9 \mathrm{~Hz}, 18 \mathrm{H}), 0.66(\mathrm{q}, J=$ $7.9 \mathrm{~Hz}, 12 \mathrm{H}), 0.62(\mathrm{q}, J=7.9 \mathrm{~Hz}, 12 \mathrm{H}) ;{ }^{13} \mathrm{C} \mathrm{NMR}\left(125 \mathrm{MHz}, \mathrm{CDCl}_{3}\right) \delta(\mathrm{ppm}) 144.85,143.54$, $142.10,140.26,135.84,135.68,135.55,135.02,134.41,133.94,131.44,131.35,131.23,130.98$, $129.99,128.79,128.29,127.77,127.58,127.54,127.22,125.57,123.65,123.32,115.36,71.83$, 71.14, 18.91, 7.11, 7.06, 6.59, 6.41. MALDI-TOF $(\mathrm{m} / z)$ calcd for $\mathrm{C}_{70} \mathrm{H}_{96} \mathrm{O}_{4} \mathrm{Si}_{4}(\mathrm{M})^{+}$1112.64, found 1112.61. IR (neat) 2954, 2875, $1076 \mathrm{~cm}^{-1}$.
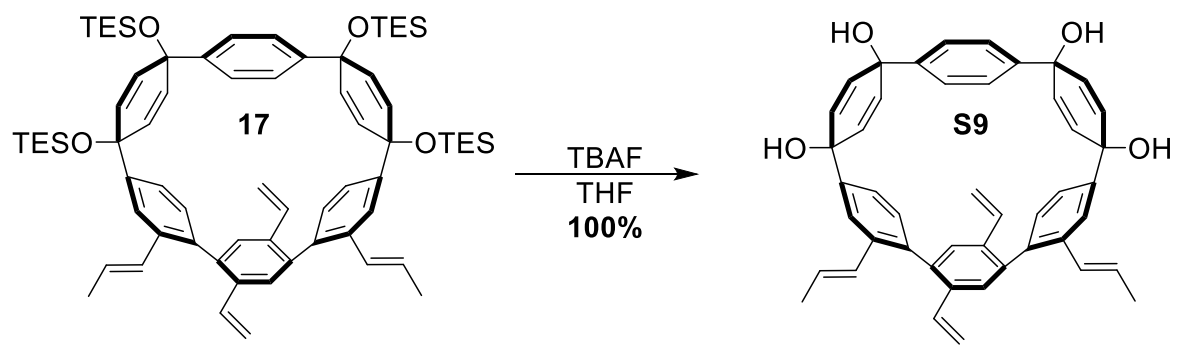

To a solution of $17(65 \mathrm{mg}, 58 \mu \mathrm{mol})$ in THF $(500 \mu \mathrm{L})$ was added TBAF $(250 \mu \mathrm{L}, 1 \mathrm{M}$ in THF) and the resulting reaction mixture was stirred for $1 \mathrm{~h}$ before it was then quenched with water $(1$ $\mathrm{mL}$ ). The organics were concentrated under reduced pressure, affording a brown solid that was filtered and washed with water $(1 \mathrm{~mL})$ and $\mathrm{CH}_{2} \mathrm{Cl}_{2}(2 \mathrm{~mL})$. The solid was dried under vacuum to 
yield $\mathbf{S 9}$ as a dark orange solid $(38 \mathrm{mg}$ ) that was used in the subsequent reaction without further purification. ${ }^{1} \mathrm{H}$ NMR $\left(300 \mathrm{MHz}, \mathrm{DMSO}-d_{6}\right) \delta(\mathrm{ppm}) 7.86(\mathrm{~s}, 2 \mathrm{H}), 7.39(\mathrm{~s}, 2 \mathrm{H}), 7.01-6.93(\mathrm{~m}$, 2H), 6.93 (s, 4H), 6.84 (dd, $J=17.5,10.8 \mathrm{~Hz}, 2 \mathrm{H}), 6.67$ (d, $J=8.3 \mathrm{~Hz}, 2 \mathrm{H}), 6.55$ (dd, $J=15.7$, $6.8 \mathrm{~Hz}, 2 \mathrm{H}), 5.98(\mathrm{~d}, J=8.3 \mathrm{~Hz}, 2 \mathrm{H}), 5.92-5.80(\mathrm{~m}, 2 \mathrm{H}), 5.76(\mathrm{~s}, 2 \mathrm{H}), 5.78-5.70(\mathrm{~m}, 2 \mathrm{H})$, $5.59(\mathrm{~d}, J=17.5 \mathrm{~Hz}, 2 \mathrm{H}), 5.46(\mathrm{dd}, J=10.1,2.2 \mathrm{~Hz}, 2 \mathrm{H}), 5.25(\mathrm{~d}, J=10.9 \mathrm{~Hz}, 2 \mathrm{H}), 3.16(\mathrm{~s}, 4 \mathrm{H})$, $1.97(\mathrm{~d}, J=6.1 \mathrm{~Hz}, 6 \mathrm{H}) ;{ }^{13} \mathrm{C}$ NMR $\left(125 \mathrm{MHz}\right.$, Acetone- $\left.d_{6}\right) \delta(\mathrm{ppm}) 145.05,144.55,143.05$, $140.90,136.93,136.50,136.20,135.09,134.92,134.75,132.02,130.76,130.42,130.08,128.77$ 126.39, 124.94, 124.26, 116.34, 70.30, 69.53, 18.87; IR (neat) 3550-3100 (br), 1264, $1026 \mathrm{~cm}^{-1}$.

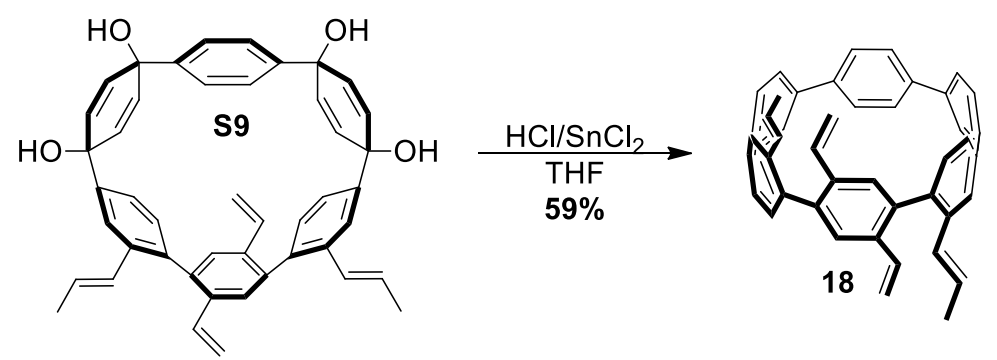

To a solution of crude $\mathbf{S 9}(38 \mathrm{mg}, 58 \mu \mathrm{mol})$ in THF $(4.8 \mathrm{~mL})$ was added a solution of tin(II) chloride dihydrate $(31 \mathrm{mg}, 139 \mu \mathrm{mol})$ and $12 \mathrm{M}$ hydrochloric acid $(23 \mu \mathrm{L})$. The reaction was stirred for $1 \mathrm{~h}$ before it was quenched with aqueous $1 \mathrm{M}$ aq sodium hydroxide $(1 \mathrm{~mL})$ and extracted into EtOAc $(2 \times 2 \mathrm{~mL})$. The combined organics were washed with brine $(1 \mathrm{~mL})$, dried over anhydrous sodium sulfate, and concentrated under vacuum to yield an orange-yellow solid. Purification on $\mathrm{SiO}_{2}\left(20 \% \mathrm{CH}_{2} \mathrm{Cl}_{2} /\right.$ hexanes $)$ afforded 18 as a yellow solid $(20 \mathrm{mg}, 59 \%) .{ }^{1} \mathrm{H}$ NMR (500 MHz, $\left.\mathrm{CDCl}_{3}\right) \delta(\mathrm{ppm}) 7.63(\mathrm{~s}, 2 \mathrm{H}), 7.48(\mathrm{~s}, 8 \mathrm{H}), 7.35$ (br, 2H), 7.18 (br, 2H), 6.77 (d, $J=8.5 \mathrm{~Hz}, 4 \mathrm{H}), 6.71-6.60(\mathrm{~m}, 4 \mathrm{H}), 6.29(\mathrm{dq}, J=13.8,6.6 \mathrm{~Hz}, 2 \mathrm{H}), 5.38(\mathrm{~s}, 2 \mathrm{H}), 5.15(\mathrm{~d}, J=$ $10.8 \mathrm{~Hz}, 2 \mathrm{H}), 1.79(\mathrm{~d}, J=6.3 \mathrm{~Hz}, 6 \mathrm{H}) ;{ }^{13} \mathrm{C}$ NMR $\left(150 \mathrm{MHz}, \mathrm{CDCl}_{3}\right) \delta(\mathrm{ppm}) 137.64,136.32$, $136.00,133.56,131.71,130.74,128.97,127.36,121.08,117.48,115.63,115.11,69.46,29.70$, 18.65. MALDI-TOF $(m / z)$ calcd for $\mathrm{C}_{46} \mathrm{H}_{36}[\mathrm{M}]^{+}$588.28, found 588.20. IR (neat) 2978, 2931, 
$1360,1088 \mathrm{~cm}^{-1}$.

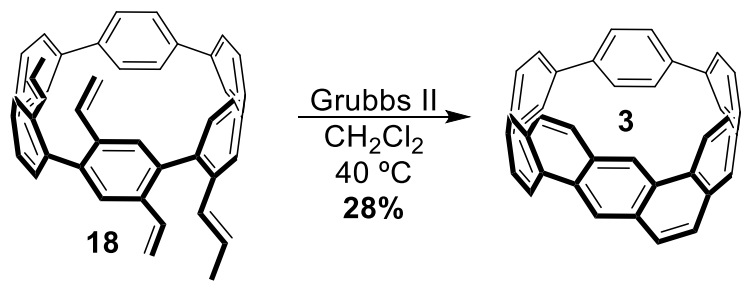

Benzo[k]tetraphene macrocycle $18(7.0 \mathrm{mg}, 12 \mu \mathrm{mol})$ and Grubbs II $(2.0 \mathrm{mg}, 2.4 \mu \mathrm{mol})$ were dissolved in $\mathrm{CH}_{2} \mathrm{Cl}_{2}(1 \mathrm{~mL})$ and the resulting solution was heated to reflux for $16 \mathrm{~h}$. After cooling to room temperature, the solvent was removed under vacuum and the resulting crude solid was purified on $\mathrm{SiO}_{2}\left(20 \% \mathrm{CH}_{2} \mathrm{Cl}_{2} /\right.$ hexanes $)$ to yield a semi-pure red solid. Additional purification by preparative recycling GPC $\left(\mathrm{CHCl}_{3}\right)$ afforded cyclo $(3,10)$-benzo[ $[k]$ tetrapheneter(p-phenylene) 3 as a red solid $(1.7 \mathrm{mg}, 28 \%) .{ }^{1} \mathrm{H}$ NMR $\left(500 \mathrm{MHz}, \mathrm{CDCl}_{3}\right) \delta(\mathrm{ppm}) 8.54(\mathrm{~s}$, 2H), $8.26(\mathrm{~d}, J=9.4 \mathrm{~Hz}, 2 \mathrm{H}), 7.86(\mathrm{~d}, J=10.1 \mathrm{~Hz}, 2 \mathrm{H}), 7.79(\mathrm{~s}, 2 \mathrm{H}), 7.75(\mathrm{~d}, J=9.4 \mathrm{~Hz}, 2 \mathrm{H})$, $7.65(\mathrm{~d}, J=9.2 \mathrm{~Hz}, 2 \mathrm{H}), 7.59-7.63$ (overlap, 6H), 7.50-7.57 (overlap, $6 \mathrm{H}) ;{ }^{13} \mathrm{C}$ NMR $(125 \mathrm{MHz}$, $\left.\mathrm{CDCl}_{3}\right) \delta(\mathrm{ppm}) 134.51,134.13,133.86,132.43,132.35,132.20,129.04,128.66,128.22,128.15$, $127.37,127.10,126.93,126.78,126.43,126.38,126.26,125.75,125.57,123.54,122.49$. MALDI-TOF $(\mathrm{m} / z)$ calcd for $\mathrm{C}_{40} \mathrm{H}_{24}[\mathrm{M}]^{+}$504.19, found 504.10. IR (neat) 3045, 2925, 1570, $1480,1259 \mathrm{~cm}^{-1}$.

2) X-ray Crystallography. Diffraction intensities were collected at $173 \mathrm{~K}$ on a Bruker Apex2 Duo CCD diffractometer with a micro-focus Incoatec $I \mu S \mathrm{Cu}$ source, $\mathrm{CuK} \alpha$ radiation, $\lambda=$ $1.54178 \AA$ A. Space groups were determined based on systematic absences (1) and intensity statistics (2). Absorption corrections were applied by SADABS. ${ }^{3}$ Structures were solved by direct methods and Fourier techniques and refined on $F^{2}$ using full matrix least-squares 
procedures. All non- $\mathrm{H}$ atoms were refined with anisotropic thermal parameters. $\mathrm{H}$ atoms in all structures were refined in calculated positions in a rigid group model. Single crystals of racemic 1 were grown from slow evaporation of a pentane/THF solution. Single crystals of $\mathbf{2}$ were grown from slow evaporation of a pentane/benzene solution. ORTEP representations (Figures S1 and S2) and relevant X-ray crystallographic data are presented below.

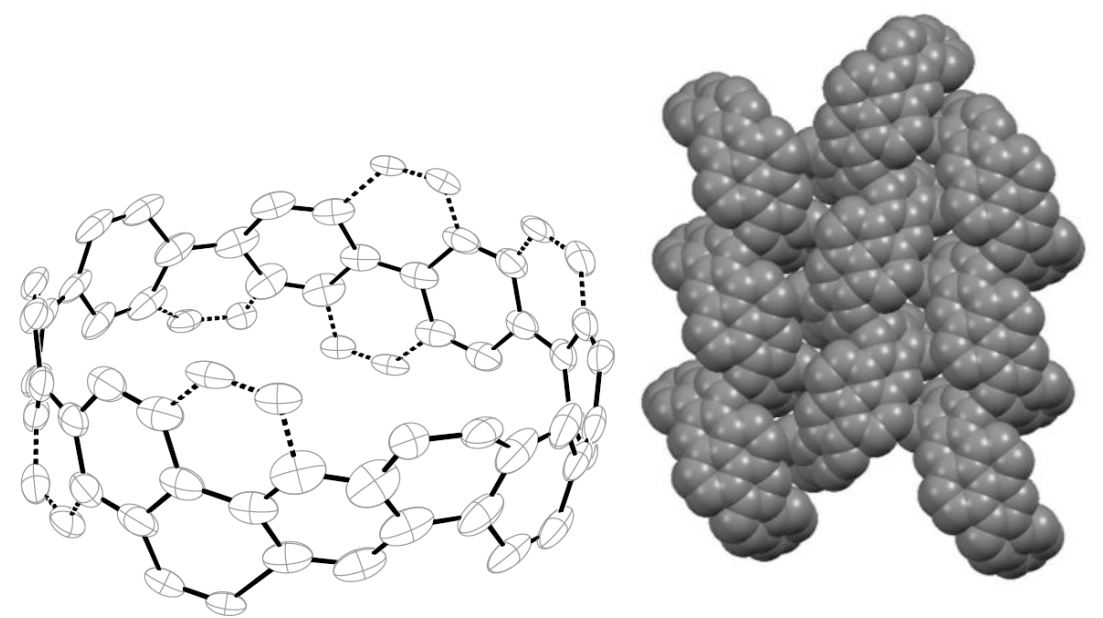

Figure S1. ORTEP representation (thermal ellipsoids shown at 30\% probability) with one possible orientation of the disordered benzo[ $k]$ tetraphene unit indicated with solid lines (dotted lines represent other possible orientations of this group) and space-filling packing model of $\mathbf{1}$. Hydrogen atoms are omitted for clarity.

Crystallographic Data for 1: $\mathrm{C}_{60} \mathrm{H}_{48} \mathrm{O}_{2}, \mathrm{M}=800.98,0.15 \times 0.12 \times 0.10 \mathrm{~mm}, \mathrm{~T}=173(2) \mathrm{K}$, Monoclinic, space group $P 2{ }_{1} / c, a=13.3012(7) \AA, b=9.9339(5) \AA, c=17.0395(6) \AA, \beta=$ 110.381(3) $, V=2110.53(18) \AA^{3}, Z=2, Z^{\prime}=0.5, D_{\mathrm{c}}=1.260 \mathrm{Mg} / \mathrm{m}^{3}, \mu=0.572 \mathrm{~mm}^{-1}, F(000)=$ $848,2 \theta_{\max }=133.28^{\circ}, 11828$ reflections, 3706 independent reflections $\left[\mathrm{R}_{\mathrm{int}}=0.0372\right], \quad \mathrm{R} 1=$ $0.1024, \mathrm{wR} 2=0.3416$ and GOF $=1.015$ for 3706 reflections (334 parameters) with $\mathrm{I}>2 \sigma(\mathrm{I}), \mathrm{R} 1$ $=0.1330, \mathrm{wR} 2=0.4100$ and $\mathrm{GOF}=1.033$ for all reflections, $\mathrm{max} / \mathrm{min}$ residual electron density $+0.314 /-0.0 .380 \mathrm{e} / \AA^{3}$.

The main molecule in $\mathbf{1}$ is disordered over several different positions having different orientation of two additional $\mathrm{C}_{6}$-rings. The structure was determined in a centro-symmetrical space group with centro-symmetrical main molecules disordered over four different positions. Two additional 
THF solvent molecules fill out a space inside the main molecule. Positions of some weak peaks (less $1 \mathrm{e}^{3}$ ) on the residual density map show that disorder of the main molecule in the crystal structure could be even more complicated than given in the final refined structure and may consist of eight positions of the main molecule with different orientations. Some of the disordered $\mathrm{C}_{6}$-rings in $\mathbf{1}$ were refined with restrictions; the distance of $1.39 \AA$ was used in the refinement as a target for the corresponding $\mathrm{C}-\mathrm{C}$ bonds.
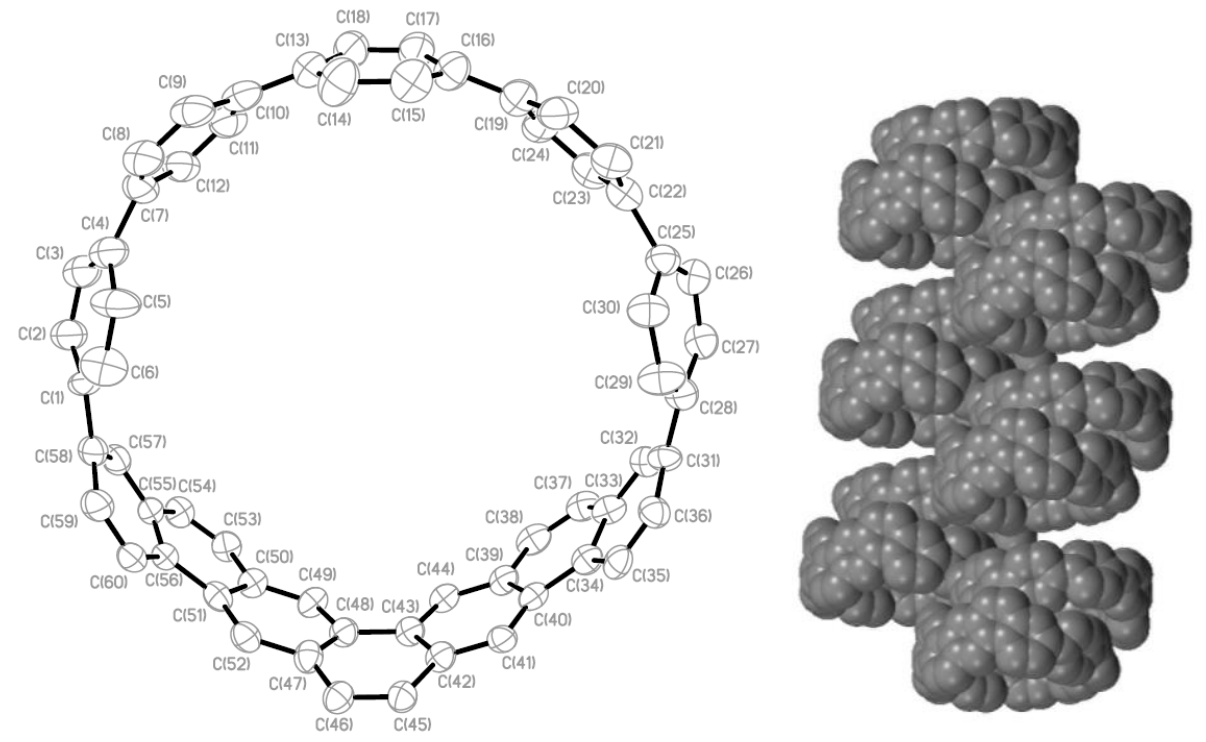

Figure S2. ORTEP representation (thermal ellipsoids shown at 30\% probability) and spacefilling packing model of $\mathbf{2}$. Hydrogen atoms are omitted for clarity.

Crystallographic Data for 2: $\mathrm{C}_{78.5} \mathrm{H}_{72},\left(\mathrm{C}_{60} \mathrm{H}_{36} \cdot\left(\mathrm{C}_{6} \mathrm{H}_{6}\right) \cdot 2.5\left(\mathrm{C}_{5} \mathrm{H}_{12}\right), \mathrm{M}=1015.36,0.10 \times 0.07 \mathrm{x}\right.$ $0.02 \mathrm{~mm}, \mathrm{~T}=173(2) \mathrm{K}$, Triclinic, space group $P-1, a=15.8435(7) \AA, b=19.3722(9) \AA, c=$ 20.9937(10) $\AA, \alpha=94.124(3)^{\circ}, \beta=90.490(3)^{\circ}, \gamma=105.769(3)^{\circ}, V=6182.3(5) \AA^{3}, Z=4, Z^{\prime}=2$, $D_{\mathrm{c}}=1.091 \mathrm{Mg} / \mathrm{m}^{3}, \mu=0.460 \mathrm{~mm}^{-1}, F(000)=2172,2 \theta_{\max }=50.256^{\circ}, 45042$ reflections, 12774 independent reflections $\left[\mathrm{R}_{\text {int }}=0.1146\right], \mathrm{R} 1=0.0807, \mathrm{wR} 2=0.2119$ and $\mathrm{GOF}=1.009$ for 12774 reflections (1165 parameters) with $\mathrm{I}>2 \sigma(\mathrm{I}), \mathrm{R} 1=0.1678, \mathrm{wR} 2=0.2404$ and $\mathrm{GOF}=1.009$ for all reflections, max/min residual electron density $+0.408 /-0.238 \mathrm{e} / \AA^{3}$.

In the crystal structure of $\mathbf{2}$ there are two benzene and five pentane solvent molecules which fill out an empty space in the packing. Two benzene molecules were located and refined and five highly disordered pentane solvent molecules were treated with SQUEEZE. ${ }^{4}$ Correction of the X- 
ray data by SQUEEZE, 557 electron/cell, is higher versus the required values of 420 electron/cell for ten pentane molecules in the full unit cells indicating that number of solvent molecules could be slightly bigger and benzene and pentane molecules could share the same disordered positions as well. Crystals of $\mathbf{2}$ suitable for data collection were very small and diffraction at high angles was very weak. Even using a strong micro-focus Incoatec $I \mu S \mathrm{Cu}$ source intensities were collected only up to $2 \theta_{\max }=50.256^{\circ}$. The collected data provided an appropriate number of measured reflections per refined parameters. All calculations were performed with the Bruker SHELXL-2013 package. $^{5}$

Table S1. Dihedral angles between adjacent rings from X-ray crystal structure of 2.

\begin{tabular}{c|c|c} 
Center C-C Bond $^{\mathbf{a}}$ & Molecule 1 (deg) $^{\mathbf{b}}$ & Molecule 2 (deg) $^{\mathbf{b}}$ \\
\hline $\mathrm{C}(1)-\mathrm{C}(58)$ & 8.9 & 28.3 \\
\hline $\mathrm{C}(4)-\mathrm{C}(7)$ & 36.5 & 39.4 \\
\hline $\mathrm{C}(10)-\mathrm{C}(13)$ & 34.0 & 23.9 \\
\hline $\mathrm{C}(16)-\mathrm{C}(19)$ & 20.4 & 25.0 \\
\hline $\mathrm{C}(22)-\mathrm{C}(25)$ & 34.8 & 38.5 \\
\hline $\mathrm{C}(28)-\mathrm{C}(31)$ & 35.6 & 25.5 \\
\hline $\mathrm{C}(34)-\mathrm{C}(40)$ & 7.2 & 0.6 \\
\hline $\mathrm{C}(43)-\mathrm{C}(48)$ & 3.1 & 2.9 \\
\hline $\mathrm{C}(51)-\mathrm{C}(56)$ & 5.3 & 1.9 \\
\hline Average & 20.6 & 20.6
\end{tabular}

${ }^{a}$ Measured dihedral angles are defined around the given central C-C bonds. ${ }^{\mathrm{b}}$ Angles are an average of the two dihedral angles defined by each $\mathrm{C}-\mathrm{C}$ bond. 


\section{3) Optical and Electrochemical Analysis.}
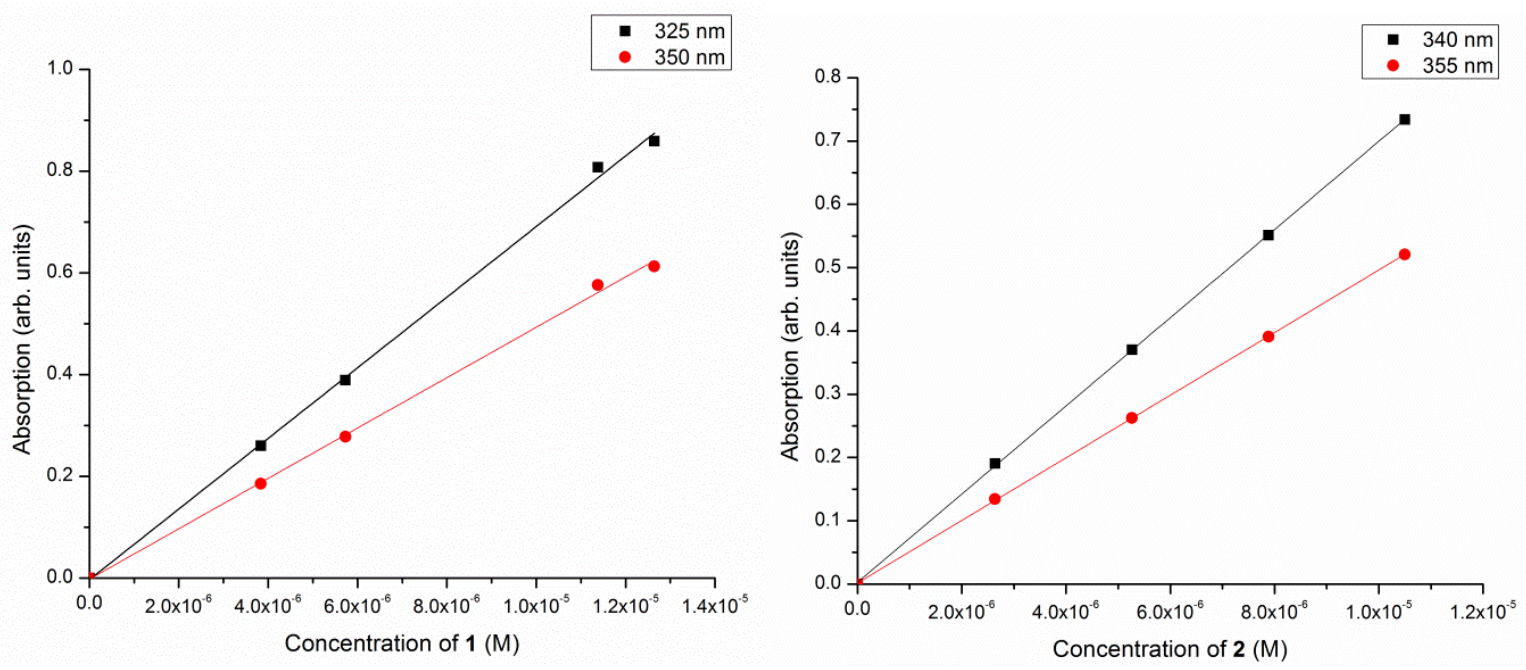

Figure S3. Left: Beer-Lambert plot of 1 at $325\left(\varepsilon=6.9 \times 10^{-4} \mathrm{M}^{-1} \mathrm{~cm}^{-1}\right)$ and $350 \mathrm{~nm}(\varepsilon=5.0 \times$ $\left.10^{-4} \mathrm{M}^{-1} \mathrm{~cm}^{-1}\right)$. Right: Beer-Lambert plot of 2 at $340\left(\varepsilon=7.0 \times 10^{-4} \mathrm{M}^{-1} \mathrm{~cm}^{-1}\right)$ and $355 \mathrm{~nm}(\varepsilon=4.9$ X $\left.10^{-4} \mathrm{M}^{-1} \mathrm{~cm}^{-1}\right)$. 

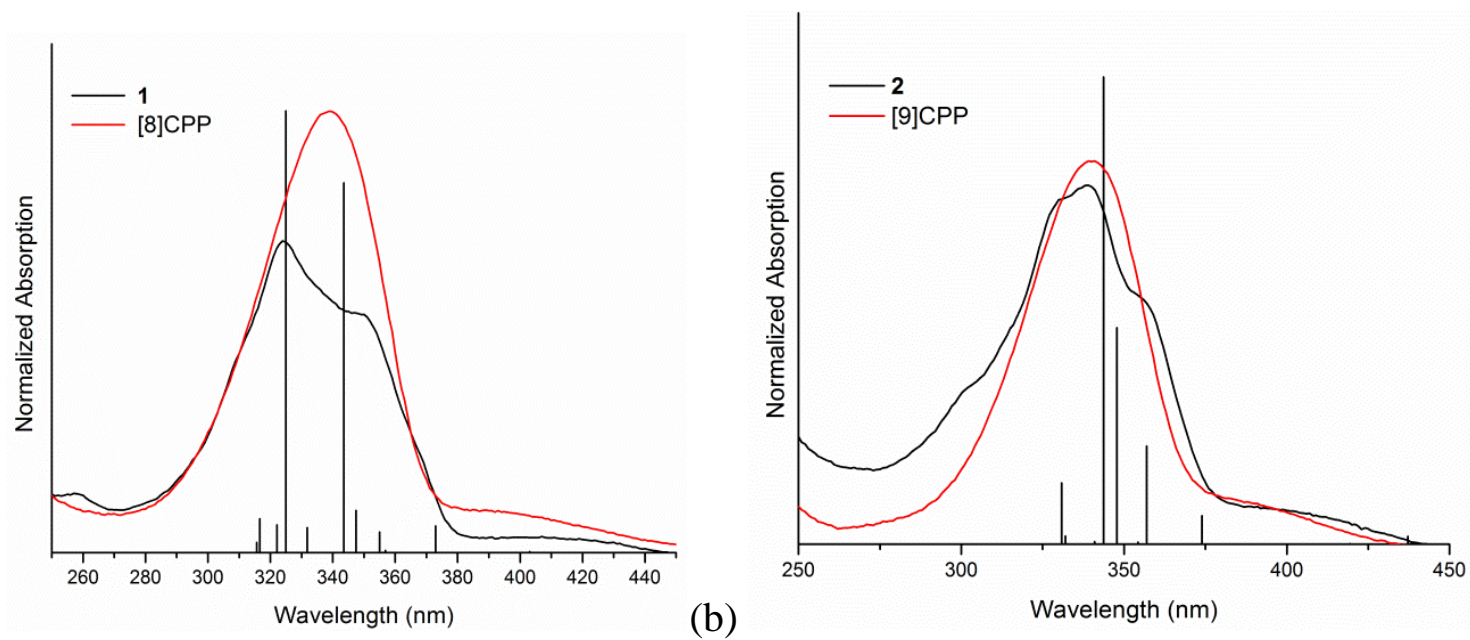

(a)

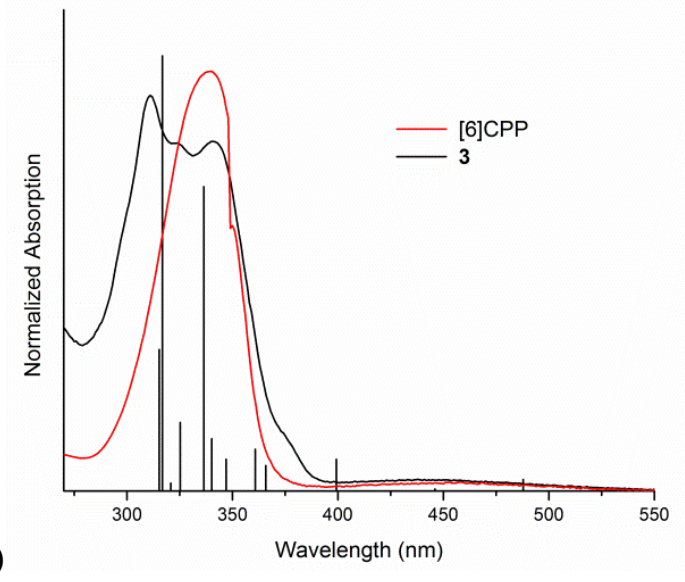

Figure S4. (a) UV-vis spectra of 1 and [8]CPP. (b) UV-vis spectra of 2 and [9]CPP (c) UV-vis spectra of $\mathbf{3}$ and [6]CPP; TD-DFT lines correspond to predicted absorptions of $\mathbf{1}-\mathbf{3}$ at the B3LYP/6-31G(d) level of theory.

Table S2. Optical predictions for 1 via TD-DFT at the B3LYP/6-31G(d) level of theory.

\begin{tabular}{|c|c|c|c|}
\hline Energy $\left(\mathrm{cm}^{-1}\right)$ & Wavelength (nm) & Osc. Strength & Major contributions \\
\hline 22181.20656 & 450.832103 & 0.0115 & HOMO $\rightarrow$ LUMO $(96 \%)$ \\
\hline 24811.39872 & 403.0405586 & 0.0033 & $\mathrm{H}-1 \rightarrow \mathrm{LUMO}(52 \%), \mathrm{HOMO} \rightarrow \mathrm{L}+1(29 \%)$ \\
\hline 26811.66752 & 372.9719531 & 0.0791 & $\mathrm{H}-1 \rightarrow \mathrm{LUMO}(32 \%), \mathrm{HOMO} \rightarrow \mathrm{L}+1(52 \%)$ \\
\hline 28019.8944 & 356.8892822 & 0.0065 & $\begin{array}{c}\mathrm{H}-3 \rightarrow \mathrm{LUMO}(48 \%), \mathrm{HOMO} \rightarrow \mathrm{L}+2(12 \%), \\
\mathrm{HOMO} \rightarrow \mathrm{L}+3(16 \%)\end{array}$ \\
\hline 28171.52768 & 354.9683252 & 0.0605 & $\mathrm{H}-2 \rightarrow \mathrm{LUMO}(64 \%), \mathrm{HOMO} \rightarrow \mathrm{L}+2(23 \%)$ \\
\hline 28778.0608 & 347.48693 & 0.1244 & $\begin{array}{c}\mathrm{H}-3 \rightarrow \mathrm{LUMO}(17 \%), \mathrm{H}-1 \rightarrow \mathrm{L}+1(31 \%) \\
\mathrm{HOMO} \rightarrow \mathrm{L}+2(20 \%), \mathrm{HOMO} \rightarrow \mathrm{L}+3(20 \%)\end{array}$ \\
\hline 29110.36352 & 343.5202722 & 1.0903 & $\begin{array}{c}\mathrm{H}-2 \rightarrow \mathrm{LUMO}(24 \%), \mathrm{HOMO} \rightarrow \mathrm{L}+2(34 \%), \\
\mathrm{HOMO} \rightarrow \mathrm{L}+3(36 \%)\end{array}$ \\
\hline 30141.1472 & 331.7723753 & 0.0736 & $\begin{array}{c}\mathrm{H}-4 \rightarrow \mathrm{LUMO}(13 \%), \mathrm{H}-2 \rightarrow \mathrm{L}+1(27 \%) \\
\mathrm{H}-1 \rightarrow \mathrm{L}+2(19 \%), \mathrm{H}-1 \rightarrow \mathrm{L}+3(10 \%) \\
\mathrm{HOMO} \rightarrow \mathrm{L}+4(15 \%)\end{array}$ \\
\hline
\end{tabular}




\begin{tabular}{c|c|c|c}
\hline 30774.2968 & 324.9464989 & 1.3025 & $\begin{array}{c}\mathrm{H}-3 \rightarrow \mathrm{LUMO}(13 \%), \mathrm{H}-1 \rightarrow \mathrm{L}+1(51 \%), \\
\mathrm{HOMO} \rightarrow \mathrm{L}+3(10 \%)\end{array}$ \\
\hline 31045.30096 & 322.1099391 & 0.0818 & $\begin{array}{c}\mathrm{H}-3 \rightarrow \mathrm{L}+1(29 \%), \mathrm{H}-1 \rightarrow \mathrm{L}+2(26 \%), \\
\mathrm{H}-1 \rightarrow \mathrm{L}+3(26 \%)\end{array}$ \\
31580.05024 & 316.6556077 & 0.0998 & \begin{tabular}{c}
$\mathrm{H}-2 \rightarrow \mathrm{L}+1(49 \%), \mathrm{HOMO} \rightarrow \mathrm{L}+4(15 \%)$ \\
\hline 31678.45056
\end{tabular} \\
315.6720049 & 0.0309 & $\begin{array}{c}\mathrm{H}-3 \rightarrow \mathrm{L}+1(44 \%), \mathrm{H}-2 \rightarrow \mathrm{L}+2(18 \%), \\
\mathrm{H}-1 \rightarrow \mathrm{L}+2(16 \%)\end{array}$
\end{tabular}

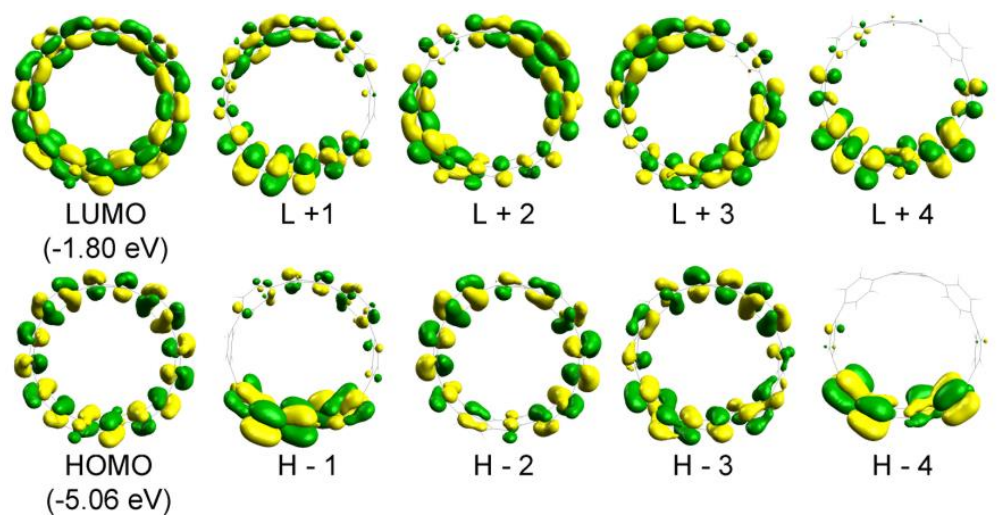

Figure S5. FMO diagrams ${ }^{6}$ for $1(\mathrm{H} / \mathrm{L}$ gap $=3.26 \mathrm{eV})(\mathrm{B} 3 \mathrm{LYP} / 6-31 \mathrm{G}(\mathrm{d})) . \mathrm{H}=\mathrm{HOMO}, \mathrm{L}=$ LUMO.

Table S3. Optical predictions for 2 via TD-DFT at the B3LYP/6-31G(d) level of theory (see Figure 3 in the main text for FMO diagrams).

\begin{tabular}{|c|c|c|c|}
\hline Energy $\left(\mathrm{cm}^{-1}\right)$ & Wavelength (nm) & Osc. Strength & Major contributions \\
\hline 22875.65472 & 437.1459581 & 0.0264 & HOMO $\rightarrow$ LUMO $(95 \%)$ \\
\hline 23841.9136 & 419.4294203 & 0.0025 & $\mathrm{H}-1 \rightarrow \mathrm{LUMO}(58 \%), \mathrm{HOMO} \rightarrow \mathrm{L}+1(29 \%)$ \\
\hline 26418.8728 & 378.5172848 & 0.0002 & $\begin{array}{c}\mathrm{H}-2 \rightarrow \text { LUMO }(18 \%), \mathrm{H}-1 \rightarrow \mathrm{L}+1(65 \%) \\
\text { HOMO } \rightarrow \mathrm{L}+2(12 \%)\end{array}$ \\
\hline 26745.5296 & 373.8942601 & 0.0963 & $\mathrm{H}-1 \rightarrow \mathrm{LUMO}(34 \%), \mathrm{HOMO} \rightarrow \mathrm{L}+1(63 \%)$ \\
\hline 27694.85072 & 361.0779528 & 0.0006 & $\begin{array}{c}\mathrm{H}-4 \rightarrow \mathrm{LUMO}(15 \%), \mathrm{H}-2 \rightarrow \mathrm{LUMO}(23 \%), \\
\mathrm{H}-1 \rightarrow \mathrm{L}+3(10 \%), \mathrm{HOMO} \rightarrow \mathrm{L}+2(25 \%) \\
\mathrm{HOMO} \rightarrow \mathrm{L}+4(11 \%)\end{array}$ \\
\hline 28016.66816 & 356.9303795 & 0.3326 & H-3 $\rightarrow$ LUMO $(93 \%)$ \\
\hline 28222.34096 & 354.3292179 & 0.0069 & $\begin{array}{c}\mathrm{H}-4 \rightarrow \text { LUMO }(15 \%), \mathrm{H}-3 \rightarrow \mathrm{L}+1(10 \%), \\
\mathrm{H}-2 \rightarrow \text { LUMO }(29 \%), \mathrm{H}-1 \rightarrow \mathrm{L}+3(10 \%), \\
\mathrm{HOMO} \rightarrow \mathrm{L}+2(20 \%), \mathrm{HOMO} \rightarrow \mathrm{L}+4(10 \%)\end{array}$ \\
\hline 28750.63776 & 347.8183713 & 0.7339 & $\mathrm{HOMO} \rightarrow \mathrm{L}+3(91 \%)$ \\
\hline 29091.81264 & 343.7393236 & 1.5827 & $\begin{array}{c}\mathrm{H}-2 \rightarrow \mathrm{LUMO}(26 \%), \mathrm{H}-1 \rightarrow \mathrm{L}+1(29 \%), \\
\mathrm{HOMO} \rightarrow \mathrm{L}+2(38 \%)\end{array}$ \\
\hline 29331.36096 & 340.9320152 & 0.0094 & $\mathrm{H}-2 \rightarrow \mathrm{L}+1(39 \%), \mathrm{H}-1 \rightarrow \mathrm{L}+2(49 \%)$ \\
\hline 30120.9832 & 331.9944749 & 0.028 & $\mathrm{H}-3 \rightarrow \mathrm{L}+1(72 \%), \mathrm{H}-1 \rightarrow \mathrm{L}+3(11 \%)$ \\
\hline
\end{tabular}




\begin{tabular}{l|l|l|l}
\hline 30230.67536 & 330.7898312 & 0.2072 & H-4 $\rightarrow$ LUMO $(25 \%), \mathrm{H}-1 \rightarrow \mathrm{L}+3(60 \%)$
\end{tabular}

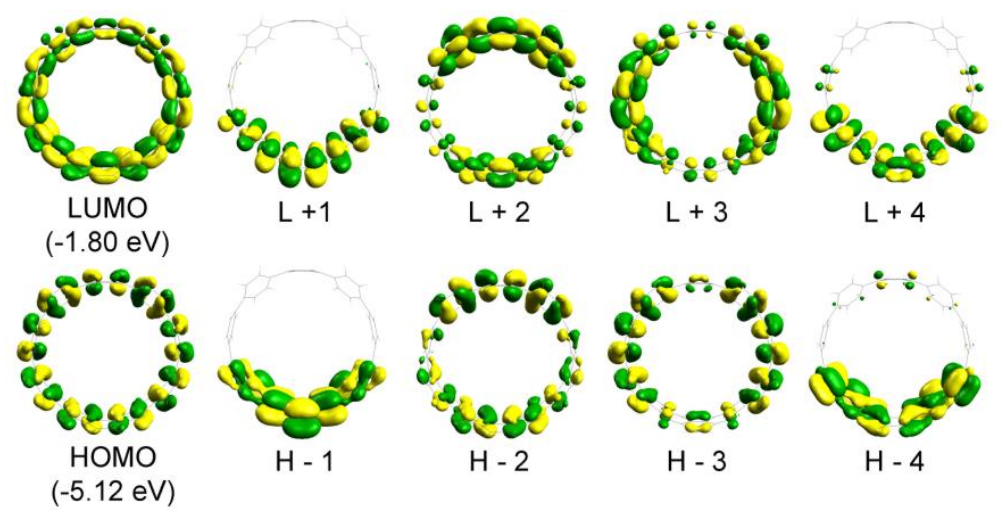

Figure S6. FMO diagrams ${ }^{6}$ for $2(\mathrm{H} / \mathrm{L}$ gap $=3.32 \mathrm{eV})(\mathrm{B} 3 \mathrm{LYP} / 6-31 \mathrm{G}(\mathrm{d})) . \mathrm{H}=\mathrm{HOMO}, \mathrm{L}=$ LUMO.

Table S4. Optical predictions for 3 via TD-DFT at the B3LYP/6-31G(d) level of theory

\begin{tabular}{|c|c|c|c|}
\hline $\operatorname{Energy}\left(\mathrm{cm}^{-1}\right)$ & Wavelength (nm) & Osc. Strength & Major contributions \\
\hline 20500.33552 & 487.79689436 & 0.0121 & HOMO $\rightarrow$ LUMO $(95 \%)$ \\
\hline 22422.368 & 445.983225322 & 0.0023 & $\mathrm{H}-1 \rightarrow \mathrm{LUMO}(63 \%), \mathrm{HOMO} \rightarrow \mathrm{L}+1(33 \%)$ \\
\hline 25050.14048 & 399.199358103 & 0.0331 & $\mathrm{H}-1 \rightarrow \mathrm{LUMO}(30 \%), \mathrm{HOMO} \rightarrow \mathrm{L}+1(59 \%)$ \\
\hline 27343.99712 & 365.71098059 & 0.0264 & $\begin{array}{c}\mathrm{H}-2 \rightarrow \mathrm{LUMO}(10 \%), \mathrm{H}-1 \rightarrow \mathrm{L}+1(48 \%) \\
\mathrm{HOMO} \rightarrow \mathrm{L}+2(22 \%)\end{array}$ \\
\hline 27720.66064 & 360.741763332 & 0.0435 & $\begin{array}{c}\mathrm{H}-2 \rightarrow \mathrm{LUMO}(20 \%), \mathrm{H}-1 \rightarrow \mathrm{L}+1(31 \%) \\
\text { HOMO } \rightarrow \mathrm{L}+2(26 \%)\end{array}$ \\
\hline 28816.77568 & 347.020086877 & 0.0331 & $\begin{array}{c}\mathrm{H}-3 \rightarrow \text { LUMO }(26 \%), \mathrm{H}-2 \rightarrow \text { LUMO }(20 \%) \\
\text { HOMO } \rightarrow \text { L }+3(44 \%)\end{array}$ \\
\hline 29398.30544 & 340.155660346 & 0.0543 & $\begin{array}{l}\mathrm{H}-4-\rightarrow \mathrm{LUMO}(18 \%), \mathrm{H}-3 \rightarrow \mathrm{LUMO}(15 \%), \\
\mathrm{H}-2 \rightarrow \mathrm{LUMO}(24 \%), \mathrm{HOMO} \rightarrow \mathrm{L}+4(26 \%)\end{array}$ \\
\hline 29723.34912 & 336.435842395 & 0.3164 & $\begin{array}{c}\text { H-2 } \rightarrow \text { LUMO }(18 \%), \mathrm{H}-1 \rightarrow \mathrm{L}+2(11 \%), \\
\mathrm{HOMO} \rightarrow \mathrm{L}+2(37 \%), \mathrm{HOMO} \rightarrow \mathrm{L}+3(14 \%)\end{array}$ \\
\hline 30752.51968 & 325.176606797 & 0.0714 & $\mathrm{H}-4 \rightarrow \mathrm{LUMO}(16 \%), \mathrm{H}-1 \rightarrow \mathrm{L}+2(63 \%)$ \\
\hline 31184.02928 & 320.676969298 & 0.0083 & $\begin{array}{c}\mathrm{H}-2 \rightarrow \mathrm{L}+1(29 \%), \mathrm{H}-1 \rightarrow \mathrm{L}+3(42 \%), \\
\mathrm{HOMO} \rightarrow \mathrm{L}+4(15 \%)\end{array}$ \\
\hline
\end{tabular}




\begin{tabular}{c|c|c|c}
\hline 31571.17808 & 316.744594537 & 0.4523 & $\begin{array}{c}\text { H-4 } \rightarrow \text { LUMO }(11 \%), \text { H-3 } \rightarrow \text { LUMO }(24 \%), \\
\text { HOMO } \rightarrow \text { L+3 }(18 \%), \text { HOMO->L+5 }(12 \%)\end{array}$ \\
\hline 31719.58512 & 315.26263544 & 0.1471 & $\begin{array}{c}\text { H-2 } \rightarrow \text { L+1 }(12 \%), \text { H-1 } \rightarrow \text { L+3 }(29 \%), \\
\text { HOMO } \rightarrow \text { L+4 }(31 \%)\end{array}$
\end{tabular}

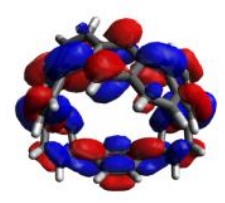

LUMO $(-1.90 \mathrm{eV})$

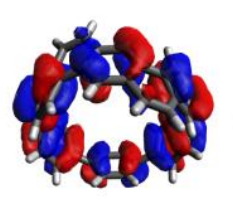

HOMO $(-4.86 \mathrm{eV})$

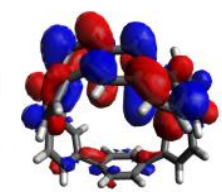

$\mathrm{L}+1$

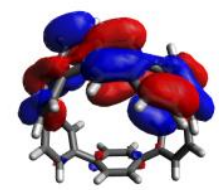

H - 1

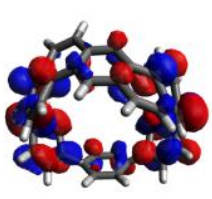

$\mathrm{L}+2$

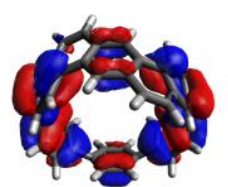

H - 2

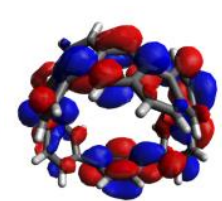

$\mathrm{L}+3$

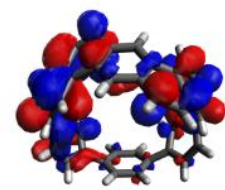

$\mathrm{L}+4$

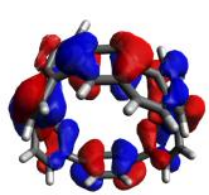

H - 3

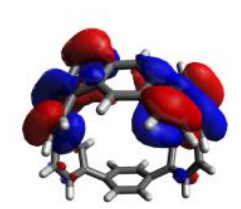

H - 4

Figure S7. FMO diagrams ${ }^{6}$ for $3(\mathrm{H} / \mathrm{L}$ gap $=2.96 \mathrm{eV})(\mathrm{B} 3 \mathrm{LYP} / 6-31 \mathrm{G}(\mathrm{d}))$. H = HOMO, L = LUMO.

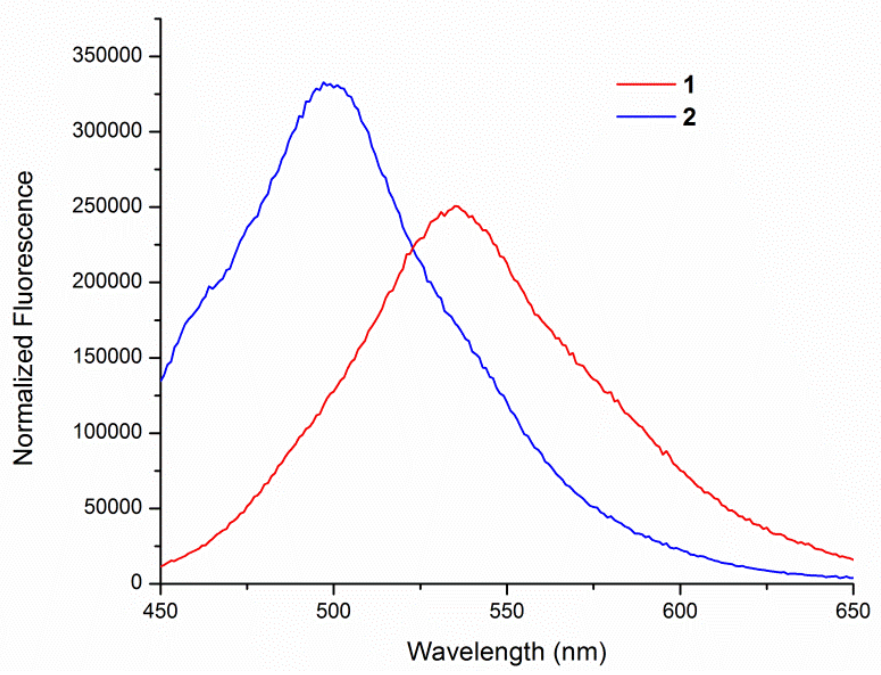

Figure S8. Fluorescence spectra of $1\left(\lambda_{\mathrm{fl}}=535 \mathrm{~nm}, \Phi=14 \pm 5 \%\right)$ and $2\left(\lambda_{\mathrm{fl}}=500 \mathrm{~nm}, \Phi=\right.$ $15 \pm 5 \%$ ) with excitation at $340 \mathrm{~nm}$. 

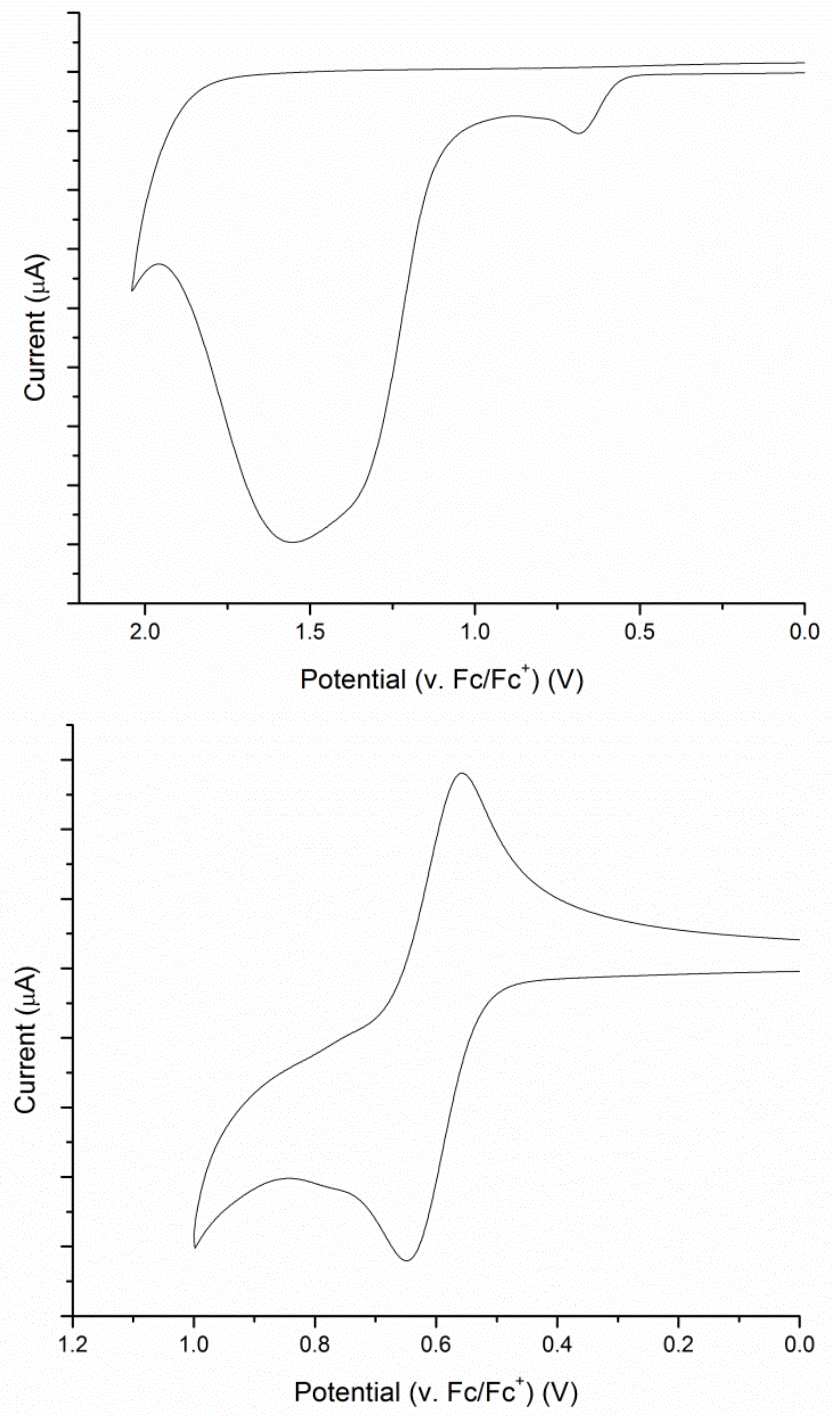

Figure S9. (Top) Oxidation of $1\left(\mathrm{CH}_{2} \mathrm{Cl}_{2}\right)$. Multiple irreversible events are observed after the initial oxidation at more positive potentials. (Bottom) Oxidation of $\mathbf{1}\left(\mathrm{CH}_{2} \mathrm{Cl}_{2}\right)$ with a narrower potential window. The initial oxidation event is reversible under these conditions: $\mathrm{E}_{1 / 2}=0.60 \mathrm{~V}$. This oxidative half-wave potential is comparable to that of [8]CPP. ${ }^{7}$ 


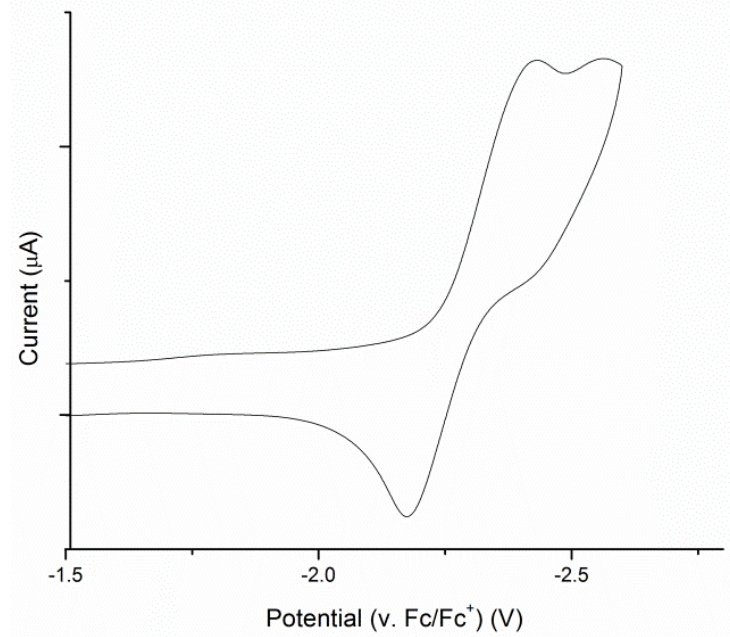

Figure S10. Reduction of 1 (THF): $\mathrm{E}_{1 / 2}=-2.30 \mathrm{~V}$. 

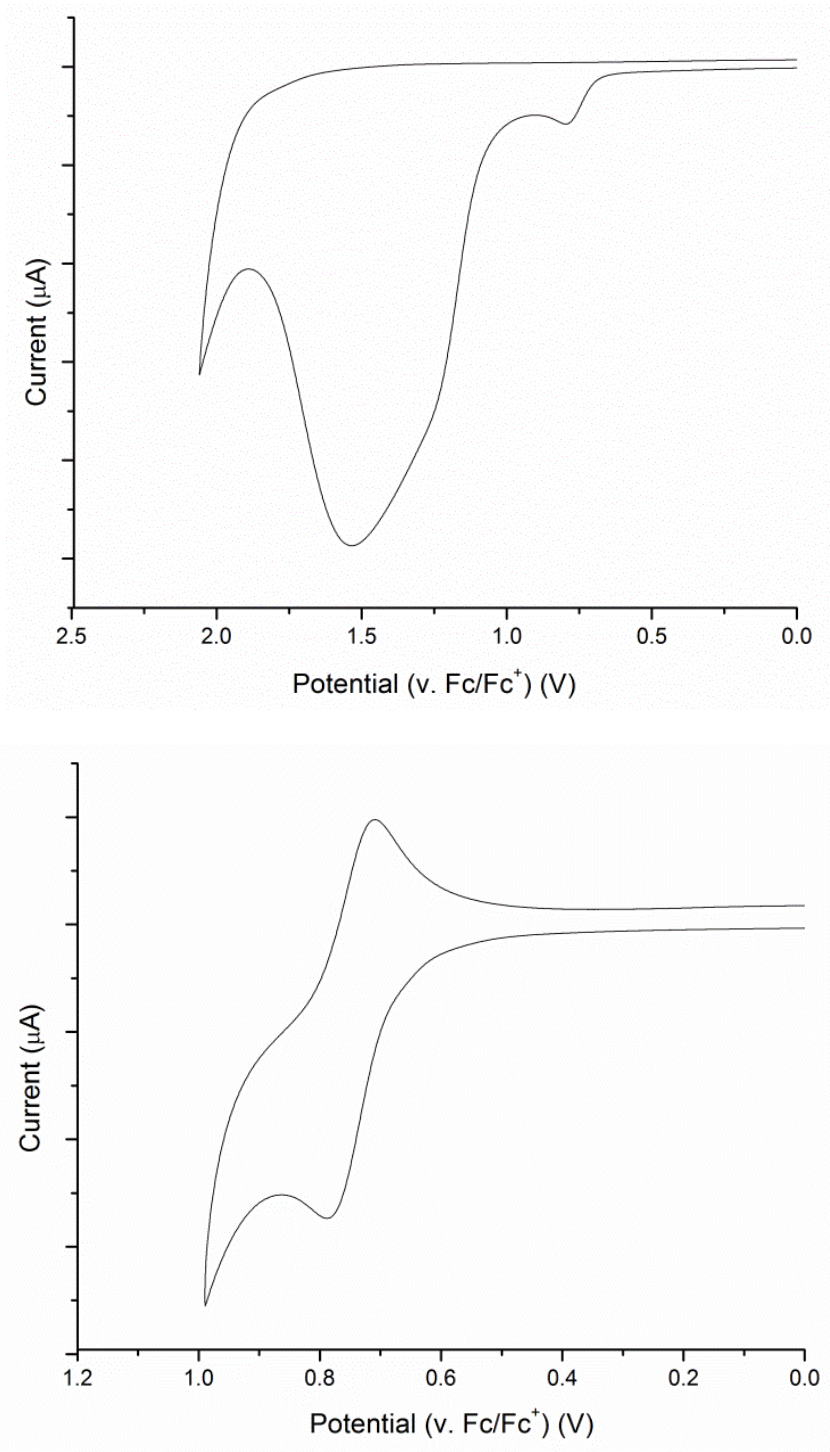

Figure S11. (Top) Oxidation of $2\left(\mathrm{CH}_{2} \mathrm{Cl}_{2}\right)$. Multiple irreversible events are observed after the initial oxidation at more positive potentials. (Bottom) Oxidation of $2\left(\mathrm{CH}_{2} \mathrm{Cl}_{2}\right.$, versus $\left.\mathrm{Fc} / \mathrm{Fc}^{+}\right)$: $\mathrm{E}_{1 / 2}=0.75 \mathrm{~V}$. This oxidative half-wave potential is comparable to that of [9]CPP. ${ }^{77}$ 


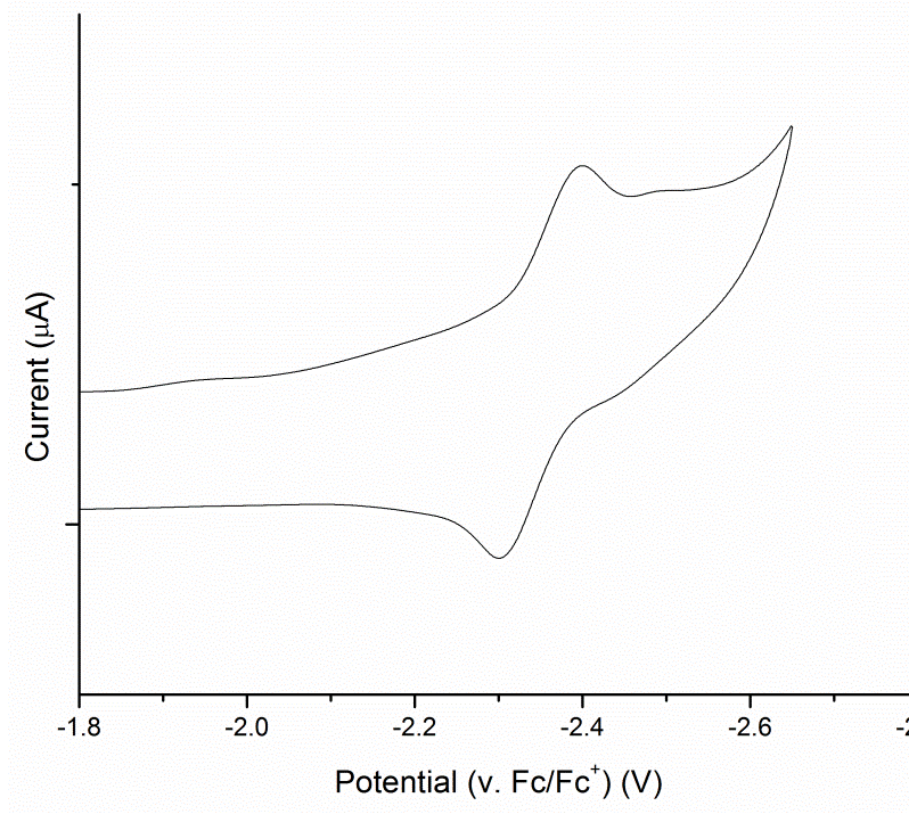

Figure S12. Reduction of $2\left(\mathrm{THF}\right.$, versus $\left.\mathrm{Fc} / \mathrm{Fc}^{+}\right)$: $\mathrm{E}_{1 / 2}=-2.34 \mathrm{~V}$.

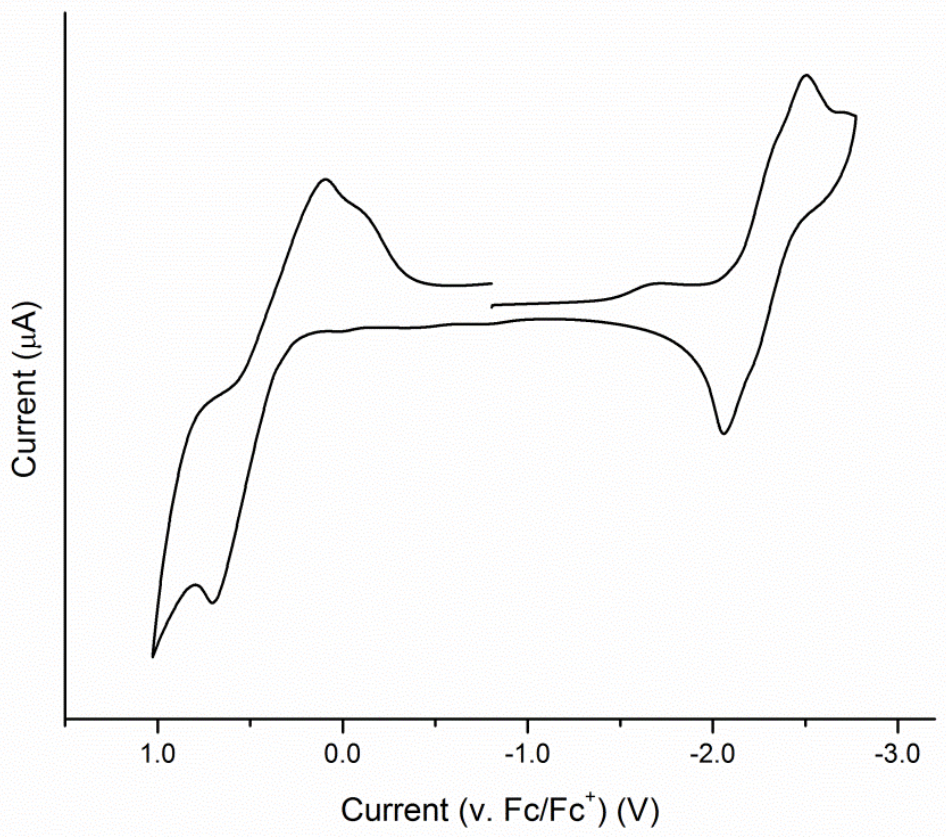

Figure S13. Oxidation and reduction of $\mathbf{3}$ (THF): $\mathrm{E}^{\mathrm{red}}{ }_{1 / 2}=-2.15 \mathrm{~V} \mathrm{E}^{\mathrm{ox}}{ }_{1 / 2}=0.40 \mathrm{~V}$. The decrease in the diameter of $\mathbf{3}$ (and hence the decrease in HOMO/LUMO gap), compared to that of $\mathbf{1}$ and $\mathbf{2}$, permitted the observation of both electrochemical events in the same solvent. 
4) Computational Analysis of Macrocycle Atropisomers. In order to study the dynamic behavior observed in the ${ }^{1} \mathrm{H}$ NMR spectra of $\mathbf{1 0}$ and $\mathbf{1 2}$, we carried out preliminary computational screening calculations for each cyclic structure. Specifically, $\mathbf{1 0}$ and $\mathbf{1 2}$ contain 3 and 4 contiguous phenyl rings, respectively, and each individual phenyl ring in the macrocycle can orient itself in two different alignments relative to the rest of the macrocycle backbone (i.e. each phenyl ring can be oriented roughly $\pm 180^{\circ}$ relative to the macrocycle backbone). These variations lead to 8 and 16 different conformations for $\mathbf{1 0}$ and 12, respectively, which we minimized without any constraints using dispersion-corrected DFT at the B97D/6-31G(d) level of theory with Gaussian09. ${ }^{8}$ Optimized geometries were confirmed with vibrational analyses. Upon comparing the ground-state energies of the various atropisomers that $\mathbf{1 0}$ can adopt, we found that $\mathbf{1 0}$ has a single, low-lying ground-state conformation that is $1.73 \mathrm{kcal} \mathrm{mol}^{-1} \mathrm{more}$ stable than the next highest energy conformation. Between the lowest energy conformation and the seven other possible conformations, the energy difference between ground-state energies is $1.73-5.10 \mathrm{kcal} \mathrm{mol}^{-1}$ (Table S5). On the other hand, $\mathbf{1 2}$ has numerous ground-state conformations that are close in energy (Table S6). Approximately one-third of the atropisomers are within $1 . \mathrm{kcal} \mathrm{mol}^{-1}$ of the lowest-energy conformation, while the highest energy atropisomer is only $3.43 \mathrm{kcal} \mathrm{mol}^{-1}$ higher in energy. Although we have not investigated the relative transition states between these interconversions within the two molecules, the computational results seem consistent with our VT-NMR experiments. That is, the more disperse ground-states of 9 lead to a single major conformation at room temperature and a sharp ${ }^{1} \mathrm{H}$ NMR spectrum. In the case of $\mathbf{1 2}$, many closely spaced ground-states lead to numerous conformations, leading to broadening in the ${ }^{1} \mathrm{H}$ NMR spectrum at room temperature. Based on our experimental RCM data, the varying 
rigidity of the vinylated macrocycles (i.e. 10 and 12) does not seem to influence the outcome of these particular reactions.

Table S5. Calculated energies of $\mathbf{1 0}$ conformers (RB97D/6-31G(d)).

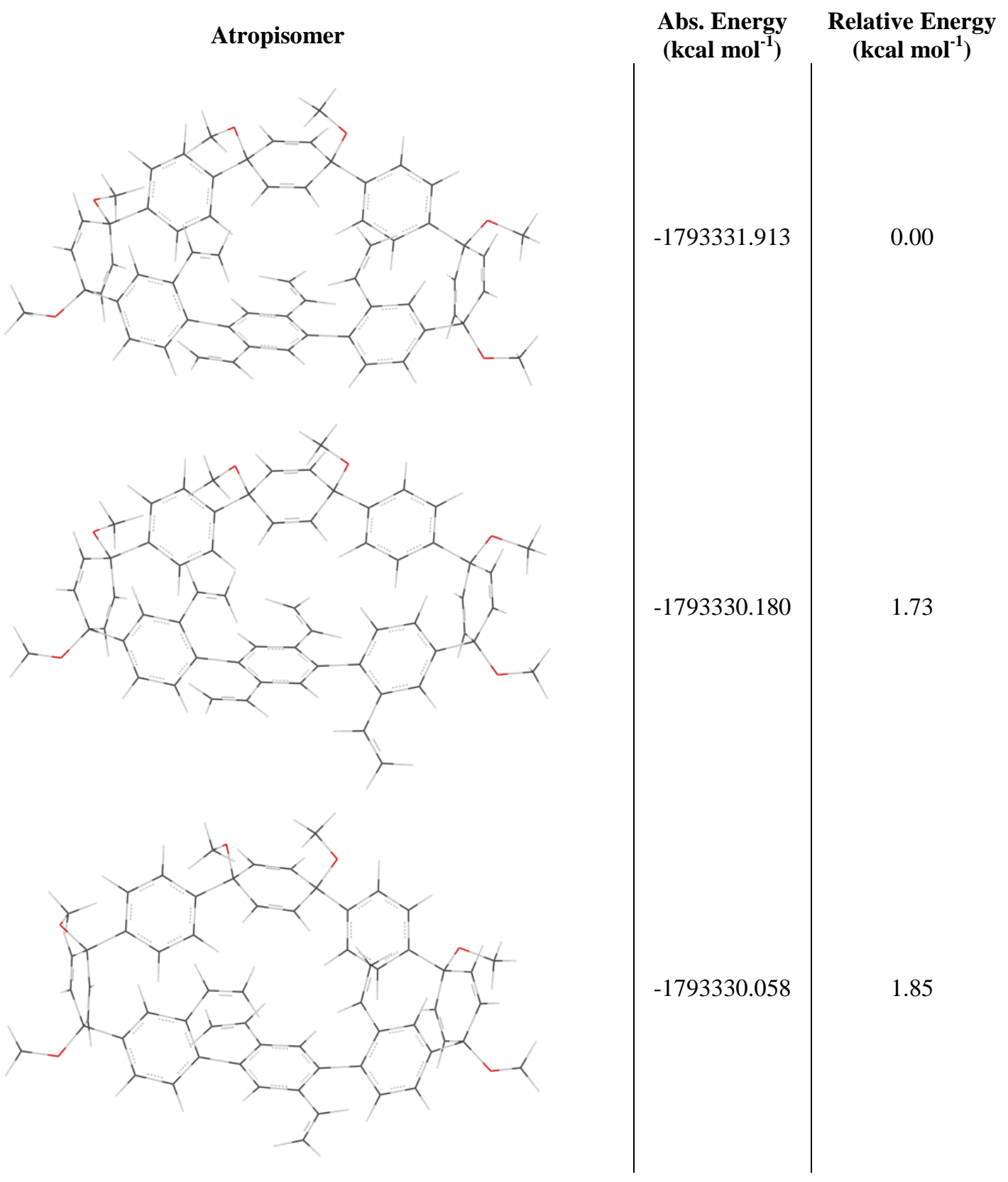



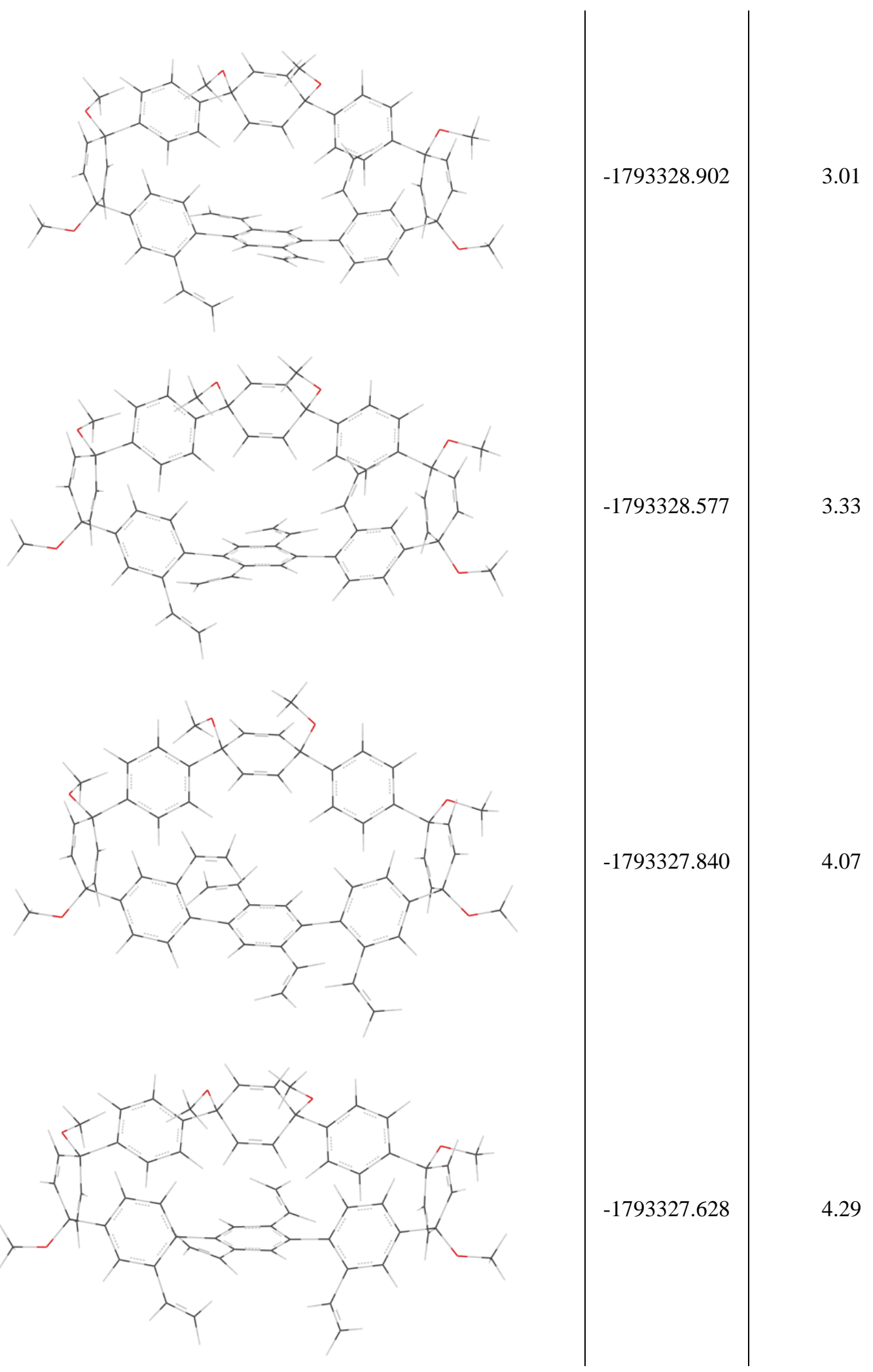

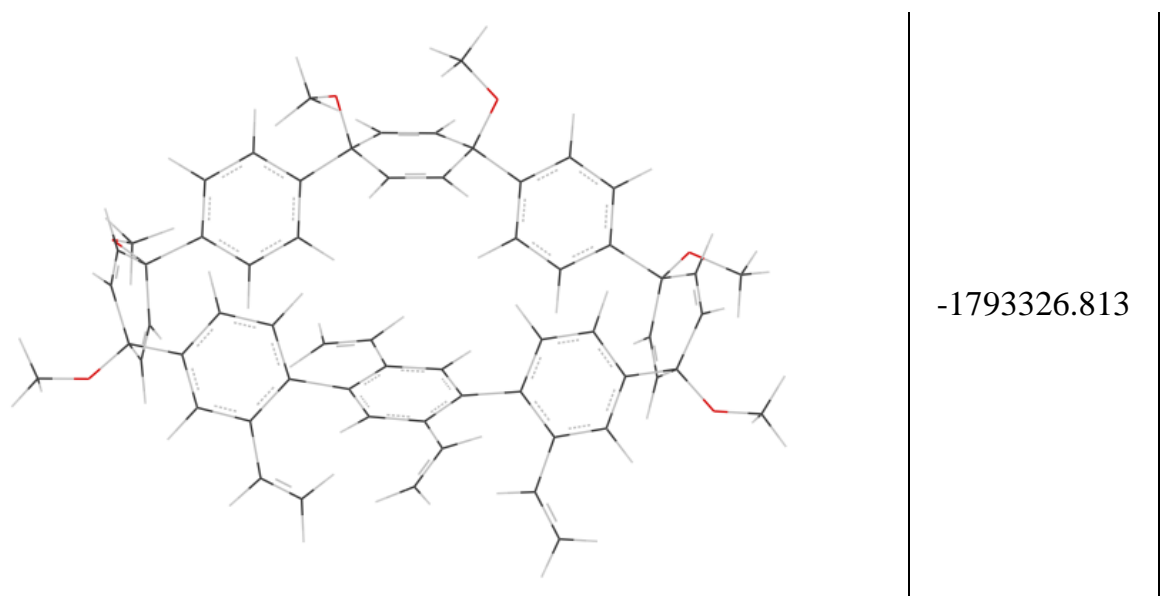

5.10

Table S6. Calculated energies of 12 conformers (RB97D/6-31G(d)).

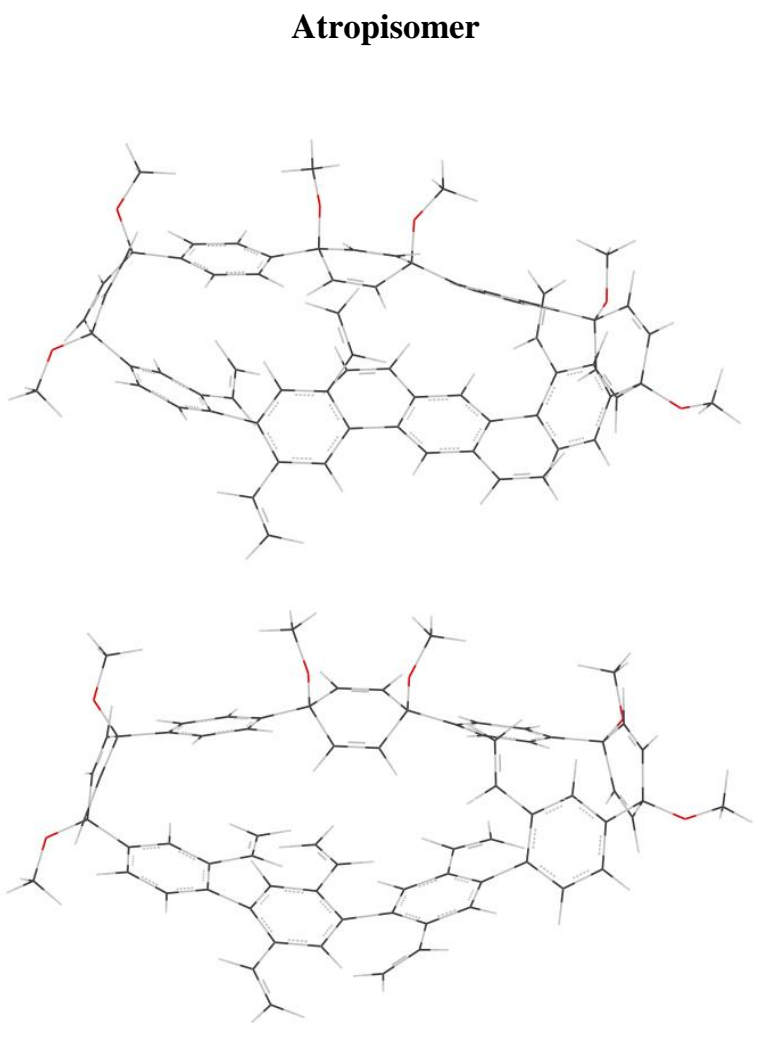

Energy (kcal mol ${ }^{-1}$ )

$-2036217.532$

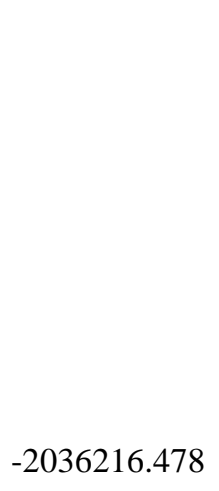

Relative Energy (kcal mol ${ }^{-1}$ )

0.00

1.05 

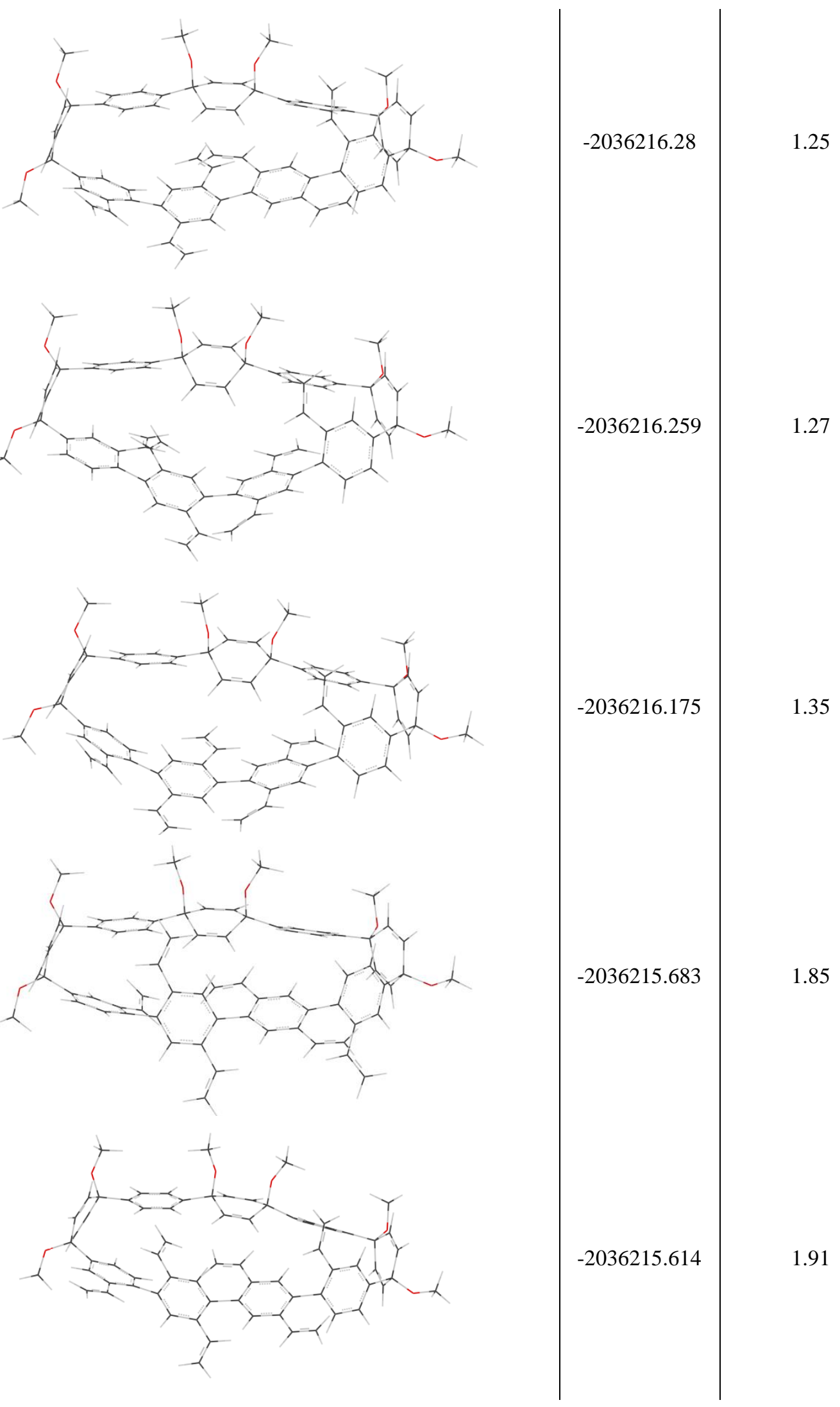

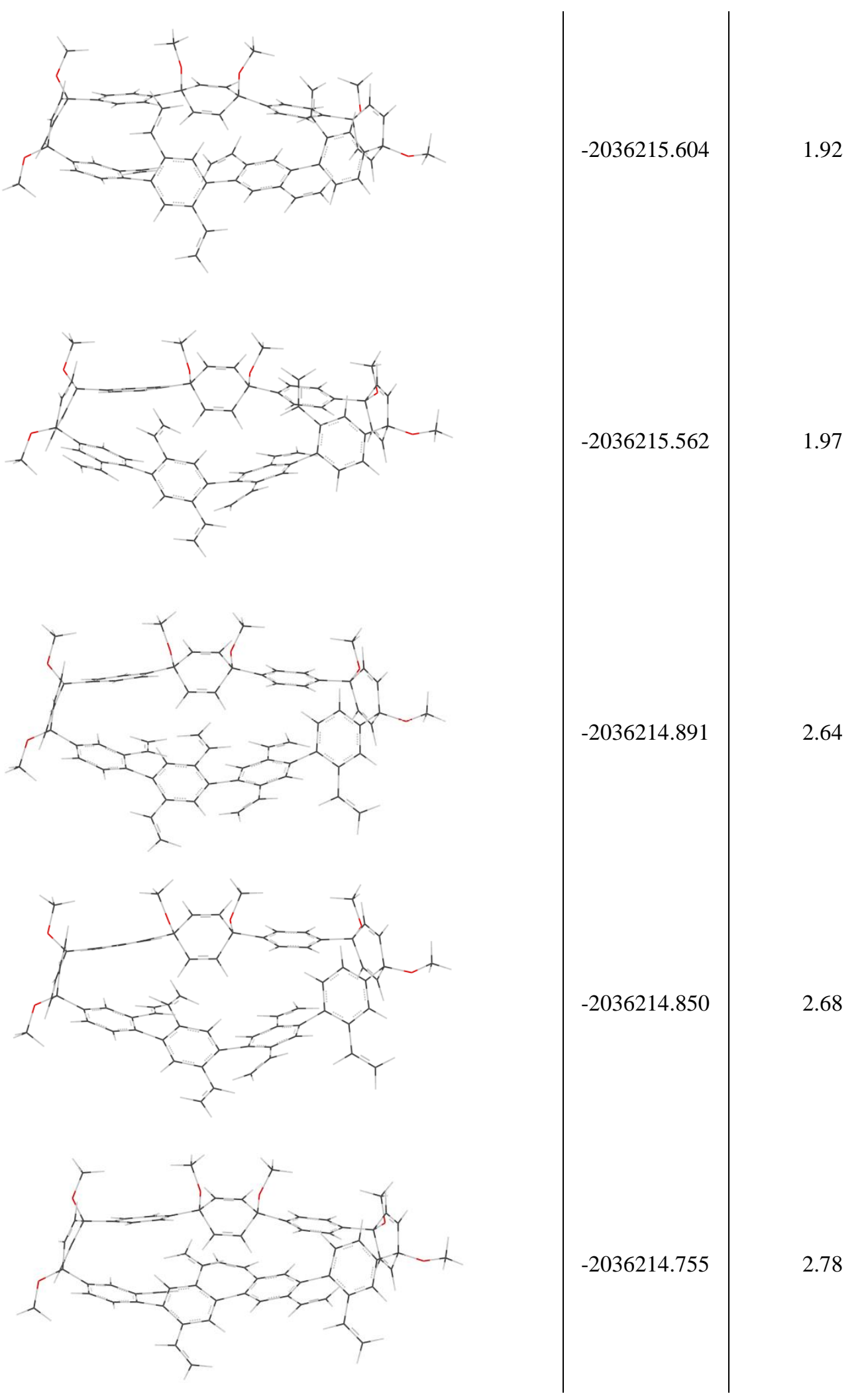

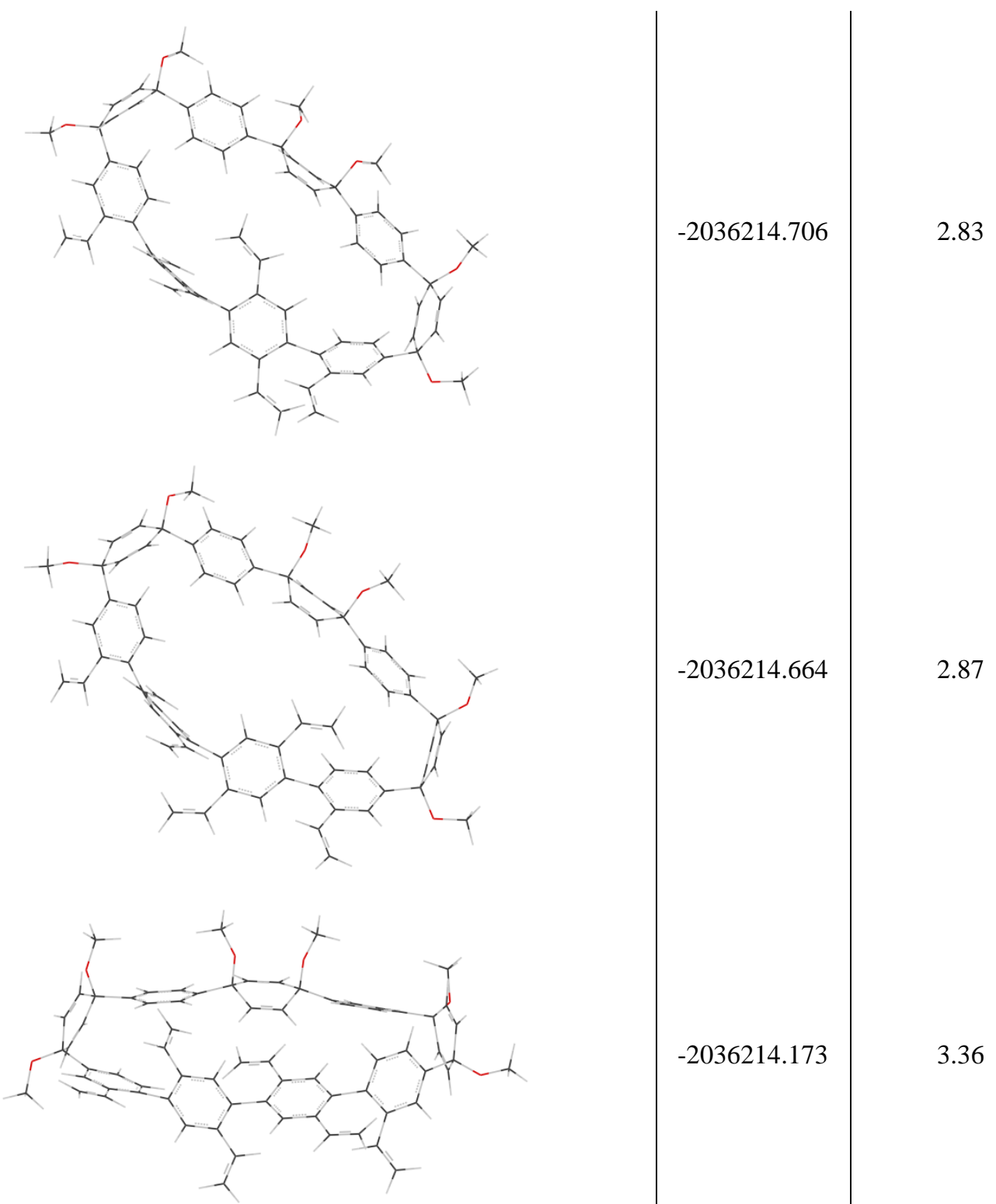

$-2036214.173$

3.36

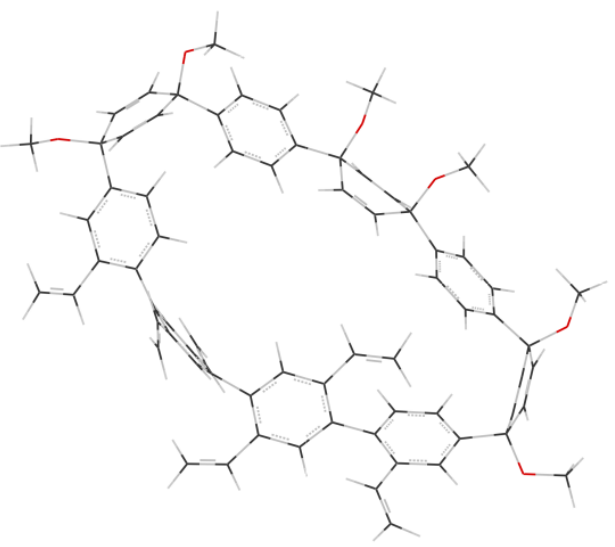

$-2036214.102$

3.43 
5) Strain Energy Analysis. All calculations were run using Gaussian09. ${ }^{8}$ Optimized geometries were confirmed with vibrational analyses. For $\mathbf{1 0}$ and 12, the lowest energy geometries were extracted from the data presented above in Tables S5 and S6.

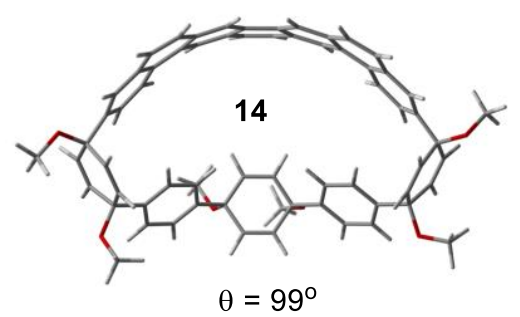

Figure S14. DFT optimized geometry of macrocycle 14 at the B3LYP/6-31G(d) level of theory. See Figure 2 in the main text for a definition of $\theta$.

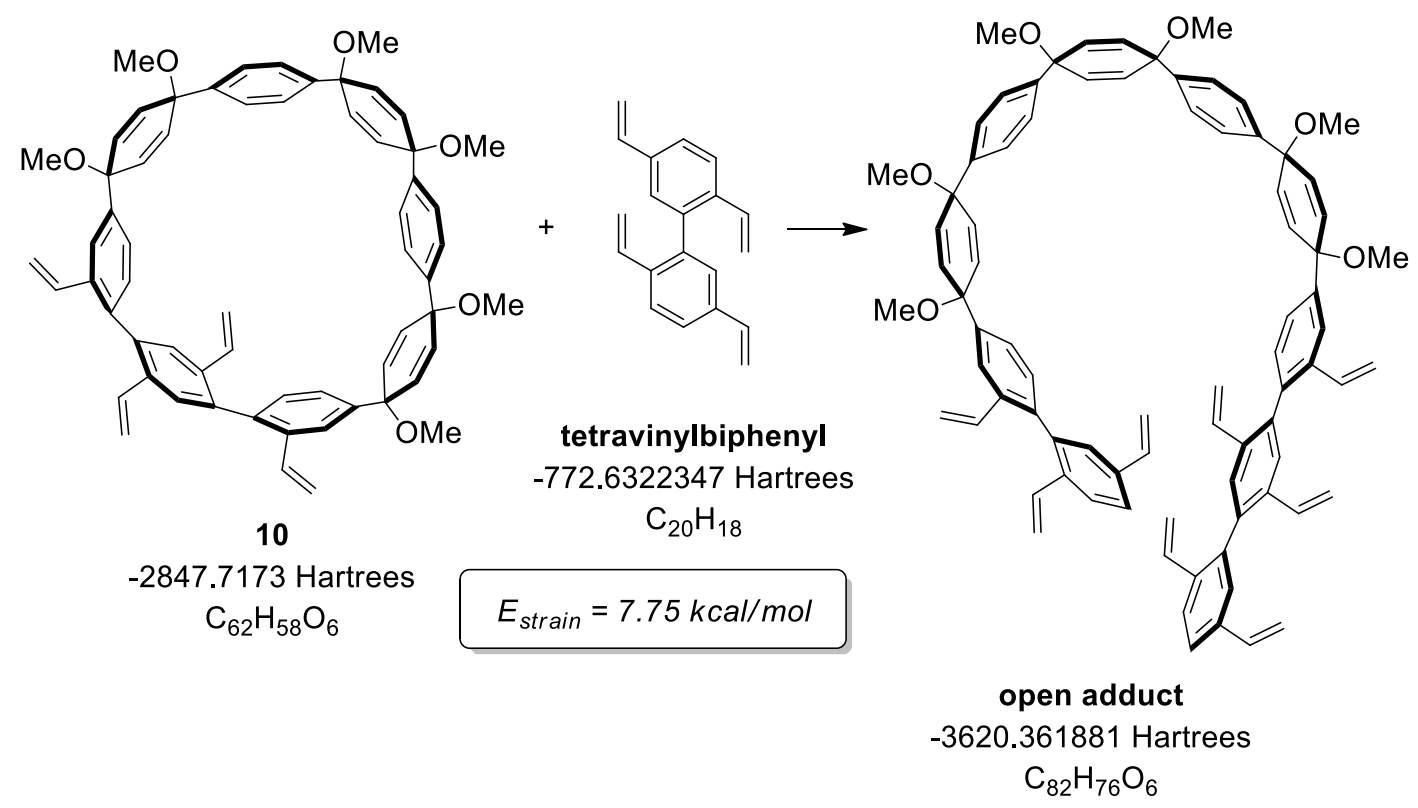

Scheme S1. Homodesmotic reaction to access strain energy of $\mathbf{1 0}$ calculated at the $\omega \mathrm{B} 97 \mathrm{D} / 6$ $31 \mathrm{G}(\mathrm{d})$ level of theory. 


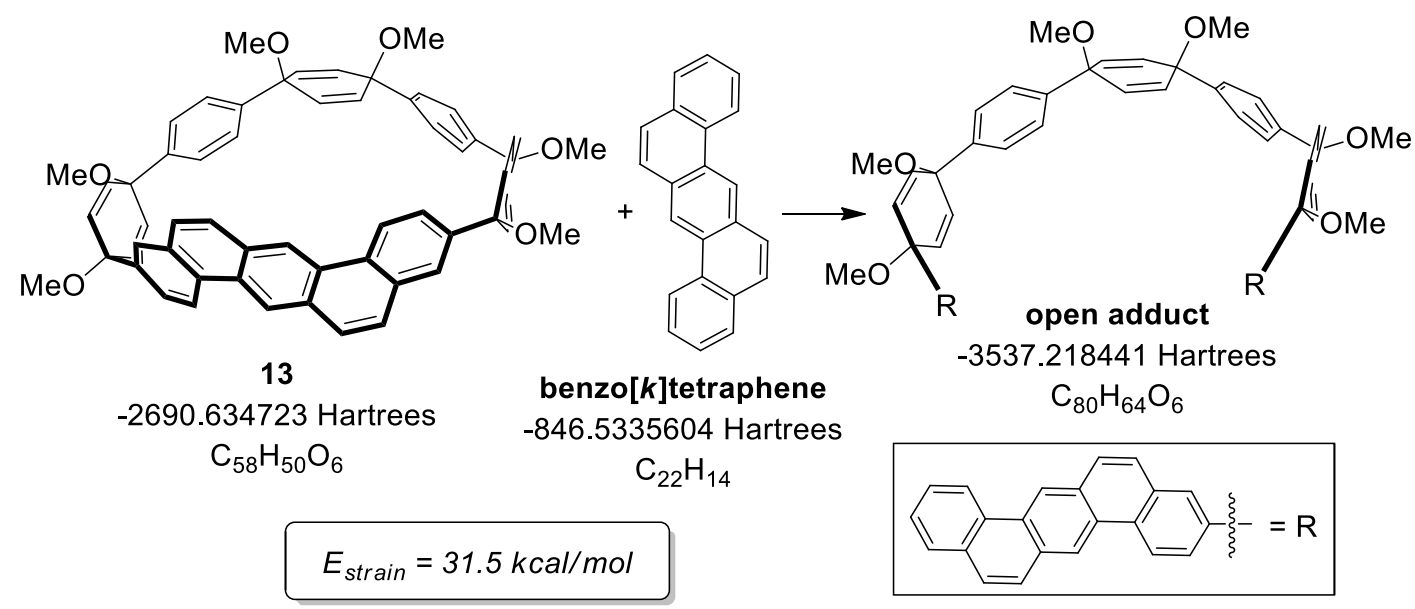

Scheme S2. Homodesmotic reaction to access strain energy of $\mathbf{1 3}$ calculated at the $\omega \mathrm{B} 97 \mathrm{D} / 6-$ $31 \mathrm{G}(\mathrm{d})$ level of theory.

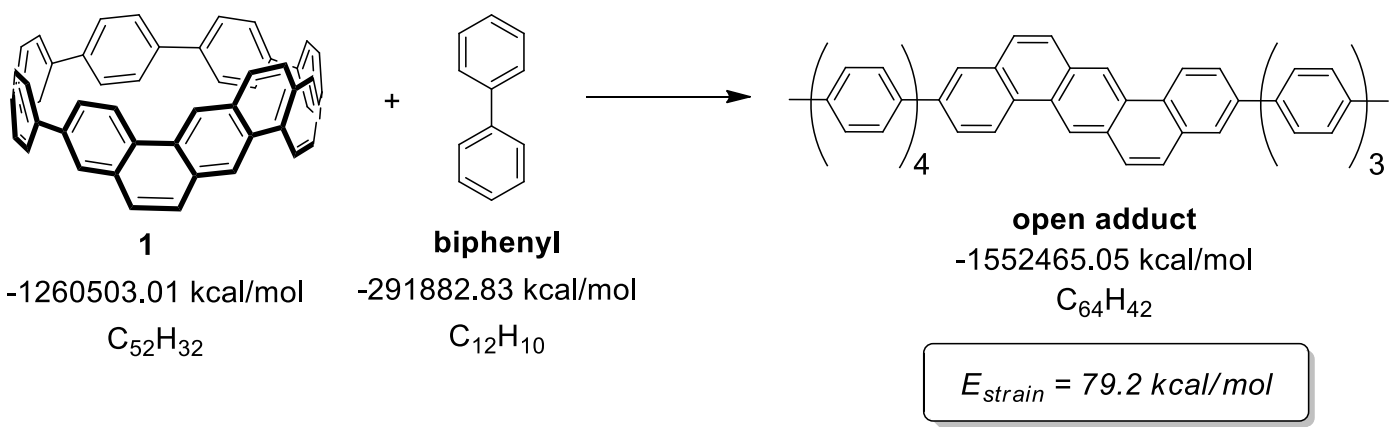

Scheme S3. Homodesmotic reaction to access strain energy of 1 calculated at the $\omega B$ 97D/6$31 \mathrm{G}(\mathrm{d})$ level of theory. 


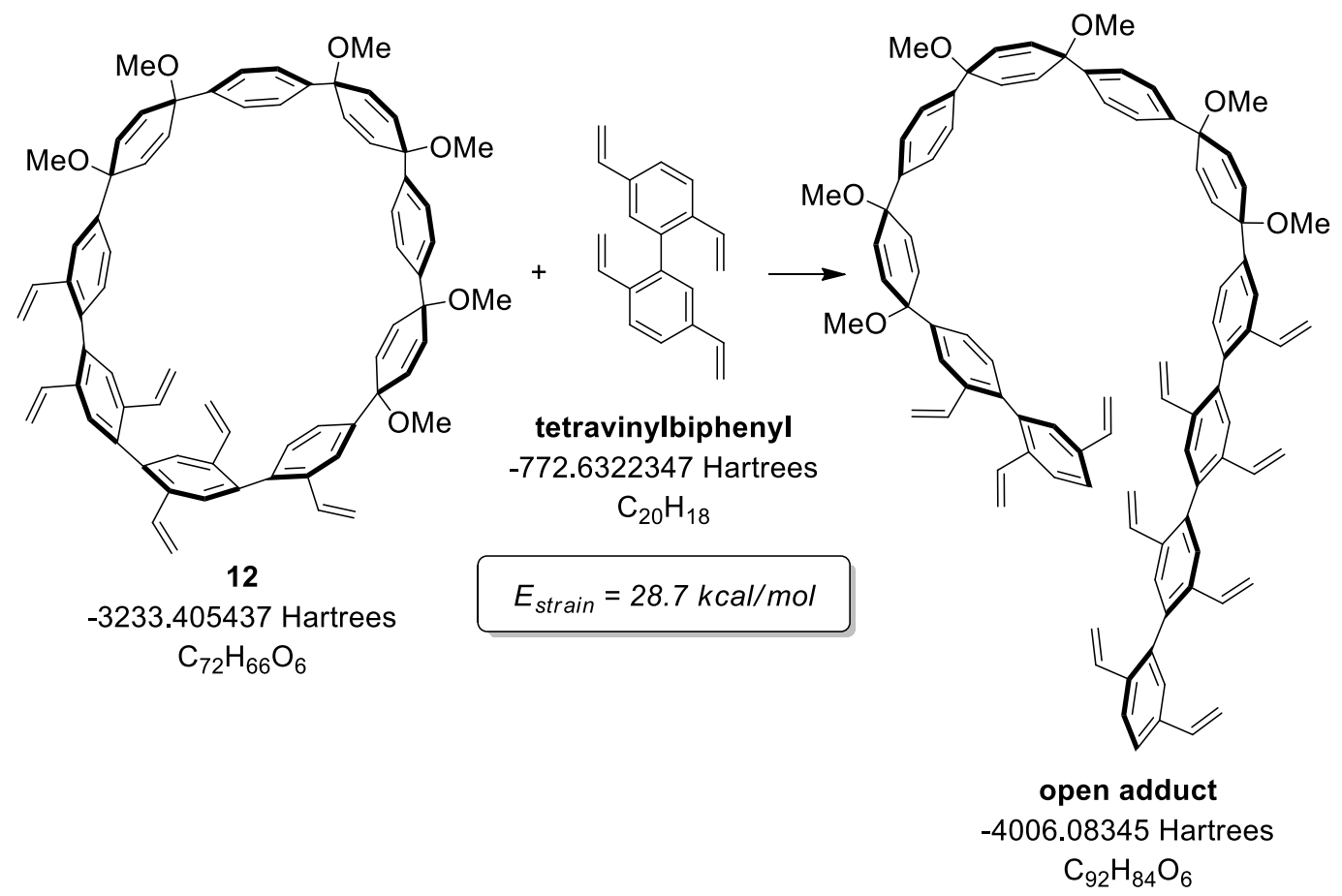

Scheme S4. Homodesmotic reaction to access strain energy of $\mathbf{1 2}$ calculated at the $\omega \mathrm{B} 97 \mathrm{D} / 6-$ $31 \mathrm{G}(\mathrm{d})$ level of theory.

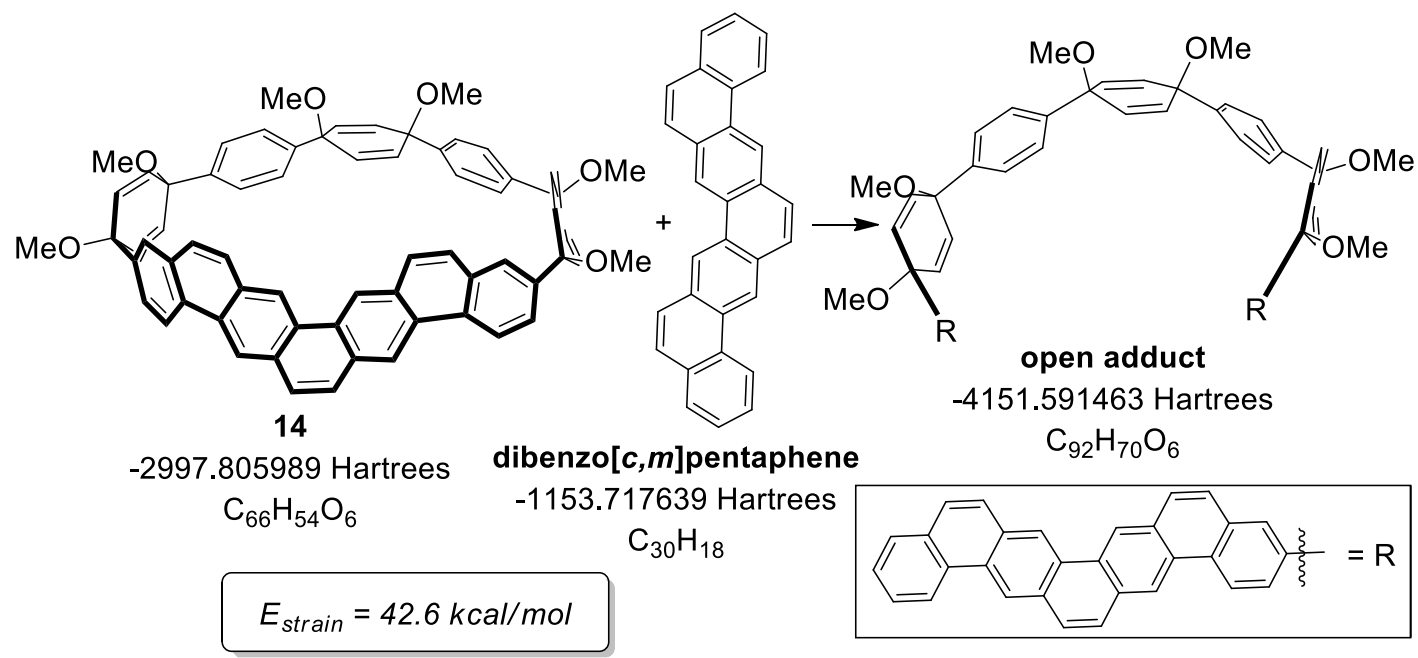

Scheme S5. Homodesmotic reaction to access strain energy of $\mathbf{1 4}$ calculated at the $\omega \mathrm{B} 97 \mathrm{D} / 6-$ $31 \mathrm{G}(\mathrm{d})$ level of theory. 


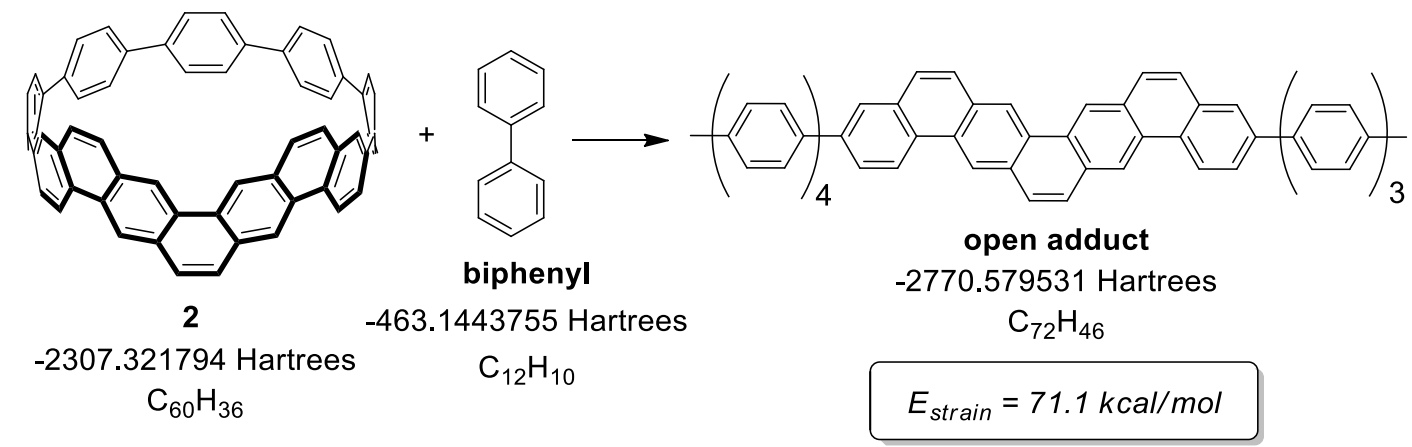

Scheme S6. Homodesmotic reaction to access strain energy of $\mathbf{2}$ calculated at the $\omega \mathrm{B} 97 \mathrm{D} / 6$ $31 \mathrm{G}(\mathrm{d})$ level of theory.

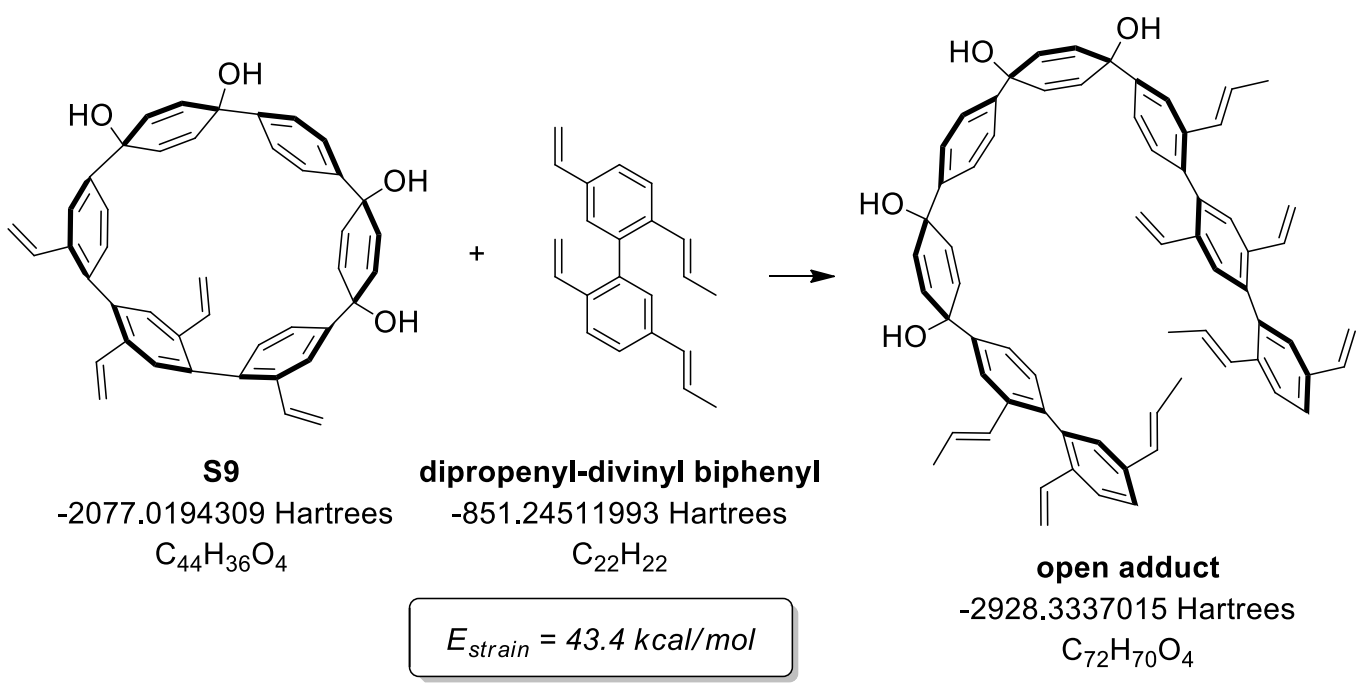

Scheme S7. Homodesmotic reaction to access strain energy of S9 calculated at the $\omega \mathrm{B} 97 \mathrm{D} / 6-$ $31 \mathrm{G}(\mathrm{d})$ level of theory. 


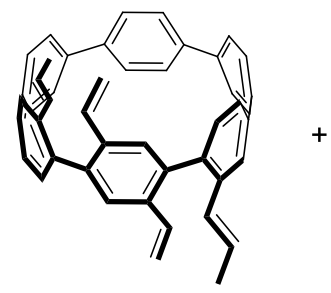

18

-1773.8048116 Hartrees

$\mathrm{C}_{46} \mathrm{H}_{36}$<smiles>c1ccc(-c2ccccc2)cc1</smiles>

biphenyl

-463.1443755 Hartrees

$\mathrm{C}_{12} \mathrm{H}_{10}$

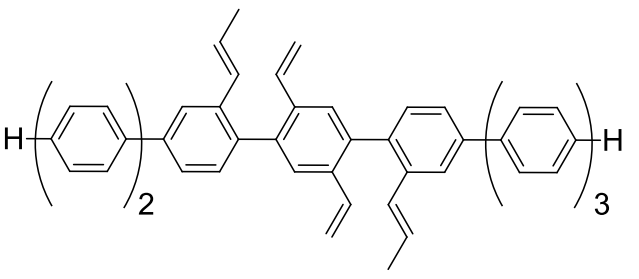

open adduct -2237.1273152 Hartrees $\mathrm{C}_{58} \mathrm{H}_{46}$

$E_{\text {strain }}=111 \mathrm{kcal} / \mathrm{mol}$

Scheme S8. Homodesmotic reaction to access strain energy of $\mathbf{1 8}$ calculated at the $\omega$ B97D/6$31 \mathrm{G}(\mathrm{d})$ level of theory.

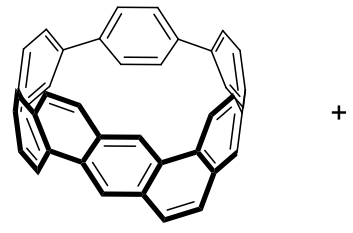

3 -1538.12170013 Hartrees

$\mathrm{C}_{40} \mathrm{H}_{24}$<smiles>c1ccc(-c2ccccc2)cc1</smiles>

biphenyl -463.1443755 Hartrees Chemical Formula: $\mathrm{C}_{12} \mathrm{H}_{10}$

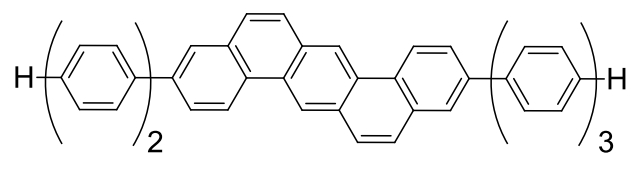

open adduct

-2001.43489615 Hartrees

$\mathrm{C}_{52} \mathrm{H}_{34}$

$E_{\text {strain }}=106 \mathrm{kcal} / \mathrm{mol}$

Scheme S9. Homodesmotic reaction to access strain energy of $\mathbf{3}$ calculated at the $\omega$ B97D/6$31 \mathrm{G}(\mathrm{d})$ level of theory. 


\section{6) VT NMR Spectra.}

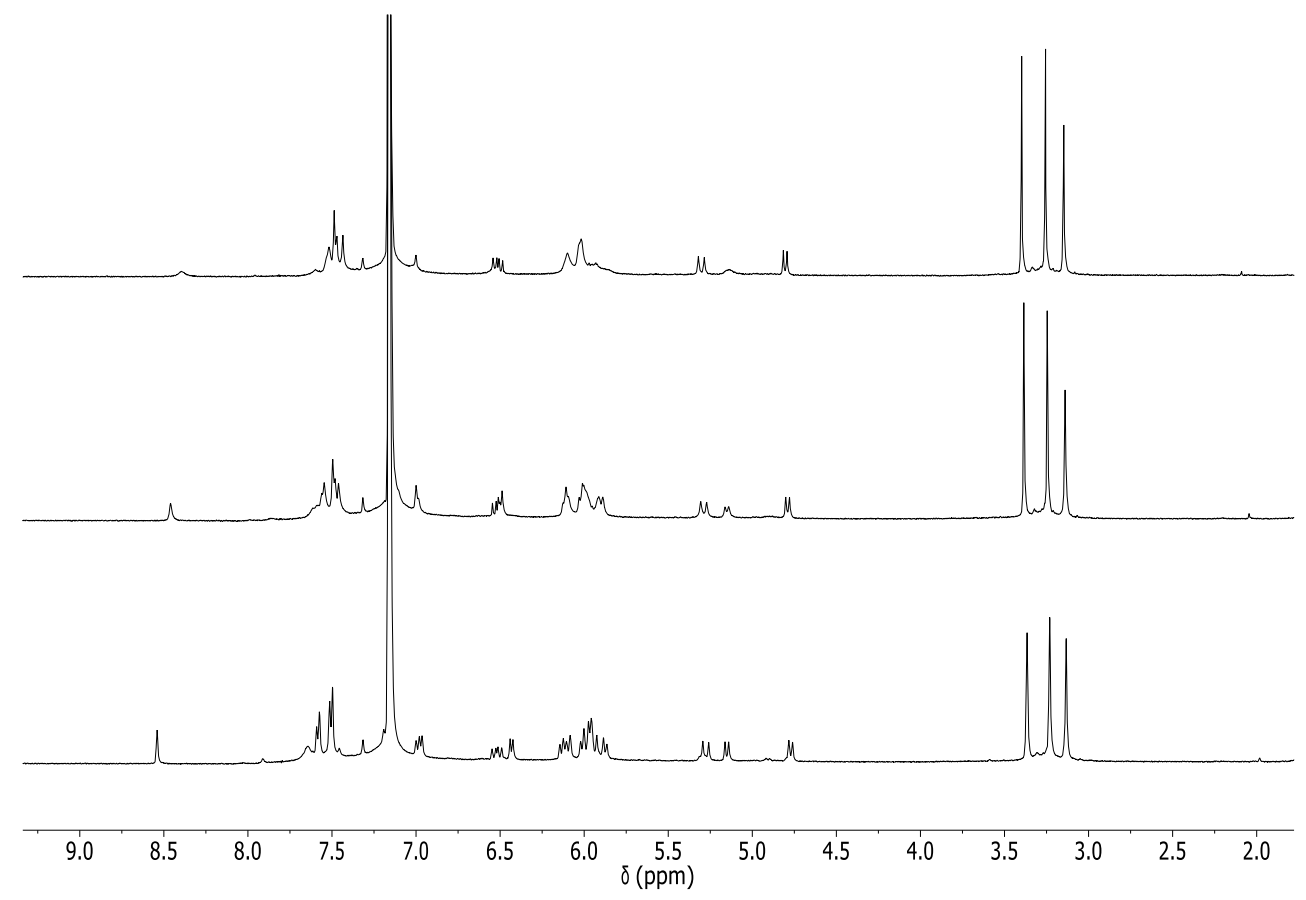

Figure S15. ${ }^{1} \mathrm{H}$ NMR spectra of 10 at $25^{\circ} \mathrm{C}, 50{ }^{\circ} \mathrm{C}$, and $70{ }^{\circ} \mathrm{C}$ (bottom $\rightarrow$ top) in $\mathrm{C}_{6} \mathrm{D}_{6}(500$ $\mathrm{MHz}$.

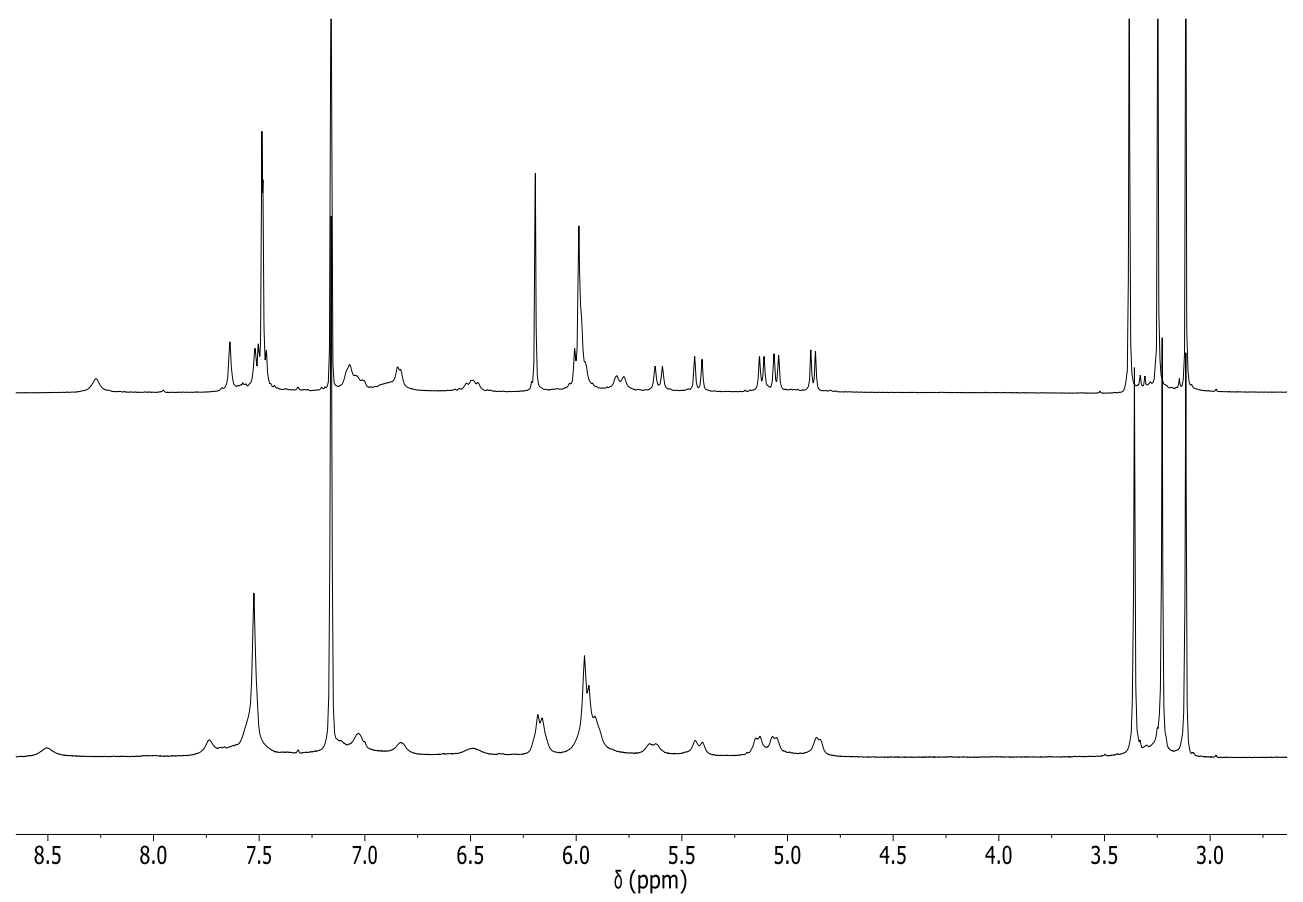

Figure S16. ${ }^{1} \mathrm{H}$ NMR spectra of 12 at $25{ }^{\circ} \mathrm{C}$ and $70{ }^{\circ} \mathrm{C}$ (bottom $\rightarrow$ top) in $\mathrm{C}_{6} \mathrm{D}_{6}(500 \mathrm{MHz})$. 


\section{7) ${ }^{1} \mathrm{H} /{ }^{13} \mathrm{C}$ NMR Spectra.}

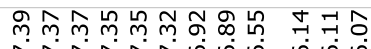

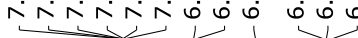
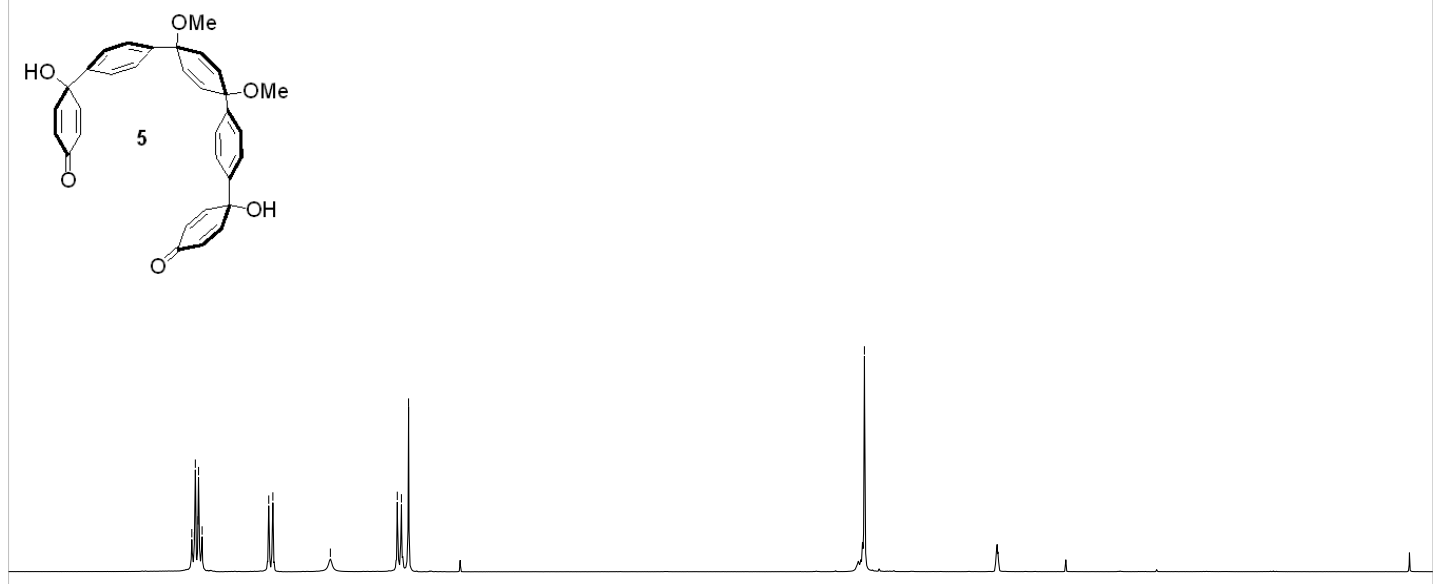

\begin{tabular}{|c|c|c|c|c|c|c|}
\hline 8.0 & 7.5 & 7.0 & 6.5 & 6.0 & 5.5 & 5.0 \\
\hline & & 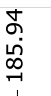 & & $\begin{array}{l}\text { م્ } \\
\text { กู }\end{array}$ & 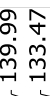 & 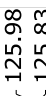 \\
\hline
\end{tabular}
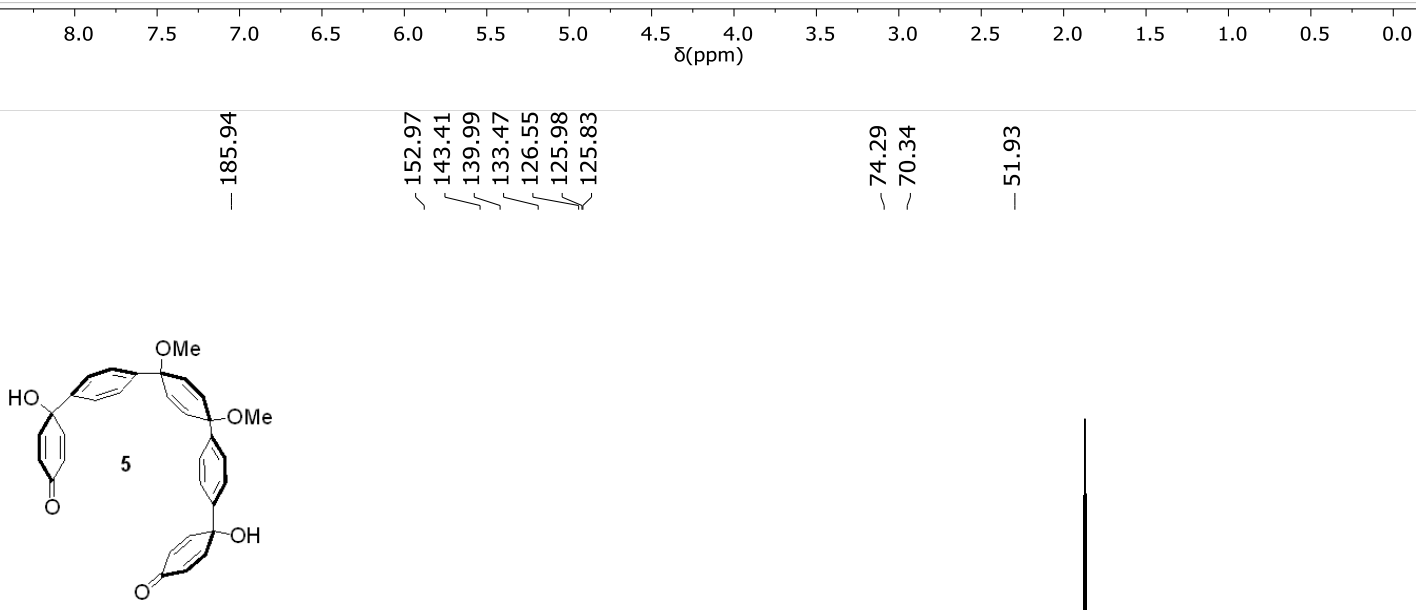

श्षे

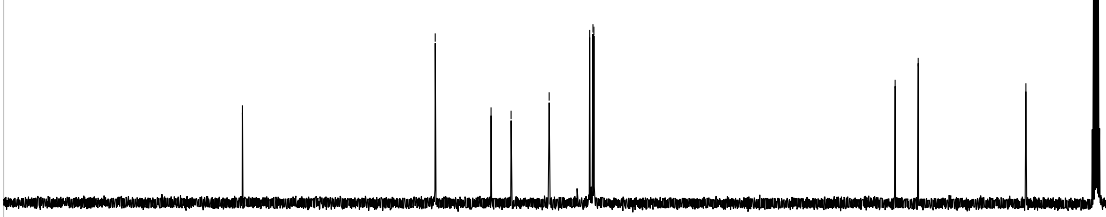

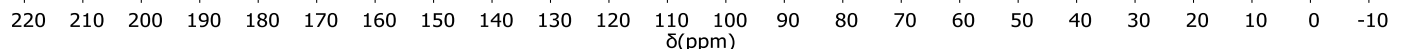



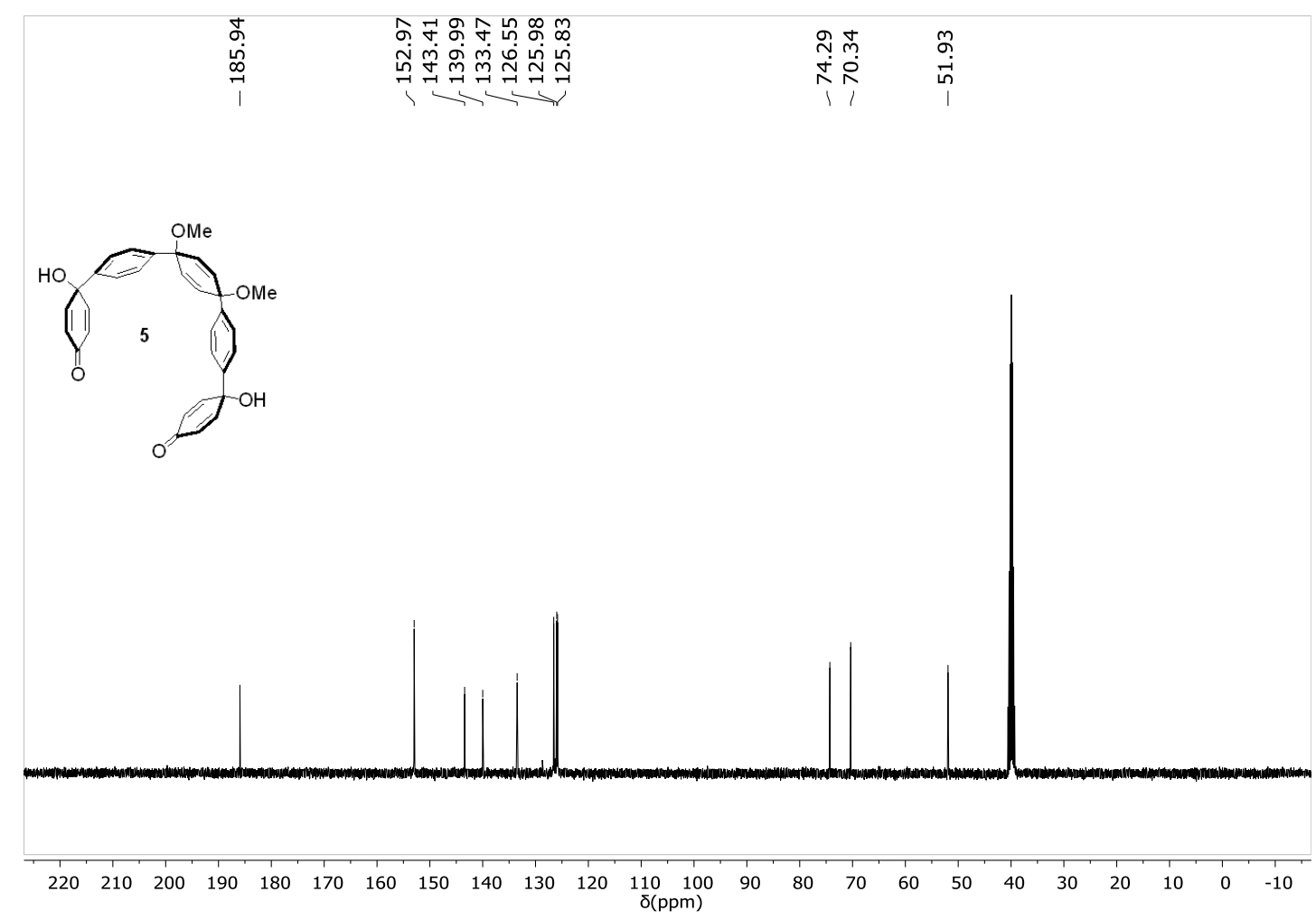

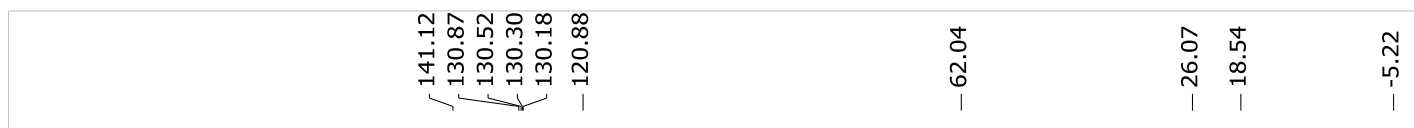
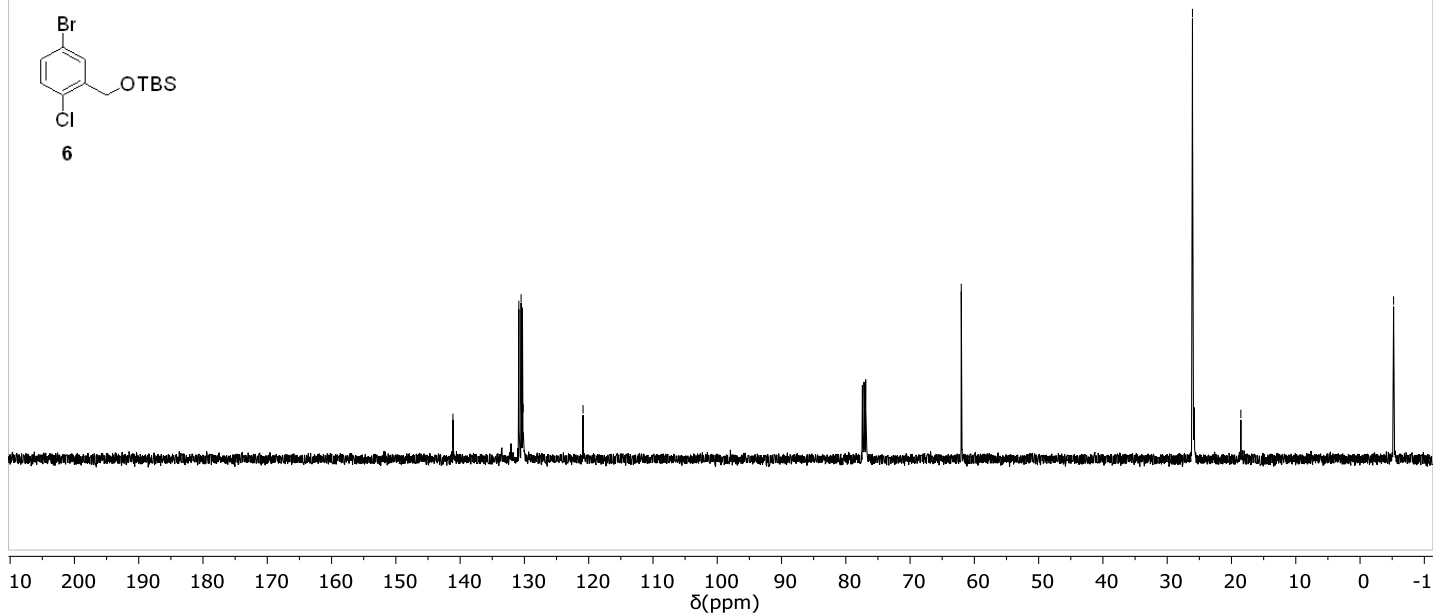

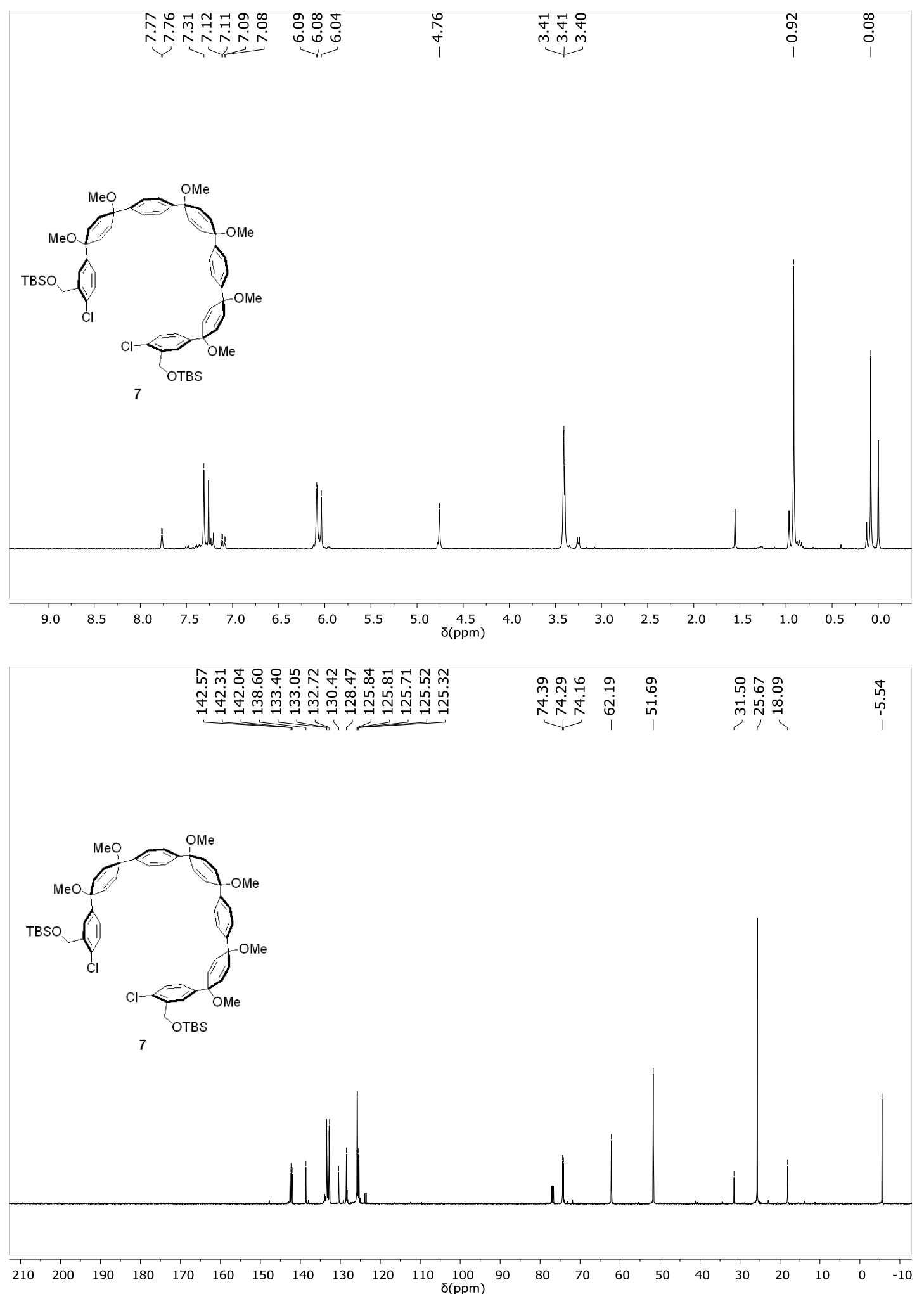


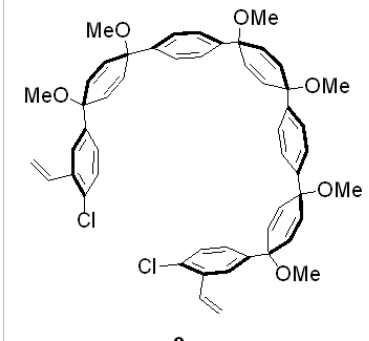

8
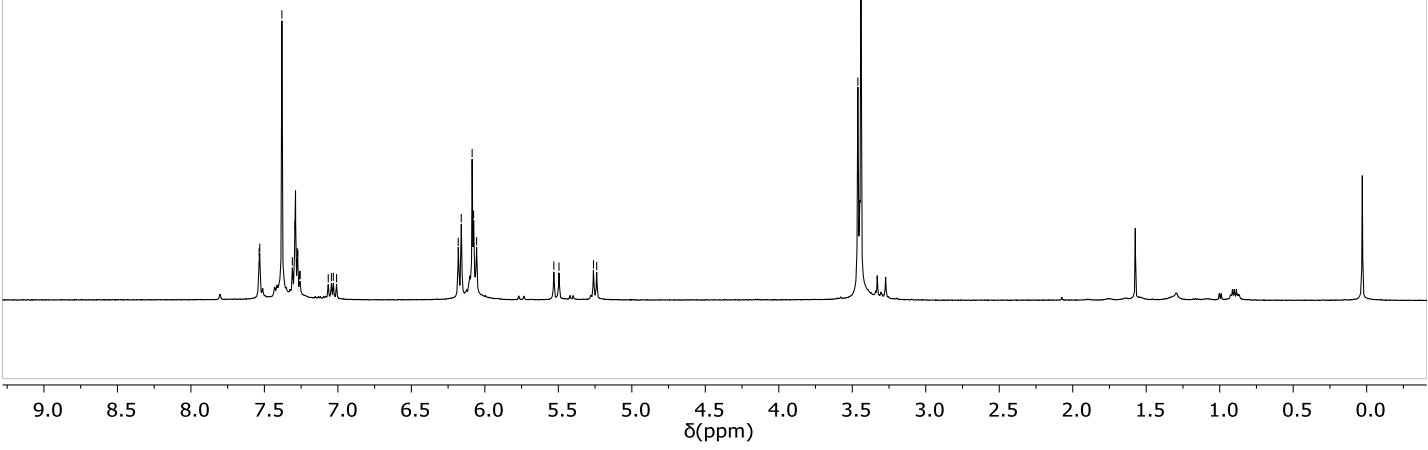

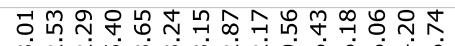

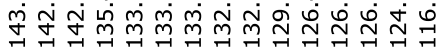

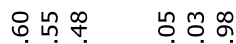

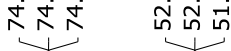
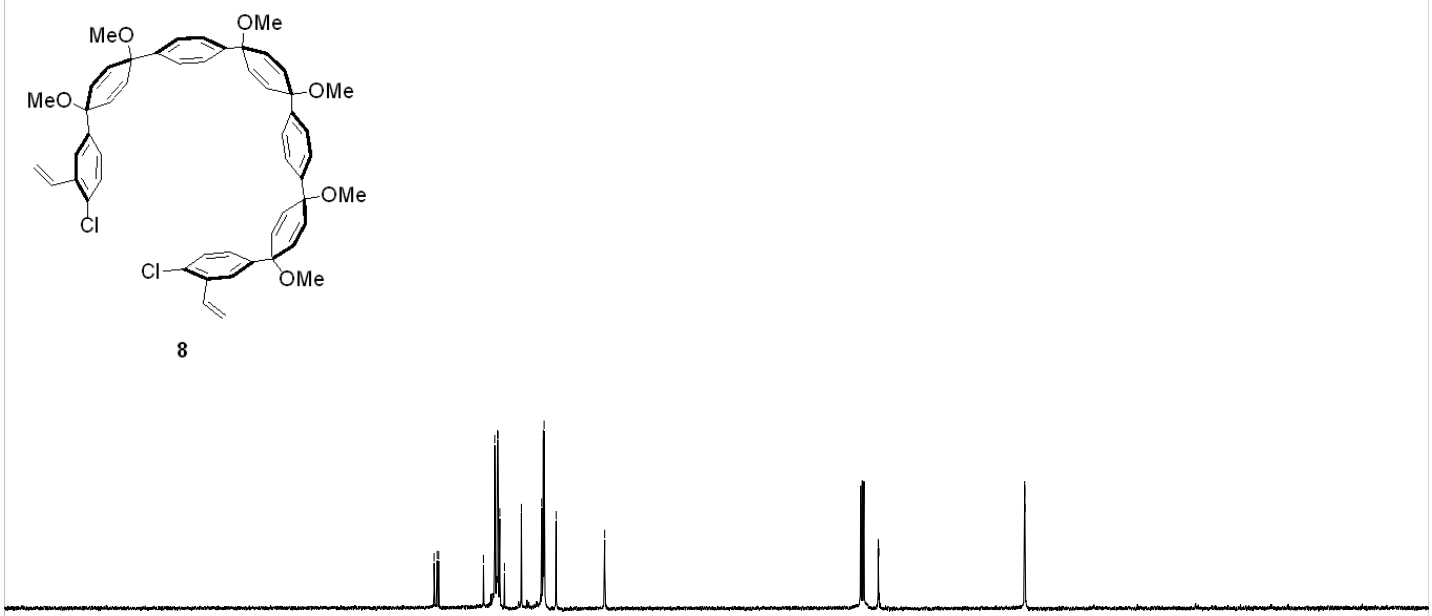

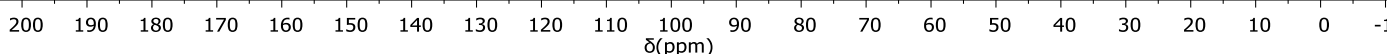




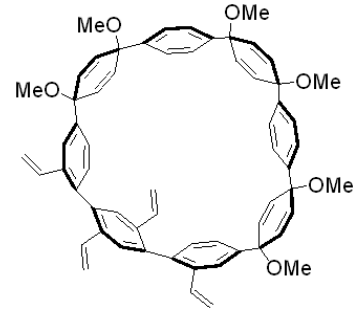

10

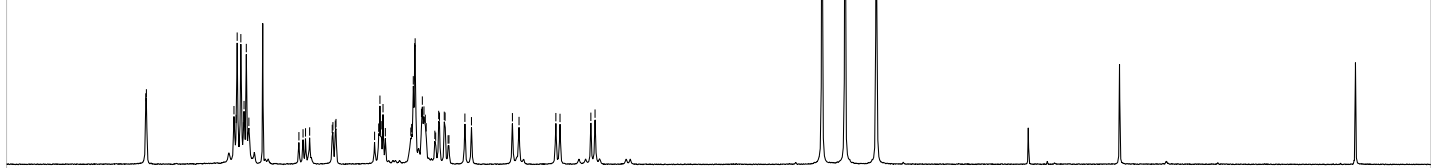

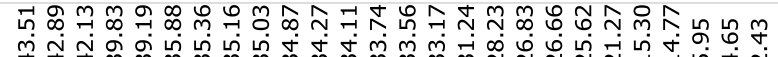

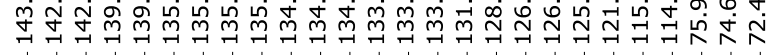

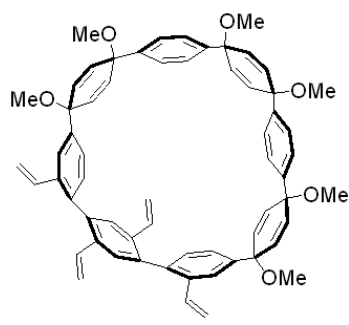

10
ㅇำ

กิ่
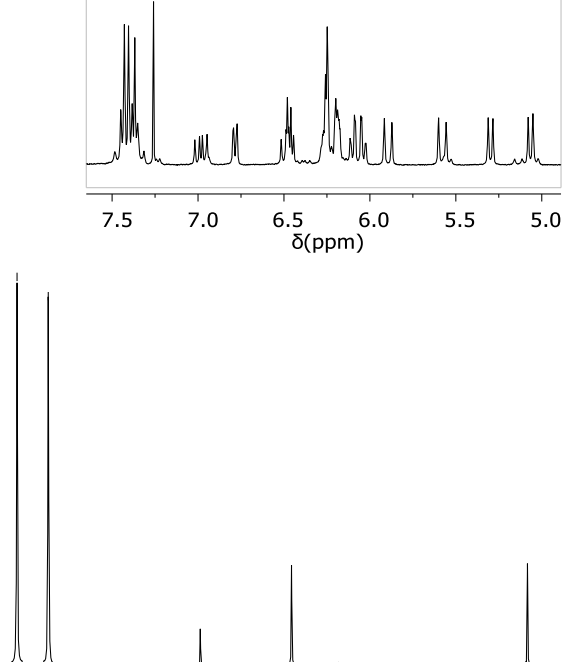

l

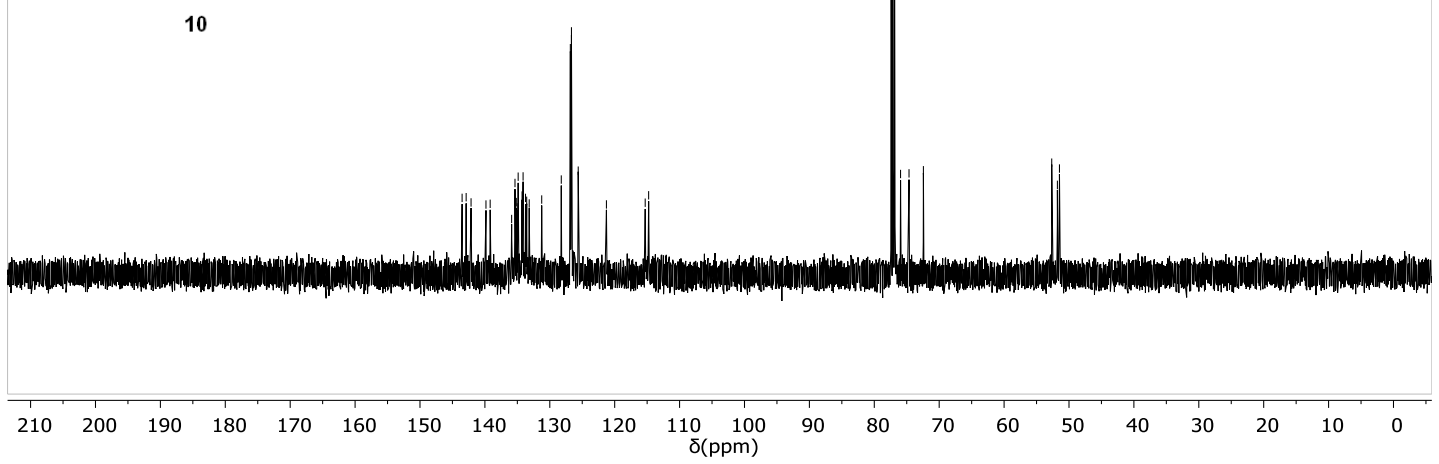




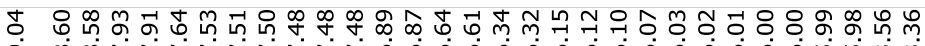

बi $\infty$ i
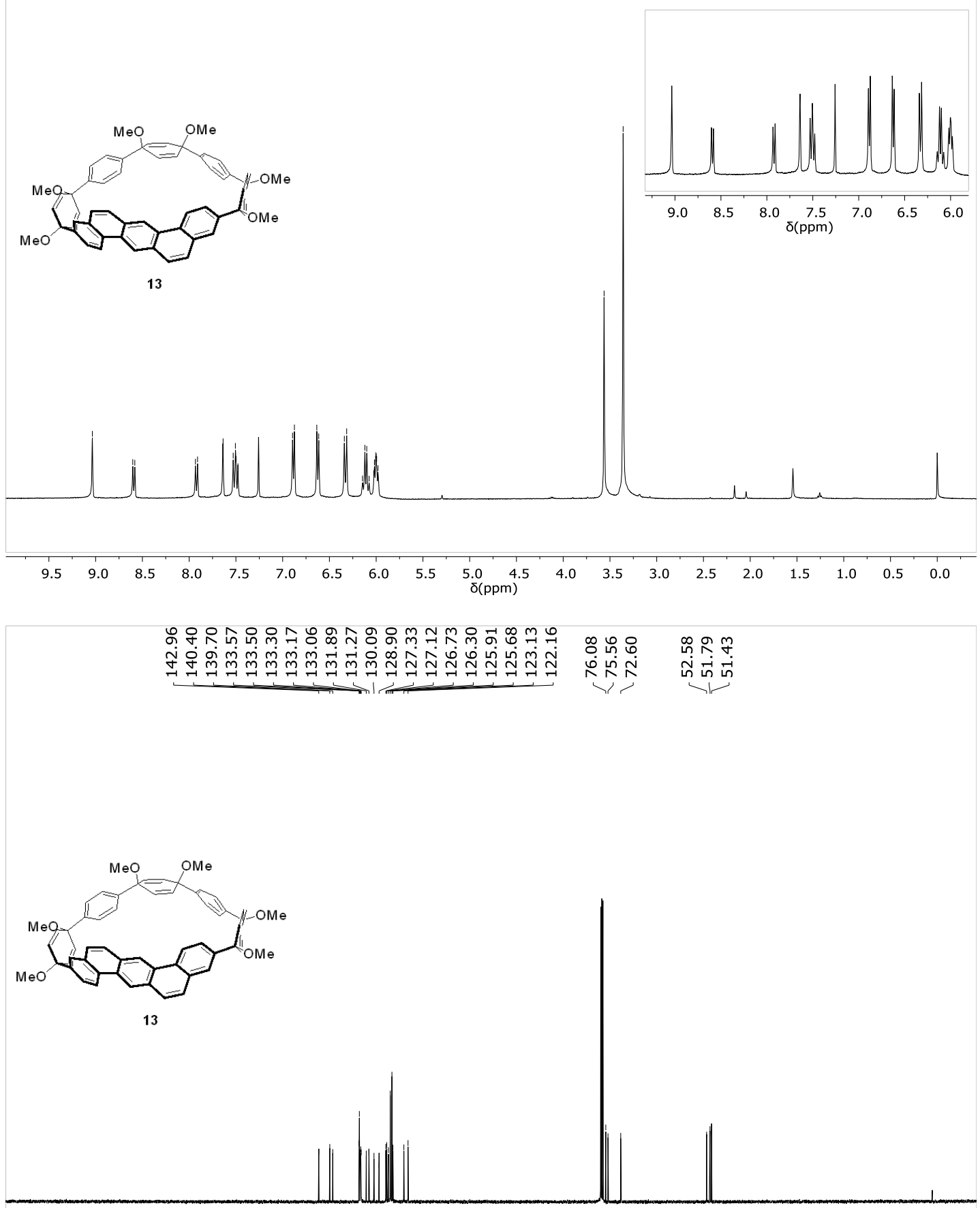

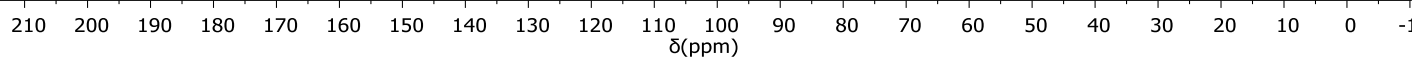




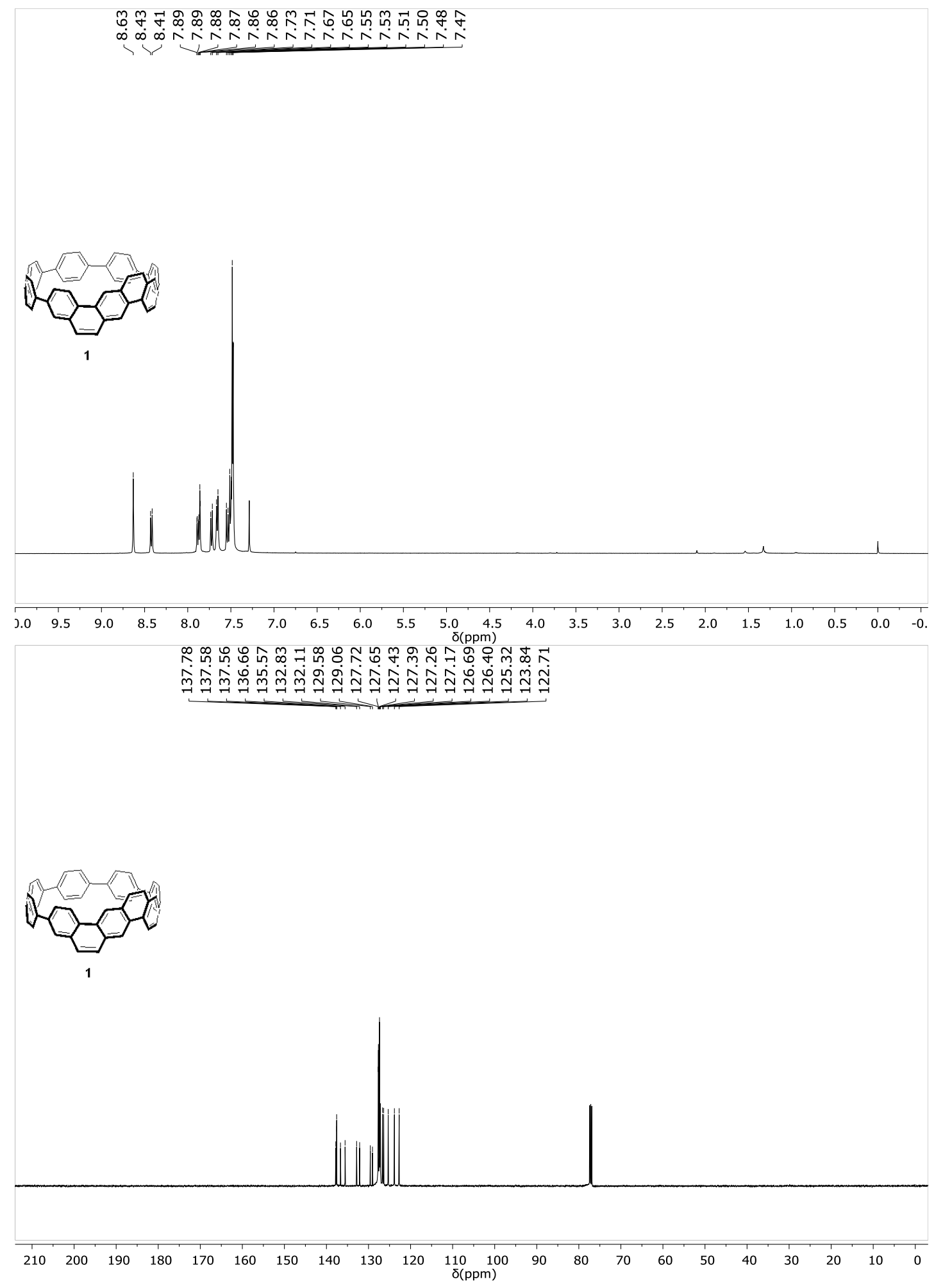




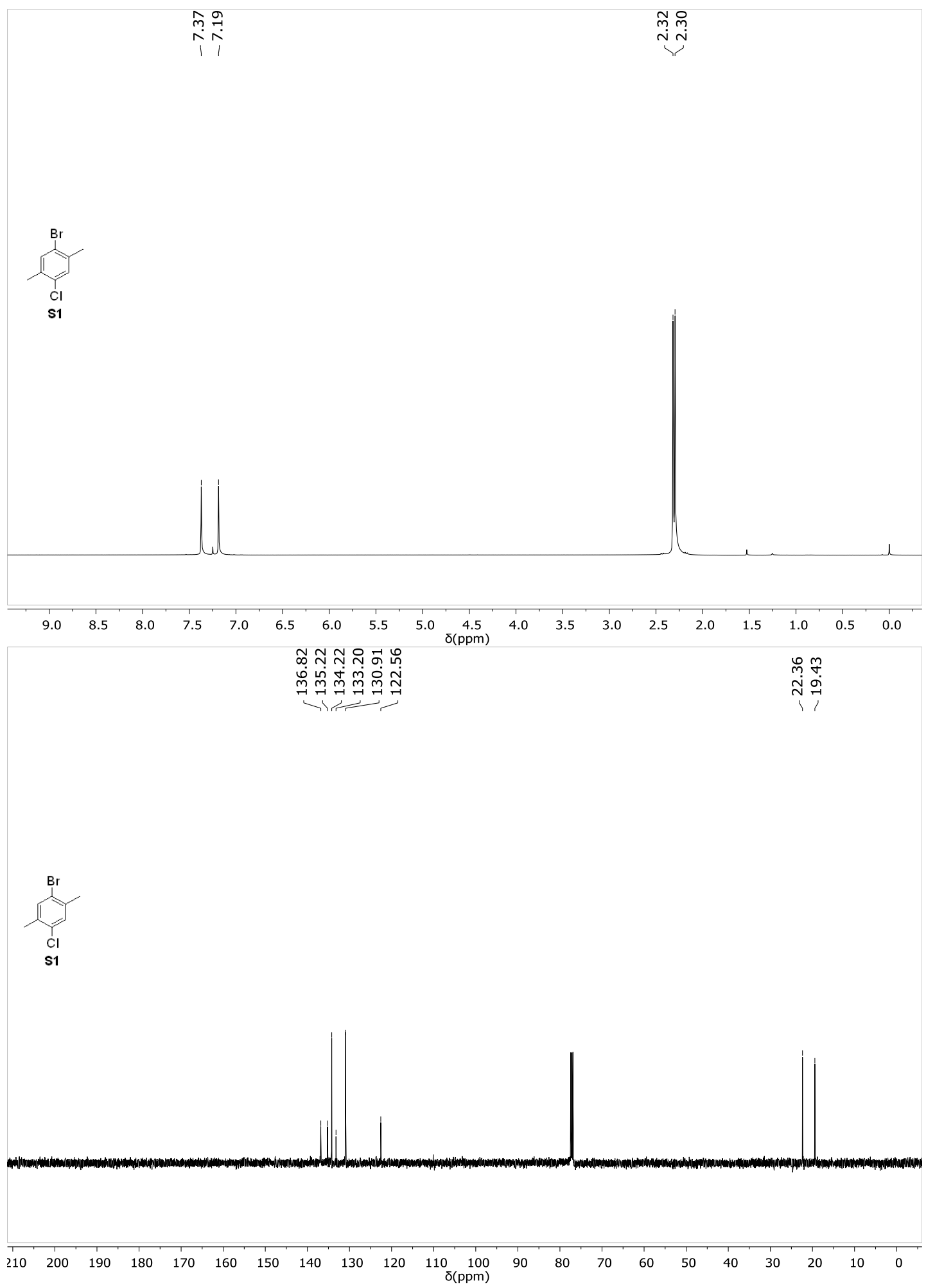




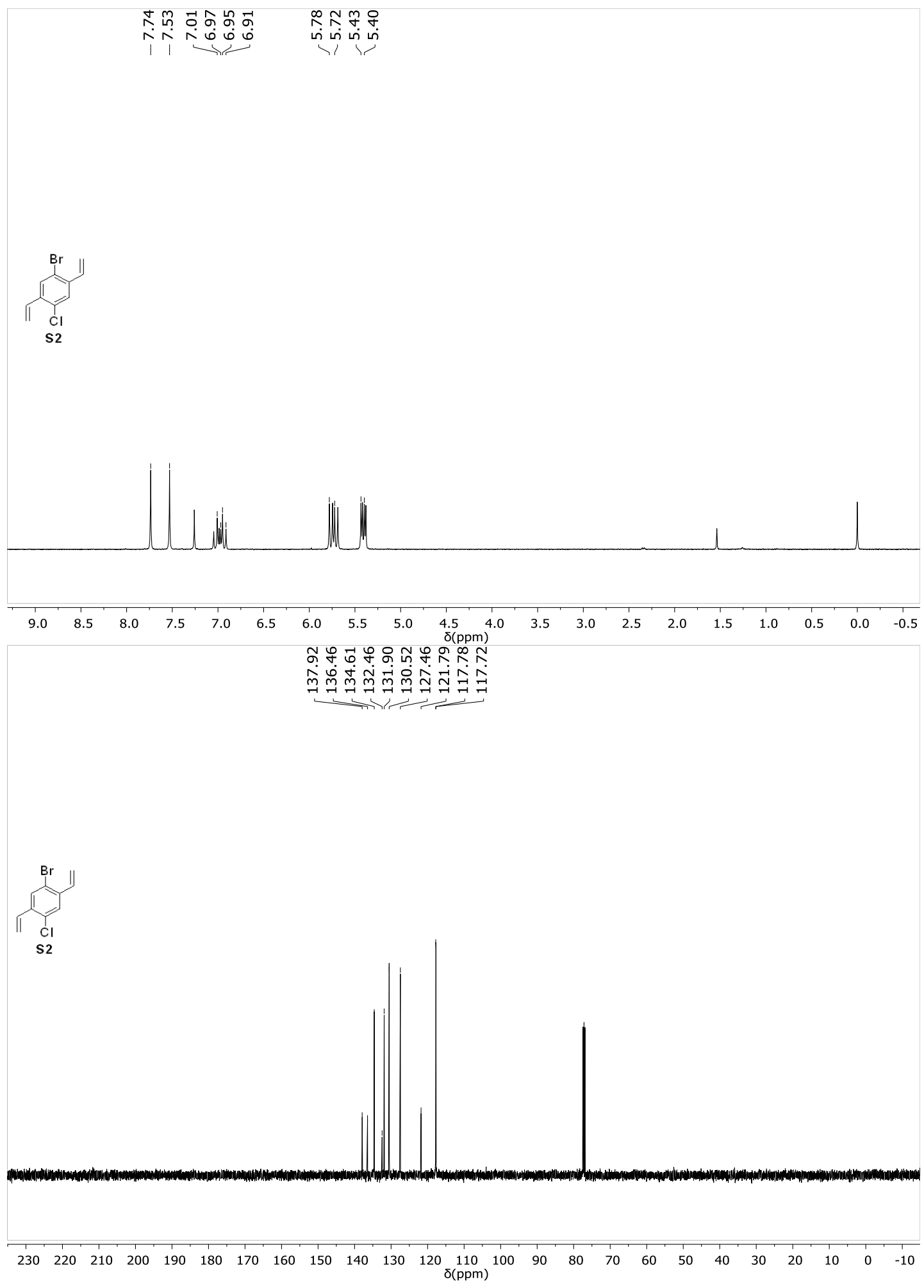




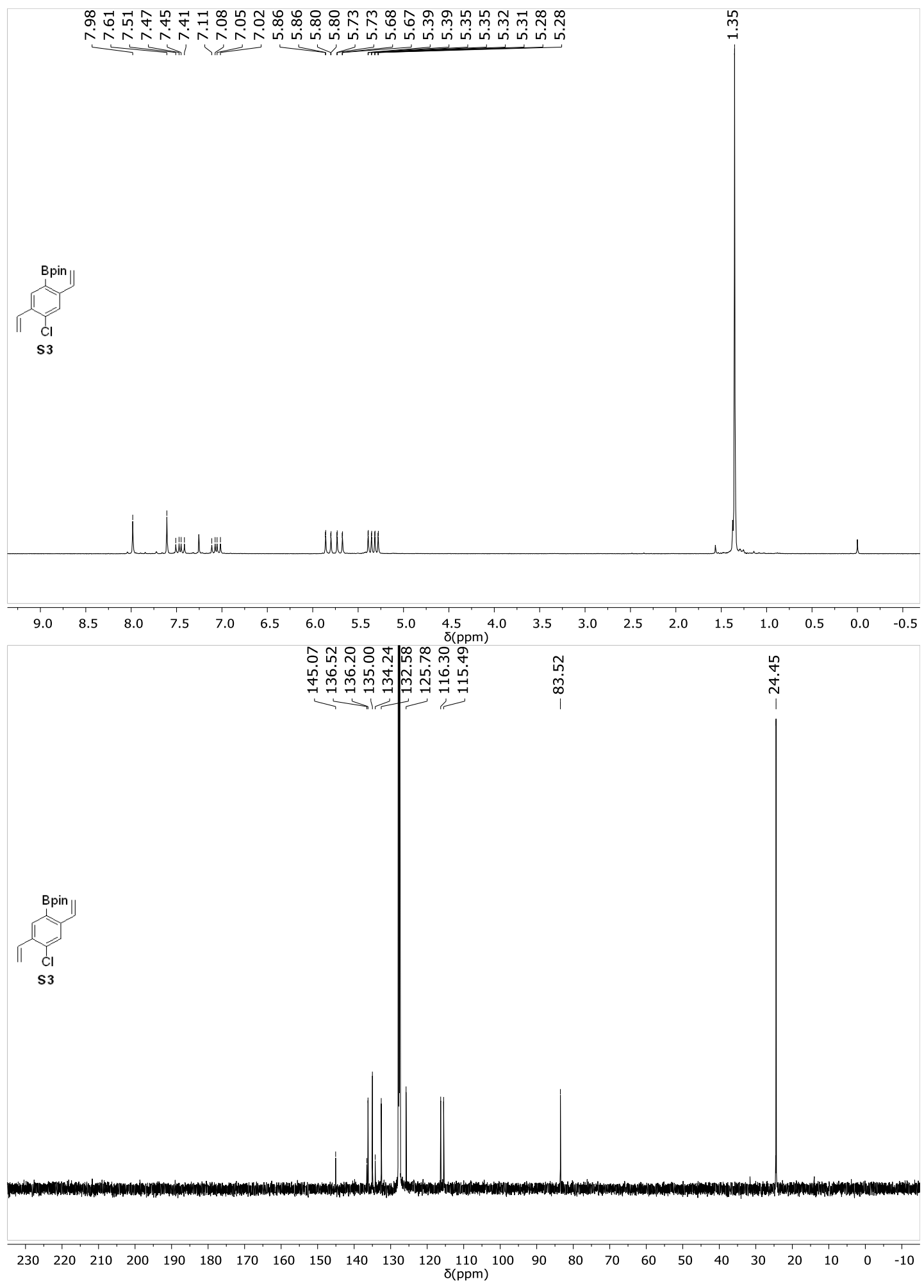




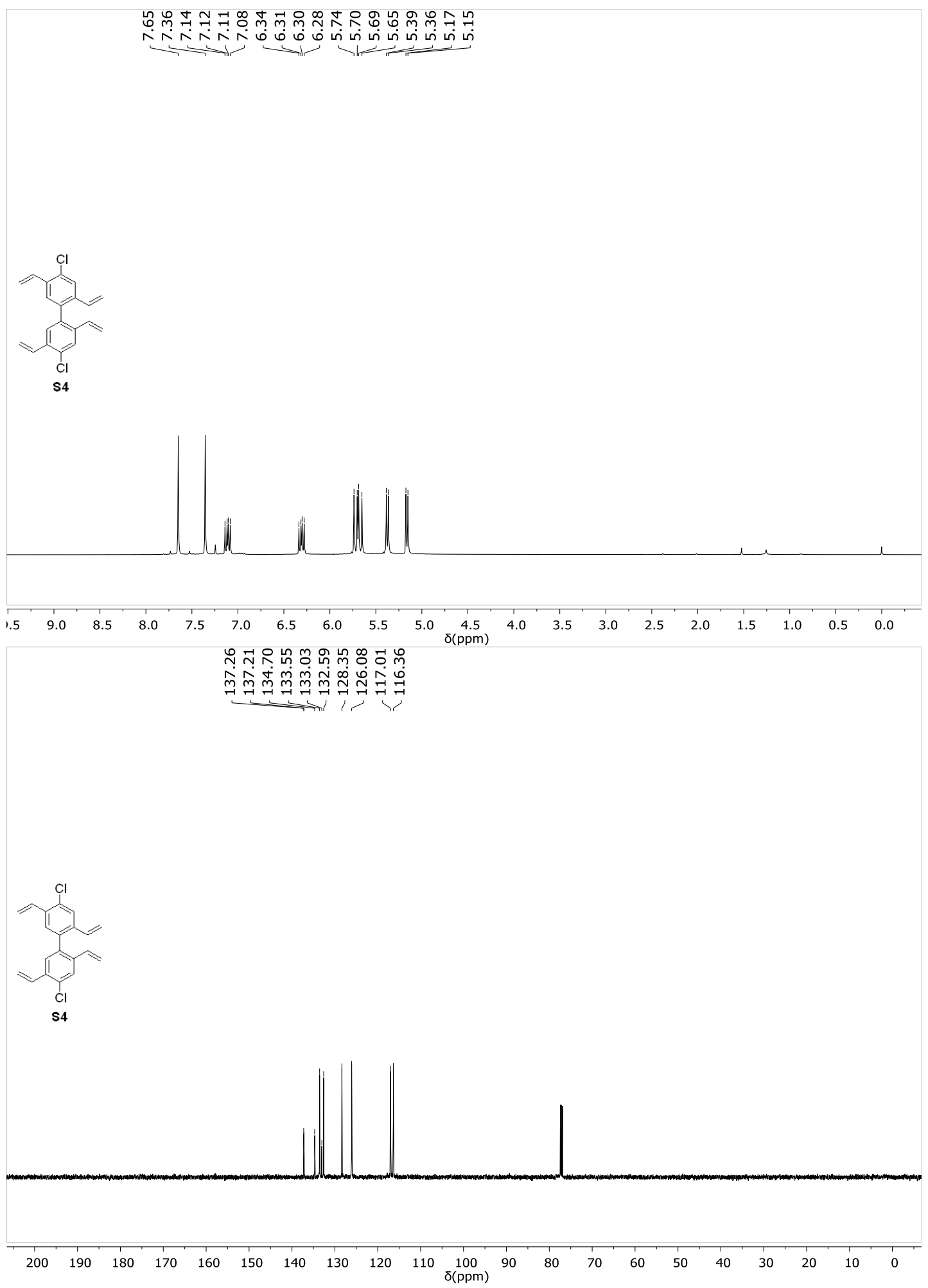




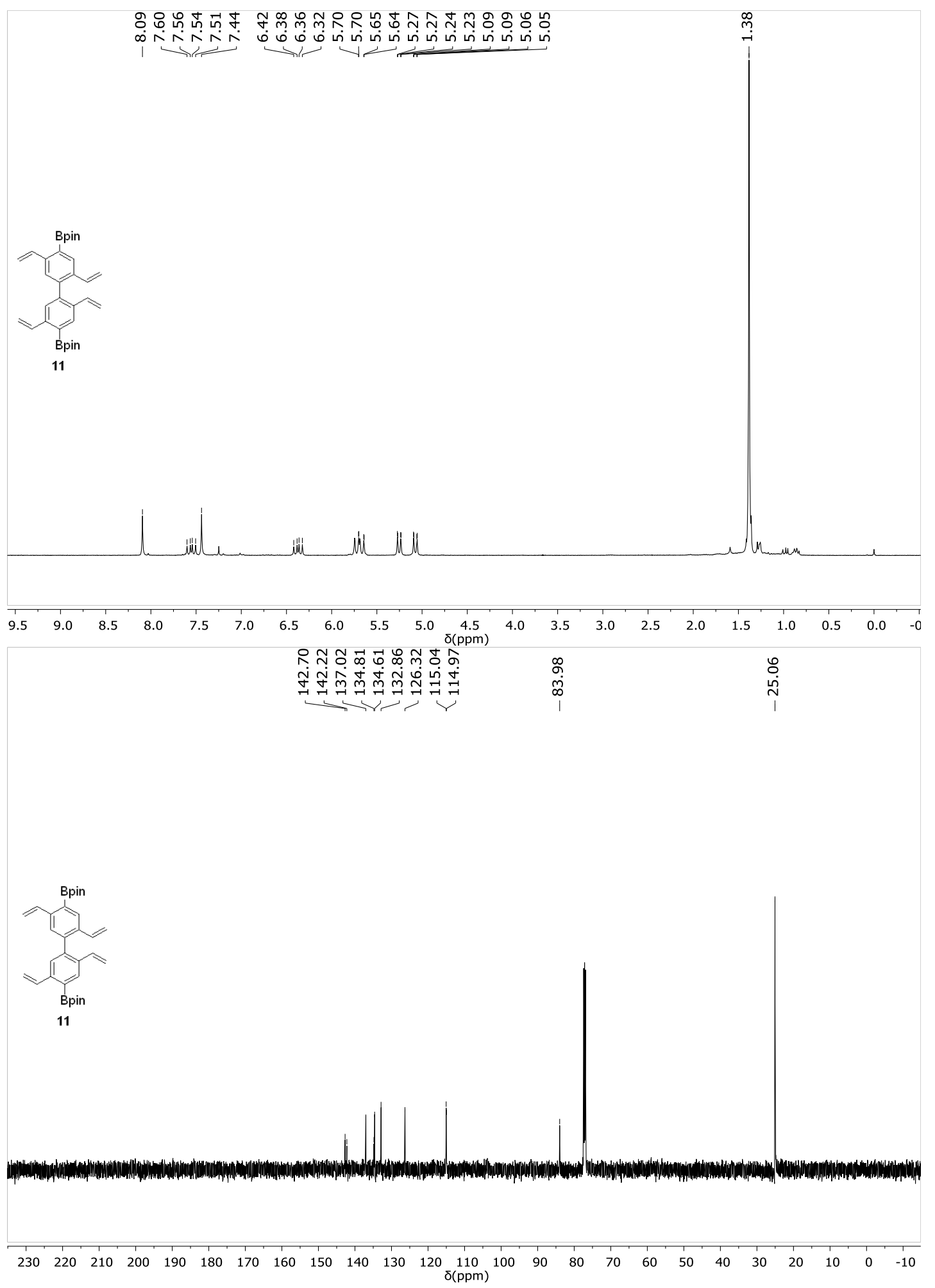




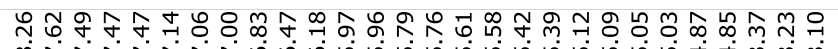

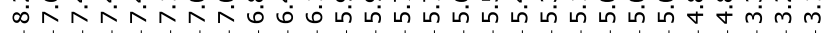

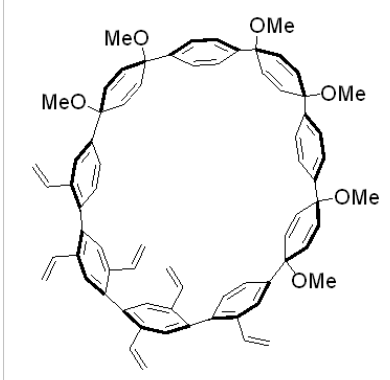

12
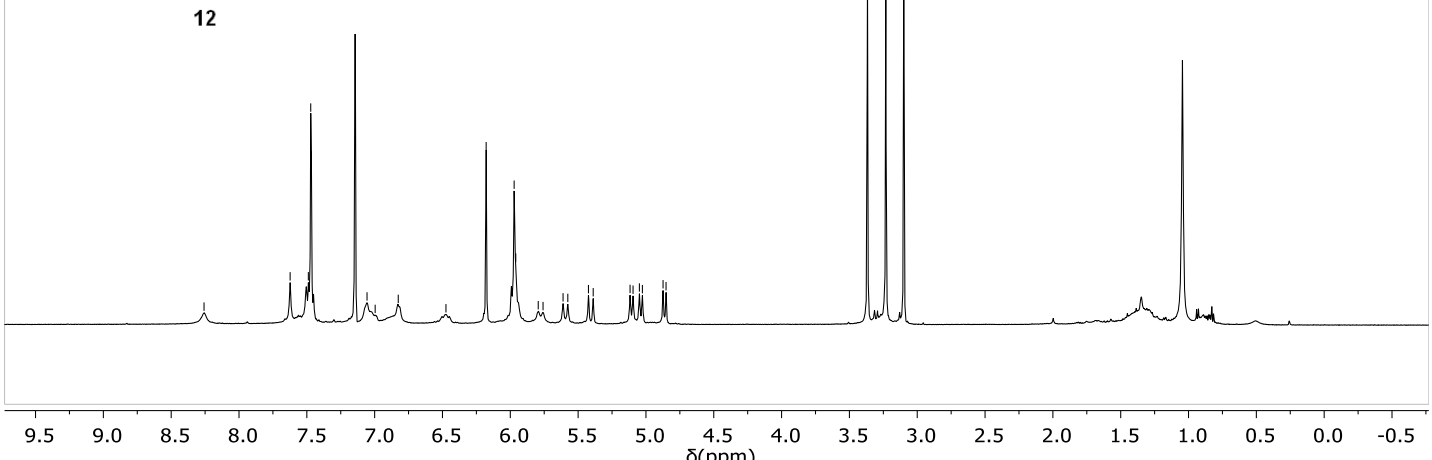

위ํ워

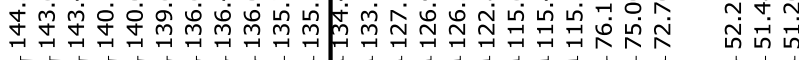

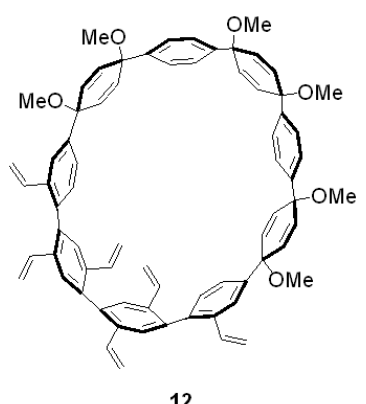

12

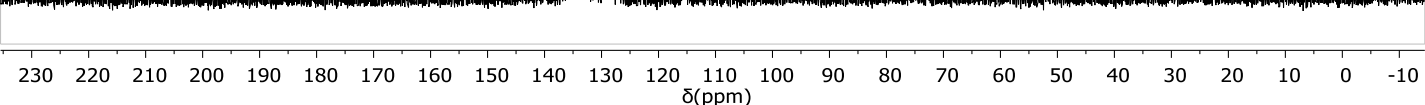




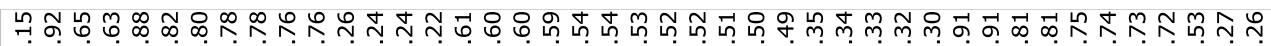

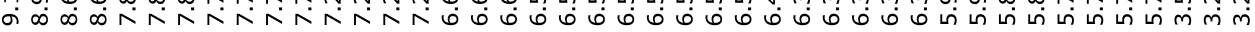

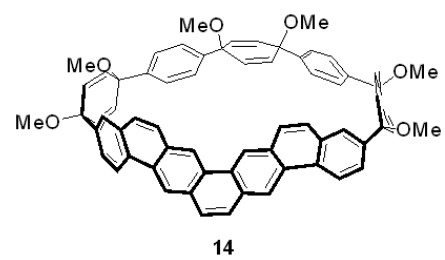
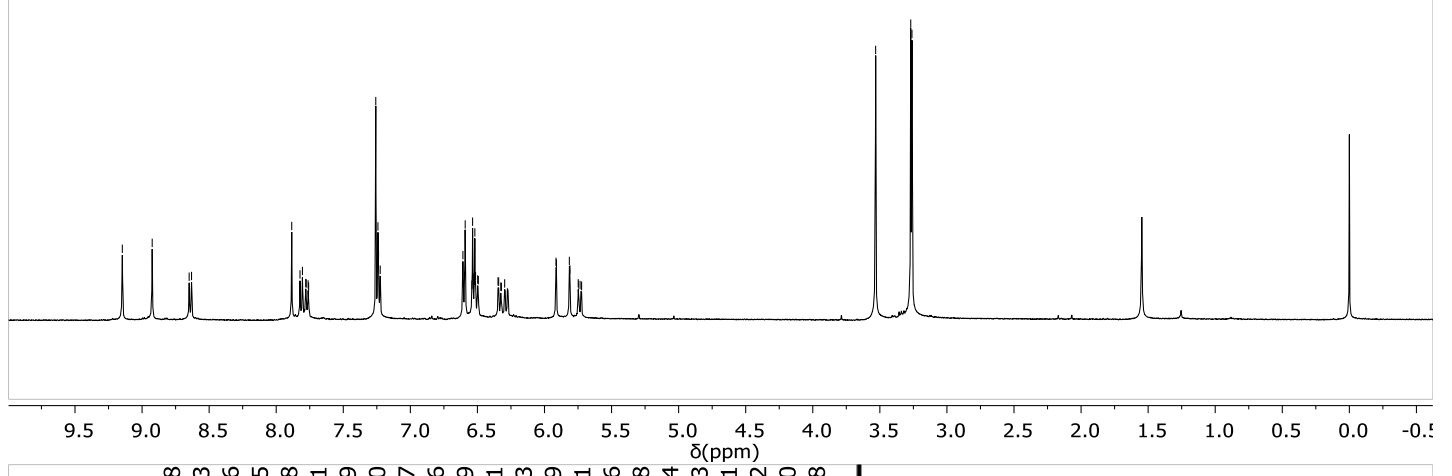

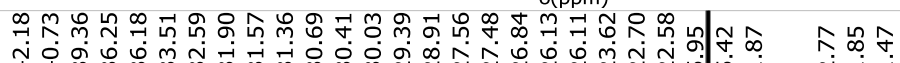

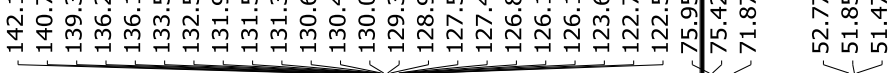

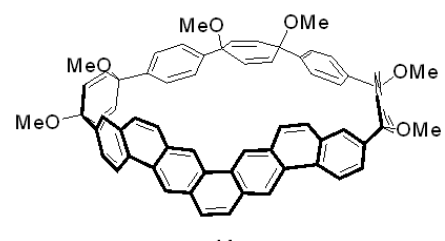

14

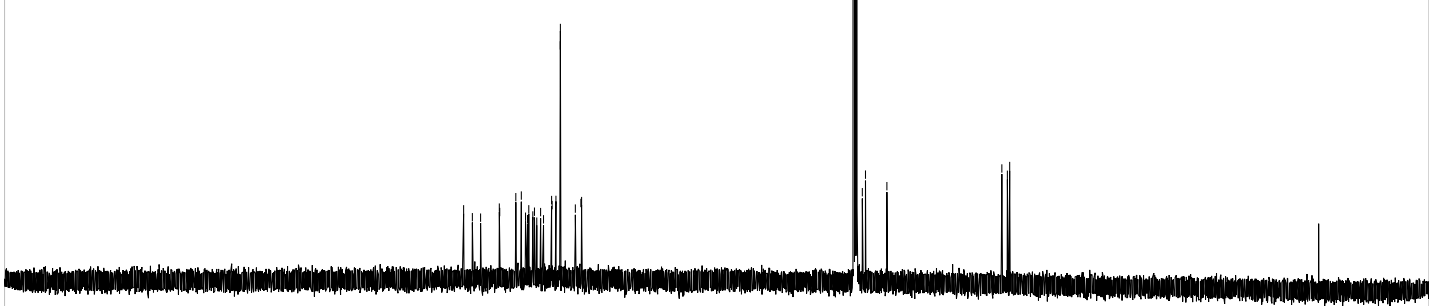

$\begin{array}{lllllllllllllllllllllll}210 & 200 & 190 & 180 & 170 & 160 & 150 & 140 & 130 & 120 & 110 & \begin{array}{c}100 \\ \delta(\mathrm{ppm})\end{array} & 90 & 80 & 70 & 60 & 50 & 40 & 30 & 20 & 10 & 0 & -10\end{array}$ 

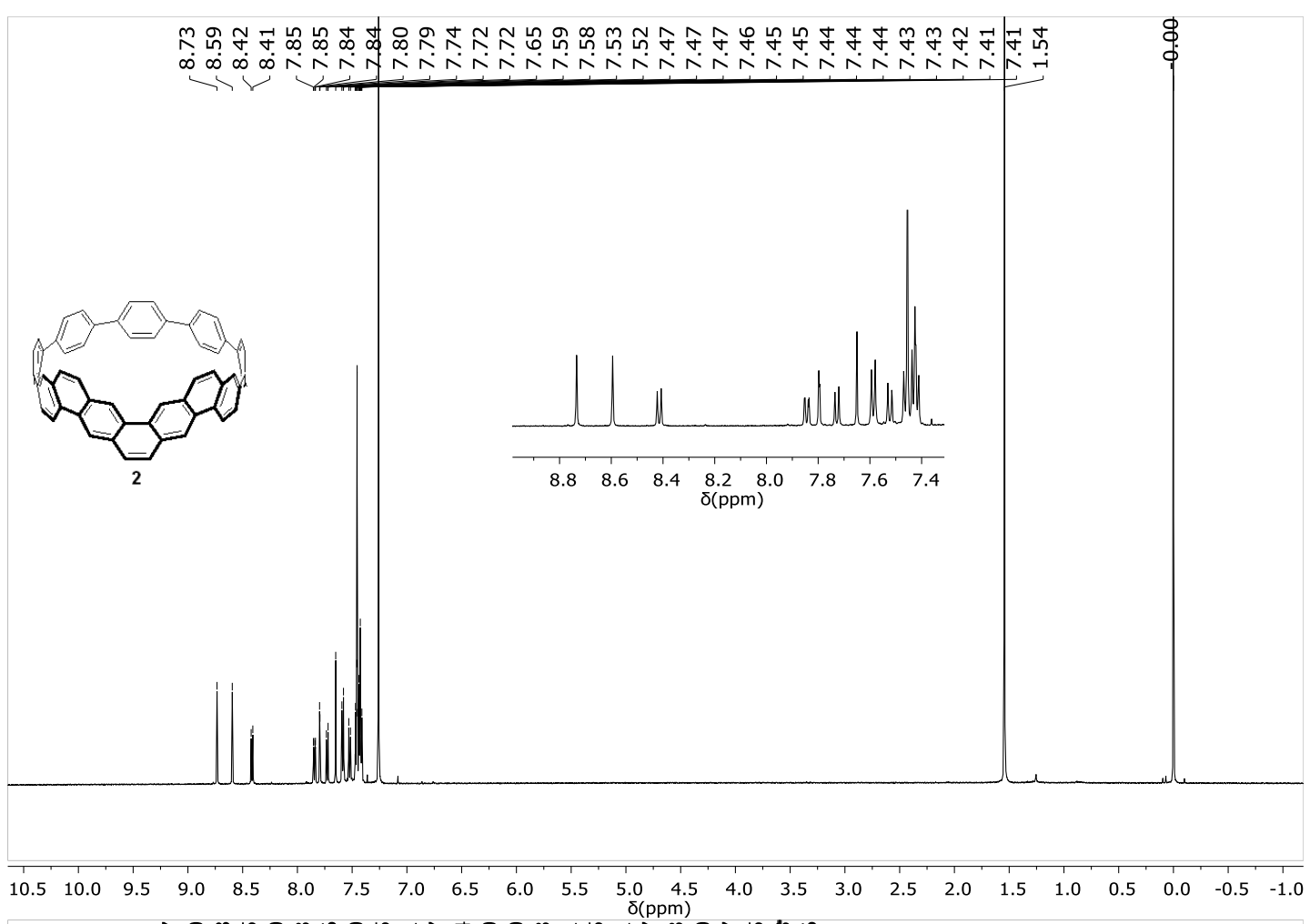

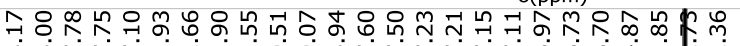

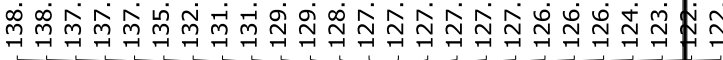
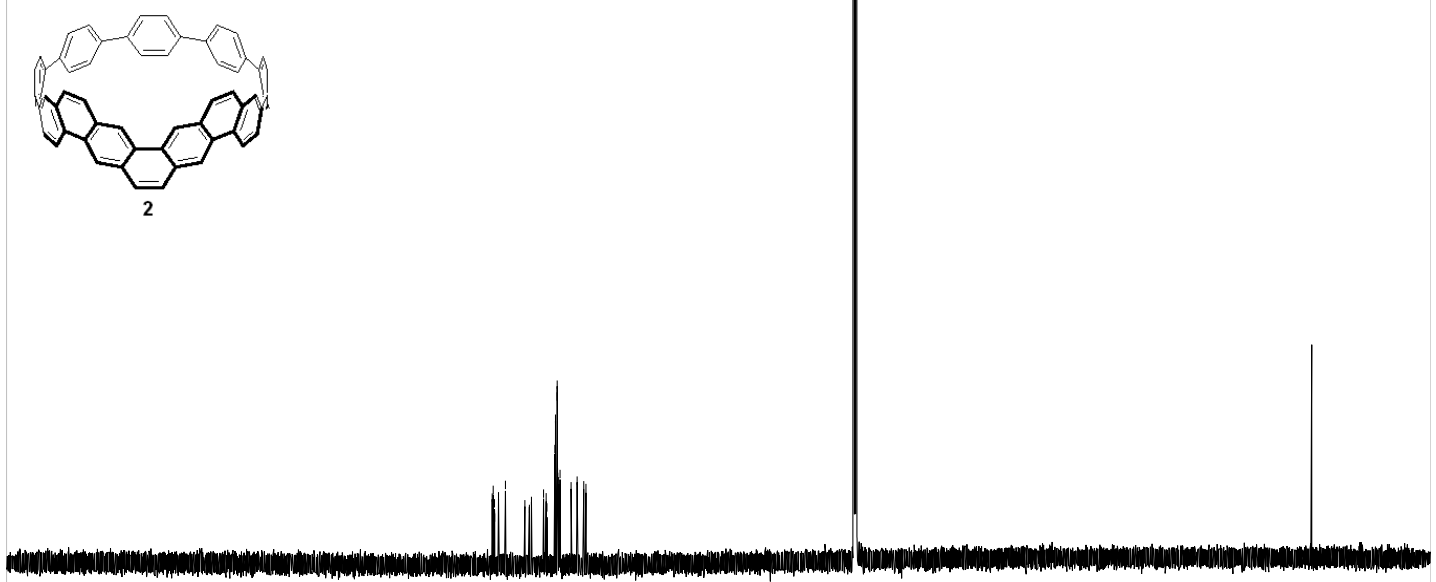

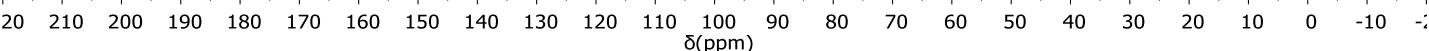



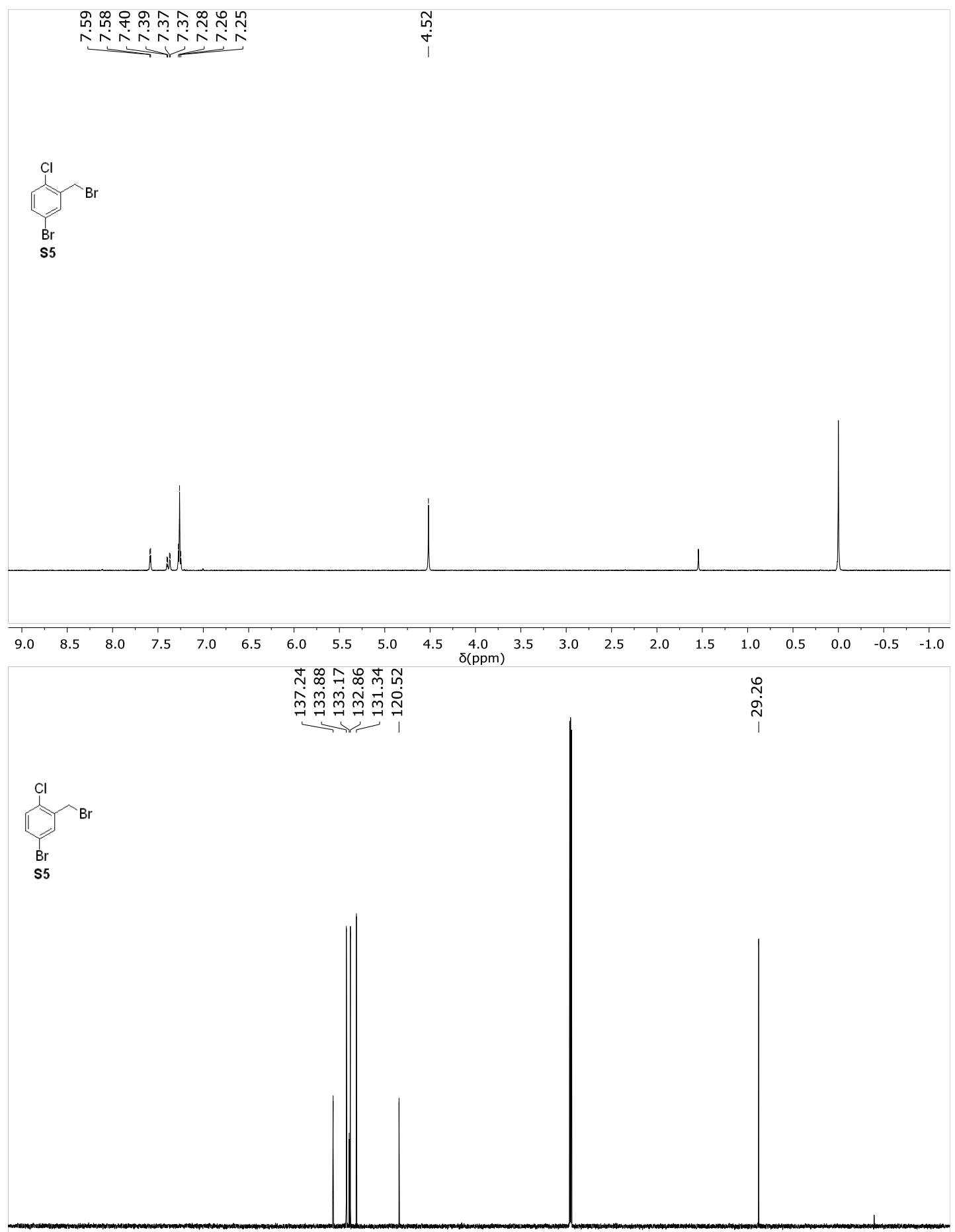

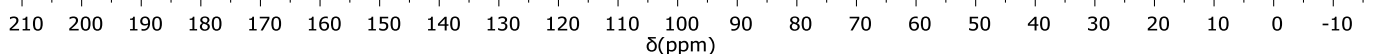




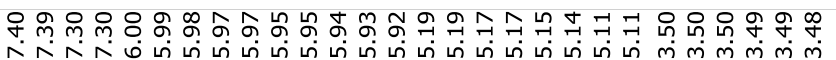
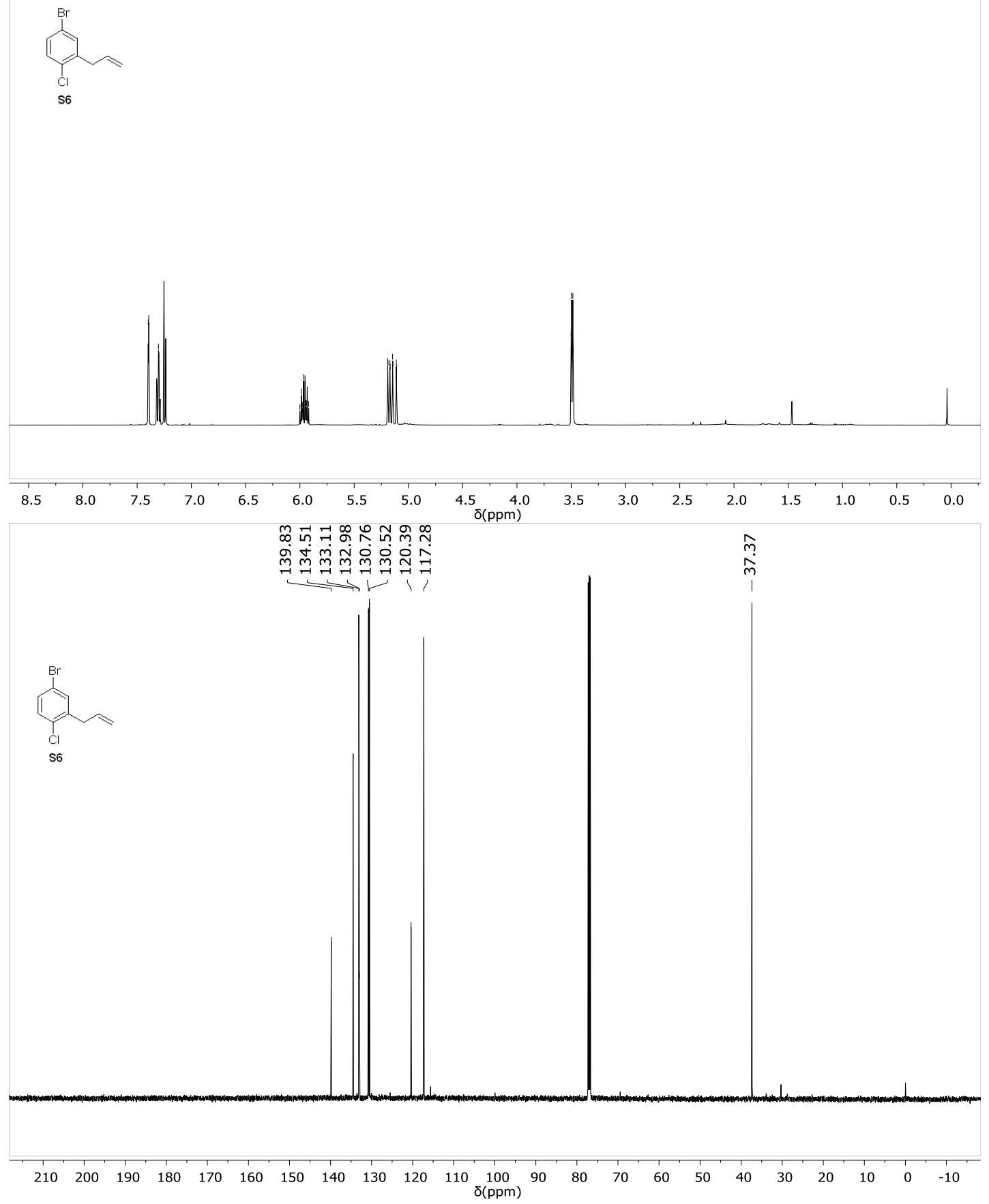

S69 

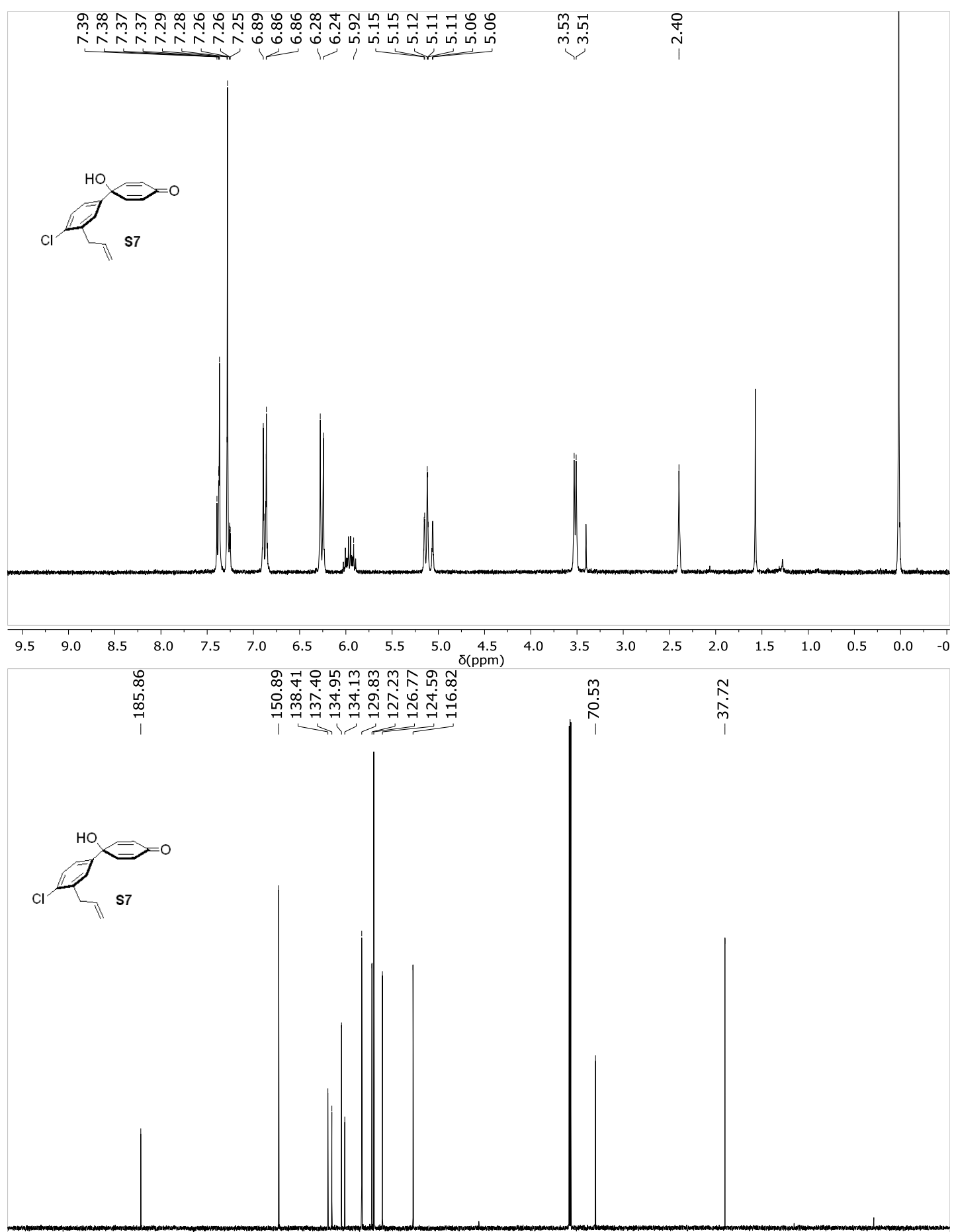

$\begin{array}{lllllllllllllllllllllll}210 & 200 & 190 & 180 & 170 & 160 & 150 & 140 & 130 & 120 & 110 & \begin{array}{c}100 \\ \delta(\mathrm{ppm})\end{array} & 90 & 80 & 70 & 60 & 50 & 40 & 30 & 20 & 10 & 0 & -10\end{array}$ 


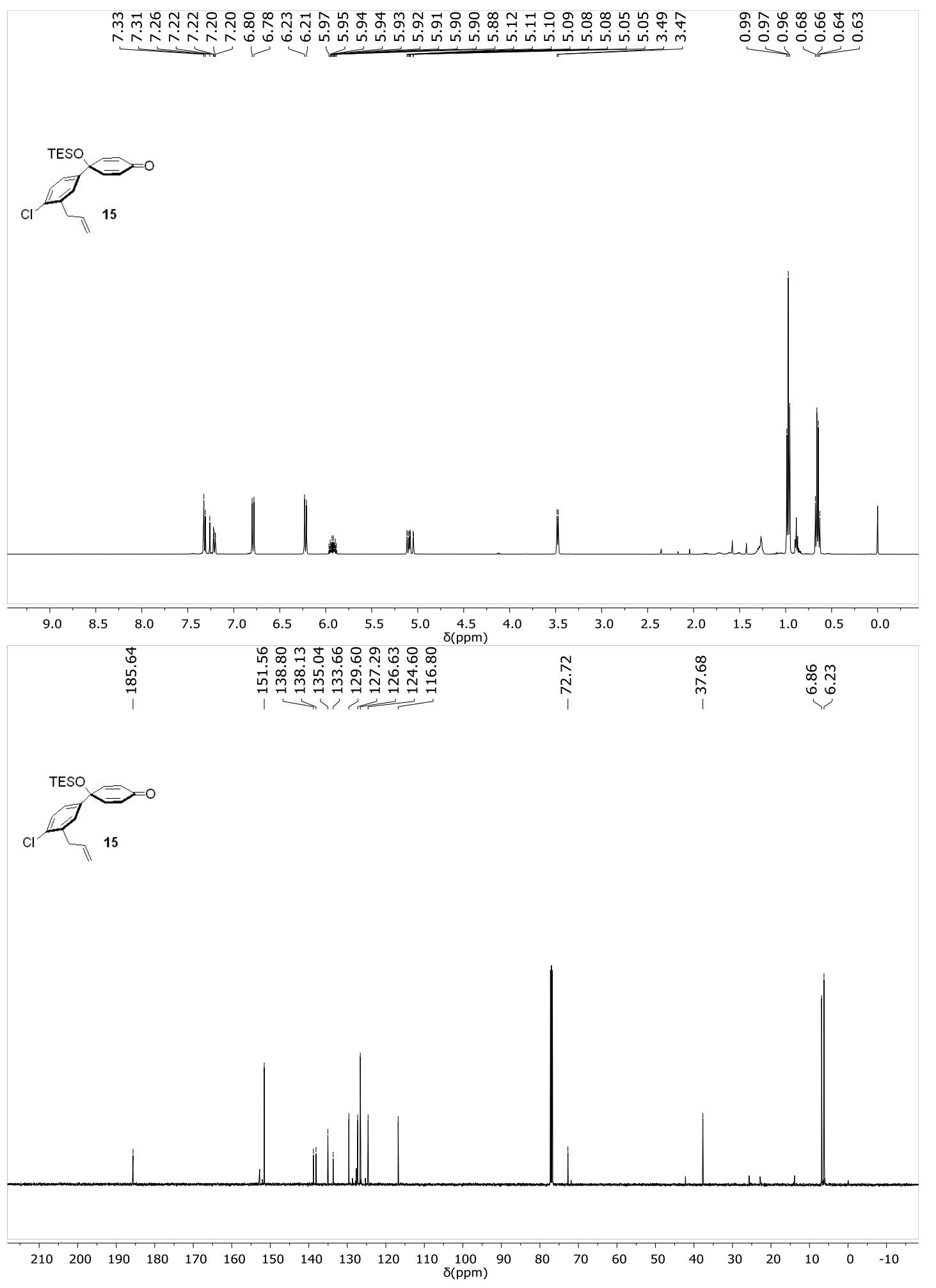




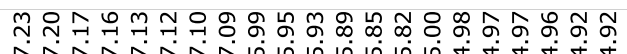

$\stackrel{\infty}{m} \stackrel{\infty}{m}$

大

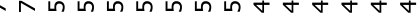
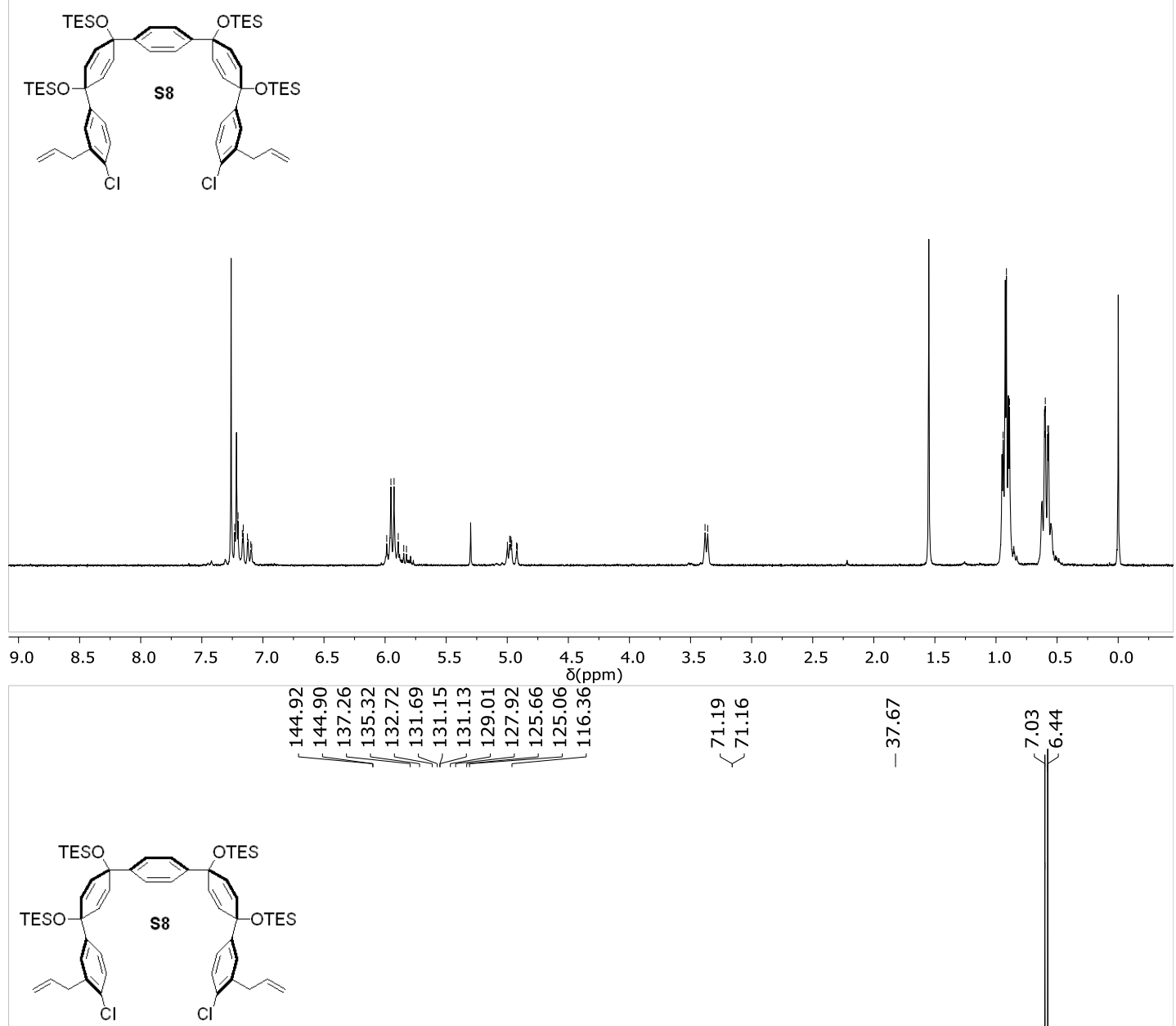

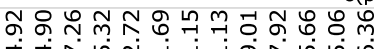

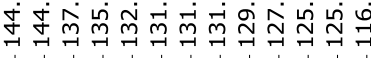

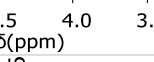

$\begin{array}{llll}5 & 3.0 & 2.5 & 2.0 \\ & & \end{array}$

仓ิ
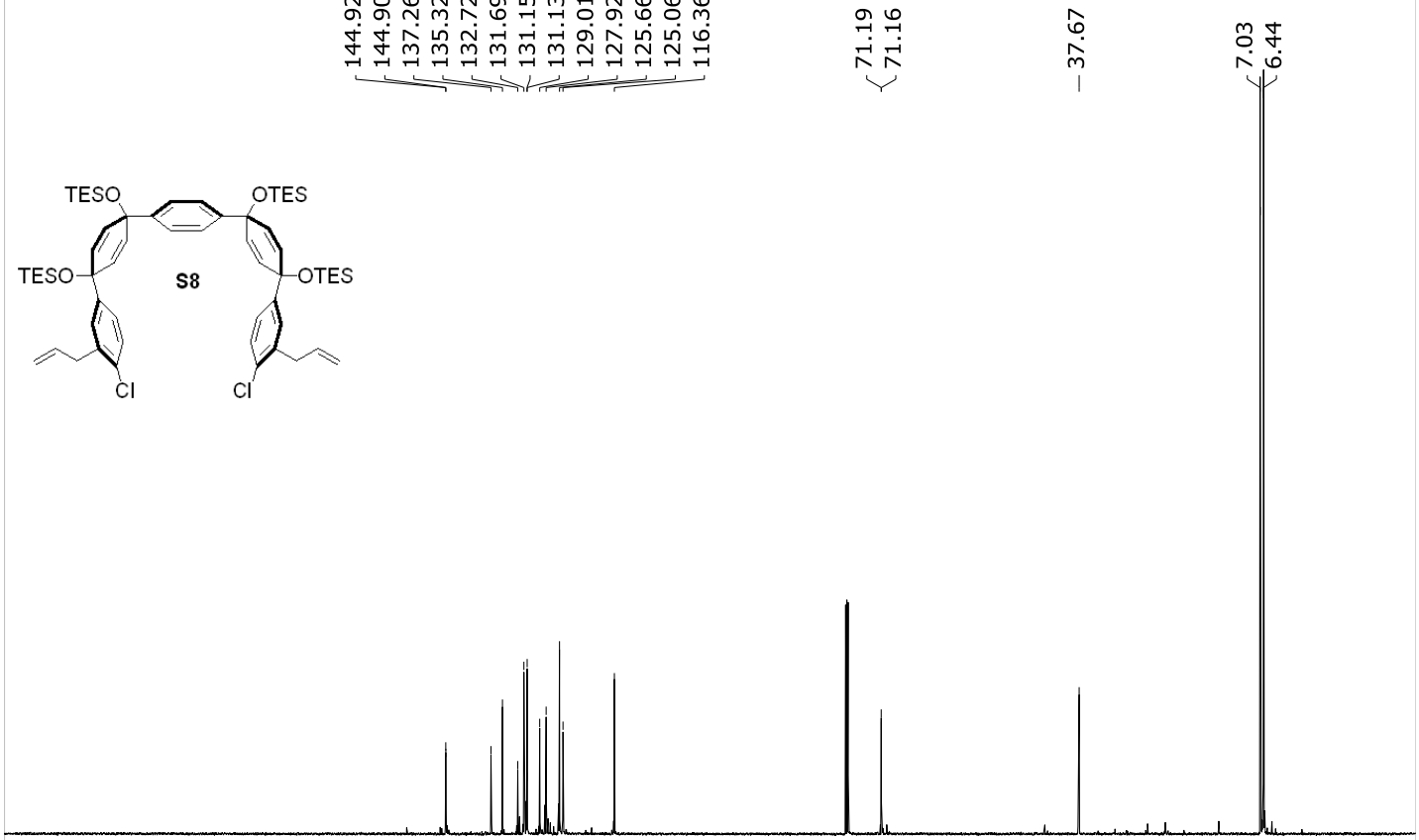

$\begin{array}{lllllllllllllllllllllll}210 & 200 & 190 & 180 & 170 & 160 & 150 & 140 & 130 & 120 & 110 & \begin{array}{c}100 \\ \delta(\mathrm{ppm})\end{array} & 90 & 80 & 70 & 60 & 50 & 40 & 30 & 20 & 10 & 0 & -10\end{array}$ 

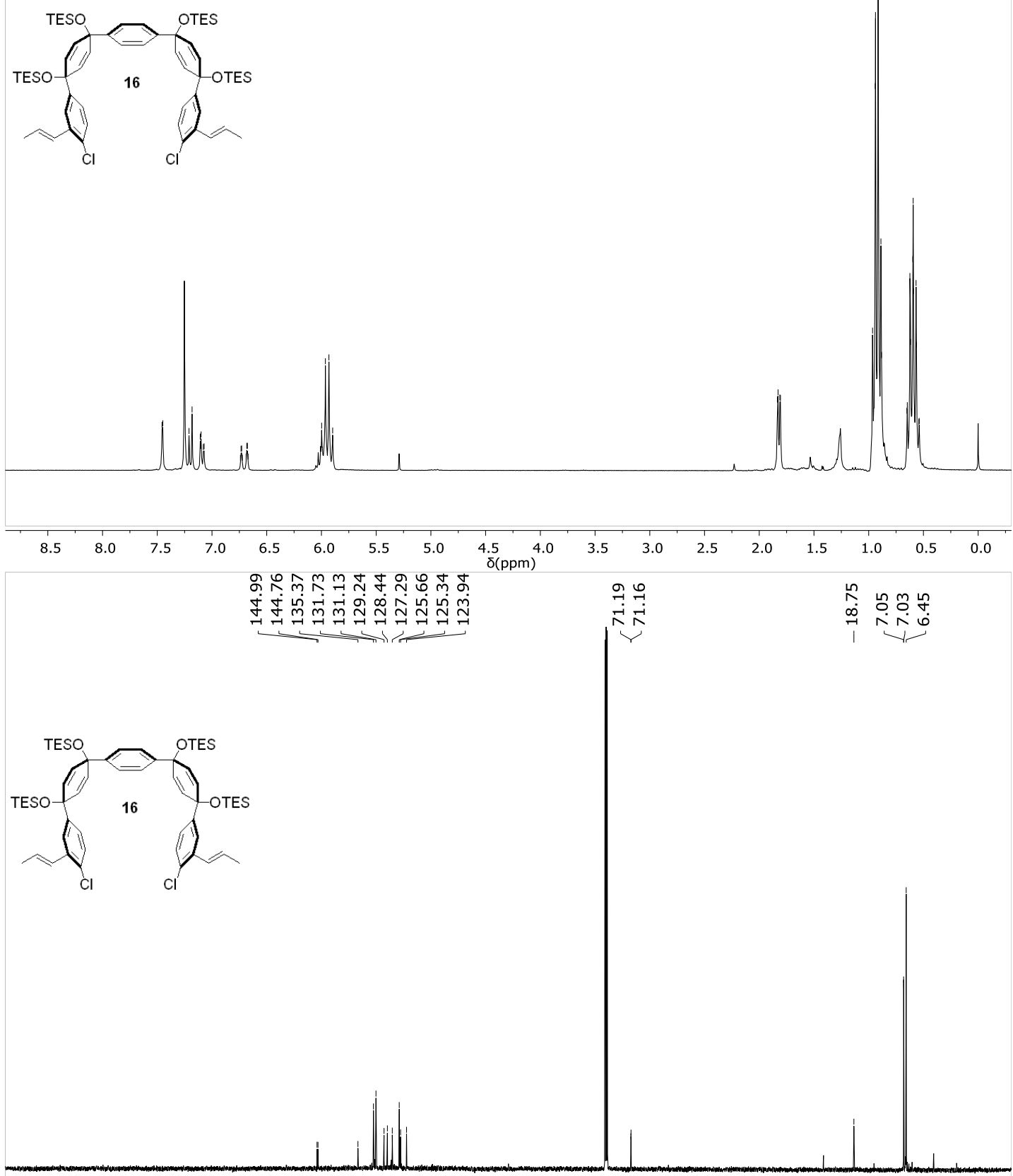

$\begin{array}{lllllllllllllllllllllll}210 & 200 & 190 & 180 & 170 & 160 & 150 & 140 & 130 & 120 & 110 & \begin{array}{c}100 \\ \delta(\mathrm{ppm})\end{array} & 90 & 80 & 70 & 60 & 50 & 40 & 30 & 20 & 10 & 0 & -10\end{array}$ 

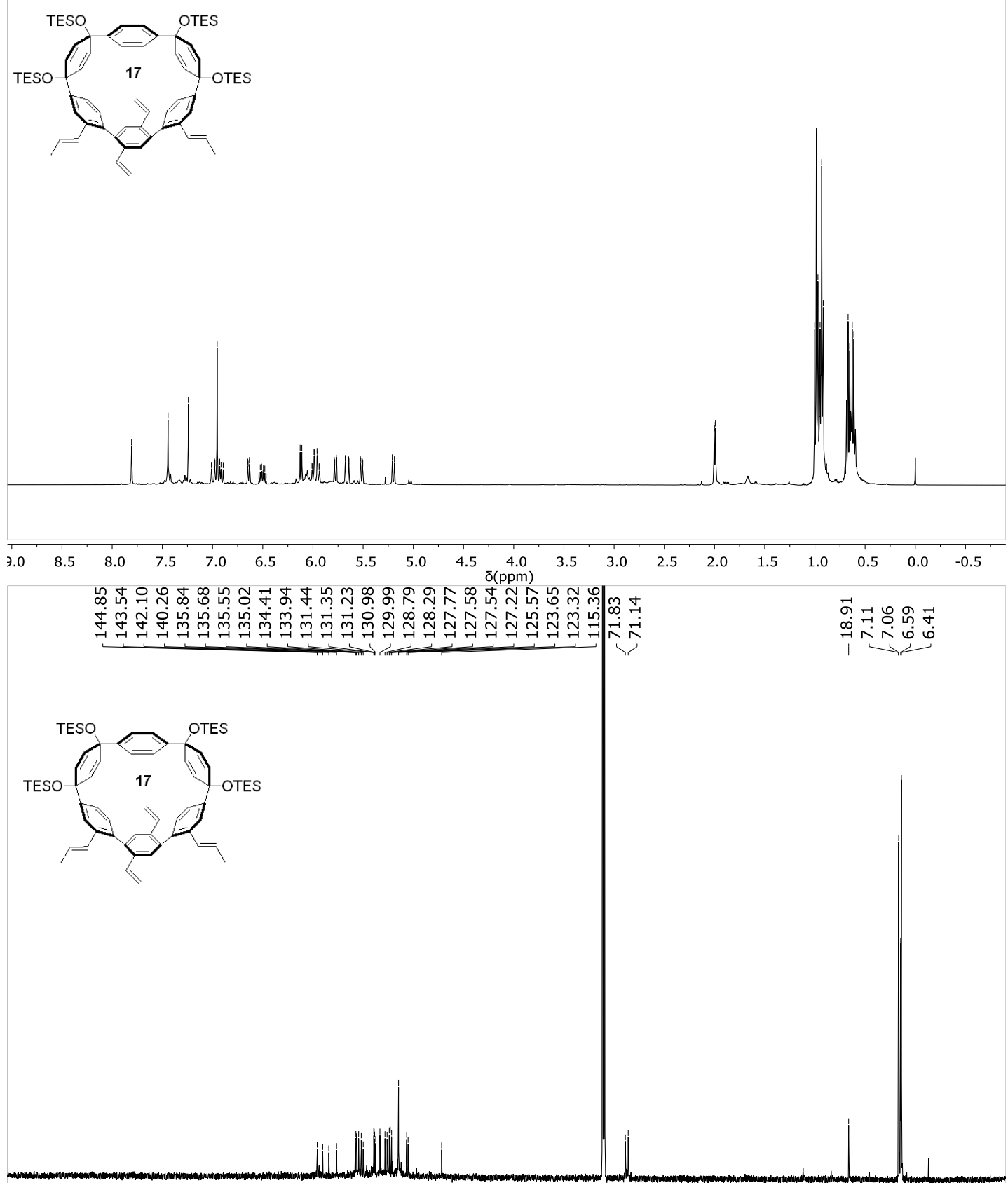

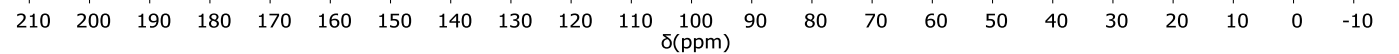



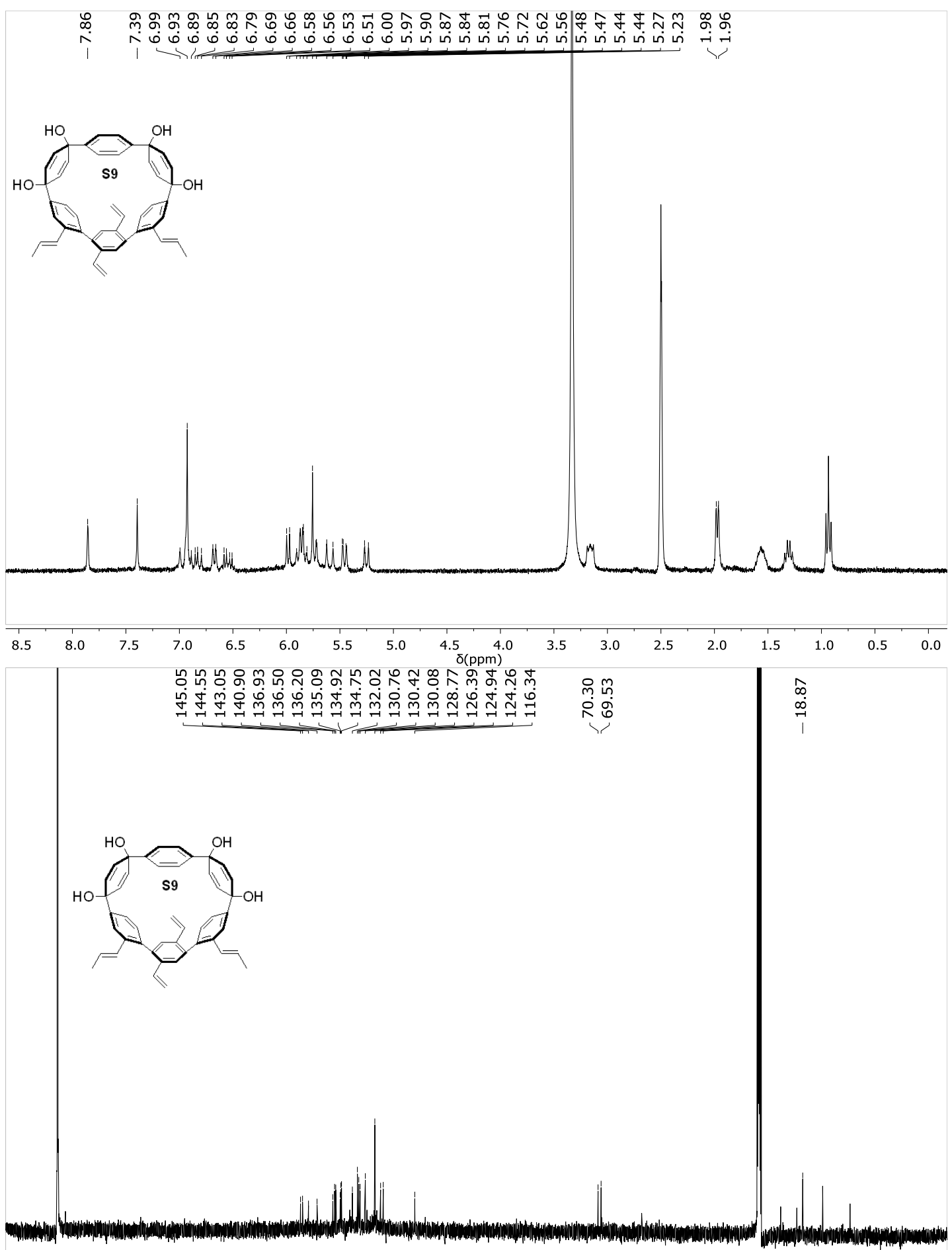

$\begin{array}{lllllllllllllllllllllll}210 & 200 & 190 & 180 & 170 & 160 & 150 & 140 & 130 & 120 & 110 & \begin{array}{c}100 \\ \delta(\mathrm{ppm})\end{array} & 90 & 80 & 70 & 60 & 50 & 40 & 30 & 20 & 10 & 0 & -10\end{array}$ 

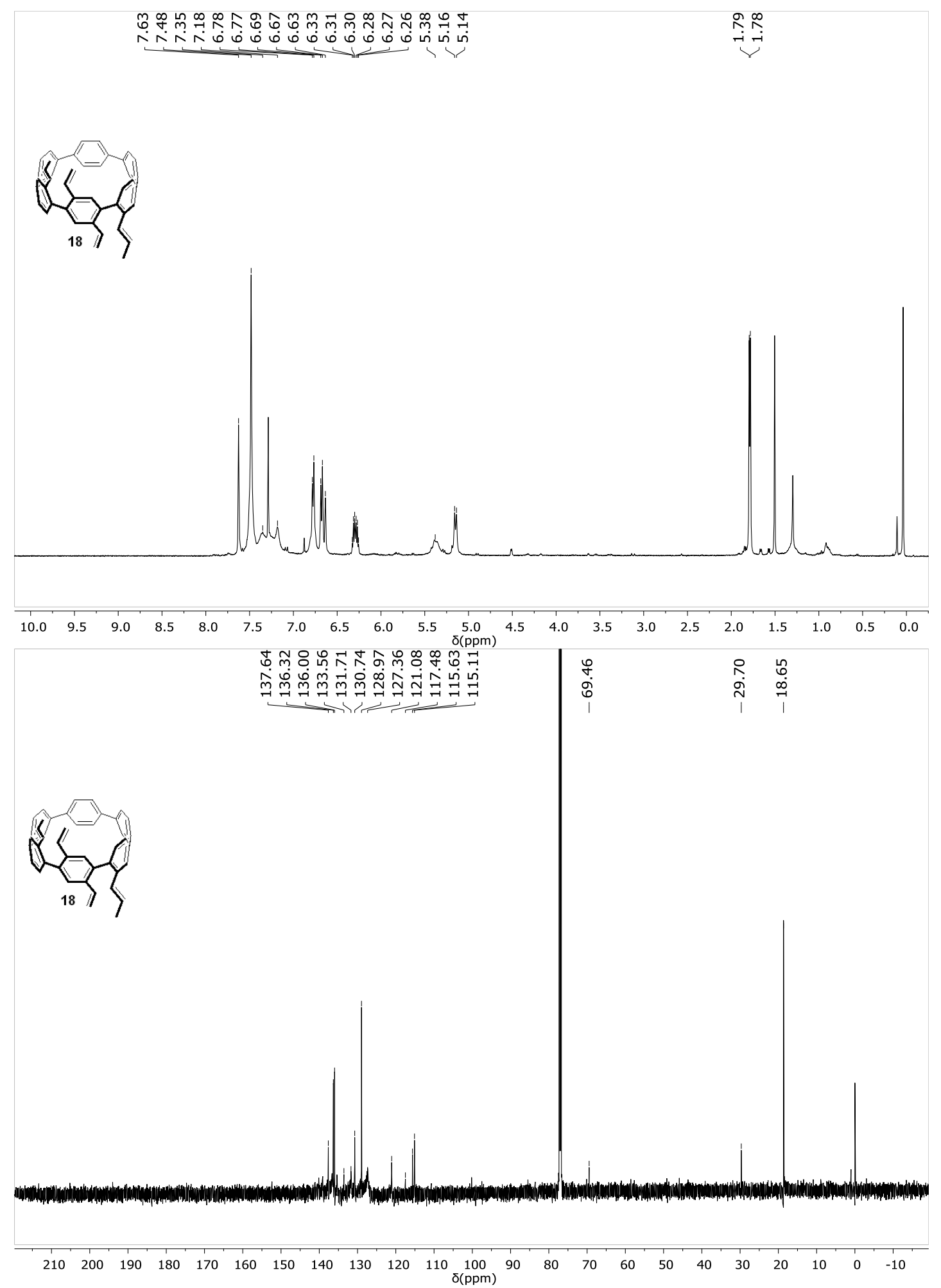


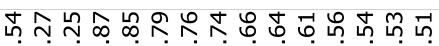

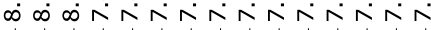

$0^{3} d$
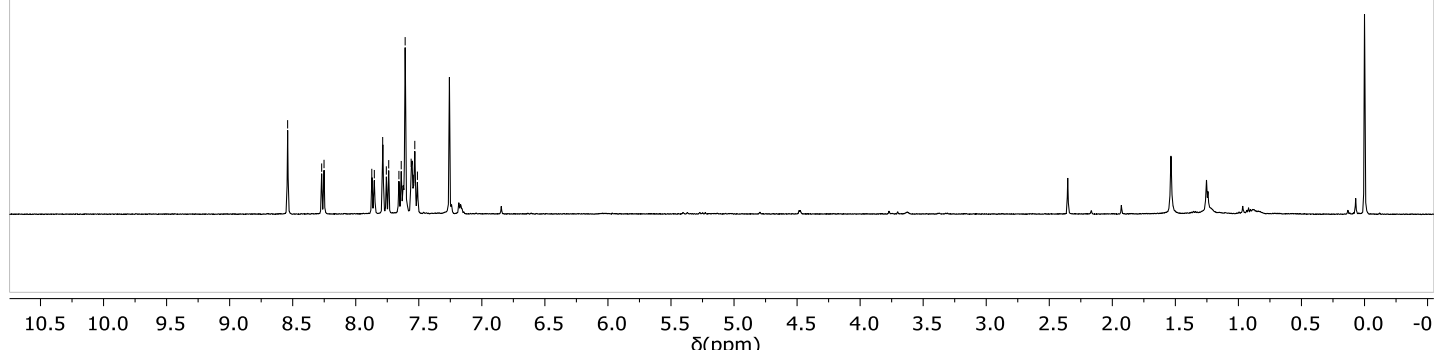

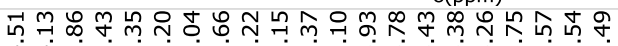

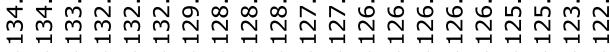
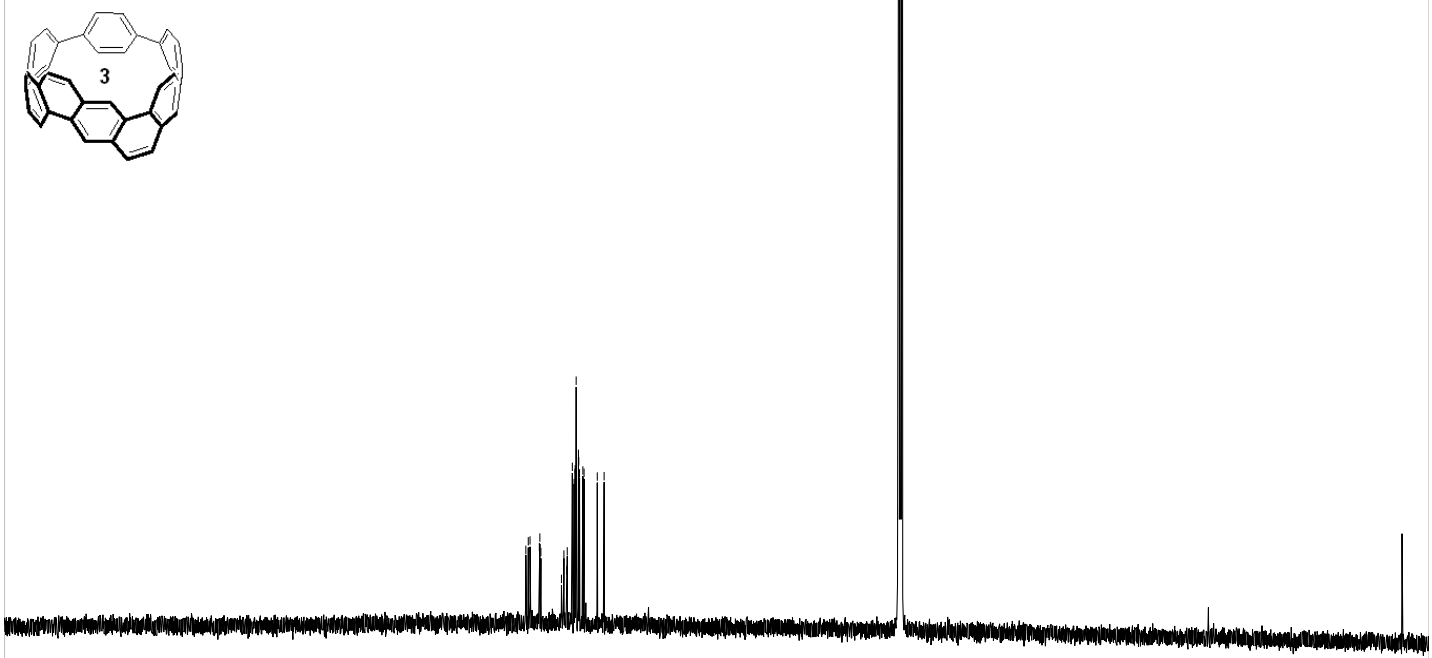

$\begin{array}{llllllllllllllllllllll}210 & 200 & 190 & 180 & 170 & 160 & 150 & 140 & 130 & 120 & \begin{array}{c}110 \\ \delta(\mathrm{ppm})\end{array} & 100 & 90 & 80 & 70 & 60 & 50 & 40 & 30 & 20 & 10 & 0\end{array}$ 


\section{8) Cartesian Coordinates of Selected Compounds.}

\begin{tabular}{|c|c|c|c|}
\hline $\mathrm{C}$ & 0.0258 & -0.029 & -0.0132 \\
\hline $\mathrm{C}$ & 1.3937 & -0.0333 & 0.0044 \\
\hline $\mathrm{C}$ & 2.119 & 1.1724 & 0.039 \\
\hline $\mathrm{C}$ & 1.4005 & 2.3345 & 0.3595 \\
\hline $\mathrm{C}$ & -0.001 & 2.3203 & 0.3219 \\
\hline $\mathrm{C}$ & -0.7016 & 1.1831 & -0.0418 \\
\hline $\mathrm{C}$ & 3.499 & 1.308 & -0.4127 \\
\hline $\mathrm{C}$ & 4.2035 & 0.2605 & -0.9969 \\
\hline $\mathrm{C}$ & 5.2874 & 0.4945 & -1.8318 \\
\hline $\mathrm{C}$ & 5.7388 & 1.8197 & -2.0591 \\
\hline $\mathrm{C}$ & 5.1924 & 2.828 & -1.2679 \\
\hline $\mathrm{C}$ & 4.0721 & 2.602 & -0.475 \\
\hline $\mathrm{C}$ & 6.5594 & 2.0765 & -3.2415 \\
\hline $\mathrm{C}$ & 6.7965 & 1.0139 & -4.1284 \\
\hline $\mathrm{C}$ & 7.0908 & 1.2782 & -5.4735 \\
\hline $\mathrm{C}$ & 7.1363 & 2.5712 & -5.9584 \\
\hline $\mathrm{C}$ & 7.2206 & 3.6012 & -4.9952 \\
\hline $\mathrm{C}$ & 6.9396 & 3.3609 & -3.6772 \\
\hline $\mathrm{C}$ & 2.1174 & 3.5714 & 0.5616 \\
\hline $\mathrm{C}$ & 3.391 & 3.6967 & 0.1737 \\
\hline $\mathrm{C}$ & 5.8671 & -0.5897 & -2.5852 \\
\hline $\mathrm{C}$ & 6.5768 & -0.3431 & -3.6887 \\
\hline $\mathrm{H}$ & -0.4972 & -0.9715 & -0.1013 \\
\hline $\mathrm{H}$ & 1.9153 & -0.9788 & -0.0589 \\
\hline $\mathrm{H}$ & -0.5202 & 3.2557 & 0.4833 \\
\hline $\mathrm{H}$ & 3.8577 & -0.7587 & -0.8914 \\
\hline $\mathrm{H}$ & 5.574 & 3.8387 & -1.3289 \\
\hline $\mathrm{H}$ & 7.46 & 4.6098 & -5.308 \\
\hline $\mathrm{H}$ & 6.9517 & 4.1891 & -2.9808 \\
\hline $\mathrm{H}$ & 1.5852 & 4.4071 & 1.0026 \\
\hline $\mathrm{H}$ & 3.9214 & 4.6348 & 0.2931 \\
\hline $\mathrm{H}$ & 5.6615 & -1.6052 & -2.2657 \\
\hline $\mathrm{H}$ & 6.9693 & -1.1511 & -4.2956 \\
\hline $\mathrm{H}$ & 7.1497 & 0.4406 & -6.1561 \\
\hline $\mathrm{C}$ & 5.0074 & 2.9743 & -9.5694 \\
\hline $\mathrm{C}$ & 5.1853 & 4.072 & -8.7285 \\
\hline $\mathrm{C}$ & 6.0475 & 4.0095 & -7.6536 \\
\hline $\mathrm{C}$ & 6.7675 & 2.8466 & -7.3719 \\
\hline $\mathrm{C}$ & 6.8017 & 1.8756 & -8.3783 \\
\hline $\mathrm{C}$ & 5.933 & 1.9346 & -9.4534 \\
\hline $\mathrm{H}$ & 4.5699 & 4.9536 & -8.8519 \\
\hline $\mathrm{H}$ & 6.0785 & 4.8429 & -6.9647 \\
\hline $\mathrm{H}$ & 7.4656 & 1.0245 & -8.2877 \\
\hline $\mathrm{H}$ & 5.9345 & 1.1301 & -10.1793 \\
\hline $\mathrm{C}$ & -3.5868 & 1.424 & -3.1119 \\
\hline $\mathrm{C}$ & -3.1767 & 2.5782 & -2.4424 \\
\hline $\mathrm{C}$ & -2.4145 & 2.5031 & -1.2957 \\
\hline $\mathrm{C}$ & -1.9925 & 1.2721 & -0.7813 \\
\hline $\mathrm{C}$ & -2.6509 & 0.1451 & -1.2858 \\
\hline
\end{tabular}




\begin{tabular}{llll}
$\mathrm{C}$ & -3.4301 & 0.2188 & -2.4256 \\
$\mathrm{H}$ & -3.38 & 3.553 & -2.8662 \\
$\mathrm{H}$ & -2.0579 & 3.4259 & -0.8596 \\
$\mathrm{H}$ & -2.5073 & -0.8234 & -0.8248 \\
$\mathrm{H}$ & -3.8717 & -0.6889 & -2.8189 \\
$\mathrm{C}$ & 0.9321 & 2.6393 & -10.6526 \\
$\mathrm{C}$ & 1.7215 & 1.4997 & -10.4876 \\
$\mathrm{C}$ & 3.088 & 1.5958 & -10.3155 \\
$\mathrm{C}$ & 3.722 & 2.8391 & -10.2985 \\
$\mathrm{C}$ & 2.9715 & 3.9377 & -10.7227 \\
$\mathrm{C}$ & 1.6026 & 3.8399 & -10.8953 \\
$\mathrm{H}$ & 1.2531 & 0.5282 & -10.3956 \\
$\mathrm{H}$ & 3.6548 & 0.7017 & -10.0899 \\
$\mathrm{H}$ & 3.4523 & 4.8991 & -10.8588 \\
$\mathrm{H}$ & 1.0409 & 4.7249 & -11.1697 \\
$\mathrm{C}$ & -3.4484 & 1.8729 & -7.3259 \\
$\mathrm{C}$ & -3.2144 & 0.6342 & -6.7272 \\
$\mathrm{C}$ & -3.4357 & 0.4403 & -5.3794 \\
$\mathrm{C}$ & -3.8818 & 1.4826 & -4.5645 \\
$\mathrm{C}$ & -4.3366 & 2.6348 & -5.2099 \\
$\mathrm{C}$ & -4.1262 & 2.8269 & -6.5644 \\
$\mathrm{H}$ & -2.7747 & -0.1694 & -7.3033 \\
$\mathrm{H}$ & -3.1619 & -0.5077 & -4.9351 \\
$\mathrm{H}$ & -4.8127 & 3.421 & -4.6358 \\
$\mathrm{H}$ & -4.4459 & 3.7555 & -7.0217 \\
$\mathrm{C}$ & -2.7331 & 2.2025 & -8.5855 \\
$\mathrm{C}$ & -2.1378 & 3.4591 & -8.7143 \\
$\mathrm{C}$ & -1.0382 & 3.6424 & -9.529 \\
$\mathrm{C}$ & -0.5 & 2.5851 & -10.2648 \\
$\mathrm{C}$ & -1.2679 & 1.4208 & -10.3457 \\
$\mathrm{C}$ & -2.3607 & 1.2323 & -9.518 \\
$\mathrm{H}$ & -2.4658 & 4.2793 & -8.0889 \\
$\mathrm{H}$ & -0.5322 & 4.5994 & -9.5189 \\
$\mathrm{H}$ & -0.9723 & 0.6243 & -11.018 \\
$\mathrm{H}$ & -2.8916 & 0.2882 & -9.5539 \\
& & & \\
\hline & &
\end{tabular}

\section{2, B3LYP/6-31G(d) (Energy $=-2308.09901152$ Hartrees $)$}

\begin{tabular}{|c|c|c|}
\hline $\mathrm{C}$ & $-4.55584-4.69222$ & 1.92284 \\
\hline $\mathrm{C}$ & $-4.63918-0.44325$ & 1.92502 \\
\hline $\mathrm{C}$ & $6.01285 \quad 2.20526$ & 1.87139 \\
\hline $\mathrm{C}$ & $7.72503-1.70043$ & 1.82794 \\
\hline $\mathrm{C}$ & $6.36325-5.75982$ & 1.81083 \\
\hline $\mathrm{C}$ & $2.76295 \quad-8.09936$ & 1.84375 \\
\hline $\mathrm{C}$ & $-1.51671 \quad-7.70879$ & 1.8904 \\
\hline $\mathrm{C}$ & $-3.83556-5.89495$ & 1.91444 \\
\hline $\mathrm{C}$ & $-5.01623-1.79139$ & 1.93072 \\
\hline $\mathrm{C}$ & $4.85878 \quad 2.9975$ & 1.87887 \\
\hline $\mathrm{C}$ & $7.51302 \quad-0.3153$ & 1.8416 \\
\hline $\mathrm{C}$ & $7.10355 \quad-4.56891$ & 1.80964 \\
\hline $\mathrm{C}$ & $4.08012-7.6163$ & 1.82878 \\
\hline $\mathrm{C}$ & $-0.1849 \quad-8.15019$ & 1.8771 \\
\hline
\end{tabular}




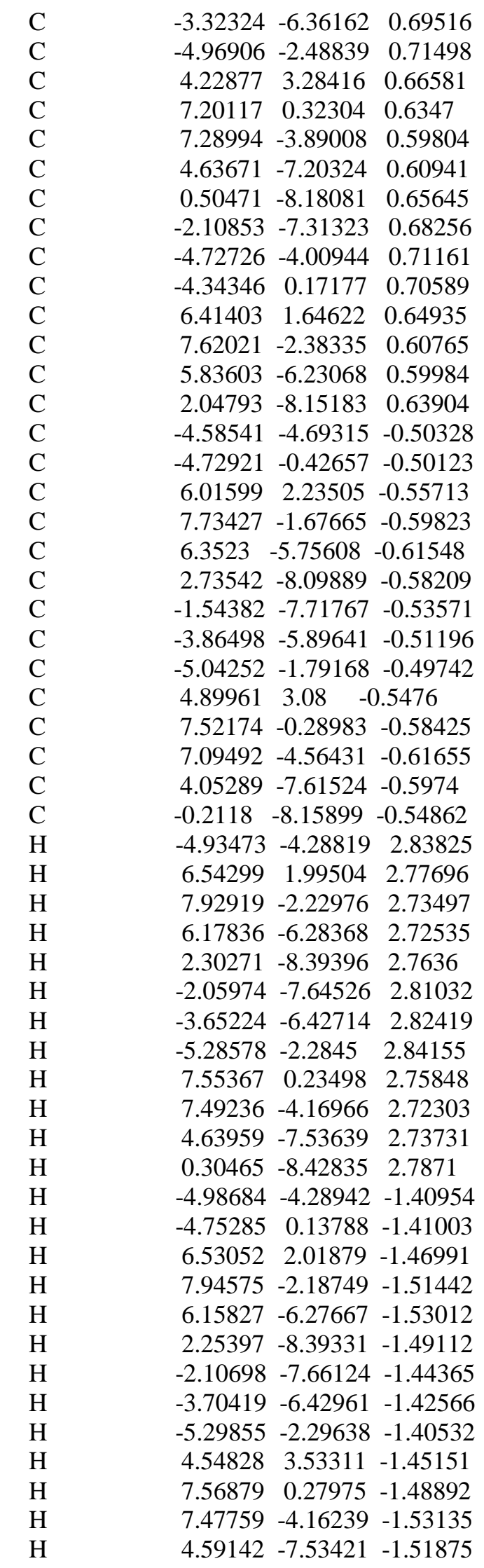




$\begin{array}{llll}\mathrm{H} & 0.25769 & -8.44419 & -1.46669 \\ \mathrm{C} & 0.68087 & 3.88659 & -0.47911 \\ \mathrm{C} & 2.07649 & 3.97343 & -0.50838 \\ \mathrm{C} & 2.77213 & 3.75227 & 0.68733 \\ \mathrm{C} & 2.12695 & 3.88487 & 1.91906 \\ \mathrm{C} & 0.72831 & 3.92914 & 1.95096 \\ \mathrm{C} & 0.03893 & 3.70869 & 0.74964 \\ \mathrm{H} & 2.59858 & 4.15961 & -1.42383 \\ \mathrm{H} & 0.20248 & 4.0829 & 2.87005 \\ \mathrm{C} & -3.1115 & 1.9897 & 1.94252 \\ \mathrm{C} & -2.06471 & 2.91958 & 1.96367 \\ \mathrm{C} & -1.40123 & 3.18717 & 0.75649 \\ \mathrm{C} & -2.0166 & 2.90867 & -0.46637 \\ \mathrm{C} & -3.14293 & 2.08014 & -0.48516 \\ \mathrm{C} & -3.52772 & 1.46779 & 0.71538 \\ \mathrm{H} & -1.75303 & 3.37734 & 2.87921 \\ \mathrm{H} & -3.66784 & 1.88565 & -1.3968 \\ \mathrm{C} & -1.42001 & 3.46095 & -1.77684 \\ \mathrm{C} & -0.14131 & 3.9243 & -1.78329 \\ \mathrm{C} & 2.95257 & 3.92093 & 3.22194 \\ \mathrm{C} & 4.24688 & 3.49977 & 3.20337 \\ \mathrm{C} & -4.48781 & 0.34433 & 3.24297 \\ \mathrm{C} & -3.76391 & 1.49696 & 3.25161 \\ \mathrm{H} & -4.9253 & -0.02554 & 4.14673 \\ \mathrm{H} & -3.6268 & 2.04137 & 4.16255 \\ \mathrm{H} & -1.99691 & 3.45482 & -2.67804 \\ \mathrm{H} & 0.29794 & 4.28566 & -2.6899 \\ \mathrm{H} & 2.50431 & 4.24901 & 4.13639 \\ \mathrm{H} & 4.82511 & 3.49246 & 4.10376 \\ & & & \end{array}$

Macrocycle 10, Relative Energy $=0.00 \mathrm{kcal} \mathrm{mol}^{-1}, R B 97 \mathrm{D} / 6-31 G(d)$

$\begin{array}{llll}\mathrm{C} & 2.88909 & 3.24002 & 0.26169 \\ \mathrm{C} & 2.82984 & 2.00675 & -0.40905 \\ \mathrm{C} & 3.98512 & 1.43259 & -0.95927 \\ \mathrm{C} & 5.22677 & 2.08448 & -0.8597 \\ \mathrm{C} & 4.11965 & 3.92506 & 0.30236 \\ \mathrm{C} & 5.27123 & 3.35319 & -0.24855 \\ \mathrm{C} & 6.54795 & 1.39779 & -1.25706 \\ \mathrm{C} & 6.74891 & -1.44643 & -0.28512 \\ \mathrm{C} & 7.22537 & 0.99582 & 0.04958 \\ \mathrm{C} & 7.31368 & -0.26887 & 0.48609 \\ \mathrm{C} & 6.33304 & 0.19781 & -2.15958 \\ \mathrm{C} & 6.44261 & -1.06819 & -1.73158 \\ \mathrm{C} & -4.88255 & 2.15341 & -1.23597 \\ \mathrm{C} & -4.83449 & 3.49934 & -0.81883 \\ \mathrm{C} & -3.69206 & 4.01463 & -0.20205 \\ \mathrm{C} & -2.56632 & 3.19778 & 0.03245 \\ \mathrm{C} & -3.73575 & 1.35897 & -1.06926\end{array}$




\begin{tabular}{|c|c|c|c|}
\hline $\mathrm{C}$ & -2.59236 & 1.87383 & -0.43628 \\
\hline $\mathrm{C}$ & -1.36773 & 3.78537 & 0.79808 \\
\hline $\mathrm{C}$ & 1.63641 & 3.82892 & 0.92475 \\
\hline $\mathrm{C}$ & -0.54818 & 2.71938 & 1.5109 \\
\hline $\mathrm{C}$ & 0.79148 & 2.74364 & 1.57157 \\
\hline $\mathrm{C}$ & -0.50718 & 4.62205 & -0.13618 \\
\hline $\mathrm{C}$ & 0.83343 & 4.631 & -0.08679 \\
\hline $\mathrm{C}$ & -6.23365 & 1.5782 & -1.72657 \\
\hline $\mathrm{C}$ & -6.84996 & -1.09902 & -0.48562 \\
\hline $\mathrm{C}$ & -7.08406 & 1.40259 & -0.47681 \\
\hline $\mathrm{C}$ & -7.34043 & 0.21507 & 0.08935 \\
\hline $\mathrm{C}$ & -6.07924 & 0.26628 & -2.4723 \\
\hline $\mathrm{C}$ & -6.37356 & -0.92101 & -1.92409 \\
\hline $\mathrm{C}$ & -3.56827 & -2.67191 & 2.05066 \\
\hline $\mathrm{C}$ & -3.63208 & -1.27244 & 1.78676 \\
\hline $\mathrm{C}$ & -4.58977 & -0.81134 & 0.85576 \\
\hline $\mathrm{C}$ & -5.43909 & -1.68455 & 0.15787 \\
\hline $\mathrm{C}$ & -4.34361 & -3.54436 & 1.26701 \\
\hline $\mathrm{C}$ & -5.26204 & -3.06819 & 0.32699 \\
\hline $\mathrm{C}$ & 2.96261 & -2.92707 & 1.93184 \\
\hline $\mathrm{C}$ & 3.2033 & -1.52656 & 1.81028 \\
\hline $\mathrm{C}$ & 4.31027 & -1.0999 & 1.04342 \\
\hline $\mathrm{C}$ & 5.15537 & -2.00855 & 0.39093 \\
\hline $\mathrm{C}$ & 3.7548 & -3.82268 & 1.18817 \\
\hline $\mathrm{C}$ & 4.83603 & -3.37866 & 0.42512 \\
\hline $\mathrm{C}$ & 0.86448 & -2.88728 & 0.96074 \\
\hline $\mathrm{C}$ & -1.97353 & -2.89174 & 1.18038 \\
\hline $\mathrm{C}$ & 0.22175 & -2.72017 & 2.19786 \\
\hline $\mathrm{C}$ & -1.18272 & -2.74431 & 2.35575 \\
\hline $\mathrm{C}$ & 0.06817 & -3.11607 & -0.20031 \\
\hline $\mathrm{C}$ & -1.3312 & -3.09604 & -0.04818 \\
\hline $\mathrm{O}$ & -7.88169 & -2.10825 & -0.40263 \\
\hline $\mathrm{C}$ & -9.05274 & -1.8127 & -1.16835 \\
\hline $\mathrm{O}$ & -6.96028 & 2.5539 & -2.51765 \\
\hline $\mathrm{C}$ & -6.31394 & 2.97717 & -3.71837 \\
\hline $\mathrm{H}$ & 1.87925 & 1.47321 & -0.4745 \\
\hline $\mathrm{H}$ & 3.92415 & 0.45037 & -1.42665 \\
\hline $\mathrm{H}$ & 4.17759 & 4.89011 & 0.80279 \\
\hline $\mathrm{H}$ & 6.22285 & 3.88255 & -0.19401 \\
\hline $\mathrm{H}$ & 7.59788 & 1.83139 & 0.64711 \\
\hline $\mathrm{H}$ & 7.75662 & -0.51083 & 1.45523 \\
\hline $\mathrm{H}$ & 6.04866 & 0.42973 & -3.18878 \\
\hline $\mathrm{H}$ & -5.71312 & 4.12884 & -0.96836 \\
\hline $\mathrm{H}$ & -3.67549 & 5.0461 & 0.15122 \\
\hline $\mathrm{H}$ & -3.751 & 0.31662 & -1.38588 \\
\hline $\mathrm{H}$ & -1.72498 & 1.22709 & -0.28875 \\
\hline
\end{tabular}




\begin{tabular}{|c|c|c|c|}
\hline $\mathrm{H}$ & -1.10983 & 1.93865 & 2.02928 \\
\hline $\mathrm{H}$ & 1.34019 & 1.99837 & 2.15246 \\
\hline $\mathrm{H}$ & -1.05198 & 5.2598 & -0.83575 \\
\hline $\mathrm{H}$ & 1.41507 & 5.28164 & -0.74356 \\
\hline $\mathrm{H}$ & -7.43485 & 2.3364 & -0.0328 \\
\hline $\mathrm{H}$ & -7.90982 & 0.13984 & 1.01855 \\
\hline $\mathrm{H}$ & -5.66884 & 0.31952 & -3.48238 \\
\hline $\mathrm{H}$ & -6.22194 & -1.85401 & -2.4724 \\
\hline $\mathrm{H}$ & -4.68645 & 0.26203 & 0.68602 \\
\hline $\mathrm{H}$ & -5.90023 & -3.76985 & -0.20777 \\
\hline $\mathrm{H}$ & 4.52853 & -0.03644 & 0.95887 \\
\hline $\mathrm{H}$ & 3.56089 & -4.89342 & 1.27506 \\
\hline $\mathrm{H}$ & 5.46837 & -4.101 & -0.08704 \\
\hline $\mathrm{H}$ & -9.53826 & -0.87925 & -0.82971 \\
\hline $\mathrm{H}$ & -8.82669 & -1.71396 & -2.24625 \\
\hline $\mathrm{H}$ & -9.73439 & -2.66032 & -1.01141 \\
\hline $\mathrm{H}$ & -6.28013 & 2.17309 & -4.47655 \\
\hline $\mathrm{H}$ & -5.28603 & 3.33533 & -3.52871 \\
\hline $\mathrm{H}$ & -6.92199 & 3.80552 & -4.10881 \\
\hline $\mathrm{O}$ & 7.65125 & -2.57343 & -0.22348 \\
\hline $\mathrm{O}$ & 7.32975 & 2.40864 & -1.94328 \\
\hline $\mathrm{O}$ & 2.14553 & 4.72036 & 1.96384 \\
\hline $\mathrm{O}$ & -1.85449 & 4.77033 & 1.77238 \\
\hline $\mathrm{C}$ & 8.6512 & 1.99196 & -2.29145 \\
\hline $\mathrm{H}$ & 9.24523 & 1.71186 & -1.40139 \\
\hline $\mathrm{H}$ & 9.11699 & 2.85584 & -2.78605 \\
\hline $\mathrm{H}$ & 8.64071 & 1.13094 & -2.98556 \\
\hline $\mathrm{C}$ & 8.91386 & -2.34485 & -0.85143 \\
\hline $\mathrm{H}$ & 6.24499 & -1.91577 & -2.39275 \\
\hline $\mathrm{H}$ & 8.80572 & -2.13558 & -1.93222 \\
\hline $\mathrm{H}$ & 9.45449 & -1.49926 & -0.38711 \\
\hline $\mathrm{H}$ & 9.49034 & -3.27009 & -0.71269 \\
\hline $\mathrm{C}$ & -2.62934 & 4.23317 & 2.8473 \\
\hline $\mathrm{H}$ & -3.47157 & 3.61829 & 2.48096 \\
\hline $\mathrm{H}$ & -2.00987 & 3.62692 & 3.53307 \\
\hline $\mathrm{H}$ & -3.02491 & 5.10229 & 3.3919 \\
\hline $\mathrm{C}$ & 1.16357 & 5.39766 & 2.75359 \\
\hline $\mathrm{H}$ & 0.45732 & 5.97433 & 2.13564 \\
\hline $\mathrm{H}$ & 0.57971 & 4.69257 & 3.37026 \\
\hline $\mathrm{H}$ & 1.7382 & 6.07236 & 3.40446 \\
\hline $\mathrm{H}$ & 0.84319 & -2.57515 & 3.08499 \\
\hline $\mathrm{H}$ & -1.95251 & -3.16872 & -0.94088 \\
\hline $\mathrm{C}$ & -1.71439 & -2.63183 & 3.7241 \\
\hline $\mathrm{H}$ & -0.97924 & -2.28913 & 4.46133 \\
\hline $\mathrm{C}$ & 0.66342 & -3.26375 & -1.5402 \\
\hline $\mathrm{H}$ & 1.67009 & -2.85852 & -1.66453 \\
\hline
\end{tabular}




$\begin{array}{lccc}\mathrm{C} & -2.94524 & -2.95907 & 4.16849 \\ \mathrm{H} & -3.72683 & -3.33557 & 3.50884 \\ \mathrm{H} & -3.19191 & -2.8596 & 5.22754 \\ \mathrm{C} & 0.07643 & -3.86931 & -2.59175 \\ \mathrm{H} & 0.57926 & -3.92456 & -3.55881 \\ \mathrm{H} & -0.90575 & -4.34225 & -2.51372 \\ \mathrm{C} & 2.30976 & -0.56265 & 2.47883 \\ \mathrm{H} & 1.41436 & -0.99875 & 2.92788 \\ \mathrm{C} & 2.48748 & 0.76914 & 2.58485 \\ \mathrm{H} & 1.74664 & 1.38866 & 3.09298 \\ \mathrm{H} & 3.36277 & 1.2828 & 2.18055 \\ \mathrm{H} & -4.27672 & -4.6165 & 1.45765 \\ \mathrm{C} & -2.7742 & -0.25749 & 2.42678 \\ \mathrm{H} & -3.13192 & 0.77085 & 2.30194 \\ \mathrm{C} & -1.60238 & -0.44187 & 3.06725 \\ \mathrm{H} & -1.15906 & -1.42752 & 3.20959 \\ \mathrm{H} & -1.05057 & 0.41336 & 3.46284\end{array}$

Macrocycle 10, Relative Energy $=1.73 \mathrm{kcal} \mathrm{mol}^{-1}, \mathrm{RB} 97 \mathrm{D} / 6-31 \mathrm{G}(\mathrm{d})$

\begin{tabular}{|c|c|c|c|}
\hline $\mathrm{C}$ & 2.91717 & 3.11154 & 0.12829 \\
\hline $\mathrm{C}$ & 2.83398 & 1.88172 & -0.54504 \\
\hline $\mathrm{C}$ & 3.97914 & 1.28198 & -1.0863 \\
\hline $\mathrm{C}$ & 5.23492 & 1.90509 & -0.98176 \\
\hline $\mathrm{C}$ & 4.16219 & 3.76876 & 0.1804 \\
\hline $\mathrm{C}$ & 5.30353 & 3.17183 & -0.36808 \\
\hline $\mathrm{C}$ & 6.54259 & 1.19603 & -1.38776 \\
\hline $\mathrm{C}$ & 6.6834 & -1.5969 & -0.28284 \\
\hline $\mathrm{C}$ & 7.26527 & 0.83624 & -0.09226 \\
\hline $\mathrm{C}$ & 7.33349 & -0.40872 & 0.40095 \\
\hline $\mathrm{C}$ & 6.29113 & -0.0391 & -2.23293 \\
\hline $\mathrm{C}$ & 6.36674 & -1.28504 & -1.74376 \\
\hline $\mathrm{C}$ & -4.8562 & 1.86763 & -1.30479 \\
\hline $\mathrm{C}$ & -4.7711 & 3.24212 & -1.00083 \\
\hline $\mathrm{C}$ & -3.62903 & 3.77104 & -0.3943 \\
\hline $\mathrm{C}$ & -2.53821 & 2.94286 & -0.05618 \\
\hline $\mathrm{C}$ & -3.7517 & 1.04983 & -1.01151 \\
\hline $\mathrm{C}$ & -2.6102 & 1.58045 & -0.38684 \\
\hline $\mathrm{C}$ & -1.33984 & 3.56536 & 0.68105 \\
\hline $\mathrm{C}$ & 1.66479 & 3.70576 & 0.78877 \\
\hline $\mathrm{C}$ & -0.48562 & 2.53957 & 1.40994 \\
\hline $\mathrm{C}$ & 0.8518 & 2.61843 & 1.47309 \\
\hline $\mathrm{C}$ & -0.5104 & 4.40564 & -0.2779 \\
\hline $\mathrm{C}$ & 0.82983 & 4.4566 & -0.23689 \\
\hline $\mathrm{C}$ & -6.19549 & 1.31121 & -1.85264 \\
\hline
\end{tabular}




\begin{tabular}{|c|c|c|c|}
\hline $\mathrm{C}$ & -6.95387 & -1.22352 & -0.42808 \\
\hline $\mathrm{C}$ & -7.16564 & 1.26495 & -0.68006 \\
\hline $\mathrm{C}$ & -7.50496 & 0.13624 & -0.04142 \\
\hline $\mathrm{C}$ & -6.04281 & -0.06096 & -2.48139 \\
\hline $\mathrm{C}$ & -6.39955 & -1.18877 & -1.8517 \\
\hline $\mathrm{C}$ & -3.17646 & -2.24381 & 1.45201 \\
\hline $\mathrm{C}$ & -3.78778 & -1.00087 & 1.77217 \\
\hline $\mathrm{C}$ & -5.06276 & -0.72611 & 1.23077 \\
\hline $\mathrm{C}$ & -5.75868 & -1.65006 & 0.44461 \\
\hline $\mathrm{C}$ & -3.93506 & -3.22056 & 0.77764 \\
\hline $\mathrm{C}$ & -5.21691 & -2.94231 & 0.29128 \\
\hline $\mathrm{C}$ & 2.61492 & -2.44707 & 1.00256 \\
\hline $\mathrm{C}$ & 3.39718 & -3.48636 & 0.42718 \\
\hline $\mathrm{C}$ & 4.74444 & -3.20681 & 0.10148 \\
\hline $\mathrm{C}$ & 5.32345 & -1.95399 & 0.35185 \\
\hline $\mathrm{C}$ & 3.22531 & -1.22293 & 1.32469 \\
\hline $\mathrm{C}$ & 4.56328 & -0.97352 & 1.01533 \\
\hline $\mathrm{C}$ & 1.12745 & -2.51477 & 1.12879 \\
\hline $\mathrm{C}$ & -1.68714 & -2.40738 & 1.47471 \\
\hline $\mathrm{C}$ & 0.50187 & -3.14721 & 2.21047 \\
\hline $\mathrm{C}$ & -0.89837 & -3.11404 & 2.42402 \\
\hline $\mathrm{C}$ & 0.32995 & -1.84756 & 0.15557 \\
\hline $\mathrm{C}$ & -1.06183 & -1.82463 & 0.36515 \\
\hline $\mathrm{O}$ & -7.95266 & -2.25591 & -0.28316 \\
\hline $\mathrm{C}$ & -9.09072 & -2.09021 & -1.13296 \\
\hline $\mathrm{O}$ & -6.80464 & 2.25139 & -2.77556 \\
\hline $\mathrm{C}$ & -6.0474 & 2.54137 & -3.95082 \\
\hline $\mathrm{H}$ & 1.87629 & 1.36473 & -0.61587 \\
\hline $\mathrm{H}$ & 3.89686 & 0.29973 & -1.55068 \\
\hline $\mathrm{H}$ & 4.24001 & 4.73103 & 0.68349 \\
\hline $\mathrm{H}$ & 6.2659 & 3.68145 & -0.30944 \\
\hline $\mathrm{H}$ & 7.69188 & 1.68579 & 0.44644 \\
\hline $\mathrm{H}$ & 7.80984 & -0.61983 & 1.36138 \\
\hline $\mathrm{H}$ & 5.99786 & 0.15149 & -3.26813 \\
\hline $\mathrm{H}$ & -5.61648 & 3.88828 & -1.24235 \\
\hline $\mathrm{H}$ & -3.58611 & 4.8294 & -0.13368 \\
\hline $\mathrm{H}$ & -3.79889 & -0.01646 & -1.23231 \\
\hline $\mathrm{H}$ & -1.78344 & 0.91829 & -0.12931 \\
\hline $\mathrm{H}$ & -1.013 & 1.74185 & 1.93561 \\
\hline $\mathrm{H}$ & 1.42408 & 1.90158 & 2.06685 \\
\hline $\mathrm{H}$ & -1.07657 & 5.01035 & -0.98996 \\
\hline $\mathrm{H}$ & 1.38722 & 5.10958 & -0.91227 \\
\hline $\mathrm{H}$ & -7.54432 & 2.23911 & -0.36414 \\
\hline $\mathrm{H}$ & -8.16678 & 0.1523 & 0.8275 \\
\hline $\mathrm{H}$ & -5.57681 & -0.10262 & -3.46747 \\
\hline $\mathrm{H}$ & -6.24245 & -2.1691 & -2.30767 \\
\hline
\end{tabular}




\begin{tabular}{|c|c|c|c|}
\hline $\mathrm{H}$ & -5.47642 & 0.27128 & 1.37845 \\
\hline $\mathrm{H}$ & -5.76853 & -3.692 & -0.27634 \\
\hline $\mathrm{H}$ & 5.33895 & -3.96325 & -0.40934 \\
\hline $\mathrm{H}$ & 2.61818 & -0.43141 & 1.76716 \\
\hline $\mathrm{H}$ & 4.98853 & 0.00688 & 1.21907 \\
\hline $\mathrm{H}$ & -9.62621 & -1.14655 & $5-0.92078$ \\
\hline $\mathrm{H}$ & -8.81129 & -2.09619 & - -2.20295 \\
\hline $\mathrm{H}$ & -9.75063 & -2.94294 & $4 \quad-0.92079$ \\
\hline $\mathrm{H}$ & -6.00666 & 1.67838 & $3-4.64095$ \\
\hline $\mathrm{H}$ & -5.01659 & 2.85734 & -3.70947 \\
\hline $\mathrm{H}$ & -6.57432 & 3.3673 & -4.44919 \\
\hline $\mathrm{O}$ & 7.51537 & -2.77307 & -0.17225 \\
\hline $\mathrm{O}$ & 7.30571 & 2.17515 & -2.13788 \\
\hline $\mathrm{O}$ & 2.16808 & 4.64735 & 1.78319 \\
\hline $\mathrm{O}$ & -1.84275 & 4.5597 & 1.64329 \\
\hline $\mathrm{C}$ & 8.61331 & 1.73712 & -2.51119 \\
\hline $\mathrm{H}$ & 9.24247 & 1.51151 & -1.62965 \\
\hline $\mathrm{H}$ & 9.05849 & 2.56861 & -3.07545 \\
\hline $\mathrm{H}$ & 8.57764 & 0.83492 & -3.15009 \\
\hline $\mathrm{C}$ & 8.77897 & -2.6547 & -0.82799 \\
\hline $\mathrm{H}$ & 6.1325 & -2.15802 & -2.35809 \\
\hline $\mathrm{H}$ & 8.66615 & -2.49111 & -1.91611 \\
\hline $\mathrm{H}$ & 9.37856 & -1.82303 & -0.41316 \\
\hline $\mathrm{H}$ & 9.29982 & -3.60632 & -0.65222 \\
\hline $\mathrm{C}$ & -2.63442 & 4.03002 & 2.70727 \\
\hline $\mathrm{H}$ & -3.51206 & 3.4732 & 2.33012 \\
\hline $\mathrm{H}$ & -2.04483 & 3.36894 & 3.36847 \\
\hline $\mathrm{H}$ & -2.97961 & 4.90041 & 3.28333 \\
\hline $\mathrm{C}$ & 1.18091 & 5.29648 & 2.58956 \\
\hline $\mathrm{H}$ & 0.4064 & 5.79052 & 1.98197 \\
\hline $\mathrm{H}$ & 0.67875 & 4.58479 & 3.26825 \\
\hline $\mathrm{H}$ & 1.73618 & 6.0412 & 3.17803 \\
\hline $\mathrm{H}$ & 1.12895 & -3.66388 & 2.94111 \\
\hline $\mathrm{H}$ & -1.69769 & -1.34451 & $1-0.37898$ \\
\hline $\mathrm{C}$ & -1.41443 & -3.78186 & 53.62841 \\
\hline $\mathrm{H}$ & -0.65675 & -4.36102 & 24.16828 \\
\hline $\mathrm{C}$ & 0.93561 & -1.23688 & -1.04079 \\
\hline $\mathrm{H}$ & 1.98817 & -1.47673 & -1.20557 \\
\hline $\mathrm{C}$ & -2.65807 & -3.74211 & 4.15256 \\
\hline $\mathrm{H}$ & -3.47578 & -3.18374 & $4 \quad 3.69857$ \\
\hline $\mathrm{H}$ & -2.87748 & -4.28409 & 5.07476 \\
\hline $\mathrm{C}$ & 0.32786 & -0.425 & -1.93181 \\
\hline $\mathrm{H}$ & 0.8802 & -0.03883 & -2.79012 \\
\hline $\mathrm{H}$ & -0.71118 & -0.10354 & $4-1.83632$ \\
\hline $\mathrm{C}$ & 2.78432 & -4.79087 & 0.11648 \\
\hline $\mathrm{H}$ & 1.69153 & -4.81274 & 0.14622 \\
\hline
\end{tabular}




$\begin{array}{llll}\mathrm{C} & 3.44129 & -5.93294 & -0.168 \\ \mathrm{H} & 2.89502 & -6.85175 & -0.38883 \\ \mathrm{H} & 4.53231 & -5.9921 & -0.17776 \\ \mathrm{H} & -3.4695 & -4.18395 & 0.56029 \\ \mathrm{C} & -3.16089 & 0.06589 & 2.5718 \\ \mathrm{H} & -3.5775 & 1.0626 & 2.39521 \\ \mathrm{C} & -2.20307 & -0.0671 & 3.51104 \\ \mathrm{H} & -1.76805 & -1.0305 & 3.77429 \\ \mathrm{H} & -1.84724 & 0.80841 & 4.05912\end{array}$

Macrocycle 10, Relative Energy $=1.85 \mathrm{kcal} \mathrm{mol}^{-1}, R B 97 D / 6-31 G(d)$

$\begin{array}{lrcc}\text { C } & 2.88909 & 3.24002 & 0.26169 \\ \mathrm{C} & 2.82984 & 2.00675 & -0.40905 \\ \mathrm{C} & 3.98512 & 1.43259 & -0.95927 \\ \mathrm{C} & 5.22677 & 2.08448 & -0.8597 \\ \mathrm{C} & 4.11965 & 3.92506 & 0.30236 \\ \mathrm{C} & 5.27123 & 3.35319 & -0.24855 \\ \mathrm{C} & 6.54795 & 1.39779 & -1.25706 \\ \mathrm{C} & 6.74891 & -1.44643 & -0.28512 \\ \mathrm{C} & 7.22537 & 0.99582 & 0.04958 \\ \mathrm{C} & 7.31368 & -0.26887 & 0.48609 \\ \mathrm{C} & 6.33304 & 0.19781 & -2.15958 \\ \mathrm{C} & 6.44261 & -1.06819 & -1.73158 \\ \mathrm{C} & -4.88255 & 2.15341 & -1.23597 \\ \mathrm{C} & -4.83449 & 3.49934 & -0.81883 \\ \mathrm{C} & -3.69206 & 4.01463 & -0.20205 \\ \mathrm{C} & -2.56632 & 3.19778 & 0.03245 \\ \mathrm{C} & -3.73575 & 1.35897 & -1.06926 \\ \mathrm{C} & -2.59236 & 1.87383 & -0.43628 \\ \mathrm{C} & -1.36773 & 3.78537 & 0.79808 \\ \mathrm{C} & 1.63641 & 3.82892 & 0.92475 \\ \mathrm{C} & -0.54818 & 2.71938 & 1.5109 \\ \mathrm{C} & 0.79148 & 2.74364 & 1.57157 \\ \mathrm{C} & -0.50718 & 4.62205 & -0.13618 \\ \mathrm{C} & 0.83343 & 4.631 & -0.08679 \\ \mathrm{C} & -6.23365 & 1.5782 & -1.72657 \\ \mathrm{C} & -6.84996 & -1.09902 & -0.48562 \\ \mathrm{C} & -7.08406 & 1.40259 & -0.47681 \\ \mathrm{C} & -7.34043 & 0.21507 & 0.08935 \\ \mathrm{C} & -6.07924 & 0.26628 & -2.4723 \\ \mathrm{C} & -6.37356 & -0.92101 & -1.92409 \\ \mathrm{C} & -3.27617 & -2.67152 & 2.13489 \\ \mathrm{C} & -3.33998 & -1.27205 & 1.87098 \\ \mathrm{C} & -4.29767 & -0.81094 & 0.93998 \\ \mathrm{C} & -5.14699 & -1.68416 & 0.24209\end{array}$




\begin{tabular}{|c|c|c|c|}
\hline $\mathrm{C}$ & -4.05151 & -3.54396 & 1.35123 \\
\hline $\mathrm{C}$ & -4.96994 & -3.0678 & 0.41121 \\
\hline $\mathrm{C}$ & 3.20651 & -2.84063 & 1.82842 \\
\hline $\mathrm{C}$ & 3.44686 & -1.44013 & 1.70605 \\
\hline $\mathrm{C}$ & 4.55388 & -1.01366 & 0.93917 \\
\hline $\mathrm{C}$ & 5.39936 & -1.92249 & 0.28742 \\
\hline $\mathrm{C}$ & 3.99909 & -3.73649 & 1.08547 \\
\hline $\mathrm{C}$ & 5.08036 & -3.29266 & 0.32238 \\
\hline $\mathrm{C}$ & 1.39809 & -3.67905 & 2.68108 \\
\hline $\mathrm{C}$ & -1.23036 & -2.77956 & 2.06082 \\
\hline $\mathrm{C}$ & 0.84144 & -3.85691 & 1.40437 \\
\hline $\mathrm{C}$ & -0.44762 & -3.40087 & 1.04598 \\
\hline $\mathrm{C}$ & 0.63988 & -2.98344 & 3.66886 \\
\hline $\mathrm{C}$ & -0.66035 & -2.56881 & 3.3235 \\
\hline $\mathrm{O}$ & -7.88169 & -2.10825 & -0.40263 \\
\hline $\mathrm{C}$ & -9.05274 & -1.8127 & -1.16835 \\
\hline $\mathrm{O}$ & -6.96028 & 2.5539 & -2.51765 \\
\hline $\mathrm{C}$ & -6.31394 & 2.97717 & -3.71837 \\
\hline $\mathrm{H}$ & 1.87925 & 1.47321 & -0.4745 \\
\hline $\mathrm{H}$ & 3.92415 & 0.45037 & -1.42665 \\
\hline $\mathrm{H}$ & 4.17759 & 4.89011 & 0.80279 \\
\hline $\mathrm{H}$ & 6.22285 & 3.88255 & -0.19401 \\
\hline $\mathrm{H}$ & 7.59788 & 1.83139 & 0.64711 \\
\hline $\mathrm{H}$ & 7.75662 & -0.51083 & 1.45523 \\
\hline $\mathrm{H}$ & 6.04866 & 0.42973 & -3.18878 \\
\hline $\mathrm{H}$ & -5.71312 & 4.12884 & -0.96836 \\
\hline $\mathrm{H}$ & -3.67549 & 5.0461 & 0.15122 \\
\hline $\mathrm{H}$ & -3.751 & 0.31662 & -1.38588 \\
\hline $\mathrm{H}$ & -1.72498 & 1.22709 & -0.28875 \\
\hline $\mathrm{H}$ & -1.10983 & 1.93865 & 2.02928 \\
\hline $\mathrm{H}$ & 1.34019 & 1.99837 & 2.15246 \\
\hline $\mathrm{H}$ & -1.05198 & 5.2598 & -0.83575 \\
\hline $\mathrm{H}$ & 1.41507 & 5.28164 & -0.74356 \\
\hline $\mathrm{H}$ & -7.43485 & 2.3364 & -0.0328 \\
\hline $\mathrm{H}$ & -7.90982 & 0.13984 & 1.01855 \\
\hline $\mathrm{H}$ & -5.66884 & 0.31952 & -3.48238 \\
\hline $\mathrm{H}$ & -6.22194 & -1.85401 & -2.4724 \\
\hline $\mathrm{H}$ & -4.39435 & 0.26243 & 0.77024 \\
\hline $\mathrm{H}$ & -5.60813 & -3.76946 & -0.12355 \\
\hline $\mathrm{H}$ & 4.77188 & 0.04981 & 0.85402 \\
\hline $\mathrm{H}$ & 3.80543 & -4.80723 & 1.17296 \\
\hline $\mathrm{H}$ & 5.713 & -4.01514 & -0.18921 \\
\hline $\mathrm{H}$ & -9.53826 & -0.87925 & -0.82971 \\
\hline $\mathrm{H}$ & -8.82669 & -1.71396 & -2.24625 \\
\hline $\mathrm{H}$ & -9.73439 & -2.66032 & -1.01141 \\
\hline $\mathrm{H}$ & -6.28013 & 2.17309 & -4.47655 \\
\hline
\end{tabular}




\begin{tabular}{|c|c|c|c|}
\hline $\mathrm{H}$ & -5.28603 & 3.33533 & -3.52871 \\
\hline $\mathrm{H}$ & -6.92199 & 3.80552 & -4.10881 \\
\hline $\mathrm{O}$ & 7.65125 & -2.57343 & -0.22348 \\
\hline $\mathrm{O}$ & 7.32975 & 2.40864 & -1.94328 \\
\hline $\mathrm{O}$ & 2.14553 & 4.72036 & 1.96384 \\
\hline $\mathrm{O}$ & -1.85449 & 4.77033 & 1.77238 \\
\hline $\mathrm{C}$ & 8.6512 & 1.99196 & -2.29145 \\
\hline $\mathrm{H}$ & 9.24523 & 1.71186 & -1.40139 \\
\hline $\mathrm{H}$ & 9.11699 & 2.85584 & -2.78605 \\
\hline $\mathrm{H}$ & 8.64071 & 1.13094 & -2.98556 \\
\hline $\mathrm{C}$ & 8.91386 & -2.34485 & -0.85143 \\
\hline $\mathrm{H}$ & 6.24499 & -1.91577 & -2.39275 \\
\hline $\mathrm{H}$ & 8.80572 & -2.13558 & -1.93222 \\
\hline $\mathrm{H}$ & 9.45449 & -1.49926 & -0.38711 \\
\hline $\mathrm{H}$ & 9.49034 & -3.27009 & -0.71269 \\
\hline $\mathrm{C}$ & -2.62934 & 4.23317 & 2.8473 \\
\hline $\mathrm{H}$ & -3.47157 & 3.61829 & 2.48096 \\
\hline $\mathrm{H}$ & -2.00987 & 3.62692 & 3.53307 \\
\hline $\mathrm{H}$ & -3.02491 & 5.10229 & 3.3919 \\
\hline $\mathrm{C}$ & 1.16357 & 5.39766 & 2.75359 \\
\hline $\mathrm{H}$ & 0.45732 & 5.97433 & 2.13564 \\
\hline $\mathrm{H}$ & 0.57971 & 4.69257 & 3.37026 \\
\hline $\mathrm{H}$ & 1.7382 & 6.07236 & 3.40446 \\
\hline $\mathrm{H}$ & 1.44333 & -4.36516 & 0.64698 \\
\hline $\mathrm{H}$ & -1.28791 & -2.12643 & 34.09724 \\
\hline $\mathrm{C}$ & -0.87074 & -3.58989 & -0.35149 \\
\hline $\mathrm{H}$ & -0.24428 & -4.2899 & -0.91624 \\
\hline $\mathrm{C}$ & 1.137 & -2.7883 & 5.04226 \\
\hline $\mathrm{H}$ & 1.92416 & -3.47167 & 5.36847 \\
\hline $\mathrm{C}$ & -1.86812 & -2.96554 & $4-1.01102$ \\
\hline $\mathrm{H}$ & -2.5197 & -2.23373 & -0.53387 \\
\hline $\mathrm{H}$ & -2.04615 & -3.17774 & $4-2.06724$ \\
\hline $\mathrm{C}$ & 0.71504 & -1.83434 & 5.89605 \\
\hline $\mathrm{H}$ & 1.12365 & -1.76521 & 6.90419 \\
\hline $\mathrm{H}$ & -0.02937 & -1.08764 & 45.60843 \\
\hline $\mathrm{C}$ & 2.55293 & -0.47604 & 2.37382 \\
\hline $\mathrm{H}$ & 1.65754 & -0.91211 & 2.82294 \\
\hline $\mathrm{C}$ & 2.73028 & 0.85586 & 2.47906 \\
\hline $\mathrm{H}$ & 1.98916 & 1.47549 & 2.98666 \\
\hline $\mathrm{H}$ & 3.60551 & 1.3695 & 2.07464 \\
\hline $\mathrm{H}$ & -3.98462 & -4.6161 & 1.54187 \\
\hline $\mathrm{C}$ & -2.48211 & -0.2571 & 2.511 \\
\hline $\mathrm{H}$ & -2.83982 & 0.77124 & 2.38616 \\
\hline $\mathrm{C}$ & -1.31028 & -0.44148 & 3.15147 \\
\hline $\mathrm{H}$ & -0.86696 & -1.42713 & 3.29381 \\
\hline $\mathrm{H}$ & -0.75847 & 0.41375 & 3.54706 \\
\hline
\end{tabular}


Macrocycle 10, Relative Energy $=3.01 \mathrm{kcal} \mathrm{mol}^{-1}, R B 97 \mathrm{D} / 6-31 G(d)$

$\begin{array}{lccl}\mathrm{C} & 2.75169 & 2.84245 & 0.15969 \\ \mathrm{C} & 2.78707 & 1.50586 & -0.26798 \\ \mathrm{C} & 3.96984 & 0.94445 & -0.77164 \\ \mathrm{C} & 5.14258 & 1.71218 & -0.87616 \\ \mathrm{C} & 3.91423 & 3.62528 & 0.00819 \\ \mathrm{C} & 5.09298 & 3.06889 & -0.49771 \\ \mathrm{C} & 6.4963 & 1.08774 & -1.27284 \\ \mathrm{C} & 6.87999 & -1.57992 & 0.04801 \\ \mathrm{C} & 7.29005 & 0.89383 & 0.01675 \\ \mathrm{C} & 7.47676 & -0.29879 & 0.60194 \\ \mathrm{C} & 6.32952 & -0.22532 & -2.0125 \\ \mathrm{C} & 6.52282 & -1.41612 & -1.42946 \\ \mathrm{C} & -5.07548 & 2.10318 & -1.34026 \\ \mathrm{C} & -5.03679 & 3.42102 & -0.84077 \\ \mathrm{C} & -3.87364 & 3.92511 & -0.25069 \\ \mathrm{C} & -2.71929 & 3.12373 & -0.1295 \\ \mathrm{C} & -3.89533 & 1.34355 & -1.30922 \\ \mathrm{C} & -2.73184 & 1.8433 & -0.7067 \\ \mathrm{C} & -1.49746 & 3.64919 & 0.64733 \\ \mathrm{C} & 1.50107 & 3.46364 & 0.79533 \\ \mathrm{C} & -0.75991 & 2.51836 & 1.35043 \\ \mathrm{C} & 0.57643 & 2.42823 & 1.40285 \\ \mathrm{C} & -0.56893 & 4.43479 & -0.26654 \\ \mathrm{C} & 0.76907 & 4.34336 & -0.20601 \\ \mathrm{C} & -6.43687 & 1.50662 & -1.77242 \\ \mathrm{C} & -6.89238 & -1.14028 & -0.41303 \\ \mathrm{C} & -7.24855 & 1.34425 & -0.49435 \\ \mathrm{C} & -7.43966 & 0.1693 & 0.12167 \\ \mathrm{C} & -6.29116 & 0.17634 & -2.48946 \\ \mathrm{C} & -6.50554 & -1.00105 & -1.8843 \\ \mathrm{C} & -2.95056 & -2.28076 & 1.03145 \\ \mathrm{C} & -3.82403 & -3.2868 & 0.52292 \\ \mathrm{C} & -5.13404 & -2.89759 & 0.16061 \\ \mathrm{C} & -5.59732 & -1.57745 & 0.29975 \\ \mathrm{C} & -3.46236 & -0.99927 & 1.28716 \\ \mathrm{C} & -4.76694 & -0.63997 & 0.93138 \\ \mathrm{C} & 2.84462 & -2.38726 & 1.44978 \\ \mathrm{C} & 3.52 & -1.23187 & 1.9334 \\ \mathrm{C} & 4.86351 & -1.03666 & 1.53956 \\ \mathrm{C} & 5.55095 & -1.95063 & 0.73738 \\ \mathrm{C} & 3.57416 & -3.35769 & 0.72961 \\ \mathrm{C} & 4.91372 & -3.16097 & 0.39037 \\ \mathrm{C} & 1.35244 & -2.48579 & 1.44762 \\ \mathrm{C} & -1.46979 & -2.42611 & 1.14 \\ \mathrm{C} & 0.75159 & -2.33807 & 0.18748 \\ & & & \\ \mathrm{n} & & & \end{array}$




\begin{tabular}{|c|c|c|}
\hline $\mathrm{C}$ & $-0.64225-2.28534$ & -0.01691 \\
\hline $\mathrm{C}$ & $0.52598-2.6362$ & 2.59238 \\
\hline $\mathrm{C}$ & $-0.87598-2.60045$ & 2.39455 \\
\hline $\mathrm{O}$ & $-7.84492-2.21006$ & $5-0.22665$ \\
\hline $\mathrm{C}$ & $-9.07159-2.03284$ & $4-0.93974$ \\
\hline $\mathrm{O}$ & $\begin{array}{ll}-7.19949 & 2.45567\end{array}$ & -2.56137 \\
\hline $\mathrm{C}$ & -6.573962 .89153 & -3.76808 \\
\hline $\mathrm{H}$ & 1.898660 .8791 & -0.17399 \\
\hline $\mathrm{H}$ & $3.98459-0.10803$ & -1.05149 \\
\hline $\mathrm{H}$ & $3.90044 \quad 4.66531$ & 0.33288 \\
\hline $\mathrm{H}$ & 5.988923 .68208 & -0.59661 \\
\hline $\mathrm{H}$ & $7.66942 \quad 1.81573$ & 0.4643 \\
\hline $\mathrm{H}$ & $\begin{array}{ll}8.00525 & -0.39342\end{array}$ & 1.55371 \\
\hline $\mathrm{H}$ & $6.00059-0.14206$ & -3.051 \\
\hline $\mathrm{H}$ & $\begin{array}{ll}-5.93712 & 4.03531\end{array}$ & -0.8969 \\
\hline $\mathrm{H}$ & -3.86264 .92996 & 0.17204 \\
\hline $\mathrm{H}$ & $-3.89507 \quad 0.33016$ & -1.7039 \\
\hline $\mathrm{H}$ & -1.843831 .2106 & -0.65781 \\
\hline $\mathrm{H}$ & $-1.38012 \quad 1.77235$ & 1.85302 \\
\hline $\mathrm{H}$ & $1.06411 \quad 1.62903$ & 1.96017 \\
\hline $\mathrm{H}$ & $-1.0591 \quad 5.12332$ & -0.95852 \\
\hline $\mathrm{H}$ & 1.40114 .95988 & -0.84919 \\
\hline $\mathrm{H}$ & $\begin{array}{ll}-7.62955 & 2.27798\end{array}$ & -0.0763 \\
\hline $\mathrm{H}$ & $-7.98097 \quad 0.105$ & 1.06823 \\
\hline $\mathrm{H}$ & $\begin{array}{ll}-5.94827 & 0.20852\end{array}$ & -3.52535 \\
\hline $\mathrm{H}$ & $-6.3531-1.9477$ & -2.40859 \\
\hline $\mathrm{H}$ & $\begin{array}{ll}-5.79969 & -3.63896\end{array}$ & $5-0.28364$ \\
\hline $\mathrm{H}$ & $\begin{array}{ll}-5.10264 & 0.38614\end{array}$ & 1.06955 \\
\hline $\mathrm{H}$ & $5.34696-0.09674$ & 1.79418 \\
\hline $\mathrm{H}$ & $3.05301-4.25528$ & 0.39062 \\
\hline $\mathrm{H}$ & $\begin{array}{lll}5.45503 & -3.90708\end{array}$ & -0.19181 \\
\hline $\mathrm{H}$ & $-9.60083-1.11606$ & $5-0.62156$ \\
\hline $\mathrm{H}$ & $-8.90932-1.97726$ & $5-2.03211$ \\
\hline $\mathrm{H}$ & $-9.68524-2.91326$ & $5-0.70332$ \\
\hline $\mathrm{H}$ & $-6.49841 \quad 2.07884$ & -4.51444 \\
\hline $\mathrm{H}$ & $-5.56522 \quad 3.30258$ & -3.58331 \\
\hline $\mathrm{H}$ & $-7.22229 \quad 3.68208$ & -4.17182 \\
\hline $\mathrm{O}$ & $7.76684-2.70353$ & 0.24565 \\
\hline $\mathrm{O}$ & $7.14785 \quad 2.0633$ & -2.12431 \\
\hline $\mathrm{O}$ & $2.04349 \quad 4.29768$ & 1.8791 \\
\hline $\mathrm{O}$ & -1.93824 .65515 & 1.62092 \\
\hline $\mathrm{C}$ & $8.47397 \quad 1.70745$ & -2.51831 \\
\hline $\mathrm{H}$ & $9.14786 \quad 1.59189$ & -1.64878 \\
\hline $\mathrm{H}$ & 8.830292 .5328 & -3.15054 \\
\hline $\mathrm{H}$ & $8.49162 \quad 0.76529$ & -3.09743 \\
\hline $\mathrm{C}$ & $9.016 \quad-2.58062$ & -0.43678 \\
\hline
\end{tabular}




$\begin{array}{lcll}\mathrm{H} & 6.35112 & -2.35087 & -1.96828 \\ \mathrm{H} & 8.88132 & -2.48821 & -1.53102 \\ \mathrm{H} & 9.59045 & -1.70361 & -0.08404 \\ \mathrm{H} & 9.57487 & -3.49981 & -0.21206 \\ \mathrm{C} & -2.69375 & 4.14698 & 2.72224 \\ \mathrm{H} & -3.54885 & 3.53198 & 2.38668 \\ \mathrm{H} & -2.06407 & 3.54919 & 3.4061 \\ \mathrm{H} & -3.07118 & 5.02985 & 3.25784 \\ \mathrm{C} & 1.08914 & 5.0182 & 2.66331 \\ \mathrm{H} & 0.42193 & 5.63873 & 2.04462 \\ \mathrm{H} & 0.46038 & 4.33991 & 3.26648 \\ \mathrm{H} & 1.69029 & 5.65462 & 3.3292 \\ \mathrm{H} & 1.40851 & -2.20951 & -0.67614 \\ \mathrm{H} & -1.53719 & -2.72321 & 3.25361 \\ \mathrm{C} & -1.0748 & -2.04659 & -1.40584 \\ \mathrm{H} & -0.23358 & -2.05448 & -2.10858 \\ \mathrm{C} & 1.13209 & -2.8196 & 3.91957 \\ \mathrm{H} & 2.21832 & -2.9427 & 3.91363 \\ \mathrm{C} & -2.28849 & -1.79893 & -1.94484 \\ \mathrm{H} & -3.21501 & -1.75508 & -1.38117 \\ \mathrm{H} & -2.36145 & -1.63134 & -3.02203 \\ \mathrm{C} & 0.49288 & -2.83547 & 5.10708 \\ \mathrm{H} & 1.04791 & -2.98419 & 6.03472 \\ \mathrm{H} & -0.58738 & -2.69976 & 5.19779 \\ \mathrm{C} & 2.81315 & -0.21126 & 2.72583 \\ \mathrm{H} & 1.74168 & -0.37379 & 2.85235 \\ \mathrm{C} & 3.34949 & 0.88593 & 3.29689 \\ \mathrm{H} & 2.71965 & 1.59208 & 3.83936 \\ \mathrm{H} & 4.40998 & 1.13519 & 3.22764 \\ \mathrm{H} & -2.78884 & -0.23919 & 1.6875 \\ \mathrm{C} & -3.45066 & -4.68746 & 0.25991 \\ \mathrm{H} & -4.2225 & -5.24748 & -0.2801 \\ \mathrm{C} & -2.33269 & -5.35891 & 0.60541 \\ \mathrm{H} & -1.50929 & -4.90781 & 1.15618 \\ \mathrm{H} & -2.22901 & -6.41114 & 0.33163 \\ & & & \end{array}$

Macrocycle 10, Relative Energy $=3.34 \mathrm{kcal} \mathrm{mol}^{-1}, \mathrm{RB} 97 \mathrm{D} / 6-31 \mathrm{G}(\mathrm{d})$

$\begin{array}{llll}\mathrm{C} & 2.88909 & 3.24002 & 0.26169 \\ \mathrm{C} & 2.82984 & 2.00675 & -0.40905 \\ \mathrm{C} & 3.98512 & 1.43259 & -0.95927 \\ \mathrm{C} & 5.22677 & 2.08448 & -0.8597 \\ \mathrm{C} & 4.11965 & 3.92506 & 0.30236 \\ \mathrm{C} & 5.27123 & 3.35319 & -0.24855 \\ \mathrm{C} & 6.54795 & 1.39779 & -1.25706 \\ \mathrm{C} & 6.74891 & -1.44643 & -0.28512\end{array}$




\begin{tabular}{|c|c|c|c|}
\hline $\mathrm{C}$ & 7.22537 & 0.99582 & 0.04958 \\
\hline $\mathrm{C}$ & 7.31368 & -0.26887 & 0.48609 \\
\hline $\mathrm{C}$ & 6.33304 & 0.19781 & -2.15958 \\
\hline $\mathrm{C}$ & 6.44261 & -1.06819 & -1.73158 \\
\hline $\mathrm{C}$ & -4.88255 & 2.15341 & -1.23597 \\
\hline $\mathrm{C}$ & -4.83449 & 3.49934 & -0.81883 \\
\hline $\mathrm{C}$ & -3.69206 & 4.01463 & -0.20205 \\
\hline $\mathrm{C}$ & -2.56632 & 3.19778 & 0.03245 \\
\hline $\mathrm{C}$ & -3.73575 & 1.35897 & -1.06926 \\
\hline $\mathrm{C}$ & -2.59236 & 1.87383 & -0.43628 \\
\hline $\mathrm{C}$ & -1.36773 & 3.78537 & 0.79808 \\
\hline $\mathrm{C}$ & 1.63641 & 3.82892 & 0.92475 \\
\hline $\mathrm{C}$ & -0.54818 & 2.71938 & 1.5109 \\
\hline $\mathrm{C}$ & 0.79148 & 2.74364 & 1.57157 \\
\hline $\mathrm{C}$ & -0.50718 & 4.62205 & -0.13618 \\
\hline $\mathrm{C}$ & 0.83343 & 4.631 & -0.08679 \\
\hline $\mathrm{C}$ & -6.23365 & 1.5782 & -1.72657 \\
\hline $\mathrm{C}$ & -6.84996 & -1.09902 & -0.48562 \\
\hline $\mathrm{C}$ & -7.08406 & 1.40259 & -0.47681 \\
\hline $\mathrm{C}$ & -7.34043 & 0.21507 & 0.08935 \\
\hline $\mathrm{C}$ & -6.07924 & 0.26628 & -2.4723 \\
\hline $\mathrm{C}$ & -6.37356 & -0.92101 & -1.92409 \\
\hline $\mathrm{C}$ & -3.06543 & -2.58135 & 1.06844 \\
\hline $\mathrm{C}$ & -3.99133 & -3.49428 & 0.48405 \\
\hline $\mathrm{C}$ & -5.24844 & -2.99821 & 0.07159 \\
\hline $\mathrm{C}$ & -5.62514 & -1.65891 & 0.2594 \\
\hline $\mathrm{C}$ & -3.49325 & -1.27267 & 1.35257 \\
\hline $\mathrm{C}$ & -4.75457 & -0.8113 & 0.96515 \\
\hline $\mathrm{C}$ & 3.20651 & -2.84063 & 1.82842 \\
\hline $\mathrm{C}$ & 3.44686 & -1.44013 & 1.70605 \\
\hline $\mathrm{C}$ & 4.55388 & -1.01366 & 0.93917 \\
\hline $\mathrm{C}$ & 5.39936 & -1.92249 & 0.28742 \\
\hline $\mathrm{C}$ & 3.99909 & -3.73649 & 1.08547 \\
\hline $\mathrm{C}$ & 5.08036 & -3.29266 & 0.32238 \\
\hline $\mathrm{C}$ & 1.24027 & -2.83725 & 0.93477 \\
\hline $\mathrm{C}$ & -1.59774 & -2.84171 & 1.15442 \\
\hline $\mathrm{C}$ & 0.59755 & -2.67014 & 2.17189 \\
\hline $\mathrm{C}$ & -0.80693 & -2.69428 & 2.32979 \\
\hline $\mathrm{C}$ & 0.44396 & -3.06604 & -0.22628 \\
\hline $\mathrm{C}$ & -0.95541 & -3.04602 & -0.07414 \\
\hline $\mathrm{O}$ & -7.88169 & -2.10825 & -0.40263 \\
\hline $\mathrm{C}$ & -9.05274 & -1.8127 & -1.16835 \\
\hline $\mathrm{O}$ & -6.96028 & 2.5539 & -2.51765 \\
\hline $\mathrm{C}$ & -6.31394 & 2.97717 & -3.71837 \\
\hline $\mathrm{H}$ & 1.87925 & 1.47321 & -0.4745 \\
\hline $\mathrm{H}$ & 3.92415 & 0.45037 & -1.42665 \\
\hline
\end{tabular}




\begin{tabular}{|c|c|c|c|}
\hline $\mathrm{H}$ & 4.17759 & 4.89011 & 0.80279 \\
\hline $\mathrm{H}$ & 6.22285 & 3.88255 & -0.19401 \\
\hline $\mathrm{H}$ & 7.59788 & 1.83139 & 0.64711 \\
\hline $\mathrm{H}$ & 7.75662 & -0.51083 & 1.45523 \\
\hline $\mathrm{H}$ & 6.04866 & 0.42973 & -3.18878 \\
\hline $\mathrm{H}$ & -5.71312 & 4.12884 & -0.96836 \\
\hline $\mathrm{H}$ & -3.67549 & 5.0461 & 0.15122 \\
\hline $\mathrm{H}$ & -3.751 & 0.31662 & -1.38588 \\
\hline $\mathrm{H}$ & -1.72498 & 1.22709 & -0.28875 \\
\hline $\mathrm{H}$ & -1.10983 & 1.93865 & 2.02928 \\
\hline $\mathrm{H}$ & 1.34019 & 1.99837 & 2.15246 \\
\hline $\mathrm{H}$ & -1.05198 & 5.2598 & -0.83575 \\
\hline $\mathrm{H}$ & 1.41507 & 5.28164 & -0.74356 \\
\hline $\mathrm{H}$ & -7.43485 & 2.3364 & -0.0328 \\
\hline $\mathrm{H}$ & -7.90982 & 0.13984 & 1.01855 \\
\hline $\mathrm{H}$ & -5.66884 & 0.31952 & -3.48238 \\
\hline $\mathrm{H}$ & -6.22194 & -1.85401 & -2.4724 \\
\hline $\mathrm{H}$ & -5.94458 & -3.67412 & -0.42723 \\
\hline $\mathrm{H}$ & -5.02372 & 0.23021 & 1.13368 \\
\hline $\mathrm{H}$ & 4.77188 & 0.04981 & 0.85402 \\
\hline $\mathrm{H}$ & 3.80543 & -4.80723 & 1.17296 \\
\hline $\mathrm{H}$ & 5.713 & -4.01514 & -0.18921 \\
\hline $\mathrm{H}$ & -9.53826 & -0.87925 & -0.82971 \\
\hline $\mathrm{H}$ & -8.82669 & -1.71396 & -2.24625 \\
\hline $\mathrm{H}$ & -9.73439 & -2.66032 & -1.01141 \\
\hline $\mathrm{H}$ & -6.28013 & 2.17309 & -4.47655 \\
\hline $\mathrm{H}$ & -5.28603 & 3.33533 & -3.52871 \\
\hline $\mathrm{H}$ & -6.92199 & 3.80552 & -4.10881 \\
\hline $\mathrm{O}$ & 7.65125 & -2.57343 & -0.22348 \\
\hline $\mathrm{O}$ & 7.32975 & 2.40864 & -1.94328 \\
\hline $\mathrm{O}$ & 2.14553 & 4.72036 & 1.96384 \\
\hline $\mathrm{O}$ & -1.85449 & 4.77033 & 1.77238 \\
\hline $\mathrm{C}$ & 8.6512 & 1.99196 & -2.29145 \\
\hline $\mathrm{H}$ & 9.24523 & 1.71186 & -1.40139 \\
\hline $\mathrm{H}$ & 9.11699 & 2.85584 & -2.78605 \\
\hline $\mathrm{H}$ & 8.64071 & 1.13094 & -2.98556 \\
\hline $\mathrm{C}$ & 8.91386 & -2.34485 & -0.85143 \\
\hline $\mathrm{H}$ & 6.24499 & -1.91577 & -2.39275 \\
\hline $\mathrm{H}$ & 8.80572 & -2.13558 & -1.93222 \\
\hline $\mathrm{H}$ & 9.45449 & -1.49926 & -0.38711 \\
\hline $\mathrm{H}$ & 9.49034 & -3.27009 & -0.71269 \\
\hline $\mathrm{C}$ & -2.62934 & 4.23317 & 2.8473 \\
\hline $\mathrm{H}$ & -3.47157 & 3.61829 & 2.48096 \\
\hline $\mathrm{H}$ & -2.00987 & 3.62692 & 3.53307 \\
\hline $\mathrm{H}$ & -3.02491 & 5.10229 & 3.3919 \\
\hline $\mathrm{C}$ & 1.16357 & 5.39766 & 2.75359 \\
\hline
\end{tabular}




$\begin{array}{llll}\mathrm{H} & 0.45732 & 5.97433 & 2.13564 \\ \mathrm{H} & 0.57971 & 4.69257 & 3.37026 \\ \mathrm{H} & 1.7382 & 6.07236 & 3.40446 \\ \mathrm{H} & 1.21899 & -2.52513 & 3.05902 \\ \mathrm{H} & -1.57671 & -3.11869 & -0.96685 \\ \mathrm{C} & -1.33859 & -2.5818 & 3.69813 \\ \mathrm{H} & -0.60344 & -2.2391 & 4.43536 \\ \mathrm{C} & 1.03921 & -3.21372 & -1.56617 \\ \mathrm{H} & 2.04588 & -2.80849 & -1.6905 \\ \mathrm{C} & -2.56944 & -2.90904 & 4.14253 \\ \mathrm{H} & -3.35103 & -3.28554 & 3.48287 \\ \mathrm{H} & -2.81611 & -2.80958 & 5.20158 \\ \mathrm{C} & 0.45222 & -3.81928 & -2.61772 \\ \mathrm{H} & 0.95506 & -3.87454 & -3.58478 \\ \mathrm{H} & -0.52995 & -4.29222 & -2.53969 \\ \mathrm{C} & 2.55293 & -0.47604 & 2.37382 \\ \mathrm{H} & 1.65754 & -0.91211 & 2.82294 \\ \mathrm{C} & 2.73028 & 0.85586 & 2.47906 \\ \mathrm{H} & 1.98916 & 1.47549 & 2.98666 \\ \mathrm{H} & 3.60551 & 1.3695 & 2.07464 \\ \mathrm{H} & -2.78856 & -0.58259 & 1.81895 \\ \mathrm{C} & -3.73006 & -4.92918 & 0.26383 \\ \mathrm{H} & -4.43786 & -5.4109 & -0.42028 \\ \mathrm{C} & -2.7913 & -5.70319 & 0.84435 \\ \mathrm{H} & -2.06683 & -5.3123 & 1.55895 \\ \mathrm{H} & -2.73638 & -6.769 & 0.61319 \\ & & & \end{array}$

Macrocycle 10, Relative Energy $=4.07 \mathrm{kcal} \mathrm{mol}^{-1}, R B 97 D / 6-31 G(d)$

$\begin{array}{lrrr}\mathrm{C} & 2.88909 & 3.24002 & 0.26169 \\ \mathrm{C} & 2.82984 & 2.00675 & -0.40905 \\ \mathrm{C} & 3.98512 & 1.43259 & -0.95927 \\ \mathrm{C} & 5.22677 & 2.08448 & -0.8597 \\ \mathrm{C} & 4.11965 & 3.92506 & 0.30236 \\ \mathrm{C} & 5.27123 & 3.35319 & -0.24855 \\ \mathrm{C} & 6.54795 & 1.39779 & -1.25706 \\ \mathrm{C} & 6.74891 & -1.44643 & -0.28512 \\ \mathrm{C} & 7.22537 & 0.99582 & 0.04958 \\ \mathrm{C} & 7.31368 & -0.26887 & 0.48609 \\ \mathrm{C} & 6.33304 & 0.19781 & -2.15958 \\ \mathrm{C} & 6.44261 & -1.06819 & -1.73158 \\ \mathrm{C} & -4.88255 & 2.15341 & -1.23597 \\ \mathrm{C} & -4.83449 & 3.49934 & -0.81883 \\ \mathrm{C} & -3.69206 & 4.01463 & -0.20205 \\ \mathrm{C} & -2.56632 & 3.19778 & 0.03245 \\ \mathrm{C} & -3.73575 & 1.35897 & -1.06926\end{array}$




\begin{tabular}{|c|c|c|c|}
\hline $\mathrm{C}$ & -2.59236 & 1.87383 & -0.43628 \\
\hline $\mathrm{C}$ & -1.36773 & 3.78537 & 0.79808 \\
\hline $\mathrm{C}$ & 1.63641 & 3.82892 & 0.92475 \\
\hline $\mathrm{C}$ & -0.54818 & 2.71938 & 1.5109 \\
\hline $\mathrm{C}$ & 0.79148 & 2.74364 & 1.57157 \\
\hline $\mathrm{C}$ & -0.50718 & 4.62205 & -0.13618 \\
\hline $\mathrm{C}$ & 0.83343 & 4.631 & -0.08679 \\
\hline $\mathrm{C}$ & -6.23365 & 1.5782 & -1.72657 \\
\hline $\mathrm{C}$ & -6.84996 & -1.09902 & -0.48562 \\
\hline $\mathrm{C}$ & -7.08406 & 1.40259 & -0.47681 \\
\hline $\mathrm{C}$ & -7.34043 & 0.21507 & 0.08935 \\
\hline $\mathrm{C}$ & -6.07924 & 0.26628 & -2.4723 \\
\hline $\mathrm{C}$ & -6.37356 & -0.92101 & -1.92409 \\
\hline $\mathrm{C}$ & -3.32053 & -2.65662 & 1.94773 \\
\hline $\mathrm{C}$ & -3.38434 & -1.25715 & 51.68382 \\
\hline $\mathrm{C}$ & -4.34203 & -0.79605 & 0.75282 \\
\hline $\mathrm{C}$ & -5.19135 & -1.66926 & 50.05493 \\
\hline $\mathrm{C}$ & -4.09587 & -3.52906 & $5 \quad 1.16407$ \\
\hline $\mathrm{C}$ & -5.0143 & -3.0529 & 0.22405 \\
\hline $\mathrm{C}$ & 2.71379 & -2.64108 & 0.83295 \\
\hline $\mathrm{C}$ & 3.60407 & -3.606 & 0.27575 \\
\hline $\mathrm{C}$ & 4.93427 & -3.21473 & 0.00567 \\
\hline $\mathrm{C}$ & 5.39936 & -1.92249 & 0.28742 \\
\hline $\mathrm{C}$ & 3.21995 & -1.38149 & 1.20706 \\
\hline $\mathrm{C}$ & 4.54212 & -1.01972 & 0.94356 \\
\hline $\mathrm{C}$ & 1.08947 & -3.59864 & 1.72824 \\
\hline $\mathrm{C}$ & -1.53898 & -2.69915 & 51.10798 \\
\hline $\mathrm{C}$ & 0.53282 & -3.77649 & 0.45153 \\
\hline $\mathrm{C}$ & -0.75623 & -3.32045 & 0.09315 \\
\hline $\mathrm{C}$ & 0.33126 & -2.90303 & 2.71602 \\
\hline $\mathrm{C}$ & -0.96896 & -2.4884 & 2.37066 \\
\hline $\mathrm{O}$ & -7.88169 & -2.10825 & -0.40263 \\
\hline $\mathrm{C}$ & -9.05274 & -1.8127 & -1.16835 \\
\hline $\mathrm{O}$ & -6.96028 & 2.5539 & -2.51765 \\
\hline $\mathrm{C}$ & -6.31394 & 2.97717 & -3.71837 \\
\hline $\mathrm{H}$ & 1.87925 & 1.47321 & -0.4745 \\
\hline $\mathrm{H}$ & 3.92415 & 0.45037 & -1.42665 \\
\hline $\mathrm{H}$ & 4.17759 & 4.89011 & 0.80279 \\
\hline $\mathrm{H}$ & 6.22285 & 3.88255 & -0.19401 \\
\hline $\mathrm{H}$ & 7.59788 & 1.83139 & 0.64711 \\
\hline $\mathrm{H}$ & 7.75662 & -0.51083 & 1.45523 \\
\hline $\mathrm{H}$ & 6.04866 & 0.42973 & -3.18878 \\
\hline $\mathrm{H}$ & -5.71312 & 4.12884 & -0.96836 \\
\hline $\mathrm{H}$ & -3.67549 & 5.0461 & 0.15122 \\
\hline $\mathrm{H}$ & -3.751 & 0.31662 & -1.38588 \\
\hline $\mathrm{H}$ & -1.72498 & 1.22709 & -0.28875 \\
\hline
\end{tabular}




\begin{tabular}{|c|c|c|c|}
\hline $\mathrm{H}$ & -1.10983 & 1.93865 & 2.02928 \\
\hline $\mathrm{H}$ & 1.34019 & 1.99837 & 2.15246 \\
\hline $\mathrm{H}$ & -1.05198 & 5.2598 & -0.83575 \\
\hline $\mathrm{H}$ & 1.41507 & 5.28164 & -0.74356 \\
\hline $\mathrm{H}$ & -7.43485 & 2.3364 & -0.0328 \\
\hline $\mathrm{H}$ & -7.90982 & 0.13984 & 1.01855 \\
\hline $\mathrm{H}$ & -5.66884 & 0.31952 & -3.48238 \\
\hline $\mathrm{H}$ & -6.22194 & -1.85401 & -2.4724 \\
\hline $\mathrm{H}$ & -4.43871 & 0.27733 & 0.58308 \\
\hline $\mathrm{H}$ & -5.65249 & -3.75456 & -0.31071 \\
\hline $\mathrm{H}$ & 5.62394 & -3.92719 & -0.44434 \\
\hline $\mathrm{H}$ & 2.53819 & -0.64903 & 1.64334 \\
\hline $\mathrm{H}$ & 4.88617 & -0.01649 & 1.18662 \\
\hline $\mathrm{H}$ & -9.53826 & -0.87925 & -0.82971 \\
\hline $\mathrm{H}$ & -8.82669 & -1.71396 & -2.24625 \\
\hline $\mathrm{H}$ & -9.73439 & -2.66032 & -1.01141 \\
\hline $\mathrm{H}$ & -6.28013 & 2.17309 & -4.47655 \\
\hline $\mathrm{H}$ & -5.28603 & 3.33533 & -3.52871 \\
\hline $\mathrm{H}$ & -6.92199 & 3.80552 & -4.10881 \\
\hline $\mathrm{O}$ & 7.65125 & -2.57343 & -0.22348 \\
\hline $\mathrm{O}$ & 7.32975 & 2.40864 & -1.94328 \\
\hline $\mathrm{O}$ & 2.14553 & 4.72036 & 1.96384 \\
\hline $\mathrm{O}$ & -1.85449 & 4.77033 & 1.77238 \\
\hline $\mathrm{C}$ & 8.6512 & 1.99196 & -2.29145 \\
\hline $\mathrm{H}$ & 9.24523 & 1.71186 & -1.40139 \\
\hline $\mathrm{H}$ & 9.11699 & 2.85584 & -2.78605 \\
\hline $\mathrm{H}$ & 8.64071 & 1.13094 & -2.98556 \\
\hline $\mathrm{C}$ & 8.91386 & -2.34485 & -0.85143 \\
\hline $\mathrm{H}$ & 6.24499 & -1.91577 & -2.39275 \\
\hline $\mathrm{H}$ & 8.80572 & -2.13558 & -1.93222 \\
\hline $\mathrm{H}$ & 9.45449 & -1.49926 & -0.38711 \\
\hline $\mathrm{H}$ & 9.49034 & -3.27009 & -0.71269 \\
\hline $\mathrm{C}$ & -2.62934 & 4.23317 & 2.8473 \\
\hline $\mathrm{H}$ & -3.47157 & 3.61829 & 2.48096 \\
\hline $\mathrm{H}$ & -2.00987 & 3.62692 & 3.53307 \\
\hline $\mathrm{H}$ & -3.02491 & 5.10229 & 3.3919 \\
\hline $\mathrm{C}$ & 1.16357 & 5.39766 & 2.75359 \\
\hline $\mathrm{H}$ & 0.45732 & 5.97433 & 2.13564 \\
\hline $\mathrm{H}$ & 0.57971 & 4.69257 & 3.37026 \\
\hline $\mathrm{H}$ & 1.7382 & 6.07236 & 3.40446 \\
\hline $\mathrm{H}$ & 1.13471 & -4.28474 & -0.30585 \\
\hline $\mathrm{H}$ & -1.59653 & -2.04602 & 3.14441 \\
\hline $\mathrm{C}$ & -1.17936 & -3.50948 & -1.30433 \\
\hline $\mathrm{H}$ & -0.5529 & -4.20948 & -1.86908 \\
\hline $\mathrm{C}$ & 0.82838 & -2.70788 & 4.08942 \\
\hline $\mathrm{H}$ & 1.61555 & -3.39126 & 4.41563 \\
\hline
\end{tabular}




$\begin{array}{llll}\mathrm{C} & -2.17673 & -2.88512 & -1.96386 \\ \mathrm{H} & -2.82831 & -2.15331 & -1.4867 \\ \mathrm{H} & -2.35477 & -3.09732 & -3.02008 \\ \mathrm{C} & 0.40642 & -1.75392 & 4.94321 \\ \mathrm{H} & 0.81554 & -1.68471 & 5.95263 \\ \mathrm{H} & -0.33798 & -1.00722 & 4.65559 \\ \mathrm{C} & 3.12863 & -4.97272 & -0.00804 \\ \mathrm{H} & 2.1272 & -5.19935 & 0.36526 \\ \mathrm{C} & 3.79441 & -5.94276 & -0.6655 \\ \mathrm{H} & 3.34531 & -6.92766 & -0.80374 \\ \mathrm{H} & 4.79159 & -5.79452 & -1.08632 \\ \mathrm{H} & -4.02898 & -4.60121 & 1.35471 \\ \mathrm{C} & -2.52646 & -0.2422 & 2.32384 \\ \mathrm{H} & -2.88418 & 0.78614 & 2.199 \\ \mathrm{C} & -1.35464 & -0.42658 & 2.96431 \\ \mathrm{H} & -0.91132 & -1.41223 & 3.10665 \\ \mathrm{H} & -0.80283 & 0.42865 & 3.35991\end{array}$

Macrocycle 10, Relative Energy $=4.29 \mathrm{kcal} \mathrm{mol}^{-1}, R B 97 \mathrm{D} / 6-31 \mathrm{G}(\mathrm{d})$

$\begin{array}{lrrl}\mathrm{C} & 2.88909 & 3.24002 & 0.26169 \\ \mathrm{C} & 2.82984 & 2.00675 & -0.40905 \\ \mathrm{C} & 3.98512 & 1.43259 & -0.95927 \\ \mathrm{C} & 5.22677 & 2.08448 & -0.8597 \\ \mathrm{C} & 4.11965 & 3.92506 & 0.30236 \\ \mathrm{C} & 5.27123 & 3.35319 & -0.24855 \\ \mathrm{C} & 6.54795 & 1.39779 & -1.25706 \\ \mathrm{C} & 6.74891 & -1.44643 & -0.28512 \\ \mathrm{C} & 7.22537 & 0.99582 & 0.04958 \\ \mathrm{C} & 7.31368 & -0.26887 & 0.48609 \\ \mathrm{C} & 6.33304 & 0.19781 & -2.15958 \\ \mathrm{C} & 6.44261 & -1.06819 & -1.73158 \\ \mathrm{C} & -4.88255 & 2.15341 & -1.23597 \\ \mathrm{C} & -4.83449 & 3.49934 & -0.81883 \\ \mathrm{C} & -3.69206 & 4.01463 & -0.20205 \\ \mathrm{C} & -2.56632 & 3.19778 & 0.03245 \\ \mathrm{C} & -3.73575 & 1.35897 & -1.06926 \\ \mathrm{C} & -2.59236 & 1.87383 & -0.43628 \\ \mathrm{C} & -1.36773 & 3.78537 & 0.79808 \\ \mathrm{C} & 1.63641 & 3.82892 & 0.92475 \\ \mathrm{C} & -0.54818 & 2.71938 & 1.5109 \\ \mathrm{C} & 0.79148 & 2.74364 & 1.57157 \\ \mathrm{C} & -0.50718 & 4.62205 & -0.13618 \\ \mathrm{C} & 0.83343 & 4.631 & -0.08679 \\ \mathrm{C} & -6.23365 & 1.5782 & -1.72657 \\ \mathrm{C} & -6.84996 & -1.09902 & -0.48562 \\ \mathrm{C} & -7.08406 & 1.40259 & -0.47681\end{array}$




\begin{tabular}{|c|c|c|c|}
\hline $\mathrm{C}$ & -7.34043 & 0.21507 & 0.08935 \\
\hline $\mathrm{C}$ & -6.07924 & 0.26628 & -2.4723 \\
\hline $\mathrm{C}$ & -6.37356 & -0.92101 & -1.92409 \\
\hline $\mathrm{C}$ & -3.06543 & -2.58135 & 1.06844 \\
\hline $\mathrm{C}$ & -3.99133 & -3.49428 & 0.48405 \\
\hline $\mathrm{C}$ & -5.24844 & -2.99821 & 0.07159 \\
\hline $\mathrm{C}$ & -5.62514 & -1.65891 & 0.2594 \\
\hline $\mathrm{C}$ & -3.49325 & -1.27267 & 1.35257 \\
\hline $\mathrm{C}$ & -4.75457 & -0.8113 & 0.96515 \\
\hline $\mathrm{C}$ & 2.71379 & -2.64108 & 0.83295 \\
\hline $\mathrm{C}$ & 3.60407 & -3.606 & 0.27575 \\
\hline $\mathrm{C}$ & 4.93427 & -3.21473 & 0.00567 \\
\hline $\mathrm{C}$ & 5.39936 & -1.92249 & 0.28742 \\
\hline $\mathrm{C}$ & 3.21995 & -1.38149 & 1.20706 \\
\hline $\mathrm{C}$ & 4.54212 & -1.01972 & 0.94356 \\
\hline $\mathrm{C}$ & 1.24321 & -3.60231 & 1.65577 \\
\hline $\mathrm{C}$ & -1.38524 & -2.70282 & 1.03552 \\
\hline $\mathrm{C}$ & 0.68656 & -3.78016 & 0.37906 \\
\hline $\mathrm{C}$ & -0.6025 & -3.32412 & 0.02068 \\
\hline $\mathrm{C}$ & 0.485 & -2.9067 & 2.64356 \\
\hline $\mathrm{C}$ & -0.81523 & -2.49207 & 2.2982 \\
\hline $\mathrm{O}$ & -7.88169 & -2.10825 & -0.40263 \\
\hline $\mathrm{C}$ & -9.05274 & -1.8127 & -1.16835 \\
\hline $\mathrm{O}$ & -6.96028 & 2.5539 & -2.51765 \\
\hline $\mathrm{C}$ & -6.31394 & 2.97717 & -3.71837 \\
\hline $\mathrm{H}$ & 1.87925 & 1.47321 & -0.4745 \\
\hline $\mathrm{H}$ & 3.92415 & 0.45037 & -1.42665 \\
\hline $\mathrm{H}$ & 4.17759 & 4.89011 & 0.80279 \\
\hline $\mathrm{H}$ & 6.22285 & 3.88255 & -0.19401 \\
\hline $\mathrm{H}$ & 7.59788 & 1.83139 & 0.64711 \\
\hline $\mathrm{H}$ & 7.75662 & -0.51083 & 1.45523 \\
\hline $\mathrm{H}$ & 6.04866 & 0.42973 & -3.18878 \\
\hline $\mathrm{H}$ & -5.71312 & 4.12884 & -0.96836 \\
\hline $\mathrm{H}$ & -3.67549 & 5.0461 & 0.15122 \\
\hline $\mathrm{H}$ & -3.751 & 0.31662 & -1.38588 \\
\hline $\mathrm{H}$ & -1.72498 & 1.22709 & -0.28875 \\
\hline $\mathrm{H}$ & -1.10983 & 1.93865 & 2.02928 \\
\hline $\mathrm{H}$ & 1.34019 & 1.99837 & 2.15246 \\
\hline $\mathrm{H}$ & -1.05198 & 5.2598 & -0.83575 \\
\hline $\mathrm{H}$ & 1.41507 & 5.28164 & -0.74356 \\
\hline $\mathrm{H}$ & -7.43485 & 2.3364 & -0.0328 \\
\hline $\mathrm{H}$ & -7.90982 & 0.13984 & 1.01855 \\
\hline $\mathrm{H}$ & -5.66884 & 0.31952 & -3.48238 \\
\hline $\mathrm{H}$ & -6.22194 & -1.85401 & -2.4724 \\
\hline $\mathrm{H}$ & -5.94458 & -3.67412 & -0.42723 \\
\hline $\mathrm{H}$ & -5.02372 & 0.23021 & 1.13368 \\
\hline
\end{tabular}




\begin{tabular}{|c|c|c|c|}
\hline $\mathrm{H}$ & 5.62394 & -3.92719 & -0.44434 \\
\hline $\mathrm{H}$ & 2.53819 & -0.64903 & 1.64334 \\
\hline $\mathrm{H}$ & 4.88617 & -0.01649 & 1.18662 \\
\hline $\mathrm{H}$ & -9.53826 & -0.87925 & -0.82971 \\
\hline $\mathrm{H}$ & -8.82669 & -1.71396 & -2.24625 \\
\hline $\mathrm{H}$ & -9.73439 & -2.66032 & -1.01141 \\
\hline $\mathrm{H}$ & -6.28013 & 2.17309 & -4.47655 \\
\hline $\mathrm{H}$ & -5.28603 & 3.33533 & -3.52871 \\
\hline $\mathrm{H}$ & -6.92199 & 3.80552 & -4.10881 \\
\hline $\mathrm{O}$ & 7.65125 & -2.57343 & -0.22348 \\
\hline $\mathrm{O}$ & 7.32975 & 2.40864 & -1.94328 \\
\hline $\mathrm{O}$ & 2.14553 & 4.72036 & 1.96384 \\
\hline $\mathrm{O}$ & -1.85449 & 4.77033 & 1.77238 \\
\hline $\mathrm{C}$ & 8.6512 & 1.99196 & -2.29145 \\
\hline $\mathrm{H}$ & 9.24523 & 1.71186 & -1.40139 \\
\hline $\mathrm{H}$ & 9.11699 & 2.85584 & -2.78605 \\
\hline $\mathrm{H}$ & 8.64071 & 1.13094 & -2.98556 \\
\hline $\mathrm{C}$ & 8.91386 & -2.34485 & -0.85143 \\
\hline $\mathrm{H}$ & 6.24499 & -1.91577 & -2.39275 \\
\hline $\mathrm{H}$ & 8.80572 & -2.13558 & -1.93222 \\
\hline $\mathrm{H}$ & 9.45449 & -1.49926 & -0.38711 \\
\hline $\mathrm{H}$ & 9.49034 & -3.27009 & -0.71269 \\
\hline $\mathrm{C}$ & -2.62934 & 4.23317 & 2.8473 \\
\hline $\mathrm{H}$ & -3.47157 & 3.61829 & 2.48096 \\
\hline $\mathrm{H}$ & -2.00987 & 3.62692 & 3.53307 \\
\hline $\mathrm{H}$ & -3.02491 & 5.10229 & 3.3919 \\
\hline $\mathrm{C}$ & 1.16357 & 5.39766 & 2.75359 \\
\hline $\mathrm{H}$ & 0.45732 & 5.97433 & 2.13564 \\
\hline $\mathrm{H}$ & 0.57971 & 4.69257 & 3.37026 \\
\hline $\mathrm{H}$ & 1.7382 & 6.07236 & 3.40446 \\
\hline $\mathrm{H}$ & 1.28845 & -4.28841 & -0.37832 \\
\hline $\mathrm{H}$ & -1.44279 & -2.04969 & 3.07194 \\
\hline $\mathrm{C}$ & -1.02562 & -3.51315 & -1.37679 \\
\hline $\mathrm{H}$ & -0.39916 & -4.21315 & -1.94155 \\
\hline $\mathrm{C}$ & 0.98212 & -2.71155 & 4.01696 \\
\hline $\mathrm{H}$ & 1.76928 & -3.39493 & 4.34317 \\
\hline $\mathrm{C}$ & -2.023 & -2.88879 & -2.03633 \\
\hline $\mathrm{H}$ & -2.67457 & -2.15698 & -1.55917 \\
\hline $\mathrm{H}$ & -2.20103 & -3.10099 & -3.09255 \\
\hline $\mathrm{C}$ & 0.56016 & -1.75759 & 4.87074 \\
\hline $\mathrm{H}$ & 0.96928 & -1.68838 & 5.88016 \\
\hline $\mathrm{H}$ & -0.18425 & -1.01089 & 4.58313 \\
\hline $\mathrm{C}$ & 3.12863 & -4.97272 & -0.00804 \\
\hline $\mathrm{H}$ & 2.1272 & -5.19935 & 0.36526 \\
\hline $\mathrm{C}$ & 3.79441 & -5.94276 & -0.6655 \\
\hline $\mathrm{H}$ & 3.34531 & -6.92766 & -0.80374 \\
\hline
\end{tabular}




$\begin{array}{llll}\mathrm{H} & 4.79159 & -5.79452 & -1.08632 \\ \mathrm{H} & -2.78856 & -0.58259 & 1.81895 \\ \mathrm{C} & -3.73006 & -4.92918 & 0.26383 \\ \mathrm{H} & -4.43786 & -5.4109 & -0.42028 \\ \mathrm{C} & -2.7913 & -5.70319 & 0.84435 \\ \mathrm{H} & -2.06683 & -5.3123 & 1.55895 \\ \mathrm{H} & -2.73638 & -6.769 & 0.61319\end{array}$

Macrocycle 10, Relative Energy $=5.10 \mathrm{kcal} \mathrm{mol}^{-1}, R B 97 \mathrm{D} / 6-31 G(d)$

\begin{tabular}{|c|c|c|c|}
\hline $\mathrm{C}$ & 2.88909 & 3.24002 & 0.26169 \\
\hline $\mathrm{C}$ & 2.82984 & 2.00675 & -0.40905 \\
\hline $\mathrm{C}$ & 3.98512 & 1.43259 & -0.95927 \\
\hline $\mathrm{C}$ & 5.22677 & 2.08448 & -0.8597 \\
\hline $\mathrm{C}$ & 4.11965 & 3.92506 & 0.30236 \\
\hline $\mathrm{C}$ & 5.27123 & 3.35319 & -0.24855 \\
\hline $\mathrm{C}$ & 6.54795 & 1.39779 & -1.25706 \\
\hline $\mathrm{C}$ & 6.74891 & -1.44643 & -0.28512 \\
\hline $\mathrm{C}$ & 7.22537 & 0.99582 & 0.04958 \\
\hline $\mathrm{C}$ & 7.31368 & -0.26887 & 0.48609 \\
\hline $\mathrm{C}$ & 6.33304 & 0.19781 & -2.15958 \\
\hline $\mathrm{C}$ & 6.44261 & -1.06819 & -1.73158 \\
\hline $\mathrm{C}$ & -4.88255 & 2.15341 & -1.23597 \\
\hline $\mathrm{C}$ & -4.83449 & 3.49934 & -0.81883 \\
\hline $\mathrm{C}$ & -3.69206 & 4.01463 & -0.20205 \\
\hline $\mathrm{C}$ & -2.56632 & 3.19778 & 0.03245 \\
\hline $\mathrm{C}$ & -3.73575 & 1.35897 & -1.06926 \\
\hline $\mathrm{C}$ & -2.59236 & 1.87383 & -0.43628 \\
\hline $\mathrm{C}$ & -1.36773 & 3.78537 & 0.79808 \\
\hline $\mathrm{C}$ & 1.63641 & 3.82892 & 0.92475 \\
\hline $\mathrm{C}$ & -0.54818 & 2.71938 & 1.5109 \\
\hline $\mathrm{C}$ & 0.79148 & 2.74364 & 1.57157 \\
\hline $\mathrm{C}$ & -0.50718 & 4.62205 & -0.13618 \\
\hline $\mathrm{C}$ & 0.83343 & 4.631 & -0.08679 \\
\hline $\mathrm{C}$ & -6.23365 & 1.5782 & -1.72657 \\
\hline $\mathrm{C}$ & -6.84996 & -1.09902 & -0.48562 \\
\hline $\mathrm{C}$ & -7.08406 & 1.40259 & -0.47681 \\
\hline $\mathrm{C}$ & -7.34043 & 0.21507 & 0.08935 \\
\hline $\mathrm{C}$ & -6.07924 & 0.26628 & -2.4723 \\
\hline $\mathrm{C}$ & -6.37356 & -0.92101 & -1.92409 \\
\hline $\mathrm{C}$ & -3.06543 & -2.58135 & 1.06844 \\
\hline $\mathrm{C}$ & -3.99133 & -3.49428 & 0.48405 \\
\hline $\mathrm{C}$ & -5.24844 & -2.99821 & 0.07159 \\
\hline $\mathrm{C}$ & -5.62514 & -1.65891 & 0.2594 \\
\hline $\mathrm{C}$ & -3.49325 & -1.27267 & 1.35257 \\
\hline $\mathrm{C}$ & -4.75457 & -0.8113 & 0.96515 \\
\hline
\end{tabular}




\begin{tabular}{|c|c|c|c|}
\hline $\mathrm{C}$ & 2.71379 & -2.64108 & 0.83295 \\
\hline $\mathrm{C}$ & 3.60407 & -3.606 & 0.27575 \\
\hline $\mathrm{C}$ & 4.93427 & -3.21473 & 0.00567 \\
\hline $\mathrm{C}$ & 5.39936 & -1.92249 & 0.28742 \\
\hline $\mathrm{C}$ & 3.21995 & -1.38149 & 1.20706 \\
\hline $\mathrm{C}$ & 4.54212 & -1.01972 & 0.94356 \\
\hline $\mathrm{C}$ & 1.24027 & -2.83725 & 0.93477 \\
\hline $\mathrm{C}$ & -1.59774 & -2.84171 & 11.15442 \\
\hline $\mathrm{C}$ & 0.59755 & -2.67014 & 2.17189 \\
\hline $\mathrm{C}$ & -0.80693 & -2.69428 & $\begin{array}{l}3.32979 \\
3\end{array}$ \\
\hline $\mathrm{C}$ & 0.44396 & -3.06604 & -0.22628 \\
\hline $\mathrm{C}$ & -0.95541 & -3.04602 & $2-0.07414$ \\
\hline $\mathrm{O}$ & -7.88169 & -2.10825 & $5-0.40263$ \\
\hline $\mathrm{C}$ & -9.05274 & -1.8127 & -1.16835 \\
\hline $\mathrm{O}$ & -6.96028 & 2.5539 & -2.51765 \\
\hline $\mathrm{C}$ & -6.31394 & 2.97717 & -3.71837 \\
\hline $\mathrm{H}$ & 1.87925 & 1.47321 & -0.4745 \\
\hline $\mathrm{H}$ & 3.92415 & 0.45037 & -1.42665 \\
\hline $\mathrm{H}$ & 4.17759 & 4.89011 & 0.80279 \\
\hline $\mathrm{H}$ & 6.22285 & 3.88255 & -0.19401 \\
\hline $\mathrm{H}$ & 7.59788 & 1.83139 & 0.64711 \\
\hline $\mathrm{H}$ & 7.75662 & -0.51083 & 31.45523 \\
\hline $\mathrm{H}$ & 6.04866 & 0.42973 & -3.18878 \\
\hline $\mathrm{H}$ & -5.71312 & 4.12884 & $4-0.96836$ \\
\hline $\mathrm{H}$ & -3.67549 & 5.0461 & 0.15122 \\
\hline $\mathrm{H}$ & -3.751 & 0.31662 & -1.38588 \\
\hline $\mathrm{H}$ & -1.72498 & 1.22709 & -0.28875 \\
\hline $\mathrm{H}$ & -1.10983 & 1.93865 & $5 \quad 2.02928$ \\
\hline $\mathrm{H}$ & 1.34019 & 1.99837 & 2.15246 \\
\hline $\mathrm{H}$ & -1.05198 & 5.2598 & -0.83575 \\
\hline $\mathrm{H}$ & 1.41507 & 5.28164 & -0.74356 \\
\hline $\mathrm{H}$ & -7.43485 & 2.3364 & -0.0328 \\
\hline $\mathrm{H}$ & -7.90982 & 0.13984 & $4 \quad 1.01855$ \\
\hline $\mathrm{H}$ & -5.66884 & 0.31952 & -3.48238 \\
\hline $\mathrm{H}$ & -6.22194 & -1.85401 & $1-2.4724$ \\
\hline $\mathrm{H}$ & -5.94458 & -3.67412 & $2-0.42723$ \\
\hline $\mathrm{H}$ & -5.02372 & 0.23021 & 1.13368 \\
\hline $\mathrm{H}$ & 5.62394 & -3.92719 & -0.44434 \\
\hline $\mathrm{H}$ & 2.53819 & -0.64903 & 31.64334 \\
\hline $\mathrm{H}$ & 4.88617 & -0.01649 & 1.18662 \\
\hline $\mathrm{H}$ & -9.53826 & -0.87925 & $5-0.82971$ \\
\hline $\mathrm{H}$ & -8.82669 & -1.71396 & $6-2.24625$ \\
\hline $\mathrm{H}$ & -9.73439 & -2.66032 & $2-1.01141$ \\
\hline $\mathrm{H}$ & -6.28013 & 2.17309 & -4.47655 \\
\hline $\mathrm{H}$ & -5.28603 & 3.33533 & $3-3.52871$ \\
\hline $\mathrm{H}$ & -6.92199 & 3.80552 & $2-4.10881$ \\
\hline
\end{tabular}




\begin{tabular}{|c|c|c|c|}
\hline $\mathrm{O}$ & 7.65125 & -2.57343 & -0.22348 \\
\hline $\mathrm{O}$ & 7.32975 & 2.40864 & -1.94328 \\
\hline $\mathrm{O}$ & 2.14553 & 4.72036 & 1.96384 \\
\hline $\mathrm{O}$ & -1.85449 & 4.77033 & 1.77238 \\
\hline $\mathrm{C}$ & 8.6512 & 1.99196 & -2.29145 \\
\hline $\mathrm{H}$ & 9.24523 & 1.71186 & -1.40139 \\
\hline $\mathrm{H}$ & 9.11699 & 2.85584 & -2.78605 \\
\hline $\mathrm{H}$ & 8.64071 & 1.13094 & -2.98556 \\
\hline $\mathrm{C}$ & 8.91386 & -2.34485 & -0.85143 \\
\hline $\mathrm{H}$ & 6.24499 & -1.91577 & -2.39275 \\
\hline $\mathrm{H}$ & 8.80572 & -2.13558 & -1.93222 \\
\hline $\mathrm{H}$ & 9.45449 & -1.49926 & -0.38711 \\
\hline $\mathrm{H}$ & 9.49034 & -3.27009 & -0.71269 \\
\hline $\mathrm{C}$ & -2.62934 & 4.23317 & 2.8473 \\
\hline $\mathrm{H}$ & -3.47157 & 3.61829 & 2.48096 \\
\hline $\mathrm{H}$ & -2.00987 & 3.62692 & 3.53307 \\
\hline $\mathrm{H}$ & -3.02491 & 5.10229 & 3.3919 \\
\hline $\mathrm{C}$ & 1.16357 & 5.39766 & 2.75359 \\
\hline $\mathrm{H}$ & 0.45732 & 5.97433 & 2.13564 \\
\hline $\mathrm{H}$ & 0.57971 & 4.69257 & 3.37026 \\
\hline $\mathrm{H}$ & 1.7382 & 6.07236 & 3.40446 \\
\hline $\mathrm{H}$ & 1.21899 & -2.52513 & 3.05902 \\
\hline $\mathrm{H}$ & -1.57671 & -3.11869 & -0.96685 \\
\hline $\mathrm{C}$ & -1.33859 & -2.5818 & 3.69813 \\
\hline $\mathrm{H}$ & -0.60344 & -2.2391 & 4.43536 \\
\hline $\mathrm{C}$ & 1.03921 & -3.21372 & -1.56617 \\
\hline $\mathrm{H}$ & 2.04588 & -2.80849 & -1.6905 \\
\hline $\mathrm{C}$ & -2.56944 & -2.90904 & 4.14253 \\
\hline $\mathrm{H}$ & -3.35103 & -3.28554 & 3.48287 \\
\hline $\mathrm{H}$ & -2.81611 & -2.80958 & 5.20158 \\
\hline $\mathrm{C}$ & 0.45222 & -3.81928 & -2.61772 \\
\hline $\mathrm{H}$ & 0.95506 & -3.87454 & -3.58478 \\
\hline $\mathrm{H}$ & -0.52995 & -4.29222 & -2.53969 \\
\hline $\mathrm{C}$ & 3.12863 & -4.97272 & -0.00804 \\
\hline $\mathrm{H}$ & 2.1272 & -5.19935 & 0.36526 \\
\hline $\mathrm{C}$ & 3.79441 & -5.94276 & -0.6655 \\
\hline $\mathrm{H}$ & 3.34531 & -6.92766 & -0.80374 \\
\hline $\mathrm{H}$ & 4.79159 & -5.79452 & -1.08632 \\
\hline $\mathrm{H}$ & -2.78856 & -0.58259 & 1.81895 \\
\hline $\mathrm{C}$ & -3.73006 & -4.92918 & 0.26383 \\
\hline $\mathrm{H}$ & -4.43786 & -5.4109 & -0.42028 \\
\hline $\mathrm{C}$ & -2.7913 & -5.70319 & 0.84435 \\
\hline $\mathrm{H}$ & -2.06683 & -5.3123 & 1.55895 \\
\hline $\mathrm{H}$ & -2.73638 & -6.769 & 0.61319 \\
\hline
\end{tabular}

Macrocycle 12, Relative Energy $=0.00 \mathrm{kcal} \mathrm{mol}^{-1}, R B 97 \mathrm{D} / 6-31 G(d)$

$\begin{array}{llll}\text { C } & 24.59729 & -24.81995 & -0.04405\end{array}$ 


\begin{tabular}{|c|c|}
\hline $\mathrm{C}$ & $24.15108-23.62601-0.64851$ \\
\hline $\mathrm{C}$ & $\begin{array}{lll}22.86946 & -23.12895 & -0.39915\end{array}$ \\
\hline $\mathrm{C}$ & $\begin{array}{lll}21.97756 & -23.81671 & 0.44821\end{array}$ \\
\hline $\mathrm{C}$ & $\begin{array}{lll}23.68026 & -25.54872 & 0.73362\end{array}$ \\
\hline $\mathrm{C}$ & $22.3838 \quad-25.05654 \quad 0.97304$ \\
\hline $\mathrm{C}$ & $20.63967 \quad-23.13036 \quad 0.81461$ \\
\hline $\mathrm{C}$ & $\begin{array}{lll}18.10966 & -24.72831 & 0.46672\end{array}$ \\
\hline $\mathrm{C}$ & $\begin{array}{lll}19.92311 & -23.81831 & 1.95955\end{array}$ \\
\hline $\mathrm{C}$ & $\begin{array}{lll}18.78125 & -24.50173 & 1.809\end{array}$ \\
\hline $\mathrm{C}$ & $\begin{array}{lll}19.75401 & -23.04983 & -0.4161\end{array}$ \\
\hline $\mathrm{C}$ & $\begin{array}{lll}18.61701 & -23.74199 & -0.56746\end{array}$ \\
\hline $\mathrm{C}$ & $\begin{array}{lll}31.26558 & -30.27337 & -0.67754\end{array}$ \\
\hline $\mathrm{C}$ & $\begin{array}{llll}31.13867 & -29.3109 & -1.70269\end{array}$ \\
\hline $\mathrm{C}$ & $30.17014-28.30934-1.62621$ \\
\hline $\mathrm{C}$ & $29.2828-28.23059-0.52863$ \\
\hline $\mathrm{C}$ & $\begin{array}{lll}30.34832 & -30.23301 & 0.38222\end{array}$ \\
\hline $\mathrm{C}$ & $\begin{array}{llll}29.36389 & -29.2304 & 0.45292\end{array}$ \\
\hline $\mathrm{C}$ & $28.40819-26.96807-0.43061$ \\
\hline $\mathrm{C}$ & $26.0732-25.23299-0.21021$ \\
\hline $\mathrm{C}$ & $27.3899-26.86788-1.56724$ \\
\hline $\mathrm{C}$ & $26.31541-26.07069-1.46766$ \\
\hline $\mathrm{C}$ & $\begin{array}{lll}27.69587 & -26.7799 & 0.90606\end{array}$ \\
\hline $\mathrm{C}$ & $\begin{array}{lll}26.62063 & -25.98311 & 1.00653\end{array}$ \\
\hline $\mathrm{C}$ & $\begin{array}{lll}32.40944 & -31.30822 & -0.78179\end{array}$ \\
\hline $\mathrm{C}$ & $31.67349-34.2101-0.36328$ \\
\hline $\mathrm{C}$ & $32.06953-32.25629-1.9153$ \\
\hline $\mathrm{C}$ & $31.75156-33.5441-1.73281$ \\
\hline $\mathrm{C}$ & $\begin{array}{lll}32.67083 & -32.06641 & 0.50913\end{array}$ \\
\hline $\mathrm{C}$ & $\begin{array}{lll}32.37969 & -33.36447 & 0.68201\end{array}$ \\
\hline $\mathrm{C}$ & $27.35942-34.11972-0.00968$ \\
\hline $\mathrm{C}$ & $\begin{array}{lll}28.13963 & -33.45526 & 0.97603\end{array}$ \\
\hline $\mathrm{C}$ & $\begin{array}{llll}29.53936 & -33.59938 & 0.91773\end{array}$ \\
\hline $\mathrm{C}$ & $\begin{array}{lll}30.16917 & -34.35787 & -0.07261\end{array}$ \\
\hline $\mathrm{C}$ & $27.98132-35.02001-0.89503$ \\
\hline $\mathrm{C}$ & $29.37325-35.14365-0.93279$ \\
\hline $\mathrm{C}$ & $\begin{array}{lll}18.86549 & -28.81588 & -0.8946\end{array}$ \\
\hline $\mathrm{C}$ & $\begin{array}{lll}19.61336 & -27.73571 & -1.44208\end{array}$ \\
\hline $\mathrm{C}$ & $\begin{array}{lll}19.39021 & -26.43768 & -0.93047\end{array}$ \\
\hline $\mathrm{C}$ & $\begin{array}{lll}18.45186 & -26.18677 & 0.08036\end{array}$ \\
\hline $\mathrm{C}$ & $\begin{array}{lll}17.96283 & -28.55906 & 0.15947\end{array}$ \\
\hline $\mathrm{C}$ & $\begin{array}{lll}17.74877 & -27.26914 & 0.64064\end{array}$ \\
\hline $\mathrm{C}$ & $20.60031-27.98033-2.51041$ \\
\hline $\mathrm{C}$ & $\begin{array}{llll}21.12209 & -27.05791 & -3.34391\end{array}$ \\
\hline $\mathrm{C}$ & $27.49794-32.58955 \quad 1.98106$ \\
\hline $\mathrm{C}$ & $\begin{array}{lll}28.10913 & -31.83598 & 2.91848\end{array}$ \\
\hline $\mathrm{C}$ & $19.91595-30.2051-0.40636$ \\
\hline
\end{tabular}




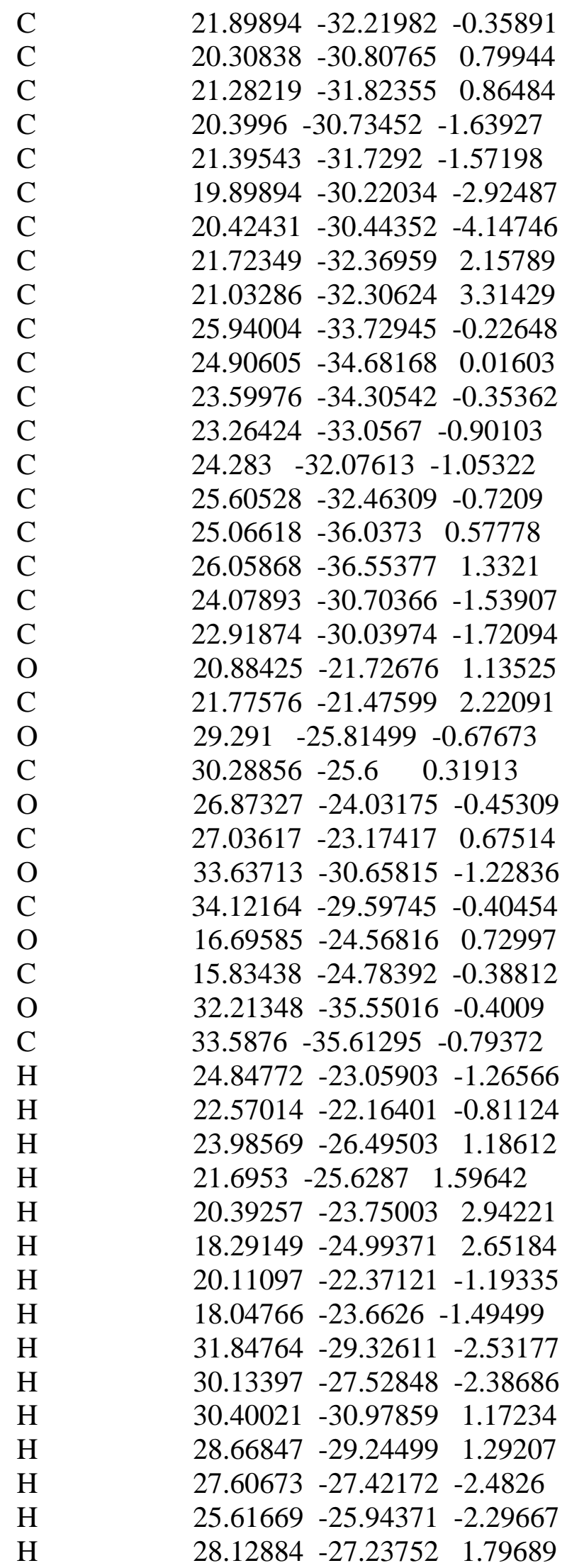




\begin{tabular}{|c|c|}
\hline $\mathrm{H}$ & $\begin{array}{lll}26.15333 & -25.78858 & 1.97422\end{array}$ \\
\hline $\mathrm{H}$ & $\begin{array}{llll}32.06485 & -31.80372 & -2.90857\end{array}$ \\
\hline $\mathrm{H}$ & $\begin{array}{llll}31.46518 & -34.17875 & -2.57391\end{array}$ \\
\hline $\mathrm{H}$ & $\begin{array}{llll}33.13564 & -31.49937 & 1.31828\end{array}$ \\
\hline $\mathrm{H}$ & $\begin{array}{lll}32.60205 & -33.87204 & 1.62424\end{array}$ \\
\hline $\mathrm{H}$ & $\begin{array}{lll}30.15273 & -33.06064 & 1.6358\end{array}$ \\
\hline $\mathrm{H}$ & $27.36484-35.55301-1.62176$ \\
\hline $\mathrm{H}$ & $29.85366-35.79552-1.66286$ \\
\hline $\mathrm{H}$ & $19.96146-25.59809-1.327$ \\
\hline $\mathrm{H}$ & $\begin{array}{lll}17.38233 & -29.3882 & 0.5657\end{array}$ \\
\hline $\mathrm{H}$ & $\begin{array}{lll}17.0087 & -27.09696 & 1.42085\end{array}$ \\
\hline $\mathrm{H}$ & $20.912 \quad-29.02208-2.62616$ \\
\hline $\mathrm{H}$ & $21.86503-27.33737-4.09308$ \\
\hline $\mathrm{H}$ & $\begin{array}{lll}20.82452 & -26.00701 & -3.31074\end{array}$ \\
\hline $\mathrm{H}$ & $26.40625 \quad-32.5734 \quad 1.95124$ \\
\hline $\mathrm{H}$ & $27.52099-31.24635 \quad 3.62395$ \\
\hline $\mathrm{H}$ & $29.1949-31.78459 \quad 3.02724$ \\
\hline $\mathrm{H}$ & $\begin{array}{lll}19.9341 & -30.38136 & 1.73026\end{array}$ \\
\hline $\mathrm{H}$ & $21.83994-32.10116-2.49597$ \\
\hline $\mathrm{H}$ & $\begin{array}{lll}19.00689 & -29.59264 & -2.85401\end{array}$ \\
\hline $\mathrm{H}$ & $\begin{array}{lll}19.95118 & -30.02979 & -5.03963\end{array}$ \\
\hline $\mathrm{H}$ & $21.33183-31.03207-4.30122$ \\
\hline $\mathrm{H}$ & $\begin{array}{lll}22.68532 & -32.88645 & 2.15173\end{array}$ \\
\hline $\mathrm{H}$ & $21.43756 \quad-32.73773 \quad 4.23146$ \\
\hline $\mathrm{H}$ & $20.04552-31.8421 \quad 3.37915$ \\
\hline $\mathrm{H}$ & $22.80057-35.04122-0.2389$ \\
\hline $\mathrm{H}$ & $26.41344-31.74523 \quad-0.88361$ \\
\hline $\mathrm{H}$ & $\begin{array}{lll}24.21207 & -36.69274 & 0.37094\end{array}$ \\
\hline $\mathrm{H}$ & $\begin{array}{lll}25.9918 & -37.58786 & 1.6732\end{array}$ \\
\hline $\mathrm{H}$ & $26.9478-35.99862 \quad 1.62471$ \\
\hline $\mathrm{H}$ & $25.01032-30.16444-1.74603$ \\
\hline $\mathrm{H}$ & $\begin{array}{llll}22.92981 & -29.00793 & -2.07773\end{array}$ \\
\hline $\mathrm{H}$ & $21.94546-30.48657-1.52179$ \\
\hline $\mathrm{H}$ & $22.76278-21.94813 \quad 2.06512$ \\
\hline $\mathrm{H}$ & $\begin{array}{llll}21.89749 & -20.38396 & 2.2561\end{array}$ \\
\hline $\mathrm{H}$ & $\begin{array}{lll}21.36225 & -21.81757 & 3.18783\end{array}$ \\
\hline $\mathrm{H}$ & $30.89459-24.75942-0.04796$ \\
\hline $\mathrm{H}$ & $29.84193 \quad-25.32565 \quad 1.29332$ \\
\hline $\mathrm{H}$ & $\begin{array}{lll}30.93841 & -26.48511 & 0.45842\end{array}$ \\
\hline $\mathrm{H}$ & $27.66275-23.64412 \quad 1.45502$ \\
\hline $\mathrm{H}$ & $\begin{array}{lll}27.54604 & -22.27785 & 0.29385\end{array}$ \\
\hline $\mathrm{H}$ & $26.06598 \quad-22.87988 \quad 1.11858$ \\
\hline $\mathrm{H}$ & $34.98674-29.18032-0.93933$ \\
\hline $\mathrm{H}$ & $\begin{array}{lll}33.36538 & -28.80513 & -0.25978\end{array}$ \\
\hline $\mathrm{H}$ & $\begin{array}{llll}34.45763 & -29.95702 & 0.58577\end{array}$ \\
\hline $\mathrm{H}$ & $\begin{array}{lll}15.90852 & -23.96797 & -1.13046\end{array}$ \\
\hline
\end{tabular}




\begin{tabular}{|c|c|}
\hline $\mathrm{H}$ & $\begin{array}{lll}16.04285 & -25.74681 & -0.88862\end{array}$ \\
\hline $\mathrm{H}$ & $\begin{array}{lll}14.81337 & -24.79948 & 0.01885\end{array}$ \\
\hline $\mathrm{H}$ & $\begin{array}{llll}33.85783 & -36.6779 & -0.77136\end{array}$ \\
\hline $\mathrm{H}$ & $33.74437-35.2114-1.81179$ \\
\hline $\mathrm{H}$ & $\begin{array}{llll}34.23787 & -35.05161 & -0.09736\end{array}$ \\
\hline
\end{tabular}

Macrocycle 12, Relative Energy $=1.05 \mathrm{kcal} \mathrm{mol}^{-1}, \mathrm{RB} 97 \mathrm{D} / 6-31 G(\mathrm{~d})$

$\begin{array}{llll}\text { C } & 24.59729 & -24.81995 & -0.04405 \\ \text { C } & 24.15108 & -23.62601 & -0.64851 \\ \text { C } & 22.86946 & -23.12895 & -0.39915 \\ \text { C } & 21.97756 & -23.81671 & 0.44821 \\ \text { C } & 23.68026 & -25.54872 & 0.73362 \\ \text { C } & 22.3838 & -25.05654 & 0.97304 \\ \text { C } & 20.63967 & -23.13036 & 0.81461 \\ \text { C } & 18.10966 & -24.72831 & 0.46672 \\ \text { C } & 19.92311 & -23.81831 & 1.95955 \\ \text { C } & 18.78125 & -24.50173 & 1.809 \\ \text { C } & 19.75401 & -23.04983 & -0.4161 \\ \text { C } & 18.61701 & -23.74199 & -0.56746 \\ \text { C } & 31.26558 & -30.27337 & -0.67754 \\ \text { C } & 31.13867 & -29.3109 & -1.70269 \\ \text { C } & 30.17014 & -28.30934 & -1.62621 \\ \text { C } & 29.2828 & -28.23059 & -0.52863 \\ \text { C } & 30.34832 & -30.23301 & 0.38222 \\ \text { C } & 29.36389 & -29.2304 & 0.45292 \\ \text { C } & 28.40819 & -26.96807 & -0.43061 \\ \text { C } & 26.0732 & -25.23299 & -0.21021 \\ \text { C } & 27.3899 & -26.86788 & -1.56724 \\ \text { C } & 26.31541 & -26.07069 & -1.46766 \\ \text { C } & 27.69587 & -26.7799 & 0.90606 \\ \text { C } & 26.62063 & -25.98311 & 1.00653 \\ \text { C } & 32.40944 & -31.30822 & -0.78179 \\ \text { C } & 31.67349 & -34.2101 & -0.36328 \\ \text { C } & 32.06953 & -32.25629 & -1.9153 \\ \text { C } & 31.75156 & -33.5441 & -1.73281 \\ \text { C } & 32.67083 & -32.06641 & 0.50913 \\ \text { C } & 32.37969 & -33.36447 & 0.68201 \\ \text { C } & 27.35942 & -34.11972 & -0.00968 \\ \text { C } & 28.13963 & -33.45526 & 0.97603 \\ \text { C } & 29.53936 & -33.59938 & 0.91773 \\ \text { C } & 30.16917 & -34.35787 & -0.07261 \\ \text { C } & 27.98132 & -35.02001 & -0.89503 \\ \text { C } & 29.37325 & -35.14365 & -0.93279 \\ \text { C } & 18.86549 & -28.81588 & -0.8946 \\ \text { C } & 19.61336 & -27.73571 & -1.44208 \\ \text { C } & 19.39021 & -26.43768 & -0.93047\end{array}$




$\begin{array}{llll}\mathrm{C} & 18.45186 & -26.18677 & 0.08036 \\ \mathrm{C} & 17.96283 & -28.55906 & 0.15947 \\ \mathrm{C} & 17.74877 & -27.26914 & 0.64064 \\ \mathrm{C} & 20.60031 & -27.98033 & -2.51041 \\ \mathrm{C} & 21.12209 & -27.05791 & -3.34391 \\ \mathrm{C} & 27.49794 & -32.58955 & 1.98106 \\ \mathrm{C} & 28.10913 & -31.83598 & 2.91848 \\ \mathrm{C} & 19.91595 & -30.2051 & -0.40636 \\ \mathrm{C} & 21.89894 & -32.21982 & -0.35891 \\ \mathrm{C} & 20.30838 & -30.80765 & 0.79944 \\ \mathrm{C} & 21.28219 & -31.82355 & 0.86484 \\ \mathrm{C} & 20.3996 & -30.73452 & -1.63927 \\ \mathrm{C} & 21.39543 & -31.7292 & -1.57198 \\ \mathrm{C} & 19.89894 & -30.22034 & -2.92487 \\ \mathrm{C} & 20.42431 & -30.44352 & -4.14746 \\ \mathrm{C} & 21.72349 & -32.36959 & 2.15789 \\ \mathrm{C} & 21.03286 & -32.30624 & 3.31429 \\ \mathrm{C} & 25.94004 & -33.72945 & -0.22648 \\ \mathrm{C} & 25.63956 & -32.43093 & -0.73463 \\ \mathrm{C} & 24.27968 & -32.06497 & -0.77193 \\ \mathrm{C} & 23.22602 & -32.90591 & -0.37931 \\ \mathrm{C} & 23.52345 & -34.23276 & 0.03729 \\ \mathrm{C} & 24.89203 & -34.59265 & 0.11523 \\ \mathrm{C} & 26.60746 & -31.41337 & -1.18927 \\ \mathrm{C} & 27.88277 & -31.55266 & -1.60756 \\ \mathrm{C} & 22.53102 & -35.25277 & 0.40609 \\ \mathrm{C} & 21.19449 & -35.2302 & 0.22423 \\ \mathrm{O} & 20.88425 & -21.72676 & 1.13525 \\ \mathrm{C} & 21.77576 & -21.47599 & 2.22091 \\ \mathrm{O} & 29.291 & -25.81499 & -0.67673 \\ \mathrm{C} & 30.28856 & -25.6 & 0.31913 \\ \mathrm{O} & 26.87327 & -24.03175 & -0.45309 \\ \mathrm{C} & 27.03617 & -23.17417 & 0.67514 \\ \mathrm{O} & 33.63713 & -30.65815 & -1.22836 \\ \mathrm{C} & 34.12164 & -29.59745 & -0.40454 \\ \mathrm{O} & 16.69585 & -24.56816 & 0.72997 \\ \mathrm{C} & 15.83438 & -24.78392 & -0.38812 \\ \mathrm{O} & 32.21348 & -35.55016 & -0.4009 \\ \mathrm{C} & 33.5876 & -35.61295 & -0.79372 \\ \mathrm{H} & 24.84772 & -23.05903 & -1.26566 \\ \mathrm{H} & 22.57014 & -22.16401 & -0.81124 \\ \mathrm{H} & 23.98569 & -26.49503 & 1.18612 \\ \mathrm{H} & 21.6953 & -25.6287 & 1.59642 \\ \mathrm{H} & 20.39257 & -23.75003 & 2.94221 \\ \mathrm{H} & 18.29149 & -24.99371 & 2.65184 \\ \mathrm{H} & 20.11097 & -22.37121 & -1.19335\end{array}$




\begin{tabular}{|c|c|}
\hline $\mathrm{H}$ & $18.04766-23.6626-1.49499$ \\
\hline $\mathrm{H}$ & $\begin{array}{llll}31.84764 & -29.32611 & -2.53177\end{array}$ \\
\hline $\mathrm{H}$ & $\begin{array}{llll}30.13397 & -27.52848 & -2.38686\end{array}$ \\
\hline $\mathrm{H}$ & $\begin{array}{lll}30.40021 & -30.97859 & 1.17234\end{array}$ \\
\hline $\mathrm{H}$ & $\begin{array}{lll}28.66847 & -29.24499 & 1.29207\end{array}$ \\
\hline $\mathrm{H}$ & $27.60673-27.42172-2.4826$ \\
\hline $\mathrm{H}$ & $\begin{array}{llll}25.61669 & -25.94371 & -2.29667\end{array}$ \\
\hline $\mathrm{H}$ & $\begin{array}{lll}28.12884 & -27.23752 & 1.79689\end{array}$ \\
\hline $\mathrm{H}$ & $\begin{array}{lll}26.15333 & -25.78858 & 1.97422\end{array}$ \\
\hline $\mathrm{H}$ & $\begin{array}{llll}32.06485 & -31.80372 & -2.90857\end{array}$ \\
\hline $\mathrm{H}$ & $\begin{array}{llll}31.46518 & -34.17875 & -2.57391\end{array}$ \\
\hline $\mathrm{H}$ & $\begin{array}{lll}33.13564 & -31.49937 & 1.31828\end{array}$ \\
\hline $\mathrm{H}$ & $\begin{array}{lll}32.60205 & -33.87204 & 1.62424\end{array}$ \\
\hline $\mathrm{H}$ & $\begin{array}{lll}30.15273 & -33.06064 & 1.6358\end{array}$ \\
\hline $\mathrm{H}$ & $\begin{array}{llll}27.36484 & -35.55301 & -1.62176\end{array}$ \\
\hline $\mathrm{H}$ & $29.85366-35.79552-1.66286$ \\
\hline $\mathrm{H}$ & $19.96146-25.59809-1.327$ \\
\hline $\mathrm{H}$ & $\begin{array}{lll}17.38233 & -29.3882 & 0.5657\end{array}$ \\
\hline $\mathrm{H}$ & $\begin{array}{lll}17.0087 & -27.09696 & 1.42085\end{array}$ \\
\hline $\mathrm{H}$ & $20.912 \quad-29.02208 \quad-2.62616$ \\
\hline $\mathrm{H}$ & $\begin{array}{lll}21.86503 & -27.33737 & -4.09308\end{array}$ \\
\hline $\mathrm{H}$ & $\begin{array}{lll}20.82452 & -26.00701 & -3.31074\end{array}$ \\
\hline $\mathrm{H}$ & $26.40625 \quad-32.5734 \quad 1.95124$ \\
\hline $\mathrm{H}$ & $27.52099-31.24635 \quad 3.62395$ \\
\hline $\mathrm{H}$ & $29.1949-31.78459 \quad 3.02724$ \\
\hline $\mathrm{H}$ & $19.9341-30.38136 \quad 1.73026$ \\
\hline $\mathrm{H}$ & $21.83994-32.10116-2.49597$ \\
\hline $\mathrm{H}$ & $\begin{array}{lll}19.00689 & -29.59264 & -2.85401\end{array}$ \\
\hline $\mathrm{H}$ & $\begin{array}{lll}19.95118 & -30.02979 & -5.03963\end{array}$ \\
\hline $\mathrm{H}$ & $21.33183-31.03207-4.30122$ \\
\hline $\mathrm{H}$ & $\begin{array}{lll}22.68532 & -32.88645 & 2.15173\end{array}$ \\
\hline $\mathrm{H}$ & $21.43756-32.73773 \quad 4.23146$ \\
\hline $\mathrm{H}$ & $\begin{array}{lll}20.04552 & -31.8421 & 3.37915\end{array}$ \\
\hline $\mathrm{H}$ & $24.02814-31.0523-1.09516$ \\
\hline $\mathrm{H}$ & $25.14736 \quad-35.58823 \quad 0.48744$ \\
\hline $\mathrm{H}$ & $26.18517-30.40223-1.22316$ \\
\hline $\mathrm{H}$ & $28.44299-30.67233-1.92589$ \\
\hline $\mathrm{H}$ & $28.40733-32.50552-1.64283$ \\
\hline $\mathrm{H}$ & $22.96642-36.14961 \quad 0.86126$ \\
\hline $\mathrm{H}$ & $\begin{array}{lll}20.58551 & -36.0791 & 0.54135\end{array}$ \\
\hline $\mathrm{H}$ & $20.67576-34.3913-0.23812$ \\
\hline $\mathrm{H}$ & $\begin{array}{lll}22.76278 & -21.94813 & 2.06512\end{array}$ \\
\hline $\mathrm{H}$ & $21.89749-20.38396 \quad 2.2561$ \\
\hline $\mathrm{H}$ & $21.36225-21.81757 \quad 3.18783$ \\
\hline $\mathrm{H}$ & $30.89459-24.75942-0.04796$ \\
\hline $\mathrm{H}$ & $\begin{array}{lll}29.84193 & -25.32565 & 1.29332\end{array}$ \\
\hline
\end{tabular}




$\begin{array}{llll}\mathrm{H} & 30.93841 & -26.48511 & 0.45842 \\ \mathrm{H} & 27.66275 & -23.64412 & 1.45502 \\ \mathrm{H} & 27.54604 & -22.27785 & 0.29385 \\ \mathrm{H} & 26.06598 & -22.87988 & 1.11858 \\ \mathrm{H} & 34.98674 & -29.18032 & -0.93933 \\ \mathrm{H} & 33.36538 & -28.80513 & -0.25978 \\ \mathrm{H} & 34.45763 & -29.95702 & 0.58577 \\ \mathrm{H} & 15.90852 & -23.96797 & -1.13046 \\ \mathrm{H} & 16.04285 & -25.74681 & -0.88862 \\ \mathrm{H} & 14.81337 & -24.79948 & 0.01885 \\ \mathrm{H} & 33.85783 & -36.6779 & -0.77136 \\ \mathrm{H} & 33.74437 & -35.2114 & -1.81179 \\ \mathrm{H} & 34.23787 & -35.05161 & -0.09736\end{array}$

Macrocycle 12, Relative Energy $=1.25 \mathrm{kcal} \mathrm{mol}^{-1}, R B 97 D / 6-31 G(d)$

$\begin{array}{llll}\text { C } & 24.59729 & -24.81995 & -0.04405 \\ \text { C } & 24.15108 & -23.62601 & -0.64851 \\ \text { C } & 22.86946 & -23.12895 & -0.39915 \\ \text { C } & 21.97756 & -23.81671 & 0.44821 \\ \text { C } & 23.68026 & -25.54872 & 0.73362 \\ \text { C } & 22.3838 & -25.05654 & 0.97304 \\ \text { C } & 20.63967 & -23.13036 & 0.81461 \\ \text { C } & 18.10966 & -24.72831 & 0.46672 \\ \text { C } & 19.92311 & -23.81831 & 1.95955 \\ \text { C } & 18.78125 & -24.50173 & 1.809 \\ \text { C } & 19.75401 & -23.04983 & -0.4161 \\ \text { C } & 18.61701 & -23.74199 & -0.56746 \\ \text { C } & 31.26558 & -30.27337 & -0.67754 \\ \text { C } & 31.13867 & -29.3109 & -1.70269 \\ \text { C } & 30.17014 & -28.30934 & -1.62621 \\ \text { C } & 29.2828 & -28.23059 & -0.52863 \\ \text { C } & 30.34832 & -30.23301 & 0.38222 \\ \text { C } & 29.36389 & -29.2304 & 0.45292 \\ \text { C } & 28.40819 & -26.96807 & -0.43061 \\ \text { C } & 26.0732 & -25.23299 & -0.21021 \\ \text { C } & 27.3899 & -26.86788 & -1.56724 \\ \text { C } & 26.31541 & -26.07069 & -1.46766 \\ \text { C } & 27.69587 & -26.7799 & 0.90606 \\ \text { C } & 26.62063 & -25.98311 & 1.00653 \\ \text { C } & 32.40944 & -31.30822 & -0.78179 \\ \text { C } & 31.67349 & -34.2101 & -0.36328 \\ \text { C } & 32.06953 & -32.25629 & -1.9153 \\ \text { C } & 31.75156 & -33.5441 & -1.73281 \\ \text { C } & 32.67083 & -32.06641 & 0.50913 \\ \text { C } & 32.37969 & -33.36447 & 0.68201\end{array}$




\begin{tabular}{|c|c|}
\hline $\mathrm{C}$ & $27.35942-34.11972-0.00968$ \\
\hline $\mathrm{C}$ & $28.13963 \quad-33.45526 \quad 0.97603$ \\
\hline $\mathrm{C}$ & $29.53936-33.59938 \quad 0.91773$ \\
\hline $\mathrm{C}$ & $\begin{array}{lll}30.16917 & -34.35787 & -0.07261\end{array}$ \\
\hline $\mathrm{C}$ & $\begin{array}{lll}27.98132 & -35.02001 & -0.89503\end{array}$ \\
\hline $\mathrm{C}$ & $29.37325-35.14365-0.93279$ \\
\hline $\mathrm{C}$ & $\begin{array}{lll}19.25375 & -28.87406 & -0.33112\end{array}$ \\
\hline $\mathrm{C}$ & $\begin{array}{lll}18.11074 & -28.59472 & 0.46969\end{array}$ \\
\hline $\mathrm{C}$ & $17.7315 \quad-27.24567 \quad 0.6505$ \\
\hline $\mathrm{C}$ & $\begin{array}{lll}18.45186 & -26.18677 & 0.08036\end{array}$ \\
\hline $\mathrm{C}$ & $19.93904-27.80313-0.94375$ \\
\hline $\mathrm{C}$ & $\begin{array}{lll}19.55555 & -26.47895 & -0.742\end{array}$ \\
\hline $\mathrm{C}$ & $\begin{array}{lll}17.3446 & -29.69599 & 1.08245\end{array}$ \\
\hline $\mathrm{C}$ & $\begin{array}{lll}16.47699 & -29.59237 & 2.10926\end{array}$ \\
\hline $\mathrm{C}$ & $27.49794 \quad-32.58955 \quad 1.98106$ \\
\hline $\mathrm{C}$ & $28.10913 \quad-31.83598 \quad 2.91848$ \\
\hline $\mathrm{C}$ & $19.91595 \quad-30.2051-0.40636$ \\
\hline $\mathrm{C}$ & $\begin{array}{lll}21.89894 & -32.21982 & -0.35891\end{array}$ \\
\hline $\mathrm{C}$ & $20.30838 \quad-30.80765 \quad 0.79944$ \\
\hline $\mathrm{C}$ & $\begin{array}{lll}21.28219 & -31.82355 & 0.86484\end{array}$ \\
\hline $\mathrm{C}$ & $20.3996-30.73452-1.63927$ \\
\hline $\mathrm{C}$ & $21.39543-31.7292-1.57198$ \\
\hline $\mathrm{C}$ & $19.89894-30.22034-2.92487$ \\
\hline $\mathrm{C}$ & $20.42431 \quad-30.44352-4.14746$ \\
\hline $\mathrm{C}$ & $\begin{array}{lll}21.72349 & -32.36959 & 2.15789\end{array}$ \\
\hline $\mathrm{C}$ & $21.03286-32.30624 \quad 3.31429$ \\
\hline $\mathrm{C}$ & $25.94004-33.72945-0.22648$ \\
\hline $\mathrm{C}$ & $24.90065 \quad-34.64136 \quad 0.12393$ \\
\hline $\mathrm{C}$ & $23.59034-34.28019-0.24652$ \\
\hline $\mathrm{C}$ & $23.25684-33.07992-0.89433$ \\
\hline $\mathrm{C}$ & $24.28359-32.13158-1.15695$ \\
\hline $\mathrm{C}$ & $25.60884-32.50694-0.82311$ \\
\hline $\mathrm{C}$ & $25.05786 \quad-35.94428 \quad 0.79966$ \\
\hline $\mathrm{C}$ & $\begin{array}{lll}26.06236 & -36.40655 & 1.57302\end{array}$ \\
\hline $\mathrm{C}$ & $24.08456-30.80455-1.75755$ \\
\hline $\mathrm{C}$ & $\begin{array}{llll}22.92855 & -30.14242 & -1.96997\end{array}$ \\
\hline $\mathrm{O}$ & $20.88425 \quad-21.72676 \quad 1.13525$ \\
\hline $\mathrm{C}$ & $\begin{array}{lll}21.77576 & -21.47599 & 2.22091\end{array}$ \\
\hline $\mathrm{O}$ & $\begin{array}{llll}29.291 & -25.81499 & -0.67673\end{array}$ \\
\hline $\mathrm{C}$ & $30.28856-25.6 \quad 0.31913$ \\
\hline $\mathrm{O}$ & $26.87327-24.03175 \quad-0.45309$ \\
\hline $\mathrm{C}$ & $\begin{array}{lll}27.03617 & -23.17417 & 0.67514\end{array}$ \\
\hline $\mathrm{O}$ & $\begin{array}{lll}33.63713 & -30.65815 & -1.22836\end{array}$ \\
\hline $\mathrm{C}$ & $\begin{array}{llll}34.12164 & -29.59745 & -0.40454\end{array}$ \\
\hline $\mathrm{O}$ & $\begin{array}{lll}16.69585 & -24.56816 & 0.72997\end{array}$ \\
\hline $\mathrm{C}$ & $15.83438-24.78392-0.38812$ \\
\hline
\end{tabular}




\begin{tabular}{|c|c|}
\hline $\mathrm{O}$ & $32.21348-35.55016-0.4009$ \\
\hline $\mathrm{C}$ & $\begin{array}{lll}33.5876 & -35.61295 & -0.79372\end{array}$ \\
\hline $\mathrm{H}$ & $24.84772-23.05903-1.26566$ \\
\hline $\mathrm{H}$ & $22.57014-22.16401-0.81124$ \\
\hline $\mathrm{H}$ & $\begin{array}{lll}23.98569 & -26.49503 & 1.18612\end{array}$ \\
\hline $\mathrm{H}$ & $\begin{array}{lll}21.6953 & -25.6287 & 1.59642\end{array}$ \\
\hline $\mathrm{H}$ & $\begin{array}{lll}20.39257 & -23.75003 & 2.94221\end{array}$ \\
\hline $\mathrm{H}$ & $\begin{array}{lll}18.29149 & -24.99371 & 2.65184\end{array}$ \\
\hline $\mathrm{H}$ & $20.11097-22.37121-1.19335$ \\
\hline $\mathrm{H}$ & $18.04766-23.6626-1.49499$ \\
\hline $\mathrm{H}$ & $\begin{array}{lll}31.84764 & -29.32611 & -2.53177\end{array}$ \\
\hline $\mathrm{H}$ & $\begin{array}{lll}30.13397 & -27.52848 & -2.38686\end{array}$ \\
\hline $\mathrm{H}$ & $\begin{array}{lll}30.40021 & -30.97859 & 1.17234\end{array}$ \\
\hline $\mathrm{H}$ & $\begin{array}{lll}28.66847 & -29.24499 & 1.29207\end{array}$ \\
\hline $\mathrm{H}$ & $27.60673-27.42172-2.4826$ \\
\hline $\mathrm{H}$ & $25.61669-25.94371-2.29667$ \\
\hline $\mathrm{H}$ & $\begin{array}{lll}28.12884 & -27.23752 & 1.79689\end{array}$ \\
\hline $\mathrm{H}$ & $26.15333-25.78858 \quad 1.97422$ \\
\hline $\mathrm{H}$ & $\begin{array}{llll}32.06485 & -31.80372 & -2.90857\end{array}$ \\
\hline $\mathrm{H}$ & $\begin{array}{lll}31.46518 & -34.17875 & -2.57391\end{array}$ \\
\hline $\mathrm{H}$ & $\begin{array}{lll}33.13564 & -31.49937 & 1.31828\end{array}$ \\
\hline $\mathrm{H}$ & $\begin{array}{lll}32.60205 & -33.87204 & 1.62424\end{array}$ \\
\hline $\mathrm{H}$ & $\begin{array}{lll}30.15273 & -33.06064 & 1.6358\end{array}$ \\
\hline $\mathrm{H}$ & $27.36484-35.55301-1.62176$ \\
\hline $\mathrm{H}$ & $29.85366-35.79552-1.66286$ \\
\hline $\mathrm{H}$ & $\begin{array}{lll}16.85178 & -27.01032 & 1.24982\end{array}$ \\
\hline $\mathrm{H}$ & $20.82233-28.02197-1.54491$ \\
\hline $\mathrm{H}$ & $20.13523-25.67221-1.18832$ \\
\hline $\mathrm{H}$ & $\begin{array}{lll}17.52709 & -30.68493 & 0.65294\end{array}$ \\
\hline $\mathrm{H}$ & $\begin{array}{lll}15.94188 & -30.4684 & 2.48013\end{array}$ \\
\hline $\mathrm{H}$ & $\begin{array}{lll}16.27863 & -28.64251 & 2.61171\end{array}$ \\
\hline $\mathrm{H}$ & $26.40625 \quad-32.5734 \quad 1.95124$ \\
\hline $\mathrm{H}$ & $\begin{array}{lll}27.52099 & -31.24635 & 3.62395\end{array}$ \\
\hline $\mathrm{H}$ & $29.1949-31.78459 \quad 3.02724$ \\
\hline $\mathrm{H}$ & $\begin{array}{lll}19.9341 & -30.38136 & 1.73026\end{array}$ \\
\hline $\mathrm{H}$ & $21.83994-32.10116-2.49597$ \\
\hline $\mathrm{H}$ & $\begin{array}{lll}19.00689 & -29.59264 & -2.85401\end{array}$ \\
\hline $\mathrm{H}$ & $\begin{array}{lll}19.95118 & -30.02979 & -5.03963\end{array}$ \\
\hline $\mathrm{H}$ & $21.33183-31.03207-4.30122$ \\
\hline $\mathrm{H}$ & $\begin{array}{lll}22.68532 & -32.88645 & 2.15173\end{array}$ \\
\hline $\mathrm{H}$ & $21.43756-32.73773 \quad 4.23146$ \\
\hline $\mathrm{H}$ & $20.04552 \quad-31.8421 \quad 3.37915$ \\
\hline $\mathrm{H}$ & $22.78517-34.99125 \quad-0.04811$ \\
\hline $\mathrm{H}$ & $26.42155-31.81812-1.06793$ \\
\hline $\mathrm{H}$ & $24.19079 \quad-36.60289 \quad 0.67204$ \\
\hline $\mathrm{H}$ & $25.99115 \quad-37.4054 \quad 2.00592$ \\
\hline
\end{tabular}




$\begin{array}{llll}\mathrm{H} & 26.96533 & -35.84077 & 1.7941 \\ \mathrm{H} & 25.01714 & -30.29935 & -2.03352 \\ \mathrm{H} & 22.94355 & -29.14642 & -2.41696 \\ \mathrm{H} & 21.95505 & -30.55567 & -1.70897 \\ \mathrm{H} & 22.76278 & -21.94813 & 2.06512 \\ \mathrm{H} & 21.89749 & -20.38396 & 2.2561 \\ \mathrm{H} & 21.36225 & -21.81757 & 3.18783 \\ \mathrm{H} & 30.89459 & -24.75942 & -0.04796 \\ \mathrm{H} & 29.84193 & -25.32565 & 1.29332 \\ \mathrm{H} & 30.93841 & -26.48511 & 0.45842 \\ \mathrm{H} & 27.66275 & -23.64412 & 1.45502 \\ \mathrm{H} & 27.54604 & -22.27785 & 0.29385 \\ \mathrm{H} & 26.06598 & -22.87988 & 1.11858 \\ \mathrm{H} & 34.98674 & -29.18032 & -0.93933 \\ \mathrm{H} & 33.36538 & -28.80513 & -0.25978 \\ \mathrm{H} & 34.45763 & -29.95702 & 0.58577 \\ \mathrm{H} & 15.90852 & -23.96797 & -1.13046 \\ \mathrm{H} & 16.04285 & -25.74681 & -0.88862 \\ \mathrm{H} & 14.81337 & -24.79948 & 0.01885 \\ \mathrm{H} & 33.85783 & -36.6779 & -0.77136 \\ \mathrm{H} & 33.74437 & -35.2114 & -1.81179 \\ \mathrm{H} & 34.23787 & -35.05161 & -0.09736\end{array}$

Macrocycle 12, Relative Energy $=1.27 \mathrm{kcal} \mathrm{mol}^{-1}, R B 97 D / 6-31 G(d)$

$\begin{array}{llll}\text { C } & 24.59729 & -24.81995 & -0.04405 \\ \mathrm{C} & 24.15108 & -23.62601 & -0.64851 \\ \mathrm{C} & 22.86946 & -23.12895 & -0.39915 \\ \mathrm{C} & 21.97756 & -23.81671 & 0.44821 \\ \mathrm{C} & 23.68026 & -25.54872 & 0.73362 \\ \mathrm{C} & 22.3838 & -25.05654 & 0.97304 \\ \mathrm{C} & 20.63967 & -23.13036 & 0.81461 \\ \mathrm{C} & 18.10966 & -24.72831 & 0.46672 \\ \mathrm{C} & 19.92311 & -23.81831 & 1.95955 \\ \mathrm{C} & 18.78125 & -24.50173 & 1.809 \\ \mathrm{C} & 19.75401 & -23.04983 & -0.4161 \\ \mathrm{C} & 18.61701 & -23.74199 & -0.56746 \\ \mathrm{C} & 31.26558 & -30.27337 & -0.67754 \\ \mathrm{C} & 31.13867 & -29.3109 & -1.70269 \\ \mathrm{C} & 30.17014 & -28.30934 & -1.62621 \\ \mathrm{C} & 29.2828 & -28.23059 & -0.52863 \\ \mathrm{C} & 30.34832 & -30.23301 & 0.38222 \\ \mathrm{C} & 29.36389 & -29.2304 & 0.45292 \\ \mathrm{C} & 28.40819 & -26.96807 & -0.43061 \\ \mathrm{C} & 26.0732 & -25.23299 & -0.21021\end{array}$




\begin{tabular}{|c|c|}
\hline $\mathrm{C}$ & $27.3899-26.86788-1.56724$ \\
\hline $\mathrm{C}$ & $\begin{array}{llll}26.31541 & -26.07069 & -1.46766\end{array}$ \\
\hline $\mathrm{C}$ & $\begin{array}{lll}27.69587 & -26.7799 & 0.90606\end{array}$ \\
\hline $\mathrm{C}$ & $\begin{array}{lll}26.62063 & -25.98311 & 1.00653\end{array}$ \\
\hline $\mathrm{C}$ & $\begin{array}{lll}32.40944 & -31.30822 & -0.78179\end{array}$ \\
\hline $\mathrm{C}$ & $\begin{array}{llll}31.67349 & -34.2101 & -0.36328\end{array}$ \\
\hline $\mathrm{C}$ & $\begin{array}{llll}32.06953 & -32.25629 & -1.9153\end{array}$ \\
\hline $\mathrm{C}$ & $\begin{array}{llll}31.75156 & -33.5441 & -1.73281\end{array}$ \\
\hline $\mathrm{C}$ & $\begin{array}{lll}32.67083 & -32.06641 & 0.50913\end{array}$ \\
\hline $\mathrm{C}$ & $\begin{array}{lll}32.37969 & -33.36447 & 0.68201\end{array}$ \\
\hline $\mathrm{C}$ & $27.35942-34.11972-0.00968$ \\
\hline $\mathrm{C}$ & $\begin{array}{lll}28.13963 & -33.45526 & 0.97603\end{array}$ \\
\hline $\mathrm{C}$ & $\begin{array}{lll}29.53936 & -33.59938 & 0.91773\end{array}$ \\
\hline $\mathrm{C}$ & $\begin{array}{lll}30.16917 & -34.35787 & -0.07261\end{array}$ \\
\hline $\mathrm{C}$ & $\begin{array}{lll}27.98132 & -35.02001 & -0.89503\end{array}$ \\
\hline $\mathrm{C}$ & $\begin{array}{lll}29.37325 & -35.14365 & -0.93279\end{array}$ \\
\hline $\mathrm{C}$ & $\begin{array}{llll}18.8971 & -28.9506 & -0.93029\end{array}$ \\
\hline $\mathrm{C}$ & $\begin{array}{llll}19.64497 & -27.87044 & -1.47777\end{array}$ \\
\hline $\mathrm{C}$ & $\begin{array}{lll}19.42182 & -26.57241 & -0.96616\end{array}$ \\
\hline $\mathrm{C}$ & $\begin{array}{lll}18.48346 & -26.32149 & 0.04467\end{array}$ \\
\hline $\mathrm{C}$ & $\begin{array}{lll}17.99444 & -28.69378 & 0.12378\end{array}$ \\
\hline $\mathrm{C}$ & $\begin{array}{lll}17.78038 & -27.40387 & 0.60495\end{array}$ \\
\hline $\mathrm{C}$ & $20.63191 \quad-28.11505 \quad-2.5461$ \\
\hline $\mathrm{C}$ & $\begin{array}{llll}21.1537 & -27.19264 & -3.3796\end{array}$ \\
\hline $\mathrm{C}$ & $\begin{array}{lll}27.49794 & -32.58955 & 1.98106\end{array}$ \\
\hline $\mathrm{C}$ & $\begin{array}{lll}28.10913 & -31.83598 & 2.91848\end{array}$ \\
\hline $\mathrm{C}$ & $\begin{array}{lll}19.00957 & -30.73731 & -0.04526\end{array}$ \\
\hline $\mathrm{C}$ & $21.43008-32.17773-0.29008$ \\
\hline $\mathrm{C}$ & $\begin{array}{lll}19.59219 & -30.96748 & -1.30169\end{array}$ \\
\hline $\mathrm{C}$ & $20.80322-31.66827-1.46586$ \\
\hline $\mathrm{C}$ & $\begin{array}{lll}19.73418 & -31.08563 & 1.13282\end{array}$ \\
\hline $\mathrm{C}$ & $\begin{array}{lll}20.92978 & -31.81288 & 0.96784\end{array}$ \\
\hline $\mathrm{C}$ & $\begin{array}{lll}19.21743 & -30.72955 & 2.46475\end{array}$ \\
\hline $\mathrm{C}$ & $\begin{array}{lll}19.65482 & -31.19191 & 3.65448\end{array}$ \\
\hline $\mathrm{C}$ & $\begin{array}{llll}21.336 & -31.95903 & -2.80618\end{array}$ \\
\hline $\mathrm{C}$ & $21.0313-31.28787-3.93532$ \\
\hline $\mathrm{C}$ & $25.94004-33.72945-0.22648$ \\
\hline $\mathrm{C}$ & $25.63956-32.43093 \quad-0.73463$ \\
\hline $\mathrm{C}$ & $\begin{array}{lll}24.27968 & -32.06497 & -0.77193\end{array}$ \\
\hline $\mathrm{C}$ & $\begin{array}{llll}23.22602 & -32.90591 & -0.37931\end{array}$ \\
\hline $\mathrm{C}$ & $\begin{array}{lll}23.52345 & -34.23276 & 0.03729\end{array}$ \\
\hline $\mathrm{C}$ & $\begin{array}{lll}24.89203 & -34.59265 & 0.11523\end{array}$ \\
\hline $\mathrm{C}$ & $\begin{array}{lll}26.60746 & -31.41337 & -1.18927\end{array}$ \\
\hline $\mathrm{C}$ & $27.88277-31.55266-1.60756$ \\
\hline $\mathrm{C}$ & $\begin{array}{lll}22.53102 & -35.25277 & 0.40609\end{array}$ \\
\hline $\mathrm{C}$ & $21.19449 \quad-35.2302 \quad 0.22423$ \\
\hline
\end{tabular}




\begin{tabular}{|c|c|}
\hline $\mathrm{O}$ & $20.88425-21.72676 \quad 1.13525$ \\
\hline $\mathrm{C}$ & $\begin{array}{lll}21.77576 & -21.47599 & 2.22091\end{array}$ \\
\hline $\mathrm{O}$ & $\begin{array}{lll}29.291 & -25.81499 & -0.67673\end{array}$ \\
\hline $\mathrm{C}$ & $30.28856-25.6$ \\
\hline $\mathrm{O}$ & $\begin{array}{lll}26.87327 & -24.03175 & -0.45309\end{array}$ \\
\hline $\mathrm{C}$ & $\begin{array}{lll}27.03617 & -23.17417 & 0.67514\end{array}$ \\
\hline $\mathrm{O}$ & $\begin{array}{lll}33.63713 & -30.65815 & -1.22836\end{array}$ \\
\hline $\mathrm{C}$ & $\begin{array}{lll}34.12164 & -29.59745 & -0.40454\end{array}$ \\
\hline $\mathrm{O}$ & $\begin{array}{lll}16.69585 & -24.56816 & 0.72997\end{array}$ \\
\hline $\mathrm{C}$ & $15.83438-24.78392-0.38812$ \\
\hline $\mathrm{O}$ & $32.21348-35.55016-0.4009$ \\
\hline $\mathrm{C}$ & $\begin{array}{lll}33.5876 & -35.61295 & -0.79372\end{array}$ \\
\hline $\mathrm{H}$ & $24.84772-23.05903-1.26566$ \\
\hline $\mathrm{H}$ & $22.57014-22.16401-0.81124$ \\
\hline $\mathrm{H}$ & $\begin{array}{lll}23.98569 & -26.49503 & 1.18612\end{array}$ \\
\hline $\mathrm{H}$ & $\begin{array}{lll}21.6953 & -25.6287 & 1.59642\end{array}$ \\
\hline $\mathrm{H}$ & $\begin{array}{lll}20.39257 & -23.75003 & 2.94221\end{array}$ \\
\hline $\mathrm{H}$ & $\begin{array}{lll}18.29149 & -24.99371 & 2.65184\end{array}$ \\
\hline $\mathrm{H}$ & $\begin{array}{lll}20.11097 & -22.37121 & -1.19335\end{array}$ \\
\hline $\mathrm{H}$ & $18.04766-23.6626-1.49499$ \\
\hline $\mathrm{H}$ & $\begin{array}{llll}31.84764 & -29.32611 & -2.53177\end{array}$ \\
\hline $\mathrm{H}$ & $\begin{array}{lll}30.13397 & -27.52848 & -2.38686\end{array}$ \\
\hline $\mathrm{H}$ & $\begin{array}{lll}30.40021 & -30.97859 & 1.17234\end{array}$ \\
\hline $\mathrm{H}$ & $\begin{array}{lll}28.66847 & -29.24499 & 1.29207\end{array}$ \\
\hline $\mathrm{H}$ & $27.60673-27.42172-2.4826$ \\
\hline $\mathrm{H}$ & $\begin{array}{lll}25.61669 & -25.94371 & -2.29667\end{array}$ \\
\hline $\mathrm{H}$ & $\begin{array}{lll}28.12884 & -27.23752 & 1.79689\end{array}$ \\
\hline $\mathrm{H}$ & $\begin{array}{lll}26.15333 & -25.78858 & 1.97422\end{array}$ \\
\hline $\mathrm{H}$ & $\begin{array}{lll}32.06485 & -31.80372 & -2.90857\end{array}$ \\
\hline $\mathrm{H}$ & $\begin{array}{llll}31.46518 & -34.17875 & -2.57391\end{array}$ \\
\hline $\mathrm{H}$ & $\begin{array}{lll}33.13564 & -31.49937 & 1.31828\end{array}$ \\
\hline $\mathrm{H}$ & $\begin{array}{lll}32.60205 & -33.87204 & 1.62424\end{array}$ \\
\hline $\mathrm{H}$ & $\begin{array}{lll}30.15273 & -33.06064 & 1.6358\end{array}$ \\
\hline $\mathrm{H}$ & $27.36484-35.55301-1.62176$ \\
\hline $\mathrm{H}$ & $29.85366-35.79552-1.66286$ \\
\hline $\mathrm{H}$ & $\begin{array}{lll}19.99307 & -25.73281 & -1.36269\end{array}$ \\
\hline $\mathrm{H}$ & $\begin{array}{lll}17.41394 & -29.52292 & 0.53001\end{array}$ \\
\hline $\mathrm{H}$ & $\begin{array}{lll}17.04031 & -27.23168 & 1.38516\end{array}$ \\
\hline $\mathrm{H}$ & $20.94361 \quad-29.1568-2.66185$ \\
\hline $\mathrm{H}$ & $21.89664-27.47209-4.12877$ \\
\hline $\mathrm{H}$ & $20.85613-26.14174 \quad-3.34643$ \\
\hline $\mathrm{H}$ & $\begin{array}{lll}26.40625 & -32.5734 & 1.95124\end{array}$ \\
\hline $\mathrm{H}$ & $\begin{array}{lll}27.52099 & -31.24635 & 3.62395\end{array}$ \\
\hline $\mathrm{H}$ & $29.1949 \quad-31.78459 \quad 3.02724$ \\
\hline $\mathrm{H}$ & $\begin{array}{llll}19.01722 & -30.70203 & -2.18891\end{array}$ \\
\hline $\mathbf{H}$ & $\begin{array}{lll}21.46843 & -32.15952 & 1.85067\end{array}$ \\
\hline
\end{tabular}




$\begin{array}{llll}\mathrm{H} & 18.391 & -30.01416 & 2.46211 \\ \mathrm{H} & 19.21109 & -30.83648 & 4.58601 \\ \mathrm{H} & 20.45356 & -31.93213 & 3.74233 \\ \mathrm{H} & 22.05592 & -32.77801 & -2.86366 \\ \mathrm{H} & 21.47285 & -31.57332 & -4.89173 \\ \mathrm{H} & 20.3524 & -30.43139 & -3.93939 \\ \mathrm{H} & 24.02814 & -31.0523 & -1.09516 \\ \mathrm{H} & 25.14736 & -35.58823 & 0.48744 \\ \mathrm{H} & 26.18517 & -30.40223 & -1.22316 \\ \mathrm{H} & 28.44299 & -30.67233 & -1.92589 \\ \mathrm{H} & 28.40733 & -32.50552 & -1.64283 \\ \mathrm{H} & 22.96642 & -36.14961 & 0.86126 \\ \mathrm{H} & 20.58551 & -36.0791 & 0.54135 \\ \mathrm{H} & 20.67576 & -34.3913 & -0.23812 \\ \mathrm{H} & 22.76278 & -21.94813 & 2.06512 \\ \mathrm{H} & 21.89749 & -20.38396 & 2.2561 \\ \mathrm{H} & 21.36225 & -21.81757 & 3.18783 \\ \mathrm{H} & 30.89459 & -24.75942 & -0.04796 \\ \mathrm{H} & 29.84193 & -25.32565 & 1.29332 \\ \mathrm{H} & 30.93841 & -26.48511 & 0.45842 \\ \mathrm{H} & 27.66275 & -23.64412 & 1.45502 \\ \mathrm{H} & 27.54604 & -22.27785 & 0.29385 \\ \mathrm{H} & 26.06598 & -22.87988 & 1.11858 \\ \mathrm{H} & 34.98674 & -29.18032 & -0.93933 \\ \mathrm{H} & 33.36538 & -28.80513 & -0.25978 \\ \mathrm{H} & 34.45763 & -29.95702 & 0.58577 \\ \mathrm{H} & 15.90852 & -23.96797 & -1.13046 \\ \mathrm{H} & 16.04285 & -25.74681 & -0.88862 \\ \mathrm{H} & 14.81337 & -24.79948 & 0.01885 \\ \mathrm{H} & 33.85783 & -36.6779 & -0.77136 \\ \mathrm{H} & 33.74437 & -35.2114 & -1.81179 \\ \mathrm{H} & 34.23787 & -35.05161 & -0.09736 \\ & & & \\ & & & \\ & 2 & & \\ \mathrm{H} & 2365\end{array}$

Macrocycle 12, Relative Energy $=1.36 \mathrm{kcal} \mathrm{mol}^{-1}, R B 97 \mathrm{D} / 6-31 G(d)$

\begin{tabular}{|c|c|}
\hline $\mathrm{C}$ & $24.59729-24.81995-0.04405$ \\
\hline $\mathrm{C}$ & $24.15108-23.62601 \quad-0.64851$ \\
\hline $\mathrm{C}$ & $\begin{array}{lll}22.86946 & -23.12895 & -0.39915\end{array}$ \\
\hline $\mathrm{C}$ & $\begin{array}{lll}21.97756 & -23.81671 & 0.44821\end{array}$ \\
\hline $\mathrm{C}$ & $\begin{array}{lll}23.68026 & -25.54872 & 0.73362\end{array}$ \\
\hline $\mathrm{C}$ & $22.3838 \quad-25.05654 \quad 0.97304$ \\
\hline $\mathrm{C}$ & $\begin{array}{lll}20.63967 & -23.13036 & 0.81461\end{array}$ \\
\hline $\mathrm{C}$ & $\begin{array}{lll}18.10966 & -24.72831 & 0.46672\end{array}$ \\
\hline $\mathrm{C}$ & $\begin{array}{lll}19.92311 & -23.81831 & 1.95955\end{array}$ \\
\hline $\mathrm{C}$ & $\begin{array}{lll}18.78125 & -24.50173 & 1.809\end{array}$ \\
\hline $\mathrm{C}$ & $\begin{array}{lll}19.75401 & -23.04983 & -0.4161\end{array}$ \\
\hline
\end{tabular}




\begin{tabular}{|c|c|}
\hline $\mathrm{C}$ & $\begin{array}{lll}18.61701 & -23.74199 & -0.56746\end{array}$ \\
\hline $\mathrm{C}$ & $\begin{array}{lll}31.26558 & -30.27337 & -0.67754\end{array}$ \\
\hline $\mathrm{C}$ & $\begin{array}{lll}31.13867 & -29.3109 & -1.70269\end{array}$ \\
\hline C & $\begin{array}{llll}30.17014 & -28.30934 & -1.62621\end{array}$ \\
\hline $\mathrm{C}$ & $29.2828-28.23059-0.52863$ \\
\hline $\mathrm{C}$ & $\begin{array}{lll}30.34832 & -30.23301 & 0.38222\end{array}$ \\
\hline $\mathrm{C}$ & $29.36389-29.2304 \quad 0.45292$ \\
\hline $\mathrm{C}$ & $\begin{array}{llll}28.40819 & -26.96807 & -0.43061\end{array}$ \\
\hline $\mathrm{C}$ & $26.0732-25.23299-0.21021$ \\
\hline $\mathrm{C}$ & $\begin{array}{llll}27.3899 & -26.86788 & -1.56724\end{array}$ \\
\hline $\mathrm{C}$ & $\begin{array}{llll}26.31541 & -26.07069 & -1.46766\end{array}$ \\
\hline $\mathrm{C}$ & $\begin{array}{lll}27.69587 & -26.7799 & 0.90606\end{array}$ \\
\hline $\mathrm{C}$ & $\begin{array}{lll}26.62063 & -25.98311 & 1.00653\end{array}$ \\
\hline $\mathrm{C}$ & $\begin{array}{llll}32.40944 & -31.30822 & -0.78179\end{array}$ \\
\hline $\mathrm{C}$ & $\begin{array}{llll}31.67349 & -34.2101 & -0.36328\end{array}$ \\
\hline $\mathrm{C}$ & $\begin{array}{llll}32.06953 & -32.25629 & -1.9153\end{array}$ \\
\hline $\mathrm{C}$ & $\begin{array}{llll}31.75156 & -33.5441 & -1.73281\end{array}$ \\
\hline $\mathrm{C}$ & $\begin{array}{lll}32.67083 & -32.06641 & 0.50913\end{array}$ \\
\hline $\mathrm{C}$ & $\begin{array}{llll}32.37969 & -33.36447 & 0.68201\end{array}$ \\
\hline $\mathrm{C}$ & $\begin{array}{lll}27.35942 & -34.11972 & -0.00968\end{array}$ \\
\hline $\mathrm{C}$ & $\begin{array}{lll}28.13963 & -33.45526 & 0.97603\end{array}$ \\
\hline $\mathrm{C}$ & $\begin{array}{llll}29.53936 & -33.59938 & 0.91773\end{array}$ \\
\hline $\mathrm{C}$ & $\begin{array}{llll}30.16917 & -34.35787 & -0.07261\end{array}$ \\
\hline $\mathrm{C}$ & $\begin{array}{llll}27.98132 & -35.02001 & -0.89503\end{array}$ \\
\hline $\mathrm{C}$ & $\begin{array}{llll}29.37325 & -35.14365 & -0.93279\end{array}$ \\
\hline $\mathrm{C}$ & $\begin{array}{lll}19.25375 & -28.87406 & -0.33112\end{array}$ \\
\hline $\mathrm{C}$ & $\begin{array}{lll}18.11074 & -28.59472 & 0.46969\end{array}$ \\
\hline $\mathrm{C}$ & $\begin{array}{lll}17.7315 & -27.24567 & 0.6505\end{array}$ \\
\hline $\mathrm{C}$ & $\begin{array}{lll}18.45186 & -26.18677 & 0.08036\end{array}$ \\
\hline $\mathrm{C}$ & $\begin{array}{lll}19.93904 & -27.80313 & -0.94375\end{array}$ \\
\hline $\mathrm{C}$ & $\begin{array}{lll}19.55555 & -26.47895 & -0.742\end{array}$ \\
\hline $\mathrm{C}$ & $\begin{array}{lll}17.3446 & -29.69599 & 1.08245\end{array}$ \\
\hline $\mathrm{C}$ & $\begin{array}{lll}16.47699 & -29.59237 & 2.10926\end{array}$ \\
\hline $\mathrm{C}$ & $\begin{array}{llll}27.49794 & -32.58955 & 1.98106\end{array}$ \\
\hline $\mathrm{C}$ & $\begin{array}{llll}28.10913 & -31.83598 & 2.91848\end{array}$ \\
\hline $\mathrm{C}$ & $19.91595-30.2051 \quad-0.40636$ \\
\hline $\mathrm{C}$ & $\begin{array}{llll}21.89894 & -32.21982 & -0.35891\end{array}$ \\
\hline $\mathrm{C}$ & $\begin{array}{llll}20.30838 & -30.80765 & 0.79944\end{array}$ \\
\hline $\mathrm{C}$ & $\begin{array}{lll}21.28219 & -31.82355 & 0.86484\end{array}$ \\
\hline $\mathrm{C}$ & $20.3996-30.73452-1.63927$ \\
\hline $\mathrm{C}$ & $21.39543-31.7292-1.57198$ \\
\hline $\mathrm{C}$ & $\begin{array}{llll}19.89894 & -30.22034 & -2.92487\end{array}$ \\
\hline $\mathrm{C}$ & $20.42431-30.44352-4.14746$ \\
\hline $\mathrm{C}$ & $\begin{array}{llll}21.72349 & -32.36959 & 2.15789\end{array}$ \\
\hline $\mathrm{C}$ & $21.03286-32.30624 \quad 3.31429$ \\
\hline $\mathrm{C}$ & $\begin{array}{llll}25.94004 & -33.72945 & -0.22648\end{array}$ \\
\hline
\end{tabular}




\begin{tabular}{|c|c|}
\hline $\mathrm{C}$ & $25.63956-32.43093-0.73463$ \\
\hline $\mathrm{C}$ & $\begin{array}{llll}24.27968 & -32.06497 & -0.77193\end{array}$ \\
\hline $\mathrm{C}$ & $\begin{array}{llll}23.22602 & -32.90591 & -0.37931\end{array}$ \\
\hline $\mathrm{C}$ & $23.52345 \quad-34.23276 \quad 0.03729$ \\
\hline $\mathrm{C}$ & $24.89203 \quad-34.59265 \quad 0.11523$ \\
\hline $\mathrm{C}$ & $\begin{array}{llll}26.60746 & -31.41337 & -1.18927\end{array}$ \\
\hline $\mathrm{C}$ & $27.88277-31.55266-1.60756$ \\
\hline $\mathrm{C}$ & $\begin{array}{llll}22.53102 & -35.25277 & 0.40609\end{array}$ \\
\hline $\mathrm{C}$ & $\begin{array}{llll}21.19449 & -35.2302 & 0.22423\end{array}$ \\
\hline $\mathrm{O}$ & $\begin{array}{lll}20.88425 & -21.72676 & 1.13525\end{array}$ \\
\hline $\mathrm{C}$ & $\begin{array}{llll}21.77576 & -21.47599 & 2.22091\end{array}$ \\
\hline $\mathrm{O}$ & $\begin{array}{lll}29.291 & -25.81499 & -0.67673\end{array}$ \\
\hline $\mathrm{C}$ & $30.28856-25.6 \quad 0.31913$ \\
\hline $\mathrm{O}$ & $\begin{array}{lll}26.87327 & -24.03175 & -0.45309\end{array}$ \\
\hline $\mathrm{C}$ & $\begin{array}{lll}27.03617 & -23.17417 & 0.67514\end{array}$ \\
\hline $\mathrm{O}$ & $\begin{array}{lll}33.63713 & -30.65815 & -1.22836\end{array}$ \\
\hline $\mathrm{C}$ & $\begin{array}{lll}34.12164 & -29.59745 & -0.40454\end{array}$ \\
\hline $\mathrm{O}$ & $\begin{array}{lll}16.69585 & -24.56816 & 0.72997\end{array}$ \\
\hline $\mathrm{C}$ & $\begin{array}{lll}15.83438 & -24.78392 & -0.38812\end{array}$ \\
\hline $\mathrm{O}$ & $32.21348-35.55016-0.4009$ \\
\hline $\mathrm{C}$ & $\begin{array}{llll}33.5876 & -35.61295 & -0.79372\end{array}$ \\
\hline $\mathrm{H}$ & $24.84772-23.05903-1.26566$ \\
\hline $\mathrm{H}$ & $22.57014-22.16401-0.81124$ \\
\hline $\mathrm{H}$ & $\begin{array}{lll}23.98569 & -26.49503 & 1.18612\end{array}$ \\
\hline $\mathrm{H}$ & $\begin{array}{lll}21.6953 & -25.6287 & 1.59642\end{array}$ \\
\hline $\mathrm{H}$ & $\begin{array}{lll}20.39257 & -23.75003 & 2.94221\end{array}$ \\
\hline $\mathrm{H}$ & $\begin{array}{lll}18.29149 & -24.99371 & 2.65184\end{array}$ \\
\hline $\mathrm{H}$ & $20.11097-22.37121-1.19335$ \\
\hline $\mathrm{H}$ & $\begin{array}{lll}18.04766 & -23.6626 & -1.49499\end{array}$ \\
\hline $\mathrm{H}$ & $\begin{array}{llll}31.84764 & -29.32611 & -2.53177\end{array}$ \\
\hline $\mathrm{H}$ & $\begin{array}{llll}30.13397 & -27.52848 & -2.38686\end{array}$ \\
\hline $\mathrm{H}$ & $\begin{array}{lll}30.40021 & -30.97859 & 1.17234\end{array}$ \\
\hline $\mathrm{H}$ & $\begin{array}{lll}28.66847 & -29.24499 & 1.29207\end{array}$ \\
\hline $\mathrm{H}$ & $27.60673-27.42172-2.4826$ \\
\hline $\mathrm{H}$ & $\begin{array}{lll}25.61669 & -25.94371 & -2.29667\end{array}$ \\
\hline $\mathrm{H}$ & $\begin{array}{lll}28.12884 & -27.23752 & 1.79689\end{array}$ \\
\hline $\mathrm{H}$ & $\begin{array}{lll}26.15333 & -25.78858 & 1.97422\end{array}$ \\
\hline $\mathrm{H}$ & $\begin{array}{lll}32.06485 & -31.80372 & -2.90857\end{array}$ \\
\hline $\mathrm{H}$ & $\begin{array}{llll}31.46518 & -34.17875 & -2.57391\end{array}$ \\
\hline $\mathrm{H}$ & $\begin{array}{lll}33.13564 & -31.49937 & 1.31828\end{array}$ \\
\hline $\mathrm{H}$ & $\begin{array}{lll}32.60205 & -33.87204 & 1.62424\end{array}$ \\
\hline $\mathrm{H}$ & $\begin{array}{lll}30.15273 & -33.06064 & 1.6358\end{array}$ \\
\hline $\mathrm{H}$ & $\begin{array}{llll}27.36484 & -35.55301 & -1.62176\end{array}$ \\
\hline $\mathrm{H}$ & $29.85366-35.79552-1.66286$ \\
\hline $\mathrm{H}$ & $\begin{array}{lll}16.85178 & -27.01032 & 1.24982\end{array}$ \\
\hline $\mathrm{H}$ & $\begin{array}{llll}20.82233 & -28.02197 & -1.54491\end{array}$ \\
\hline
\end{tabular}




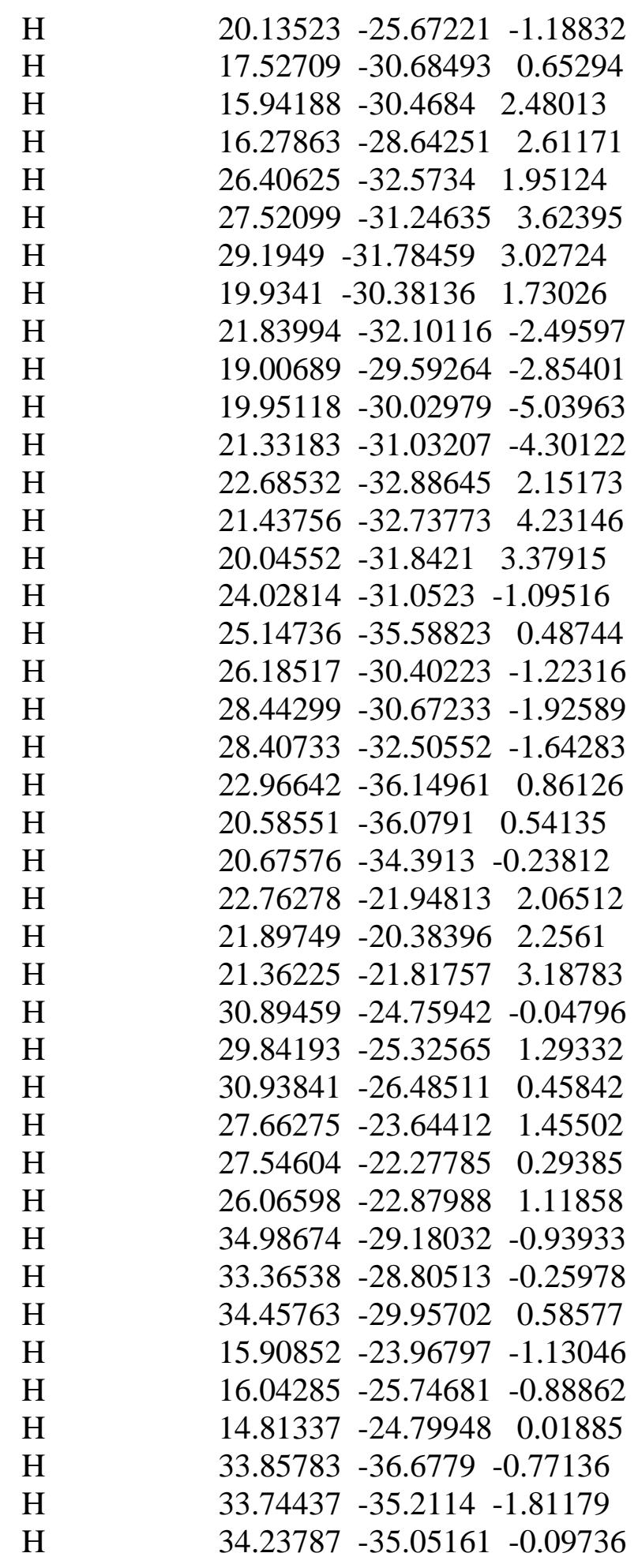

Macrocycle 12, Relative Energy $=1.85 \mathrm{kcal} \mathrm{mol}^{-1}, \mathrm{RB} 97 \mathrm{D} / 6-31 \mathrm{G}(\mathrm{d})$

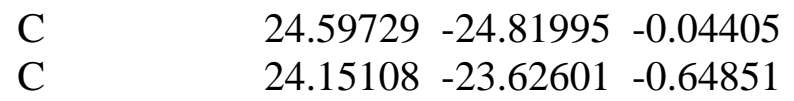




\begin{tabular}{|c|c|}
\hline $\mathrm{C}$ & $22.86946-23.12895 \quad-0.39915$ \\
\hline $\mathrm{C}$ & $\begin{array}{lll}21.97756 & -23.81671 & 0.44821\end{array}$ \\
\hline $\mathrm{C}$ & $\begin{array}{lll}23.68026 & -25.54872 & 0.73362\end{array}$ \\
\hline $\mathrm{C}$ & $22.3838 \quad-25.05654 \quad 0.97304$ \\
\hline $\mathrm{C}$ & $\begin{array}{lll}20.63967 & -23.13036 & 0.81461\end{array}$ \\
\hline $\mathrm{C}$ & $\begin{array}{lll}18.10966 & -24.72831 & 0.46672\end{array}$ \\
\hline $\mathrm{C}$ & $19.92311-23.81831 \quad 1.95955$ \\
\hline $\mathrm{C}$ & $\begin{array}{lll}18.78125 & -24.50173 & 1.809\end{array}$ \\
\hline $\mathrm{C}$ & $19.75401-23.04983-0.4161$ \\
\hline $\mathrm{C}$ & $\begin{array}{lll}18.61701 & -23.74199 & -0.56746\end{array}$ \\
\hline $\mathrm{C}$ & $31.26558-30.27337-0.67754$ \\
\hline $\mathrm{C}$ & $\begin{array}{lll}31.13867 & -29.3109 & -1.70269\end{array}$ \\
\hline $\mathrm{C}$ & $\begin{array}{lll}30.17014 & -28.30934 & -1.62621\end{array}$ \\
\hline $\mathrm{C}$ & $29.2828-28.23059-0.52863$ \\
\hline $\mathrm{C}$ & $\begin{array}{lll}30.34832 & -30.23301 & 0.38222\end{array}$ \\
\hline $\mathrm{C}$ & $\begin{array}{lll}29.36389 & -29.2304 & 0.45292\end{array}$ \\
\hline $\mathrm{C}$ & $28.40819-26.96807-0.43061$ \\
\hline $\mathrm{C}$ & $26.0732-25.23299-0.21021$ \\
\hline $\mathrm{C}$ & $27.3899-26.86788-1.56724$ \\
\hline $\mathrm{C}$ & $26.31541-26.07069-1.46766$ \\
\hline $\mathrm{C}$ & $\begin{array}{lll}27.69587 & -26.7799 & 0.90606\end{array}$ \\
\hline $\mathrm{C}$ & $\begin{array}{lll}26.62063 & -25.98311 & 1.00653\end{array}$ \\
\hline $\mathrm{C}$ & $32.40944-31.30822-0.78179$ \\
\hline $\mathrm{C}$ & $\begin{array}{llll}31.67349 & -34.2101 & -0.36328\end{array}$ \\
\hline $\mathrm{C}$ & $32.06953-32.25629-1.9153$ \\
\hline $\mathrm{C}$ & $31.75156-33.5441-1.73281$ \\
\hline $\mathrm{C}$ & $\begin{array}{lll}32.67083 & -32.06641 & 0.50913\end{array}$ \\
\hline $\mathrm{C}$ & $\begin{array}{lll}32.37969 & -33.36447 & 0.68201\end{array}$ \\
\hline $\mathrm{C}$ & $\begin{array}{lll}27.44392 & -34.89492 & 0.79994\end{array}$ \\
\hline $\mathrm{C}$ & $28.1397-35.7371-0.18366$ \\
\hline $\mathrm{C}$ & $29.40454-35.36033-0.67523$ \\
\hline $\mathrm{C}$ & $\begin{array}{lll}30.16917 & -34.35787 & -0.07261\end{array}$ \\
\hline $\mathrm{C}$ & $28.32874 \quad-33.9336 \quad 1.45524$ \\
\hline $\mathrm{C}$ & $29.58352-33.58139 \quad 0.94961$ \\
\hline $\mathrm{C}$ & $\begin{array}{lll}18.92511 & -29.05274 & -0.9604\end{array}$ \\
\hline $\mathrm{C}$ & $\begin{array}{lll}19.68386 & -27.97353 & -1.49465\end{array}$ \\
\hline $\mathrm{C}$ & $19.4585-26.6763-0.98198$ \\
\hline $\mathrm{C}$ & $\begin{array}{lll}18.50779 & -26.42519 & 0.0172\end{array}$ \\
\hline $\mathrm{C}$ & $\begin{array}{lll}18.00955 & -28.79598 & 0.0825\end{array}$ \\
\hline $\mathrm{C}$ & $\begin{array}{lll}17.79365 & -27.5068 & 0.56482\end{array}$ \\
\hline $\mathrm{C}$ & $20.68393-28.21833-2.55066$ \\
\hline $\mathrm{C}$ & $21.21983-27.2952-3.37436$ \\
\hline $\mathrm{C}$ & $27.38171-36.84823-0.78546$ \\
\hline $\mathrm{C}$ & $27.76491-37.63599-1.81151$ \\
\hline $\mathrm{C}$ & $\begin{array}{llll}20.45483 & -30.32812 & -0.21987\end{array}$ \\
\hline $\mathrm{C}$ & $21.5336-32.91778-0.57127$ \\
\hline
\end{tabular}




\begin{tabular}{|c|c|}
\hline $\mathrm{C}$ & $20.40418-30.97139-1.46673$ \\
\hline $\mathrm{C}$ & $20.94146-32.25492-1.68704$ \\
\hline $\mathrm{C}$ & $21.20996-30.91665 \quad 0.83732$ \\
\hline $\mathrm{C}$ & $21.72084 \quad-32.21278 \quad 0.6261$ \\
\hline $\mathrm{C}$ & $21.40254 \quad-30.20219 \quad 2.11024$ \\
\hline $\mathrm{C}$ & $\begin{array}{lll}21.87497 & -30.7158 & 3.26503\end{array}$ \\
\hline $\mathrm{C}$ & $\begin{array}{llll}20.79341 & -32.92651 & -2.98778\end{array}$ \\
\hline $\mathrm{C}$ & $\begin{array}{llll}20.53978 & -32.31577 & -4.1629\end{array}$ \\
\hline $\mathrm{C}$ & $26.10087-34.7834-0.33205$ \\
\hline $\mathrm{C}$ & $24.61213-35.31162-0.10093$ \\
\hline $\mathrm{C}$ & $\begin{array}{lll}23.52616 & -34.41997 & 0.00012\end{array}$ \\
\hline $\mathrm{C}$ & $23.60845-33.04402-0.26731$ \\
\hline $\mathrm{C}$ & $24.86924-32.48479-0.61406$ \\
\hline $\mathrm{C}$ & $25.95597 \quad-33.3833-0.75654$ \\
\hline $\mathrm{C}$ & $\begin{array}{lll}24.33519 & -36.72507 & 0.22218\end{array}$ \\
\hline $\mathrm{C}$ & $25.17999-37.71972 \quad 0.56533$ \\
\hline $\mathrm{C}$ & $\begin{array}{llll}25.13259 & -31.05778 & -0.84996\end{array}$ \\
\hline $\mathrm{C}$ & $24.33775-30.00073-0.58387$ \\
\hline $\mathrm{O}$ & $20.88425 \quad-21.72676 \quad 1.13525$ \\
\hline $\mathrm{C}$ & $\begin{array}{lll}21.77576 & -21.47599 & 2.22091\end{array}$ \\
\hline $\mathrm{O}$ & $29.291 \quad-25.81499-0.67673$ \\
\hline $\mathrm{C}$ & $\begin{array}{lll}30.28856 & -25.6 & 0.31913\end{array}$ \\
\hline $\mathrm{O}$ & $\begin{array}{lll}26.87327 & -24.03175 & -0.45309\end{array}$ \\
\hline $\mathrm{C}$ & $\begin{array}{lll}27.03617 & -23.17417 & 0.67514\end{array}$ \\
\hline $\mathrm{O}$ & $\begin{array}{llll}33.63713 & -30.65815 & -1.22836\end{array}$ \\
\hline $\mathrm{C}$ & $\begin{array}{lll}34.12164 & -29.59745 & -0.40454\end{array}$ \\
\hline $\mathrm{O}$ & $\begin{array}{lll}16.69585 & -24.56816 & 0.72997\end{array}$ \\
\hline $\mathrm{C}$ & $15.83438-24.78392-0.38812$ \\
\hline $\mathrm{O}$ & $32.21348-35.55016-0.4009$ \\
\hline $\mathrm{C}$ & $33.5876-35.61295-0.79372$ \\
\hline $\mathrm{H}$ & $24.84772-23.05903-1.26566$ \\
\hline $\mathrm{H}$ & $\begin{array}{lll}22.57014 & -22.16401 & -0.81124\end{array}$ \\
\hline $\mathrm{H}$ & $\begin{array}{lll}23.98569 & -26.49503 & 1.18612\end{array}$ \\
\hline $\mathrm{H}$ & $\begin{array}{lll}21.6953 & -25.6287 & 1.59642\end{array}$ \\
\hline $\mathrm{H}$ & $\begin{array}{lll}20.39257 & -23.75003 & 2.94221\end{array}$ \\
\hline $\mathrm{H}$ & $\begin{array}{lll}18.29149 & -24.99371 & 2.65184\end{array}$ \\
\hline $\mathrm{H}$ & $\begin{array}{lll}20.11097 & -22.37121 & -1.19335\end{array}$ \\
\hline $\mathrm{H}$ & $\begin{array}{llll}18.04766 & -23.6626 & -1.49499\end{array}$ \\
\hline $\mathrm{H}$ & $\begin{array}{lll}31.84764 & -29.32611 & -2.53177\end{array}$ \\
\hline $\mathrm{H}$ & $\begin{array}{lll}30.13397 & -27.52848 & -2.38686\end{array}$ \\
\hline $\mathrm{H}$ & $\begin{array}{lll}30.40021 & -30.97859 & 1.17234\end{array}$ \\
\hline $\mathrm{H}$ & $\begin{array}{lll}28.66847 & -29.24499 & 1.29207\end{array}$ \\
\hline $\mathrm{H}$ & $27.60673-27.42172-2.4826$ \\
\hline $\mathrm{H}$ & $\begin{array}{lll}25.61669 & -25.94371 & -2.29667\end{array}$ \\
\hline $\mathrm{H}$ & $\begin{array}{lll}28.12884 & -27.23752 & 1.79689\end{array}$ \\
\hline $\mathrm{H}$ & $\begin{array}{lll}26.15333 & -25.78858 & 1.97422\end{array}$ \\
\hline
\end{tabular}




\begin{tabular}{|c|c|}
\hline $\mathrm{H}$ & $\begin{array}{llll}32.06485 & -31.80372 & -2.90857\end{array}$ \\
\hline $\mathrm{H}$ & $\begin{array}{llll}31.46518 & -34.17875 & -2.57391\end{array}$ \\
\hline $\mathrm{H}$ & $\begin{array}{lll}33.13564 & -31.49937 & 1.31828\end{array}$ \\
\hline $\mathrm{H}$ & $\begin{array}{lll}32.60205 & -33.87204 & 1.62424\end{array}$ \\
\hline $\mathrm{H}$ & $29.82215-35.90275-1.51989$ \\
\hline $\mathrm{H}$ & $\begin{array}{lll}27.91259 & -33.39585 & 2.30959\end{array}$ \\
\hline $\mathrm{H}$ & $\begin{array}{lll}30.14227 & -32.74954 & 1.37924\end{array}$ \\
\hline $\mathrm{H}$ & $20.03782-25.8375-1.36835$ \\
\hline $\mathrm{H}$ & $\begin{array}{lll}17.42089 & -29.62433 & 0.47848\end{array}$ \\
\hline $\mathrm{H}$ & $\begin{array}{lll}17.04398 & -27.33442 & 1.33576\end{array}$ \\
\hline $\mathrm{H}$ & $20.9935-29.2608 \quad-2.66554$ \\
\hline $\mathrm{H}$ & $21.97159-27.57496-4.11456$ \\
\hline $\mathrm{H}$ & $\begin{array}{llll}20.92549 & -26.24337 & -3.34183\end{array}$ \\
\hline $\mathrm{H}$ & $26.40159-37.03608-0.34161$ \\
\hline $\mathrm{H}$ & $27.10362-38.4207 \quad-2.18325$ \\
\hline $\mathrm{H}$ & $28.7259-37.52774-2.31958$ \\
\hline $\mathrm{H}$ & $19.81399-30.50872-2.25783$ \\
\hline $\mathrm{H}$ & $22.23813-32.7207 \quad 1.44104$ \\
\hline $\mathrm{H}$ & $\begin{array}{lll}21.12447 & -29.14525 & 2.09192\end{array}$ \\
\hline $\mathrm{H}$ & $22.00053-30.08232 \quad 4.14476$ \\
\hline $\mathrm{H}$ & $22.14454 \quad-31.76874 \quad 3.37585$ \\
\hline $\mathrm{H}$ & $20.92738-34.01016-2.98069$ \\
\hline $\mathrm{H}$ & $\begin{array}{llll}20.44291 & -32.89357 & -5.08374\end{array}$ \\
\hline $\mathrm{H}$ & $20.43839-31.23076-4.24623$ \\
\hline $\mathrm{H}$ & $\begin{array}{lll}22.54962 & -34.82732 & 0.27161\end{array}$ \\
\hline $\mathrm{H}$ & $\begin{array}{llll}26.92155 & -32.98441 & -1.07817\end{array}$ \\
\hline $\mathrm{H}$ & $23.2658 \quad-36.96644 \quad 0.21671$ \\
\hline $\mathrm{H}$ & $24.78123 \quad-38.71044 \quad 0.78812$ \\
\hline $\mathrm{H}$ & $26.25992 \quad-37.6013 \quad 0.62921$ \\
\hline $\mathrm{H}$ & $26.12323-30.85454-1.27233$ \\
\hline $\mathrm{H}$ & $24.68266-28.98857-0.80437$ \\
\hline $\mathrm{H}$ & $23.34602-30.10356-0.14513$ \\
\hline $\mathrm{H}$ & $\begin{array}{lll}22.76278 & -21.94813 & 2.06512\end{array}$ \\
\hline $\mathrm{H}$ & $\begin{array}{lll}21.89749 & -20.38396 & 2.2561\end{array}$ \\
\hline $\mathrm{H}$ & $\begin{array}{lll}21.36225 & -21.81757 & 3.18783\end{array}$ \\
\hline $\mathrm{H}$ & $\begin{array}{lll}30.89459 & -24.75942 & -0.04796\end{array}$ \\
\hline $\mathrm{H}$ & $\begin{array}{lll}29.84193 & -25.32565 & 1.29332\end{array}$ \\
\hline $\mathrm{H}$ & $\begin{array}{lll}30.93841 & -26.48511 & 0.45842\end{array}$ \\
\hline $\mathrm{H}$ & $27.66275-23.64412 \quad 1.45502$ \\
\hline $\mathrm{H}$ & $27.54604-22.27785 \quad 0.29385$ \\
\hline $\mathrm{H}$ & $\begin{array}{lll}26.06598 & -22.87988 & 1.11858\end{array}$ \\
\hline $\mathrm{H}$ & $34.98674-29.18032-0.93933$ \\
\hline $\mathrm{H}$ & $\begin{array}{lll}33.36538 & -28.80513 & -0.25978\end{array}$ \\
\hline $\mathrm{H}$ & $\begin{array}{llll}34.45763 & -29.95702 & 0.58577\end{array}$ \\
\hline $\mathrm{H}$ & $\begin{array}{lll}15.90852 & -23.96797 & -1.13046\end{array}$ \\
\hline $\mathrm{H}$ & $\begin{array}{lll}16.04285 & -25.74681 & -0.88862\end{array}$ \\
\hline
\end{tabular}




$\begin{array}{llll}\mathrm{H} & 14.81337 & -24.79948 & 0.01885 \\ \mathrm{H} & 33.85783 & -36.6779 & -0.77136 \\ \mathrm{H} & 33.74437 & -35.2114 & -1.81179 \\ \mathrm{H} & 34.23787 & -35.05161 & -0.09736\end{array}$

Macrocycle 12, Relative Energy $=1.92 \mathrm{kcal} \mathrm{mol}^{-1}, \mathrm{RB} 97 \mathrm{D} / 6-31 \mathrm{G}(\mathrm{d})$

\begin{tabular}{|c|c|}
\hline $\mathrm{C}$ & $24.59729-24.81995-0.04405$ \\
\hline $\mathrm{C}$ & $24.15108-23.62601 \quad-0.64851$ \\
\hline $\mathrm{C}$ & $22.86946-23.12895 \quad-0.39915$ \\
\hline $\mathrm{C}$ & $\begin{array}{llll}21.97756 & -23.81671 & 0.44821\end{array}$ \\
\hline $\mathrm{C}$ & $\begin{array}{lll}23.68026 & -25.54872 & 0.73362\end{array}$ \\
\hline $\mathrm{C}$ & $22.3838 \quad-25.05654 \quad 0.97304$ \\
\hline $\mathrm{C}$ & $\begin{array}{lll}20.63967 & -23.13036 & 0.81461\end{array}$ \\
\hline $\mathrm{C}$ & $\begin{array}{lll}18.10966 & -24.72831 & 0.46672\end{array}$ \\
\hline $\mathrm{C}$ & $\begin{array}{lll}19.92311 & -23.81831 & 1.95955\end{array}$ \\
\hline $\mathrm{C}$ & $\begin{array}{lll}18.78125 & -24.50173 & 1.809\end{array}$ \\
\hline $\mathrm{C}$ & $\begin{array}{lll}19.75401 & -23.04983 & -0.4161\end{array}$ \\
\hline $\mathrm{C}$ & $\begin{array}{lll}18.61701 & -23.74199 & -0.56746\end{array}$ \\
\hline $\mathrm{C}$ & $\begin{array}{lll}31.26558 & -30.27337 & -0.67754\end{array}$ \\
\hline $\mathrm{C}$ & $\begin{array}{llll}31.13867 & -29.3109 & -1.70269\end{array}$ \\
\hline $\mathrm{C}$ & $\begin{array}{lll}30.17014 & -28.30934 & -1.62621\end{array}$ \\
\hline $\mathrm{C}$ & $29.2828-28.23059-0.52863$ \\
\hline $\mathrm{C}$ & $\begin{array}{lll}30.34832 & -30.23301 & 0.38222\end{array}$ \\
\hline $\mathrm{C}$ & $\begin{array}{lll}29.36389 & -29.2304 & 0.45292\end{array}$ \\
\hline $\mathrm{C}$ & $\begin{array}{lll}28.40819 & -26.96807 & -0.43061\end{array}$ \\
\hline $\mathrm{C}$ & $26.0732-25.23299-0.21021$ \\
\hline $\mathrm{C}$ & $27.3899-26.86788-1.56724$ \\
\hline $\mathrm{C}$ & $26.31541-26.07069-1.46766$ \\
\hline $\mathrm{C}$ & $\begin{array}{lll}27.69587 & -26.7799 & 0.90606\end{array}$ \\
\hline $\mathrm{C}$ & $\begin{array}{lll}26.62063 & -25.98311 & 1.00653\end{array}$ \\
\hline $\mathrm{C}$ & $\begin{array}{lll}32.40944 & -31.30822 & -0.78179\end{array}$ \\
\hline $\mathrm{C}$ & $\begin{array}{llll}31.67349 & -34.2101 & -0.36328\end{array}$ \\
\hline $\mathrm{C}$ & $\begin{array}{llll}32.06953 & -32.25629 & -1.9153\end{array}$ \\
\hline $\mathrm{C}$ & $\begin{array}{llll}31.75156 & -33.5441 & -1.73281\end{array}$ \\
\hline $\mathrm{C}$ & $\begin{array}{lll}32.67083 & -32.06641 & 0.50913\end{array}$ \\
\hline $\mathrm{C}$ & $\begin{array}{lll}32.37969 & -33.36447 & 0.68201\end{array}$ \\
\hline $\mathrm{C}$ & $27.35942-34.11972-0.00968$ \\
\hline $\mathrm{C}$ & $\begin{array}{lll}28.13963 & -33.45526 & 0.97603\end{array}$ \\
\hline $\mathrm{C}$ & $\begin{array}{lll}29.53936 & -33.59938 & 0.91773\end{array}$ \\
\hline $\mathrm{C}$ & $\begin{array}{lll}30.16917 & -34.35787 & -0.07261\end{array}$ \\
\hline $\mathrm{C}$ & $\begin{array}{lll}27.98132 & -35.02001 & -0.89503\end{array}$ \\
\hline $\mathrm{C}$ & $\begin{array}{lll}29.37325 & -35.14365 & -0.93279\end{array}$ \\
\hline $\mathrm{C}$ & $\begin{array}{lll}19.25375 & -28.87406 & -0.33112\end{array}$ \\
\hline $\mathrm{C}$ & $\begin{array}{lll}18.11074 & -28.59472 & 0.46969\end{array}$ \\
\hline $\mathrm{C}$ & $\begin{array}{lll}17.7315 & -27.24567 & 0.6505\end{array}$ \\
\hline
\end{tabular}




$\begin{array}{llll}\text { C } & 18.45186 & -26.18677 & 0.08036 \\ \text { C } & 19.93904 & -27.80313 & -0.94375 \\ \text { C } & 19.55555 & -26.47895 & -0.742 \\ \text { C } & 17.3446 & -29.69599 & 1.08245 \\ \text { C } & 16.47699 & -29.59237 & 2.10926 \\ \text { C } & 27.49794 & -32.58955 & 1.98106 \\ \text { C } & 28.10913 & -31.83598 & 2.91848 \\ \text { C } & 20.22452 & -30.46098 & -0.45241 \\ \text { C } & 20.61515 & -33.25897 & -0.56309 \\ \text { C } & 20.15887 & -31.15436 & -1.67139 \\ \text { C } & 20.36062 & -32.54501 & -1.77158 \\ \text { C } & 20.66181 & -31.1493 & 0.71775 \\ \text { C } & 20.83385 & -32.54467 & 0.62339 \\ \text { C } & 20.87886 & -30.42203 & 1.97941 \\ \text { C } & 21.05251 & -30.95859 & 3.20518 \\ \text { C } & 20.2009 & -33.24435 & -3.05621 \\ \text { C } & 20.26369 & -32.67189 & -4.27547 \\ \text { C } & 25.34831 & -33.6361 & -0.26059 \\ \text { C } & 24.30892 & -34.548 & 0.08982 \\ \text { C } & 22.99861 & -34.18684 & -0.28063 \\ \text { C } & 22.66511 & -32.98657 & -0.92844 \\ \text { C } & 23.69186 & -32.03823 & -1.19105 \\ \text { C } & 25.01711 & -32.41359 & -0.85721 \\ \text { C } & 24.46614 & -35.85093 & 0.76555 \\ \text { C } & 25.47063 & -36.3132 & 1.53891 \\ \text { C } & 23.49283 & -30.71119 & -1.79165 \\ \text { C } & 22.33682 & -30.04907 & -2.00407 \\ \text { O } & 20.88425 & -21.72676 & 1.13525 \\ \text { C } & 21.77576 & -21.47599 & 2.22091 \\ \text { O } & 29.291 & -25.81499 & -0.67673 \\ \text { C } & 30.28856 & -25.6 & 0.31913 \\ \text { O } & 26.87327 & -24.03175 & -0.45309 \\ \text { C } & 27.03617 & -23.17417 & 0.67514 \\ \text { O } & 33.63713 & -30.65815 & -1.22836 \\ \text { C } & 34.12164 & -29.59745 & -0.40454 \\ \text { O } & 16.69585 & -24.56816 & 0.72997 \\ \text { C } & 15.83438 & -24.78392 & -0.38812 \\ \text { O } & 32.21348 & -35.55016 & -0.4009 \\ \text { C } & 33.5876 & -35.61295 & -0.79372 \\ \text { H } & 24.84772 & -23.05903 & -1.26566 \\ \text { H } & 22.57014 & -22.16401 & -0.81124 \\ \text { H } & 23.98569 & -26.49503 & 1.18612 \\ \text { H } & 21.6953 & -25.6287 & 1.59642 \\ \text { H } & 20.39257 & -23.75003 & 2.94221 \\ \text { H } & 18.29149 & -24.99371 & 2.65184 \\ \text { H } & 20.11097 & -22.37121 & -1.19335\end{array}$




\begin{tabular}{|c|c|}
\hline $\mathrm{H}$ & $18.04766-23.6626-1.49499$ \\
\hline $\mathrm{H}$ & $\begin{array}{llll}31.84764 & -29.32611 & -2.53177\end{array}$ \\
\hline $\mathrm{H}$ & $\begin{array}{llll}30.13397 & -27.52848 & -2.38686\end{array}$ \\
\hline $\mathrm{H}$ & $\begin{array}{lll}30.40021 & -30.97859 & 1.17234\end{array}$ \\
\hline $\mathrm{H}$ & $\begin{array}{lll}28.66847 & -29.24499 & 1.29207\end{array}$ \\
\hline $\mathrm{H}$ & $27.60673-27.42172-2.4826$ \\
\hline $\mathrm{H}$ & $25.61669-25.94371 \quad-2.29667$ \\
\hline $\mathrm{H}$ & $\begin{array}{lll}28.12884 & -27.23752 & 1.79689\end{array}$ \\
\hline $\mathrm{H}$ & $\begin{array}{lll}26.15333 & -25.78858 & 1.97422\end{array}$ \\
\hline $\mathrm{H}$ & $\begin{array}{llll}32.06485 & -31.80372 & -2.90857\end{array}$ \\
\hline $\mathrm{H}$ & $\begin{array}{llll}31.46518 & -34.17875 & -2.57391\end{array}$ \\
\hline $\mathrm{H}$ & $\begin{array}{lll}33.13564 & -31.49937 & 1.31828\end{array}$ \\
\hline $\mathrm{H}$ & $\begin{array}{lll}32.60205 & -33.87204 & 1.62424\end{array}$ \\
\hline $\mathrm{H}$ & $\begin{array}{lll}30.15273 & -33.06064 & 1.6358\end{array}$ \\
\hline $\mathrm{H}$ & $\begin{array}{llll}27.36484 & -35.55301 & -1.62176\end{array}$ \\
\hline $\mathrm{H}$ & $29.85366-35.79552-1.66286$ \\
\hline $\mathrm{H}$ & $\begin{array}{lll}16.85178 & -27.01032 & 1.24982\end{array}$ \\
\hline $\mathrm{H}$ & $20.82233-28.02197-1.54491$ \\
\hline $\mathrm{H}$ & $20.13523-25.67221-1.18832$ \\
\hline $\mathrm{H}$ & $\begin{array}{lll}17.52709 & -30.68493 & 0.65294\end{array}$ \\
\hline $\mathrm{H}$ & $\begin{array}{lll}15.94188 & -30.4684 & 2.48013\end{array}$ \\
\hline $\mathrm{H}$ & $\begin{array}{lll}16.27863 & -28.64251 & 2.61171\end{array}$ \\
\hline $\mathrm{H}$ & $26.40625 \quad-32.5734 \quad 1.95124$ \\
\hline $\mathrm{H}$ & $27.52099-31.24635 \quad 3.62395$ \\
\hline $\mathrm{H}$ & $29.1949-31.78459 \quad 3.02724$ \\
\hline $\mathrm{H}$ & $19.8135-30.61135-2.55121$ \\
\hline $\mathrm{H}$ & $\begin{array}{lll}21.09461 & -33.11087 & 1.51844\end{array}$ \\
\hline $\mathrm{H}$ & $\begin{array}{lll}20.89538 & -29.33306 & 1.88645\end{array}$ \\
\hline $\mathrm{H}$ & $21.23236-30.31939 \quad 4.07124$ \\
\hline $\mathrm{H}$ & $\begin{array}{lll}21.01755 & -32.03508 & 3.38841\end{array}$ \\
\hline $\mathrm{H}$ & $\begin{array}{lll}20.04179 & -34.32279 & -2.99325\end{array}$ \\
\hline $\mathrm{H}$ & $20.13116-33.26768-5.18026$ \\
\hline $\mathrm{H}$ & $20.46366-31.60582-4.40952$ \\
\hline $\mathrm{H}$ & $22.19344-34.8979-0.08222$ \\
\hline $\mathrm{H}$ & $25.82982-31.72477-1.10203$ \\
\hline $\mathrm{H}$ & $\begin{array}{lll}23.59906 & -36.50954 & 0.63793\end{array}$ \\
\hline $\mathrm{H}$ & $25.39942 \quad-37.31204 \quad 1.97181$ \\
\hline $\mathrm{H}$ & $26.3736-35.74741 \quad 1.76$ \\
\hline $\mathrm{H}$ & $24.42541 \quad-30.206 \quad-2.06762$ \\
\hline $\mathrm{H}$ & $\begin{array}{llll}22.35182 & -29.05307 & -2.45107\end{array}$ \\
\hline $\mathrm{H}$ & $21.36332-30.46232-1.74307$ \\
\hline $\mathrm{H}$ & $\begin{array}{lll}22.76278 & -21.94813 & 2.06512\end{array}$ \\
\hline $\mathrm{H}$ & $21.89749-20.38396 \quad 2.2561$ \\
\hline $\mathrm{H}$ & $21.36225-21.81757 \quad 3.18783$ \\
\hline $\mathrm{H}$ & $30.89459-24.75942-0.04796$ \\
\hline $\mathrm{H}$ & $\begin{array}{lll}29.84193 & -25.32565 & 1.29332\end{array}$ \\
\hline
\end{tabular}




$\begin{array}{llll}\mathrm{H} & 30.93841 & -26.48511 & 0.45842 \\ \mathrm{H} & 27.66275 & -23.64412 & 1.45502 \\ \mathrm{H} & 27.54604 & -22.27785 & 0.29385 \\ \mathrm{H} & 26.06598 & -22.87988 & 1.11858 \\ \mathrm{H} & 34.98674 & -29.18032 & -0.93933 \\ \mathrm{H} & 33.36538 & -28.80513 & -0.25978 \\ \mathrm{H} & 34.45763 & -29.95702 & 0.58577 \\ \mathrm{H} & 15.90852 & -23.96797 & -1.13046 \\ \mathrm{H} & 16.04285 & -25.74681 & -0.88862 \\ \mathrm{H} & 14.81337 & -24.79948 & 0.01885 \\ \mathrm{H} & 33.85783 & -36.6779 & -0.77136 \\ \mathrm{H} & 33.74437 & -35.2114 & -1.81179 \\ \mathrm{H} & 34.23787 & -35.05161 & -0.09736\end{array}$

Macrocycle 12, Relative Energy $=1.93 \mathrm{kcal} \mathrm{mol}^{-1}, \mathrm{RB} 97 \mathrm{D} / 6-31 \mathrm{G}(\mathrm{d})$

$\begin{array}{llll}\text { C } & 24.59729 & -24.81995 & -0.04405 \\ \mathrm{C} & 24.15108 & -23.62601 & -0.64851 \\ \mathrm{C} & 22.86946 & -23.12895 & -0.39915 \\ \mathrm{C} & 21.97756 & -23.81671 & 0.44821 \\ \mathrm{C} & 23.68026 & -25.54872 & 0.73362 \\ \mathrm{C} & 22.3838 & -25.05654 & 0.97304 \\ \mathrm{C} & 20.63967 & -23.13036 & 0.81461 \\ \mathrm{C} & 18.10966 & -24.72831 & 0.46672 \\ \mathrm{C} & 19.92311 & -23.81831 & 1.95955 \\ \mathrm{C} & 18.78125 & -24.50173 & 1.809 \\ \mathrm{C} & 19.75401 & -23.04983 & -0.4161 \\ \mathrm{C} & 18.61701 & -23.74199 & -0.56746 \\ \mathrm{C} & 31.26558 & -30.27337 & -0.67754 \\ \mathrm{C} & 31.13867 & -29.3109 & -1.70269 \\ \mathrm{C} & 30.17014 & -28.30934 & -1.62621 \\ \mathrm{C} & 29.2828 & -28.23059 & -0.52863 \\ \mathrm{C} & 30.34832 & -30.23301 & 0.38222 \\ \mathrm{C} & 29.36389 & -29.2304 & 0.45292 \\ \mathrm{C} & 28.40819 & -26.96807 & -0.43061 \\ \mathrm{C} & 26.0732 & -25.23299 & -0.21021 \\ \mathrm{C} & 27.3899 & -26.86788 & -1.56724 \\ \mathrm{C} & 26.31541 & -26.07069 & -1.46766 \\ \mathrm{C} & 27.69587 & -26.7799 & 0.90606 \\ \mathrm{C} & 26.62063 & -25.98311 & 1.00653 \\ \mathrm{C} & 32.40944 & -31.30822 & -0.78179 \\ \mathrm{C} & 31.67349 & -34.2101 & -0.36328 \\ \mathrm{C} & 32.06953 & -32.25629 & -1.9153 \\ \mathrm{C} & 31.75156 & -33.5441 & -1.73281 \\ \mathrm{C} & 32.67083 & -32.06641 & 0.50913 \\ \mathrm{C} & 32.37969 & -33.36447 & 0.68201\end{array}$




\begin{tabular}{|c|c|}
\hline $\mathrm{C}$ & $27.35942-34.11972-0.00968$ \\
\hline $\mathrm{C}$ & $28.13963 \quad-33.45526 \quad 0.97603$ \\
\hline $\mathrm{C}$ & $29.53936-33.59938 \quad 0.91773$ \\
\hline $\mathrm{C}$ & $\begin{array}{lll}30.16917 & -34.35787 & -0.07261\end{array}$ \\
\hline $\mathrm{C}$ & $\begin{array}{lll}27.98132 & -35.02001 & -0.89503\end{array}$ \\
\hline $\mathrm{C}$ & $29.37325-35.14365-0.93279$ \\
\hline $\mathrm{C}$ & $\begin{array}{llll}18.8971 & -28.9506 & -0.93029\end{array}$ \\
\hline $\mathrm{C}$ & $\begin{array}{llll}19.64497 & -27.87044 & -1.47777\end{array}$ \\
\hline $\mathrm{C}$ & $\begin{array}{lll}19.42182 & -26.57241 & -0.96616\end{array}$ \\
\hline $\mathrm{C}$ & $\begin{array}{lll}18.48346 & -26.32149 & 0.04467\end{array}$ \\
\hline $\mathrm{C}$ & $\begin{array}{lll}17.99444 & -28.69378 & 0.12378\end{array}$ \\
\hline $\mathrm{C}$ & $\begin{array}{lll}17.78038 & -27.40387 & 0.60495\end{array}$ \\
\hline $\mathrm{C}$ & $20.63191 \quad-28.11505-2.5461$ \\
\hline $\mathrm{C}$ & $\begin{array}{llll}21.1537 & -27.19264 & -3.3796\end{array}$ \\
\hline $\mathrm{C}$ & $27.49794 \quad-32.58955 \quad 1.98106$ \\
\hline $\mathrm{C}$ & $28.10913 \quad-31.83598 \quad 2.91848$ \\
\hline $\mathrm{C}$ & $\begin{array}{lll}19.00957 & -30.73731 & -0.04526\end{array}$ \\
\hline $\mathrm{C}$ & $21.43008-32.17773-0.29008$ \\
\hline $\mathrm{C}$ & $19.59219-30.96748-1.30169$ \\
\hline $\mathrm{C}$ & $\begin{array}{lll}20.80322 & -31.66827 & -1.46586\end{array}$ \\
\hline $\mathrm{C}$ & $\begin{array}{lll}19.73418 & -31.08563 & 1.13282\end{array}$ \\
\hline $\mathrm{C}$ & $20.92978 \quad-31.81288 \quad 0.96784$ \\
\hline $\mathrm{C}$ & $\begin{array}{lll}19.21743 & -30.72955 & 2.46475\end{array}$ \\
\hline $\mathrm{C}$ & $\begin{array}{lll}19.65482 & -31.19191 & 3.65448\end{array}$ \\
\hline $\mathrm{C}$ & $\begin{array}{llll}21.336 & -31.95903 & -2.80618\end{array}$ \\
\hline $\mathrm{C}$ & $21.0313-31.28787 \quad-3.93532$ \\
\hline $\mathrm{C}$ & $25.94004-33.72945-0.22648$ \\
\hline $\mathrm{C}$ & $\begin{array}{lll}24.9048 & -34.67491 & 0.03635\end{array}$ \\
\hline $\mathrm{C}$ & $23.59779-34.30146-0.33355$ \\
\hline $\mathrm{C}$ & $\begin{array}{lll}23.26288 & -33.06107 & -0.89995\end{array}$ \\
\hline $\mathrm{C}$ & $24.28336-32.08574-1.07288$ \\
\hline $\mathrm{C}$ & $25.60618-32.47055-0.7402$ \\
\hline $\mathrm{C}$ & $25.06413-36.0215 \quad 0.61966$ \\
\hline $\mathrm{C}$ & $\begin{array}{lll}26.05876 & -36.528 & 1.3779\end{array}$ \\
\hline $\mathrm{C}$ & $24.08049-30.72095-1.58037$ \\
\hline $\mathrm{C}$ & $22.92113-30.05715 \quad-1.76793$ \\
\hline $\mathrm{O}$ & $20.88425 \quad-21.72676 \quad 1.13525$ \\
\hline $\mathrm{C}$ & $\begin{array}{lll}21.77576 & -21.47599 & 2.22091\end{array}$ \\
\hline $\mathrm{O}$ & $\begin{array}{llll}29.291 & -25.81499 & -0.67673\end{array}$ \\
\hline $\mathrm{C}$ & $30.28856-25.6$ \\
\hline $\mathrm{O}$ & $26.87327-24.03175 \quad-0.45309$ \\
\hline $\mathrm{C}$ & $\begin{array}{lll}27.03617 & -23.17417 & 0.67514\end{array}$ \\
\hline $\mathrm{O}$ & $\begin{array}{lll}33.63713 & -30.65815 & -1.22836\end{array}$ \\
\hline $\mathrm{C}$ & $\begin{array}{llll}34.12164 & -29.59745 & -0.40454\end{array}$ \\
\hline $\mathrm{O}$ & $\begin{array}{lll}16.69585 & -24.56816 & 0.72997\end{array}$ \\
\hline $\mathrm{C}$ & $15.83438-24.78392-0.38812$ \\
\hline
\end{tabular}




\begin{tabular}{|c|c|}
\hline $\mathrm{O}$ & $\begin{array}{lll}32.21348 & -35.55016 & -0.4009\end{array}$ \\
\hline $\mathrm{C}$ & $\begin{array}{lll}33.5876 & -35.61295 & -0.79372\end{array}$ \\
\hline $\mathrm{H}$ & $24.84772-23.05903-1.26566$ \\
\hline $\mathrm{H}$ & $22.57014-22.16401-0.81124$ \\
\hline $\mathrm{H}$ & $23.98569-26.49503 \quad 1.18612$ \\
\hline $\mathrm{H}$ & $\begin{array}{lll}21.6953 & -25.6287 & 1.59642\end{array}$ \\
\hline $\mathrm{H}$ & $\begin{array}{lll}20.39257 & -23.75003 & 2.94221\end{array}$ \\
\hline $\mathrm{H}$ & $\begin{array}{lll}18.29149 & -24.99371 & 2.65184\end{array}$ \\
\hline $\mathrm{H}$ & $\begin{array}{lll}20.11097 & -22.37121 & -1.19335\end{array}$ \\
\hline $\mathrm{H}$ & $\begin{array}{llll}18.04766 & -23.6626 & -1.49499\end{array}$ \\
\hline $\mathrm{H}$ & $\begin{array}{llll}31.84764 & -29.32611 & -2.53177\end{array}$ \\
\hline $\mathrm{H}$ & $\begin{array}{lll}30.13397 & -27.52848 & -2.38686\end{array}$ \\
\hline $\mathrm{H}$ & $\begin{array}{lll}30.40021 & -30.97859 & 1.17234\end{array}$ \\
\hline $\mathrm{H}$ & $\begin{array}{lll}28.66847 & -29.24499 & 1.29207\end{array}$ \\
\hline $\mathrm{H}$ & $27.60673-27.42172-2.4826$ \\
\hline $\mathrm{H}$ & $\begin{array}{lll}25.61669 & -25.94371 & -2.29667\end{array}$ \\
\hline $\mathrm{H}$ & $28.12884 \quad-27.23752 \quad 1.79689$ \\
\hline $\mathrm{H}$ & $26.15333-25.78858 \quad 1.97422$ \\
\hline $\mathrm{H}$ & $\begin{array}{lll}32.06485 & -31.80372 & -2.90857\end{array}$ \\
\hline $\mathrm{H}$ & $\begin{array}{lll}31.46518 & -34.17875 & -2.57391\end{array}$ \\
\hline $\mathrm{H}$ & $\begin{array}{llll}33.13564 & -31.49937 & 1.31828\end{array}$ \\
\hline $\mathrm{H}$ & $\begin{array}{lll}32.60205 & -33.87204 & 1.62424\end{array}$ \\
\hline $\mathrm{H}$ & $30.15273-33.06064 \quad 1.6358$ \\
\hline $\mathrm{H}$ & $27.36484-35.55301-1.62176$ \\
\hline $\mathrm{H}$ & $29.85366-35.79552-1.66286$ \\
\hline $\mathrm{H}$ & $\begin{array}{lll}19.99307 & -25.73281 & -1.36269\end{array}$ \\
\hline $\mathrm{H}$ & $\begin{array}{lll}17.41394 & -29.52292 & 0.53001\end{array}$ \\
\hline $\mathrm{H}$ & $\begin{array}{lll}17.04031 & -27.23168 & 1.38516\end{array}$ \\
\hline $\mathrm{H}$ & $20.94361-29.1568 \quad-2.66185$ \\
\hline $\mathrm{H}$ & $\begin{array}{llll}21.89664 & -27.47209 & -4.12877\end{array}$ \\
\hline $\mathrm{H}$ & $20.85613-26.14174 \quad-3.34643$ \\
\hline $\mathrm{H}$ & $26.40625 \quad-32.5734 \quad 1.95124$ \\
\hline $\mathrm{H}$ & $\begin{array}{lll}27.52099 & -31.24635 & 3.62395\end{array}$ \\
\hline $\mathrm{H}$ & $29.1949-31.78459 \quad 3.02724$ \\
\hline $\mathrm{H}$ & $\begin{array}{lll}19.01722 & -30.70203 & -2.18891\end{array}$ \\
\hline $\mathrm{H}$ & $\begin{array}{lll}21.46843 & -32.15952 & 1.85067\end{array}$ \\
\hline $\mathrm{H}$ & $\begin{array}{lll}18.391 & -30.01416 & 2.46211\end{array}$ \\
\hline $\mathrm{H}$ & $\begin{array}{lll}19.21109 & -30.83648 \quad 4.58601\end{array}$ \\
\hline $\mathrm{H}$ & $\begin{array}{lll}20.45356 & -31.93213 & 3.74233\end{array}$ \\
\hline $\mathrm{H}$ & $\begin{array}{llll}22.05592 & -32.77801 & -2.86366\end{array}$ \\
\hline $\mathrm{H}$ & $21.47285-31.57332-4.89173$ \\
\hline $\mathrm{H}$ & $20.3524 \quad-30.43139 \quad-3.93939$ \\
\hline $\mathrm{H}$ & $\begin{array}{llll}22.79731 & -35.03322 & -0.20311\end{array}$ \\
\hline $\mathrm{H}$ & $26.41536-31.75754-0.91837$ \\
\hline $\mathrm{H}$ & $24.20746 \quad-36.67807 \quad 0.4275$ \\
\hline $\mathrm{H}$ & $\begin{array}{lll}25.99088 & -37.55616 & 1.73631\end{array}$ \\
\hline
\end{tabular}




$\begin{array}{llll}\mathrm{H} & 26.95058 & -35.97038 & 1.65731 \\ \mathrm{H} & 25.01226 & -30.18759 & -1.80039 \\ \mathrm{H} & 22.93313 & -29.0314 & -2.14173 \\ \mathrm{H} & 21.94766 & -30.49815 & -1.55705 \\ \mathrm{H} & 22.76278 & -21.94813 & 2.06512 \\ \mathrm{H} & 21.89749 & -20.38396 & 2.2561 \\ \mathrm{H} & 21.36225 & -21.81757 & 3.18783 \\ \mathrm{H} & 30.89459 & -24.75942 & -0.04796 \\ \mathrm{H} & 29.84193 & -25.32565 & 1.29332 \\ \mathrm{H} & 30.93841 & -26.48511 & 0.45842 \\ \mathrm{H} & 27.66275 & -23.64412 & 1.45502 \\ \mathrm{H} & 27.54604 & -22.27785 & 0.29385 \\ \mathrm{H} & 26.06598 & -22.87988 & 1.11858 \\ \mathrm{H} & 34.98674 & -29.18032 & -0.93933 \\ \mathrm{H} & 33.36538 & -28.80513 & -0.25978 \\ \mathrm{H} & 34.45763 & -29.95702 & 0.58577 \\ \mathrm{H} & 15.90852 & -23.96797 & -1.13046 \\ \mathrm{H} & 16.04285 & -25.74681 & -0.88862 \\ \mathrm{H} & 14.81337 & -24.79948 & 0.01885 \\ \mathrm{H} & 33.85783 & -36.6779 & -0.77136 \\ \mathrm{H} & 33.74437 & -35.2114 & -1.81179 \\ \mathrm{H} & 34.23787 & -35.05161 & -0.09736\end{array}$

Macrocycle 12, Relative Energy $=1.97 \mathrm{kcal} \mathrm{mol}^{-1}, R B 97 D / 6-31 G(d)$

$\begin{array}{llll}\mathrm{C} & 24.59729 & -24.81995 & -0.04405 \\ \mathrm{C} & 24.15108 & -23.62601 & -0.64851 \\ \mathrm{C} & 22.86946 & -23.12895 & -0.39915 \\ \mathrm{C} & 21.97756 & -23.81671 & 0.44821 \\ \mathrm{C} & 23.68026 & -25.54872 & 0.73362 \\ \mathrm{C} & 22.3838 & -25.05654 & 0.97304 \\ \mathrm{C} & 20.63967 & -23.13036 & 0.81461 \\ \mathrm{C} & 18.10966 & -24.72831 & 0.46672 \\ \mathrm{C} & 19.92311 & -23.81831 & 1.95955 \\ \mathrm{C} & 18.78125 & -24.50173 & 1.809 \\ \mathrm{C} & 19.75401 & -23.04983 & -0.4161 \\ \mathrm{C} & 18.61701 & -23.74199 & -0.56746 \\ \mathrm{C} & 31.26558 & -30.27337 & -0.67754 \\ \mathrm{C} & 31.13867 & -29.3109 & -1.70269 \\ \mathrm{C} & 30.17014 & -28.30934 & -1.62621 \\ \mathrm{C} & 29.2828 & -28.23059 & -0.52863 \\ \mathrm{C} & 30.34832 & -30.23301 & 0.38222 \\ \mathrm{C} & 29.36389 & -29.2304 & 0.45292 \\ \mathrm{C} & 28.40819 & -26.96807 & -0.43061\end{array}$




\begin{tabular}{|c|c|}
\hline $\mathrm{C}$ & $\begin{array}{lll}26.0732 & -25.23299 & -0.21021\end{array}$ \\
\hline $\mathrm{C}$ & $27.3899-26.86788-1.56724$ \\
\hline $\mathrm{C}$ & $26.31541-26.07069-1.46766$ \\
\hline $\mathrm{C}$ & $27.69587-26.7799 \quad 0.90606$ \\
\hline $\mathrm{C}$ & $\begin{array}{lll}26.62063 & -25.98311 & 1.00653\end{array}$ \\
\hline $\mathrm{C}$ & $\begin{array}{lll}32.40944 & -31.30822 & -0.78179\end{array}$ \\
\hline $\mathrm{C}$ & $31.67349-34.2101-0.36328$ \\
\hline $\mathrm{C}$ & $32.06953-32.25629-1.9153$ \\
\hline $\mathrm{C}$ & $31.75156-33.5441-1.73281$ \\
\hline $\mathrm{C}$ & $\begin{array}{lll}32.67083 & -32.06641 & 0.50913\end{array}$ \\
\hline $\mathrm{C}$ & $\begin{array}{lll}32.37969 & -33.36447 & 0.68201\end{array}$ \\
\hline $\mathrm{C}$ & $27.35942-34.11972-0.00968$ \\
\hline $\mathrm{C}$ & $28.13963-33.45526 \quad 0.97603$ \\
\hline $\mathrm{C}$ & $\begin{array}{llll}29.53936 & -33.59938 & 0.91773\end{array}$ \\
\hline $\mathrm{C}$ & $\begin{array}{lll}30.16917 & -34.35787 & -0.07261\end{array}$ \\
\hline $\mathrm{C}$ & $27.98132-35.02001-0.89503$ \\
\hline $\mathrm{C}$ & $29.37325 \quad-35.14365-0.93279$ \\
\hline $\mathrm{C}$ & $19.25375-28.87406-0.33112$ \\
\hline $\mathrm{C}$ & $\begin{array}{lll}18.11074 & -28.59472 & 0.46969\end{array}$ \\
\hline $\mathrm{C}$ & $\begin{array}{lll}17.7315 & -27.24567 & 0.6505\end{array}$ \\
\hline $\mathrm{C}$ & $\begin{array}{lll}18.45186 & -26.18677 & 0.08036\end{array}$ \\
\hline $\mathrm{C}$ & $19.93904-27.80313-0.94375$ \\
\hline $\mathrm{C}$ & $19.55555-26.47895-0.742$ \\
\hline $\mathrm{C}$ & $\begin{array}{lll}17.3446 & -29.69599 & 1.08245\end{array}$ \\
\hline $\mathrm{C}$ & $\begin{array}{lll}16.47699 & -29.59237 & 2.10926\end{array}$ \\
\hline $\mathrm{C}$ & $27.49794-32.58955 \quad 1.98106$ \\
\hline $\mathrm{C}$ & $\begin{array}{lll}28.10913 & -31.83598 & 2.91848\end{array}$ \\
\hline $\mathrm{C}$ & $19.29256-30.43336-0.19352$ \\
\hline $\mathrm{C}$ & $\begin{array}{llll}21.79618 & -31.73096 & -0.39777\end{array}$ \\
\hline $\mathrm{C}$ & $19.89272-30.65815-1.44264$ \\
\hline $\mathrm{C}$ & $\begin{array}{lll}21.14429 & -31.28852 & -1.58699\end{array}$ \\
\hline $\mathrm{C}$ & $20.03232-30.70906 \quad 0.99434$ \\
\hline $\mathrm{C}$ & $\begin{array}{lll}21.2701 & -31.36679 & 0.84979\end{array}$ \\
\hline $\mathrm{C}$ & $19.49007 \quad-30.35287 \quad 2.31605$ \\
\hline $\mathrm{C}$ & $\begin{array}{lll}19.94999 & -30.75932 & 3.51764\end{array}$ \\
\hline $\mathrm{C}$ & $21.69866-31.57877-2.91864$ \\
\hline $\mathrm{C}$ & $21.34674-30.96555-4.06684$ \\
\hline $\mathrm{C}$ & $25.94004-33.72945-0.22648$ \\
\hline $\mathrm{C}$ & $25.63956-32.43093-0.73463$ \\
\hline $\mathrm{C}$ & $24.27968-32.06497 \quad-0.77193$ \\
\hline $\mathrm{C}$ & $23.22602-32.90591-0.37931$ \\
\hline $\mathrm{C}$ & $\begin{array}{lll}23.52345 & -34.23276 & 0.03729\end{array}$ \\
\hline $\mathrm{C}$ & $24.89203 \quad-34.59265 \quad 0.11523$ \\
\hline $\mathrm{C}$ & $26.60746-31.41337-1.18927$ \\
\hline $\mathrm{C}$ & $27.88277-31.55266-1.60756$ \\
\hline $\mathrm{C}$ & $\begin{array}{lll}22.53102 & -35.25277 & 0.40609\end{array}$ \\
\hline
\end{tabular}




\begin{tabular}{|c|c|}
\hline $\mathrm{C}$ & $21.19449-35.2302 \quad 0.22423$ \\
\hline $\mathrm{O}$ & $20.88425 \quad-21.72676 \quad 1.13525$ \\
\hline $\mathrm{C}$ & $\begin{array}{lll}21.77576 & -21.47599 & 2.22091\end{array}$ \\
\hline $\mathrm{O}$ & $\begin{array}{lll}29.291 & -25.81499 & -0.67673\end{array}$ \\
\hline $\mathrm{C}$ & $30.28856-25.6$ \\
\hline $\mathrm{O}$ & $\begin{array}{lll}26.87327 & -24.03175 & -0.45309\end{array}$ \\
\hline $\mathrm{C}$ & $\begin{array}{lll}27.03617 & -23.17417 & 0.67514\end{array}$ \\
\hline $\mathrm{O}$ & $33.63713-30.65815-1.22836$ \\
\hline $\mathrm{C}$ & $\begin{array}{lll}34.12164 & -29.59745 & -0.40454\end{array}$ \\
\hline $\mathrm{O}$ & $\begin{array}{lll}16.69585 & -24.56816 & 0.72997\end{array}$ \\
\hline $\mathrm{C}$ & $\begin{array}{lll}15.83438 & -24.78392 & -0.38812\end{array}$ \\
\hline $\mathrm{O}$ & $32.21348-35.55016-0.4009$ \\
\hline $\mathrm{C}$ & $\begin{array}{lll}33.5876 & -35.61295 & -0.79372\end{array}$ \\
\hline $\mathrm{H}$ & $24.84772-23.05903-1.26566$ \\
\hline $\mathrm{H}$ & $22.57014-22.16401-0.81124$ \\
\hline $\mathrm{H}$ & $23.98569-26.49503 \quad 1.18612$ \\
\hline $\mathrm{H}$ & $21.6953-25.6287 \quad 1.59642$ \\
\hline $\mathrm{H}$ & $\begin{array}{lll}20.39257 & -23.75003 & 2.94221\end{array}$ \\
\hline $\mathrm{H}$ & $\begin{array}{lll}18.29149 & -24.99371 & 2.65184\end{array}$ \\
\hline $\mathrm{H}$ & $\begin{array}{lll}20.11097 & -22.37121 & -1.19335\end{array}$ \\
\hline $\mathrm{H}$ & $\begin{array}{llll}18.04766 & -23.6626 & -1.49499\end{array}$ \\
\hline $\mathrm{H}$ & $\begin{array}{lll}31.84764 & -29.32611 & -2.53177\end{array}$ \\
\hline $\mathrm{H}$ & $\begin{array}{lll}30.13397 & -27.52848 & -2.38686\end{array}$ \\
\hline $\mathrm{H}$ & $\begin{array}{lll}30.40021 & -30.97859 & 1.17234\end{array}$ \\
\hline $\mathrm{H}$ & $\begin{array}{lll}28.66847 & -29.24499 & 1.29207\end{array}$ \\
\hline $\mathrm{H}$ & $27.60673-27.42172-2.4826$ \\
\hline $\mathrm{H}$ & $\begin{array}{lll}25.61669 & -25.94371 & -2.29667\end{array}$ \\
\hline $\mathrm{H}$ & $28.12884 \quad-27.23752 \quad 1.79689$ \\
\hline $\mathrm{H}$ & $26.15333-25.78858 \quad 1.97422$ \\
\hline $\mathrm{H}$ & $\begin{array}{lll}32.06485 & -31.80372 & -2.90857\end{array}$ \\
\hline $\mathrm{H}$ & $\begin{array}{llll}31.46518 & -34.17875 & -2.57391\end{array}$ \\
\hline $\mathrm{H}$ & $\begin{array}{llll}33.13564 & -31.49937 & 1.31828\end{array}$ \\
\hline $\mathrm{H}$ & $\begin{array}{lll}32.60205 & -33.87204 & 1.62424\end{array}$ \\
\hline $\mathrm{H}$ & $30.15273-33.06064 \quad 1.6358$ \\
\hline $\mathrm{H}$ & $27.36484-35.55301-1.62176$ \\
\hline $\mathrm{H}$ & $29.85366-35.79552-1.66286$ \\
\hline $\mathrm{H}$ & $\begin{array}{lll}16.85178 & -27.01032 & 1.24982\end{array}$ \\
\hline $\mathrm{H}$ & $\begin{array}{llll}20.82233 & -28.02197 & -1.54491\end{array}$ \\
\hline $\mathrm{H}$ & $20.13523-25.67221-1.18832$ \\
\hline $\mathrm{H}$ & $\begin{array}{lll}17.52709 & -30.68493 & 0.65294\end{array}$ \\
\hline $\mathrm{H}$ & $\begin{array}{lll}15.94188 & -30.4684 & 2.48013\end{array}$ \\
\hline $\mathrm{H}$ & $\begin{array}{lll}16.27863 & -28.64251 & 2.61171\end{array}$ \\
\hline $\mathrm{H}$ & $26.40625 \quad-32.5734 \quad 1.95124$ \\
\hline $\mathrm{H}$ & $27.52099 \quad-31.24635 \quad 3.62395$ \\
\hline $\mathrm{H}$ & $29.1949-31.78459 \quad 3.02724$ \\
\hline $\mathrm{H}$ & $19.3062-30.44915 \quad-2.33737$ \\
\hline
\end{tabular}




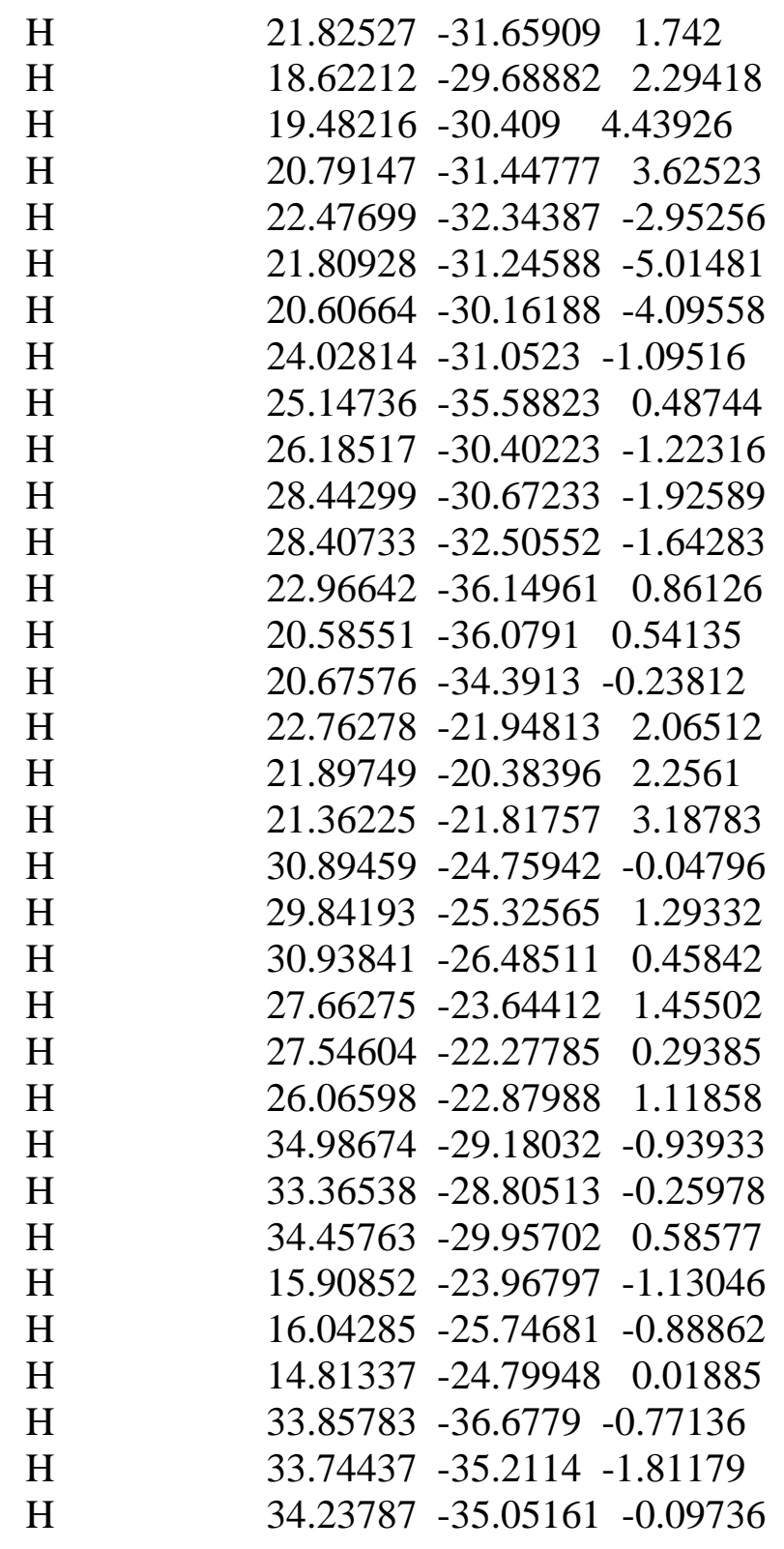

Macrocycle 12, Relative Energy $=2.64 \mathrm{kcal} \mathrm{mol}^{-1}, R B 97 \mathrm{D} / 6-31 G(d)$

$\begin{array}{llll}\mathrm{C} & 24.59729 & -24.81995 & -0.04405 \\ \mathrm{C} & 24.15108 & -23.62601 & -0.64851 \\ \mathrm{C} & 22.86946 & -23.12895 & -0.39915 \\ \mathrm{C} & 21.97756 & -23.81671 & 0.44821 \\ \mathrm{C} & 23.68026 & -25.54872 & 0.73362 \\ \mathrm{C} & 22.3838 & -25.05654 & 0.97304 \\ \mathrm{C} & 20.63967 & -23.13036 & 0.81461 \\ \mathrm{C} & 18.10966 & -24.72831 & 0.46672 \\ \mathrm{C} & 19.92311 & -23.81831 & 1.95955 \\ \mathrm{C} & 18.78125 & -24.50173 & 1.809\end{array}$




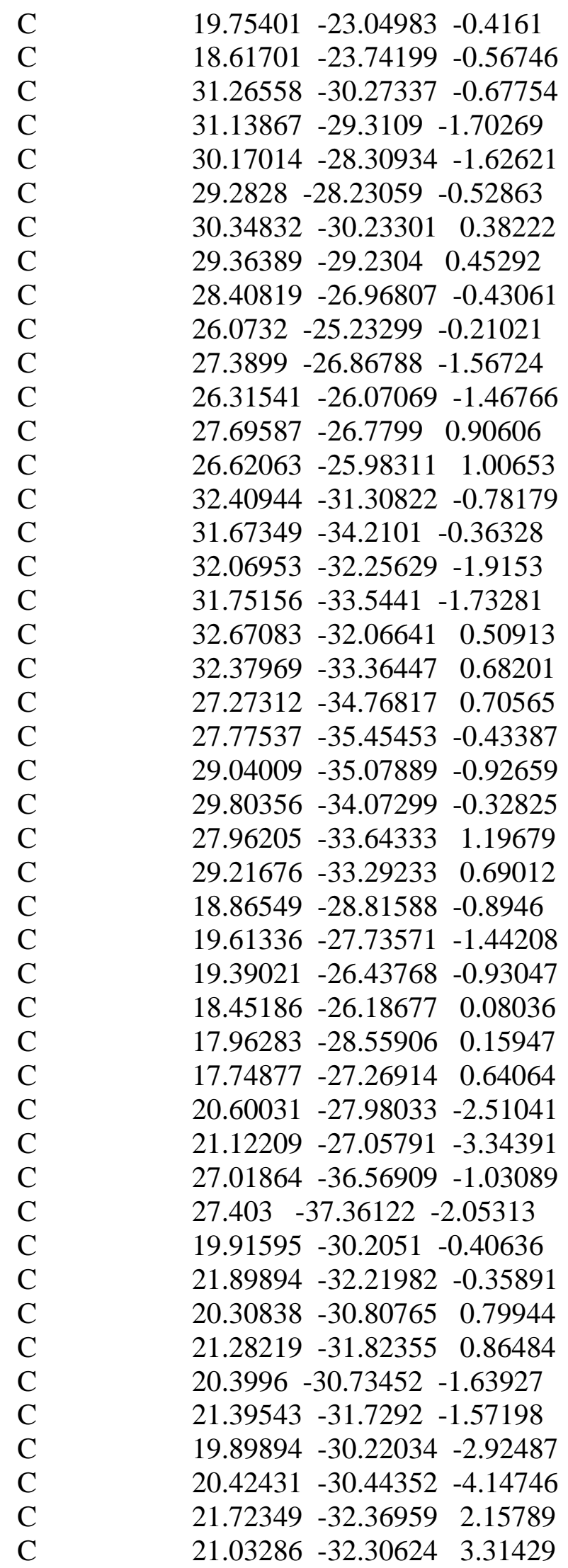




\begin{tabular}{|c|c|}
\hline $\mathrm{C}$ & $25.94004-33.72945-0.22648$ \\
\hline $\mathrm{C}$ & $25.63956-32.43093-0.73463$ \\
\hline $\mathrm{C}$ & $24.27968-32.06497 \quad-0.77193$ \\
\hline $\mathrm{C}$ & $\begin{array}{llll}23.22602 & -32.90591 & -0.37931\end{array}$ \\
\hline $\mathrm{C}$ & $23.52345 \quad-34.23276 \quad 0.03729$ \\
\hline $\mathrm{C}$ & $24.89203 \quad-34.59265 \quad 0.11523$ \\
\hline $\mathrm{C}$ & $\begin{array}{llll}26.60746 & -31.41337 & -1.18927\end{array}$ \\
\hline $\mathrm{C}$ & $27.88277-31.55266-1.60756$ \\
\hline $\mathrm{C}$ & $\begin{array}{llll}22.53102 & -35.25277 & 0.40609\end{array}$ \\
\hline $\mathrm{C}$ & $\begin{array}{lll}21.19449 & -35.2302 & 0.22423\end{array}$ \\
\hline $\mathrm{O}$ & $20.88425-21.72676 \quad 1.13525$ \\
\hline $\mathrm{C}$ & $\begin{array}{lll}21.77576 & -21.47599 & 2.22091\end{array}$ \\
\hline $\mathrm{O}$ & $\begin{array}{llll}29.291 & -25.81499 & -0.67673\end{array}$ \\
\hline $\mathrm{C}$ & $30.28856-25.6$ \\
\hline $\mathrm{O}$ & $26.87327-24.03175-0.45309$ \\
\hline $\mathrm{C}$ & $\begin{array}{lll}27.03617 & -23.17417 & 0.67514\end{array}$ \\
\hline $\mathrm{O}$ & $33.63713-30.65815-1.22836$ \\
\hline $\mathrm{C}$ & $\begin{array}{llll}34.12164 & -29.59745 & -0.40454\end{array}$ \\
\hline $\mathrm{O}$ & $\begin{array}{lll}16.69585 & -24.56816 & 0.72997\end{array}$ \\
\hline $\mathrm{C}$ & $15.83438-24.78392-0.38812$ \\
\hline $\mathrm{O}$ & $\begin{array}{llll}32.21348 & -35.55016 & -0.4009\end{array}$ \\
\hline $\mathrm{C}$ & $\begin{array}{lll}33.5876 & -35.61295 & -0.79372\end{array}$ \\
\hline $\mathrm{H}$ & $24.84772-23.05903-1.26566$ \\
\hline $\mathrm{H}$ & $22.57014-22.16401-0.81124$ \\
\hline $\mathrm{H}$ & $\begin{array}{lll}23.98569 & -26.49503 & 1.18612\end{array}$ \\
\hline $\mathrm{H}$ & $\begin{array}{lll}21.6953 & -25.6287 & 1.59642\end{array}$ \\
\hline $\mathrm{H}$ & $\begin{array}{lll}20.39257 & -23.75003 & 2.94221\end{array}$ \\
\hline $\mathrm{H}$ & $\begin{array}{lll}18.29149 & -24.99371 & 2.65184\end{array}$ \\
\hline $\mathrm{H}$ & $\begin{array}{lll}20.11097 & -22.37121 & -1.19335\end{array}$ \\
\hline $\mathrm{H}$ & $\begin{array}{llll}18.04766 & -23.6626 & -1.49499\end{array}$ \\
\hline $\mathrm{H}$ & $\begin{array}{llll}31.84764 & -29.32611 & -2.53177\end{array}$ \\
\hline $\mathrm{H}$ & $\begin{array}{llll}30.13397 & -27.52848 & -2.38686\end{array}$ \\
\hline $\mathrm{H}$ & $\begin{array}{lll}30.40021 & -30.97859 & 1.17234\end{array}$ \\
\hline $\mathrm{H}$ & $\begin{array}{lll}28.66847 & -29.24499 & 1.29207\end{array}$ \\
\hline $\mathrm{H}$ & $27.60673-27.42172-2.4826$ \\
\hline $\mathrm{H}$ & $25.61669-25.94371-2.29667$ \\
\hline $\mathrm{H}$ & $28.12884 \quad-27.23752 \quad 1.79689$ \\
\hline $\mathrm{H}$ & $26.15333 \quad-25.78858 \quad 1.97422$ \\
\hline $\mathrm{H}$ & $\begin{array}{llll}32.06485 & -31.80372 & -2.90857\end{array}$ \\
\hline $\mathrm{H}$ & $\begin{array}{llll}31.46518 & -34.17875 & -2.57391\end{array}$ \\
\hline $\mathrm{H}$ & $\begin{array}{lll}33.13564 & -31.49937 & 1.31828\end{array}$ \\
\hline $\mathrm{H}$ & $\begin{array}{lll}32.60205 & -33.87204 & 1.62424\end{array}$ \\
\hline $\mathrm{H}$ & $29.45857-35.62483-1.76856$ \\
\hline $\mathrm{H}$ & $\begin{array}{lll}27.54504 & -33.10202 & 2.04847\end{array}$ \\
\hline $\mathrm{H}$ & $29.77457 \quad-32.45802 \quad 1.11616$ \\
\hline $\mathrm{H}$ & $\begin{array}{llll}19.96146 & -25.59809 & -1.327\end{array}$ \\
\hline
\end{tabular}




\begin{tabular}{|c|c|}
\hline $\mathrm{H}$ & $\begin{array}{lll}17.38233 & -29.3882 & 0.5657\end{array}$ \\
\hline $\mathrm{H}$ & $\begin{array}{lll}17.0087 & -27.09696 & 1.42085\end{array}$ \\
\hline $\mathrm{H}$ & $20.912-29.02208-2.62616$ \\
\hline $\mathrm{H}$ & $21.86503-27.33737-4.09308$ \\
\hline $\mathrm{H}$ & $\begin{array}{lll}20.82452 & -26.00701 & -3.31074\end{array}$ \\
\hline $\mathrm{H}$ & $26.03848-36.75577-0.58661$ \\
\hline $\mathrm{H}$ & $26.74258-38.14823 \quad-2.42155$ \\
\hline $\mathrm{H}$ & $28.36413-37.25446-2.56126$ \\
\hline $\mathrm{H}$ & $\begin{array}{lll}19.9341 & -30.38136 & 1.73026\end{array}$ \\
\hline $\mathrm{H}$ & $\begin{array}{llll}21.83994 & -32.10116 & -2.49597\end{array}$ \\
\hline $\mathrm{H}$ & $\begin{array}{lll}19.00689 & -29.59264 & -2.85401\end{array}$ \\
\hline $\mathrm{H}$ & $\begin{array}{lll}19.95118 & -30.02979 & -5.03963\end{array}$ \\
\hline $\mathrm{H}$ & $21.33183-31.03207-4.30122$ \\
\hline $\mathrm{H}$ & $\begin{array}{lll}22.68532 & -32.88645 & 2.15173\end{array}$ \\
\hline $\mathrm{H}$ & $21.43756-32.73773 \quad 4.23146$ \\
\hline $\mathrm{H}$ & $\begin{array}{lll}20.04552 & -31.8421 & 3.37915\end{array}$ \\
\hline $\mathrm{H}$ & $24.02814-31.0523-1.09516$ \\
\hline $\mathrm{H}$ & $\begin{array}{lll}25.14736 & -35.58823 & 0.48744\end{array}$ \\
\hline $\mathrm{H}$ & $26.18517-30.40223-1.22316$ \\
\hline $\mathrm{H}$ & $28.44299-30.67233-1.92589$ \\
\hline $\mathrm{H}$ & $28.40733-32.50552-1.64283$ \\
\hline $\mathrm{H}$ & $22.96642-36.14961 \quad 0.86126$ \\
\hline $\mathrm{H}$ & $20.58551 \quad-36.0791 \quad 0.54135$ \\
\hline $\mathrm{H}$ & $20.67576-34.3913-0.23812$ \\
\hline $\mathrm{H}$ & $\begin{array}{lll}22.76278 & -21.94813 & 2.06512\end{array}$ \\
\hline $\mathrm{H}$ & $\begin{array}{lll}21.89749 & -20.38396 & 2.2561\end{array}$ \\
\hline $\mathrm{H}$ & $\begin{array}{lll}21.36225 & -21.81757 & 3.18783\end{array}$ \\
\hline $\mathrm{H}$ & $\begin{array}{lll}30.89459 & -24.75942 & -0.04796\end{array}$ \\
\hline $\mathrm{H}$ & $\begin{array}{lll}29.84193 & -25.32565 & 1.29332\end{array}$ \\
\hline $\mathrm{H}$ & $\begin{array}{lll}30.93841 & -26.48511 & 0.45842\end{array}$ \\
\hline $\mathrm{H}$ & $27.66275-23.64412 \quad 1.45502$ \\
\hline $\mathrm{H}$ & $\begin{array}{lll}27.54604 & -22.27785 & 0.29385\end{array}$ \\
\hline $\mathrm{H}$ & $\begin{array}{lll}26.06598 & -22.87988 & 1.11858\end{array}$ \\
\hline $\mathrm{H}$ & $\begin{array}{lll}34.98674 & -29.18032 & -0.93933\end{array}$ \\
\hline $\mathrm{H}$ & $\begin{array}{lll}33.36538 & -28.80513 & -0.25978\end{array}$ \\
\hline $\mathrm{H}$ & $\begin{array}{lll}34.45763 & -29.95702 & 0.58577\end{array}$ \\
\hline $\mathrm{H}$ & $\begin{array}{lll}15.90852 & -23.96797 & -1.13046\end{array}$ \\
\hline $\mathrm{H}$ & $\begin{array}{lll}16.04285 & -25.74681 & -0.88862\end{array}$ \\
\hline $\mathrm{H}$ & $\begin{array}{lll}14.81337 & -24.79948 & 0.01885\end{array}$ \\
\hline $\mathrm{H}$ & $\begin{array}{llll}33.85783 & -36.6779 & -0.77136\end{array}$ \\
\hline $\mathrm{H}$ & $33.74437-35.2114-1.81179$ \\
\hline $\mathrm{H}$ & $\begin{array}{lll}34.23787 & -35.05161 & -0.09736\end{array}$ \\
\hline
\end{tabular}

Macrocycle 12, Relative Energy $=2.68 \mathrm{kcal} \mathrm{mol}^{-1}, R B 97 \mathrm{D} / 6-31 G(d)$

C $\quad 24.59729-24.81995-0.04405$ 


\begin{tabular}{|c|c|}
\hline $\mathrm{C}$ & $24.15108-23.62601-0.64851$ \\
\hline $\mathrm{C}$ & $22.86946-23.12895 \quad-0.39915$ \\
\hline $\mathrm{C}$ & $\begin{array}{lll}21.97756 & -23.81671 & 0.44821\end{array}$ \\
\hline $\mathrm{C}$ & $\begin{array}{lll}23.68026 & -25.54872 & 0.73362\end{array}$ \\
\hline $\mathrm{C}$ & $22.3838 \quad-25.05654 \quad 0.97304$ \\
\hline $\mathrm{C}$ & $\begin{array}{llll}20.63967 & -23.13036 & 0.81461\end{array}$ \\
\hline $\mathrm{C}$ & $\begin{array}{lll}18.10966 & -24.72831 & 0.46672\end{array}$ \\
\hline $\mathrm{C}$ & $\begin{array}{lll}19.92311 & -23.81831 & 1.95955\end{array}$ \\
\hline $\mathrm{C}$ & $\begin{array}{lll}18.78125 & -24.50173 & 1.809\end{array}$ \\
\hline $\mathrm{C}$ & $19.75401-23.04983-0.4161$ \\
\hline $\mathrm{C}$ & $18.61701-23.74199-0.56746$ \\
\hline $\mathrm{C}$ & $\begin{array}{lll}31.26558 & -30.27337 & -0.67754\end{array}$ \\
\hline $\mathrm{C}$ & $\begin{array}{lll}31.13867 & -29.3109 & -1.70269\end{array}$ \\
\hline $\mathrm{C}$ & $30.17014-28.30934-1.62621$ \\
\hline $\mathrm{C}$ & $29.2828-28.23059-0.52863$ \\
\hline $\mathrm{C}$ & $30.34832-30.23301 \quad 0.38222$ \\
\hline $\mathrm{C}$ & $\begin{array}{lll}29.36389 & -29.2304 & 0.45292\end{array}$ \\
\hline $\mathrm{C}$ & $28.40819-26.96807-0.43061$ \\
\hline $\mathrm{C}$ & $26.0732-25.23299-0.21021$ \\
\hline $\mathrm{C}$ & $27.3899-26.86788-1.56724$ \\
\hline $\mathrm{C}$ & $26.31541-26.07069-1.46766$ \\
\hline $\mathrm{C}$ & $27.69587-26.7799 \quad 0.90606$ \\
\hline $\mathrm{C}$ & $26.62063-25.98311 \quad 1.00653$ \\
\hline $\mathrm{C}$ & $32.40944-31.30822-0.78179$ \\
\hline $\mathrm{C}$ & $31.67349-34.2101-0.36328$ \\
\hline $\mathrm{C}$ & $32.06953-32.25629-1.9153$ \\
\hline $\mathrm{C}$ & $31.75156-33.5441 \quad-1.73281$ \\
\hline $\mathrm{C}$ & $\begin{array}{lll}32.67083 & -32.06641 & 0.50913\end{array}$ \\
\hline $\mathrm{C}$ & $\begin{array}{lll}32.37969 & -33.36447 & 0.68201\end{array}$ \\
\hline $\mathrm{C}$ & $\begin{array}{lll}27.21835 & -34.72153 & 0.67028\end{array}$ \\
\hline $\mathrm{C}$ & $\begin{array}{llll}27.72286 & -35.41747 & -0.46242\end{array}$ \\
\hline $\mathrm{C}$ & $28.98737-35.04413-0.95745$ \\
\hline $\mathrm{C}$ & $29.7485-34.0314-0.36771$ \\
\hline $\mathrm{C}$ & $27.90484-33.59099 \quad 1.15166$ \\
\hline $\mathrm{C}$ & $29.15938 \quad-33.24244 \quad 0.6429$ \\
\hline $\mathrm{C}$ & $\begin{array}{lll}18.86549 & -28.81588 & -0.8946\end{array}$ \\
\hline $\mathrm{C}$ & $\begin{array}{lll}19.61336 & -27.73571 & -1.44208\end{array}$ \\
\hline $\mathrm{C}$ & $\begin{array}{llll}19.39021 & -26.43768 & -0.93047\end{array}$ \\
\hline $\mathrm{C}$ & $\begin{array}{lll}18.45186 & -26.18677 & 0.08036\end{array}$ \\
\hline $\mathrm{C}$ & $\begin{array}{llll}17.96283 & -28.55906 & 0.15947\end{array}$ \\
\hline $\mathrm{C}$ & $\begin{array}{lll}17.74877 & -27.26914 & 0.64064\end{array}$ \\
\hline $\mathrm{C}$ & $20.60031-27.98033-2.51041$ \\
\hline $\mathrm{C}$ & $21.12209-27.05791 \quad-3.34391$ \\
\hline $\mathrm{C}$ & $26.96866-36.53882-1.04982$ \\
\hline $\mathrm{C}$ & $\begin{array}{llll}27.35536 & -37.33965 & -2.06438\end{array}$ \\
\hline $\mathrm{C}$ & $19.29256-30.43336-0.19352$ \\
\hline
\end{tabular}




\begin{tabular}{|c|c|}
\hline $\mathrm{C}$ & $\begin{array}{llll}21.79618 & -31.73096 & -0.39777\end{array}$ \\
\hline $\mathrm{C}$ & $\begin{array}{lll}19.89272 & -30.65815 & -1.44264\end{array}$ \\
\hline $\mathrm{C}$ & $21.14429-31.28852-1.58699$ \\
\hline $\mathrm{C}$ & $20.03232-30.70906 \quad 0.99434$ \\
\hline $\mathrm{C}$ & $\begin{array}{lll}21.2701 & -31.36679 & 0.84979\end{array}$ \\
\hline $\mathrm{C}$ & $\begin{array}{lll}19.49007 & -30.35287 & 2.31605\end{array}$ \\
\hline $\mathrm{C}$ & $\begin{array}{lll}19.94999 & -30.75932 & 3.51764\end{array}$ \\
\hline $\mathrm{C}$ & $\begin{array}{llll}21.69866 & -31.57877 & -2.91864\end{array}$ \\
\hline $\mathrm{C}$ & $\begin{array}{llll}21.34674 & -30.96555 & -4.06684\end{array}$ \\
\hline $\mathrm{C}$ & $\begin{array}{llll}25.94004 & -33.72945 & -0.22648\end{array}$ \\
\hline $\mathrm{C}$ & $\begin{array}{llll}25.63956 & -32.43093 & -0.73463\end{array}$ \\
\hline $\mathrm{C}$ & $\begin{array}{llll}24.27968 & -32.06497 & -0.77193\end{array}$ \\
\hline $\mathrm{C}$ & $23.22602-32.90591 \quad-0.37931$ \\
\hline $\mathrm{C}$ & $\begin{array}{lll}23.52345 & -34.23276 & 0.03729\end{array}$ \\
\hline $\mathrm{C}$ & $\begin{array}{llll}24.89203 & -34.59265 & 0.11523\end{array}$ \\
\hline $\mathrm{C}$ & $26.60746-31.41337-1.18927$ \\
\hline $\mathrm{C}$ & $27.88277-31.55266-1.60756$ \\
\hline $\mathrm{C}$ & $\begin{array}{lll}22.53102 & -35.25277 & 0.40609\end{array}$ \\
\hline $\mathrm{C}$ & $21.19449 \quad-35.2302 \quad 0.22423$ \\
\hline $\mathrm{O}$ & $\begin{array}{lll}20.88425 & -21.72676 & 1.13525\end{array}$ \\
\hline $\mathrm{C}$ & $\begin{array}{lll}21.77576 & -21.47599 & 2.22091\end{array}$ \\
\hline $\mathrm{O}$ & $29.291-25.81499-0.67673$ \\
\hline $\mathrm{C}$ & $30.28856-25.6$ \\
\hline $\mathrm{O}$ & $26.87327-24.03175-0.45309$ \\
\hline $\mathrm{C}$ & $\begin{array}{lll}27.03617 & -23.17417 & 0.67514\end{array}$ \\
\hline $\mathrm{O}$ & $33.63713-30.65815-1.22836$ \\
\hline $\mathrm{C}$ & $\begin{array}{lll}34.12164 & -29.59745 & -0.40454\end{array}$ \\
\hline $\mathrm{O}$ & $\begin{array}{lll}16.69585 & -24.56816 & 0.72997\end{array}$ \\
\hline $\mathrm{C}$ & $\begin{array}{lll}15.83438 & -24.78392 & -0.38812\end{array}$ \\
\hline $\mathrm{O}$ & $32.21348-35.55016-0.4009$ \\
\hline $\mathrm{C}$ & $33.5876-35.61295-0.79372$ \\
\hline $\mathrm{H}$ & $24.84772-23.05903-1.26566$ \\
\hline $\mathrm{H}$ & $22.57014-22.16401-0.81124$ \\
\hline $\mathrm{H}$ & $\begin{array}{lll}23.98569 & -26.49503 & 1.18612\end{array}$ \\
\hline $\mathrm{H}$ & $\begin{array}{lll}21.6953 & -25.6287 & 1.59642\end{array}$ \\
\hline $\mathrm{H}$ & $\begin{array}{llll}20.39257 & -23.75003 & 2.94221\end{array}$ \\
\hline $\mathrm{H}$ & $\begin{array}{lll}18.29149 & -24.99371 & 2.65184\end{array}$ \\
\hline $\mathrm{H}$ & $20.11097-22.37121-1.19335$ \\
\hline $\mathrm{H}$ & $18.04766-23.6626-1.49499$ \\
\hline $\mathrm{H}$ & $\begin{array}{llll}31.84764 & -29.32611 & -2.53177\end{array}$ \\
\hline $\mathrm{H}$ & $\begin{array}{llll}30.13397 & -27.52848 & -2.38686\end{array}$ \\
\hline $\mathrm{H}$ & $\begin{array}{lll}30.40021 & -30.97859 & 1.17234\end{array}$ \\
\hline $\mathrm{H}$ & $\begin{array}{llll}28.66847 & -29.24499 & 1.29207\end{array}$ \\
\hline $\mathrm{H}$ & $27.60673-27.42172-2.4826$ \\
\hline $\mathrm{H}$ & $\begin{array}{llll}25.61669 & -25.94371 & -2.29667\end{array}$ \\
\hline$T$ & $28.12884-27.23752 \quad 1.796$ \\
\hline
\end{tabular}




\begin{tabular}{|c|c|}
\hline $\mathrm{H}$ & $26.15333-25.78858 \quad 1.97422$ \\
\hline $\mathrm{H}$ & $\begin{array}{lll}32.06485 & -31.80372 & -2.90857\end{array}$ \\
\hline $\mathrm{H}$ & $\begin{array}{lll}31.46518 & -34.17875 & -2.57391\end{array}$ \\
\hline $\mathrm{H}$ & $\begin{array}{lll}33.13564 & -31.49937 & 1.31828\end{array}$ \\
\hline $\mathrm{H}$ & $\begin{array}{lll}32.60205 & -33.87204 & 1.62424\end{array}$ \\
\hline $\mathrm{H}$ & $29.40758-35.59705-1.79398$ \\
\hline $\mathrm{H}$ & $27.48609-33.04261 \quad 1.99794$ \\
\hline $\mathrm{H}$ & $29.71533-32.40324 \quad 1.06174$ \\
\hline $\mathrm{H}$ & $\begin{array}{llll}19.96146 & -25.59809 & -1.327\end{array}$ \\
\hline $\mathrm{H}$ & $\begin{array}{lll}17.38233 & -29.3882 & 0.5657\end{array}$ \\
\hline $\mathrm{H}$ & $\begin{array}{lll}17.0087 & -27.09696 & 1.42085\end{array}$ \\
\hline $\mathrm{H}$ & $20.912-29.02208-2.62616$ \\
\hline $\mathrm{H}$ & $\begin{array}{llll}21.86503 & -27.33737 & -4.09308\end{array}$ \\
\hline $\mathrm{H}$ & $20.82452-26.00701-3.31074$ \\
\hline $\mathrm{H}$ & $25.98843-36.72315-0.60473$ \\
\hline $\mathrm{H}$ & $\begin{array}{lll}26.69668 & -38.13119 & -2.42612\end{array}$ \\
\hline $\mathrm{H}$ & $28.31676-37.23586-2.57261$ \\
\hline $\mathrm{H}$ & $\begin{array}{lll}19.3062 & -30.44915 & -2.33737\end{array}$ \\
\hline $\mathrm{H}$ & $\begin{array}{lll}21.82527 & -31.65909 & 1.742\end{array}$ \\
\hline $\mathrm{H}$ & $\begin{array}{lll}18.62212 & -29.68882 & 2.29418\end{array}$ \\
\hline $\mathrm{H}$ & $19.48216-30.409 \quad 4.43926$ \\
\hline $\mathrm{H}$ & $20.79147 \quad-31.44777 \quad 3.62523$ \\
\hline $\mathrm{H}$ & $\begin{array}{llll}22.47699 & -32.34387 & -2.95256\end{array}$ \\
\hline $\mathrm{H}$ & $21.80928-31.24588 \quad-5.01481$ \\
\hline $\mathrm{H}$ & $20.60664-30.16188-4.09558$ \\
\hline $\mathrm{H}$ & $24.02814-31.0523-1.09516$ \\
\hline $\mathrm{H}$ & $\begin{array}{lll}25.14736 & -35.58823 & 0.48744\end{array}$ \\
\hline $\mathrm{H}$ & $26.18517-30.40223-1.22316$ \\
\hline $\mathrm{H}$ & $28.44299-30.67233-1.92589$ \\
\hline $\mathrm{H}$ & $28.40733-32.50552-1.64283$ \\
\hline $\mathrm{H}$ & $22.96642 \quad-36.14961 \quad 0.86126$ \\
\hline $\mathrm{H}$ & $\begin{array}{lll}20.58551 & -36.0791 & 0.54135\end{array}$ \\
\hline $\mathrm{H}$ & $20.67576-34.3913-0.23812$ \\
\hline $\mathrm{H}$ & $\begin{array}{lll}22.76278 & -21.94813 & 2.06512\end{array}$ \\
\hline $\mathrm{H}$ & $21.89749-20.38396 \quad 2.2561$ \\
\hline $\mathrm{H}$ & $\begin{array}{lll}21.36225 & -21.81757 & 3.18783\end{array}$ \\
\hline $\mathrm{H}$ & $\begin{array}{lll}30.89459 & -24.75942 & -0.04796\end{array}$ \\
\hline $\mathrm{H}$ & $\begin{array}{lll}29.84193 & -25.32565 & 1.29332\end{array}$ \\
\hline $\mathrm{H}$ & $\begin{array}{lll}30.93841 & -26.48511 & 0.45842\end{array}$ \\
\hline $\mathrm{H}$ & $27.66275-23.64412 \quad 1.45502$ \\
\hline $\mathrm{H}$ & $\begin{array}{lll}27.54604 & -22.27785 & 0.29385\end{array}$ \\
\hline $\mathrm{H}$ & $\begin{array}{lll}26.06598 & -22.87988 & 1.11858\end{array}$ \\
\hline $\mathrm{H}$ & $\begin{array}{lll}34.98674 & -29.18032 & -0.93933\end{array}$ \\
\hline $\mathrm{H}$ & $\begin{array}{lll}33.36538 & -28.80513 & -0.25978\end{array}$ \\
\hline $\mathrm{H}$ & $\begin{array}{lll}34.45763 & -29.95702 & 0.58577\end{array}$ \\
\hline $\mathrm{H}$ & $\begin{array}{lll}15.90852 & -23.96797 & -1.13046\end{array}$ \\
\hline
\end{tabular}




$\begin{array}{llll}\mathrm{H} & 16.04285 & -25.74681 & -0.88862 \\ \mathrm{H} & 14.81337 & -24.79948 & 0.01885 \\ \mathrm{H} & 33.85783 & -36.6779 & -0.77136 \\ \mathrm{H} & 33.74437 & -35.2114 & -1.81179 \\ \mathrm{H} & 34.23787 & -35.05161 & -0.09736\end{array}$

Macrocycle 12, Relative Energy $=2.78 \mathrm{kcal} \mathrm{mol}^{-1}, R B 97 D / 6-31 G(d)$

\begin{tabular}{|c|c|}
\hline $\mathrm{C}$ & $24.59729-24.81995-0.04405$ \\
\hline $\mathrm{C}$ & $24.15108-23.62601-0.64851$ \\
\hline $\mathrm{C}$ & $22.86946-23.12895 \quad-0.39915$ \\
\hline $\mathrm{C}$ & $21.97756-23.81671 \quad 0.44821$ \\
\hline $\mathrm{C}$ & $23.68026-25.54872 \quad 0.73362$ \\
\hline $\mathrm{C}$ & $22.3838 \quad-25.05654 \quad 0.97304$ \\
\hline $\mathrm{C}$ & $20.63967 \quad-23.13036 \quad 0.81461$ \\
\hline $\mathrm{C}$ & $\begin{array}{lll}18.10966 & -24.72831 & 0.46672\end{array}$ \\
\hline $\mathrm{C}$ & $\begin{array}{lll}19.92311 & -23.81831 & 1.95955\end{array}$ \\
\hline $\mathrm{C}$ & $18.78125-24.50173 \quad 1.809$ \\
\hline $\mathrm{C}$ & $\begin{array}{llll}19.75401 & -23.04983 & -0.4161\end{array}$ \\
\hline $\mathrm{C}$ & $\begin{array}{lll}18.61701 & -23.74199 & -0.56746\end{array}$ \\
\hline $\mathrm{C}$ & $\begin{array}{lll}31.26558 & -30.27337 & -0.67754\end{array}$ \\
\hline $\mathrm{C}$ & $\begin{array}{llll}31.13867 & -29.3109 & -1.70269\end{array}$ \\
\hline $\mathrm{C}$ & $\begin{array}{llll}30.17014 & -28.30934 & -1.62621\end{array}$ \\
\hline $\mathrm{C}$ & $29.2828-28.23059-0.52863$ \\
\hline $\mathrm{C}$ & $\begin{array}{lll}30.34832 & -30.23301 & 0.38222\end{array}$ \\
\hline $\mathrm{C}$ & $\begin{array}{lll}29.36389 & -29.2304 & 0.45292\end{array}$ \\
\hline $\mathrm{C}$ & $28.40819-26.96807-0.43061$ \\
\hline $\mathrm{C}$ & $26.0732-25.23299-0.21021$ \\
\hline $\mathrm{C}$ & $27.3899-26.86788-1.56724$ \\
\hline $\mathrm{C}$ & $26.31541-26.07069-1.46766$ \\
\hline $\mathrm{C}$ & $27.69587 \quad-26.7799 \quad 0.90606$ \\
\hline $\mathrm{C}$ & $\begin{array}{lll}26.62063 & -25.98311 & 1.00653\end{array}$ \\
\hline $\mathrm{C}$ & $\begin{array}{lll}32.40944 & -31.30822 & -0.78179\end{array}$ \\
\hline $\mathrm{C}$ & $\begin{array}{lll}31.67349 & -34.2101 & -0.36328\end{array}$ \\
\hline $\mathrm{C}$ & $32.06953-32.25629-1.9153$ \\
\hline $\mathrm{C}$ & $\begin{array}{llll}31.75156 & -33.5441 & -1.73281\end{array}$ \\
\hline $\mathrm{C}$ & $\begin{array}{lll}32.67083 & -32.06641 & 0.50913\end{array}$ \\
\hline $\mathrm{C}$ & $\begin{array}{lll}32.37969 & -33.36447 & 0.68201\end{array}$ \\
\hline $\mathrm{C}$ & $\begin{array}{lll}27.27312 & -34.76817 & 0.70565\end{array}$ \\
\hline $\mathrm{C}$ & $27.77537 \quad-35.45453 \quad-0.43387$ \\
\hline $\mathrm{C}$ & $\begin{array}{lll}29.04009 & -35.07889 & -0.92659\end{array}$ \\
\hline $\mathrm{C}$ & $29.80356-34.07299-0.32825$ \\
\hline $\mathrm{C}$ & $\begin{array}{lll}27.96205 & -33.64333 & 1.19679\end{array}$ \\
\hline $\mathrm{C}$ & $29.21676-33.29233 \quad 0.69012$ \\
\hline $\mathrm{C}$ & $\begin{array}{lll}18.86549 & -28.81588 & -0.8946\end{array}$ \\
\hline $\mathrm{C}$ & $19.61336-27.73571-1.44208$ \\
\hline
\end{tabular}




\begin{tabular}{|c|c|}
\hline$c$ & $\begin{array}{lll}19.39021 & -26.43768 & -0.93047\end{array}$ \\
\hline $\mathrm{C}$ & $\begin{array}{lll}18.45186 & -26.18677 & 0.08036\end{array}$ \\
\hline $\mathrm{C}$ & $\begin{array}{lll}17.96283 & -28.55906 & 0.15947\end{array}$ \\
\hline $\mathrm{C}$ & $\begin{array}{lll}17.74877 & -27.26914 & 0.64064\end{array}$ \\
\hline $\mathrm{C}$ & $\begin{array}{lll}20.60031 & -27.98033 & -2.51041\end{array}$ \\
\hline $\mathrm{C}$ & $\begin{array}{lll}21.12209 & -27.05791 & -3.34391\end{array}$ \\
\hline $\mathrm{C}$ & $27.01864-36.56909-1.03089$ \\
\hline $\mathrm{C}$ & $27.403-37.36122-2.05313$ \\
\hline $\mathrm{C}$ & $19.91595-30.2051-0.40636$ \\
\hline $\mathrm{C}$ & $\begin{array}{llll}21.89894 & -32.21982 & -0.35891\end{array}$ \\
\hline $\mathrm{C}$ & $\begin{array}{lll}20.30838 & -30.80765 & 0.79944\end{array}$ \\
\hline $\mathrm{C}$ & $21.28219 \quad-31.82355 \quad 0.86484$ \\
\hline $\mathrm{C}$ & $\begin{array}{llll}20.3996 & -30.73452 & -1.63927\end{array}$ \\
\hline $\mathrm{C}$ & $\begin{array}{lll}21.39543 & -31.7292 & -1.57198\end{array}$ \\
\hline $\mathrm{C}$ & $\begin{array}{lll}19.89894 & -30.22034 & -2.92487\end{array}$ \\
\hline $\mathrm{C}$ & $20.42431-30.44352-4.14746$ \\
\hline $\mathrm{C}$ & $\begin{array}{llll}21.72349 & -32.36959 & 2.15789\end{array}$ \\
\hline $\mathrm{C}$ & $21.03286-32.30624 \quad 3.31429$ \\
\hline $\mathrm{C}$ & $25.79076-34.6502-0.41162$ \\
\hline $\mathrm{C}$ & $24.54983 \quad-35.19898 \quad 0.02835$ \\
\hline $\mathrm{C}$ & $23.46385 \quad-34.30734 \quad 0.1294$ \\
\hline $\mathrm{C}$ & $23.54614-32.93139-0.13803$ \\
\hline $\mathrm{C}$ & $\begin{array}{llll}24.80693 & -32.37215 & -0.48478\end{array}$ \\
\hline $\mathrm{C}$ & $\begin{array}{llll}25.89366 & -33.27066 & -0.62726\end{array}$ \\
\hline $\mathrm{C}$ & $24.27288-36.61243 \quad 0.35146$ \\
\hline $\mathrm{C}$ & $\begin{array}{llll}25.11768 & -37.60709 & 0.69461\end{array}$ \\
\hline $\mathrm{C}$ & $25.07028-30.94514 \quad-0.72067$ \\
\hline $\mathrm{C}$ & $\begin{array}{lll}24.27544 & -29.8881 & -0.45459\end{array}$ \\
\hline $\mathrm{O}$ & $20.88425-21.72676 \quad 1.13525$ \\
\hline $\mathrm{C}$ & $\begin{array}{lll}21.77576 & -21.47599 & 2.22091\end{array}$ \\
\hline $\mathrm{O}$ & $29.291-25.81499-0.67673$ \\
\hline $\mathrm{C}$ & $30.28856-25.6$ \\
\hline $\mathrm{O}$ & $\begin{array}{lll}26.87327 & -24.03175 & -0.45309\end{array}$ \\
\hline $\mathrm{C}$ & $\begin{array}{lll}27.03617 & -23.17417 & 0.67514\end{array}$ \\
\hline $\mathrm{O}$ & $33.63713-30.65815-1.22836$ \\
\hline $\mathrm{C}$ & $\begin{array}{lll}34.12164 & -29.59745 & -0.40454\end{array}$ \\
\hline $\mathrm{O}$ & $\begin{array}{lll}16.69585 & -24.56816 & 0.72997\end{array}$ \\
\hline $\mathrm{C}$ & $\begin{array}{lll}15.83438 & -24.78392 & -0.38812\end{array}$ \\
\hline $\mathrm{O}$ & $32.21348-35.55016-0.4009$ \\
\hline $\mathrm{C}$ & $33.5876-35.61295-0.79372$ \\
\hline $\mathrm{H}$ & $24.84772-23.05903-1.26566$ \\
\hline $\mathrm{H}$ & $22.57014-22.16401-0.81124$ \\
\hline $\mathrm{H}$ & $\begin{array}{lll}23.98569 & -26.49503 & 1.18612\end{array}$ \\
\hline $\mathrm{H}$ & $\begin{array}{lll}21.6953 & -25.6287 & 1.59642\end{array}$ \\
\hline $\mathrm{H}$ & $\begin{array}{lll}20.39257 & -23.75003 & 2.94221\end{array}$ \\
\hline $\mathrm{H}$ & $\begin{array}{lll}18.29149 & -24.99371 & 2.65184\end{array}$ \\
\hline
\end{tabular}




\begin{tabular}{|c|c|}
\hline $\mathrm{H}$ & $20.11097-22.37121-1.19335$ \\
\hline $\mathrm{H}$ & $18.04766-23.6626-1.49499$ \\
\hline $\mathrm{H}$ & $\begin{array}{llll}31.84764 & -29.32611 & -2.53177\end{array}$ \\
\hline $\mathrm{H}$ & $\begin{array}{llll}30.13397 & -27.52848 & -2.38686\end{array}$ \\
\hline $\mathrm{H}$ & $\begin{array}{lll}30.40021 & -30.97859 & 1.17234\end{array}$ \\
\hline $\mathrm{H}$ & $\begin{array}{lll}28.66847 & -29.24499 & 1.29207\end{array}$ \\
\hline $\mathrm{H}$ & $27.60673-27.42172-2.4826$ \\
\hline $\mathrm{H}$ & $25.61669-25.94371-2.29667$ \\
\hline $\mathrm{H}$ & $\begin{array}{lll}28.12884 & -27.23752 & 1.79689\end{array}$ \\
\hline $\mathrm{H}$ & $\begin{array}{lll}26.15333 & -25.78858 & 1.97422\end{array}$ \\
\hline $\mathrm{H}$ & $\begin{array}{llll}32.06485 & -31.80372 & -2.90857\end{array}$ \\
\hline $\mathrm{H}$ & $\begin{array}{llll}31.46518 & -34.17875 & -2.57391\end{array}$ \\
\hline $\mathrm{H}$ & $\begin{array}{llll}33.13564 & -31.49937 & 1.31828\end{array}$ \\
\hline $\mathrm{H}$ & $\begin{array}{lll}32.60205 & -33.87204 & 1.62424\end{array}$ \\
\hline $\mathrm{H}$ & $29.45857-35.62483-1.76856$ \\
\hline $\mathrm{H}$ & $27.54504-33.10202 \quad 2.04847$ \\
\hline $\mathrm{H}$ & $29.77457-32.45802 \quad 1.11616$ \\
\hline $\mathrm{H}$ & $19.96146-25.59809-1.327$ \\
\hline $\mathrm{H}$ & $\begin{array}{llll}17.38233 & -29.3882 & 0.5657\end{array}$ \\
\hline $\mathrm{H}$ & $\begin{array}{lll}17.0087 & -27.09696 & 1.42085\end{array}$ \\
\hline $\mathrm{H}$ & $20.912 \quad-29.02208-2.62616$ \\
\hline $\mathrm{H}$ & $21.86503-27.33737-4.09308$ \\
\hline $\mathrm{H}$ & $\begin{array}{lll}20.82452 & -26.00701 & -3.31074\end{array}$ \\
\hline $\mathrm{H}$ & $\begin{array}{llll}26.03848 & -36.75577 & -0.58661\end{array}$ \\
\hline $\mathrm{H}$ & $26.74258-38.14823-2.42155$ \\
\hline $\mathrm{H}$ & $28.36413-37.25446-2.56126$ \\
\hline $\mathrm{H}$ & $19.9341-30.38136 \quad 1.73026$ \\
\hline $\mathrm{H}$ & $21.83994-32.10116-2.49597$ \\
\hline $\mathrm{H}$ & $19.00689-29.59264-2.85401$ \\
\hline $\mathrm{H}$ & $\begin{array}{lll}19.95118 & -30.02979 & -5.03963\end{array}$ \\
\hline $\mathrm{H}$ & $21.33183-31.03207-4.30122$ \\
\hline $\mathrm{H}$ & $\begin{array}{lll}22.68532 & -32.88645 & 2.15173\end{array}$ \\
\hline $\mathrm{H}$ & $21.43756-32.73773 \quad 4.23146$ \\
\hline $\mathrm{H}$ & $20.04552-31.8421 \quad 3.37915$ \\
\hline $\mathrm{H}$ & $\begin{array}{llll}22.48731 & -34.71469 & 0.40089\end{array}$ \\
\hline $\mathrm{H}$ & $26.85924-32.87178-0.94889$ \\
\hline $\mathrm{H}$ & $\begin{array}{lll}23.20349 & -36.85381 & 0.34599\end{array}$ \\
\hline $\mathrm{H}$ & $24.71892-38.5978 \quad 0.9174$ \\
\hline $\mathrm{H}$ & $\begin{array}{lll}26.19761 & -37.48867 & 0.75849\end{array}$ \\
\hline $\mathrm{H}$ & $26.06092-30.7419-1.14305$ \\
\hline $\mathrm{H}$ & $24.62035-28.87594-0.67509$ \\
\hline $\mathrm{H}$ & $\begin{array}{lll}23.28371 & -29.99092 & -0.01585\end{array}$ \\
\hline $\mathrm{H}$ & $22.76278-21.94813 \quad 2.06512$ \\
\hline $\mathrm{H}$ & $21.89749-20.38396 \quad 2.2561$ \\
\hline $\mathrm{H}$ & $\begin{array}{lll}21.36225 & -21.81757 & 3.18783\end{array}$ \\
\hline $\mathrm{H}$ & $30.89459-24.75942-0.04796$ \\
\hline
\end{tabular}




$\begin{array}{llll}\mathrm{H} & 29.84193 & -25.32565 & 1.29332 \\ \mathrm{H} & 30.93841 & -26.48511 & 0.45842 \\ \mathrm{H} & 27.66275 & -23.64412 & 1.45502 \\ \mathrm{H} & 27.54604 & -22.27785 & 0.29385 \\ \mathrm{H} & 26.06598 & -22.87988 & 1.11858 \\ \mathrm{H} & 34.98674 & -29.18032 & -0.93933 \\ \mathrm{H} & 33.36538 & -28.80513 & -0.25978 \\ \mathrm{H} & 34.45763 & -29.95702 & 0.58577 \\ \mathrm{H} & 15.90852 & -23.96797 & -1.13046 \\ \mathrm{H} & 16.04285 & -25.74681 & -0.88862 \\ \mathrm{H} & 14.81337 & -24.79948 & 0.01885 \\ \mathrm{H} & 33.85783 & -36.6779 & -0.77136 \\ \mathrm{H} & 33.74437 & -35.2114 & -1.81179 \\ \mathrm{H} & 34.23787 & -35.05161 & -0.09736\end{array}$

Macrocycle 12, Relative Energy $=2.83 \mathrm{kcal} \mathrm{mol}^{-1}, R B 97 D / 6-31 G(d)$

$\begin{array}{llll}\mathrm{C} & 24.59729 & -24.81995 & -0.04405 \\ \mathrm{C} & 24.15108 & -23.62601 & -0.64851 \\ \mathrm{C} & 22.86946 & -23.12895 & -0.39915 \\ \mathrm{C} & 21.97756 & -23.81671 & 0.44821 \\ \mathrm{C} & 23.68026 & -25.54872 & 0.73362 \\ \mathrm{C} & 22.3838 & -25.05654 & 0.97304 \\ \mathrm{C} & 20.63967 & -23.13036 & 0.81461 \\ \mathrm{C} & 18.10966 & -24.72831 & 0.46672 \\ \mathrm{C} & 19.92311 & -23.81831 & 1.95955 \\ \mathrm{C} & 18.78125 & -24.50173 & 1.809 \\ \mathrm{C} & 19.75401 & -23.04983 & -0.4161 \\ \mathrm{C} & 18.61701 & -23.74199 & -0.56746 \\ \mathrm{C} & 31.26558 & -30.27337 & -0.67754 \\ \mathrm{C} & 31.13867 & -29.3109 & -1.70269 \\ \mathrm{C} & 30.17014 & -28.30934 & -1.62621 \\ \mathrm{C} & 29.2828 & -28.23059 & -0.52863 \\ \mathrm{C} & 30.34832 & -30.23301 & 0.38222 \\ \mathrm{C} & 29.36389 & -29.2304 & 0.45292 \\ \mathrm{C} & 28.40819 & -26.96807 & -0.43061 \\ \mathrm{C} & 26.0732 & -25.23299 & -0.21021 \\ \mathrm{C} & 27.3899 & -26.86788 & -1.56724 \\ \mathrm{C} & 26.31541 & -26.07069 & -1.46766 \\ \mathrm{C} & 27.69587 & -26.7799 & 0.90606 \\ \mathrm{C} & 26.62063 & -25.98311 & 1.00653 \\ \mathrm{C} & 32.40944 & -31.30822 & -0.78179 \\ \mathrm{C} & 31.67349 & -34.2101 & -0.36328 \\ \mathrm{C} & 32.06953 & -32.25629 & -1.9153 \\ \mathrm{C} & 31.75156 & -33.5441 & -1.73281 \\ \mathrm{C} & 32.67083 & -32.06641 & 0.50913\end{array}$




$\begin{array}{llll}\text { C } & 32.37969 & -33.36447 & 0.68201 \\ \text { C } & 27.44392 & -34.89492 & 0.79994 \\ \text { C } & 28.1397 & -35.7371 & -0.18366 \\ \text { C } & 29.40454 & -35.36033 & -0.67523 \\ \text { C } & 30.16917 & -34.35787 & -0.07261 \\ \text { C } & 28.32874 & -33.9336 & 1.45524 \\ \text { C } & 29.58352 & -33.58139 & 0.94961 \\ \text { C } & 19.25375 & -28.87406 & -0.33112 \\ \text { C } & 18.11074 & -28.59472 & 0.46969 \\ \text { C } & 17.7315 & -27.24567 & 0.6505 \\ \text { C } & 18.45186 & -26.18677 & 0.08036 \\ \text { C } & 19.93904 & -27.80313 & -0.94375 \\ \text { C } & 19.55555 & -26.47895 & -0.742 \\ \text { C } & 17.3446 & -29.69599 & 1.08245 \\ \text { C } & 16.47699 & -29.59237 & 2.10926 \\ \text { C } & 27.38171 & -36.84823 & -0.78546 \\ \text { C } & 27.76491 & -37.63599 & -1.81151 \\ \text { C } & 19.91595 & -30.2051 & -0.40636 \\ \text { C } & 21.89894 & -32.21982 & -0.35891 \\ \text { C } & 20.30838 & -30.80765 & 0.79944 \\ \text { C } & 21.28219 & -31.82355 & 0.86484 \\ \text { C } & 20.3996 & -30.73452 & -1.63927 \\ \text { C } & 21.39543 & -31.7292 & -1.57198 \\ \text { C } & 19.89894 & -30.22034 & -2.92487 \\ \text { C } & 20.42431 & -30.44352 & -4.14746 \\ \text { C } & 21.72349 & -32.36959 & 2.15789 \\ \text { C } & 21.03286 & -32.30624 & 3.31429 \\ \text { C } & 26.10087 & -34.7834 & -0.33205 \\ \text { C } & 24.61213 & -35.31162 & -0.10093 \\ \text { C } & 23.52616 & -34.41997 & 0.00012 \\ \text { C } & 23.60845 & -33.04402 & -0.26731 \\ \text { C } & 24.86924 & -32.48479 & -0.61406 \\ \text { C } & 25.95597 & -33.3833 & -0.75654 \\ \text { C } & 24.33519 & -36.72507 & 0.22218 \\ \text { C } & 25.17999 & -37.71972 & 0.56533 \\ \text { C } & 25.13259 & -31.05778 & -0.84996 \\ \text { C } & 24.33775 & -30.00073 & -0.58387 \\ \text { O } & 20.88425 & -21.72676 & 1.13525 \\ \text { C } & 21.77576 & -21.47599 & 2.22091 \\ \text { O } & 29.291 & -25.81499 & -0.67673 \\ \text { C } & 30.28856 & -25.6 & 0.31913 \\ \text { O } & 26.87327 & -24.03175 & -0.45309 \\ \text { C } & 27.03617 & -23.17417 & 0.67514 \\ \text { O } & 33.63713 & -30.65815 & -1.22836 \\ \text { C } & 34.12164 & -29.59745 & -0.40454 \\ \text { O } & 16.69585 & -24.56816 & 0.72997\end{array}$




\begin{tabular}{|c|c|}
\hline $\mathrm{C}$ & $15.83438-24.78392-0.38812$ \\
\hline $\mathrm{O}$ & $32.21348-35.55016-0.4009$ \\
\hline $\mathrm{C}$ & $\begin{array}{lll}33.5876 & -35.61295 & -0.79372\end{array}$ \\
\hline $\mathrm{H}$ & $24.84772-23.05903-1.26566$ \\
\hline $\mathrm{H}$ & $22.57014-22.16401-0.81124$ \\
\hline $\mathrm{H}$ & $\begin{array}{lll}23.98569 & -26.49503 & 1.18612\end{array}$ \\
\hline $\mathrm{H}$ & $\begin{array}{lll}21.6953 & -25.6287 & 1.59642\end{array}$ \\
\hline $\mathrm{H}$ & $\begin{array}{lll}20.39257 & -23.75003 & 2.94221\end{array}$ \\
\hline $\mathrm{H}$ & $\begin{array}{lll}18.29149 & -24.99371 & 2.65184\end{array}$ \\
\hline $\mathrm{H}$ & $\begin{array}{lll}20.11097 & -22.37121 & -1.19335\end{array}$ \\
\hline $\mathrm{H}$ & $\begin{array}{llll}18.04766 & -23.6626 & -1.49499\end{array}$ \\
\hline $\mathrm{H}$ & $\begin{array}{lll}31.84764 & -29.32611 & -2.53177\end{array}$ \\
\hline $\mathrm{H}$ & $\begin{array}{lll}30.13397 & -27.52848 & -2.38686\end{array}$ \\
\hline $\mathrm{H}$ & $\begin{array}{lll}30.40021 & -30.97859 & 1.17234\end{array}$ \\
\hline $\mathrm{H}$ & $\begin{array}{lll}28.66847 & -29.24499 & 1.29207\end{array}$ \\
\hline $\mathrm{H}$ & $27.60673-27.42172-2.4826$ \\
\hline $\mathrm{H}$ & $\begin{array}{llll}25.61669 & -25.94371 & -2.29667\end{array}$ \\
\hline $\mathrm{H}$ & $\begin{array}{lll}28.12884 & -27.23752 & 1.79689\end{array}$ \\
\hline $\mathrm{H}$ & $26.15333 \quad-25.78858 \quad 1.97422$ \\
\hline $\mathrm{H}$ & $\begin{array}{lll}32.06485 & -31.80372 & -2.90857\end{array}$ \\
\hline $\mathrm{H}$ & $\begin{array}{lll}31.46518 & -34.17875 & -2.57391\end{array}$ \\
\hline $\mathrm{H}$ & $\begin{array}{llll}33.13564 & -31.49937 & 1.31828\end{array}$ \\
\hline $\mathrm{H}$ & $\begin{array}{lll}32.60205 & -33.87204 & 1.62424\end{array}$ \\
\hline $\mathrm{H}$ & $29.82215-35.90275-1.51989$ \\
\hline $\mathrm{H}$ & $\begin{array}{lll}27.91259 & -33.39585 & 2.30959\end{array}$ \\
\hline $\mathrm{H}$ & $\begin{array}{lll}30.14227 & -32.74954 & 1.37924\end{array}$ \\
\hline $\mathrm{H}$ & $\begin{array}{lll}16.85178 & -27.01032 & 1.24982\end{array}$ \\
\hline $\mathrm{H}$ & $\begin{array}{lll}20.82233 & -28.02197 & -1.54491\end{array}$ \\
\hline $\mathrm{H}$ & $20.13523-25.67221-1.18832$ \\
\hline $\mathrm{H}$ & $\begin{array}{lll}17.52709 & -30.68493 & 0.65294\end{array}$ \\
\hline $\mathrm{H}$ & $\begin{array}{lll}15.94188 & -30.4684 & 2.48013\end{array}$ \\
\hline $\mathrm{H}$ & $\begin{array}{lll}16.27863 & -28.64251 & 2.61171\end{array}$ \\
\hline $\mathrm{H}$ & $26.40159-37.03608-0.34161$ \\
\hline $\mathrm{H}$ & $\begin{array}{lll}27.10362 & -38.4207 & -2.18325\end{array}$ \\
\hline $\mathrm{H}$ & $28.7259-37.52774-2.31958$ \\
\hline $\mathrm{H}$ & $\begin{array}{lll}19.9341 & -30.38136 & 1.73026\end{array}$ \\
\hline $\mathrm{H}$ & $\begin{array}{llll}21.83994 & -32.10116 & -2.49597\end{array}$ \\
\hline $\mathrm{H}$ & $\begin{array}{lll}19.00689 & -29.59264 & -2.85401\end{array}$ \\
\hline $\mathrm{H}$ & $\begin{array}{lll}19.95118 & -30.02979 & -5.03963\end{array}$ \\
\hline $\mathrm{H}$ & $21.33183-31.03207-4.30122$ \\
\hline $\mathrm{H}$ & $\begin{array}{lll}22.68532 & -32.88645 & 2.15173\end{array}$ \\
\hline $\mathrm{H}$ & $21.43756-32.73773 \quad 4.23146$ \\
\hline $\mathrm{H}$ & $\begin{array}{lll}20.04552 & -31.8421 & 3.37915\end{array}$ \\
\hline $\mathrm{H}$ & $\begin{array}{llll}22.54962 & -34.82732 & 0.27161\end{array}$ \\
\hline $\mathrm{H}$ & $\begin{array}{lll}26.92155 & -32.98441 & -1.07817\end{array}$ \\
\hline $\mathrm{H}$ & $23.2658 \quad-36.96644 \quad 0.21671$ \\
\hline
\end{tabular}




$\begin{array}{llll}\mathrm{H} & 24.78123 & -38.71044 & 0.78812 \\ \mathrm{H} & 26.25992 & -37.6013 & 0.62921 \\ \mathrm{H} & 26.12323 & -30.85454 & -1.27233 \\ \mathrm{H} & 24.68266 & -28.98857 & -0.80437 \\ \mathrm{H} & 23.34602 & -30.10356 & -0.14513 \\ \mathrm{H} & 22.76278 & -21.94813 & 2.06512 \\ \mathrm{H} & 21.89749 & -20.38396 & 2.2561 \\ \mathrm{H} & 21.36225 & -21.81757 & 3.18783 \\ \mathrm{H} & 30.89459 & -24.75942 & -0.04796 \\ \mathrm{H} & 29.84193 & -25.32565 & 1.29332 \\ \mathrm{H} & 30.93841 & -26.48511 & 0.45842 \\ \mathrm{H} & 27.66275 & -23.64412 & 1.45502 \\ \mathrm{H} & 27.54604 & -22.27785 & 0.29385 \\ \mathrm{H} & 26.06598 & -22.87988 & 1.11858 \\ \mathrm{H} & 34.98674 & -29.18032 & -0.93933 \\ \mathrm{H} & 33.36538 & -28.80513 & -0.25978 \\ \mathrm{H} & 34.45763 & -29.95702 & 0.58577 \\ \mathrm{H} & 15.90852 & -23.96797 & -1.13046 \\ \mathrm{H} & 16.04285 & -25.74681 & -0.88862 \\ \mathrm{H} & 14.81337 & -24.79948 & 0.01885 \\ \mathrm{H} & 33.85783 & -36.6779 & -0.77136 \\ \mathrm{H} & 33.74437 & -35.2114 & -1.81179 \\ \mathrm{H} & 34.23787 & -35.05161 & -0.09736\end{array}$

Macrocycle 12, Relative Energy $=2.87 \mathrm{kcal} \mathrm{mol}^{-1}, R B 97 \mathrm{D} / 6-31 G(d)$

$\begin{array}{llll}\text { C } & 24.59729 & -24.81995 & -0.04405 \\ \mathrm{C} & 24.15108 & -23.62601 & -0.64851 \\ \mathrm{C} & 22.86946 & -23.12895 & -0.39915 \\ \mathrm{C} & 21.97756 & -23.81671 & 0.44821 \\ \mathrm{C} & 23.68026 & -25.54872 & 0.73362 \\ \mathrm{C} & 22.3838 & -25.05654 & 0.97304 \\ \mathrm{C} & 20.63967 & -23.13036 & 0.81461 \\ \mathrm{C} & 18.10966 & -24.72831 & 0.46672 \\ \mathrm{C} & 19.92311 & -23.81831 & 1.95955 \\ \mathrm{C} & 18.78125 & -24.50173 & 1.809 \\ \mathrm{C} & 19.75401 & -23.04983 & -0.4161 \\ \mathrm{C} & 18.61701 & -23.74199 & -0.56746 \\ \mathrm{C} & 31.26558 & -30.27337 & -0.67754 \\ \mathrm{C} & 31.13867 & -29.3109 & -1.70269 \\ \mathrm{C} & 30.17014 & -28.30934 & -1.62621 \\ \mathrm{C} & 29.2828 & -28.23059 & -0.52863 \\ \mathrm{C} & 30.34832 & -30.23301 & 0.38222 \\ \mathrm{C} & 29.36389 & -29.2304 & 0.45292 \\ \mathrm{C} & 28.40819 & -26.96807 & -0.43061 \\ \mathrm{C} & 26.0732 & -25.23299 & -0.21021\end{array}$




\begin{tabular}{|c|c|}
\hline $\mathrm{C}$ & $27.3899-26.86788-1.56724$ \\
\hline $\mathrm{C}$ & $26.31541-26.07069-1.46766$ \\
\hline $\mathrm{C}$ & $\begin{array}{lll}27.69587 & -26.7799 & 0.90606\end{array}$ \\
\hline $\mathrm{C}$ & $\begin{array}{lll}26.62063 & -25.98311 & 1.00653\end{array}$ \\
\hline $\mathrm{C}$ & $\begin{array}{lll}32.40944 & -31.30822 & -0.78179\end{array}$ \\
\hline $\mathrm{C}$ & $\begin{array}{llll}31.67349 & -34.2101 & -0.36328\end{array}$ \\
\hline $\mathrm{C}$ & $32.06953-32.25629-1.9153$ \\
\hline $\mathrm{C}$ & $31.75156-33.5441-1.73281$ \\
\hline $\mathrm{C}$ & $\begin{array}{lll}32.67083 & -32.06641 & 0.50913\end{array}$ \\
\hline $\mathrm{C}$ & $\begin{array}{lll}32.37969 & -33.36447 & 0.68201\end{array}$ \\
\hline $\mathrm{C}$ & $\begin{array}{lll}27.44392 & -34.89492 & 0.79994\end{array}$ \\
\hline $\mathrm{C}$ & $28.1397-35.7371-0.18366$ \\
\hline $\mathrm{C}$ & $29.40454-35.36033-0.67523$ \\
\hline $\mathrm{C}$ & $\begin{array}{lll}30.16917 & -34.35787 & -0.07261\end{array}$ \\
\hline $\mathrm{C}$ & $28.32874 \quad-33.9336 \quad 1.45524$ \\
\hline $\mathrm{C}$ & $29.58352-33.58139 \quad 0.94961$ \\
\hline $\mathrm{C}$ & $19.25375-28.87406-0.33112$ \\
\hline $\mathrm{C}$ & $\begin{array}{lll}18.11074 & -28.59472 & 0.46969\end{array}$ \\
\hline $\mathrm{C}$ & $\begin{array}{lll}17.7315 & -27.24567 & 0.6505\end{array}$ \\
\hline $\mathrm{C}$ & $\begin{array}{lll}18.45186 & -26.18677 & 0.08036\end{array}$ \\
\hline $\mathrm{C}$ & $19.93904-27.80313-0.94375$ \\
\hline $\mathrm{C}$ & $\begin{array}{lll}19.55555 & -26.47895 & -0.742\end{array}$ \\
\hline $\mathrm{C}$ & $\begin{array}{lll}17.3446 & -29.69599 & 1.08245\end{array}$ \\
\hline $\mathrm{C}$ & $\begin{array}{lll}16.47699 & -29.59237 & 2.10926\end{array}$ \\
\hline $\mathrm{C}$ & $27.38171-36.84823-0.78546$ \\
\hline $\mathrm{C}$ & $27.76491-37.63599-1.81151$ \\
\hline $\mathrm{C}$ & $19.91595-30.2051-0.40636$ \\
\hline $\mathrm{C}$ & $\begin{array}{llll}21.89894 & -32.21982 & -0.35891\end{array}$ \\
\hline $\mathrm{C}$ & $\begin{array}{lll}20.30838 & -30.80765 & 0.79944\end{array}$ \\
\hline $\mathrm{C}$ & $\begin{array}{lll}21.28219 & -31.82355 & 0.86484\end{array}$ \\
\hline $\mathrm{C}$ & $20.3996-30.73452-1.63927$ \\
\hline $\mathrm{C}$ & $21.39543-31.7292-1.57198$ \\
\hline $\mathrm{C}$ & $19.89894-30.22034-2.92487$ \\
\hline $\mathrm{C}$ & $20.42431-30.44352-4.14746$ \\
\hline $\mathrm{C}$ & $\begin{array}{lll}21.72349 & -32.36959 & 2.15789\end{array}$ \\
\hline $\mathrm{C}$ & $\begin{array}{lll}21.03286 & -32.30624 & 3.31429\end{array}$ \\
\hline $\mathrm{C}$ & $26.18512-33.85616-0.23025$ \\
\hline $\mathrm{C}$ & $25.88464-32.55763 \quad-0.7384$ \\
\hline $\mathrm{C}$ & $24.52476-32.19167-0.7757$ \\
\hline $\mathrm{C}$ & $23.4711-33.03261-0.38308$ \\
\hline $\mathrm{C}$ & $\begin{array}{lll}23.76853 & -34.35946 & 0.03352\end{array}$ \\
\hline $\mathrm{C}$ & $25.13711-34.71936 \quad 0.11146$ \\
\hline $\mathrm{C}$ & $26.85254-31.54008-1.19304$ \\
\hline $\mathrm{C}$ & $28.12785-31.67936-1.61133$ \\
\hline $\mathrm{C}$ & $22.7761 \quad-35.37947 \quad 0.40232$ \\
\hline $\mathrm{C}$ & $\begin{array}{lll}21.43957 & -35.3569 & 0.22046\end{array}$ \\
\hline
\end{tabular}




\begin{tabular}{|c|c|}
\hline $\mathrm{O}$ & $20.88425-21.72676 \quad 1.13525$ \\
\hline $\mathrm{C}$ & $\begin{array}{lll}21.77576 & -21.47599 & 2.22091\end{array}$ \\
\hline $\mathrm{O}$ & $\begin{array}{lll}29.291 & -25.81499 & -0.67673\end{array}$ \\
\hline $\mathrm{C}$ & $30.28856-25.6$ \\
\hline $\mathrm{O}$ & $\begin{array}{lll}26.87327 & -24.03175 & -0.45309\end{array}$ \\
\hline $\mathrm{C}$ & $\begin{array}{lll}27.03617 & -23.17417 & 0.67514\end{array}$ \\
\hline $\mathrm{O}$ & $\begin{array}{lll}33.63713 & -30.65815 & -1.22836\end{array}$ \\
\hline $\mathrm{C}$ & $\begin{array}{lll}34.12164 & -29.59745 & -0.40454\end{array}$ \\
\hline $\mathrm{O}$ & $\begin{array}{lll}16.69585 & -24.56816 & 0.72997\end{array}$ \\
\hline $\mathrm{C}$ & $15.83438-24.78392-0.38812$ \\
\hline $\mathrm{O}$ & $32.21348-35.55016-0.4009$ \\
\hline $\mathrm{C}$ & $\begin{array}{lll}33.5876 & -35.61295 & -0.79372\end{array}$ \\
\hline $\mathrm{H}$ & $24.84772-23.05903-1.26566$ \\
\hline $\mathrm{H}$ & $22.57014-22.16401-0.81124$ \\
\hline $\mathrm{H}$ & $\begin{array}{lll}23.98569 & -26.49503 & 1.18612\end{array}$ \\
\hline $\mathrm{H}$ & $\begin{array}{lll}21.6953 & -25.6287 & 1.59642\end{array}$ \\
\hline $\mathrm{H}$ & $\begin{array}{lll}20.39257 & -23.75003 & 2.94221\end{array}$ \\
\hline $\mathrm{H}$ & $\begin{array}{lll}18.29149 & -24.99371 & 2.65184\end{array}$ \\
\hline $\mathrm{H}$ & $\begin{array}{lll}20.11097 & -22.37121 & -1.19335\end{array}$ \\
\hline $\mathrm{H}$ & $18.04766-23.6626-1.49499$ \\
\hline $\mathrm{H}$ & $\begin{array}{llll}31.84764 & -29.32611 & -2.53177\end{array}$ \\
\hline $\mathrm{H}$ & $\begin{array}{lll}30.13397 & -27.52848 & -2.38686\end{array}$ \\
\hline $\mathrm{H}$ & $\begin{array}{lll}30.40021 & -30.97859 & 1.17234\end{array}$ \\
\hline $\mathrm{H}$ & $\begin{array}{lll}28.66847 & -29.24499 & 1.29207\end{array}$ \\
\hline $\mathrm{H}$ & $27.60673-27.42172-2.4826$ \\
\hline $\mathrm{H}$ & $\begin{array}{lll}25.61669 & -25.94371 & -2.29667\end{array}$ \\
\hline $\mathrm{H}$ & $\begin{array}{lll}28.12884 & -27.23752 & 1.79689\end{array}$ \\
\hline $\mathrm{H}$ & $\begin{array}{lll}26.15333 & -25.78858 & 1.97422\end{array}$ \\
\hline $\mathrm{H}$ & $\begin{array}{lll}32.06485 & -31.80372 & -2.90857\end{array}$ \\
\hline $\mathrm{H}$ & $\begin{array}{llll}31.46518 & -34.17875 & -2.57391\end{array}$ \\
\hline $\mathrm{H}$ & $\begin{array}{lll}33.13564 & -31.49937 & 1.31828\end{array}$ \\
\hline $\mathrm{H}$ & $\begin{array}{lll}32.60205 & -33.87204 & 1.62424\end{array}$ \\
\hline $\mathrm{H}$ & $29.82215-35.90275-1.51989$ \\
\hline $\mathrm{H}$ & $\begin{array}{lll}27.91259 & -33.39585 & 2.30959\end{array}$ \\
\hline $\mathrm{H}$ & $\begin{array}{lll}30.14227 & -32.74954 & 1.37924\end{array}$ \\
\hline $\mathrm{H}$ & $\begin{array}{lll}16.85178 & -27.01032 & 1.24982\end{array}$ \\
\hline $\mathrm{H}$ & $\begin{array}{llll}20.82233 & -28.02197 & -1.54491\end{array}$ \\
\hline $\mathrm{H}$ & $\begin{array}{lll}20.13523 & -25.67221 & -1.18832\end{array}$ \\
\hline $\mathrm{H}$ & $\begin{array}{lll}17.52709 & -30.68493 & 0.65294\end{array}$ \\
\hline $\mathrm{H}$ & $\begin{array}{lll}15.94188 & -30.4684 & 2.48013\end{array}$ \\
\hline $\mathrm{H}$ & $\begin{array}{lll}16.27863 & -28.64251 & 2.61171\end{array}$ \\
\hline $\mathrm{H}$ & $26.40159-37.03608-0.34161$ \\
\hline $\mathrm{H}$ & $27.10362-38.4207 \quad-2.18325$ \\
\hline $\mathrm{H}$ & $28.7259-37.52774-2.31958$ \\
\hline $\mathrm{H}$ & $\begin{array}{lll}19.9341 & -30.38136 & 1.73026\end{array}$ \\
\hline $\mathrm{H}$ & $21.83994-32.10116-2.49597$ \\
\hline
\end{tabular}




$\begin{array}{llll}\mathrm{H} & 19.00689 & -29.59264 & -2.85401 \\ \mathrm{H} & 19.95118 & -30.02979 & -5.03963 \\ \mathrm{H} & 21.33183 & -31.03207 & -4.30122 \\ \mathrm{H} & 22.68532 & -32.88645 & 2.15173 \\ \mathrm{H} & 21.43756 & -32.73773 & 4.23146 \\ \mathrm{H} & 20.04552 & -31.8421 & 3.37915 \\ \mathrm{H} & 24.27322 & -31.179 & -1.09893 \\ \mathrm{H} & 25.39244 & -35.71493 & 0.48367 \\ \mathrm{H} & 26.43025 & -30.52894 & -1.22693 \\ \mathrm{H} & 28.68807 & -30.79904 & -1.92966 \\ \mathrm{H} & 28.65241 & -32.63222 & -1.64659 \\ \mathrm{H} & 23.2115 & -36.27631 & 0.85749 \\ \mathrm{H} & 20.83059 & -36.2058 & 0.53758 \\ \mathrm{H} & 20.92084 & -34.518 & -0.24189 \\ \mathrm{H} & 22.76278 & -21.94813 & 2.06512 \\ \mathrm{H} & 21.89749 & -20.38396 & 2.2561 \\ \mathrm{H} & 21.36225 & -21.81757 & 3.18783 \\ \mathrm{H} & 30.89459 & -24.75942 & -0.04796 \\ \mathrm{H} & 29.84193 & -25.32565 & 1.29332 \\ \mathrm{H} & 30.93841 & -26.48511 & 0.45842 \\ \mathrm{H} & 27.66275 & -23.64412 & 1.45502 \\ \mathrm{H} & 27.54604 & -22.27785 & 0.29385 \\ \mathrm{H} & 26.06598 & -22.87988 & 1.11858 \\ \mathrm{H} & 34.98674 & -29.18032 & -0.93933 \\ \mathrm{H} & 33.36538 & -28.80513 & -0.25978 \\ \mathrm{H} & 34.45763 & -29.95702 & 0.58577 \\ \mathrm{H} & 15.90852 & -23.96797 & -1.13046 \\ \mathrm{H} & 16.04285 & -25.74681 & -0.88862 \\ \mathrm{H} & 14.81337 & -24.79948 & 0.01885 \\ \mathrm{H} & 33.85783 & -36.6779 & -0.77136 \\ \mathrm{H} & 33.74437 & -35.2114 & -1.81179 \\ \mathrm{H} & 34.23787 & -35.05161 & -0.09736 \\ & & & \\ & & & \\ & 2 & & \\ \mathrm{H} & 2536\end{array}$

Macrocycle 12, Relative Energy $=3.36 \mathrm{kcal} \mathrm{mol}^{-1}, R B 97 \mathrm{D} / 6-31 G(d)$

$\begin{array}{llll}\mathrm{C} & 24.59729 & -24.81995 & -0.04405 \\ \mathrm{C} & 24.15108 & -23.62601 & -0.64851 \\ \mathrm{C} & 22.86946 & -23.12895 & -0.39915 \\ \mathrm{C} & 21.97756 & -23.81671 & 0.44821 \\ \mathrm{C} & 23.68026 & -25.54872 & 0.73362 \\ \mathrm{C} & 22.3838 & -25.05654 & 0.97304 \\ \mathrm{C} & 20.63967 & -23.13036 & 0.81461 \\ \mathrm{C} & 18.10966 & -24.72831 & 0.46672 \\ \mathrm{C} & 19.92311 & -23.81831 & 1.95955 \\ \mathrm{C} & 18.78125 & -24.50173 & 1.809 \\ \mathrm{C} & 19.75401 & -23.04983 & -0.4161 \\ \mathrm{C} & 18.61701 & -23.74199 & -0.56746\end{array}$




\begin{tabular}{|c|c|}
\hline C & $\begin{array}{lll}31.26558 & -30.27337 & -0.67754\end{array}$ \\
\hline $\mathrm{C}$ & $\begin{array}{llll}31.13867 & -29.3109 & -1.70269\end{array}$ \\
\hline $\mathrm{C}$ & $\begin{array}{llll}30.17014 & -28.30934 & -1.62621\end{array}$ \\
\hline $\mathrm{C}$ & $29.2828-28.23059-0.52863$ \\
\hline $\mathrm{C}$ & $\begin{array}{lll}30.34832 & -30.23301 & 0.38222\end{array}$ \\
\hline $\mathrm{C}$ & $29.36389 \quad-29.2304 \quad 0.45292$ \\
\hline $\mathrm{C}$ & $\begin{array}{llll}28.40819 & -26.96807 & -0.43061\end{array}$ \\
\hline $\mathrm{C}$ & $26.0732-25.23299-0.21021$ \\
\hline $\mathrm{C}$ & $27.3899-26.86788-1.56724$ \\
\hline $\mathrm{C}$ & $\begin{array}{llll}26.31541 & -26.07069 & -1.46766\end{array}$ \\
\hline $\mathrm{C}$ & $\begin{array}{lll}27.69587 & -26.7799 & 0.90606\end{array}$ \\
\hline $\mathrm{C}$ & $\begin{array}{lll}26.62063 & -25.98311 & 1.00653\end{array}$ \\
\hline $\mathrm{C}$ & $\begin{array}{llll}32.40944 & -31.30822 & -0.78179\end{array}$ \\
\hline $\mathrm{C}$ & $\begin{array}{llll}31.67349 & -34.2101 & -0.36328\end{array}$ \\
\hline $\mathrm{C}$ & $\begin{array}{llll}32.06953 & -32.25629 & -1.9153\end{array}$ \\
\hline $\mathrm{C}$ & $\begin{array}{llll}31.75156 & -33.5441 & -1.73281\end{array}$ \\
\hline $\mathrm{C}$ & $\begin{array}{lll}32.67083 & -32.06641 & 0.50913\end{array}$ \\
\hline $\mathrm{C}$ & $\begin{array}{llll}32.37969 & -33.36447 & 0.68201\end{array}$ \\
\hline $\mathrm{C}$ & $\begin{array}{llll}27.44392 & -34.89492 & 0.79994\end{array}$ \\
\hline $\mathrm{C}$ & $28.1397-35.7371-0.18366$ \\
\hline $\mathrm{C}$ & $29.40454-35.36033-0.67523$ \\
\hline $\mathrm{C}$ & $\begin{array}{llll}30.16917 & -34.35787 & -0.07261\end{array}$ \\
\hline $\mathrm{C}$ & $28.32874 \quad-33.9336 \quad 1.45524$ \\
\hline $\mathrm{C}$ & $\begin{array}{llll}29.58352 & -33.58139 & 0.94961\end{array}$ \\
\hline $\mathrm{C}$ & $\begin{array}{lll}19.25375 & -28.87406 & -0.33112\end{array}$ \\
\hline $\mathrm{C}$ & $\begin{array}{lll}18.11074 & -28.59472 & 0.46969\end{array}$ \\
\hline $\mathrm{C}$ & $\begin{array}{lll}17.7315 & -27.24567 & 0.6505\end{array}$ \\
\hline $\mathrm{C}$ & $\begin{array}{lll}18.45186 & -26.18677 & 0.08036\end{array}$ \\
\hline $\mathrm{C}$ & $\begin{array}{lll}19.93904 & -27.80313 & -0.94375\end{array}$ \\
\hline $\mathrm{C}$ & $\begin{array}{lll}19.55555 & -26.47895 & -0.742\end{array}$ \\
\hline $\mathrm{C}$ & $\begin{array}{lll}17.3446 & -29.69599 & 1.08245\end{array}$ \\
\hline $\mathrm{C}$ & $\begin{array}{lll}16.47699 & -29.59237 & 2.10926\end{array}$ \\
\hline $\mathrm{C}$ & $\begin{array}{llll}27.38171 & -36.84823 & -0.78546\end{array}$ \\
\hline $\mathrm{C}$ & $\begin{array}{llll}27.76491 & -37.63599 & -1.81151\end{array}$ \\
\hline $\mathrm{C}$ & $\begin{array}{lll}20.45483 & -30.32812 & -0.21987\end{array}$ \\
\hline $\mathrm{C}$ & $21.5336-32.91778-0.57127$ \\
\hline $\mathrm{C}$ & $20.40418-30.97139-1.46673$ \\
\hline $\mathrm{C}$ & $\begin{array}{llll}20.94146 & -32.25492 & -1.68704\end{array}$ \\
\hline $\mathrm{C}$ & $\begin{array}{llll}21.20996 & -30.91665 & 0.83732\end{array}$ \\
\hline $\mathrm{C}$ & $\begin{array}{lll}21.72084 & -32.21278 & 0.6261\end{array}$ \\
\hline $\mathrm{C}$ & $21.40254 \quad-30.20219 \quad 2.11024$ \\
\hline $\mathrm{C}$ & $\begin{array}{lll}21.87497 & -30.7158 & 3.26503\end{array}$ \\
\hline $\mathrm{C}$ & $\begin{array}{llll}20.79341 & -32.92651 & -2.98778\end{array}$ \\
\hline $\mathrm{C}$ & $20.53978-32.31577 \quad-4.1629$ \\
\hline $\mathrm{C}$ & $26.10087-34.7834-0.33205$ \\
\hline$C$ & \\
\hline
\end{tabular}




\begin{tabular}{|c|c|}
\hline $\mathrm{C}$ & $\begin{array}{lll}23.52616 & -34.41997 & 0.00012\end{array}$ \\
\hline $\mathrm{C}$ & $\begin{array}{llll}23.60845 & -33.04402 & -0.26731\end{array}$ \\
\hline $\mathrm{C}$ & $24.86924-32.48479-0.61406$ \\
\hline $\mathrm{C}$ & $25.95597 \quad-33.3833-0.75654$ \\
\hline $\mathrm{C}$ & $\begin{array}{lll}24.33519 & -36.72507 & 0.22218\end{array}$ \\
\hline $\mathrm{C}$ & $\begin{array}{lll}25.17999 & -37.71972 & 0.56533\end{array}$ \\
\hline $\mathrm{C}$ & $25.13259-31.05778-0.84996$ \\
\hline $\mathrm{C}$ & $24.33775-30.00073-0.58387$ \\
\hline $\mathrm{O}$ & $20.88425 \quad-21.72676 \quad 1.13525$ \\
\hline $\mathrm{C}$ & $\begin{array}{lll}21.77576 & -21.47599 & 2.22091\end{array}$ \\
\hline $\mathrm{O}$ & $29.291 \quad-25.81499-0.67673$ \\
\hline $\mathrm{C}$ & $\begin{array}{lll}30.28856 & -25.6 & 0.31913\end{array}$ \\
\hline $\mathrm{O}$ & $\begin{array}{lll}26.87327 & -24.03175 & -0.45309\end{array}$ \\
\hline $\mathrm{C}$ & $\begin{array}{lll}27.03617 & -23.17417 & 0.67514\end{array}$ \\
\hline $\mathrm{O}$ & $\begin{array}{lll}33.63713 & -30.65815 & -1.22836\end{array}$ \\
\hline $\mathrm{C}$ & $\begin{array}{lll}34.12164 & -29.59745 & -0.40454\end{array}$ \\
\hline $\mathrm{O}$ & $\begin{array}{lll}16.69585 & -24.56816 & 0.72997\end{array}$ \\
\hline $\mathrm{C}$ & $\begin{array}{lll}15.83438 & -24.78392 & -0.38812\end{array}$ \\
\hline $\mathrm{O}$ & $32.21348 \quad-35.55016-0.4009$ \\
\hline $\mathrm{C}$ & $\begin{array}{lll}33.5876 & -35.61295 & -0.79372\end{array}$ \\
\hline $\mathrm{H}$ & $24.84772-23.05903-1.26566$ \\
\hline $\mathrm{H}$ & $22.57014-22.16401-0.81124$ \\
\hline $\mathrm{H}$ & $\begin{array}{lll}23.98569 & -26.49503 & 1.18612\end{array}$ \\
\hline $\mathrm{H}$ & $\begin{array}{lll}21.6953 & -25.6287 & 1.59642\end{array}$ \\
\hline $\mathrm{H}$ & $\begin{array}{lll}20.39257 & -23.75003 & 2.94221\end{array}$ \\
\hline $\mathrm{H}$ & $\begin{array}{lll}18.29149 & -24.99371 & 2.65184\end{array}$ \\
\hline $\mathrm{H}$ & $\begin{array}{lll}20.11097 & -22.37121 & -1.19335\end{array}$ \\
\hline $\mathrm{H}$ & $18.04766-23.6626-1.49499$ \\
\hline $\mathrm{H}$ & $\begin{array}{lll}31.84764 & -29.32611 & -2.53177\end{array}$ \\
\hline $\mathrm{H}$ & $\begin{array}{lll}30.13397 & -27.52848 & -2.38686\end{array}$ \\
\hline $\mathrm{H}$ & $\begin{array}{lll}30.40021 & -30.97859 & 1.17234\end{array}$ \\
\hline $\mathrm{H}$ & $\begin{array}{lll}28.66847 & -29.24499 & 1.29207\end{array}$ \\
\hline $\mathrm{H}$ & $27.60673-27.42172-2.4826$ \\
\hline $\mathrm{H}$ & $\begin{array}{lll}25.61669 & -25.94371 & -2.29667\end{array}$ \\
\hline $\mathrm{H}$ & $\begin{array}{lll}28.12884 & -27.23752 & 1.79689\end{array}$ \\
\hline $\mathrm{H}$ & $\begin{array}{lll}26.15333 & -25.78858 & 1.97422\end{array}$ \\
\hline $\mathrm{H}$ & $\begin{array}{lll}32.06485 & -31.80372 & -2.90857\end{array}$ \\
\hline $\mathrm{H}$ & $\begin{array}{llll}31.46518 & -34.17875 & -2.57391\end{array}$ \\
\hline $\mathrm{H}$ & $\begin{array}{lll}33.13564 & -31.49937 & 1.31828\end{array}$ \\
\hline $\mathrm{H}$ & $\begin{array}{lll}32.60205 & -33.87204 & 1.62424\end{array}$ \\
\hline $\mathrm{H}$ & $29.82215-35.90275-1.51989$ \\
\hline $\mathrm{H}$ & $\begin{array}{lll}27.91259 & -33.39585 & 2.30959\end{array}$ \\
\hline $\mathrm{H}$ & $\begin{array}{lll}30.14227 & -32.74954 & 1.37924\end{array}$ \\
\hline $\mathrm{H}$ & $\begin{array}{lll}16.85178 & -27.01032 & 1.24982\end{array}$ \\
\hline $\mathrm{H}$ & $\begin{array}{llll}20.82233 & -28.02197 & -1.54491\end{array}$ \\
\hline $\mathrm{H}$ & $20.13523-25.67221-1.18832$ \\
\hline
\end{tabular}




\begin{tabular}{|c|c|}
\hline $\mathrm{H}$ & $\begin{array}{lll}17.52709 & -30.68493 & 0.65294\end{array}$ \\
\hline $\mathrm{H}$ & $15.94188-30.4684 \quad 2.48013$ \\
\hline $\mathrm{H}$ & $\begin{array}{lll}16.27863 & -28.64251 & 2.61171\end{array}$ \\
\hline $\mathrm{H}$ & $26.40159-37.03608-0.34161$ \\
\hline $\mathrm{H}$ & $27.10362-38.4207 \quad-2.18325$ \\
\hline $\mathrm{H}$ & $\begin{array}{lll}28.7259 & -37.52774 & -2.31958\end{array}$ \\
\hline $\mathrm{H}$ & $\begin{array}{lll}19.81399 & -30.50872 & -2.25783\end{array}$ \\
\hline $\mathrm{H}$ & $\begin{array}{lll}22.23813 & -32.7207 & 1.44104\end{array}$ \\
\hline $\mathrm{H}$ & $\begin{array}{lll}21.12447 & -29.14525 & 2.09192\end{array}$ \\
\hline $\mathrm{H}$ & $22.00053-30.08232 \quad 4.14476$ \\
\hline $\mathrm{H}$ & $22.14454 \quad-31.76874 \quad 3.37585$ \\
\hline $\mathrm{H}$ & $20.92738-34.01016-2.98069$ \\
\hline $\mathrm{H}$ & $\begin{array}{lll}20.44291 & -32.89357 & -5.08374\end{array}$ \\
\hline $\mathrm{H}$ & $20.43839-31.23076-4.24623$ \\
\hline $\mathrm{H}$ & $\begin{array}{lll}22.54962 & -34.82732 & 0.27161\end{array}$ \\
\hline $\mathrm{H}$ & $\begin{array}{lll}26.92155 & -32.98441 & -1.07817\end{array}$ \\
\hline $\mathrm{H}$ & $23.2658 \quad-36.96644 \quad 0.21671$ \\
\hline $\mathrm{H}$ & $\begin{array}{lll}24.78123 & -38.71044 & 0.78812\end{array}$ \\
\hline $\mathrm{H}$ & $\begin{array}{lll}26.25992 & -37.6013 & 0.62921\end{array}$ \\
\hline $\mathrm{H}$ & $26.12323-30.85454-1.27233$ \\
\hline $\mathrm{H}$ & $24.68266-28.98857-0.80437$ \\
\hline $\mathrm{H}$ & $23.34602-30.10356-0.14513$ \\
\hline $\mathrm{H}$ & $\begin{array}{lll}22.76278 & -21.94813 & 2.06512\end{array}$ \\
\hline $\mathrm{H}$ & $\begin{array}{lll}21.89749 & -20.38396 & 2.2561\end{array}$ \\
\hline $\mathrm{H}$ & $\begin{array}{lll}21.36225 & -21.81757 & 3.18783\end{array}$ \\
\hline $\mathrm{H}$ & $\begin{array}{lll}30.89459 & -24.75942 & -0.04796\end{array}$ \\
\hline $\mathrm{H}$ & $\begin{array}{lll}29.84193 & -25.32565 & 1.29332\end{array}$ \\
\hline $\mathrm{H}$ & $\begin{array}{lll}30.93841 & -26.48511 & 0.45842\end{array}$ \\
\hline $\mathrm{H}$ & $27.66275-23.64412 \quad 1.45502$ \\
\hline $\mathrm{H}$ & $\begin{array}{lll}27.54604 & -22.27785 & 0.29385\end{array}$ \\
\hline $\mathrm{H}$ & $\begin{array}{lll}26.06598 & -22.87988 & 1.11858\end{array}$ \\
\hline $\mathrm{H}$ & $\begin{array}{lll}34.98674 & -29.18032 & -0.93933\end{array}$ \\
\hline $\mathrm{H}$ & $\begin{array}{lll}33.36538 & -28.80513 & -0.25978\end{array}$ \\
\hline $\mathrm{H}$ & $\begin{array}{lll}34.45763 & -29.95702 & 0.58577\end{array}$ \\
\hline $\mathrm{H}$ & $\begin{array}{lll}15.90852 & -23.96797 & -1.13046\end{array}$ \\
\hline $\mathrm{H}$ & $\begin{array}{lll}16.04285 & -25.74681 & -0.88862\end{array}$ \\
\hline $\mathrm{H}$ & $\begin{array}{lll}14.81337 & -24.79948 & 0.01885\end{array}$ \\
\hline $\mathrm{H}$ & $\begin{array}{llll}33.85783 & -36.6779 & -0.77136\end{array}$ \\
\hline $\mathrm{H}$ & $33.74437-35.2114-1.81179$ \\
\hline $\mathrm{H}$ & $\begin{array}{lll}34.23787 & -35.05161 & -0.09736\end{array}$ \\
\hline
\end{tabular}

Macrocycle 12, Relative Energy $=3.43 \mathrm{kcal} \mathrm{mol}^{-1}, \mathrm{RB} 97 \mathrm{D} / 6-31 \mathrm{G}(\mathrm{d})$
C $\quad 24.59729-24.81995-0.04405$
C $\quad 24.15108-23.62601-0.64851$
$\begin{array}{llll}\text { C } & 22.86946 & -23.12895 & -0.39915\end{array}$
$\begin{array}{llll}\text { C } & 21.97756 & -23.81671 & 0.44821\end{array}$ 


\begin{tabular}{|c|c|}
\hline 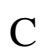 & $\begin{array}{lll}23.68026 & -25.54872 & 0.73362\end{array}$ \\
\hline$C$ & $22.3838-25.05654 \quad 0.97304$ \\
\hline $\mathrm{C}$ & $\begin{array}{llll}20.63967 & -23.13036 & 0.81461\end{array}$ \\
\hline $\mathrm{C}$ & $\begin{array}{lll}18.10966 & -24.72831 & 0.46672\end{array}$ \\
\hline $\mathrm{C}$ & $\begin{array}{llll}19.92311 & -23.81831 & 1.95955\end{array}$ \\
\hline $\mathrm{C}$ & $\begin{array}{lll}18.78125 & -24.50173 & 1.809\end{array}$ \\
\hline $\mathrm{C}$ & $\begin{array}{lll}19.75401 & -23.04983 & -0.4161\end{array}$ \\
\hline $\mathrm{C}$ & $\begin{array}{lll}18.61701 & -23.74199 & -0.56746\end{array}$ \\
\hline $\mathrm{C}$ & $\begin{array}{lll}31.26558 & -30.27337 & -0.67754\end{array}$ \\
\hline $\mathrm{C}$ & $\begin{array}{lll}31.13867 & -29.3109 & -1.70269\end{array}$ \\
\hline $\mathrm{C}$ & $\begin{array}{llll}30.17014 & -28.30934 & -1.62621\end{array}$ \\
\hline $\mathrm{C}$ & $29.2828-28.23059-0.52863$ \\
\hline $\mathrm{C}$ & $\begin{array}{lll}30.34832 & -30.23301 & 0.38222\end{array}$ \\
\hline $\mathrm{C}$ & $\begin{array}{lll}29.36389 & -29.2304 & 0.45292\end{array}$ \\
\hline $\mathrm{C}$ & $\begin{array}{llll}28.40819 & -26.96807 & -0.43061\end{array}$ \\
\hline $\mathrm{C}$ & $26.0732-25.23299-0.21021$ \\
\hline $\mathrm{C}$ & $\begin{array}{lll}27.3899 & -26.86788 & -1.56724\end{array}$ \\
\hline $\mathrm{C}$ & $26.31541-26.07069-1.46766$ \\
\hline $\mathrm{C}$ & $\begin{array}{lll}27.69587 & -26.7799 & 0.90606\end{array}$ \\
\hline $\mathrm{C}$ & $\begin{array}{lll}26.62063 & -25.98311 & 1.00653\end{array}$ \\
\hline $\mathrm{C}$ & $\begin{array}{llll}32.40944 & -31.30822 & -0.78179\end{array}$ \\
\hline $\mathrm{C}$ & $\begin{array}{lll}31.67349 & -34.2101 & -0.36328\end{array}$ \\
\hline $\mathrm{C}$ & $\begin{array}{llll}32.06953 & -32.25629 & -1.9153\end{array}$ \\
\hline $\mathrm{C}$ & $\begin{array}{llll}31.75156 & -33.5441 & -1.73281\end{array}$ \\
\hline $\mathrm{C}$ & $\begin{array}{lll}32.67083 & -32.06641 & 0.50913\end{array}$ \\
\hline $\mathrm{C}$ & $\begin{array}{llll}32.37969 & -33.36447 & 0.68201\end{array}$ \\
\hline $\mathrm{C}$ & $\begin{array}{lll}27.21835 & -34.72153 & 0.67028\end{array}$ \\
\hline $\mathrm{C}$ & $\begin{array}{llll}27.72286 & -35.41747 & -0.46242\end{array}$ \\
\hline $\mathrm{C}$ & $\begin{array}{lll}28.98737 & -35.04413 & -0.95745\end{array}$ \\
\hline $\mathrm{C}$ & $29.7485-34.0314-0.36771$ \\
\hline $\mathrm{C}$ & $27.90484-33.59099 \quad 1.15166$ \\
\hline $\mathrm{C}$ & $29.15938 \quad-33.24244 \quad 0.6429$ \\
\hline $\mathrm{C}$ & $19.25375-28.87406-0.33112$ \\
\hline $\mathrm{C}$ & $\begin{array}{lll}18.11074 & -28.59472 & 0.46969\end{array}$ \\
\hline $\mathrm{C}$ & $\begin{array}{lll}17.7315 & -27.24567 & 0.6505\end{array}$ \\
\hline $\mathrm{C}$ & $\begin{array}{llll}18.45186 & -26.18677 & 0.08036\end{array}$ \\
\hline $\mathrm{C}$ & $\begin{array}{lll}19.93904 & -27.80313 & -0.94375\end{array}$ \\
\hline $\mathrm{C}$ & $\begin{array}{lll}19.55555 & -26.47895 & -0.742\end{array}$ \\
\hline $\mathrm{C}$ & $\begin{array}{lll}17.3446 & -29.69599 & 1.08245\end{array}$ \\
\hline $\mathrm{C}$ & $\begin{array}{lll}16.47699 & -29.59237 & 2.10926\end{array}$ \\
\hline $\mathrm{C}$ & $\begin{array}{llll}26.96866 & -36.53882 & -1.04982\end{array}$ \\
\hline $\mathrm{C}$ & $\begin{array}{llll}27.35536 & -37.33965 & -2.06438\end{array}$ \\
\hline $\mathrm{C}$ & $19.29256-30.43336-0.19352$ \\
\hline $\mathrm{C}$ & $\begin{array}{llll}21.79618 & -31.73096 & -0.39777\end{array}$ \\
\hline $\mathrm{C}$ & $\begin{array}{lll}19.89272 & -30.65815 & -1.44264\end{array}$ \\
\hline$C$ & $21.14429-31.28$ \\
\hline
\end{tabular}




\begin{tabular}{|c|c|}
\hline C & $\begin{array}{llll}20.03232 & -30.70906 & 0.99434\end{array}$ \\
\hline $\mathrm{C}$ & $21.2701 \quad-31.36679 \quad 0.84979$ \\
\hline $\mathrm{C}$ & $\begin{array}{lll}19.49007 & -30.35287 & 2.31605\end{array}$ \\
\hline $\mathrm{C}$ & $\begin{array}{lll}19.94999 & -30.75932 & 3.51764\end{array}$ \\
\hline $\mathrm{C}$ & $\begin{array}{llll}21.69866 & -31.57877 & -2.91864\end{array}$ \\
\hline $\mathrm{C}$ & $\begin{array}{llll}21.34674 & -30.96555 & -4.06684\end{array}$ \\
\hline $\mathrm{C}$ & $25.94004-33.72945-0.22648$ \\
\hline $\mathrm{C}$ & $\begin{array}{llll}25.63956 & -32.43093 & -0.73463\end{array}$ \\
\hline $\mathrm{C}$ & $\begin{array}{llll}24.27968 & -32.06497 & -0.77193\end{array}$ \\
\hline $\mathrm{C}$ & $23.22602-32.90591-0.37931$ \\
\hline $\mathrm{C}$ & $\begin{array}{llll}23.52345 & -34.23276 & 0.03729\end{array}$ \\
\hline $\mathrm{C}$ & $\begin{array}{llll}24.89203 & -34.59265 & 0.11523\end{array}$ \\
\hline $\mathrm{C}$ & $26.60746-31.41337-1.18927$ \\
\hline $\mathrm{C}$ & $\begin{array}{lll}27.88277 & -31.55266 & -1.60756\end{array}$ \\
\hline $\mathrm{C}$ & $\begin{array}{lll}22.53102 & -35.25277 & 0.40609\end{array}$ \\
\hline $\mathrm{C}$ & $21.19449 \quad-35.2302 \quad 0.22423$ \\
\hline $\mathrm{O}$ & $20.88425 \quad-21.72676 \quad 1.13525$ \\
\hline $\mathrm{C}$ & $\begin{array}{llll}21.77576 & -21.47599 & 2.22091\end{array}$ \\
\hline $\mathrm{O}$ & $29.291-25.81499 \quad-0.67673$ \\
\hline $\mathrm{C}$ & $30.28856-25.6$ \\
\hline $\mathrm{O}$ & $26.87327-24.03175-0.45309$ \\
\hline $\mathrm{C}$ & $\begin{array}{lll}27.03617 & -23.17417 & 0.67514\end{array}$ \\
\hline $\mathrm{O}$ & $33.63713-30.65815-1.22836$ \\
\hline $\mathrm{C}$ & $\begin{array}{llll}34.12164 & -29.59745 & -0.40454\end{array}$ \\
\hline $\mathrm{O}$ & $\begin{array}{llll}16.69585 & -24.56816 & 0.72997\end{array}$ \\
\hline $\mathrm{C}$ & $\begin{array}{lll}15.83438 & -24.78392 & -0.38812\end{array}$ \\
\hline $\mathrm{O}$ & $32.21348-35.55016-0.4009$ \\
\hline $\mathrm{C}$ & $\begin{array}{llll}33.5876 & -35.61295 & -0.79372\end{array}$ \\
\hline $\mathrm{H}$ & $24.84772-23.05903-1.26566$ \\
\hline $\mathrm{H}$ & $22.57014-22.16401-0.81124$ \\
\hline $\mathrm{H}$ & $\begin{array}{lll}23.98569 & -26.49503 & 1.18612\end{array}$ \\
\hline $\mathrm{H}$ & $\begin{array}{lll}21.6953 & -25.6287 & 1.59642\end{array}$ \\
\hline $\mathrm{H}$ & $\begin{array}{llll}20.39257 & -23.75003 & 2.94221\end{array}$ \\
\hline $\mathrm{H}$ & $\begin{array}{lll}18.29149 & -24.99371 & 2.65184\end{array}$ \\
\hline $\mathrm{H}$ & $20.11097-22.37121-1.19335$ \\
\hline $\mathrm{H}$ & $18.04766-23.6626-1.49499$ \\
\hline $\mathrm{H}$ & $\begin{array}{llll}31.84764 & -29.32611 & -2.53177\end{array}$ \\
\hline $\mathrm{H}$ & $\begin{array}{llll}30.13397 & -27.52848 & -2.38686\end{array}$ \\
\hline $\mathrm{H}$ & $\begin{array}{lll}30.40021 & -30.97859 & 1.17234\end{array}$ \\
\hline $\mathrm{H}$ & $\begin{array}{llll}28.66847 & -29.24499 & 1.29207\end{array}$ \\
\hline $\mathrm{H}$ & $27.60673-27.42172-2.4826$ \\
\hline $\mathrm{H}$ & $25.61669-25.94371-2.29667$ \\
\hline $\mathrm{H}$ & $\begin{array}{llll}28.12884 & -27.23752 & 1.79689\end{array}$ \\
\hline $\mathrm{H}$ & $\begin{array}{lll}26.15333 & -25.78858 & 1.97422\end{array}$ \\
\hline $\mathrm{H}$ & $\begin{array}{llll}32.06485 & -31.80372 & -2.90857\end{array}$ \\
\hline 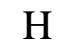 & $31.46518-34.17875$ \\
\hline
\end{tabular}




\begin{tabular}{|c|c|c|}
\hline $\mathrm{H}$ & $33.13564-31.49937$ & 1.31828 \\
\hline $\mathrm{H}$ & $32.60205-33.87204$ & 1.62424 \\
\hline $\mathrm{H}$ & $29.40758-35.59705$ & -1.79398 \\
\hline $\mathrm{H}$ & $27.48609-33.04261$ & 1.99794 \\
\hline $\mathrm{H}$ & $29.71533-32.40324$ & 1.06174 \\
\hline $\mathrm{H}$ & $16.85178-27.01032$ & 1.24982 \\
\hline $\mathrm{H}$ & $20.82233-28.02197$ & -1.54491 \\
\hline $\mathrm{H}$ & $20.13523-25.67221$ & -1.18832 \\
\hline $\mathrm{H}$ & $17.52709-30.68493$ & 0.65294 \\
\hline $\mathrm{H}$ & $15.94188-30.4684$ & 2.48013 \\
\hline $\mathrm{H}$ & $16.27863-28.64251$ & 2.61171 \\
\hline $\mathrm{H}$ & $25.98843-36.72315$ & -0.60473 \\
\hline $\mathrm{H}$ & $26.69668-38.13119$ & -2.42612 \\
\hline $\mathrm{H}$ & $28.31676-37.23586$ & -2.57261 \\
\hline $\mathrm{H}$ & $19.3062-30.44915$ & -2.33737 \\
\hline $\mathrm{H}$ & $21.82527-31.65909$ & 1.742 \\
\hline $\mathrm{H}$ & $18.62212-29.68882$ & 2.29418 \\
\hline $\mathrm{H}$ & $19.48216-30.409$ & 4.43926 \\
\hline $\mathrm{H}$ & $20.79147-31.44777$ & 3.62523 \\
\hline $\mathrm{H}$ & $22.47699-32.34387$ & -2.95256 \\
\hline $\mathrm{H}$ & $21.80928-31.24588$ & -5.01481 \\
\hline $\mathrm{H}$ & $20.60664-30.16188$ & -4.09558 \\
\hline $\mathrm{H}$ & $24.02814-31.0523$ & -1.09516 \\
\hline $\mathrm{H}$ & $25.14736-35.58823$ & 0.48744 \\
\hline $\mathrm{H}$ & $26.18517-30.40223$ & -1.22316 \\
\hline $\mathrm{H}$ & $28.44299-30.67233$ & -1.92589 \\
\hline $\mathrm{H}$ & $28.40733-32.50552$ & -1.64283 \\
\hline $\mathrm{H}$ & $22.96642-36.14961$ & 0.86126 \\
\hline $\mathrm{H}$ & $20.58551 \quad-36.0791$ & 0.54135 \\
\hline $\mathrm{H}$ & $20.67576-34.3913$ & -0.23812 \\
\hline $\mathrm{H}$ & $22.76278-21.94813$ & 2.06512 \\
\hline $\mathrm{H}$ & $21.89749-20.38396$ & 2.2561 \\
\hline $\mathrm{H}$ & $21.36225-21.81757$ & 3.18783 \\
\hline $\mathrm{H}$ & $30.89459-24.75942$ & -0.04796 \\
\hline $\mathrm{H}$ & $29.84193-25.32565$ & 1.29332 \\
\hline $\mathrm{H}$ & $30.93841-26.48511$ & 0.45842 \\
\hline $\mathrm{H}$ & $27.66275-23.64412$ & 1.45502 \\
\hline $\mathrm{H}$ & $27.54604-22.27785$ & 0.29385 \\
\hline $\mathrm{H}$ & $26.06598-22.87988$ & 1.11858 \\
\hline $\mathrm{H}$ & $34.98674-29.18032$ & -0.93933 \\
\hline $\mathrm{H}$ & $33.36538-28.80513$ & -0.25978 \\
\hline $\mathrm{H}$ & $34.45763-29.95702$ & 0.58577 \\
\hline $\mathrm{H}$ & $15.90852-23.96797$ & -1.13046 \\
\hline $\mathrm{H}$ & $16.04285-25.74681$ & -0.88862 \\
\hline $\mathrm{H}$ & $14.81337-24.79948$ & 0.01885 \\
\hline $\mathrm{H}$ & $33.85783-36.6779$ & -0.77136 \\
\hline
\end{tabular}




$$
\begin{array}{llll}
\mathrm{H} & 33.74437 & -35.2114 & -1.81179 \\
\mathrm{H} & 34.23787 & -35.05161 & -0.09736
\end{array}
$$

tetravinylbiphenyl, $\omega B 97 D / 6-31 G(d)($ Energy $=-772.6322347$ Hartrees $)$
C $\quad-7.91414100 \quad 2.79032700 \quad-1.03339000$
C $\quad-6.51942500 \quad 2.82167100 \quad-0.98557900$
$\begin{array}{llll}\text { C } & -5.79815900 & 2.38070600 & 0.12586800\end{array}$
C $\quad-6.49243500 \quad 1.88470100 \quad 1.24529200$
C $\quad-7.89366400 \quad 1.86648000 \quad 1.20171000$
C $\quad-8.59329800 \quad 2.30132900 \quad 0.08998000$
$\mathrm{H} \quad-5.96858500 \quad 3.19301000 \quad-1.84636500$
$\mathrm{H} \quad-8.44100900 \quad 1.52775100 \quad 2.07648600$
$\mathrm{H} \quad-9.67863600 \quad 2.27379500 \quad 0.10510200$
$\begin{array}{llll}\text { C } & -4.30785100 & 2.39389100 & 0.07679600\end{array}$
C $\quad-3.58260500 \quad 3.57835600 \quad-0.16695200$
$\begin{array}{llll}\text { C } & -3.62488200 & 1.18810000 & 0.23074700\end{array}$
$\begin{array}{llll}\text { C } & -2.18741600 & 3.49548200 & -0.24541000\end{array}$
$\begin{array}{llll}\text { C } & -2.23226400 & 1.10970100 & 0.16222400\end{array}$
$\mathrm{H} \quad-4.20509200 \quad 0.28329000 \quad 0.38554800$
C $\quad-1.52405500 \quad 2.28756400 \quad-0.09719800$
$\mathrm{H} \quad-1.61406600 \quad 4.40537200 \quad-0.39607300$
$\mathrm{H} \quad-0.43884300 \quad 2.25905400 \quad-0.15691600$
$\begin{array}{llll}\text { C } & -8.59639300 & 3.27309400 & -2.24675800\end{array}$
$\mathrm{H} \quad-7.92992700 \quad 3.64871700 \quad-3.02234200$
C $\quad-5.77387300 \quad 1.42720400 \quad 2.45180900$
$\mathrm{H} \quad-4.82525900 \quad 1.91808900 \quad 2.65837000$
C $\quad-4.26715000 \quad 4.87948100 \quad-0.30575400$
$\begin{array}{llll}\mathrm{H} & -5.20392000 & 4.98595000 & 0.23721800\end{array}$ 


$\begin{array}{llll}\mathrm{C} & -1.50543000 & -0.15695800 & 0.36016400 \\ \mathrm{H} & -0.49108100 & -0.17857500 & -0.03710900 \\ \mathrm{C} & -1.97004300 & -1.23602200 & 0.99164200 \\ \mathrm{H} & -2.95808400 & -1.26463900 & 1.44388400 \\ \mathrm{H} & -1.36411300 & -2.13101600 & 1.09004000 \\ \mathrm{C} & -3.83354500 & 5.90242100 & -1.04413700 \\ \mathrm{H} & -2.93071300 & 5.84099400 & -1.64679100 \\ \mathrm{H} & -4.38055600 & 6.83925100 & -1.07924400 \\ \mathrm{C} & -6.19279300 & 0.46417300 & 3.27467900 \\ \mathrm{H} & -7.10814400 & -0.09382900 & 3.09338300 \\ \mathrm{H} & -5.62209000 & 0.19366900 & 4.15751500 \\ \mathrm{C} & -9.90988500 & 3.29150400 & -2.47805300 \\ \mathrm{H} & -10.30465300 & 3.67422000 & -3.41359300 \\ \mathrm{H} & -10.64050300 & 2.93261400 & -1.75845000\end{array}$

10 open adduct, $\omega B 97 D / 6-31 G(d)($ Energy $=-3620.361881$ Hartrees $)$

$\begin{array}{llll}\text { C } & 29.58021800 & -12.74064200 & -1.21368800 \\ \text { C } & 29.36293400 & -13.52931600 & -0.08340600 \\ \text { C } & 28.97758500 & -12.95894300 & 1.12406700 \\ \text { C } & 28.76848300 & -11.58440800 & 1.22764700 \\ \text { C } & 29.37748100 & -11.36435800 & -1.11119200 \\ \text { C } & 28.95797100 & -10.80025500 & 0.08827500 \\ \text { C } & 28.32132000 & -10.89401100 & 2.52967500 \\ \text { C } & 25.92706000 & -12.36558400 & 3.43381600 \\ \text { C } & 28.34757700 & -11.82440500 & 3.72035900 \\ \text { C } & 27.26364500 & -12.46436500 & 4.13996000 \\ \text { C } & 26.91568200 & -10.36115500 & 2.34495800\end{array}$




\begin{tabular}{|c|c|c|c|}
\hline $\mathrm{C}$ & 25.83404200 & -11.01786600 & 2.75057300 \\
\hline $\mathrm{C}$ & 35.02886400 & -9.72434000 & -5.43343200 \\
\hline $\mathrm{C}$ & 35.27558100 & -11.04482700 & -5.80452000 \\
\hline $\mathrm{C}$ & 34.33384400 & -12.03856600 & -5.55843600 \\
\hline $\mathrm{C}$ & 33.12905300 & -11.74009200 & -4.92398700 \\
\hline $\mathrm{C}$ & 33.84065400 & -9.43337700 & -4.76101300 \\
\hline $\mathrm{C}$ & 32.90365500 & -10.42736300 & -4.50732400 \\
\hline $\mathrm{C}$ & 32.11657300 & -12.85585900 & -4.64347100 \\
\hline $\mathrm{C}$ & 30.06793000 & -13.41443100 & -2.50164400 \\
\hline $\mathrm{C}$ & 32.43714200 & -13.50055900 & -3.31626000 \\
\hline $\mathrm{C}$ & 31.53666700 & -13.74220500 & -2.37262300 \\
\hline $\mathrm{C}$ & 30.70182700 & -12.32497300 & -4.66907100 \\
\hline $\mathrm{C}$ & 29.80138100 & -12.56469400 & -3.72235800 \\
\hline $\mathrm{C}$ & 36.08012900 & -8.62499100 & -5.67181500 \\
\hline $\mathrm{C}$ & 35.85969700 & -6.01264900 & -4.15993600 \\
\hline $\mathrm{C}$ & 36.76437900 & -8.35156900 & -4.35620900 \\
\hline $\mathrm{C}$ & 36.65571400 & -7.21051400 & -3.68718200 \\
\hline $\mathrm{C}$ & 35.43777300 & -7.37200200 & -6.22585300 \\
\hline $\mathrm{C}$ & 35.35727900 & -6.22066000 & -5.56667700 \\
\hline $\mathrm{C}$ & 32.67984700 & -5.66740000 & -1.23867400 \\
\hline $\mathrm{C}$ & 34.00741300 & -5.36621800 & -0.88224300 \\
\hline $\mathrm{C}$ & 34.99881700 & -5.44119900 & -1.86421200 \\
\hline $\mathrm{C}$ & 34.70504400 & -5.78930300 & -3.18034700 \\
\hline $\mathrm{C}$ & 32.37709900 & -5.94327300 & -2.56994600 \\
\hline $\mathrm{C}$ & 33.37345100 & -6.00212000 & -3.53439100 \\
\hline $\mathrm{C}$ & 26.19277300 & -15.67823500 & 0.63829000 \\
\hline $\mathrm{C}$ & 26.26226500 & -15.90524900 & 2.02921900 \\
\hline
\end{tabular}




\begin{tabular}{|c|c|c|c|}
\hline $\mathrm{C}$ & 26.07218400 & -14.82804100 & 2.89563400 \\
\hline $\mathrm{C}$ & 25.87000100 & -13.52964300 & 2.42663900 \\
\hline $\mathrm{C}$ & 25.86944900 & -14.40171300 & 0.18046000 \\
\hline $\mathrm{C}$ & 25.72074400 & -13.33532500 & 1.05761700 \\
\hline $\mathrm{C}$ & 26.53453600 & -17.25652200 & 2.56416200 \\
\hline $\mathrm{C}$ & 27.18502400 & -17.51649700 & 3.69938700 \\
\hline $\mathrm{C}$ & 34.35000500 & -5.03498700 & 0.51589000 \\
\hline $\mathrm{C}$ & 35.36000300 & -4.24993100 & 0.89212400 \\
\hline $\mathrm{C}$ & 26.61035800 & -16.71542500 & -0.34468300 \\
\hline $\mathrm{C}$ & 27.89138400 & -17.24919200 & -0.21281000 \\
\hline $\mathrm{C}$ & 28.44586600 & -18.12637000 & -1.14459600 \\
\hline C & 25.82885400 & -17.08251700 & -1.45510200 \\
\hline $\mathrm{C}$ & 26.39220200 & -17.94480900 & -2.40183100 \\
\hline $\mathrm{C}$ & 24.46032300 & -16.55868000 & -1.62925900 \\
\hline $\mathrm{C}$ & 23.86342900 & -16.33964000 & -2.80212300 \\
\hline $\mathrm{C}$ & 29.78545000 & -18.70621900 & -0.92692500 \\
\hline $\mathrm{C}$ & 30.35527000 & -18.87844600 & 0.26694200 \\
\hline $\mathrm{C}$ & 31.58771500 & -5.72105800 & -0.22736400 \\
\hline $\mathrm{C}$ & 31.05282000 & -6.94401600 & 0.22530200 \\
\hline $\mathrm{C}$ & 29.97577700 & -6.88521600 & 1.12714600 \\
\hline $\mathrm{C}$ & 29.46815000 & -5.68468900 & 1.59320300 \\
\hline $\mathrm{C}$ & 30.01477900 & -4.46821000 & 1.17010200 \\
\hline $\mathrm{C}$ & 31.06750100 & -4.52008900 & 0.25326800 \\
\hline $\mathrm{C}$ & 31.51875800 & -8.29539700 & -0.15297600 \\
\hline $\mathrm{C}$ & 32.62068600 & -8.70567600 & -0.78952000 \\
\hline $\mathrm{C}$ & 29.53354100 & -3.15613000 & 1.63550000 \\
\hline $\mathrm{C}$ & 28.61310600 & -2.92528900 & 2.57316300 \\
\hline
\end{tabular}




\begin{tabular}{|c|c|c|c|}
\hline $\mathrm{O}$ & 29.12061400 & -9.73009500 & 2.75865100 \\
\hline C & 30.49718800 & -9.95081100 & 2.98775500 \\
\hline $\mathrm{O}$ & 32.28371200 & -13.92104200 & -5.58809900 \\
\hline $\mathrm{C}$ & 31.85049800 & -13.65921900 & -6.90494300 \\
\hline $\mathrm{O}$ & 29.44358300 & -14.69573000 & -2.63171900 \\
\hline $\mathrm{C}$ & 28.07434600 & -14.67253500 & -2.97279300 \\
\hline $\mathrm{O}$ & 37.12203300 & -9.10086800 & -6.52309700 \\
\hline $\mathrm{C}$ & 36.83337900 & -9.18541600 & -7.90088200 \\
\hline $\mathrm{O}$ & 24.95575600 & -12.52014400 & 4.46189100 \\
\hline $\mathrm{C}$ & 23.61789300 & -12.64629600 & 4.03930200 \\
\hline $\mathrm{O}$ & 36.65350900 & -4.82842900 & -4.08485600 \\
\hline $\mathrm{C}$ & 37.83511800 & -4.85482100 & -4.85687300 \\
\hline $\mathrm{H}$ & 29.48592400 & -14.60552200 & -0.16116200 \\
\hline $\mathrm{H}$ & 28.80983800 & -13.59976900 & 1.98372900 \\
\hline $\mathrm{H}$ & 29.55218200 & -10.71914600 & -1.96786100 \\
\hline $\mathrm{H}$ & 28.78285000 & -9.72988000 & 0.14319000 \\
\hline $\mathrm{H}$ & 29.29955500 & -11.98062100 & 4.21759600 \\
\hline $\mathrm{H}$ & 27.29142300 & -13.13640200 & 4.99272700 \\
\hline $\mathrm{H}$ & 26.84424500 & -9.40238900 & 1.83873500 \\
\hline $\mathrm{H}$ & 24.84531500 & -10.60561000 & 2.57173500 \\
\hline $\mathrm{H}$ & 36.21478200 & -11.29678200 & -6.28521700 \\
\hline $\mathrm{H}$ & 34.52852700 & -13.06155000 & -5.86517800 \\
\hline $\mathrm{H}$ & 33.65104400 & -8.42375200 & -4.41186300 \\
\hline $\mathrm{H}$ & 31.99132500 & -10.17655200 & -3.97212700 \\
\hline $\mathrm{H}$ & 33.47308800 & -13.80167000 & -3.18630900 \\
\hline $\mathrm{H}$ & 31.82006200 & -14.24920300 & -1.45414100 \\
\hline $\mathrm{H}$ & 30.43114000 & -11.70957200 & -5.52441100 \\
\hline
\end{tabular}




\begin{tabular}{|c|c|c|c|}
\hline $\mathrm{H}$ & 28.80109100 & -12.14875200 & -3.81916000 \\
\hline $\mathrm{H}$ & 37.34556400 & -9.18212300 & -3.96479000 \\
\hline $\mathrm{H}$ & 37.15217000 & -7.08719600 & -2.72759900 \\
\hline $\mathrm{H}$ & 35.00357700 & -7.45200200 & -7.21917300 \\
\hline $\mathrm{H}$ & 34.88694100 & -5.35287900 & -6.02488700 \\
\hline $\mathrm{H}$ & 36.03205200 & -5.25580700 & -1.58800700 \\
\hline $\mathrm{H}$ & 31.34606500 & -6.15021500 & -2.84126900 \\
\hline $\mathrm{H}$ & 33.11044100 & -6.24131900 & -4.55964200 \\
\hline $\mathrm{H}$ & 26.09736300 & -15.00504800 & 3.96719300 \\
\hline $\mathrm{H}$ & 25.79345900 & -14.22902800 & -0.88835500 \\
\hline $\mathrm{H}$ & 25.55552700 & -12.33930700 & 0.66168100 \\
\hline $\mathrm{H}$ & 26.18006300 & -18.08919600 & 1.95993000 \\
\hline $\mathrm{H}$ & 27.32859200 & -18.53617900 & 4.04260100 \\
\hline $\mathrm{H}$ & 27.60418200 & -16.72774700 & 4.31938900 \\
\hline $\mathrm{H}$ & 33.70097000 & -5.46174500 & 1.27756600 \\
\hline $\mathrm{H}$ & 35.55832300 & -4.05895300 & 1.94202900 \\
\hline $\mathrm{H}$ & 36.01155800 & -3.75926900 & 0.17324900 \\
\hline $\mathrm{H}$ & 28.49261400 & -16.93618000 & 0.63529600 \\
\hline $\mathrm{H}$ & 25.80023300 & -18.24300100 & -3.26274100 \\
\hline $\mathrm{H}$ & 23.92268000 & -16.32242800 & -0.71298600 \\
\hline $\mathrm{H}$ & 22.84538600 & -15.96639200 & -2.85166400 \\
\hline $\mathrm{H}$ & 24.36770600 & -16.51037900 & -3.74996600 \\
\hline $\mathrm{H}$ & 30.31902700 & -19.03675000 & -1.81433100 \\
\hline 11 & 31.34593000 & -19.31301200 & 0.35422300 \\
\hline $\mathrm{H}$ & 29.85386700 & -18.61573700 & 1.19548200 \\
\hline $\mathrm{H}$ & 29.53156200 & -7.81152000 & 1.48501500 \\
\hline $\mathrm{H}$ & 31.50626300 & -3.59229400 & -0.10711700 \\
\hline
\end{tabular}




\begin{tabular}{|c|c|c|c|}
\hline $\mathrm{H}$ & 30.84409500 & -9.07830700 & 0.18528700 \\
\hline $\mathrm{H}$ & 32.77336400 & -9.77002000 & -0.94753800 \\
\hline $\mathrm{H}$ & 33.39618500 & -8.04711900 & -1.16150900 \\
\hline $\mathrm{H}$ & 30.00245600 & -2.30044700 & 1.15080800 \\
\hline $\mathrm{H}$ & 28.33445200 & -1.91095700 & 2.83987000 \\
\hline $\mathrm{H}$ & 28.10818700 & -3.72278900 & 3.11129000 \\
\hline $\mathrm{H}$ & 30.92625200 & -10.65212700 & 2.26047700 \\
\hline $\mathrm{H}$ & 30.98870400 & -8.98216600 & 2.87412300 \\
\hline $\mathrm{H}$ & 30.68790900 & -10.32049900 & 4.00315000 \\
\hline $\mathrm{H}$ & 32.22486900 & -14.48256500 & -7.51708200 \\
\hline $\mathrm{H}$ & 30.75506100 & -13.63807100 & -6.97529200 \\
\hline $\mathrm{H}$ & 32.25599200 & -12.71463500 & -7.29336100 \\
\hline $\mathrm{H}$ & 27.92639100 & -14.39146500 & -4.02327100 \\
\hline $\mathrm{H}$ & 27.70240700 & -15.68600600 & -2.82857200 \\
\hline $\mathrm{H}$ & 27.50836000 & -13.98769300 & -2.32691900 \\
\hline $\mathrm{H}$ & 37.60815000 & -9.82079400 & -8.33635300 \\
\hline $\mathrm{H}$ & 35.85374000 & -9.64283400 & -8.09623200 \\
\hline $\mathrm{H}$ & 36.87300200 & -8.20193900 & -8.38716500 \\
\hline $\mathrm{H}$ & 23.23546800 & -11.72648600 & 3.57540300 \\
\hline $\mathrm{H}$ & 23.48683400 & -13.47619200 & 3.33280600 \\
\hline $\mathrm{H}$ & 23.03362400 & -12.84669400 & 4.93957800 \\
\hline $\mathrm{H}$ & 38.31143200 & -3.88274000 & -4.71469600 \\
\hline $\mathrm{H}$ & 38.52279600 & -5.64652900 & -4.53240200 \\
\hline $\mathrm{H}$ & 37.62414500 & -5.00084900 & -5.92481600 \\
\hline $\mathrm{H}$ & 28.63763600 & -5.70138000 & 2.29237400 \\
\hline $\mathrm{C}$ & 27.68905300 & -18.44092600 & -2.28989500 \\
\hline $\mathrm{C}$ & 28.21900900 & -19.31176600 & -3.38219300 \\
\hline
\end{tabular}




$\begin{array}{llll}\text { C } & 29.18906000 & -18.84078300 & -4.29476500 \\ \text { C } & 27.70332200 & -20.59767600 & -3.51497800 \\ \text { C } & 29.62631500 & -19.72026700 & -5.29143000 \\ \text { C } & 28.12233100 & -21.46200300 & -4.53138900 \\ \text { H } & 26.96828100 & -20.93259800 & -2.78850800 \\ \text { C } & 29.10608600 & -21.00013800 & -5.41089600 \\ \text { H } & 30.40394500 & -19.39605600 & -5.97652900 \\ \text { C } & 29.73668500 & -17.47271700 & -4.19235600 \\ \text { H } & 29.70774000 & -16.99839300 & -3.21531100 \\ \text { C } & 30.25039100 & -16.76162500 & -5.19810700 \\ \text { H } & 30.28612000 & -17.13786200 & -6.21871700 \\ \text { H } & 30.65797600 & -15.77025300 & -5.02090900 \\ \text { C } & 27.58222400 & -22.82275500 & -4.69557800 \\ \text { H } & 28.15320700 & -23.46345300 & -5.36704100 \\ \text { C } & 26.48209100 & -23.31943100 & -4.12771600 \\ \text { H } & 25.84831900 & -22.73261200 & -3.46831000 \\ H & 26.17114100 & -24.34213400 & -4.31474500 \\ H & 29.47132900 & -21.65669100 & -6.19695500\end{array}$

13, $\omega B 97 D / 6-31 G(d)($ Energy $=-2690.634723$ Hartrees $)$
$\mathrm{C}$
$\begin{array}{lll}-2.73865900 & 2.83155800 & -0.15142600\end{array}$
$\begin{array}{llll}\text { C } & -2.73735500 & 1.55666600 & 0.40841400\end{array}$
$\begin{array}{llll}\text { C } & -3.90134900 & 1.01613000 & 0.94978500\end{array}$
$\begin{array}{llll}\text { C } & -5.09076800 & 1.74214400 & 0.95126400\end{array}$
$\begin{array}{llll}\text { C } & -3.92116000 & 3.57522700 & -0.11420100\end{array}$
$\begin{array}{llll}\text { C } & -5.07945900 & 3.03824000 & 0.43015000\end{array}$
$\begin{array}{llll}\text { C } & -6.42144300 & 1.13648300 & 1.41635300\end{array}$ 


\begin{tabular}{|c|c|c|c|}
\hline $\mathrm{C}$ & -6.76277500 & -1.65521300 & 0.40151400 \\
\hline $\mathrm{C}$ & -7.22938600 & 0.78319400 & 0.18023900 \\
\hline $\mathrm{C}$ & -7.38950000 & -0.45718400 & -0.26963200 \\
\hline $\mathrm{C}$ & -6.21067200 & -0.08262500 & 2.28000900 \\
\hline $\mathrm{C}$ & -6.37910600 & -1.32224200 & 1.83338400 \\
\hline $\mathrm{C}$ & 5.02875600 & 1.48911500 & 1.13662500 \\
\hline $\mathrm{C}$ & 5.01122100 & 2.83506100 & 0.75993100 \\
\hline $\mathrm{C}$ & 3.87245200 & 3.40284600 & 0.20899400 \\
\hline $\mathrm{C}$ & 2.71852100 & 2.64124900 & -0.00000900 \\
\hline $\mathrm{C}$ & 3.86339900 & 0.74171700 & 0.97544800 \\
\hline $\mathrm{C}$ & 2.72329400 & 1.30924500 & 0.40693800 \\
\hline $\mathrm{C}$ & 1.51098200 & 3.29654700 & -0.67850600 \\
\hline $\mathrm{C}$ & -1.48004200 & 3.42373400 & -0.78525100 \\
\hline $\mathrm{C}$ & 0.66052200 & 2.28924500 & -1.42305300 \\
\hline $\mathrm{C}$ & -0.66562600 & 2.35708000 & -1.48258400 \\
\hline $\mathrm{C}$ & 0.67793900 & 4.07463600 & 0.31851600 \\
\hline $\mathrm{C}$ & -0.64916900 & 4.13419900 & 0.26450300 \\
\hline $\mathrm{C}$ & 6.35686200 & 0.87463900 & 1.62203600 \\
\hline $\mathrm{C}$ & 6.78692900 & -1.86441100 & 0.48404200 \\
\hline $\mathrm{C}$ & 7.20312100 & 0.59430600 & 0.39845900 \\
\hline $\mathrm{C}$ & 7.39119300 & -0.61753600 & -0.11255000 \\
\hline $\mathrm{C}$ & 6.14039700 & -0.39126100 & 2.41556900 \\
\hline $\mathrm{C}$ & 6.35533300 & -1.60314700 & 1.91660000 \\
\hline $\mathrm{C}$ & 2.88157500 & -2.89788900 & -1.07400400 \\
\hline $\mathrm{C}$ & 3.60949100 & -3.79146400 & -0.26061400 \\
\hline $\mathrm{C}$ & 4.89651200 & -3.50857100 & 0.13586900 \\
\hline $\mathrm{C}$ & 5.52094500 & -2.30660500 & -0.25189000 \\
\hline
\end{tabular}




\begin{tabular}{|c|c|c|c|}
\hline $\mathrm{C}$ & 3.56372300 & -1.77356000 & -1.58739400 \\
\hline $\mathrm{C}$ & 4.87434300 & -1.49350500 & -1.15649800 \\
\hline $\mathrm{C}$ & -2.82324800 & -2.63881300 & -1.12903500 \\
\hline $\mathrm{C}$ & -3.53078600 & -3.55173000 & -0.31190300 \\
\hline $\mathrm{C}$ & -4.85001600 & -3.26222400 & 0.07655800 \\
\hline $\mathrm{C}$ & -5.46545900 & -2.08008300 & -0.29500100 \\
\hline $\mathrm{C}$ & -3.50812100 & -1.50380800 & -1.59233300 \\
\hline $\mathrm{C}$ & -4.79212800 & -1.22346500 & -1.17825800 \\
\hline $\mathrm{C}$ & -1.39441300 & -2.83784800 & -1.34736300 \\
\hline $\mathrm{C}$ & 1.44389300 & -3.01300000 & -1.29631100 \\
\hline $\mathrm{C}$ & -0.60818100 & -1.94649300 & -2.07351900 \\
\hline $\mathrm{C}$ & 0.78497300 & -2.00393000 & -2.05444900 \\
\hline $\mathrm{C}$ & -0.74117100 & -3.92274300 & -0.69711300 \\
\hline $\mathrm{C}$ & 0.65267900 & -3.99161300 & -0.69454200 \\
\hline $\mathrm{O}$ & 7.69083000 & -2.96002700 & 0.42039400 \\
\hline $\mathrm{C}$ & 8.88977500 & -2.77494000 & 1.14160700 \\
\hline $\mathrm{O}$ & 7.11316500 & 1.83656300 & 2.35499300 \\
\hline $\mathrm{C}$ & 6.56239200 & 2.26397200 & 3.58109900 \\
\hline $\mathrm{H}$ & -1.82703600 & 0.96216600 & 0.40435300 \\
\hline $\mathrm{H}$ & -3.87948800 & 0.00642200 & 1.34585000 \\
\hline $\mathrm{H}$ & -3.93435000 & 4.56934000 & -0.54478200 \\
\hline $\mathrm{H}$ & -5.99117000 & 3.62678400 & 0.44914900 \\
\hline $\mathrm{H}$ & -7.64632900 & 1.63367700 & -0.35513700 \\
\hline $\mathrm{H}$ & -7.93312600 & -0.65936800 & -1.18985500 \\
\hline $\mathrm{H}$ & -5.86749300 & 0.11136500 & 3.29355900 \\
\hline $\mathrm{H}$ & 5.90662300 & 3.43399000 & 0.89714100 \\
\hline $\mathrm{H}$ & 3.87918300 & 4.44306200 & -0.10134000 \\
\hline
\end{tabular}




\begin{tabular}{|c|c|c|c|}
\hline $\mathrm{H}$ & 3.84507100 & -0.30456900 & 1.26270400 \\
\hline $\mathrm{F}$ & 1.83898600 & 0.69315100 & 0.26702000 \\
\hline & 1.19123600 & 1.50662200 & -1.95723400 \\
\hline & -1.22890300 & 1.64764800 & -2.08517700 \\
\hline & 1.23289500 & 4.64289400 & 1.06060800 \\
\hline & -1.20513900 & 4.75485400 & 0.96275300 \\
\hline & 7.63379300 & 1.47781200 & -0.06539900 \\
\hline $\mathrm{H}$ & 7.97428600 & -0.75970200 & -1.01943900 \\
\hline $\mathrm{H}$ & 5.74912200 & -0.28105600 & 3.42324600 \\
\hline $\mathrm{H}$ & 6.15681300 & -2.49306600 & 2.50994000 \\
\hline $\mathrm{H}$ & 5.42841500 & -4.19515700 & 0.78675600 \\
\hline $\mathrm{H}$ & 5.33701800 & -0.56816300 & -1.48554300 \\
\hline $\mathrm{H}$ & -5.37239500 & -3.95513400 & 0.73056200 \\
\hline $\mathrm{H}$ & -3.01145300 & -0.78497200 & -2.23434200 \\
\hline $\mathrm{H}$ & -5.25825300 & -0.29770400 & -1.49493200 \\
\hline $\mathrm{H}$ & 9.47370300 & -1.92922100 & 0.75484700 \\
\hline $\mathrm{H}$ & 8.70449800 & -2.60324000 & 2.21072300 \\
\hline $\mathrm{H}$ & 9.46448700 & -3.69502300 & 1.01964100 \\
\hline $\mathrm{H}$ & 6.70241100 & 1.51856700 & 4.37526900 \\
\hline $\mathrm{H}$ & 5.49322500 & 2.49771400 & 3.49315200 \\
\hline $\mathrm{H}$ & 7.10071300 & 3.17244000 & 3.85997100 \\
\hline 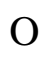 & -7.62972200 & -2.78177400 & 0.36275100 \\
\hline $\mathrm{O}$ & -7.08081500 & 2.16373100 & 2.15247900 \\
\hline C & -1.97166300 & 4.36562000 & -1.74654700 \\
\hline $\mathrm{C}$ & 1.98382700 & 4.31119400 & -1.58431800 \\
\hline C & -8.36422800 & 1.82151600 & 2.62466100 \\
\hline $\mathrm{H}$ & -9.04521600 & 1.54229700 & 1.80882900 \\
\hline
\end{tabular}




\begin{tabular}{|c|c|c|c|}
\hline $\mathrm{H}$ & -8.75287500 & 2.71016600 & 3.12586300 \\
\hline 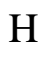 & -8.33020100 & 0.99037300 & 3.34226200 \\
\hline $\mathrm{C}$ & -8.84953200 & -2.60495800 & 1.04826900 \\
\hline 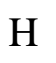 & -6.17401100 & -2.18163100 & 2.46833800 \\
\hline $\mathrm{H}$ & -8.69550600 & -2.39846100 & 2.11643400 \\
\hline $\mathrm{H}$ & -9.44378300 & -1.78525100 & 0.62182800 \\
\hline $\mathrm{H}$ & -9.40010800 & -3.54135400 & 0.93977600 \\
\hline $\mathrm{C}$ & 2.63405200 & 3.84081400 & -2.74390000 \\
\hline $\mathrm{H}$ & 3.42399000 & 3.11390400 & -2.50895500 \\
\hline $\mathrm{H}$ & 1.92615300 & 3.38443700 & -3.44910800 \\
\hline $\mathrm{H}$ & 3.08770300 & 4.71505800 & -3.21580900 \\
\hline $\mathrm{C}$ & -1.01171600 & 5.08464400 & -2.48981600 \\
\hline $\mathrm{H}$ & -0.33058600 & 5.65632000 & -1.85029700 \\
\hline $\mathrm{H}$ & -0.40742300 & 4.42494300 & -3.12530000 \\
\hline $\mathrm{H}$ & -1.58479200 & 5.76689300 & -3.12151700 \\
\hline $\mathrm{H}$ & 3.14387600 & -4.70201400 & 0.10112900 \\
\hline $\mathrm{H}$ & -1.06812100 & -1.13199800 & -2.62435200 \\
\hline $\mathrm{H}$ & 1.11314000 & -4.80313700 & -0.13956600 \\
\hline $\mathrm{C}$ & 1.56263100 & -0.98717200 & -2.71419800 \\
\hline $\mathrm{H}$ & 1.04645800 & -0.29168800 & -3.37170700 \\
\hline $\mathrm{C}$ & -1.52881000 & -4.89222500 & 0.02401200 \\
\hline $\mathrm{H}$ & -1.02076100 & -5.75140800 & 0.45402600 \\
\hline $\mathrm{C}$ & 2.88556700 & -0.86375700 & -2.47510300 \\
\hline $\mathrm{H}$ & 3.45921500 & -0.05588200 & -2.92180300 \\
\hline $\mathrm{C}$ & -2.85618400 & -4.72000100 & 0.19937700 \\
\hline $\mathrm{H}$ & -3.43668100 & -5.43820900 & 0.77235700 \\
\hline
\end{tabular}

benzo[k]tetraphene, $\omega B 97 D / 6-31 G(d)($ Energy $=-846.5335604$ Hartrees $)$ 


\begin{tabular}{|c|c|c|c|}
\hline $\mathrm{C}$ & -4.31612300 & 1.37161000 & -0.47272800 \\
\hline $\mathrm{C}$ & -2.90955100 & 1.32048700 & -0.41538700 \\
\hline $\mathrm{C}$ & -2.17562700 & 2.49768700 & -0.13518200 \\
\hline $\mathrm{C}$ & -2.89237900 & 3.69114200 & 0.08032200 \\
\hline $\mathrm{C}$ & -4.27084100 & 3.72158900 & 0.02022100 \\
\hline $\mathrm{C}$ & -4.99297600 & 2.55273700 & -0.25918500 \\
\hline $\mathrm{H}$ & -4.86267500 & 0.45714100 & -0.68927900 \\
\hline $\mathrm{H}$ & -2.36331400 & 4.61248800 & 0.29799400 \\
\hline $\mathrm{H}$ & -4.79562400 & 4.65688800 & 0.18994600 \\
\hline $\mathrm{H}$ & -6.07735900 & 2.57949700 & -0.30722900 \\
\hline $\mathrm{C}$ & -0.71606100 & 2.42803100 & -0.08117600 \\
\hline $\mathrm{C}$ & 0.09273600 & 3.53180500 & 0.18377100 \\
\hline $\mathrm{C}$ & -0.07262100 & 1.17786200 & -0.30795700 \\
\hline $\mathrm{C}$ & 1.48517600 & 3.44472600 & 0.23151400 \\
\hline $\mathrm{H}$ & -0.35156300 & 4.50592100 & 0.36218900 \\
\hline $\mathrm{C}$ & 1.31981900 & 1.09078600 & -0.26021300 \\
\hline $\mathrm{C}$ & 2.12861500 & 2.19456200 & 0.00475200 \\
\hline $\mathrm{H}$ & 1.76411400 & 0.11667100 & -0.43863100 \\
\hline $\mathrm{C}$ & 3.58817500 & 2.12490600 & 0.05880500 \\
\hline $\mathrm{C}$ & 4.30494200 & 0.93144400 & -0.15664000 \\
\hline $\mathrm{C}$ & 4.32208200 & 3.30210700 & 0.33902100 \\
\hline $\mathrm{C}$ & 5.68339800 & 0.90100400 & -0.09646200 \\
\hline $\mathrm{H}$ & 3.77589600 & 0.01009000 & -0.37431600 \\
\hline $\mathrm{C}$ & 5.72864900 & 3.25099400 & 0.39644300 \\
\hline $\mathrm{C}$ & 6.40551500 & 2.06986300 & 0.18296900 \\
\hline $\mathrm{H}$ & 6.20819900 & -0.03429400 & -0.26613600 \\
\hline $\mathrm{H}$ & 6.27518400 & 4.16547300 & 0.61300400 \\
\hline
\end{tabular}




$\begin{array}{llll}\mathrm{H} & 7.48989500 & 2.04310000 & 0.23107200 \\ \mathrm{C} & 2.28010000 & 4.61310500 & 0.51150800 \\ \mathrm{C} & 3.62709000 & 4.54449300 & 0.56311100 \\ \mathrm{H} & 1.76740000 & 5.55639800 & 0.68044100 \\ \mathrm{H} & 4.21699900 & 5.43233800 & 0.77535000 \\ \mathrm{C} & -2.21455000 & 0.07809500 & -0.63952100 \\ \mathrm{H} & -2.80442900 & -0.80964600 & -0.85228400 \\ \mathrm{C} & -0.86757100 & 0.00947600 & -0.58792200 \\ \mathrm{H} & -0.35485600 & -0.93370900 & -0.75742100\end{array}$

13 open adduct, $\omega B 97 D / 6-31 G(d)($ Energy $=-3537.218441$ Hartrees $)$

$\begin{array}{llll}\text { C } & 5.86823400 & -4.23515100 & -0.20488500\end{array}$

$\begin{array}{llll}\text { C } & 4.84307800 & -3.60171300 & -0.91167600\end{array}$

$\begin{array}{llll}\text { C } & 3.71962600 & -4.30830100 & -1.31938500\end{array}$

C $\quad 3.60212500 \quad-5.67202400 \quad-1.04692700$

$\begin{array}{llll}\text { C } & 5.75179200 & -5.59696000 & 0.06010400\end{array}$

$\begin{array}{llll}\text { C } & 4.63084500 & -6.30805100 & -0.35802900\end{array}$

$\begin{array}{llll}\text { C } & 2.34059000 & -6.43271100 & -1.46610700\end{array}$

$\begin{array}{llll}\text { C } & 0.03337600 & -5.15602500 & -2.94644200\end{array}$

$\begin{array}{llll}\text { C } & 1.14930600 & -5.80026800 & -0.79210200\end{array}$

C $\quad 0.14587600 \quad-5.22097300 \quad-1.44138000$

$\begin{array}{llll}\text { C } & 2.19985600 & -6.42545700 & -2.97134100\end{array}$

$\begin{array}{llll}\text { C } & 1.18170800 & -5.86884400 & -3.62135200\end{array}$

$\begin{array}{llll}\text { C } & 6.59564000 & 2.23958400 & -3.15068600\end{array}$

$\begin{array}{llll}\text { C } & 7.81927700 & 2.46588700 & -2.51743600\end{array}$

$\begin{array}{llll}\text { C } & 8.40608900 & 1.48309000 & -1.72687200\end{array}$

$\begin{array}{lllll}\text { C } & 7.77155400 & 0.25612100 & -1.54064300\end{array}$ 


\begin{tabular}{|c|c|c|c|}
\hline $\mathrm{C}$ & 6.02213200 & 0.97247600 & -3.04882900 \\
\hline $\mathrm{C}$ & 6.59724700 & -0.00162700 & -2.24510800 \\
\hline $\mathrm{C}$ & 8.28573200 & -0.78993000 & -0.54641600 \\
\hline $\mathrm{C}$ & 7.09759300 & -3.45011000 & 0.24675600 \\
\hline $\mathrm{C}$ & 7.22202300 & -0.96315400 & 0.52080800 \\
\hline $\mathrm{C}$ & 6.68532800 & -2.13210300 & 0.85410000 \\
\hline $\mathrm{C}$ & 8.57193600 & -2.08860500 & -1.26197400 \\
\hline $\mathrm{C}$ & 8.03746200 & -3.25599600 & -0.92160500 \\
\hline $\mathrm{C}$ & 5.85967100 & 3.41813400 & -3.81379800 \\
\hline $\mathrm{C}$ & 2.99154400 & 3.57173900 & -2.91291700 \\
\hline $\mathrm{C}$ & 5.36936600 & 4.31065800 & -2.69206500 \\
\hline $\mathrm{C}$ & 4.10777900 & 4.36488400 & -2.27893800 \\
\hline $\mathrm{C}$ & 4.69921500 & 2.93943600 & -4.65190500 \\
\hline $\mathrm{C}$ & 3.43805200 & 2.99278200 & -4.23786500 \\
\hline $\mathrm{O}$ & 1.83972700 & 4.38387000 & -3.09485900 \\
\hline $\mathrm{C}$ & 2.00099800 & 5.46556400 & -3.98759100 \\
\hline $\mathrm{O}$ & 6.76804800 & 4.23602600 & -4.54733300 \\
\hline $\mathrm{C}$ & 7.40186300 & 3.62586500 & -5.64779000 \\
\hline $\mathrm{H}$ & 4.90515400 & -2.53592800 & -1.11735900 \\
\hline $\mathrm{H}$ & 2.90922000 & -3.78384000 & -1.81778300 \\
\hline $\mathrm{H}$ & 6.53806600 & -6.09826600 & 0.61163400 \\
\hline $\mathrm{H}$ & 4.54400600 & -7.36414900 & -0.13596100 \\
\hline $\mathrm{H}$ & 1.17009400 & -5.83064000 & 0.29251200 \\
\hline $\mathrm{H}$ & -0.67064400 & -4.75725000 & -0.89268500 \\
\hline $\mathrm{H}$ & 2.99866600 & -6.93033300 & -3.51046600 \\
\hline $\mathrm{H}$ & 8.30355900 & 3.43179600 & -2.63112900 \\
\hline $\mathrm{H}$ & 9.35618600 & 1.67291400 & -1.23945000 \\
\hline
\end{tabular}




\begin{tabular}{|c|c|c|c|}
\hline $\mathrm{H}$ & 5.08229500 & 0.76088200 & -3.54668100 \\
\hline $\mathrm{H}$ & 6.11490600 & -0.97033400 & -2.14886400 \\
\hline $\mathrm{H}$ & 6.87424300 & -0.05180800 & 1.00191700 \\
\hline $\mathrm{H}$ & 5.92388400 & -2.18759900 & 1.62970400 \\
\hline H & 9.28068200 & -2.01409500 & -2.08259400 \\
\hline H & 8.30073500 & -4.16587400 & -1.45513300 \\
\hline H & 6.14799500 & 4.89109500 & -2.20395300 \\
\hline $\mathrm{H}$ & 3.81904800 & 4.99410300 & -1.44051100 \\
\hline $\mathrm{H}$ & 4.93508800 & 2.46287300 & -5.60047600 \\
\hline $\mathrm{H}$ & 2.63117200 & 2.58637400 & -4.83712700 \\
\hline $\mathrm{H}$ & 2.74000000 & 6.19024300 & -3.61964000 \\
\hline $\mathrm{H}$ & 2.30776700 & 5.12922400 & -4.98572100 \\
\hline $\mathrm{H}$ & 1.02370300 & 5.94655300 & -4.06393300 \\
\hline $\mathrm{H}$ & 6.71720600 & 3.49158100 & -6.49618900 \\
\hline $\mathrm{H}$ & 7.83858100 & 2.65307800 & -5.38453600 \\
\hline $\mathrm{H}$ & 8.20231100 & 4.30251400 & -5.95477300 \\
\hline $\mathrm{O}$ & -1.20937600 & -5.72961300 & -3.37029200 \\
\hline $\mathrm{O}$ & 2.52157800 & -7.76978800 & -1.00686300 \\
\hline $\mathrm{O}$ & 7.74028500 & -4.25773500 & 1.23643800 \\
\hline $\mathrm{O}$ & 9.54950800 & -0.39682500 & -0.00337500 \\
\hline C & 1.44042700 & -8.64322200 & -1.22861900 \\
\hline $\mathrm{H}$ & 0.52383400 & -8.29335000 & -0.73626500 \\
\hline $\mathrm{H}$ & 1.73330200 & -9.60339600 & -0.79889600 \\
\hline $\mathrm{H}$ & 1.23403000 & -8.77729200 & -2.29908200 \\
\hline $\mathrm{H}$ & 1.12714600 & -5.90695600 & -4.70751000 \\
\hline C & 9.53723600 & 0.38754000 & 1.16814000 \\
\hline & 8.94943500 & 1.30940000 & 1.05462000 \\
\hline
\end{tabular}




\begin{tabular}{|c|c|c|c|}
\hline $\mathrm{H}$ & 9.15292100 & -0.17198300 & 2.03088000 \\
\hline $\mathrm{H}$ & 10.57867000 & 0.65711100 & 1.35703700 \\
\hline $\mathrm{C}$ & 8.86349900 & -3.67930600 & 1.86614300 \\
\hline $\mathrm{H}$ & 9.56862700 & -3.24935000 & 1.14378800 \\
\hline $\mathrm{H}$ & 8.57093600 & -2.89400100 & 2.57563500 \\
\hline $\mathrm{H}$ & 9.35266100 & -4.48898500 & 2.41178900 \\
\hline C & 1.79594900 & 0.09302800 & -0.56947200 \\
\hline C & 3.12908500 & 0.54385800 & -0.58215400 \\
\hline C & 3.49067500 & 1.68088600 & -1.27197900 \\
\hline $\mathrm{C}$ & 2.55052800 & 2.40124900 & -2.02846200 \\
\hline $\mathrm{C}$ & 1.23465400 & 1.98369000 & -2.01772900 \\
\hline $\mathrm{C}$ & 0.83635800 & 0.85954100 & -1.26960900 \\
\hline $\mathrm{C}$ & 1.36416500 & -1.10118200 & 0.15112700 \\
\hline $\mathrm{C}$ & 2.24872300 & -1.97554800 & 0.77781500 \\
\hline $\mathrm{C}$ & 1.81647100 & -3.10978000 & 1.46737900 \\
\hline $\mathrm{C}$ & 0.42398900 & -3.38295100 & 1.57965000 \\
\hline $\mathrm{C}$ & -0.46011000 & -2.52036300 & 0.93268100 \\
\hline $\mathrm{C}$ & -0.02572800 & -1.40813700 & 0.21133500 \\
\hline $\mathrm{H}$ & 3.89753200 & 0.01259000 & -0.02835200 \\
\hline $\mathrm{H}$ & 4.51963800 & 2.01815600 & -1.23316800 \\
\hline $\mathrm{H}$ & 0.49065500 & 2.53682600 & -2.58253000 \\
\hline $\mathrm{H}$ & 3.31903600 & -1.80774100 & 0.71683500 \\
\hline $\mathrm{H}$ & -1.52936600 & -2.70787900 & 0.96065700 \\
\hline 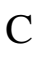 & -0.76919700 & -6.89933200 & 3.71174900 \\
\hline $\mathrm{C}$ & 0.56533700 & -6.59321000 & 3.55572500 \\
\hline C & 0.96919200 & -5.43136200 & 2.86862500 \\
\hline $\mathrm{C}$ & -0.00926500 & -4.56598400 & 2.32062900 \\
\hline
\end{tabular}




\begin{tabular}{|c|c|c|c|}
\hline $\mathrm{C}$ & -1.36652000 & -4.89988500 & 2.50002400 \\
\hline $\mathrm{C}$ & -1.74184400 & -6.04003800 & 3.18079100 \\
\hline $\mathrm{H}$ & 1.32989800 & -7.25081200 & 3.96157500 \\
\hline $\mathrm{H}$ & -2.14140700 & -4.25045800 & 2.10719200 \\
\hline $\mathrm{C}$ & -0.55453200 & 0.49624300 & -1.20025300 \\
\hline $\mathrm{H}$ & -1.26886500 & 1.10692800 & -1.74342700 \\
\hline $\mathrm{C}$ & -0.96676200 & -0.56982500 & -0.48408900 \\
\hline $\mathrm{H}$ & -2.02154400 & -0.82871000 & -0.44016000 \\
\hline $\mathrm{C}$ & 2.77079800 & -4.02476000 & 2.03754900 \\
\hline $\mathrm{H}$ & 3.82895500 & -3.82569800 & 1.89204400 \\
\hline $\mathrm{C}$ & 2.36861200 & -5.13141700 & 2.69767400 \\
\hline $\mathrm{H}$ & 3.09906100 & -5.82853100 & 3.09885000 \\
\hline $\mathrm{C}$ & -1.41509800 & -7.07658900 & -3.01415300 \\
\hline $\mathrm{H}$ & -1.42294100 & -7.22080200 & -1.92570400 \\
\hline $\mathrm{H}$ & -0.65139300 & -7.73673900 & -3.44678100 \\
\hline $\mathrm{H}$ & -2.39171000 & -7.34928700 & -3.41965200 \\
\hline $\mathrm{C}$ & -1.25948900 & -3.02512000 & -3.30166600 \\
\hline $\mathrm{C}$ & -0.05380200 & -3.69535100 & -3.39266000 \\
\hline $\mathrm{C}$ & 1.06474000 & -3.00075800 & -3.87925300 \\
\hline $\mathrm{C}$ & 0.95106300 & -1.69725200 & -4.31873500 \\
\hline $\mathrm{C}$ & -0.28128800 & -1.02339500 & -4.28949300 \\
\hline $\mathrm{C}$ & -1.39102900 & -1.69345200 & -3.72771500 \\
\hline $\mathrm{H}$ & -2.13071000 & -3.54442500 & -2.91485100 \\
\hline $\mathrm{H}$ & 2.02817600 & -3.49888000 & -3.92960400 \\
\hline $\mathrm{H}$ & 1.83963400 & -1.18977600 & -4.67938700 \\
\hline $\mathrm{C}$ & -0.45583700 & 0.33052300 & -4.80243900 \\
\hline $\mathrm{C}$ & -1.67981700 & 1.01348600 & -4.55406600 \\
\hline
\end{tabular}




$\begin{array}{lrrr}\mathrm{C} & 0.50187400 & 0.97763100 & -5.57957600 \\ \mathrm{C} & -1.82317000 & 2.34463900 & -4.95143500 \\ \mathrm{C} & 0.32288500 & 2.28037200 & -6.04788800 \\ \mathrm{H} & 1.40802400 & 0.45508000 & -5.87189600 \\ \mathrm{C} & -0.83211900 & 3.01843900 & -5.66543000 \\ \mathrm{H} & -2.76164800 & 2.83948000 & -4.72048000 \\ \mathrm{C} & -0.94217100 & 4.41888100 & -6.06673800 \\ \mathrm{C} & 0.03880500 & 4.96735000 & -6.92682000 \\ \mathrm{C} & -1.97314800 & 5.26271300 & -5.61008200 \\ \mathrm{C} & -0.05204400 & 6.31681000 & -7.31833000 \\ \mathrm{C} & -2.04058300 & 6.58598400 & -5.99752800 \\ \mathrm{H} & -2.72033400 & 4.88215800 & -4.92189300 \\ \mathrm{C} & -1.07639300 & 7.11973600 & -6.86448600 \\ \mathrm{H} & 0.70853100 & 6.72052600 & -7.98191500 \\ \mathrm{C} & -2.64207900 & -0.99345800 & -3.57455000 \\ \mathrm{C} & -2.76904800 & 0.30357800 & -3.92930000 \\ \mathrm{C} & 1.28778700 & 2.88203100 & -6.93292400 \\ \mathrm{C} & 1.14176000 & 4.15129300 & -7.36785100 \\ \mathrm{H} & -3.70790300 & 0.83002800 & -3.77812400 \\ \mathrm{H} & -3.47920500 & -1.52976200 & -3.13528500 \\ \mathrm{H} & 2.13733100 & 2.28287300 & -7.25161900 \\ \mathrm{H} & 1.87076100 & 4.59131000 & -8.04318000 \\ \mathrm{H} & -2.84176200 & 7.21642500 & -5.62385500 \\ \mathrm{H} & -1.13308000 & 8.16057800 & -7.16806900 \\ \mathrm{H} & -1.06650200 & -7.79812400 & 4.24350000 \\ \mathrm{H} & -2.79583700 & -6.26916000 & 3.30658400 \\ \mathrm{H} & & & \\ \mathrm{H} & & & \end{array}$




\begin{tabular}{|c|c|c|}
\hline $\mathrm{C}$ & $-2.75938-4.47731$ & 1.30882 \\
\hline $\mathrm{C}$ & $-3.71639-3.48653$ & 1.40409 \\
\hline $\mathrm{C}$ & $-4.26723-2.88926$ & 0.24781 \\
\hline $\mathrm{C}$ & $-4.01394-3.53267$ & -0.99624 \\
\hline $\mathrm{C}$ & $-3.06794-4.57779$ & -1.06317 \\
\hline $\mathrm{C}$ & $-2.31707-4.95916$ & 0.04978 \\
\hline $\mathrm{C}$ & $-4.82577-1.54102$ & 0.24555 \\
\hline $\mathrm{C}$ & $-4.88618-0.74136$ & 1.3932 \\
\hline $\mathrm{C}$ & $-4.89294 \quad 0.65646$ & 1.31825 \\
\hline $\mathrm{C}$ & $-4.91695 \quad 1.30141$ & 0.03664 \\
\hline $\mathrm{C}$ & $-5.15255 \quad 0.49276$ & -1.08417 \\
\hline $\mathrm{C}$ & $\begin{array}{ll}-5.081 & -0.90557\end{array}$ & -1.01457 \\
\hline $\mathrm{C}$ & $-4.44361 \quad 2.68005$ & -0.05695 \\
\hline $\mathrm{C}$ & $-3.9434 \quad 3.30906$ & 1.11746 \\
\hline $\mathrm{C}$ & $-3.03104 \quad 4.37782$ & 1.00008 \\
\hline $\mathrm{C}$ & $-2.59443 \quad 4.84288$ & -0.2392 \\
\hline $\mathrm{C}$ & $-3.3215 \quad 4.39733$ & -1.37478 \\
\hline $\mathrm{C}$ & $-4.21521 \quad 3.34718$ & -1.28426 \\
\hline $\mathrm{C}$ & $-4.5677 \quad-2.96819$ & -2.20403 \\
\hline $\mathrm{C}$ & $-5.07314-1.7075$ & -2.21319 \\
\hline $\mathrm{C}$ & $-4.67013 \quad 1.44618$ & 2.5028 \\
\hline $\mathrm{C}$ & $-4.20177 \quad 2.71585$ & 2.4065 \\
\hline $\mathrm{H}$ & $-2.25059-4.79283$ & 32.21387 \\
\hline $\mathrm{H}$ & $-3.94302-3.08352$ & 2.3857 \\
\hline $\mathrm{H}$ & $-2.82999-4.9798$ & -2.04466 \\
\hline $\mathrm{H}$ & $-4.76845-1.18752$ & 2.37609 \\
\hline $\mathrm{H}$ & $\begin{array}{lll}-5.24174 & 0.94104\end{array}$ & -2.06951 \\
\hline $\mathrm{H}$ & $-3.11558 \quad 4.82815$ & -2.35036 \\
\hline $\mathrm{H}$ & $-4.67449 \quad 2.97794$ & -2.19614 \\
\hline $\mathrm{H}$ & $-4.48339-3.53627$ & -3.12744 \\
\hline $\mathrm{H}$ & $-5.39971-1.25001$ & -3.14412 \\
\hline
\end{tabular}




\begin{tabular}{|c|c|c|c|}
\hline $\mathrm{H}$ & -4.78675 & 0.96942 & $2 \quad 3.47307$ \\
\hline $\mathrm{H}$ & -3.93442 & 3.27809 & $9 \quad 3.29795$ \\
\hline U & -2.53282 & 24.70659 & 91.90758 \\
\hline $\mathrm{C}$ & 1.61153 & 5.4888 & -0.28452 \\
\hline $\mathrm{C}$ & 0.91889 & 5.16599 & -1.46448 \\
\hline $\mathrm{C}$ & -0.47027 & 5.14288 & $8-1.50175$ \\
\hline $\mathrm{C}$ & -1.23878 & 5.44523 & $3-0.36259$ \\
\hline $\mathrm{C}$ & -0.54644 & 6.00583 & $\begin{array}{ll}3 & 0.72994\end{array}$ \\
\hline $\mathrm{C}$ & 0.84545 & 6.02112 & 20.77098 \\
\hline $\mathrm{H}$ & 1.466 & 4.77168 & -2.3155 \\
\hline $\mathrm{H}$ & -0.9501 & 4.72933 & -2.38264 \\
\hline $\mathrm{H}$ & -1.09795 & 6.35455 & $\begin{array}{ll}5 & 1.59898\end{array}$ \\
\hline $\mathrm{H}$ & 1.34175 & 6.37683 & 31.67042 \\
\hline $\mathrm{C}$ & 1.91501 & -5.41503 & $3-0.01686$ \\
\hline $\mathrm{C}$ & 1.19917 & -5.17205 & $5-1.2029$ \\
\hline $\mathrm{C}$ & -0.18916 & -5.21068 & $8-1.22685$ \\
\hline $\mathrm{C}$ & -0.93576 & -5.5033 & -0.06996 \\
\hline $\mathrm{C}$ & -0.20928 & -5.98977 & $\begin{array}{ll}7 & 1.03579\end{array}$ \\
\hline $\mathrm{C}$ & 1.18278 & -5.94049 & $\begin{array}{ll}9 & 1.06467\end{array}$ \\
\hline $\mathrm{H}$ & 1.72109 & -4.78702 & $\begin{array}{lll}2 & -2.07374\end{array}$ \\
\hline $\mathrm{H}$ & -0.69313 & $3-4.85107$ & $7-2.11783$ \\
\hline $\mathrm{H}$ & -0.73555 & -6.33025 & $\begin{array}{ll}5 & 1.92333\end{array}$ \\
\hline $\mathrm{H}$ & 1.70113 & -6.23615 & $5 \quad 1.97335$ \\
\hline $\mathrm{C}$ & 5. & $.93357 \quad 0$ & 0.04119 \\
\hline $\mathrm{C}$ & 4.27151 & 3.31686 & $6 \quad 1.18161$ \\
\hline $\mathrm{C}$ & 3.28606 & 4.29578 & 1.11279 \\
\hline $\mathrm{C}$ & 2.97955 & 4.93716 & $5-0.10082$ \\
\hline $\mathrm{C}$ & 3.86334 & 4.71724 & $4-1.17511$ \\
\hline $\mathrm{C}$ & 4.85155 & 3.73879 & - -1.10514 \\
\hline $\mathrm{H}$ & 4.36381 & 2.73819 & $9 \quad 2.09574$ \\
\hline $\mathrm{H}$ & 2.64297 & 4.44699 & $\begin{array}{ll}9 & 1.9747\end{array}$ \\
\hline
\end{tabular}




\begin{tabular}{|c|c|c|c|}
\hline $\mathrm{H}$ & 3.72446 & 5.26111 & -2.10602 \\
\hline $\mathbf{H}$ & 5.46267 & 3.54426 & -1.98273 \\
\hline $\mathrm{C}$ & 5.16268 & -2.67292 & 0.1737 \\
\hline $\mathrm{C}$ & 4.46165 & -3.04479 & 1.33493 \\
\hline $\mathrm{C}$ & 3.53093 & -4.07792 & 1.31582 \\
\hline $\mathrm{C}$ & 3.25414 & -4.78745 & 0.13345 \\
\hline $\mathrm{C}$ & 4.12015 & -4.56808 & $3-0.95528$ \\
\hline $\mathrm{C}$ & 5.05456 & -3.536 & -0.93452 \\
\hline $\mathrm{H}$ & 4.52536 & -2.42187 & $7 \quad 2.22198$ \\
\hline $\mathrm{H}$ & 2.90049 & -4.22536 & $6 \quad 2.18766$ \\
\hline $\mathrm{H}$ & 4.00672 & -5.15886 & $6-1.86071$ \\
\hline $\mathrm{H}$ & 5.65087 & -3.34807 & $\begin{array}{ll}7 & -1.82364\end{array}$ \\
\hline $\mathrm{C}$ & 5.67176 & -1.28067 & $7 \quad 0.05989$ \\
\hline $\mathrm{C}$ & 5.46091 & -0.5838 & -1.14362 \\
\hline $\mathrm{C}$ & 5.4201 & 0.8052 & -1.17629 \\
\hline $\mathrm{C}$ & 5.58813 & 1.56885 & -0.00719 \\
\hline $\mathrm{C}$ & 6.01891 & 0.87871 & 1.14254 \\
\hline $\mathrm{C}$ & 6.06005 & -0.5131 & 1.17522 \\
\hline $\mathrm{H}$ & 5.17295 & -1.13086 & $\begin{array}{ll}6 & -2.03618\end{array}$ \\
\hline $\mathrm{H}$ & 5.10175 & 1.29241 & $1-2.09284$ \\
\hline $\mathrm{H}$ & 6.25215 & 1.4332 & 2.04789 \\
\hline 11 & 6.32449 & -1.00963 & $\begin{array}{ll}3 & 2.10528\end{array}$ \\
\hline
\end{tabular}

biphenyl, $\omega B 97 D / 6-31 G(d)($ Energy $=-463.1443755$ Hartrees $)$
C
$3.56375-0.00035-0.00012$
C
$2.8595-1.13878-0.39644$
C
$1.46541-1.13773-0.39666$
C
$\begin{array}{lll}0.74287 & 0.00039 & 0.00011\end{array}$
C
$\begin{array}{lll}1.46601 & 1.13813 & 0.39671\end{array}$
C
$\begin{array}{lll}2.86014 & 1.13839 & 0.39635\end{array}$
$\mathrm{H}$
$3.39651-2.02833-0.71551$ 


$\begin{array}{llll}\mathrm{H} & 0.92763 & -2.01997 & -0.73285 \\ \mathrm{H} & 0.92888 & 2.02077 & 0.73284 \\ \mathrm{H} & 3.3976 & 2.02771 & 0.71531 \\ \mathrm{C} & -0.74287 & 0.0004 & -0.00006 \\ \mathrm{C} & -1.46543 & -1.13772 & 0.3967 \\ \mathrm{C} & -2.85951 & -1.13876 & 0.39644 \\ \mathrm{C} & -3.56375 & -0.00035 & 0.00005 \\ \mathrm{C} & -2.86012 & 1.1384 & -0.39636 \\ \mathrm{C} & -1.466 & 1.13813 & -0.39668 \\ \mathrm{H} & -0.92765 & -2.01996 & 0.73288 \\ \mathrm{H} & -3.39653 & -2.02832 & 0.7155 \\ \mathrm{H} & -4.65042 & -0.00065 & 0.00012 \\ \mathrm{H} & -3.39757 & 2.02772 & -0.71532 \\ \mathrm{H} & -0.92885 & 2.02078 & -0.73279 \\ \mathrm{H} & 4.65042 & -0.00064 & -0.00031 \\ & & & \end{array}$

1 open adduct, $\omega B 97 D / 6-31 G(d)$ (Energy = -2463.395471 Hartrees)
C $\quad-1.10511 \quad 1.2807 \quad-0.20774$
C $\quad-2.48718 \quad 1.29218-0.20548$
C $\quad-3.23867 \quad 0.1084-0.03913$
C $\quad-2.51778-1.10953 \quad 0.12597$
C $\quad-1.10843-1.09645 \quad 0.12132$
C $\quad-0.37898 \quad 0.0774 \quad-0.04314$
C $\quad-4.69794 \quad 0.08005 \quad-0.02922$
C $\quad-5.49226 \quad 1.22058-0.18308$
$\begin{array}{llll}\text { C } & -6.8921 & 1.173 & -0.1703\end{array}$
$\begin{array}{llll}\text { C } & -7.56371 & -0.08263 & 0.00552\end{array}$
C $\quad-6.76938-1.22312 \quad 0.15964$
C $\quad-5.36956-1.17543 \quad 0.14763$
$\begin{array}{llll}\text { C } & -9.02299 & -0.11106 & 0.01554\end{array}$
$\begin{array}{llll}\text { C } & -9.74394 & 1.10743 & -0.14494\end{array}$ 


\begin{tabular}{|c|c|}
\hline $\mathrm{C}$ & $\begin{array}{lll}-11.15334 & 1.09467 & -0.13777\end{array}$ \\
\hline $\mathrm{C}$ & $\begin{array}{lll}-11.88256 & -0.07977 & 0.02313\end{array}$ \\
\hline $\mathrm{C}$ & $\begin{array}{llll}-11.15644 & -1.28395 & 0.18114\end{array}$ \\
\hline $\mathrm{C}$ & $\begin{array}{lll}-9.77435 & -1.29547 & 0.17765\end{array}$ \\
\hline $\mathrm{C}$ & $-3.23654-2.3441 \quad 0.30473$ \\
\hline $\mathrm{C}$ & $\begin{array}{llll}-4.59373 & -2.37511 & 0.31342\end{array}$ \\
\hline $\mathrm{C}$ & $\begin{array}{llll}-7.66799 & 2.37305 & -0.33313\end{array}$ \\
\hline $\mathrm{C}$ & $\begin{array}{llll}-9.02519 & 2.34228 & -0.32187\end{array}$ \\
\hline $\mathrm{H}$ & $\begin{array}{lll}-0.5664 & 2.21031 & -0.3669\end{array}$ \\
\hline $\mathrm{H}$ & $\begin{array}{llll}-2.99452 & 2.24084 & -0.34618\end{array}$ \\
\hline $\mathrm{H}$ & $\begin{array}{lll}-0.58589 & -2.03657 & 0.27951\end{array}$ \\
\hline $\mathrm{H}$ & $\begin{array}{llll}-5.02889 & 2.19295 & -0.31887\end{array}$ \\
\hline $\mathrm{H}$ & $\begin{array}{lll}-7.23275 & -2.19537 & 0.29628\end{array}$ \\
\hline $\mathrm{H}$ & $\begin{array}{lll}-11.69519 & -2.21418 & 0.33649\end{array}$ \\
\hline $\mathrm{H}$ & $\begin{array}{lll}-9.26696 & -2.24481 & 0.31353\end{array}$ \\
\hline $\mathrm{H}$ & $-2.66257 \quad-3.2587 \quad 0.43234$ \\
\hline $\mathrm{H}$ & $-5.12363 \quad-3.3151 \quad 0.44782$ \\
\hline $\mathrm{H}$ & $\begin{array}{llll}-7.13817 & 3.31321 & -0.46666\end{array}$ \\
\hline $\mathrm{H}$ & $\begin{array}{llll}-9.59919 & 3.25728 & -0.4465\end{array}$ \\
\hline $\mathrm{H}$ & $\begin{array}{llll}-11.67614 & 2.03545 & -0.29117\end{array}$ \\
\hline $\mathrm{C}$ & $\begin{array}{lll}-16.21409 & -0.06415 & 0.02995\end{array}$ \\
\hline $\mathrm{C}$ & $\begin{array}{lll}-15.48982 & -1.12172 & -0.54518\end{array}$ \\
\hline $\mathrm{C}$ & $\begin{array}{lll}-14.09859 & -1.12746 & -0.54604\end{array}$ \\
\hline $\mathrm{C}$ & $\begin{array}{lll}-13.36533 & -0.07517 & 0.0279\end{array}$ \\
\hline $\mathrm{C}$ & $\begin{array}{lll}-14.08964 & 0.98154 & 0.60517\end{array}$ \\
\hline $\mathrm{C}$ & $\begin{array}{lll}-15.48068 & 0.98734 & 0.60504\end{array}$ \\
\hline $\mathrm{H}$ & $-16.02483-1.96006-0.98255$ \\
\hline $\mathrm{H}$ & $-13.57169-1.95353-1.01553$ \\
\hline $\mathrm{H}$ & $\begin{array}{lll}-13.55453 & 1.80155 & 1.07593\end{array}$ \\
\hline $\mathrm{H}$ & $\begin{array}{lll}-16.00854 & 1.82924 & 1.04425\end{array}$ \\
\hline $\mathrm{C}$ & $\begin{array}{llll}3.9531 & 0.06074 & -0.03692\end{array}$ \\
\hline
\end{tabular}




$\begin{array}{llll}\mathrm{C} & 3.22683 & 1.12839 & 0.51783 \\ \mathrm{C} & 1.83581 & 1.13452 & 0.51382 \\ \mathrm{C} & 1.10355 & 0.07281 & -0.04438 \\ \mathrm{C} & 1.82977 & -0.99362 & -0.60164 \\ \mathrm{C} & 3.22057 & -1.00005 & -0.59705 \\ \mathrm{H} & 3.75956 & 1.94842 & 0.99124 \\ \mathrm{H} & 1.30815 & 1.95951 & 0.98429 \\ \mathrm{H} & 1.29649 & -1.81217 & -1.07693 \\ \mathrm{H} & 3.74886 & -1.82398 & -1.06866 \\ \mathrm{C} & 14.10037 & 0.01151 & 0.00617 \\ \mathrm{C} & 14.82318 & -1.1787 & 0.19495 \\ \mathrm{C} & 16.21425 & -1.185 & 0.20328 \\ \mathrm{C} & 16.95002 & -0.00197 & 0.02111 \\ \mathrm{C} & 16.22742 & 1.18792 & -0.16894 \\ \mathrm{C} & 14.83633 & 1.19478 & -0.17523 \\ \mathrm{H} & 14.28748 & -2.11559 & 0.31998 \\ \mathrm{H} & 16.73992 & -2.12096 & 0.37075 \\ \mathrm{H} & 16.76367 & 2.11886 & -0.33077 \\ \mathrm{H} & 14.31082 & 2.13667 & -0.30581 \\ \mathrm{C} & 8.28505 & 0.03962 & -0.02028 \\ \mathrm{C} & 7.54917 & -1.14493 & 0.15348 \\ \mathrm{C} & 6.15824 & -1.13801 & 0.14836 \\ \mathrm{C} & 5.4355 & 0.05368 & -0.0319 \\ \mathrm{C} & 6.17137 & 1.23813 & -0.20646 \\ \mathrm{C} & 7.56231 & 1.23132 & -0.20021 \\ \mathrm{H} & 8.07499 & -2.08755 & 0.27735 \\ \mathrm{H} & 5.62222 & -2.07008 & 0.30431 \\ \mathrm{H} & 5.64588 & 2.17546 & -0.36671 \\ \mathrm{H} & 8.09836 & 2.16873 & -0.31969 \\ \mathrm{C} & 9.76741 & 0.03235 & -0.01378 \\ & 10.48913 & -0.82688 & 0.83243\end{array}$




\begin{tabular}{llll} 
C & 11.88007 & -0.83329 & 0.83895 \\
$\mathrm{C}$ & 12.61788 & 0.01856 & -0.00094 \\
$\mathrm{C}$ & 11.89599 & 0.87743 & -0.84746 \\
$\mathrm{C}$ & 10.50503 & 0.88446 & -0.85349 \\
$\mathrm{H}$ & 9.95291 & -1.47351 & 1.52152 \\
$\mathrm{H}$ & 12.40358 & -1.48465 & 1.5333 \\
$\mathrm{H}$ & 12.43204 & 1.52377 & -1.53691 \\
$\mathrm{H}$ & 9.98134 & 1.53622 & -1.54738 \\
$\mathrm{C}$ & -17.69835 & -0.05827 & 0.03098 \\
$\mathrm{C}$ & -18.42419 & -0.55236 & -1.06667 \\
$\mathrm{C}$ & -19.81811 & -0.54722 & -1.06595 \\
$\mathrm{C}$ & -20.51938 & -0.04661 & 0.03255 \\
$\mathrm{C}$ & -19.81278 & 0.44818 & 1.13026 \\
$\mathrm{C}$ & -18.41886 & 0.44178 & 1.12943 \\
$\mathrm{H}$ & -17.88952 & -0.91887 & -1.93859 \\
$\mathrm{H}$ & -20.35737 & -0.92708 & -1.92986 \\
$\mathrm{H}$ & -21.606 & -0.0421 & 0.03314 \\
$\mathrm{H}$ & -20.34791 & 0.83252 & 1.99475 \\
$\mathrm{H}$ & -17.8802 & 0.80393 & 2.00072 \\
$\mathrm{C}$ & 18.43419 & -0.00891 & 0.02935 \\
$\mathrm{C}$ & 19.15568 & -1.0762 & -0.53292 \\
$\mathrm{C}$ & 20.54957 & -1.08333 & -0.52458 \\
$\mathrm{C}$ & 21.25527 & -0.02201 & 0.04521 \\
$\mathrm{C}$ & 20.5531 & 1.04584 & 0.60709 \\
$\mathrm{C}$ & 19.15921 & 1.05167 & 0.59975 \\
$\mathrm{H}$ & 18.61778 & -1.89428 & -1.00398 \\
$\mathrm{H}$ & 21.08544 & -1.91608 & -0.9727 \\
$\mathrm{H}$ & 22.34188 & -0.02707 & 0.05132 \\
$\mathrm{H}$ & 21.09161 & 1.87359 & 1.06127 \\
$\mathrm{H}$ & 18.62366 & 1.8747 & 1.0648 \\
\hline & & &
\end{tabular}

12 open adduct, $\omega B 97 D / 6-31 G(d)($ Energy $=-4006.08345$ Hartrees) 


\begin{tabular}{|c|c|c|c|}
\hline $\mathrm{C}$ & 29.69520600 & -12.28729000 & -0.84704900 \\
\hline $\mathrm{C}$ & 29.42547500 & -13.10159800 & 0.25299600 \\
\hline $\mathrm{C}$ & 28.77978400 & -12.59618600 & 1.37661400 \\
\hline $\mathrm{C}$ & 28.37448600 & -11.26319800 & 1.42879500 \\
\hline $\mathrm{C}$ & 29.28890300 & -10.95298300 & -0.79910400 \\
\hline $\mathrm{C}$ & 28.62978400 & -10.45340500 & 0.31890900 \\
\hline $\mathrm{C}$ & 27.63201000 & -10.65307100 & 2.63450600 \\
\hline $\mathrm{C}$ & 25.22066900 & -12.32005900 & 3.05009400 \\
\hline $\mathrm{C}$ & 27.50346800 & -11.62399200 & 3.78568200 \\
\hline $\mathrm{C}$ & 26.40972900 & -12.34752500 & 3.98584800 \\
\hline $\mathrm{C}$ & 26.25037400 & -10.22593200 & 2.18641500 \\
\hline $\mathrm{C}$ & 25.16569900 & -10.97309300 & 2.36335200 \\
\hline $\mathrm{C}$ & 35.13848000 & -9.03800600 & -5.48268200 \\
\hline $\mathrm{C}$ & 35.53386400 & -10.37524400 & -5.55540300 \\
\hline $\mathrm{C}$ & 34.68529300 & -11.38958200 & -5.12987700 \\
\hline $\mathrm{C}$ & 33.42129300 & -11.09690900 & -4.61351100 \\
\hline $\mathrm{C}$ & 33.88299000 & -8.74711700 & -4.95417700 \\
\hline $\mathrm{C}$ & 33.03585400 & -9.76126600 & -4.52113100 \\
\hline $\mathrm{C}$ & 32.51888700 & -12.23126800 & -4.12010400 \\
\hline $\mathrm{C}$ & 30.45619500 & -12.87155700 & -2.04305700 \\
\hline $\mathrm{C}$ & 32.85729400 & -12.57035500 & -2.68457300 \\
\hline $\mathrm{C}$ & 31.94363100 & -12.85232400 & -1.76316800 \\
\hline $\mathrm{C}$ & 31.05506200 & -11.87309200 & -4.25918300 \\
\hline $\mathrm{C}$ & 30.14292100 & -12.14254200 & -3.33182600 \\
\hline $\mathrm{C}$ & 36.12683300 & -7.92401900 & -5.87330100 \\
\hline $\mathrm{C}$ & 35.87584400 & -5.34815500 & -4.31706300 \\
\hline $\mathrm{C}$ & 36.93816100 & -7.60245800 & -4.64260100 \\
\hline
\end{tabular}




\begin{tabular}{|c|c|c|c|}
\hline $\mathrm{C}$ & 36.82016200 & -6.47535800 & -3.95251500 \\
\hline $\mathrm{C}$ & 35.42659500 & -6.68732300 & -6.39162300 \\
\hline $\mathrm{C}$ & 35.33698800 & -5.54641500 & -5.71447600 \\
\hline $\mathrm{C}$ & 32.80348500 & -5.67858600 & -1.28530000 \\
\hline $\mathrm{C}$ & 34.12111900 & -5.34349200 & -0.92210200 \\
\hline $\mathrm{C}$ & 35.07247400 & -5.17772900 & -1.93166900 \\
\hline $\mathrm{C}$ & 34.74817500 & -5.32099200 & -3.27952600 \\
\hline $\mathrm{C}$ & 32.45727500 & -5.71489200 & -2.63301300 \\
\hline $\mathrm{C}$ & 33.41396800 & -5.53026200 & -3.62208800 \\
\hline $\mathrm{C}$ & 26.22612200 & -15.61520500 & 0.40987600 \\
\hline $\mathrm{C}$ & 25.96542500 & -15.84369700 & 1.77789500 \\
\hline $\mathrm{C}$ & 25.54819400 & -14.77284600 & 2.56869600 \\
\hline $\mathrm{C}$ & 25.43046200 & -13.47920400 & 2.05878600 \\
\hline $\mathrm{C}$ & 25.98917500 & -14.34628800 & -0.11714400 \\
\hline $\mathrm{C}$ & 25.60966900 & -13.28419000 & 0.69380300 \\
\hline $\mathrm{C}$ & 26.13345200 & -17.18904100 & 2.36827000 \\
\hline $\mathrm{C}$ & 26.50434700 & -17.43534500 & 3.62570300 \\
\hline $\mathrm{C}$ & 34.49890600 & -5.23877200 & 0.50163300 \\
\hline $\mathrm{C}$ & 35.47161300 & -4.46319400 & 0.98128200 \\
\hline $\mathrm{C}$ & 26.90089400 & -16.63875700 & -0.43560600 \\
\hline $\mathrm{C}$ & 28.12193000 & -17.13859400 & 0.01605900 \\
\hline $\mathrm{C}$ & 28.91372100 & -18.00045600 & -0.74272000 \\
\hline $\mathrm{C}$ & 26.43484200 & -17.02294400 & -1.70587000 \\
\hline $\mathrm{C}$ & 27.23835000 & -17.87062100 & -2.47653100 \\
\hline $\mathrm{C}$ & 25.14451800 & -16.52974200 & -2.22590800 \\
\hline $\mathrm{C}$ & 24.87182900 & -16.30562100 & -3.51252700 \\
\hline $\mathrm{C}$ & 30.17422500 & -18.54016400 & -0.19640200 \\
\hline
\end{tabular}




\begin{tabular}{|c|c|c|c|}
\hline $\mathrm{C}$ & 30.42860900 & -18.70737300 & 1.10240700 \\
\hline $\mathrm{C}$ & 31.77598700 & -6.04158300 & -0.27001600 \\
\hline $\mathrm{C}$ & 31.48626200 & -7.38731600 & 0.03291800 \\
\hline $\mathrm{C}$ & 30.46837600 & -7.62636400 & 0.96845800 \\
\hline $\mathrm{C}$ & 29.75902400 & -6.61041300 & 1.58284600 \\
\hline $\mathrm{C}$ & 30.05702600 & -5.27294100 & 1.29876500 \\
\hline $\mathrm{C}$ & 31.06870500 & -5.02251000 & 0.36699800 \\
\hline $\mathrm{C}$ & 32.13343800 & -8.58838600 & -0.53765100 \\
\hline $\mathrm{C}$ & 33.22367000 & -8.72974500 & -1.29821100 \\
\hline $\mathrm{C}$ & 29.35930000 & -4.13782500 & 1.92604400 \\
\hline $\mathrm{C}$ & 28.46677300 & -4.19779900 & 2.91574700 \\
\hline $\mathrm{O}$ & 28.27883800 & -9.43987700 & 3.03421500 \\
\hline $\mathrm{C}$ & 29.54856600 & -9.58822300 & 3.63276400 \\
\hline $\mathrm{O}$ & 32.82409900 & -13.43661900 & -4.83493800 \\
\hline $\mathrm{C}$ & 32.40159500 & -13.47820700 & -6.18058200 \\
\hline $\mathrm{O}$ & 30.14425400 & -14.26333100 & -2.17221500 \\
\hline $\mathrm{C}$ & 28.85185100 & -14.54091800 & -2.67052400 \\
\hline $\mathrm{O}$ & 37.08288100 & -8.41180600 & -6.81359700 \\
\hline $\mathrm{C}$ & 36.60283200 & -8.70198400 & -8.10752700 \\
\hline $\mathrm{O}$ & 24.08950500 & -12.54963700 & 3.88228100 \\
\hline $\mathrm{C}$ & 22.85986400 & -12.73389700 & 3.21981700 \\
\hline $\mathrm{O}$ & 36.53243200 & -4.08757200 & -4.20433100 \\
\hline $\mathrm{C}$ & 37.67358000 & -3.94267700 & -5.02269000 \\
\hline $\mathrm{H}$ & 29.70924200 & -14.14895800 & 0.21468100 \\
\hline $\mathrm{H}$ & 28.56266200 & -13.26106000 & 2.20676200 \\
\hline $\mathrm{H}$ & 29.49484500 & -10.29009900 & -1.63570200 \\
\hline $\mathrm{H}$ & 28.31641700 & -9.41404700 & 0.33114000 \\
\hline
\end{tabular}




\begin{tabular}{|c|c|c|c|}
\hline $\mathrm{H}$ & 28.35666700 & -11.74344900 & 4.44545200 \\
\hline $\mathrm{H}$ & 26.33544100 & -13.05051400 & 4.81053800 \\
\hline $\mathrm{H}$ & 26.20152900 & -9.26385200 & 1.68395600 \\
\hline $\mathrm{H}$ & 24.20402400 & -10.63368300 & 1.98968600 \\
\hline $\mathrm{H}$ & 36.51490700 & -10.61680400 & -5.95261200 \\
\hline $\mathrm{H}$ & 34.99644600 & -12.42699800 & -5.20394300 \\
\hline $\mathrm{H}$ & 33.56456000 & -7.71706800 & -4.85706800 \\
\hline I & 32.07439700 & -9.50056100 & -4.08732900 \\
\hline 1 & 33.91759100 & -12.63216600 & -2.45415000 \\
\hline 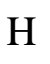 & 32.23941600 & -13.14770600 & -0.76010400 \\
\hline $\mathrm{H}$ & 30.75790000 & -11.38070800 & -5.18247900 \\
\hline $\mathrm{F}$ & 29.10256500 & -11.87680200 & -3.50468000 \\
\hline 1 & 37.61656900 & -8.39013000 & -4.32611600 \\
\hline $\mathrm{H}$ & 37.40789800 & -6.32123500 & -3.05111600 \\
\hline $\mathrm{F}$ & 34.96432500 & -6.76664700 & -7.37220400 \\
\hline $\mathrm{F}$ & 34.82922500 & -4.68556700 & -6.14506900 \\
\hline $\mathrm{H}$ & 36.10293200 & -4.98065400 & -1.65351800 \\
\hline $\mathrm{H}$ & 31.42950300 & -5.93583100 & -2.90604200 \\
\hline $\mathrm{H}$ & 33.11995200 & -5.59437300 & -4.66437100 \\
\hline $\mathrm{H}$ & 25.31972200 & -14.94841300 & 3.61624900 \\
\hline $\mathrm{H}$ & 26.16266500 & -14.17318900 & -1.17408800 \\
\hline $\mathrm{H}$ & 25.51411500 & -12.29226600 & 0.26583300 \\
\hline $\mathrm{H}$ & 25.94722000 & -18.02891000 & 1.70183300 \\
\hline $\mathrm{H}$ & 26.58486000 & -18.45173600 & 3.99820800 \\
\hline $\mathrm{H}$ & 26.75088700 & -16.63846200 & 4.32296900 \\
\hline $\mathrm{H}$ & 33.90889800 & -5.83612400 & 1.19329500 \\
\hline $\mathrm{H}$ & 35.69824900 & -4.44238800 & 2.04259400 \\
\hline
\end{tabular}




\begin{tabular}{|c|c|c|c|}
\hline $\mathrm{H}$ & 36.06304300 & -3.81240000 & 0.34184800 \\
\hline $\mathrm{H}$ & 28.48680100 & -16.80720500 & 0.98332600 \\
\hline $\mathrm{H}$ & 26.88880700 & -18.18080500 & -3.45736200 \\
\hline $\mathrm{H}$ & 24.37896300 & -16.32115000 & -1.48102400 \\
\hline $\mathrm{H}$ & 23.89294400 & -15.95658100 & -3.82588900 \\
\hline $\mathrm{H}$ & 25.61423300 & -16.44826900 & -4.29388800 \\
\hline $\mathrm{H}$ & 30.92486900 & -18.84391800 & -0.92189200 \\
\hline $\mathrm{H}$ & 31.37893600 & -19.10988400 & 1.43824900 \\
\hline $\mathrm{H}$ & 29.69850200 & -18.46926600 & 1.87232600 \\
\hline $\mathrm{H}$ & 30.22423000 & -8.65427200 & 1.21111000 \\
\hline $\mathrm{H}$ & 31.31975700 & -3.99332700 & 0.11997200 \\
\hline $\mathrm{H}$ & 31.62115400 & -9.50912500 & -0.25740300 \\
\hline $\mathrm{H}$ & 33.52658600 & -9.72870300 & -1.59950600 \\
\hline $\mathrm{H}$ & 33.84505800 & -7.91408700 & -1.64787700 \\
\hline $\mathrm{H}$ & 29.62506300 & -3.15985500 & 1.52610000 \\
\hline $\mathrm{H}$ & 28.00987100 & -3.29542000 & 3.30893100 \\
\hline $\mathrm{H}$ & 28.16097100 & -5.13400700 & 3.37472400 \\
\hline $\mathrm{H}$ & 30.18660600 & -10.28533700 & 3.07185400 \\
\hline $\mathrm{H}$ & 30.01629000 & -8.60204000 & 3.62408100 \\
\hline $\mathrm{H}$ & 29.47396300 & -9.92579200 & 4.67423000 \\
\hline $\mathrm{H}$ & 32.86808300 & -14.36324800 & -6.61835500 \\
\hline $\mathrm{H}$ & 31.31112500 & -13.57888100 & -6.25895700 \\
\hline $\mathrm{H}$ & 32.72484700 & -12.59007800 & -6.74107600 \\
\hline $\mathrm{H}$ & 28.78813600 & -14.35170300 & -3.74962400 \\
\hline $\mathrm{H}$ & 28.66840500 & -15.59883700 & -2.49140700 \\
\hline $\mathrm{H}$ & 28.08492200 & -13.95301600 & -2.14996300 \\
\hline $\mathrm{H}$ & 37.35718000 & -9.33422200 & -8.58135400 \\
\hline
\end{tabular}




\begin{tabular}{|c|c|c|c|}
\hline $\mathrm{H}$ & 35.64971100 & -9.24726000 & -8.08391500 \\
\hline $\mathrm{H}$ & 36.48122000 & -7.79289900 & -8.71118200 \\
\hline $\mathrm{H}$ & 22.50759900 & -11.81673100 & 2.72805600 \\
\hline $\mathrm{H}$ & 22.91227900 & -13.53627000 & 2.47247200 \\
\hline $\mathrm{H}$ & 22.13709200 & -13.01014300 & 3.99001000 \\
\hline $\mathrm{H}$ & 38.05038600 & -2.93345400 & -4.84535900 \\
\hline $\mathrm{H}$ & 38.45584900 & -4.67076700 & -4.77102300 \\
\hline $\mathrm{H}$ & 37.43179200 & -4.05598200 & -6.08825400 \\
\hline $\mathrm{H}$ & 28.96390300 & -6.87521400 & 2.27440100 \\
\hline $\mathrm{C}$ & 28.47542200 & -18.33601800 & -2.03796700 \\
\hline $\mathrm{C}$ & 29.29707800 & -19.17891900 & -2.95692400 \\
\hline $\mathrm{C}$ & 30.41387900 & -18.64430100 & -3.63126800 \\
\hline $\mathrm{C}$ & 28.93435300 & -20.50328000 & -3.17530700 \\
\hline $\mathrm{C}$ & 31.12981900 & -19.48823300 & -4.48168700 \\
\hline $\mathrm{C}$ & 29.63790400 & -21.34003200 & -4.04812800 \\
\hline $\mathrm{H}$ & 28.09063000 & -20.90702500 & -2.62270600 \\
\hline $\mathrm{C}$ & 30.76606200 & -20.81560000 & -4.70616300 \\
\hline $\mathrm{H}$ & 32.01355300 & -19.10045500 & -4.98012200 \\
\hline $\mathrm{C}$ & 31.56917400 & -21.63284000 & -5.66009300 \\
\hline $\mathrm{C}$ & 30.94778800 & -22.15263400 & -6.79728300 \\
\hline $\mathrm{C}$ & 32.94438000 & -21.85684700 & -5.46258800 \\
\hline $\mathrm{C}$ & 31.64258100 & -22.88660200 & -7.76008700 \\
\hline $\mathrm{H}$ & 29.88586000 & -21.96552600 & -6.93806500 \\
\hline $\mathrm{C}$ & 33.63675600 & -22.61141900 & -6.42009600 \\
\hline $\mathrm{C}$ & 33.00820200 & -23.11329300 & -7.54610500 \\
\hline $\mathrm{H}$ & 34.68675300 & -22.83318000 & -6.25326400 \\
\hline $\mathrm{H}$ & 33.58097400 & -23.70894000 & -8.25041100 \\
\hline
\end{tabular}




\begin{tabular}{|c|c|c|}
\hline 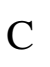 & $30.82631500-17.23792500$ & -3.44514100 \\
\hline [ & $30.63687300-16.79064700$ & -2.47251900 \\
\hline $\mathrm{C}$ & $31.39426600-16.47235300$ & -4.37815600 \\
\hline $\mathrm{H}$ & $31.57977500-16.83905200$ & -5.38611200 \\
\hline $\mathrm{H}$ & $31.67863100-15.44725400$ & -4.15805100 \\
\hline $\mathrm{C}$ & $29.22995800-22.74727500$ & -4.23054500 \\
\hline 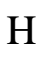 & $30.02721100-23.44708500$ & -4.47200900 \\
\hline $\mathrm{C}$ & $27.98047200-23.20544700$ & -4.13798000 \\
\hline I & $27.13510300-22.54973300$ & -3.94451600 \\
\hline [ & $27.76134600-24.26054400$ & -4.26743900 \\
\hline $\mathrm{C}$ & $33.63651000-21.35064400$ & -4.26075300 \\
\hline $\mathrm{H}$ & $33.02705900-21.25084500$ & -3.36518000 \\
\hline $\mathrm{C}$ & $34.92236700-21.00018100$ & -4.20526400 \\
\hline $\mathrm{H}$ & $35.56983000-21.02991200$ & -5.07821200 \\
\hline $\mathrm{H}$ & $35.36780400-20.65001200$ & -3.27962300 \\
\hline S & $30.91405300-23.38910900$ & -8.93791700 \\
\hline 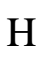 & $29.83262400-23.26580100$ & -8.89041400 \\
\hline $\mathrm{C}$ & $31.44046500-23.95003500$ & -10.02760500 \\
\hline $\mathrm{H}$ & $32.50976700-24.09075700$ & -10.15929100 \\
\hline $\mathrm{H}$ & $30.80602600-24.28670600$ & -10.84112700 \\
\hline
\end{tabular}

14, B3LYP/6-31G(d) (Energy = -2998.71776081 Hartrees $)$
C $\quad-3.5535 \quad 0.23596-0.68208$
C $\quad-3.4013-1.00886-0.06522$
C $\quad-4.52773 \quad-1.79005 \quad 0.23581$
C $\quad-5.81553-1.35258-0.12244$ 


\begin{tabular}{|c|c|c|c|}
\hline $\mathrm{C}$ & -4.84442 & 0.72527 & -0.94651 \\
\hline $\mathrm{C}$ & -5.97583 & -0.07939 & -0.69081 \\
\hline $\mathrm{C}$ & -7.01601 & -2.31368 & 0.11369 \\
\hline $\mathrm{C}$ & -7.74245 & -5.21773 & 0.45232 \\
\hline $\mathrm{C}$ & -7.07255 & -3.28833 & -1.08186 \\
\hline $\mathrm{C}$ & -7.38423 & -4.61005 & -0.93088 \\
\hline $\mathrm{C}$ & -6.78566 & -3.06967 & 1.42982 \\
\hline $\mathrm{C}$ & -7.13082 & -4.37115 & 1.58242 \\
\hline $\mathrm{C}$ & 4.64879 & 0.54925 & 0.26992 \\
\hline $\mathrm{C}$ & 4.36045 & 1.84169 & -0.20025 \\
\hline $\mathrm{C}$ & 3.04019 & 2.20877 & -0.49829 \\
\hline $\mathrm{C}$ & 2.01585 & 1.26733 & -0.37761 \\
\hline $\mathrm{C}$ & 3.60068 & -0.33416 & 0.5622 \\
\hline $\mathrm{C}$ & 2.28437 & 0.01511 & 0.20766 \\
\hline $\mathrm{C}$ & 0.60204 & 1.59098 & -0.91819 \\
\hline $\mathrm{C}$ & -2.29545 & 1.04771 & -1.08477 \\
\hline $\mathrm{C}$ & 0.06157 & 0.30977 & -1.56112 \\
\hline $\mathrm{C}$ & -1.25932 & 0.05691 & -1.63497 \\
\hline $\mathrm{C}$ & -0.35251 & 2.05789 & 0.20984 \\
\hline $\mathrm{C}$ & -1.70254 & 1.80895 & 0.13183 \\
\hline $\mathrm{C}$ & 6.12706 & 0.12391 & 0.41296 \\
\hline $\mathrm{C}$ & 6.65033 & -2.87623 & 0.41333 \\
\hline $\mathrm{C}$ & 6.40225 & -0.69549 & -0.86048 \\
\hline
\end{tabular}




\begin{tabular}{|c|c|c|c|}
\hline $\mathrm{C}$ & 6.32881 & -2.05593 & -0.85427 \\
\hline $\mathrm{C}$ & 6.37786 & -0.70496 & 1.69201 \\
\hline $\mathrm{C}$ & 6.42812 & -2.05498 & 1.69622 \\
\hline $\mathrm{O}$ & 8.0195 & -3.24952 & 0.29148 \\
\hline $\mathrm{C}$ & 8.80967 & -2.06659 & 0.19137 \\
\hline $\mathrm{O}$ & 6.95186 & 1.29064 & 0.49528 \\
\hline $\mathrm{C}$ & 6.60664 & 2.02499 & 1.67471 \\
\hline $\mathrm{H}$ & -2.41957 & -1.37064 & 0.17355 \\
\hline $\mathrm{H}$ & -4.40228 & -2.73287 & 0.73272 \\
\hline $\mathrm{H}$ & -4.96612 & 1.70466 & -1.35966 \\
\hline $\mathrm{H}$ & -6.95792 & 0.28016 & -0.92999 \\
\hline $\mathrm{H}$ & -6.83933 & -2.91608 & -2.0594 \\
\hline $\mathrm{H}$ & -7.36149 & -5.2395 & -1.78861 \\
\hline $\mathrm{H}$ & -6.32583 & -2.55911 & 2.24876 \\
\hline $\mathrm{H}$ & 5.15173 & 2.54724 & -0.33378 \\
\hline $\mathrm{H}$ & 2.82346 & 3.20318 & -0.83382 \\
\hline $\mathrm{H}$ & 3.80118 & -1.27618 & 1.03125 \\
\hline $\mathrm{H}$ & 1.48486 & -0.67304 & 0.37796 \\
\hline $\mathrm{H}$ & 0.75147 & -0.40424 & -1.96169 \\
\hline $\mathrm{H}$ & -1.5917 & -0.85209 & -2.08843 \\
\hline $\mathrm{H}$ & 0.04658 & 2.57838 & 1.05065 \\
\hline $\mathrm{H}$ & -2.34564 & 2.14041 & 0.91962 \\
\hline $\mathrm{H}$ & 6.63057 & -0.18363 & -1.77366 \\
\hline
\end{tabular}




\begin{tabular}{|c|c|c|c|}
\hline $\mathrm{H}$ & 6.06766 & -2.57195 & -1.75937 \\
\hline $\mathrm{H}$ & 6.50768 & -0.18586 & 2.61806 \\
\hline $\mathrm{H}$ & 6.31536 & -2.57342 & 2.62454 \\
\hline $\mathrm{H}$ & 8.51681 & -1.51835 & -0.679 \\
\hline $\mathrm{H}$ & 8.65856 & -1.46306 & 1.06246 \\
\hline $\mathrm{H}$ & 9.84369 & -2.33232 & 0.11585 \\
\hline $\mathrm{H}$ & 6.7532 & 1.40624 & 2.53614 \\
\hline $\mathrm{H}$ & 5.57996 & 2.32348 & 1.62193 \\
\hline $\mathrm{H}$ & 7.22713 & 2.89269 & 1.74917 \\
\hline $\mathrm{O}$ & -9.16654 & -5.30229 & 0.57558 \\
\hline $\mathrm{O}$ & -8.25303 & -1.61301 & 0.21416 \\
\hline $\mathrm{O}$ & -2.64791 & 1.99277 & -2.10411 \\
\hline $\mathrm{O}$ & 0.69452 & 2.62773 & -1.90178 \\
\hline $\mathrm{C}$ & -9.27848 & -2.5843 & 0.4326 \\
\hline $\mathrm{H}$ & -9.29757 & -3.27611 & -0.38366 \\
\hline $\mathrm{H}$ & -10.22636 & $6 \begin{array}{ll}6 & -2.09321\end{array}$ & 10.50472 \\
\hline $\mathrm{H}$ & -9.08034 & -3.11119 & 1.3426 \\
\hline $\mathrm{H}$ & -6.94433 & -4.83791 & 2.51845 \\
\hline $\mathrm{C}$ & 1.61188 & 2.26869 & -2.94071 \\
\hline $\mathrm{H}$ & 2.58691 & 2.12603 & -2.52324 \\
\hline $\mathrm{H}$ & 1.28676 & 1.36473 & -3.40749 \\
\hline $\mathrm{H}$ & 1.6494 & 3.05386 & -3.66961 \\
\hline $\mathrm{C}$ & -1.5149 & 2.78637 & -2.4833 \\
\hline
\end{tabular}




\begin{tabular}{|c|c|c|c|}
\hline $\mathrm{H}$ & -1.15841 & 3.33141 & -1.63476 \\
\hline $\mathrm{H}$ & -0.73861 & 2.14876 & -2.8504 \\
\hline $\mathrm{H}$ & -1.80345 & 3.47471 & -3.25276 \\
\hline $\mathrm{C}$ & 3.94412 & -6.22503 & 0.92542 \\
\hline $\mathrm{C}$ & 4.92096 & -6.32167 & -0.07942 \\
\hline $\mathrm{C}$ & 5.8633 & -5.27886 & -0.24323 \\
\hline $\mathrm{C}$ & 5.76148 & -4.12804 & 0.55972 \\
\hline $\mathrm{C}$ & 4.82065 & -4.07486 & 1.57726 \\
\hline $\mathrm{C}$ & 3.98438 & -5.14882 & 1.8201 \\
\hline $\mathrm{C}$ & 2.7993 & -7.25064 & 1.07253 \\
\hline $\mathrm{C}$ & 2.54766 & -8.25406 & 0.10551 \\
\hline $\mathrm{C}$ & 1.29278 & -8.91329 & 0.14644 \\
\hline $\mathrm{C}$ & 0.45601 & -8.69509 & 1.23738 \\
\hline $\mathrm{C}$ & 0.85887 & -7.94997 & 2.323 \\
\hline $\mathrm{C}$ & 1.95666 & -7.13534 & 2.18909 \\
\hline $\mathrm{H}$ & 4.9471 & -7.17663 & -0.7202 \\
\hline $\mathrm{H}$ & 6.63735 & -5.35894 & -0.97717 \\
\hline $\mathrm{H}$ & 4.75219 & -3.19603 & 2.18939 \\
\hline $\mathrm{H}$ & 3.26986 & -8.48229 & -0.65147 \\
\hline $\mathrm{H}$ & 0.29834 & -7.9745 & 3.23417 \\
\hline $\mathrm{C}$ & -3.65814 & -9.05215 & 1.02486 \\
\hline $\mathrm{C}$ & -2.92466 & -9.61446 & -0.05687 \\
\hline C & -1.5148 & -9.66168 & 0.0628 \\
\hline
\end{tabular}




\begin{tabular}{|c|c|c|c|}
\hline $\mathrm{C}$ & -0.96795 & -9.19103 & 1.23965 \\
\hline $\mathrm{C}$ & -1.71355 & -9.09559 & 2.40642 \\
\hline $\mathrm{C}$ & -3.07961 & -8.99149 & 2.2993 \\
\hline $\mathrm{C}$ & -5.0531 & -8.41498 & 0.86105 \\
\hline $\mathrm{C}$ & -5.68534 & -8.28584 & -0.39254 \\
\hline $\mathrm{C}$ & -6.70495 & -7.3321 & -0.52741 \\
\hline $\mathrm{C}$ & -7.05192 & -6.57508 & 0.58819 \\
\hline $\mathrm{C}$ & -6.70678 & -6.99203 & 1.86477 \\
\hline $\mathrm{C}$ & -5.66724 & -7.88616 & 2.00603 \\
\hline $\mathrm{H}$ & -3.41698 & -9.95126 & -0.9432 \\
\hline $\mathrm{H}$ & -1.23848 & -9.06378 & 3.36613 \\
\hline $\mathrm{H}$ & -5.38024 & -8.87325 & -1.23071 \\
\hline $\mathrm{H}$ & -7.18618 & -7.16018 & -1.47096 \\
\hline $\mathrm{H}$ & -7.21047 & -6.59568 & 2.72101 \\
\hline $\mathrm{C}$ & 3.10815 & -5.12464 & 3.0805 \\
\hline $\mathrm{H}$ & 3.22466 & -4.34393 & 3.80114 \\
\hline $\mathrm{C}$ & 2.19133 & -6.08381 & 3.27455 \\
\hline $\mathrm{H}$ & 1.60917 & -6.09777 & 4.17225 \\
\hline $\mathrm{C}$ & 0.78955 & -9.83271 & -0.99692 \\
\hline $\mathrm{H}$ & 1.46675 & -10.18638 & $8-1.74712$ \\
\hline $\mathrm{C}$ & -0.55041 & -10.16117 & $\begin{array}{ll}7 & -1.05521\end{array}$ \\
\hline $\mathrm{H}$ & -0.92706 & -10.73597 & $\begin{array}{ll}7 & -1.87668\end{array}$ \\
\hline $\mathrm{C}$ & -3.9304 & -8.74649 & 3.56007 \\
\hline
\end{tabular}




$$
\begin{aligned}
& \mathrm{H} \quad-3.53626 \quad-8.95323 \quad 4.53254 \\
& \begin{array}{llll}
\text { C } & -5.16838 & -8.23383 & 3.41748
\end{array} \\
& \mathrm{H} \quad-5.77918 \quad-8.04443 \quad 4.27329 \\
& \text { C } \quad-9.66776-6.19533-0.41926 \\
& \text { H } \quad-8.95765-6.93962-0.1104 \\
& \text { H } \quad \begin{array}{llll}
-9.39938 & -5.49228 & -1.17284
\end{array} \\
& \begin{array}{llll}
\mathrm{H} & -10.6477 & -6.15104 & 0.03717
\end{array}
\end{aligned}
$$

14, $\omega B 97 D / 6-31 G(d)$ (Energy = -2997.805989 Hartrees $)$
$\begin{array}{llll}\text { C } & -2.76918800 & -2.14007000 & 0.44685000\end{array}$
C $\quad-3.00249700 \quad-1.19995800 \quad-0.54967100$
C $\quad-4.17238800 \quad-1.24377700 \quad-1.31190600$
C $\quad-5.12175700 \quad-2.24058600 \quad-1.11141900$
C $\quad-3.72959700 \quad-3.13906700 \quad 0.65196400$
C $\quad-4.87559600 \quad-3.19785300 \quad-0.12178900$
C $\quad-6.43071100 \quad-2.33097900-1.90961000$
C $\quad-7.95655500 \quad 0.13048300 \quad-1.33088700$
C $\quad-7.61368500 \quad-2.29724900 \quad-0.95521300$
C $\quad-8.31775400 \quad-1.19825600 \quad-0.70563600$
$\begin{array}{llll}\text { C } & -6.56528300 & -1.21017100 & -2.91300300\end{array}$
C $\quad-7.27016600 \quad-0.11402300 \quad-2.66093600$
C $\quad 5.15291800 \quad-2.26760900 \quad-1.05932700$
C $\quad 4.83748100 \quad-3.29063300 \quad-0.15739900$
C $\quad 3.66179300 \quad-3.25490600 \quad 0.57241400$
C $\quad 2.74022100 \quad-2.21091500 \quad 0.41176500$
C $\quad 4.24133100 \quad-1.22848000 \quad-1.21859200$ 


\begin{tabular}{|c|c|c|c|}
\hline $\mathrm{C}$ & 3.04537300 & -1.20394300 & -0.49729100 \\
\hline $\mathrm{C}$ & 1.48407300 & -2.22725800 & 1.29116400 \\
\hline $\mathrm{C}$ & -1.52129800 & -2.16112300 & 1.32971200 \\
\hline $\mathrm{C}$ & 0.67104800 & -0.94965100 & 1.22792200 \\
\hline $\mathrm{C}$ & -0.66044800 & -0.92475300 & 1.22962100 \\
\hline $\mathrm{C}$ & 0.61862700 & -3.42677900 & 0.97434500 \\
\hline $\mathrm{C}$ & -0.70874300 & -3.40043700 & 1.01519600 \\
\hline $\mathrm{C}$ & 6.49628300 & -2.34767200 & -1.81238100 \\
\hline $\mathrm{C}$ & 7.93818600 & 0.17239300 & -1.22583200 \\
\hline $\mathrm{C}$ & 7.62902100 & -2.25897400 & -0.80973600 \\
\hline $\mathrm{C}$ & 8.28457900 & -1.13370700 & -0.55014200 \\
\hline $\mathrm{C}$ & 6.63538100 & -1.24246600 & -2.83320800 \\
\hline $\mathrm{C}$ & 7.28390100 & -0.11418600 & -2.56803700 \\
\hline $\mathrm{O}$ & 9.08116100 & 1.00163200 & -1.38879100 \\
\hline $\mathrm{C}$ & 10.10307300 & 0.44354100 & -2.18602500 \\
\hline $\mathrm{O}$ & 6.65489500 & -3.63955000 & -2.39785800 \\
\hline $\mathrm{C}$ & 5.67510500 & -4.03366900 & -3.33164500 \\
\hline $\mathrm{H}$ & -2.27937100 & -0.41278800 & -0.74150900 \\
\hline $\mathrm{H}$ & -4.34534700 & -0.47129800 & -2.05421600 \\
\hline $\mathrm{H}$ & -3.57560800 & -3.86972500 & 1.43978300 \\
\hline $\mathrm{H}$ & -5.59099400 & -3.99800800 & 0.04225000 \\
\hline $\mathrm{H}$ & -7.84684600 & -3.23612800 & -0.45851900 \\
\hline $\mathrm{H}$ & -9.13145100 & -1.21775200 & 0.01377000 \\
\hline $\mathrm{H}$ & -6.02905700 & -1.33508800 & -3.85026100 \\
\hline $\mathrm{H}$ & 5.52535400 & -4.12216300 & -0.03276700 \\
\hline $\mathrm{H}$ & 3.45715600 & -4.03369600 & 1.30116200 \\
\hline $\mathrm{H}$ & 4.46672300 & -0.40728300 & -1.89130000 \\
\hline
\end{tabular}




\begin{tabular}{|c|c|c|c|}
\hline $\mathrm{H}$ & 2.35794400 & -0.37826600 & -0.65303300 \\
\hline $\mathrm{H}$ & 1.21751000 & -0.00910300 & 1.24939900 \\
\hline $\mathrm{H}$ & -1.18236100 & 0.02833800 & 1.26662600 \\
\hline $\mathrm{H}$ & 1.14235400 & -4.35750200 & 0.77343200 \\
\hline $\mathrm{H}$ & -1.27318400 & -4.31304800 & 0.84321700 \\
\hline $\mathrm{H}$ & 7.85958300 & -3.18577000 & -0.29208500 \\
\hline $\mathrm{H}$ & 9.06460200 & -1.09569500 & 0.20628400 \\
\hline $\mathrm{H}$ & 6.13375300 & -1.37439900 & -3.78758600 \\
\hline $\mathrm{H}$ & 7.33227300 & 0.68976000 & -3.29884200 \\
\hline $\mathrm{H}$ & 10.51010100 & -0.47752100 & -1.74747200 \\
\hline $\mathrm{H}$ & 9.75544600 & 0.21424900 & -3.20263700 \\
\hline $\mathrm{H}$ & 10.89390200 & 1.19425200 & -2.23902700 \\
\hline $\mathrm{H}$ & 5.76301500 & -3.49001800 & -4.28213100 \\
\hline $\mathrm{H}$ & 4.65761600 & -3.90536800 & -2.94089000 \\
\hline $\mathrm{H}$ & 5.85011900 & -5.09399500 & -3.52534800 \\
\hline O & -9.09686500 & 0.92137300 & -1.64242100 \\
\hline $\mathrm{C}$ & -6.37161900 & -3.59633200 & -2.56691400 \\
\hline $\mathrm{C}$ & -2.06926500 & -2.24578700 & 2.66099900 \\
\hline $\mathrm{O}$ & 1.90633900 & -2.49004300 & 2.64709100 \\
\hline C & -7.50454800 & -3.91271400 & -3.34535300 \\
\hline $\mathrm{H}$ & -8.42286500 & -3.95952900 & -2.74379000 \\
\hline $\mathrm{H}$ & -7.31252300 & -4.89563000 & -3.78025300 \\
\hline $\mathrm{H}$ & -7.66432800 & -3.18688100 & -4.15403800 \\
\hline $\mathrm{H}$ & -7.34454200 & 0.69841000 & -3.37805700 \\
\hline $\mathrm{C}$ & 2.67864700 & -1.47897800 & 3.24864400 \\
\hline $\mathrm{H}$ & 3.53174900 & -1.18494900 & 2.62053600 \\
\hline $\mathrm{H}$ & 2.07519400 & -0.58908600 & 3.46845700 \\
\hline
\end{tabular}




\begin{tabular}{|c|c|c|c|}
\hline $\mathrm{H}$ & 3.05491300 & -1.89503300 & 4.18608100 \\
\hline $\mathrm{C}$ & -1.14463700 & -2.28315900 & 3.72773800 \\
\hline $\mathrm{H}$ & -0.39720100 & -3.07471000 & 3.60707500 \\
\hline $\mathrm{H}$ & -0.61160300 & -1.32933900 & 3.83950100 \\
\hline $\mathrm{F}$ & -1.73902100 & -2.47222800 & 4.62501100 \\
\hline$C$ & 4.73724100 & 2.25675800 & 0.84689000 \\
\hline $\mathrm{C}$ & 5.33686200 & 1.12190300 & 1.41687400 \\
\hline $\mathrm{C}$ & 6.39536000 & 0.48851900 & 0.80329600 \\
\hline $\mathrm{C}$ & 6.93487500 & 0.98257800 & -0.39406600 \\
\hline $\mathrm{C}$ & 6.44233200 & 2.17428100 & -0.89453100 \\
\hline $\mathrm{C}$ & 5.33350600 & 2.81039200 & -0.31057400 \\
\hline $\mathrm{C}$ & 3.45393200 & 2.77633400 & 1.30372600 \\
\hline $\mathrm{C}$ & 2.75833700 & 2.24220600 & 2.39075200 \\
\hline $\mathrm{C}$ & 1.40710400 & 2.50799200 & 2.60203300 \\
\hline $\mathrm{C}$ & 0.71150400 & 3.36971200 & 1.70875100 \\
\hline $\mathrm{C}$ & 1.45638400 & 4.05632400 & 0.75485300 \\
\hline $\mathrm{C}$ & 2.80585200 & 3.77549400 & 0.52882200 \\
\hline $\mathrm{H}$ & 4.92722500 & 0.68082400 & 2.31864100 \\
\hline $\mathrm{H}$ & 6.77310900 & -0.43450800 & 1.22705100 \\
\hline $\mathrm{H}$ & 6.87200900 & 2.59928000 & -1.79781500 \\
\hline $\mathrm{H}$ & 3.24261800 & 1.53333200 & 3.05475200 \\
\hline $\mathrm{H}$ & 0.97422800 & 4.76284200 & 0.08628700 \\
\hline $\mathrm{C}$ & -3.46887600 & 2.67333200 & 1.26830000 \\
\hline $\mathrm{C}$ & -2.75612500 & 2.11445300 & 2.33081700 \\
\hline $\mathrm{C}$ & -1.42013200 & 2.43167600 & 2.56485300 \\
\hline C & -0.75160500 & 3.34705900 & 1.70524600 \\
\hline $\mathrm{C}$ & -1.51844200 & 4.04758000 & 0.77962900 \\
\hline
\end{tabular}




\begin{tabular}{|c|c|c|c|}
\hline $\mathrm{C}$ & -2.85895000 & 3.73375100 & 0.54474100 \\
\hline $\mathrm{C}$ & -4.74188200 & 2.14271900 & 0.79808700 \\
\hline $\mathrm{C}$ & -5.29515500 & 0.95628900 & 1.30634500 \\
\hline $\mathrm{C}$ & -6.36665700 & 0.34878100 & 0.68858800 \\
\hline $\mathrm{C}$ & -6.94844900 & 0.90652300 & -0.45971500 \\
\hline $\mathrm{C}$ & -6.48874000 & 2.13456700 & -0.90196500 \\
\hline $\mathrm{C}$ & -5.37778300 & 2.75543400 & -0.30794400 \\
\hline $\mathrm{H}$ & -3.21012500 & 1.35417600 & 2.95863600 \\
\hline $\mathrm{H}$ & -1.06341100 & 4.80611000 & 0.14994900 \\
\hline $\mathrm{H}$ & -4.83248700 & 0.44771600 & 2.14477900 \\
\hline $\mathrm{H}$ & -6.71145100 & -0.60931700 & 1.05911200 \\
\hline $\mathrm{H}$ & -6.94722100 & 2.59842900 & -1.77220300 \\
\hline $\mathrm{C}$ & 4.72709500 & 3.95354300 & -0.94713400 \\
\hline $\mathrm{H}$ & 5.24149400 & 4.40791200 & -1.78976200 \\
\hline $\mathrm{C}$ & 3.51623600 & 4.40487400 & -0.55599300 \\
\hline $\mathrm{H}$ & 3.03551300 & 5.22803400 & -1.07806200 \\
\hline $\mathrm{C}$ & 0.67115900 & 1.81244000 & 3.63477100 \\
\hline $\mathrm{H}$ & 1.22999800 & 1.27807300 & 4.39811200 \\
\hline $\mathrm{C}$ & -0.67508000 & 1.76041500 & 3.60571100 \\
\hline $\mathrm{H}$ & -1.22558500 & 1.17119200 & 4.33404200 \\
\hline $\mathrm{C}$ & -3.60053300 & 4.40239200 & -0.49458900 \\
\hline $\mathrm{H}$ & -3.14766700 & 5.26400000 & -0.97808100 \\
\hline $\mathrm{C}$ & -4.80892600 & 3.94672100 & -0.88834900 \\
\hline $\mathrm{H}$ & -5.34884000 & 4.43561500 & -1.69503800 \\
\hline $\mathrm{C}$ & -9.82608400 & 1.41707600 & -0.54339200 \\
\hline $\mathrm{H}$ & -9.18790500 & 1.97657500 & 0.15271500 \\
\hline $\mathrm{H}$ & -10.34078600 & 0.62035300 & 0.01117000 \\
\hline
\end{tabular}


$\mathrm{H}$ $-10.57991200 \quad 2.09078800 \quad-0.95600800$

dibenzo[c,m]pentaphene, $\omega B 97 D / 6-31 G(d)($ Energy = -1153.717639 Hartrees)
C $\quad-4.28569800 \quad 1.33067600 \quad-0.69065600$
C $\quad-2.88182900 \quad 1.28860600 \quad-0.57711600$
C $\quad-2.15957800 \quad 2.48600600 \quad-0.35731300$
C $\quad-2.88480600 \quad 3.69030700 \quad-0.25996800$
C $\quad-4.25963700 \quad 3.71127300 \quad-0.37436800$
$\begin{array}{llll}\text { C } & -4.97030700 & 2.52195600 & -0.59182000\end{array}$
$\begin{array}{llll}\mathrm{H} & -4.82288300 & 0.40071600 & -0.85909500\end{array}$
$\mathrm{H} \quad-2.36476300 \quad 4.62728700 \quad-0.09341800$
$\mathrm{H} \quad-4.79120000 \quad 4.65476000 \quad-0.29523700$
$\mathrm{H} \quad-6.05206100 \quad 2.54179000 \quad-0.68288600$
C $\quad-0.70415200 \quad 2.42514700 \quad-0.24324700$
C $\quad 0.09426400 \quad 3.54930200 \quad-0.02453000$
$\begin{array}{llll}\text { C } & -0.05201500 & 1.16612200 & -0.35880000\end{array}$
$\begin{array}{llll}\text { C } & 1.48174600 & 3.47075300 & 0.07973300\end{array}$
$\mathrm{H} \quad-0.35853700 \quad 4.53094200 \quad 0.07303300$
C $\quad 1.34002800 \quad 1.08913100 \quad-0.25466000$
$\begin{array}{llll}\text { C } & 2.13380100 & 2.21076100 & -0.03790500\end{array}$
$\mathrm{H} \quad 1.79340900 \quad 0.10736900 \quad-0.35047400$
$\begin{array}{llll}\text { C } & 3.59388300 & 2.15143000 & 0.07502300\end{array}$
C $\quad 4.31891700 \quad 0.96904000 \quad-0.02788300$
$\begin{array}{llll}\text { C } & 4.31985000 & 3.35491400 & 0.30175700\end{array}$
$\begin{array}{llll}\text { C } & 5.71195200 & 0.93260100 & 0.08522600\end{array}$
$\mathrm{H} \quad 3.80922000 \quad 0.02638600 \quad-0.20155700$
$\begin{array}{llll}\text { C } & 5.70845400 & 3.32018300 & 0.41556400\end{array}$ 


\begin{tabular}{|c|c|c|c|}
\hline $\mathrm{C}$ & 6.43781800 & 2.13428800 & 0.31537100 \\
\hline $\mathrm{H}$ & 6.21764300 & 4.26325000 & 0.58733000 \\
\hline $\mathrm{C}$ & 2.26621900 & 4.66178900 & 0.30799100 \\
\hline $\mathrm{C}$ & 3.60871300 & 4.60679500 & 0.41322800 \\
\hline $\mathrm{H}$ & 1.74407800 & 5.61104100 & 0.39407400 \\
\hline $\mathrm{H}$ & 4.18607700 & 5.51136100 & 0.58520100 \\
\hline $\mathrm{C}$ & -2.17828400 & 0.03655800 & -0.68535000 \\
\hline $\mathrm{H}$ & -2.75850200 & -0.86683300 & -0.85392000 \\
\hline $\mathrm{C}$ & -0.83332300 & -0.02228400 & -0.58245000 \\
\hline $\mathrm{H}$ & -0.31323300 & -0.97281100 & -0.66719800 \\
\hline $\mathrm{C}$ & 7.89288000 & 2.07531700 & 0.43488200 \\
\hline $\mathrm{C}$ & 8.68768900 & 3.21546200 & 0.66869100 \\
\hline $\mathrm{C}$ & 8.54143400 & 0.82303600 & 0.31391900 \\
\hline $\mathrm{C}$ & 10.05997800 & 3.12300300 & 0.77798200 \\
\hline $\mathrm{H}$ & 8.22360100 & 4.19053100 & 0.76881200 \\
\hline $\mathrm{C}$ & 9.94388000 & 0.74946600 & 0.42765100 \\
\hline $\mathrm{C}$ & 10.69761500 & 1.87982300 & 0.65657200 \\
\hline $\mathrm{H}$ & 10.64622800 & 4.01882400 & 0.95933900 \\
\hline $\mathrm{H}$ & 10.42535300 & -0.22035000 & 0.33116500 \\
\hline $\mathrm{H}$ & 11.77745000 & 1.80918600 & 0.74455600 \\
\hline $\mathrm{C}$ & 6.41997800 & -0.31531800 & -0.03041400 \\
\hline $\mathrm{H}$ & 5.84475600 & -1.22023100 & -0.20776900 \\
\hline $\mathrm{C}$ & 7.76485600 & -0.36685700 & 0.07717000 \\
\hline $\mathrm{H}$ & 8.28980500 & -1.31420700 & -0.01263600 \\
\hline
\end{tabular}

14 open adduct, $\omega B 97 D / 6-31 G(d)($ Energy $=-2307.321794$ Hartrees $)$

$\begin{array}{llll}\text { C } & 5.30475100 & -3.40482600 & -0.03115600\end{array}$ 

C $\quad 3.97813800 \quad-3.15118000 \quad-0.37715100$
C $\quad 3.04896800 \quad-4.18377800 \quad-0.43680800$
C $\quad 3.43069600 \quad-5.49841500 \quad-0.16285700$
C $\quad 5.68969400 \quad-4.72125900 \quad 0.22133300$
C $\quad 4.76368800 \quad-5.75565800 \quad 0.15466400$
C $\quad 2.41311400 \quad-6.64391700 \quad-0.20634500$
C $\quad-0.04514000 \quad-6.35820600 \quad-1.92408600$
$\begin{array}{llll}\text { C } & 1.08801500 & -6.15236900 & 0.29870900\end{array}$
C $\quad 0.00788700 \quad-6.00824500 \quad-0.45637900$
C $\quad 2.30292800 \quad-7.18519400 \quad-1.61482600$
$\begin{array}{llll}\text { C } & 1.21449000 & -7.06479800 & -2.36958000\end{array}$
$\begin{array}{llll}\text { C } & 4.37384600 & 2.97699800 & -3.83138800\end{array}$
C $\quad 5.67741500 \quad 3.33094100 \quad-3.47991500$
C $\quad 6.42957200 \quad 2.52998800 \quad-2.62714800$
C $\quad 5.88257400 \quad 1.37085100 \quad-2.07744600$
C $\quad 3.85285400 \quad 1.78347300 \quad-3.32916500$
C $\quad 4.59185500 \quad 1.00050500 \quad-2.45140600$
C $\quad 6.67773400 \quad 0.48343500 \quad-1.11481900$
C $\quad 6.32339000 \quad-2.27080000 \quad 0.05209400$
$\begin{array}{llll}\text { C } & 5.84664800 & 0.18742400 & 0.11797200\end{array}$
C $\quad 5.69276900 \quad-1.02825200 \quad 0.63086500$
C $\quad 7.08242400 \quad-0.78900800 \quad-1.82547400$
C $\quad 6.91240400 \quad-2.00389600 \quad-1.31533900$
C $\quad 3.52799400 \quad 3.94420100 \quad-4.68344500$
C $\quad 0.72649000 \quad 4.03653500 \quad-3.64266800$
C $\quad 3.00950900 \quad 5.03584900 \quad-3.77153700$
$\begin{array}{llll}\text { C } & 1.76526600 & 5.07926900 & -3.30675900\end{array}$ 


\begin{tabular}{|c|c|c|c|}
\hline $\mathrm{C}$ & 2.38175400 & 3.22297100 & -5.34527200 \\
\hline $\mathrm{C}$ & 1.14039200 & 3.26514500 & -4.87758200 \\
\hline $\mathrm{O}$ & -0.55406900 & 4.62601300 & -3.82725000 \\
\hline $\mathrm{C}$ & -0.64543900 & 5.48891600 & -4.94249300 \\
\hline $\mathrm{O}$ & 4.35261000 & 4.63337600 & -5.62223000 \\
\hline $\mathrm{C}$ & 4.92107300 & 3.84725700 & -6.64352800 \\
\hline $\mathrm{H}$ & 3.64943100 & -2.13333500 & -0.57189900 \\
\hline $\mathrm{H}$ & 2.01376100 & -3.95136800 & -0.66847700 \\
\hline $\mathrm{H}$ & 6.71701300 & -4.92857700 & 0.49741900 \\
\hline $\mathrm{H}$ & 5.06710100 & -6.77148200 & 0.38076300 \\
\hline $\mathrm{H}$ & 1.08367200 & -5.88621900 & 1.34630200 \\
\hline $\mathrm{H}$ & -0.91464800 & -5.61244900 & -0.03703200 \\
\hline $\mathrm{H}$ & 3.19302000 & -7.68661900 & -1.98886900 \\
\hline $\mathrm{H}$ & 6.10577000 & 4.24246700 & -3.88644700 \\
\hline $\mathrm{H}$ & 7.45005800 & 2.80512100 & -2.38266800 \\
\hline $\mathrm{H}$ & 2.84894700 & 1.47300100 & -3.59729100 \\
\hline $\mathrm{H}$ & 4.15424200 & 0.08906600 & -2.05107900 \\
\hline $\mathrm{H}$ & 5.36853500 & 1.04192600 & 0.59227100 \\
\hline $\mathrm{H}$ & 5.11414800 & -1.17858700 & 1.53880000 \\
\hline $\mathrm{H}$ & 7.56113100 & -0.64744800 & -2.79088300 \\
\hline $\mathrm{H}$ & 7.25285100 & -2.88525100 & -1.85302200 \\
\hline $\mathrm{H}$ & 3.74718500 & 5.78386700 & -3.49318000 \\
\hline $\mathrm{H}$ & 1.44691500 & 5.86401100 & -2.62462400 \\
\hline $\mathrm{H}$ & 2.61297900 & 2.58468700 & -6.19246700 \\
\hline $\mathrm{H}$ & 0.34916700 & 2.68360900 & -5.33829000 \\
\hline $\mathrm{H}$ & 0.03858400 & 6.34388000 & -4.85245700 \\
\hline $\mathrm{H}$ & -0.42828600 & 4.96863200 & -5.88390700 \\
\hline
\end{tabular}




\begin{tabular}{|c|c|c|c|}
\hline $\mathrm{H}$ & -1.67527800 & 5.85070900 & -4.96990400 \\
\hline $\mathrm{H}$ & 4.18627300 & 3.58395200 & -7.41653600 \\
\hline $\mathrm{H}$ & 5.37285900 & 2.92585000 & -6.25176900 \\
\hline $\mathrm{H}$ & 5.70340200 & 4.45608300 & -7.10239600 \\
\hline $\mathrm{O}$ & -1.18706800 & -7.17553200 & -2.18706300 \\
\hline $\mathrm{O}$ & 2.92336300 & -7.65290700 & 0.66358500 \\
\hline $\mathrm{O}$ & 7.35629400 & -2.74164300 & 0.92560900 \\
\hline $\mathrm{O}$ & 7.92278700 & 1.10224800 & -0.76930600 \\
\hline $\mathrm{C}$ & 2.06162700 & -8.74840700 & 0.88076100 \\
\hline $\mathrm{H}$ & 1.13781500 & -8.44990300 & 1.39399200 \\
\hline $\mathrm{H}$ & 2.60967500 & -9.44349600 & 1.51988700 \\
\hline $\mathrm{H}$ & 1.79417400 & -9.25196900 & -0.05887800 \\
\hline $\mathrm{H}$ & 1.18723400 & -7.46205400 & -3.38213100 \\
\hline $\mathrm{C}$ & 7.87670100 & 2.08509800 & 0.24143500 \\
\hline $\mathrm{H}$ & 7.10612900 & 2.84557300 & 0.05195300 \\
\hline $\mathrm{H}$ & 7.70267300 & 1.64071500 & 1.23023900 \\
\hline $\mathrm{H}$ & 8.85577000 & 2.56929700 & 0.23871200 \\
\hline $\mathrm{C}$ & 8.37780600 & -1.81662200 & 1.23219400 \\
\hline $\mathrm{H}$ & 8.77894000 & -1.32560900 & 0.33762700 \\
\hline $\mathrm{H}$ & 8.02641100 & -1.03930500 & 1.92382400 \\
\hline $\mathrm{H}$ & 9.16878000 & -2.39300200 & 1.71704600 \\
\hline $\mathrm{C}$ & 0.43897300 & 0.82831600 & -0.71430400 \\
\hline $\mathrm{C}$ & 1.46661900 & 1.78324400 & -0.63839400 \\
\hline $\mathrm{C}$ & 1.52717400 & 2.84242300 & -1.51826200 \\
\hline $\mathrm{C}$ & 0.56424500 & 2.99818100 & -2.52746400 \\
\hline $\mathrm{C}$ & -0.48952600 & 2.10372400 & -2.57860100 \\
\hline C & -0.57559300 & 1.02456900 & -1.68127200 \\
\hline
\end{tabular}




\begin{tabular}{|c|c|c|c|}
\hline $\mathrm{C}$ & 0.38642800 & -0.34923500 & 0.14476200 \\
\hline $\mathrm{C}$ & 1.39519400 & -0.67422500 & 1.05381500 \\
\hline $\mathrm{C}$ & 1.33743000 & -1.81450800 & 1.85153300 \\
\hline $\mathrm{C}$ & 0.21138500 & -2.68003700 & 1.76994900 \\
\hline $\mathrm{C}$ & -0.78159300 & -2.37307800 & 0.84544800 \\
\hline $\mathrm{C}$ & -0.71750100 & -1.23888800 & 0.03159200 \\
\hline $\mathrm{H}$ & 2.24613800 & 1.69360600 & 0.11054100 \\
\hline $\mathrm{H}$ & 2.35372300 & 3.54063600 & -1.45050100 \\
\hline $\mathrm{H}$ & -1.25830000 & 2.22095000 & -3.33782100 \\
\hline $\mathrm{H}$ & 2.28160300 & -0.05179400 & 1.13327200 \\
\hline $\mathrm{H}$ & -1.62864100 & -3.03846500 & 0.70862600 \\
\hline $\mathrm{C}$ & 0.38844400 & -6.42212000 & 3.87870500 \\
\hline $\mathrm{C}$ & 1.42404800 & -5.48781000 & 3.95543000 \\
\hline $\mathrm{C}$ & 1.35117400 & -4.24429900 & 3.33082900 \\
\hline $\mathrm{C}$ & 0.18857100 & -3.89194700 & 2.58833100 \\
\hline $\mathrm{C}$ & -0.89089000 & -4.76904200 & 2.61745600 \\
\hline $\mathrm{C}$ & -0.81233800 & -6.02510200 & 3.22653800 \\
\hline $\mathrm{C}$ & 0.49545800 & -7.77866200 & 4.40827000 \\
\hline $\mathrm{C}$ & 1.67166000 & -8.27277400 & 5.00679500 \\
\hline $\mathrm{C}$ & 1.74604900 & -9.56706900 & 5.47836100 \\
\hline $\mathrm{C}$ & 0.64190200 & -10.42535300 & 5.36963900 \\
\hline $\mathrm{C}$ & -0.51740200 & -9.96937300 & 4.78113400 \\
\hline $\mathrm{C}$ & -0.61210400 & -8.65229100 & 4.28951500 \\
\hline $\mathrm{H}$ & 2.34187700 & -5.72829600 & 4.48174300 \\
\hline $\mathrm{H}$ & -1.81894600 & -4.51053500 & 2.11624200 \\
\hline $\mathrm{H}$ & 2.54577000 & -7.63712000 & 5.09348100 \\
\hline $\mathrm{H}$ & 2.66576100 & -9.92227700 & 5.93315000 \\
\hline
\end{tabular}




\begin{tabular}{|c|c|c|c|}
\hline $\mathrm{H}$ & -1.37837100 & -10.62621800 & 4.68519500 \\
\hline$C$ & -1.67774000 & 0.10109900 & -1.75242100 \\
\hline$U$ & -2.45247300 & 0.28503200 & -2.48924300 \\
\hline$C$ & -1.74765700 & -0.97257300 & -0.93755400 \\
\hline $\mathrm{H}$ & -2.57808600 & -1.66866600 & -1.01484700 \\
\hline $\mathrm{C}$ & 2.43826800 & -2.15894300 & 2.71942900 \\
\hline$H$ & 3.27195500 & -1.46559200 & 2.78547800 \\
\hline$C$ & 2.46036300 & -3.32223000 & 3.39811300 \\
\hline $\mathrm{H}$ & 3.31911000 & -3.59428800 & 4.00526600 \\
\hline $\mathrm{C}$ & -1.91648800 & -6.94545200 & 3.14738000 \\
\hline $\mathrm{H}$ & -2.82855400 & -6.61422600 & 2.65768200 \\
\hline $\mathrm{C}$ & -1.82120600 & -8.19355400 & 3.65449500 \\
\hline $\mathrm{H}$ & -2.65712000 & -8.88457000 & 3.58336600 \\
\hline $\mathrm{C}$ & -1.22977300 & -8.38314700 & -1.45887700 \\
\hline $\mathrm{H}$ & -1.25196300 & -8.21150200 & -0.37416000 \\
\hline $\mathrm{H}$ & -0.37109800 & -9.02861700 & -1.68886700 \\
\hline $\mathrm{H}$ & -2.14844700 & -8.88882000 & -1.76333700 \\
\hline $\mathrm{C}$ & -1.53775100 & -4.55004500 & -2.86321700 \\
\hline $\mathrm{C}$ & -0.26604600 & -5.07173600 & -2.72169500 \\
\hline $\mathrm{C}$ & 0.81637200 & -4.36399300 & -3.27100600 \\
\hline $\mathrm{C}$ & 0.61177400 & -3.20172200 & -3.98354700 \\
\hline $\mathrm{C}$ & -0.67932300 & -2.67919400 & -4.17460100 \\
\hline $\mathrm{C}$ & -1.76362300 & -3.35394000 & -3.56771800 \\
\hline $\mathrm{H}$ & -2.38313600 & -5.08134300 & -2.43659300 \\
\hline $\mathrm{H}$ & 1.82625400 & -4.73727700 & -3.13468000 \\
\hline $\mathrm{H}$ & 1.47555800 & -2.68305600 & -4.38555700 \\
\hline $\mathrm{C}$ & -0.93876900 & -1.47789400 & -4.95864100 \\
\hline
\end{tabular}




\begin{tabular}{|c|c|c|c|}
\hline $\mathrm{C}$ & -2.24848500 & -0.92439800 & -4.96365900 \\
\hline $\mathrm{C}$ & 0.02943500 & -0.86597300 & -5.75636500 \\
\hline $\mathrm{C}$ & -2.48872400 & 0.28143900 & -5.62866500 \\
\hline $\mathrm{C}$ & -0.23394400 & 0.29254200 & -6.48405400 \\
\hline $\mathrm{H}$ & 1.01369400 & -1.31296700 & -5.85626700 \\
\hline $\mathrm{C}$ & -1.49988500 & 0.93075500 & -6.36203500 \\
\hline $\mathrm{H}$ & -3.49417800 & 0.69025000 & -5.58733700 \\
\hline $\mathrm{C}$ & -1.70733300 & 2.22168700 & -7.02087700 \\
\hline $\mathrm{C}$ & -0.70507700 & 2.72069500 & -7.89992900 \\
\hline $\mathrm{C}$ & -2.82238400 & 3.01841000 & -6.78721700 \\
\hline $\mathrm{C}$ & -0.88337900 & 3.95102700 & -8.52955100 \\
\hline $\mathrm{C}$ & -2.99251500 & 4.26241300 & -7.40137100 \\
\hline $\mathrm{H}$ & -3.58234100 & 2.69836600 & -6.08101300 \\
\hline $\mathrm{C}$ & -2.01050800 & 4.74603300 & -8.30992300 \\
\hline $\mathrm{H}$ & -0.09188200 & 4.29377600 & -9.18820800 \\
\hline $\mathrm{C}$ & -2.20261900 & 6.05084400 & -8.93753600 \\
\hline $\mathrm{C}$ & -3.35087900 & 6.80942400 & -8.60638700 \\
\hline $\mathrm{C}$ & -1.29015500 & 6.59492200 & -9.86343100 \\
\hline $\mathrm{C}$ & -3.54726300 & 8.07242800 & -9.19927800 \\
\hline $\mathrm{C}$ & -1.50069000 & 7.83301700 & -10.43422800 \\
\hline $\mathrm{H}$ & -0.40249500 & 6.03878500 & -10.14455100 \\
\hline $\mathrm{C}$ & -2.63864100 & 8.58206700 & -10.10072400 \\
\hline $\mathrm{H}$ & -4.43460000 & 8.64151900 & -8.93363400 \\
\hline $\mathrm{H}$ & -0.78112400 & 8.22641700 & -11.14589000 \\
\hline $\mathrm{H}$ & -2.80150100 & 9.55586000 & -10.55221300 \\
\hline $\mathrm{C}$ & -3.09001400 & -2.79812800 & -3.66429900 \\
\hline $\mathrm{C}$ & -3.31621700 & -1.62705300 & -4.29879200 \\
\hline
\end{tabular}




$\begin{array}{llll}\mathrm{C} & 0.75182700 & 0.83897500 & -7.38804500 \\ \mathrm{C} & 0.51495800 & 1.96979100 & -8.08439400 \\ \mathrm{C} & -4.14304600 & 5.07244800 & -7.09867200 \\ \mathrm{C} & -4.31022600 & 6.28547700 & -7.66829100 \\ \mathrm{H} & -4.31595300 & -1.20233200 & -4.34107100 \\ \mathrm{H} & -3.90787700 & -3.33599300 & -3.19144100 \\ \mathrm{H} & 1.69182600 & 0.30549200 & -7.50337800 \\ \mathrm{H} & 1.25998600 & 2.36233200 & -8.77156500 \\ \mathrm{H} & -4.87405700 & 4.68624100 & -6.39337900 \\ \mathrm{H} & -5.18017700 & 6.89180800 & -7.43006800 \\ \mathrm{H} & 0.70358500 & -11.44263000 & 5.74404400\end{array}$

2, $\omega B 97 D / 6-31 G(d)($ Energy $=-2307.321794$ Hartrees $)$
$\begin{array}{llll}\text { C } & -0.28478 & 6.79495 & 0.81167\end{array}$
$\begin{array}{llll}\text { C } & 3.55638 & 4.71216 & 1.05007\end{array}$
$\begin{array}{llll}\text { C } & 1.42826 & -5.90788 & 0.98489\end{array}$
$\begin{array}{llll}\text { C } & -2.80282 & -6.25342 & 0.87432\end{array}$
$\begin{array}{llll}\text { C } & -5.4946 & -2.78857 & 1.25271\end{array}$
$\begin{array}{llll}\text { C } & -6.71995 & 1.28826 & 1.20026\end{array}$
$\begin{array}{lllll}\text { C } & -3.96699 & 4.70994 & 1.19555\end{array}$
$\begin{array}{lllll}\text { C } & -1.66783 & 6.64483 & 0.87492\end{array}$
$\begin{array}{llll}\mathrm{C} & 2.43905 & 5.57017 & 0.98346\end{array}$
$\begin{array}{llll}\text { C } & 2.67789 & -5.25735 & 1.05173\end{array}$
$\begin{array}{llll}\text { C } & -1.46681 & -6.64117 & 0.81208\end{array}$
$\begin{array}{llll}\text { C } & -4.72952 & -3.94851 & 1.19387\end{array}$
$\begin{array}{llll}\text { C } & -6.84202 & -0.09915 & 1.19955\end{array}$
$\begin{array}{llll}\text { C } & -4.92328 & 3.70165 & 1.25442\end{array}$
$\begin{array}{llll}\text { C } & -2.38562 & 6.05161 & -0.1811\end{array}$
$\begin{array}{llll}\text { C } & 1.88671 & 5.97135 & -0.231\end{array}$ 


\begin{tabular}{|c|c|c|c|}
\hline $\mathrm{C}$ & 3.34396 & -4.88842 & -0.15103 \\
\hline $\mathrm{C}$ & -0.66581 & -6.32653 & -0.30364 \\
\hline $\mathrm{C}$ & -4.59569 & -4.67311 & -0.00367 \\
\hline $\mathrm{C}$ & -6.52734 & -0.85893 & 0.05678 \\
\hline $\mathrm{C}$ & -5.67005 & 3.33713 & 0.12 \\
\hline $\mathrm{C}$ & -3.70888 & 5.4003 & -0.00191 \\
\hline $\mathrm{C}$ & 0.4488 & $6.3456-$ & -0.30431 \\
\hline $\mathrm{C}$ & 4.14723 & 4.23205 & -0.15279 \\
\hline $\mathrm{C}$ & 0.81507 & -6.20741 & -0.22971 \\
\hline $\mathrm{C}$ & -3.40619 & -5.54506 & $5-0.18233$ \\
\hline $\mathrm{C}$ & -6.16579 & -2.29887 & 0.11819 \\
\hline $\mathrm{C}$ & -6.27746 & 1.98234 & 0.05802 \\
\hline $\mathrm{C}$ & -0.29647 & 5.95055 & -1.43007 \\
\hline $\mathrm{C}$ & 3.74075 & 4.85324 & -1.35764 \\
\hline $\mathrm{C}$ & 1.61299 & -6.07134 & -1.39609 \\
\hline $\mathrm{C}$ & -2.66678 & -5.43088 & -1.37206 \\
\hline $\mathrm{C}$ & -6.19791 & -3.13686 & $5-1.01306$ \\
\hline $\mathrm{C}$ & -6.15394 & 1.23796 & -1.12861 \\
\hline $\mathrm{C}$ & -4.5954 & 5.17454 & -1.07182 \\
\hline $\mathrm{C}$ & -1.67787 & 5.81007 & -1.37112 \\
\hline $\mathrm{C}$ & 2.6485 & 5.69822 & -1.39761 \\
\hline $\mathrm{C}$ & 2.83583 & -5.42964 & -1.35588 \\
\hline $\mathrm{C}$ & -1.33072 & -5.80903 & -1.43012 \\
\hline $\mathrm{C}$ & -5.42898 & -4.29577 & -1.07368 \\
\hline $\mathrm{C}$ & -6.27575 & -0.14654 & -1.12933 \\
\hline $\mathrm{C}$ & -5.55513 & 4.16789 & -1.01124 \\
\hline $\mathrm{H}$ & 0.23757 & 7.19886 & 1.67477 \\
\hline $\mathrm{H}$ & 0.87373 & -6.01727 & 1.91257 \\
\hline $\mathrm{H}$ & -3.36587 & -6.44407 & $7 \quad 1.78449$ \\
\hline $\mathrm{H}$ & -5.45116 & -2.18214 & $4 \quad 2.15246$ \\
\hline $\mathrm{H}$ & -6.91109 & 1.83355 & 2.12087 \\
\hline
\end{tabular}




\begin{tabular}{|c|c|c|c|}
\hline 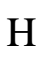 & -3.3139 & 4.86057 & 2.05017 \\
\hline $\mathrm{H}$ & -2.1888 & 6.93046 & 1.78529 \\
\hline $\mathrm{H}$ & 1.91246 & $5 \quad 5.77475$ & 1.9113 \\
\hline $\mathrm{H}$ & -1.023 & -7.12925 & 1.67565 \\
\hline $\mathrm{H}$ & -4.11294 & $4-4.21113$ & 2.04852 \\
\hline $\mathrm{H}$ & -7.12538 & $8-0.60358$ & 2.11969 \\
\hline $\mathrm{H}$ & -4.98641 & 13.09676 & 2.15404 \\
\hline $\mathrm{H}$ & 0.21606 & $5 \quad 5.60621$ & -2.32233 \\
\hline $\mathrm{H}$ & 4.23929 & 4.60675 & -2.29001 \\
\hline $\mathrm{H}$ & 1.22916 & $5-6.41094$ & -2.3537 \\
\hline 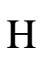 & -3.09533 & $3-4.90355$ & -2.21904 \\
\hline $\mathrm{H}$ & -6.77841 & $1 \quad-2.84314$ & -1.8837 \\
\hline $\mathrm{H}$ & -5.82045 & $5 \quad 1.73049$ & -2.03695 \\
\hline $\mathbf{H}$ & -4.48742 & $2 \quad 5.74782$ & -1.98903 \\
\hline $\mathrm{H}$ & -2.19191 & 15.36489 & -2.21773 \\
\hline 11 & 2.32965 & 6.09952 & -2.3552 \\
\hline H & 3.36998 & -5.27403 & -2.28811 \\
\hline H & -0.76589 & $9-5.55942$ & -2.32235 \\
\hline $\mathbf{H}$ & -5.42311 & $1-4.87902$ & -1.99095 \\
\hline $\mathrm{H}$ & -6.03349 & $9-0.68864$ & -2.03835 \\
\hline $\mathrm{H}$ & -6.17791 & 13.98005 & -1.88196 \\
\hline $\mathrm{C}$ & 5.38779 & -1.89967 & -1.20396 \\
\hline$C$ & 4.8425 & -3.18832 & -1.24815 \\
\hline $\mathrm{C}$ & 4.3371 & -3.82017 & -0.10192 \\
\hline C & 4.63718 & -3.21985 & 1.16455 \\
\hline C & 5.24316 & -1.95641 & 1.20981 \\
\hline $\mathrm{C}$ & 5.50296 & -1.21855 & 0.05198 \\
\hline $\mathrm{H}$ & 4.68697 & $7-3.63265$ & -2.22688 \\
\hline $\mathrm{H}$ & 5.36788 & -1.50011 & 2.18714 \\
\hline C & 5.12972 & 2.36404 & 1.16321 \\
\hline $\mathrm{C}$ & 5.50551 & 1.0142 & 1.20902 \\
\hline
\end{tabular}




$\begin{array}{llll}\text { C } & 5.63172 & 0.24154 & 0.05156 \\ \mathrm{C} & 5.637 & 0.93157 & -1.20473 \\ \mathrm{C} & 5.32516 & 2.29565 & -1.24953 \\ \mathrm{C} & 4.93849 & 3.00678 & -0.10351 \\ \mathrm{H} & 5.54967 & 0.54377 & 2.18659 \\ \mathrm{H} & 5.249 & 2.75981 & -2.22846 \\ \mathrm{C} & 5.79659 & 0.17143 & -2.42261 \\ \mathrm{C} & 5.67773 & -1.17981 & -2.42222 \\ \mathrm{C} & 4.14038 & -3.82833 & 2.37124 \\ \mathrm{C} & 3.18947 & -4.79562 & 2.31739 \\ \mathrm{C} & 3.98046 & 4.16909 & 2.31577 \\ \mathrm{C} & 4.74784 & 3.05073 & 2.3698 \\ \mathrm{H} & 3.61054 & 4.63232 & 3.22727 \\ \mathrm{H} & 5.00562 & 2.6007 & 3.32552 \\ \mathrm{H} & 5.93178 & 0.71406 & -3.3552 \\ \mathrm{H} & 5.71618 & -1.73828 & -3.35446 \\ \mathrm{H} & 4.47231 & -3.42955 & 3.32683 \\ \mathrm{H} & 2.74389 & -5.18642 & 3.22895\end{array}$

2 open adduct, $\omega B 97 D / 6-31 G(d)$ (Energy = -2770.579531 Hartrees)
C
$\begin{array}{lll}-4.10516 & -0.82253 & 0.72028\end{array}$
C
$\begin{array}{lll}0.24683 & -0.99019 & 0.00471\end{array}$
C
$13.16583-1.06916-0.00507$
C
$17.49886-0.96637-0.71971$
C
$21.84653-0.45795-1.1539$
$\begin{array}{llll}\text { C } & -12.77159 & -0.96002 & 0.70798\end{array}$
C $\quad-8.44764-0.28447 \quad 1.14506$
$\begin{array}{llll}\text { C } & -5.49588 & -0.84729 & 0.71686\end{array}$
$\begin{array}{llll}\text { C } & -1.163 & -0.99723 & 0.00493\end{array}$
$\begin{array}{llll}\text { C } & 11.75617 & -1.04788 & -0.00419\end{array}$ 

C $\quad 16.10835-0.92577-0.72332$
C $\quad 20.45318-0.41662-1.15655$
$\begin{array}{llll}\text { C } & -14.16257 & -0.97735 & 0.70923\end{array}$

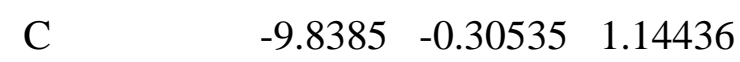
$\begin{array}{llll}\text { C } & -6.24066 & 0.10348 & -0.00179\end{array}$
$\begin{array}{llll}\text { C } & -1.90926 & 0.17725 & 0.00218\end{array}$
$\begin{array}{llll}\text { C } & 11.06616 & 0.19905 & -0.00161\end{array}$
$\begin{array}{llll}\text { C } & 15.40625 & 0.05714 & -0.00548\end{array}$
$\begin{array}{llll}\text { C } & 19.73759 & -0.06728 & 0.00175\end{array}$
$\begin{array}{llll}\text { C } & -14.90439 & -0.01925 & -0.00248\end{array}$
$\begin{array}{lllll}\text { C } & -10.57275 & 0.03684 & -0.00414\end{array}$
$\begin{array}{llll}\text { C } & -7.72293 & 0.07935 & -0.00288\end{array}$
$\begin{array}{llll}\text { C } & -3.39176 & 0.15238 & 0.0025\end{array}$
$\begin{array}{llll}\text { C } & 0.94942 & 0.24971 & 0.00095\end{array}$
$\begin{array}{llll}\text { C } & 13.92397 & 0.09764 & -0.0043\end{array}$
$\begin{array}{llll}\text { C } & 18.25397 & -0.02454 & -0.00076\end{array}$
$\begin{array}{llll}\text { C } & 22.55752 & -0.14937 & 0.00727\end{array}$
$\begin{array}{llll}\text { C } & -12.05507 & 0.01681 & -0.00416\end{array}$
$\begin{array}{llll}\text { C } & -4.13605 & 1.10348 & -0.71578\end{array}$
$\begin{array}{llll}\text { C } & 0.18034 & 1.4342 & -0.00202\end{array}$
$\begin{array}{llll}\text { C } & 13.22828 & 1.33024 & -0.00092\end{array}$
$\begin{array}{llll}\text { C } & 17.55185 & 0.95986 & 0.71467\end{array}$
$\begin{array}{llll}\text { C } & 21.86117 & 0.20026 & 1.16568\end{array}$
$\begin{array}{llll}\text { C } & -12.79693 & 0.97478 & -0.71595\end{array}$
C $\quad-8.45718 \quad 0.42054-1.1517$
$\begin{array}{llll}\text { C } & -5.52692 & 1.07958 & -0.71773\end{array}$
C $\quad-1.20109 \quad 1.40275-0.0022$
$\begin{array}{llll}\text { C } & 11.84718 & 1.37569 & -0.00035\end{array}$
$\begin{array}{llll}\text { C } & 16.16114 & 0.99997 & 0.71245\end{array}$
$\begin{array}{llll}\text { C } & 20.46779 & 0.24016 & 1.16278\end{array}$
$\begin{array}{lllll}\text { C } & -14.18784 & 0.95691 & -0.71559\end{array}$ 


\begin{tabular}{|c|c|}
\hline $\mathrm{C}$ & $\begin{array}{lll}-9.848 & 0.39987 & -1.15231\end{array}$ \\
\hline $\mathrm{H}$ & $\begin{array}{llll}-3.56188 & -1.56076 & 1.30345\end{array}$ \\
\hline $\mathrm{H}$ & $\begin{array}{lll}13.66485 & -2.03467 & 0.02179\end{array}$ \\
\hline $\mathrm{H}$ & $\begin{array}{lll}18.00875 & -1.75175 & -1.27064\end{array}$ \\
\hline $\mathrm{H}$ & $22.37798 \quad-0.72296-2.06427$ \\
\hline $\mathrm{H}$ & $\begin{array}{llll}-12.2307 & -1.72768 & 1.25436\end{array}$ \\
\hline $\mathrm{H}$ & $\begin{array}{lll}-7.91451 & -0.51841 & 2.06236\end{array}$ \\
\hline $\mathrm{H}$ & $\begin{array}{lll}-6.01414 & -1.62726 & 1.26762\end{array}$ \\
\hline $\mathrm{H}$ & $-1.67168-1.95771 \quad-0.02101$ \\
\hline $\mathrm{H}$ & $15.55664-1.65772-1.30651$ \\
\hline $\mathrm{H}$ & $19.91193-0.63147 \quad-2.07382$ \\
\hline $\mathrm{H}$ & $-14.68345-1.73616 \quad 1.28646$ \\
\hline $\mathrm{H}$ & $\begin{array}{lll}-10.36548 & -0.55532 & 2.06098\end{array}$ \\
\hline $\mathrm{H}$ & $\begin{array}{lll}-3.61791 & 1.86241 & -1.29531\end{array}$ \\
\hline $\mathrm{H}$ & $\begin{array}{lll}0.67364 & 2.4005 & 0.00575\end{array}$ \\
\hline $\mathrm{H}$ & $\begin{array}{lll}13.79053 & 2.25916 & -0.028\end{array}$ \\
\hline $\mathrm{H}$ & $18.10398 \quad 1.71575 \quad 1.26617$ \\
\hline $\mathrm{H}$ & $22.40358 \quad 0.43406 \quad 2.07813$ \\
\hline $\mathrm{H}$ & $\begin{array}{llll}-12.27621 & 1.75582 & -1.26292\end{array}$ \\
\hline $\mathrm{H}$ & $\begin{array}{llll}-7.93036 & 0.67037 & -2.06845\end{array}$ \\
\hline $\mathrm{H}$ & $\begin{array}{llll}-6.06988 & 1.84238 & -1.26874\end{array}$ \\
\hline $\mathrm{H}$ & $\begin{array}{llll}-1.75384 & 2.33737 & 0.02363\end{array}$ \\
\hline $\mathrm{H}$ & $\begin{array}{lll}11.36367 & 2.34694 & -0.00892\end{array}$ \\
\hline $\mathrm{H}$ & $\begin{array}{lll}15.65161 & 1.76484 & 1.29186\end{array}$ \\
\hline $\mathrm{H}$ & $19.93628 \quad 0.48616 \quad 2.0779$ \\
\hline $\mathrm{H}$ & $\begin{array}{llll}-14.72843 & 1.70223 & -1.29224\end{array}$ \\
\hline $\mathrm{H}$ & $\begin{array}{lll}-10.38138 & 0.63422 & -2.06936\end{array}$ \\
\hline $\mathrm{C}$ & $\begin{array}{llll}7.44262 & 1.36465 & -0.00092\end{array}$ \\
\hline $\mathrm{C}$ & $\begin{array}{llll}8.84155 & 1.37806 & -0.00135\end{array}$ \\
\hline $\mathrm{C}$ & $\begin{array}{lll}9.60753 & 0.20611 & -0.00073\end{array}$ \\
\hline $\mathrm{C}$ & $\begin{array}{lll}8.90447 & -1.04282 & 0.00112\end{array}$ \\
\hline
\end{tabular}




\begin{tabular}{|c|c|}
\hline $\mathrm{C}$ & $\begin{array}{lll}7.50193 & -1.05436 & 0.00152\end{array}$ \\
\hline $\mathrm{C}$ & $\begin{array}{lll}6.73952 & 0.11501 & 0.00026\end{array}$ \\
\hline $\mathrm{H}$ & $\begin{array}{llll}9.3304 & 2.34743 & -0.00223\end{array}$ \\
\hline $\mathrm{H}$ & $\begin{array}{lll}7.0129 & -2.02377 & 0.00294\end{array}$ \\
\hline $\mathrm{C}$ & $\begin{array}{lll}3.0985 & -1.01378 & 0.00043\end{array}$ \\
\hline $\mathrm{C}$ & $\begin{array}{llll}4.50085 & -1.03937 & 0.00033\end{array}$ \\
\hline $\mathrm{C}$ & $\begin{array}{lll}5.27491 & 0.12233 & 0.00025\end{array}$ \\
\hline $\mathrm{C}$ & $\begin{array}{llll}4.58437 & 1.37898 & -0.00015\end{array}$ \\
\hline $\mathrm{C}$ & $\begin{array}{lll}3.18565 & 1.4064 & 0.00002\end{array}$ \\
\hline $\mathrm{C}$ & $\begin{array}{lll}2.40802 & 0.24215 & 0.0006\end{array}$ \\
\hline $\mathrm{H}$ & $\begin{array}{lll}4.98019 & -2.01362 & 0.00016\end{array}$ \\
\hline $\mathrm{H}$ & $2.70656 \quad 2.38063-0.00035$ \\
\hline $\mathrm{C}$ & $\begin{array}{llll}5.34156 & 2.60511 & -0.00097\end{array}$ \\
\hline $\mathrm{C}$ & $6.69776 \quad 2.5983 \quad-0.00151$ \\
\hline $\mathrm{C}$ & $\begin{array}{llll}9.64803 & -2.27308 & 0.00366\end{array}$ \\
\hline $\mathrm{C}$ & $\begin{array}{llll}11.00621 & -2.27606 & 0.00209\end{array}$ \\
\hline $\mathrm{C}$ & $\begin{array}{llll}0.98439 & -2.22586 & 0.00025\end{array}$ \\
\hline $\mathrm{C}$ & $2.34254-2.23647 \quad-0.00082$ \\
\hline $\mathrm{H}$ & $0.42502-3.15824-0.00106$ \\
\hline $\mathrm{H}$ & $2.88714-3.17767-0.00259$ \\
\hline $\mathrm{H}$ & $4.79448 \quad 3.54482-0.00129$ \\
\hline $\mathrm{H}$ & $7.25424 \quad 3.53248 \quad-0.00231$ \\
\hline $\mathrm{H}$ & $\begin{array}{llll}9.09398 & -3.20874 & 0.00678\end{array}$ \\
\hline $\mathrm{H}$ & $\begin{array}{lll}11.55621 & -3.214 & 0.00435\end{array}$ \\
\hline $\mathrm{C}$ & $\begin{array}{lll}-18.49337 & -1.26236 & -0.00859\end{array}$ \\
\hline $\mathrm{C}$ & $\begin{array}{lll}-17.10223 & -1.2465 & -0.01016\end{array}$ \\
\hline $\mathrm{C}$ & $\begin{array}{lll}-16.38704 & -0.03689 & -0.00081\end{array}$ \\
\hline $\mathrm{C}$ & $\begin{array}{lll}-17.1301 & 1.15578 & 0.01031\end{array}$ \\
\hline $\mathrm{C}$ & $\begin{array}{lll}-18.5212 & 1.13955 & 0.01167\end{array}$ \\
\hline $\mathrm{C}$ & $\begin{array}{lll}-19.23615 & -0.06986 & 0.00221\end{array}$ \\
\hline $\mathrm{H}$ & $\begin{array}{lll}-19.01369 & -2.21612 & 0.00158\end{array}$ \\
\hline
\end{tabular}




$\begin{array}{llll}\mathrm{H} & -16.56072 & -2.18804 & -0.03717 \\ \mathrm{H} & -16.61042 & 2.10957 & 0.03636 \\ \mathrm{H} & -19.06341 & 2.08104 & 0.00271 \\ \mathrm{C} & -22.82688 & -1.05799 & -0.74119 \\ \mathrm{C} & -21.43299 & -1.04127 & -0.74267 \\ \mathrm{C} & -20.72028 & -0.08701 & 0.00363 \\ \mathrm{C} & -21.4535 & 0.85049 & 0.7513 \\ \mathrm{C} & -22.8474 & 0.83479 & 0.75258 \\ \mathrm{C} & -23.54101 & -0.1198 & 0.00641 \\ \mathrm{H} & -23.35611 & -1.80048 & -1.33285 \\ \mathrm{H} & -20.88823 & -1.75882 & -1.34994 \\ \mathrm{H} & -20.92437 & 1.58058 & 1.35748 \\ \mathrm{H} & -23.39259 & 1.56482 & 1.34527 \\ \mathrm{H} & -24.62757 & -0.13245 & 0.0075 \\ \mathrm{H} & 23.64369 & -0.18104 & 0.00939\end{array}$

S9, $\omega B 97 D / 6-31 G(d)($ Energy $=-2077.0194309$ Hartrees $)$

$\begin{array}{llll}\mathrm{O} & 3.88400800 & 0.45060700 & 3.08472100 \\ \mathrm{C} & 4.81377500 & -0.62021300 & 2.93876100 \\ \mathrm{C} & 6.05758000 & -0.13039600 & 2.22470800 \\ \mathrm{C} & 6.62316900 & -0.74540900 & 1.18882700 \\ \mathrm{C} & 4.17854300 & -1.76536400 & 2.18485600 \\ \mathrm{C} & 4.74547400 & -2.38432400 & 1.15023100 \\ \mathrm{C} & 6.07061200 & -1.98780700 & 0.53418000 \\ \mathrm{O} & 5.85082300 & -1.70660200 & -0.85078600 \\ \mathrm{C} & 7.07224900 & -3.14753100 & 0.58764300 \\ \mathrm{C} & 7.63007900 & -3.55987100 & 1.79986000 \\ \mathrm{C} & 8.49729000 & -4.64686400 & 1.85389500\end{array}$




\begin{tabular}{|c|c|c|c|}
\hline $\mathrm{C}$ & 8.86342200 & -5.33245300 & 0.69273900 \\
\hline $\mathrm{C}$ & 9.85488100 & -6.50237700 & 0.72885300 \\
\hline $\mathrm{O}$ & 9.62324700 & -7.25602400 & -0.46638600 \\
\hline $\mathrm{C}$ & 11.28499500 & -6.00148400 & 0.69723400 \\
\hline $\mathrm{C}$ & 12.19124000 & -6.24036900 & 1.64425100 \\
\hline $\mathrm{C}$ & 11.89526000 & -6.98507900 & 2.92508200 \\
\hline $\mathrm{C}$ & 11.81340000 & -6.04497900 & 4.12745200 \\
\hline $\mathrm{C}$ & 12.03858600 & -6.47882700 & 5.43360400 \\
\hline $\mathrm{C}$ & 11.61246000 & -5.69875800 & 6.51098100 \\
\hline $\mathrm{C}$ & 11.25834000 & -4.78787900 & 3.93303400 \\
\hline $\mathrm{C}$ & 10.77133600 & -4.02619300 & 4.98798900 \\
\hline $\mathrm{C}$ & 9.96354200 & -2.83567200 & 4.63234200 \\
\hline $\mathrm{C}$ & 10.34446000 & -1.86843000 & 3.79922200 \\
\hline $\mathrm{C}$ & 9.47843200 & -0.70362500 & 3.42994800 \\
\hline $\mathrm{C}$ & 10.91815200 & -4.50291700 & 6.31430600 \\
\hline $\mathrm{C}$ & 10.10429500 & -3.76176400 & 7.32647200 \\
\hline $\mathrm{C}$ & 8.91954600 & -4.33278100 & 7.84472300 \\
\hline $\mathrm{C}$ & 8.76842300 & -5.78402600 & 8.05666600 \\
\hline $\mathrm{C}$ & 7.98197400 & -6.33103400 & 8.98482000 \\
\hline $\mathrm{C}$ & 7.80367000 & -3.49901000 & 7.95772700 \\
\hline $\mathrm{C}$ & 7.82269700 & -2.16285100 & 7.53994000 \\
\hline $\mathrm{C}$ & 6.67143000 & -1.70990100 & 6.69160500 \\
\hline $\mathrm{C}$ & 6.33081100 & -2.66921200 & 5.72462800 \\
\hline $\mathrm{C}$ & 5.61960300 & -2.35433400 & 4.58205300 \\
\hline $\mathrm{C}$ & 5.24715500 & -1.03721100 & 4.34250000 \\
\hline
\end{tabular}




\begin{tabular}{|c|c|c|c|}
\hline $\mathrm{C}$ & 5.43866200 & -0.10494900 & 5.36190500 \\
\hline $\mathrm{C}$ & 6.10415700 & -0.42303600 & 6.55897800 \\
\hline $\mathrm{C}$ & 6.19629100 & 0.61818000 & 7.59642300 \\
\hline $\mathrm{C}$ & 6.47313600 & 0.42308200 & 8.88827100 \\
\hline $\mathrm{C}$ & 6.58085700 & 1.51663000 & 9.90385600 \\
\hline $\mathrm{C}$ & 9.08564100 & -1.53729800 & 7.44330500 \\
\hline $\mathrm{C}$ & 9.26703200 & -0.10844600 & 7.12650200 \\
\hline $\mathrm{C}$ & 10.27187400 & 0.63592000 & 7.58848200 \\
\hline $\mathrm{C}$ & 10.20802300 & -2.37232200 & 7.35378900 \\
\hline $\mathrm{O}$ & 12.91354300 & -7.94180100 & 3.19441300 \\
\hline $\mathrm{C}$ & 10.53907900 & -7.66149100 & 2.84819000 \\
\hline $\mathrm{C}$ & 9.62643400 & -7.41242700 & 1.91192600 \\
\hline $\mathrm{C}$ & 8.32308800 & -4.90464600 & -0.52005600 \\
\hline $\mathrm{C}$ & 7.43175000 & -3.83542800 & -0.57081800 \\
\hline $\mathrm{H}$ & 6.49753700 & 0.77184300 & 2.64448800 \\
\hline $\mathrm{H}$ & 7.52397200 & -0.34773700 & 0.72829100 \\
\hline $\mathrm{H}$ & 3.20956000 & -2.08465100 & 2.56207200 \\
\hline $\mathrm{H}$ & 4.24478600 & -3.21229600 & 0.65354500 \\
\hline $\mathrm{H}$ & 7.39333700 & -3.02895100 & 2.71718700 \\
\hline $\mathrm{H}$ & 8.88969600 & -4.96125600 & 2.81577600 \\
\hline $\mathrm{H}$ & 11.54823900 & -5.44179400 & -0.19762000 \\
\hline $\mathrm{H}$ & 13.20816300 & -5.86643400 & 1.54992300 \\
\hline $\mathrm{H}$ & 12.50266100 & -7.44378300 & 5.60824600 \\
\hline $\mathrm{H}$ & 11.77088800 & -6.05877600 & 7.52389200 \\
\hline $\mathrm{H}$ & 11.08373700 & -4.42893200 & 2.92581400 \\
\hline
\end{tabular}




\begin{tabular}{|c|c|c|c|}
\hline $\mathrm{H}$ & 8.96757700 & -2.78139700 & 5.06614200 \\
\hline $\mathrm{H}$ & 11.34691100 & -1.89534700 & 3.37123000 \\
\hline $\mathrm{H}$ & 9.93715800 & 0.24096900 & 3.74793800 \\
\hline U & 8.49107000 & -0.78037500 & 3.89850700 \\
\hline $\mathrm{H}$ & 9.33321200 & -0.64883100 & 2.34477700 \\
\hline 11 & 9.35970800 & -6.43077100 & 7.41267600 \\
\hline $\mathrm{H}$ & 7.40415500 & -5.72472200 & 9.67820800 \\
\hline $\mathrm{H}$ & 7.90056400 & -7.40818200 & 9.09063500 \\
\hline $\mathrm{H}$ & 6.84011900 & -3.96832400 & 8.13447300 \\
\hline $\mathrm{H}$ & 6.72711600 & -3.67400500 & 5.81980400 \\
\hline $\mathrm{H}$ & 5.43548600 & -3.12359800 & 3.84054500 \\
\hline $\mathrm{H}$ & 5.07276400 & 0.90807300 & 5.21973100 \\
\hline $\mathrm{H}$ & 5.99161800 & 1.63378600 & 7.25561500 \\
\hline $\mathrm{H}$ & 6.65898200 & -0.58751200 & 9.24812600 \\
\hline $\mathrm{H}$ & 6.36846600 & 2.49654100 & 9.46485300 \\
\hline $\mathrm{H}$ & 7.59204200 & 1.54446100 & 10.32858100 \\
\hline $\mathrm{H}$ & 5.88737200 & 1.35409400 & 10.73772100 \\
\hline $\mathrm{H}$ & 8.53142100 & 0.34189600 & 6.46633900 \\
\hline $\mathrm{H}$ & 11.01293800 & 0.23468800 & 8.27576200 \\
\hline $\mathrm{H}$ & 10.37724500 & 1.67845600 & 7.30517800 \\
\hline $\mathrm{H}$ & 11.13603300 & -1.91918100 & 7.02128200 \\
\hline $\mathrm{H}$ & 10.32744100 & -8.34644800 & 3.66661900 \\
\hline $\mathrm{H}$ & 8.65577800 & -7.90142100 & 1.93467200 \\
\hline $\mathrm{H}$ & 8.58637600 & -5.42613400 & -1.43296800 \\
\hline $\mathrm{H}$ & 7.01305600 & -3.52813900 & -1.52155900 \\
\hline
\end{tabular}




$\begin{array}{llll}\mathrm{H} & 5.19693400 & -0.99731800 & -0.88474900 \\ \mathrm{H} & 3.70860000 & 0.79226100 & 2.19866300 \\ \mathrm{H} & 12.86983400 & -8.60465500 & 2.49383100 \\ \mathrm{H} & 10.28327700 & -7.96086400 & -0.47488100\end{array}$

Dipropenyl-divinyl biphenyl, $\omega B 97 D / 6-31 G(d)$ (Energy = -851.24511993 Hartrees)

$\begin{array}{llll}\mathrm{C} & 3.56375 & -0.00035 & -0.00012 \\ \mathrm{C} & 2.8595 & -1.13878 & -0.39644 \\ \mathrm{C} & 1.46541 & -1.13773 & -0.39666 \\ \mathrm{C} & 0.74287 & 0.00039 & 0.00011 \\ \mathrm{C} & 1.46601 & 1.13813 & 0.39671 \\ \mathrm{C} & 2.86014 & 1.13839 & 0.39635 \\ \mathrm{H} & 3.39651 & -2.02833 & -0.71551 \\ \mathrm{H} & 0.92888 & 2.02077 & 0.73284 \\ \mathrm{C} & -0.74287 & 0.0004 & -0.00006 \\ \mathrm{C} & -1.46543 & -1.13772 & 0.3967 \\ \mathrm{C} & -2.85951 & -1.13876 & 0.39644 \\ \mathrm{C} & -3.56375 & -0.00035 & 0.00005 \\ \mathrm{C} & -2.86012 & 1.1384 & -0.39636 \\ \mathrm{C} & -1.466 & 1.13813 & -0.39668 \\ \mathrm{H} & -0.92765 & -2.01996 & 0.73288 \\ \mathrm{H} & -4.65042 & -0.00065 & 0.00012 \\ \mathrm{H} & -3.39757 & 2.02772 & -0.71532 \\ \mathrm{H} & 4.65042 & -0.00064 & -0.00031 \\ \mathrm{C} & -3.62036 & -2.39907 & 0.84848 \\ \mathrm{H} & -3.07719 & -3.26667 & 1.16015 \\ \mathrm{C} & -4.97548 & -2.41306 & 0.85289 \\ \mathrm{H} & -5.52411 & -1.5485 & 0.54231 \\ \mathrm{C} & -5.72835 & -3.67759 & 1.30645 \\ \mathrm{H} & -5.45812 & -3.91107 & 2.3151 \\ \mathrm{H} & -6.78274 & -3.50419 & 1.25071 \\ \mathrm{H} & -5.46731 & -4.49613 & 0.66868 \\ \mathrm{C} & -0.70467 & 2.38915 & -0.87306 \\ \mathrm{H} & -1.24751 & 3.25098 & -1.20092 \\ \mathrm{C} & 0.70319 & -2.38816 & -0.87316 \\ \mathrm{H} & -2.2454 & -3.25029 & -1.20121 \\ \mathrm{C} & -1.65195 & -2.40034 & -0.8784 \\ \mathrm{H} & -1.40984 & -3.65711 & -0.41177 \\ \mathrm{C} & -1.46498 & -3.66416 & 0.65678 \\ \mathrm{H} & \mathrm{H} & -3.64923 & -0.81991 \\ \mathrm{H} & & & \end{array}$




$\begin{array}{llll}\mathrm{H} & -0.89222 & -4.53114 & -0.74798 \\ \mathrm{C} & 3.6216 & 2.39837 & 0.84825 \\ \mathrm{H} & 4.69156 & 2.39991 & 0.85798 \\ \mathrm{C} & 0.65045 & 2.40226 & -0.87846 \\ \mathrm{H} & 1.19604 & 1.5481 & -1.22144 \\ \mathrm{H} & 1.17661 & 3.26974 & -0.53856 \\ \mathrm{C} & 2.9412 & 3.50521 & 1.23365 \\ \mathrm{H} & 1.87124 & 3.50966 & 1.22603 \\ \mathrm{H} & 3.47579 & 4.37765 & 1.54662\end{array}$

S9 open adduct, $\omega B 97 D / 6-31 G(d)$ (Energy $=-2928.3337015$ Hartrees)

$\begin{array}{llll}\text { O } & 6.91325200 & -4.59124500 & -10.64188300\end{array}$

C $\quad 8.31519600 \quad-4.51429700-10.37078700$

$\begin{array}{llll}\text { C } & 9.05472300 & -4.11510200 & -11.62376500\end{array}$

$\begin{array}{llll}\text { C } & 10.00783600 & -4.84355800 & -12.19431800\end{array}$

$\begin{array}{llll}\text { C } & 8.71032500 & -5.89663500 & -9.91355100\end{array}$

$\begin{array}{llll}\text { C } & 9.66591600 & -6.62461900 & -10.48138100\end{array}$

C $\quad 10.46116800 \quad-6.19235400-11.69180700$

$\begin{array}{llll}\text { O } & 10.27352700 & -7.15404000 & -12.73609500\end{array}$

C $\quad 11.94626300 \quad-6.20245300-11.34337900$

$\begin{array}{llll}\text { C } & 12.52916100 & -5.10301000 & -10.72142100\end{array}$

C $\quad 13.87727300 \quad-5.10156800-10.38858200$

C $\quad 14.67953100 \quad-6.20901400-10.65392400$

$\begin{array}{llll}\text { C } & 16.18911900 & -6.19788400 & -10.37462200\end{array}$

$\begin{array}{llll}\text { O } & 16.40711500 & -7.11944600 & -9.30921900\end{array}$

$\begin{array}{llll}\text { C } & 16.95581900 & -6.64837400 & -11.60904300\end{array}$

C $\quad 17.42858800 \quad-5.78681400-12.50305700$

$\begin{array}{llll}\text { C } & 17.18957000 & -4.29821500 & -12.36615800\end{array}$

$\begin{array}{llll}\text { C } & 15.87481900 & -3.87607500 & -13.04642000\end{array}$ 

C $\quad 15.51920900 \quad-2.52426100-13.06842100$
C $\quad 14.28491800 \quad-2.14090400 \quad-13.57062400$
C $\quad 14.99161500 \quad-4.80076500-13.58687600$
C $\quad 13.74840700 \quad-4.43434200 \quad-14.11387300$
C $\quad 12.84545200 \quad-5.48782300 \quad-14.61738400$
C $\quad 13.23881500 \quad-6.62836300 \quad-15.18868300$
C $\quad 12.30566300 \quad-7.72450100 \quad-15.60096700$
$\begin{array}{llll}\text { C } & 8.73262900 & 1.22038600 & -4.79802400\end{array}$
$\begin{array}{llll}\text { C } & 7.92211900 & 2.45195300 & -4.73878200\end{array}$
$\begin{array}{llll}\text { C } & 7.34078800 & 3.04316400 & -5.78331700\end{array}$
$\begin{array}{llll}\text { C } & 8.51769800 & 0.26322300 & -5.79250700\end{array}$
$\begin{array}{llll}\text { C } & 9.29530900 & -0.88564500 & -5.90189000\end{array}$
C $\quad 9.07662900 \quad-1.78751700 \quad-7.06790800$
C $\quad 7.88525400 \quad-2.51126600 \quad-7.15297700$
C $\quad 7.62891000 \quad-3.37083500 \quad-8.21393700$
C $\quad 8.57427900 \quad-3.52495400 \quad-9.22793400$
C $\quad 9.75679300 \quad-2.79763700 \quad-9.14962800$
$\begin{array}{llll}\text { C } & 10.03074000 & -1.92005200 & -8.09643700\end{array}$
C $\quad 11.31766700 \quad-1.19095400 \quad-8.14399400$
$\begin{array}{llll}\text { C } & 11.54778800 & 0.07087300 & -7.77100500\end{array}$
$\begin{array}{llll}\text { C } & 12.88149000 & 0.74570800 & -7.86506000\end{array}$
C $\quad 10.28888500 \quad-1.14393600 \quad-4.94018000$
C $\quad 11.10934700 \quad-2.36718000 \quad-5.01355300$
C $\quad 12.36638100 \quad-2.47736600 \quad-4.58224600$
C $\quad 10.47708900 \quad-0.20718900 \quad-3.92205500$ 


\begin{tabular}{|c|c|c|c|}
\hline $\mathrm{O}$ & 18.27777000 & -3.54740200 & -12.89591500 \\
\hline $\mathrm{C}$ & 17.11567400 & -3.94939600 & -10.89313600 \\
\hline $\mathrm{C}$ & 16.66214600 & -4.81120300 & -9.99006300 \\
\hline $\mathrm{C}$ & 14.08168400 & -7.33019400 & -11.23408000 \\
\hline $\mathrm{C}$ & 12.73626600 & -7.32691300 & -11.58393600 \\
\hline $\mathrm{H}$ & 8.77672900 & -3.15648900 & -12.06016900 \\
\hline $\mathrm{H}$ & 10.49345100 & -4.48622500 & -13.09745300 \\
\hline $\mathrm{H}$ & 8.15871600 & -6.27154100 & -9.05512900 \\
\hline $\mathrm{H}$ & 9.91666000 & -7.61025700 & -10.09662900 \\
\hline $\mathrm{H}$ & 11.92552800 & -4.22985800 & -10.49925500 \\
\hline $\mathrm{H}$ & 14.30909600 & -4.20903600 & -9.94991900 \\
\hline $\mathrm{H}$ & 17.08929300 & -7.72123500 & -11.72287400 \\
\hline $\mathrm{H}$ & 17.96369800 & -6.12020100 & -13.38896000 \\
\hline $\mathrm{H}$ & 16.20387700 & -1.77765700 & -12.67642200 \\
\hline $\mathrm{H}$ & 14.00645700 & -1.09085300 & -13.56698900 \\
\hline $\mathrm{H}$ & 15.22956400 & -5.85573700 & -13.53798400 \\
\hline $\mathrm{H}$ & 11.77870900 & -5.32479500 & -14.49047700 \\
\hline $\mathrm{H}$ & 14.30066700 & -6.80191200 & -15.36557100 \\
\hline $\mathrm{H}$ & 12.36071500 & -7.91352200 & -16.68041600 \\
\hline $\mathrm{H}$ & 11.27352200 & -7.48422900 & -15.33298900 \\
\hline $\mathrm{H}$ & 12.56986600 & -8.66301300 & -15.09788700 \\
\hline $\mathrm{H}$ & 7.82451400 & 2.90828500 & -3.75605100 \\
\hline $\mathrm{H}$ & 7.44358300 & 2.66355000 & -6.79684800 \\
\hline $\mathrm{H}$ & 6.75297900 & 3.94685000 & -5.65824800 \\
\hline $\mathrm{H}$ & 7.73118000 & 0.42555700 & -6.52458300 \\
\hline
\end{tabular}




\begin{tabular}{|c|c|c|c|}
\hline $\mathrm{H}$ & 7.15276600 & -2.40396100 & -6.35737400 \\
\hline $\mathrm{H}$ & 6.69889900 & -3.92909400 & -8.25672400 \\
\hline $\mathrm{H}$ & 10.48254100 & -2.87927400 & -9.95199200 \\
\hline $\mathrm{H}$ & 12.14412300 & -1.75816400 & -8.57604000 \\
\hline $\mathrm{H}$ & 10.73668400 & 0.66927200 & -7.36121100 \\
\hline $\mathrm{H}$ & 13.64257700 & 0.07981200 & -8.28397800 \\
\hline $\mathrm{H}$ & 13.22008800 & 1.07056200 & -6.87300400 \\
\hline $\mathrm{H}$ & 12.82713000 & 1.64390900 & -8.49284500 \\
\hline $\mathrm{H}$ & 10.63767500 & -3.22821400 & -5.48268800 \\
\hline $\mathrm{H}$ & 12.90672800 & -1.63781800 & -4.15215100 \\
\hline $\mathrm{H}$ & 12.90439700 & -3.41653400 & -4.66249700 \\
\hline $\mathrm{H}$ & 11.22807700 & -0.39686800 & -3.15988700 \\
\hline $\mathrm{H}$ & 17.42873900 & -2.94322300 & -10.62987400 \\
\hline $\mathrm{H}$ & 16.57143400 & -4.54760100 & -8.93956400 \\
\hline $\mathrm{H}$ & 14.67822100 & -8.21510900 & -11.43600100 \\
\hline $\mathrm{H}$ & 12.29618900 & -8.19134800 & -12.06808800 \\
\hline $\mathrm{C}$ & 13.37625700 & -3.07803800 & -14.07853300 \\
\hline $\mathrm{C}$ & 12.05079900 & -2.62063700 & -14.58162500 \\
\hline $\mathrm{C}$ & 11.67507500 & -2.92791700 & -15.89129300 \\
\hline $\mathrm{C}$ & 11.17231300 & -1.87432900 & -13.77139000 \\
\hline $\mathrm{C}$ & 10.45143600 & -2.53311400 & -16.43577000 \\
\hline $\mathrm{H}$ & 12.36498300 & -3.50120200 & -16.50530700 \\
\hline $\mathrm{C}$ & 9.94231400 & -1.48127500 & -14.31801300 \\
\hline $\mathrm{C}$ & 9.58743400 & -1.79239700 & -15.61872900 \\
\hline $\mathrm{H}$ & 9.24285400 & -0.93245800 & -13.69353900 \\
\hline
\end{tabular}



$\mathrm{H} \quad 8.62728100 \quad-1.45674600 \quad-15.99873900$
C $\quad 11.49862900 \quad-1.54747700 \quad-12.36881400$
C $\quad 11.07351300 \quad-0.46346400 \quad-11.71675300$
$\mathrm{H} \quad 10.47061400 \quad 0.30000100-12.20287900$
$\mathrm{H} \quad 11.31764500 \quad-0.29974100 \quad-10.67164100$
$\mathrm{H} \quad 12.14079200 \quad-2.25816800 \quad-11.85165100$
C $\quad 10.13353600 \quad-2.91196600 \quad-17.82251100$
$\mathrm{H} \quad 10.95724200 \quad-3.36865400 \quad-18.37223200$
C $\quad 8.96150700 \quad-2.76824200 \quad-18.44691700$
$\mathrm{H} \quad 8.11412300 \quad-2.33315200-17.91792400$
C $\quad 8.70208800 \quad-3.17629900-19.86499000$
H $\quad 9.59548600 \quad-3.60648400 \quad-20.32812200$
$\mathrm{H} \quad 7.89703700 \quad-3.91940200-19.91958200$
$\begin{array}{llll}\text { C } & 9.74484400 & 0.97539400 & -3.85115800\end{array}$
$\begin{array}{llll}\text { C } & 10.08111000 & 1.96767200 & -2.78965600\end{array}$
C $\quad 10.70686600 \quad 3.18673900 \quad-3.12505200$
C $\quad 9.77794400 \quad 1.68266000 \quad-1.46206000$
$\begin{array}{llll}\text { C } & 10.99441400 & 4.08478600 & -2.09336500\end{array}$
$\begin{array}{llll}\text { C } & 10.06836800 & 2.58206900 & -0.43005900\end{array}$
$\begin{array}{llll}\mathrm{H} & 9.28032400 & 0.74273200 & -1.24029800\end{array}$
$\begin{array}{llll}\text { C } & 10.67142300 & 3.79554000 & -0.77512600\end{array}$
$\mathrm{H} \quad 11.50524200 \quad 5.01441300 \quad-2.32740300$
$\begin{array}{llll}\mathrm{H} & 10.91267400 & 4.51368900 & 0.00473900\end{array}$
$\begin{array}{llll}\text { C } & 11.06137600 & 3.48050700 & -4.52576500\end{array}$
$\begin{array}{llll}\mathrm{H} & 11.31571300 & 2.61984300 & -5.14394500\end{array}$ 


\begin{tabular}{llll}
$\mathrm{C}$ & 11.05337800 & 4.69080000 & -5.08819600 \\
$\mathrm{H}$ & 10.74559200 & 5.55340100 & -4.49663900 \\
$\mathrm{C}$ & 11.40456900 & 4.95870600 & -6.51952300 \\
$\mathrm{H}$ & 11.70770500 & 4.04109200 & -7.03396400 \\
$\mathrm{H}$ & 10.54814000 & 5.38475500 & -7.05649300 \\
$\mathrm{C}$ & 9.76981500 & 2.29909100 & 0.98474400 \\
$\mathrm{H}$ & 9.86377300 & 3.15514200 & 1.65236800 \\
$\mathrm{C}$ & 9.42343600 & 1.12277800 & 1.50971500 \\
$\mathrm{H}$ & 9.33309300 & 0.21793800 & 0.91483700 \\
$\mathrm{H}$ & 9.22577300 & 1.02293300 & 2.57204700 \\
$\mathrm{H}$ & 6.60327100 & -3.68852400 & -10.79693300 \\
$\mathrm{H}$ & 18.17661200 & -3.53571300 & -13.85621100 \\
$\mathrm{H}$ & 17.36057900 & -7.14053500 & -9.15038800 \\
$\mathrm{H}$ & 9.32053100 & -7.19958000 & -12.88929500 \\
$\mathrm{H}$ & 8.38352600 & -2.31785500 & -20.46929900 \\
$\mathrm{H}$ & 12.22389400 & 5.68366100 & -6.59922400 \\
\hline & & & \\
\hline
\end{tabular}

\begin{tabular}{lrrr}
\multicolumn{1}{l}{ 18, $\omega B 97 D / 6-31 G(d)($ Energy $=-1773.8048116$ Hartrees $)$} \\
C & -18.72574900 & 6.89881700 & 11.10900700 \\
C & -19.37281700 & 5.87607300 & 10.22586500 \\
C & -19.04579500 & 4.58553200 & 10.08029900 \\
C & -17.97244400 & 3.85004900 & 10.76979900 \\
C & -18.17525300 & 2.47751500 & 11.02434000 \\
C & -17.27389000 & 1.71577100 & 11.76813300 \\
C & -16.25410600 & 2.41193900 & 12.42969800
\end{tabular}




$\begin{array}{llll}\mathrm{C} & -15.97788300 & 3.72031200 & 12.09508600 \\ \mathrm{C} & -16.73683000 & 4.43115600 & 11.14919400 \\ \mathrm{C} & -15.95442000 & 5.49501000 & 10.43837300 \\ \mathrm{C} & -15.83718900 & 5.56808800 & 9.03114600 \\ \mathrm{C} & -16.83402900 & 5.03880100 & 8.07754500 \\ \mathrm{C} & -17.16006300 & 5.63413200 & 6.93082500 \\ \mathrm{C} & -14.61067800 & 5.97726800 & 8.49405500 \\ \mathrm{C} & -13.46487900 & 6.19197700 & 9.26816600 \\ \mathrm{C} & -12.16554000 & 5.66787900 & 8.72579900 \\ \mathrm{C} & -12.31381600 & 4.47848700 & 7.99583500 \\ \mathrm{C} & -11.30144800 & 3.54803700 & 7.87943600 \\ \mathrm{C} & -10.09519500 & 3.73228400 & 8.56358600 \\ \mathrm{C} & -9.46302500 & 2.44852300 & 8.98887500 \\ \mathrm{C} & -9.35727900 & 1.35235800 & 8.12134400 \\ \mathrm{C} & -9.58067900 & 0.05904000 & 8.57999000 \\ \mathrm{C} & -9.49148300 & 2.17059100 & 10.35923800 \\ \mathrm{C} & -9.73413300 & 0.88036900 & 10.81238400 \\ \mathrm{C} & -9.94381900 & -0.17766200 & 9.91583100 \\ \mathrm{C} & -10.91041100 & -1.25205800 & 10.29754000 \\ \mathrm{C} & -11.76140600 & -1.79204500 & 9.32107800 \\ \mathrm{C} & -13.05067500 & -2.20356700 & 9.63116600 \\ \mathrm{C} & -13.56367500 & -2.04898500 & 10.92682200 \\ \mathrm{C} & -14.99648400 & -1.69540800 & 11.16218700 \\ \mathrm{C} & -15.72992000 & -1.14498800 & 10.10087200 \\ \mathrm{C} & -16.71323800 & -0.18991700 & 10.32299500\end{array}$




\begin{tabular}{|c|c|c|c|}
\hline $\mathrm{C}$ & -16.98871600 & 0.25831400 & 11.61890400 \\
\hline $\mathrm{C}$ & -16.48271200 & -0.51065500 & 12.67640700 \\
\hline $\mathrm{C}$ & -15.51234100 & -1.48047800 & 12.45055600 \\
\hline $\mathrm{C}$ & -12.61669800 & -1.80495400 & 11.93325500 \\
\hline $\mathrm{C}$ & -11.31256100 & -1.43817700 & 11.62777300 \\
\hline $\mathrm{C}$ & -9.85393000 & 5.00642500 & 9.08176900 \\
\hline $\mathrm{C}$ & -10.84248600 & 6.01015300 & 9.10549100 \\
\hline $\mathrm{C}$ & -10.45291600 & 7.35595200 & 9.55354700 \\
\hline $\mathrm{C}$ & -11.12261700 & 8.48396800 & 9.30293500 \\
\hline $\mathrm{C}$ & -10.72399600 & 9.83322200 & 9.81054400 \\
\hline $\mathrm{C}$ & -13.69007800 & 6.44479600 & 10.64348200 \\
\hline $\mathrm{C}$ & -12.63241400 & 6.80313900 & 11.61157100 \\
\hline $\mathrm{C}$ & -12.78976800 & 7.68649600 & 12.59728800 \\
\hline $\mathrm{C}$ & -14.95060900 & 6.14588100 & 11.16790400 \\
\hline $\mathrm{H}$ & -17.97416600 & 7.47730200 & 10.55598300 \\
\hline $\mathrm{H}$ & -18.21776400 & 6.43941000 & 11.96213900 \\
\hline $\mathrm{H}$ & -19.47304400 & 7.60515700 & 11.48528200 \\
\hline $\mathrm{H}$ & -20.21634600 & 6.23178700 & 9.63647000 \\
\hline $\mathrm{H}$ & -19.67214300 & 3.98242300 & 9.42374300 \\
\hline $\mathrm{H}$ & -19.04374900 & 1.99742200 & 10.57799500 \\
\hline $\mathrm{H}$ & -15.54633600 & 1.86885200 & 13.04738900 \\
\hline $\mathrm{H}$ & -15.04550800 & 4.13922200 & 12.45438700 \\
\hline $\mathrm{H}$ & -17.29521400 & 4.08993400 & 8.33621000 \\
\hline $\mathrm{H}$ & -16.74306100 & 6.59800700 & 6.64893600 \\
\hline $\mathrm{H}$ & -17.87023800 & 5.18464700 & 6.24391400 \\
\hline
\end{tabular}




\begin{tabular}{|c|c|c|c|}
\hline $\mathrm{H}$ & -14.49821000 & 5.89835600 & 7.41781100 \\
\hline $\mathrm{H}$ & -13.29740300 & 4.18508400 & 7.64921500 \\
\hline $\mathrm{H}$ & -11.52487100 & 2.58447000 & 7.43345600 \\
\hline $\mathrm{H}$ & -9.24307900 & 1.52177800 & 7.05376400 \\
\hline $\mathrm{H}$ & -9.60542300 & -0.74942000 & 7.85488400 \\
\hline $\mathrm{H}$ & -9.53608600 & 2.99146500 & 11.06971600 \\
\hline $\mathrm{H}$ & -9.94955800 & 0.75157900 & 11.86750300 \\
\hline $\mathrm{H}$ & -11.48330500 & -1.75127300 & 8.27274600 \\
\hline $\mathrm{H}$ & -13.70216400 & -2.50702800 & 8.81713600 \\
\hline $\mathrm{H}$ & -15.41260500 & -1.30869500 & 9.07671100 \\
\hline $\mathrm{H}$ & -17.11586100 & 0.35435800 & 9.47320200 \\
\hline U & -16.74173700 & -0.25334200 & 13.70033100 \\
\hline $\mathrm{H}$ & -15.06230700 & -1.97175900 & 13.30858800 \\
\hline $\mathrm{H}$ & -12.92752500 & -1.74496500 & 12.97149000 \\
\hline $\mathrm{H}$ & -10.65557900 & -1.15210500 & 12.44367500 \\
\hline $\mathrm{H}$ & -8.88737800 & 5.22774800 & 9.52975900 \\
\hline $\mathrm{H}$ & -9.51786800 & 7.41668300 & 10.11210400 \\
\hline $\mathrm{H}$ & -12.04652000 & 8.43684600 & 8.72811200 \\
\hline $\mathrm{H}$ & -10.66926500 & 10.56876500 & 8.99954500 \\
\hline Н & -9.75411600 & 9.80302800 & 10.31696400 \\
\hline $\mathrm{H}$ & -11.47015600 & 10.19922600 & 10.52784700 \\
\hline $\mathrm{H}$ & -11.68380700 & 6.28470100 & 11.50483800 \\
\hline $\mathrm{H}$ & -13.71350100 & 8.24724700 & 12.71924700 \\
\hline $\mathrm{H}$ & -11.99386000 & 7.88682700 & 13.30766000 \\
\hline H & -15.06627200 & 6.23033500 & 12.24355000 \\
\hline
\end{tabular}




\begin{tabular}{|c|c|c|c|}
\hline $\mathrm{C}$ & -10.88878700 & 11.33354500 & 13.81328700 \\
\hline $\mathrm{C}$ & -12.31210600 & 11.50257300 & 14.25366400 \\
\hline $\mathrm{C}$ & -12.98605700 & 10.69666700 & 15.07704000 \\
\hline $\mathrm{C}$ & -12.44269500 & 9.45709700 & 15.69347800 \\
\hline $\mathrm{C}$ & -12.26859200 & 9.40900700 & 17.07804300 \\
\hline $\mathrm{C}$ & -11.71815900 & 8.29769800 & 17.72010400 \\
\hline $\mathrm{C}$ & -11.34105400 & 7.20274000 & 16.93645400 \\
\hline $\mathrm{C}$ & -11.52297800 & 7.22727600 & 15.56135400 \\
\hline $\mathrm{C}$ & -12.08114100 & 8.33737800 & 14.92153400 \\
\hline $\mathrm{C}$ & -12.26947900 & 8.30677900 & 13.44368800 \\
\hline $\mathrm{C}$ & -13.54651600 & 8.35213200 & 12.85493400 \\
\hline $\mathrm{C}$ & -14.76363200 & 8.39059200 & 13.68759600 \\
\hline $\mathrm{C}$ & -15.91820900 & 8.95998400 & 13.33839100 \\
\hline $\mathrm{C}$ & -13.63301600 & 8.32404000 & 11.46125900 \\
\hline $\mathrm{C}$ & -12.50628100 & 8.29708900 & 10.64300000 \\
\hline $\mathrm{C}$ & -12.68956900 & 8.27382600 & 9.16338100 \\
\hline $\mathrm{C}$ & -13.25932900 & 7.13011700 & 8.59788000 \\
\hline $\mathrm{C}$ & -13.42979200 & 6.99846100 & 7.22738300 \\
\hline $\mathrm{C}$ & -13.01509100 & 8.01915500 & 6.36784000 \\
\hline $\mathrm{C}$ & -13.17215100 & 7.89332800 & 4.89784500 \\
\hline $\mathrm{C}$ & -13.56364500 & 8.98593400 & 4.11702500 \\
\hline $\mathrm{C}$ & -13.71487200 & 8.86458300 & 2.74230100 \\
\hline $\mathrm{C}$ & -12.93808400 & 6.67547300 & 4.25084200 \\
\hline $\mathrm{C}$ & -13.09445200 & 6.55272700 & 2.87689800 \\
\hline
\end{tabular}




\begin{tabular}{|c|c|c|c|}
\hline $\mathrm{C}$ & -13.48622000 & 7.64529000 & 2.09597500 \\
\hline $\mathrm{C}$ & -13.66026300 & 7.51222700 & 0.62896000 \\
\hline $\mathrm{C}$ & -14.72374300 & 8.13931500 & -0.02901300 \\
\hline $\mathrm{C}$ & -14.89133600 & 8.01207700 & -1.40132700 \\
\hline $\mathrm{C}$ & -14.00394600 & 7.24989400 & -2.16805100 \\
\hline $\mathrm{C}$ & -11.15182200 & 8.25545400 & 21.99579800 \\
\hline $\mathrm{C}$ & -12.31131900 & 8.79470100 & 21.42870000 \\
\hline $\mathrm{C}$ & -12.49489500 & 8.80943900 & 20.05248300 \\
\hline $\mathrm{C}$ & -11.52614900 & 8.28428700 & 19.19106500 \\
\hline $\mathrm{C}$ & -10.36668500 & 7.74506500 & 19.75793700 \\
\hline $\mathrm{C}$ & -10.18279700 & 7.73137000 & 21.13380400 \\
\hline $\mathrm{C}$ & -12.94117700 & 6.62199600 & -1.51006000 \\
\hline $\mathrm{C}$ & -12.77111500 & 6.75235900 & -0.13840500 \\
\hline $\mathrm{C}$ & -12.45260500 & 9.16334700 & 6.93413300 \\
\hline $\mathrm{C}$ & -12.29925700 & 9.33020500 & 8.31539500 \\
\hline $\mathrm{C}$ & -11.73718800 & 10.61937600 & 8.77121400 \\
\hline $\mathrm{C}$ & -12.01813200 & 11.28725100 & 9.89325100 \\
\hline $\mathrm{C}$ & -11.44427300 & 12.63198300 & 10.22300500 \\
\hline $\mathrm{C}$ & -11.22919200 & 8.24783400 & 11.23107500 \\
\hline $\mathrm{C}$ & -10.01032700 & 8.21414400 & 10.40169800 \\
\hline $\mathrm{C}$ & -8.82883000 & 8.71902200 & 10.75901800 \\
\hline $\mathrm{C}$ & -11.14633000 & 8.22795000 & 12.62454900 \\
\hline $\mathrm{H}$ & -10.84062400 & 10.99124100 & 12.77194000 \\
\hline $\mathrm{H}$ & -10.35866300 & 10.59977600 & 14.42700200 \\
\hline $\mathrm{H}$ & -10.35202400 & 12.28736500 & 13.87302200 \\
\hline
\end{tabular}




\begin{tabular}{|c|c|c|c|}
\hline $\mathrm{H}$ & -12.83256700 & 12.37460900 & 13.85993200 \\
\hline $\mathrm{H}$ & -14.01324600 & 10.94946800 & 15.33423800 \\
\hline $\mathrm{H}$ & -12.52993800 & 10.28693600 & 17.66383000 \\
\hline $\mathrm{H}$ & -10.93126800 & 6.31513800 & 17.40995400 \\
\hline $\mathrm{H}$ & -11.24909900 & 6.36136200 & 14.96469500 \\
\hline $\mathrm{H}$ & -14.68226000 & 7.92464900 & 14.66775000 \\
\hline $\mathrm{H}$ & -16.04471000 & 9.47986400 & 12.39209200 \\
\hline $\mathrm{H}$ & -16.77807600 & 8.93399400 & 14.00045000 \\
\hline $\mathrm{H}$ & -14.61294300 & 8.32076300 & 10.99177100 \\
\hline $\mathrm{H}$ & -13.56556400 & 6.32352900 & 9.25843400 \\
\hline $\mathrm{H}$ & -13.89589700 & 6.10411600 & 6.82424100 \\
\hline $\mathrm{H}$ & -13.76784100 & 9.94010100 & 4.59455300 \\
\hline $\mathrm{H}$ & -14.00317400 & 9.73454200 & 2.15867600 \\
\hline $\mathrm{H}$ & -12.61662400 & 5.81424100 & 4.82996300 \\
\hline $\mathrm{H}$ & -12.92658800 & 5.58903600 & 2.40423100 \\
\hline $\mathrm{H}$ & -15.43899300 & 8.72207200 & 0.54473800 \\
\hline $\mathrm{H}$ & -15.71741800 & 8.52283100 & -1.88842300 \\
\hline $\mathrm{H}$ & -13.09418800 & 9.17916200 & 22.07659500 \\
\hline $\mathrm{H}$ & -13.41779400 & 9.20771000 & 19.63997600 \\
\hline $\mathrm{H}$ & -9.58369500 & 7.36085200 & 19.11003300 \\
\hline $\mathrm{H}$ & -9.25847800 & 7.33573300 & 21.54552800 \\
\hline $\mathrm{H}$ & -12.24703600 & 6.01000200 & -2.07926600 \\
\hline $\mathrm{H}$ & -11.92567700 & 6.26860600 & 0.34289200 \\
\hline $\mathrm{H}$ & -12.11302200 & 9.96127600 & 6.27783800 \\
\hline $\mathrm{H}$ & -11.06297100 & 11.09141700 & 8.05512900 \\
\hline
\end{tabular}




\begin{tabular}{|c|c|c|c|}
\hline $\mathrm{H}$ & -12.70850500 & 10.86705700 & 10.62179900 \\
\hline $\mathrm{H}$ & -12.24004000 & 13.37713700 & 10.34650700 \\
\hline $\mathrm{H}$ & -10.76513700 & 12.98292800 & 9.43963300 \\
\hline $\mathrm{H}$ & -10.88838600 & 12.60426000 & 11.16758300 \\
\hline $\mathrm{H}$ & -10.11495400 & 7.76112400 & 9.41803200 \\
\hline $\mathrm{H}$ & -8.67989200 & 9.22825200 & 11.70813800 \\
\hline $\mathrm{H}$ & -7.96930000 & 8.65472900 & 10.09935900 \\
\hline $\mathrm{H}$ & -10.16863500 & 8.15800600 & 13.09373100 \\
\hline $\mathrm{C}$ & -10.57398400 & 8.21435100 & 26.24831800 \\
\hline $\mathrm{C}$ & -11.13609300 & 9.32671400 & 25.62825800 \\
\hline $\mathrm{C}$ & -11.32305900 & 9.33979500 & 24.25032900 \\
\hline $\mathrm{C}$ & -10.95413200 & 8.24066000 & 23.46668800 \\
\hline $\mathrm{C}$ & -10.39192500 & 7.12799900 & 24.10237200 \\
\hline $\mathrm{C}$ & -10.20236900 & 7.11464200 & 25.47989700 \\
\hline $\mathrm{H}$ & -10.42622600 & 8.20431000 & 27.32409600 \\
\hline $\mathrm{H}$ & -11.42242000 & 10.19267800 & 26.21807500 \\
\hline $\mathrm{H}$ & -11.73895900 & 10.22198700 & 23.77148100 \\
\hline $\mathrm{H}$ & -10.12217000 & 6.25576900 & 23.51312500 \\
\hline $\mathrm{H}$ & -9.77003000 & 6.23882500 & 25.95483900 \\
\hline $\mathrm{C}$ & -14.51741800 & 6.83544400 & -6.40900000 \\
\hline $\mathrm{C}$ & -15.62193100 & 6.79574100 & -5.56236700 \\
\hline $\mathrm{C}$ & -15.45545200 & 6.93065800 & -4.18841000 \\
\hline $\mathrm{C}$ & -14.18276900 & 7.10945600 & -3.63473600 \\
\hline $\mathrm{C}$ & -13.08092000 & 7.15007200 & -4.49675500 \\
\hline $\mathrm{C}$ & -13.24602200 & 7.01317900 & -5.87057400 \\
\hline
\end{tabular}




$\begin{array}{llll}\mathrm{H} & -14.64724000 & 6.72714600 & -7.48155800 \\ \mathrm{H} & -16.61688300 & 6.64998200 & -5.97278800 \\ \mathrm{H} & -16.31964000 & 6.87297500 & -3.53222500 \\ \mathrm{H} & -12.08820800 & 7.31279900 & -4.08617500 \\ \mathrm{H} & -12.37896900 & 7.05298600 & -6.52357500\end{array}$

3, $\omega B 97 D / 6-31 G(d)($ Energy $=-1538.12170013$ Hartrees $)$

$\begin{array}{llll}\text { C } & -3.72139 & -2.35148 & -0.60767 \\ \text { C } & -2.5466 & -3.07611 & -0.82705 \\ \text { C } & -1.66534 & -3.13038 & 0.26147 \\ \text { C } & -2.16133 & -3.17601 & 1.5684 \\ \text { C } & -3.38812 & -2.53993 & 1.79263 \\ \text { C } & -3.97903 & -1.9043 & 0.69023 \\ \text { C } & -4.74777 & -0.75759 & 0.84747 \\ \text { C } & -4.94357 & -0.19033 & 2.11593 \\ \text { C } & -5.00585 & 1.20419 & 2.15445 \\ \text { C } & -5.05393 & 1.89035 & 0.94049 \\ \text { C } & -4.56265 & 3.18976 & 0.90166 \\ \text { C } & -4.03862 & 3.73041 & 2.07802 \\ \text { C } & -4.43841 & 3.22646 & 3.32491 \\ \text { C } & -4.94156 & 1.91385 & 3.36447 \\ \text { C } & -3.05375 & 4.71498 & 1.96141 \\ \text { C } & -2.57757 & 4.93333 & 0.66162 \\ \text { C } & -1.0689 & 5.13864 & 0.4315 \\ \text { C } & -0.5642 & 5.20506 & -0.87348 \\ \text { C } & 0.69469 & 4.62419 & -1.09269 \\ \text { C } & 1.33052 & 4.04141 & 0.01525 \\ \text { C } & 2.20629 & 2.77954 & -0.16623 \\ \text { C } & 2.36523 & 2.21077 & -1.43899 \\ \text { C } & 2.52278 & 0.81625 & -1.51054 \\ \text { C } & 2.50944 & 0.1004 & -0.30371 \\ \text { C } & 1.93852 & -1.33639 & -0.26049 \\ \text { C } & 1.42832 & -1.9254 & -1.42803 \\ \text { C } & 0.3317 & -2.78826 & -1.27986 \\ \text { C } & -0.15242 & -2.97333 & 0.02152 \\ \text { C } & 0.72068 & -2.87399 & 1.11225 \\ \text { C } & 1.81713 & -2.01024 & 0.96517 \\ \text { C } & 2.90405 & 0.73617 & 0.88319 \\ \text { C } & 2.74578 & 2.13057 & 0.95484 \\ \text { C } & 1.07063 & 4.53689 & 1.30252 \\ & & & \end{array}$




$\begin{array}{llll}\mathrm{C} & -0.18851 & 5.11647 & 1.5207 \\ \mathrm{C} & -3.43963 & 4.81737 & -0.43355 \\ \mathrm{C} & -4.49485 & 3.90646 & -0.30281 \\ \mathrm{C} & -5.48339 & 1.24734 & -0.23054 \\ \mathrm{C} & -5.22719 & -0.12375 & -0.29928 \\ \mathrm{C} & -5.36486 & -0.84236 & -1.49795 \\ \mathrm{C} & -4.58211 & -1.99967 & -1.65849 \\ \mathrm{H} & -2.3072 & -3.49792 & -1.78085 \\ \mathrm{H} & -1.61009 & -3.6286 & 2.36602 \\ \mathrm{H} & -3.82821 & -2.5002 & 2.76716 \\ \mathrm{H} & -4.97864 & -0.78362 & 3.00569 \\ \mathrm{H} & -4.32856 & 3.80431 & 4.21872 \\ \mathrm{H} & -5.22855 & 1.45962 & 4.28976 \\ \mathrm{H} & -2.64367 & 5.21434 & 2.81434 \\ \mathrm{H} & -1.13095 & 5.63086 & -1.67494 \\ \mathrm{H} & 1.12722 & 4.58544 & -2.07064 \\ \mathrm{H} & 2.3225 & 2.80929 & -2.32494 \\ \mathrm{H} & 2.60401 & 0.31607 & -2.45298 \\ \mathrm{H} & 1.82557 & -1.69028 & -2.39337 \\ \mathrm{H} & -0.14201 & -3.24086 & -2.12586 \\ \mathrm{H} & 0.53449 & -3.39005 & 2.03089 \\ \mathrm{H} & 2.50167 & -1.83739 & 1.76927 \\ \mathrm{H} & 3.2689 & 0.17674 & 1.7192 \\ \mathrm{H} & 2.9854 & 2.66969 & 1.84751 \\ \mathrm{H} & 1.7814 & 4.43434 & 2.09575 \\ \mathrm{H} & -0.47856 & 5.47652 & 2.48565 \\ \mathrm{H} & -3.27398 & 5.35754 & -1.3422 \\ \mathrm{H} & -5.18585 & 3.73189 & -1.10099 \\ \mathrm{H} & -5.92127 & 1.7833 & -1.04656 \\ \mathrm{H} & -6.01315 & -0.50104 & -2.27774 \\ \mathrm{H} & -4.61392 & -2.56804 & -2.5645 \\ & & & \\ & & & \end{array}$

3 open adduct, $\omega B 97 D / 6-31 G(d)$ (Energy $=-2001.43489615$ Hartrees)

$\begin{array}{llll}\mathrm{C} & -3.0298 & -1.5702 & -0.59755 \\ \mathrm{C} & -2.14946 & -2.62867 & -0.86376 \\ \mathrm{C} & -1.06333 & -2.8281 & 0.01252 \\ \mathrm{C} & -1.09732 & -2.30638 & 1.30955 \\ \mathrm{C} & -2.06357 & -1.35496 & 1.6177 \\ \mathrm{C} & -2.90541 & -0.89256 & 0.6041 \\ \mathrm{C} & -3.56554 & 0.29375 & 0.80713 \\ \mathrm{C} & -3.43407 & 0.88734 & 2.03625 \\ \mathrm{C} & -3.72801 & 2.22257 & 2.17882 \\ \mathrm{C} & -4.21243 & 2.96843 & 1.08965 \\ \mathrm{C} & -4.2034 & 4.39266 & 1.20257 \\ \mathrm{C} & -3.79792 & 4.97615 & 2.42512\end{array}$




\begin{tabular}{|c|c|c|c|}
\hline $\mathrm{C}$ & -3.54102 & 4.18082 & 3.54801 \\
\hline $\mathrm{C}$ & -3.48951 & 2.80269 & 3.41358 \\
\hline $\mathrm{C}$ & -3.61948 & 6.36255 & 2.53363 \\
\hline $\mathrm{C}$ & -3.75627 & 7.18787 & 1.41344 \\
\hline $\mathrm{C}$ & -3.23821 & 8.65556 & 1.54232 \\
\hline $\mathrm{C}$ & -3.16634 & 9.51776 & 0.42777 \\
\hline $\mathrm{C}$ & -2.48879 & 10.75556 & $\begin{array}{ll}6 & 0.54003\end{array}$ \\
\hline $\mathrm{C}$ & -1.9168 & 11.15158 & 1.76536 \\
\hline $\mathrm{C}$ & 5.77208 & -3.84057 & -4.66787 \\
\hline $\mathrm{C}$ & 4.5641 & -4.41969 & -5.03539 \\
\hline $\mathrm{C}$ & 3.57318 & -4.61585 & -4.0889 \\
\hline $\mathrm{C}$ & 3.77193 & -4.21796 & -2.76771 \\
\hline $\mathrm{C}$ & 2.53221 & -4.19489 & -1.8514 \\
\hline $\mathrm{C}$ & 1.29212 & -4.50466 & -2.43132 \\
\hline $\mathrm{C}$ & 0.1171 & -4.17942 & -1.75619 \\
\hline $\mathrm{C}$ & 0.18772 & -3.55145 & -0.51901 \\
\hline $\mathrm{C}$ & 1.40293 & -3.52541 & 0.1928 \\
\hline $\mathrm{C}$ & 2.60089 & -3.83159 & -0.49509 \\
\hline $\mathrm{C}$ & 5.05187 & -3.81838 & -2.32985 \\
\hline $\mathrm{C}$ & 6.06518 & -3.60406 & -3.29929 \\
\hline $\mathrm{C}$ & -2.13998 & 10.36097 & $\begin{array}{ll}7 & 2.91235\end{array}$ \\
\hline $\mathrm{C}$ & -2.79255 & 9.11605 & 2.79852 \\
\hline $\mathrm{C}$ & -4.30864 & 6.64506 & 0.22616 \\
\hline $\mathrm{C}$ & -4.54641 & 5.24468 & 0.12793 \\
\hline $\mathrm{C}$ & -4.65013 & 2.26866 & -0.07585 \\
\hline $\mathrm{C}$ & -4.29815 & 0.89584 & -0.21429 \\
\hline $\mathrm{C}$ & -4.62929 & 0.11796 & -1.34975 \\
\hline $\mathrm{C}$ & -4.00105 & -1.14316 & -1.53325 \\
\hline $\mathrm{H}$ & -2.27121 & -3.2437 & -1.73564 \\
\hline $\mathrm{H}$ & -0.38641 & -2.61295 & 2.0391 \\
\hline $\mathrm{H}$ & -2.14612 & -0.96411 & 2.60816 \\
\hline $\mathrm{H}$ & -3.07914 & 0.31769 & 2.86727 \\
\hline $\mathrm{H}$ & -3.36759 & 4.62945 & 4.50315 \\
\hline $\mathrm{H}$ & -3.2498 & 2.18449 & 4.25213 \\
\hline $\mathrm{H}$ & -3.34881 & 6.78894 & 3.47813 \\
\hline $\mathrm{H}$ & -3.60868 & 9.23185 & -0.50645 \\
\hline $\mathrm{H}$ & -2.40668 & 11.39169 & $9-0.31835$ \\
\hline $\mathrm{H}$ & 4.39891 & -4.70471 & -6.05563 \\
\hline $\mathrm{H}$ & 2.64329 & -5.05379 & -4.37595 \\
\hline $\mathrm{H}$ & 1.2406 & -4.96863 & -3.39161 \\
\hline $\mathrm{H}$ & -0.83131 & -4.38925 & -2.19476 \\
\hline $\mathrm{H}$ & 1.42093 & -3.24553 & 1.22686 \\
\hline $\mathrm{H}$ & 3.54684 & -3.76955 & 0.00113 \\
\hline $\mathrm{H}$ & 5.2452 & -3.66306 & -1.28759 \\
\hline $\mathrm{H}$ & 7.03698 & -3.26057 & -3.00798 \\
\hline $\mathrm{H}$ & -1.80304 & 10.70195 & $\begin{array}{ll}5 & 3.87098\end{array}$ \\
\hline
\end{tabular}




$\begin{array}{llll}\mathrm{H} & -2.94273 & 8.50959 & 3.67576 \\ \mathrm{H} & -4.53909 & 7.28711 & -0.60186 \\ \mathrm{H} & -4.96686 & 4.83157 & -0.76561 \\ \mathrm{H} & -5.20934 & 2.76726 & -0.83858 \\ \mathrm{H} & -5.3323 & 0.47965 & -2.07752 \\ \mathrm{H} & -4.24023 & -1.75286 & -2.38331 \\ \mathrm{C} & -0.43884 & 12.80317 & 3.06653 \\ \mathrm{C} & 0.41404 & 13.91909 & 3.13191 \\ \mathrm{C} & 0.65957 & 14.67862 & 1.98416 \\ \mathrm{C} & 0.0479 & 14.34011 & 0.77207 \\ \mathrm{C} & -0.80188 & 13.22009 & 0.70108 \\ \mathrm{C} & -1.02923 & 12.43132 & 1.84712 \\ \mathrm{H} & -0.63093 & 12.22763 & 3.94549 \\ \mathrm{H} & 0.87979 & 14.18698 & 4.06075 \\ \mathrm{H} & 1.31911 & 15.52434 & 2.0339 \\ \mathrm{H} & 0.22815 & 14.93336 & -0.10344 \\ \mathrm{H} & -1.26746 & 12.96217 & -0.22717 \\ \mathrm{C} & 6.72292 & -3.47176 & -5.82178 \\ \mathrm{C} & 6.29721 & -3.76355 & -7.12281 \\ \mathrm{C} & 7.08112 & -3.39795 & -8.22013 \\ \mathrm{C} & 8.31001 & -2.75729 & -8.01274 \\ \mathrm{C} & 8.75718 & -2.48733 & -6.70426 \\ \mathrm{C} & 7.95773 & -2.8486 & -5.60684 \\ \mathrm{H} & 5.36497 & -4.26686 & -7.28061 \\ \mathrm{H} & 6.73834 & -3.60785 & -9.21493 \\ \mathrm{H} & 8.90883 & -2.47285 & -8.85075 \\ \mathrm{H} & 9.70341 & -2.00373 & -6.54729 \\ \mathrm{H} & 8.29311 & -2.64759 & -4.61335\end{array}$

\section{0) References.}

(1) Pangborn, A. B.; Giardello, M. A.; Grubbs, R. H.; Rosen, R. K.; Timmers, F. J. Organometallics 1996, 15, 1518.

(2) Darzi, E. R.; Sisto, T. J.; Jasti, R. J. Org. Chem. 2012, 77, 6624.

(3) Sheldrick, G. M.; Bruker AXS: Madison, WI, 1998.

(4) Sluis, P. V. d.; Spek, A. L. Acta. Cryst. A. 1990, A46, 194.

(5) Sheldrick, G. M. Acta. Cryst. A. 2008, A64, 112.

(6) Hanwell, M.; Curtis, D.; Lonie, D.; Vandermeersch, T.; Zurek, E.; Hutchison, G. J. Chem. Inf. 2012, 4, 17.

(7) Darzi, E. R.; Jasti, R. Chem. Soc. Rev. 2015, 44, 6401.

(8) Full Reference 33: Frisch, M. J.; Trucks, G. W.; Schlegel, H. B.; Scuseria, G. E.; Robb, M. A.; Cheeseman, J. R.; Scalmani, G.; Barone, V.; Mennucci, B.; Petersson, G. A.; Nakatsuji, H.; Caricato, M.; Li, X.; Hratchian, H. P.; Izmaylov, A. F.; Bloino, J.; Zheng, G.; Sonnenberg, J. L.; Hada, M.; Ehara, M.; Toyota, K.; 
Fukuda, R.; Hasegawa, J.; Ishida, M.; Nakajima, T.; Honda, Y.; Kitao, O.; Nakai, H.; Vreven, T.; Montgomery, J. A.; Peralta, J. E.; Ogliaro, F.; Bearpark, M.; Heyd, J. J.; Brothers, E.; Kudin, K. N.; Staroverov, V. N.; Kobayashi, R.; Normand, J.; Raghavachari, K.; Rendell, A.; Burant, J. C.; Iyengar, S. S.; Tomasi, J.; Cossi, M.; Rega, N.; Millam, J. M.; Klene, M.; Knox, J. E.; Cross, J. B.; Bakken, V.; Adamo, C.; Jaramillo, J.; Gomperts, R.; Stratmann, R. E.; Yazyev, O.; Austin, A. J.; Cammi, R.; Pomelli, C.; Ochterski, J. W.; Martin, R. L.; Morokuma, K.; Zakrzewski, V. G.; Voth, G. A.; Salvador, P.; Dannenberg, J. J.; Dapprich, S.; Daniels, A. D.; Farkas; Foresman, J. B.; Ortiz, J. V.; Cioslowski, J.; Fox, D. J. Wallingford CT, 2009. 